\title{
ENTROPIC AND ENTHALPIC DRIVING FORCES ON MORPHOLOGY IN POLYMER GRAFTED PARTICLE FILLED NANOCOMPOSITES
}

\author{
By \\ TYLER B. MARTIN \\ B.S. Clarkson University, 2011
}

A thesis submitted to the

Faculty of the Graduate School of the

University of Colorado in partial fulfillment

of the requirement for the degree of

Doctor of Philosophy

Department of Chemical and Biological Engineering

2016 
This thesis entitled:

\section{Entropic And Enthalpic Driving Forces On Morphology}

In Polymer Grafted Particle Filled Nanocomposites

written by Tyler B. Martin

has been approved for the Department of Chemical and Biological Engineering

Arthi Jayaraman, Ph.D.

Yifu Ding, Ph.D.

Date

The final copy of this thesis has been examined by the signatories, and we find that both the content and the form meet acceptable presentation standards of scholarly work in the above mentioned discipline. 


\section{ABSTRACT \\ Martin, Tyler Biron (Ph.D. Chemical and Biological Engineering) \\ Entropic and Enthalpic Driving Forces on Morphology in Polymer Grafted Particle Filled Nanocomposites \\ Thesis directed by Professor Arthi Jayaraman}

Controlling polymer nanocomposite (PNC) morphology is an essential step towards designing PNCs with target macroscopic properties for specific applications. One strategy to control PNC morphology is to modify the surface of the nanoscale filler (nanoparticles) with polymer chains. By tailoring the properties of the grafted and matrix polymers, the effective filler-filler interactions and the PNC morphology can be tuned. The goal of this thesis is to elucidate how physical and chemical design parameters of polymer-grafted particle based PNC impact morphology, in order to engineer new polymer nanocomposite materials.

First, we use Monte Carlo simulations to show how the assembly of copolymer grafted particles in implicit solvent is affected by various grafted layer properties: grafted polymer chemistry, particle-diameter, particle concentration, grafting density, and graft sequence. Despite our focus on isotropically grafted particles, anisotropic particle assembly appears in the simulations over much of the parameter space. We highlight how the blockiness of the graft polymer sequence (number of contiguous like monomers) tunes cluster anisotropy when the outerblock monomers are highly solvo-phobic.

Second, we study homopolymer grafted particles in chemically-identical matrix homopolymer. We examine how polydispersity and flexibility of graft and matrix polymers affect the mixing of the graft and matrix chains, and in turn the dispersion and aggregation of the grafted particles in the matrix. Increasing graft polydispersity or decreasing graft and matrix flexibility 
increases the mixing of the graft and matrix chains (i.e. grafted layer wetting) and stabilizes the dispersed morphology of the composites.

Finally, we show that for composites where the graft and matrix chains are chemicallydissimilar, the wetting-dewetting and dispersion-aggregation transitions are distinct transitions, unlike what is generally assumed for the chemically identical case. Using temperature, graft-matrix $\chi$, or polymer composition, the degree of wetting of the grafted layer by matrix chains is tuned in the dispersed state. The ability to tune wetting in the dispersed state is not present for chemicallyidentical composites and reveals the possibility of greater macroscopic property control in composites where the graft and matrix chains are chemically-dissimilar than chemically-identical. 
For my parents and brothers 


\section{ACKNOWLEDGEMENTS}

From the start, I want to thank my advisor, Professor Arthi Jayaraman, for guiding and mentoring me throughout graduate school. Her great wisdom, unmatched enthusiasm, and staunch belief in my ability to succeed, even when I didn't believe it myself, are primary reasons I have been able to complete this thesis. I would also like to thank my committee members for their helpful comments and criticisms during the construction of this thesis: Professor Christopher Bowman, Professor Jennifer Cha, Professor Yifu Ding, Professor Matthew Glaser, and Professor Ryan Hayward.

I thank all past and present Jayaraman group members for scientific and personal guidance: Postdoctoral researchers Dr. Nitish Nair, Dr. Eric Jankowski, Dr. Francesca Stanzione, and Dr. Ahmadreza Ghobadi, and fellow graduate students Arezou Seifpour, Robert Elder, Hilary Marsh, and Carla Estridge. I would also like to thank my friends and classmates at CU for helping to make graduate school a more enjoyable experience. The aphorism "it takes a village" is quite applicable here and I thank all those named and unnamed who have helped me along the way.

I want to acknowledge my funding sources: National Science Foundation GRFP Award Number DGE 1144083, Teets Family Endowed Graduate Fellowship, and the GAANN Fellowship program.

Finally, I want to thank my family for their unwavering belief in me long before I started graduate school. My parents have shown me what it means to be a compassionate, hardworking, and proud adult. My brothers have helped me understand the value of both inner strength and selfconfidence. And of course I need to thank the rest of my family, especially those no longer with us, for their contributions to my success. 


\section{TABLE OF CONTENTS}

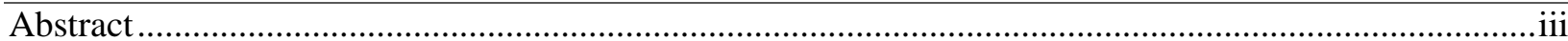

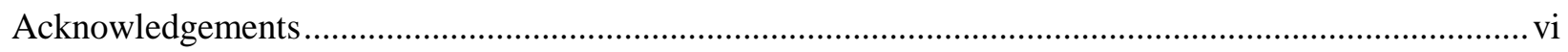

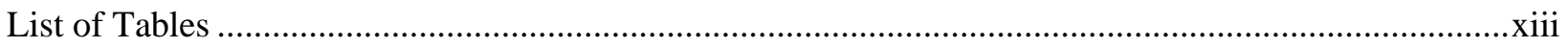

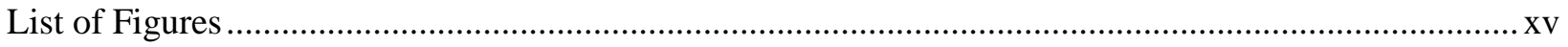

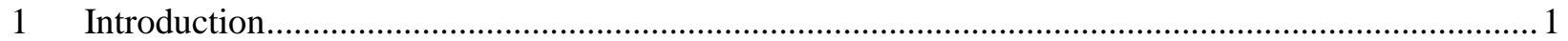

2 Effect of Blockiness in Grafted Monomer Sequences on Assembly of Copolymer Grafted Nanoparticles: A Monte Carlo Simulation Study ....................................................................... 9

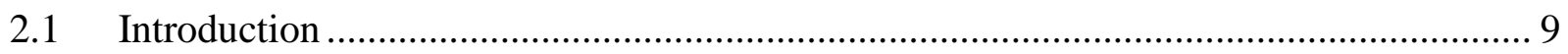

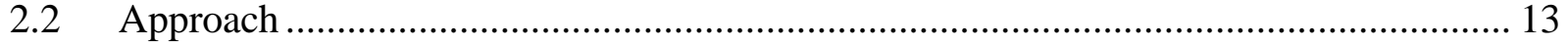

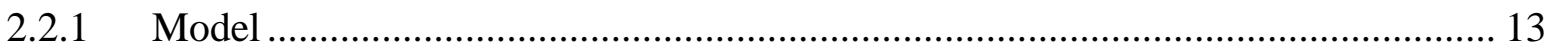

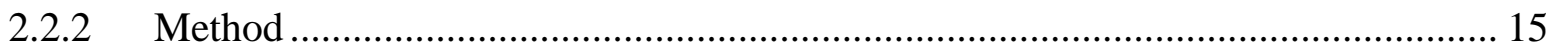

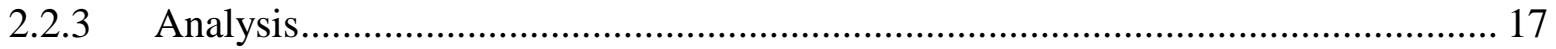

2.2.4 Driving Forces for Assembly of Grafted Nanoparticles ................................... 20

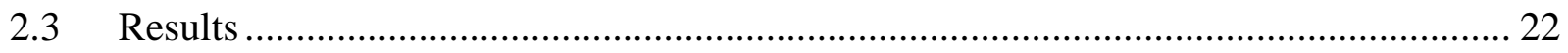

2.3.1 Effects of Monomer Sequence on Grafted Chain Conformations and Monomer

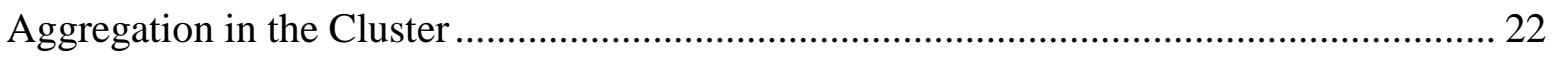

2.3.2 Effect of Monomer Sequence on Nanoparticle Arrangement in the Cluster .......... 30

2.3.3 Effects of Monomer-Particle Interactions on Assembly................................... 37

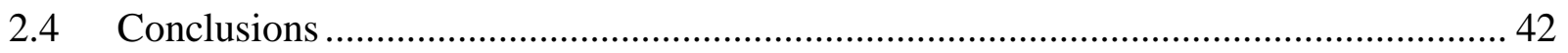

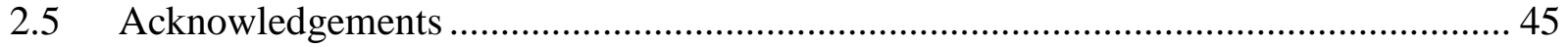

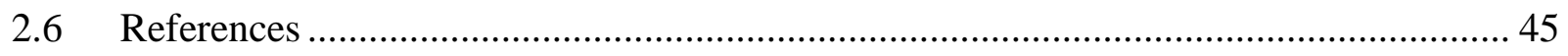

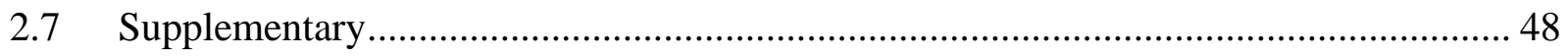

2.7.1 Types of Monomer Contacts in Clusters/Dispersions of Copolymer Grafted

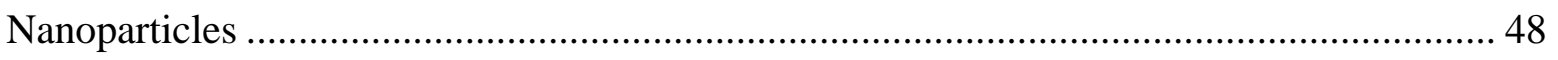

2.7.2 Effects of Monomer Sequence on Grafted Chain Conformations and Monomer Aggregation in the Cluster for $D=4 d$ and $D=12 d$ with and without Monomer-Particle

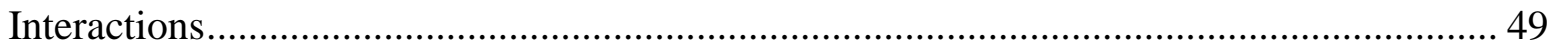

2.7.3 Discussion of Lattice Effects and the Alternating Sequence ............................. 58

2.7.4 Tabulated Data for the number of $A$ and B Domains for $D=4 d$ and $D=12 d$........ 61

2.7.5 Additional Results Showing Effect of Graft Length to Particle Size Ratio on Nanoparticle Assembly and Chain Conformations...................................................... 65 
2.7.6 Effect of Monomer Sequence on Nanoparticle Arrangement in the Cluster for $D=4 d$ and $\mathrm{D}=12 \mathrm{~d}$ with and without Monomer-Particle Interactions

2.7.7 Relative Strength of Monomer-Particle and Monomer-Monomer Interactions for D$4 \mathrm{~d}$ and $\mathrm{D}=12 \mathrm{~d}$.

3 Polydispersity For Tuning the Potential of Mean Force Between Polymer Grafted Nanoparticles in a

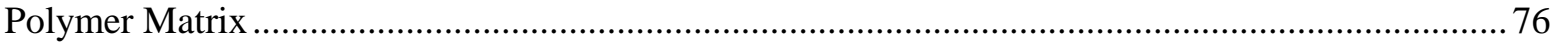

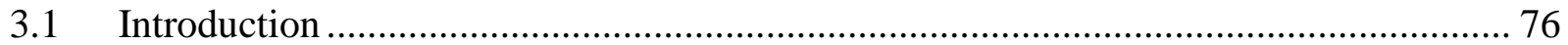

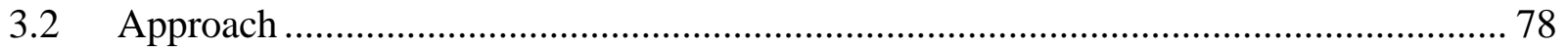

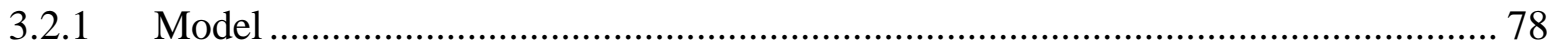

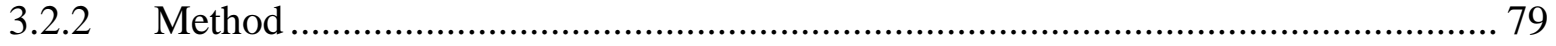

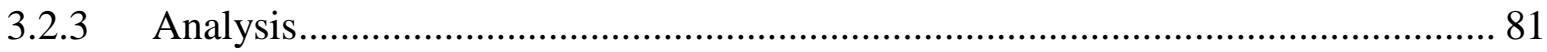

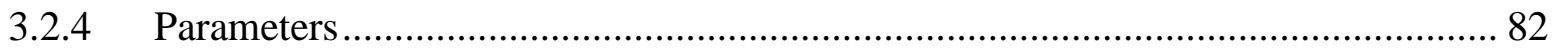

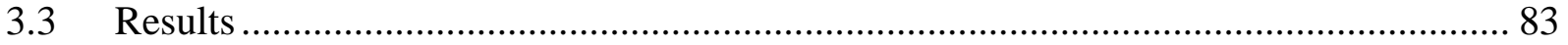

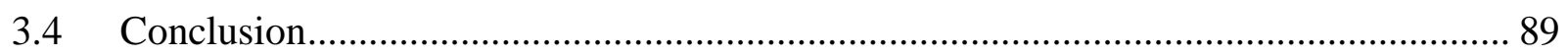

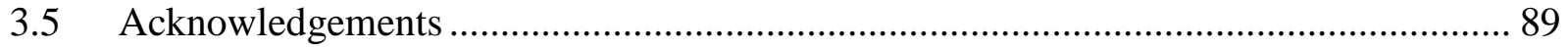

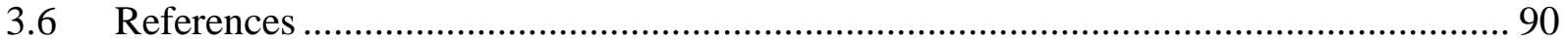

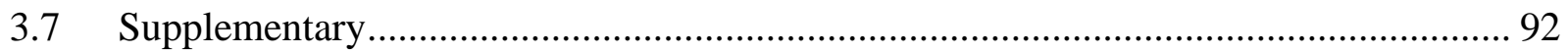

3.7.1 Effect of matrix length on the potential of mean force between monodisperse grafted

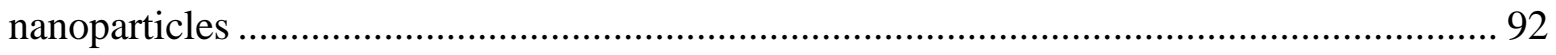

3.7.2 Dataset for concentration of end monomers at the particle surface and penetration depth of matrix into the grafted layer 93

3.7.3 Effect of incremental increases in PDI on the intermediate-range attractive PMF well 94

3.7.4 Effect of log-normal chain length distribution on the potential of mean force between

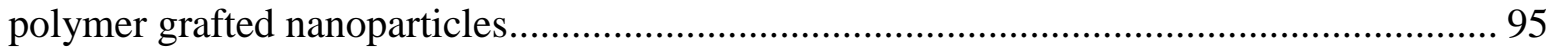

3.7.5 Comparison of log-normal and bidisperse chain length distributions ..................... 96

3.7.6 Effect of lowered curvature on potential of mean force between polymer grafted

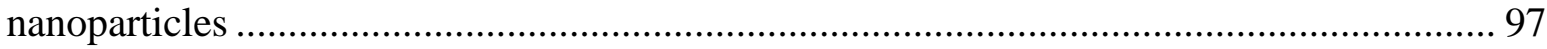

4 Polydisperse Homopolymer Grafts Stabilize Dispersions of Nanoparticles in a Chemically Identical Homopolymer Matrix: An Integrated Theory And Simulation Study ...............................................98

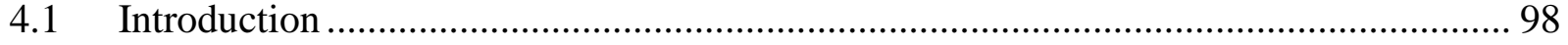

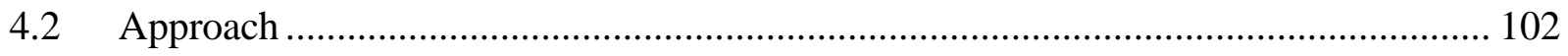

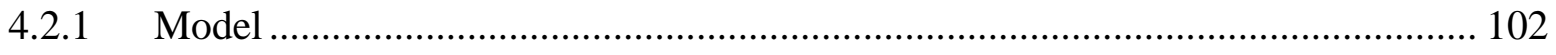




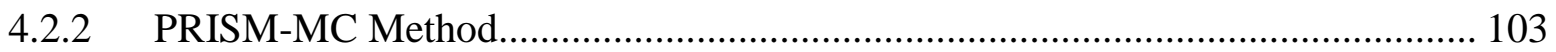

4.2.3 Brownian Dynamics Method ....................................................................... 107

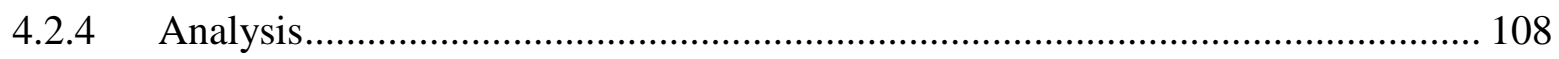

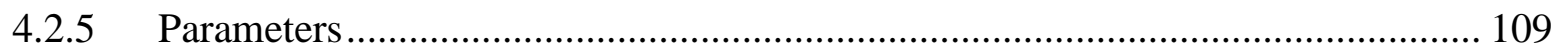

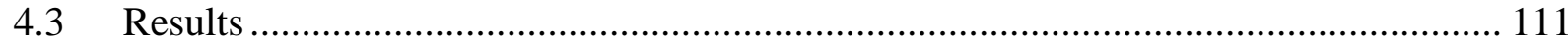

4.3.1 Effect of Graft Length Polydispersity on Effective Interactions and Wetting...... 111

4.3.2 Effect of Increasing Matrix Packing Fraction........................................................ 119

4.3.3 Effect of Particle Diameter or Curvature ............................................................. 122

4.3.4 Effect of Distribution of Chain Lengths …………………............................ 126

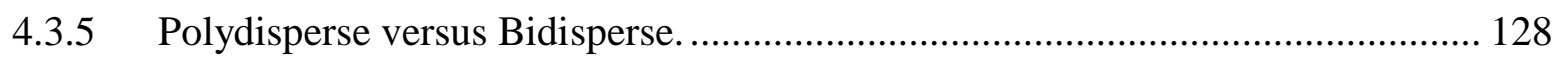

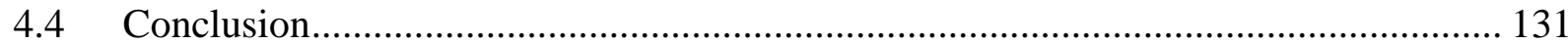

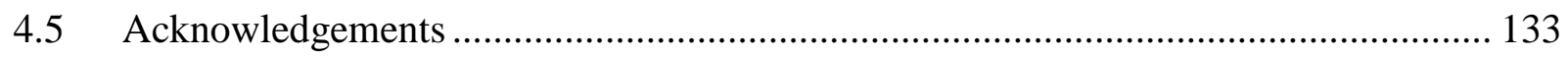

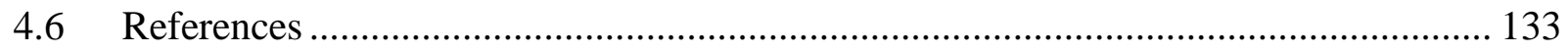

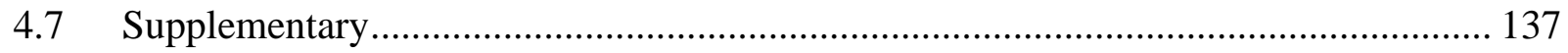

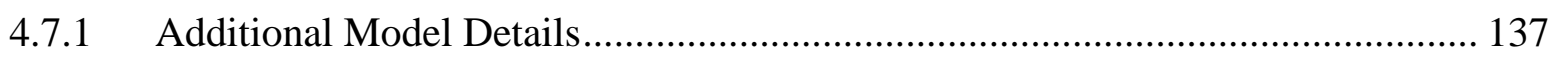

4.7.2 Brownian Dynamics Simulation Results ........................................................ 139

4.7.3 Additional PRISM-MC Results .................................................................. 143

5 Identifying the ideal characteristics of the grafted polymer chain length distribution for maximizing dispersion of polymer grafted nanoparticles in a polymer matrix ................................................... 148

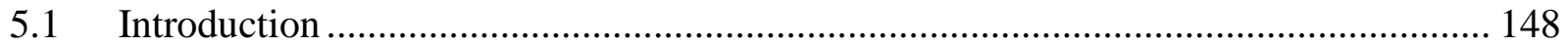

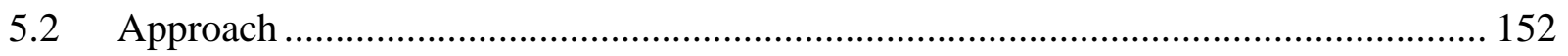

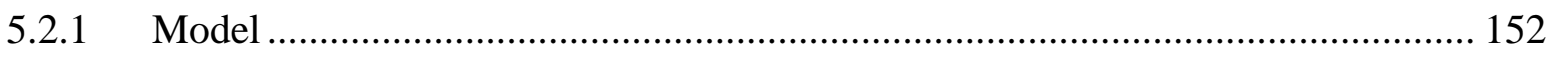

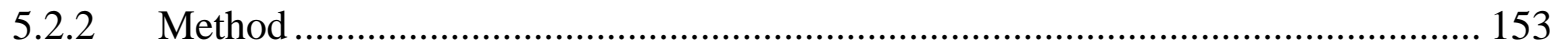

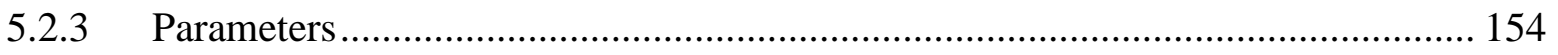

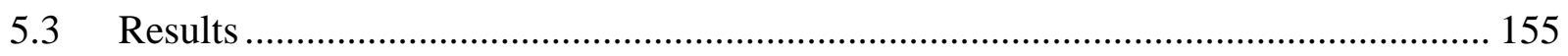

5.3.1 Effect of particle-particle attraction on polydispersity- and bidispersity- stabilized

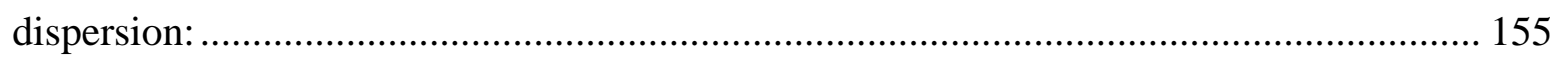

5.3.2 Role of Short and Long Chains on the Potential of Mean Force ......................... 160

5.3.3 Features of an Ideal Graft Length Distribution for Maximizing Particle Dispersion: 165

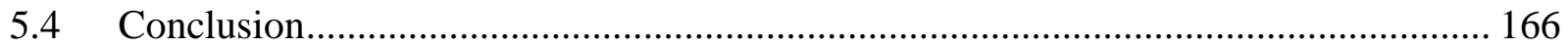

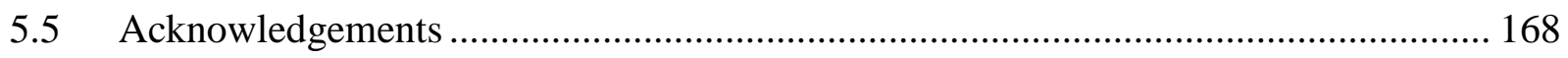




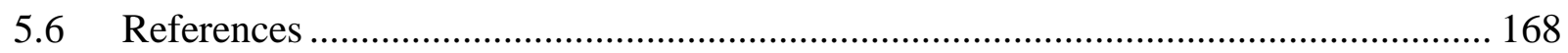

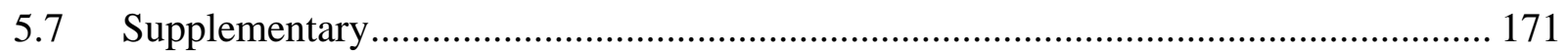

5.7.1 Colloid Lennard Jones Potential ........................................................................ 171

5.7.2 Schematic of terminology used to describe the potential of mean force .............. 172

5.7.3 Effect of Particle-Particle attraction on the Potential of Mean Force and Grafted

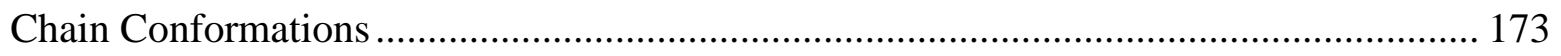

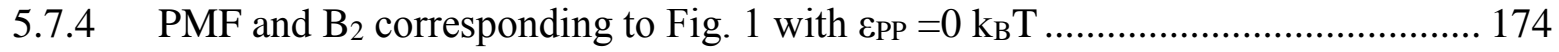

5.7.5 Log-Normal versus Bidisperse Monomer Concentration Profiles......................... 175

5.7.6 Comparison of Bidisperse PMF to Direct Addition of PMFs for Systems 4 and 5 176

5.7.7 Tables of Number of Long Chains for Bidisperse and Log-Normal Distributions 177

6 Effect of Matrix Bidispersity on the Morphology of Polymer Grafted Nanoparticle Filled Polymer

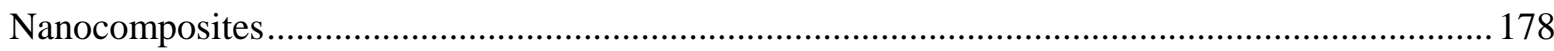

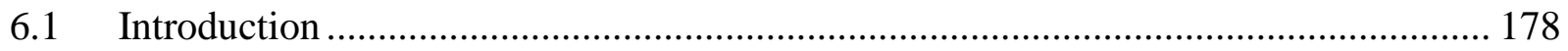

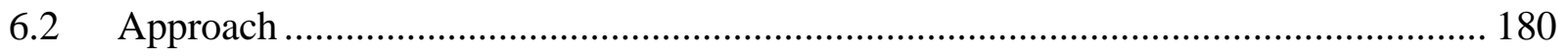

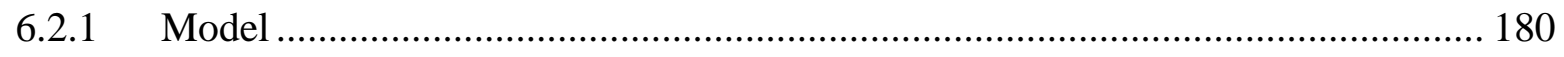

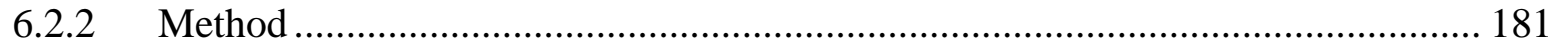

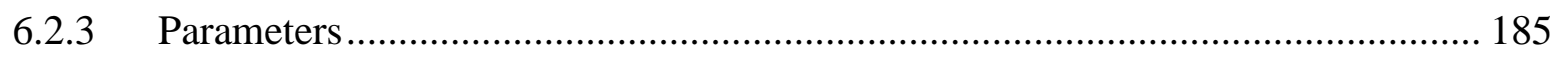

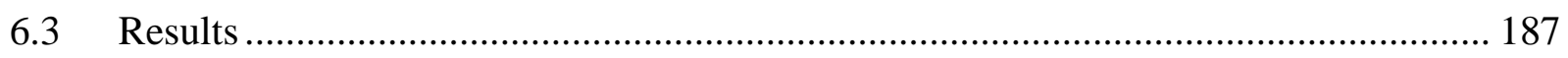

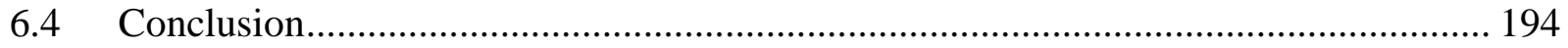

6.5 Acknowledgements ........................................................................................... 194

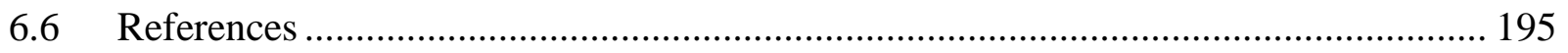

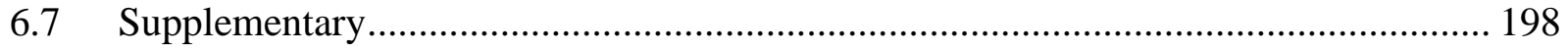

6.7.1 Internal Distances Calculation Showing Relaxation of Chains ............................ 198

6.7.2 Pair correlation function data showing variation in individual trials................... 199

6.7.3 Simulation Snaphots ………...................................................................... 200

6.7.4 Comparison of Brownian Dynamics Simulations and Liquid-State Theory

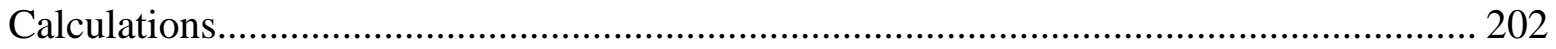

6.7.5 Variation in Short-Matrix Preferential Wetting with Varying Filler-Fraction ..... 203

6.7.6 Effect of matrix bidispersity on the average brush height of the grafted particles 204

6.7.7 Data tables for $\Delta \mathrm{S}_{\text {wetting }}$ Calculation.................................................................... 205 
6.7.8 Liquid-State Theory Results for the Particle-Particle Correlation Function and Potential of Mean Force between Grafted Particles ......................................................... 206

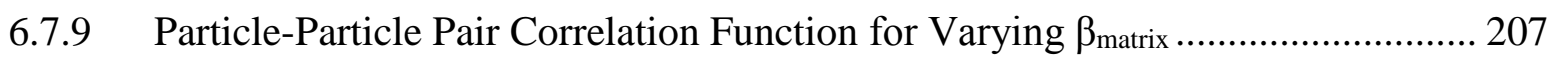

7 Decreasing Polymer Flexibility Improves Wetting and Dispersion of Polymer Grafted Particles in a Chemically Identical Polymer Matrix........................................................................................... 208

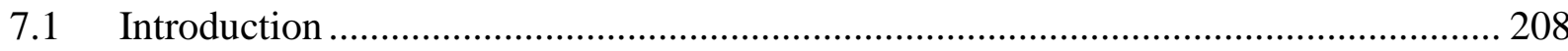

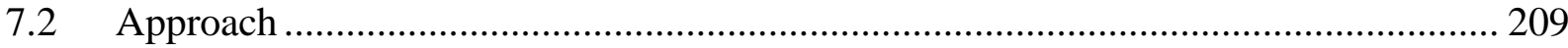

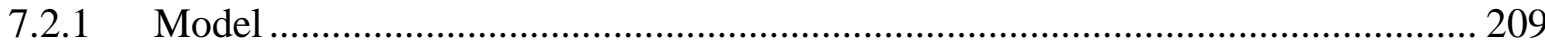

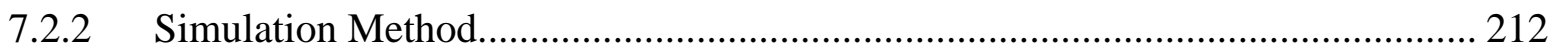

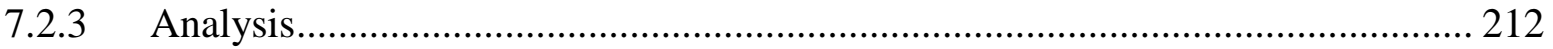

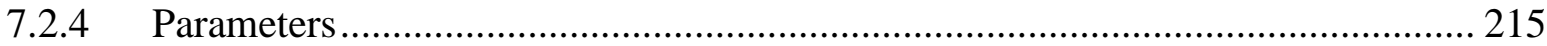

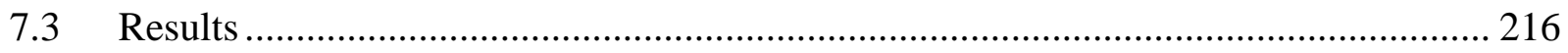

7.3.1 Effect of polymer flexibility on wetting of the grafted layer by matrix ............... 216

7.3.2 Effect of polymer flexibility on trends of varying grafting density, graft and matrix

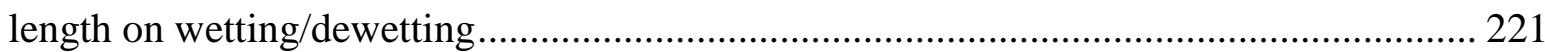

7.3.3 Effect of polymer flexibility on particle assembly/dispersion at finite filler fraction: 223

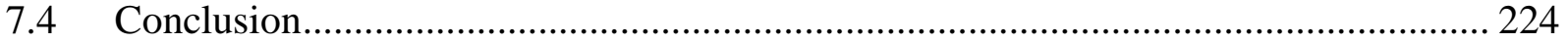

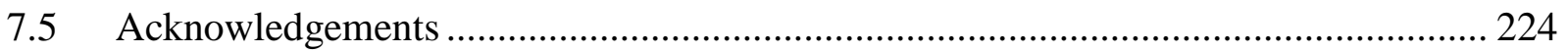

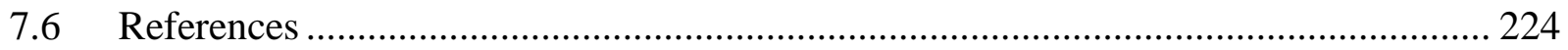

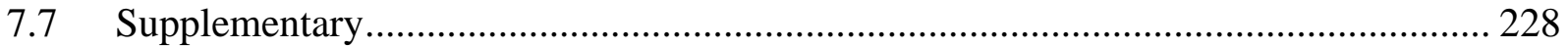

7.7.1 Effect of polymer flexibility on wetting of the grafted polymer layer by matrix

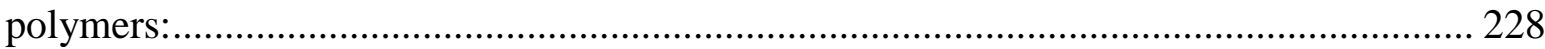

7.7.2 Effect of polymer flexibility on trends of varying grafting density and varying matrix

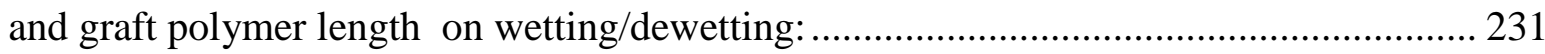

8 Wetting-Dewetting and Dispersion-Aggregation Transitions are Distinct for Polymer Grafted Nanoparticles in Chemically Dissimilar Polymer Matrix .............................................................2. 234

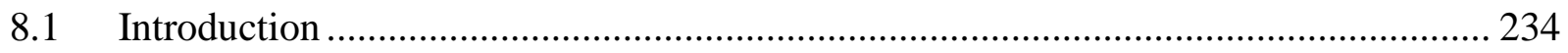

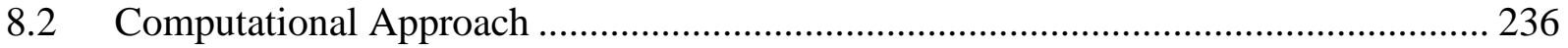

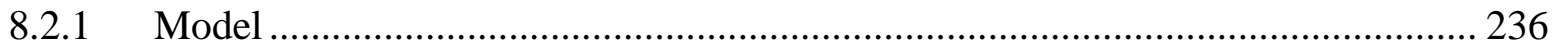

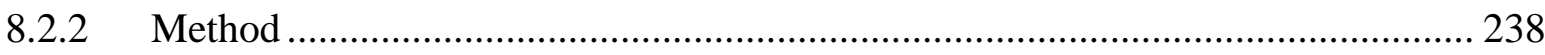

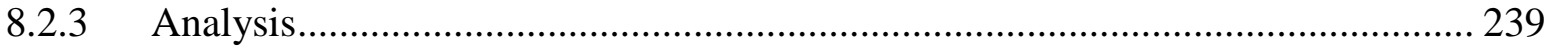

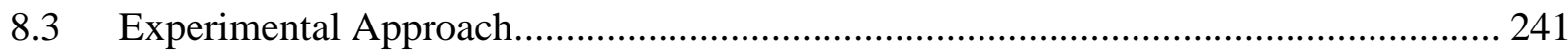


8.3.1 Experimental preparation of polymer-grafted nanoparticles ............................ 241

8.3.2 Experimental preparation of nanocomposites............................................. 242

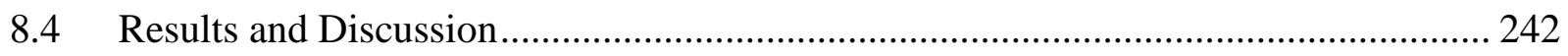

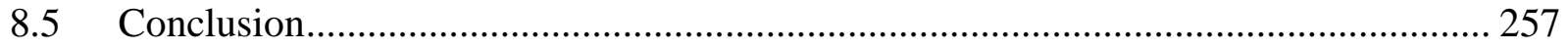

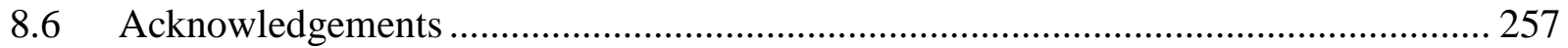

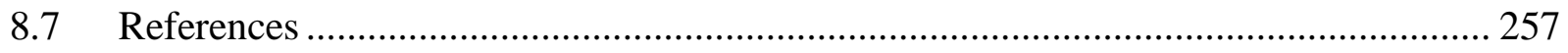

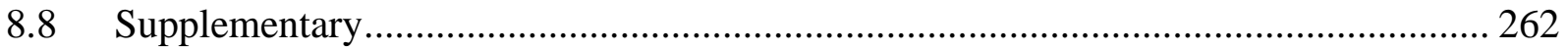

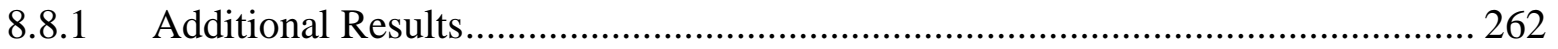

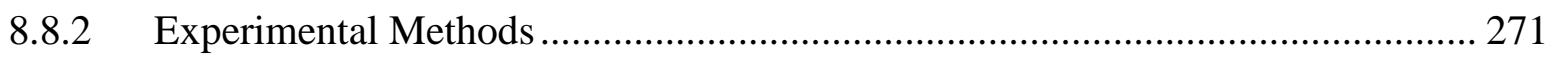

9 Tuning the Wetting-Dewetting and Dispersion-Aggregation Transitions in Polymer Nanocomposites using Composition of Graft and Matrix Polymers .......................................................................282

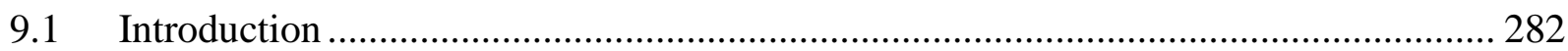

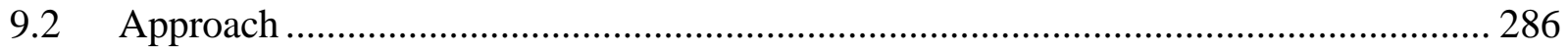

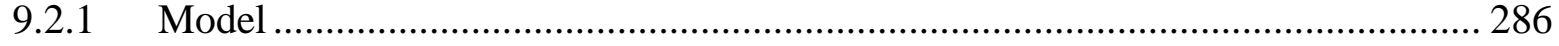

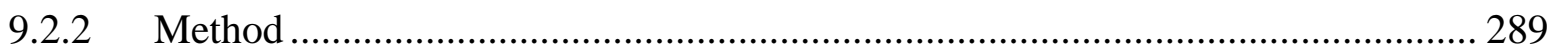

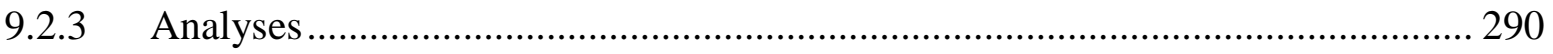

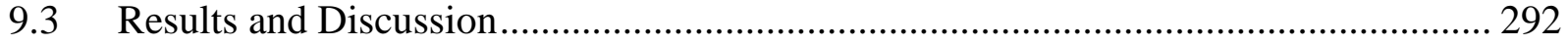

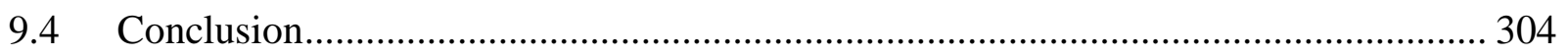

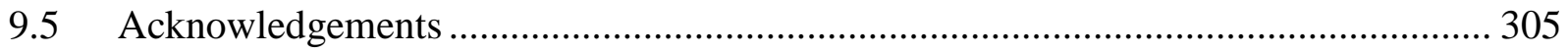

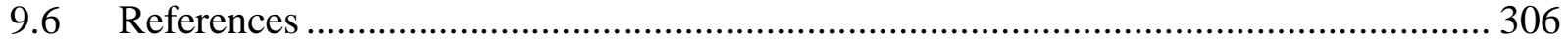

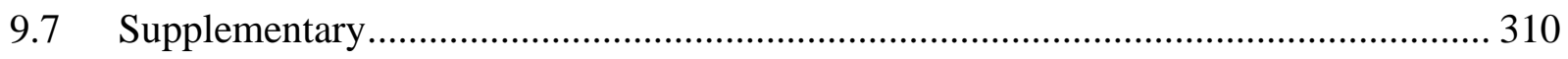

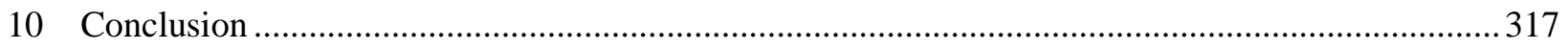

10.1 Copolymer Grafted Nanoparticles in Implicit Solvent........................................ 317

10.2 Homopolymer Grafted Nanoparticles in Chemically Identical Polymer Matrix ..... 318

10.3 Homopolymer Grafted Nanoparticles in Chemically Dissimilar Polymer Matrix ... 320

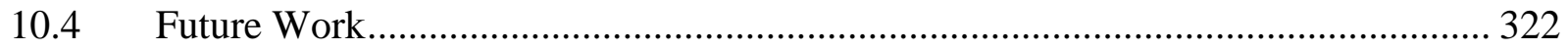

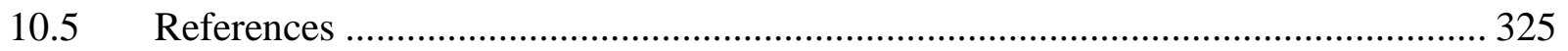

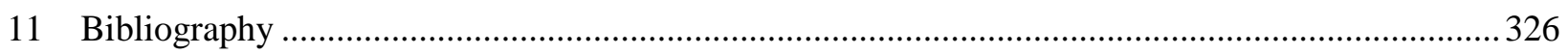




\section{LIST OF TABLES}

Table 2.1: List of monomer-monomer and (in kT). Negative values of $\varepsilon$ values represent attractive interactions while positive values represent repulsive interactions, in the square well potential form. Each of these interactions is studied without monomer-particle interactions, with particle to A-particle attraction of $1 \mathrm{kT}$, or with B-particle attraction $1 \mathrm{kT}$. 14

Table 2.2: Interaction sets and figure parts

Table 2.3: Number of A Domains for copolymer grafted nanoparticle of varying sequences and monomer interactions as specified in Table 1 and $\mathrm{D}=4 \mathrm{~d}, \mathrm{Ngraft}=24$, and $\mathrm{Ng}=6$. Note that all of these interaction sets are in the absence of particle monomer interactions. 61

Table 2.4: Number of B Domains for copolymer grafted nanoparticle of varying sequences and monomer interactions as specified in Table 1 and $\mathrm{D}=4 \mathrm{~d}, \mathrm{Ngraft}=24$, and $\mathrm{Ng}=6$. Note that all of these interaction sets are in the absence of particle monomer interactions.

Table 2.5: Number of A Domains for copolymer grafted nanoparticle of varying sequences and monomer interactions as specified in Table 1 and $\mathrm{D}=12 \mathrm{~d}, \mathrm{Ngraft}=24$, and $\mathrm{Ng}=6$. Note that all of these interaction sets are in the absence of particle monomer interactions. 63

Table 2.6: Number of B Domains for copolymer grafted nanoparticle of varying sequences and monomer interactions as specified in Table 1 and $\mathrm{D}=12 \mathrm{~d}, \mathrm{Ngraft}=24$, and $\mathrm{Ng}=6$. Note that all of these interaction sets are in the absence of particle monomer interactions.

Table 3.1: Tabulated data for the end monomer concentration at contact $\left(\varphi_{c}\right)$ and the penetration depth of the matrix into the grafted layer $(\lambda)$ in units of $d$, for varying PDI, matrix volume fraction $(\eta)$, grafting density $(\sigma)$, and matrix length $\left(\mathrm{N}_{\text {matrix }}\right)$.

Table 4.1: Minimum values for the potentials of mean force, PMF (in units of kT), versus PDI between nanoparticles of size $\mathrm{D}=8 \mathrm{~d}$ and $\mathrm{D}=5 \mathrm{~d}$ ( grafted with polydisperse chains with $\mathrm{N}_{\mathrm{g}, \mathrm{avg}}=20$ at a grafting density of $\boldsymbol{\sigma}=\mathbf{0 . 2 5}$ chains $/ \mathrm{d}^{2}$, in a dense solution $(\boldsymbol{\eta}=\mathbf{0 . 1})$ of monodisperse homopolymers with $\mathbf{N}_{\text {matrix }}=\mathbf{4 0}$. 125 
Table 5.1: Number of Chains $w / N>N_{\text {graft,avg }}$ for Bidisperse Distributions at $\sigma=0.65$ chains $/ \mathrm{d}^{2} 177$

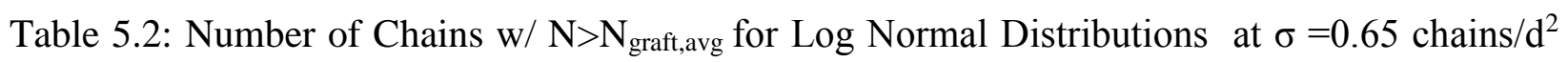
and $\mathrm{N}_{\text {graft,avg }}=20$.

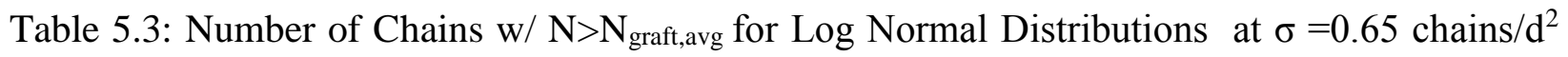
and $\mathrm{N}_{\text {graft,avg }}=14$

Table 6.1: Wetted and Dewetted Monomer Counts used for $\Delta \mathrm{S}_{\text {wetting }}$ Calculation 205

Table 6.2: Example $\Delta \mathrm{S}_{\text {wetting }}$ Calculation for Chains of in Monodisperse and Bidisperse Matrices 205

Table 7.1: Expected persistence lengths $\mathrm{L}_{\mathrm{p}}$, (in units of $\sigma$ ), of graft and matrix chains at given grafting density and chain lengths calculated using three methods

Table 7.2: Average percentage of matrix beads that wet the grafted layer (\% Wet Matrix) as a function of graft and matrix lengths, grafting density and flexibility.

Table 7.3: Average end-end distance of the matrix polymers for varying matrix and graft lengths, grafting densities, and flexibilities. The graft and matrix end-to-end distances are defined as the average over all of the graft and matrix chains respectively. The "wet matrix" end-to-end distance is defined as the average over any chain that has any bead within the grafted layer "brush" height, $\left\langle\mathrm{H}_{\mathrm{B}}{ }^{2}\right\rangle^{0.5}$, and the "unwet matrix" end-to-end distance is the average over the remainder of the chains. The standard deviation of the independent snapshots is shown. 228

Table 7.4: Average radii of gyration of the graft chains. The standard deviation for all values is $<0.1 \sigma$. 230

Table 8.1: Values of the Scattering Length Densities. 281 


\section{LIST OF FIGURES}

Figure 2.1: Schematic of the monomer sequences used in this study, in decreasing order of blockiness from top to bottom. 13

Figure 2.2: Average radius of gyration of a grafted chain $\left\langle\mathrm{R}_{\mathrm{g}, \text { chain }}\right\rangle^{0.5}$, average number of total AA contacts, total $\mathrm{BB}$ contacts and total $\mathrm{AB}$ contacts as a function of graft monomer sequence for copolymer grafted nanoparticles of size $D=4 d$ with six grafts of length $N_{\text {graft }}=24$. The $x$ - axis indices correspond to the graft sequence $\left(A_{1} B_{1}\right)_{12},\left(A_{2} B_{2}\right)_{6},\left(A_{3} B_{3}\right)_{4},\left(A_{6} B_{6}\right)_{2}$, and $\left(A_{12} B_{12}\right)_{1}$. The interaction sets correspond to the plot letters as follows: lines on plot a, g, m, and s correspond to interaction sets 1 (blue triangles) and 2 (black circles), lines on plot $\mathrm{b}, \mathrm{h}, \mathrm{n}$, and $\mathrm{t}$ correspond to interaction sets 3 (blue triangles) and 4 (black circles), lines on plot c, i, o, and u correspond to interaction sets 5 (blue triangles) and 6 (black circles), lines on plot $\mathrm{d}, \mathrm{j}, \mathrm{p}$, and $\mathrm{v}$ correspond to interaction sets 7 (blue triangles) and 8 (black circles), lines on plot e, k, q, and w correspond to interaction sets 9 (blue triangles) and 10 (black circles), and lines on plot $\mathrm{f}, \mathrm{l}, \mathrm{r}$, and $\mathrm{x}$ correspond to interaction sets 11 (blue triangles) and 12 (black circles). The black lines with circle markers represent monomer-monomer attraction $=1 \mathrm{kT}$, while the blue triangles with triangle markers represent monomer-monomer attraction $=0.5 \mathrm{kT}$. All monomer-particle interactions are maintained as hard sphere interactions 22

Figure 2.3: (a-e) Snapshots of clusters for systems with Interaction Set 4 and no monomer-particle attraction (best viewed in color). The plots show the average number of A domains (f-k) and B domains (l-q) for copolymer grafted nanoparticles of size $D=4 d$ each with six grafts of length $\mathrm{N}_{\text {graft }}=24$. The interaction sets correspond to the plot letters as follows: The interaction sets to the plot letters as follows: lines on plot $\mathrm{f}$ and 1 correspond to interaction sets 1 (blue triangles) and 2 (black circles), lines on plot $\mathrm{g}$ and $\mathrm{m}$ correspond to interaction sets 3 (blue triangles) and 4 (black circles), lines on plot $\mathrm{h}$ and $\mathrm{n}$ correspond to interaction sets 5 (blue triangles) and 6 (black circles), lines on plot $\mathrm{i}$ and o correspond to interaction sets 7 (blue triangles) and 8 (black circles), lines on plot $\mathrm{j}$ and $\mathrm{p}$ correspond to interaction sets 9 (blue triangles) and 10 (black circle), and lines on plot $\mathrm{k}$ and $\mathrm{q}$ correspond to interaction sets 11 (blue triangles) and 12 (black circles). The black circles represent monomer-monomer attraction $=1 \mathrm{kT}$, while the blue triangles represent monomermonomer attraction $=0.5 \mathrm{kT}$. All monomer-particle interactions are maintained as hard sphere interactions. 26 
Figure 2.4: Average height of A-monomers (top row) and B-monomers (bottom row) from the particle centers as functions of monomer sequences for copolymer grafted nanoparticles of size $\mathrm{D}=4 \mathrm{~d}$ with six grafts of length $\mathrm{N}_{\text {graft }}=24$. The interaction sets correspond to the plot letters as follows: lines on plot a and g correspond to interaction sets 1 (blue triangles) and 2 (black circles), lines on plot $\mathrm{b}$ and $\mathrm{h}$ correspond to interaction sets 3 (blue triangles) and 4 (black circles), lines on plot $\mathrm{c}$ and $\mathrm{i}$ correspond to interaction sets 5 (blue triangles) and 6 (black circles), lines on plot $\mathrm{d}$ and $\mathrm{j}$ correspond to interaction sets 7 (blue triangles) and 8 (black circles), lines on plot e and $\mathrm{k}$ correspond to interaction sets 9 (blue triangles) and 10 (black circles), and lines on plot $\mathrm{f}$ and 1 correspond to interaction sets 11 (blue triangles) and 12 (black circles). The black circles represent monomer-monomer attraction=1kT, while the blue triangles represent monomer-monomer attraction $=0.5 \mathrm{kT}$. All monomer-particle interactions are maintained as hard sphere interactions.

Figure 2.5: Average coordination number $\langle\mathrm{Z}\rangle$, average number of particles per cluster $\langle\mathrm{N}\rangle$, and average radius of gyration of a cluster $\left\langle\mathrm{R}_{\mathrm{g} \text {,cluster }}^{0.5}\right.$ as a function of monomer sequences for copolymer grafted nanoparticles of size $D=4 d$ with six grafts of length $N_{\text {graft }}=24$. The interaction sets correspond to the plot letters as follows: lines on plot a, g, and $\mathrm{m}$ correspond to interaction sets 1 (blue triangles) and 2 (black circle), lines on plot b, h, and $\mathrm{n}$ correspond to interaction sets 3 (blue triangles) and 4 (black circle), lines on plot c, i, and o correspond to interaction sets 5 (blue triangles) and 6 (black circle), lines on plot d, j, and p correspond to interaction sets 7 (blue triangles) and 8 (black circle), lines on plot e, k, and q correspond to interaction sets 9 (blue triangles) and 10 (black circle), and lines on plot f, l, and r correspond to interaction sets 11 (blue triangles) and 12 (black circle). The black circles represent monomer-monomer attraction=1kT, while the blue triangles represent monomer-monomer attraction=0.5kT. All monomer-particle interactions are maintained as hard sphere interactions. 30

Figure 2.6: Relative shape anisotropy (RSA) as a function of monomer sequence on copolymer grafted nanoparticles for copolymer grafted nanoparticles of size $\mathrm{D}=4 \mathrm{~d}$ with six grafts of length $\mathrm{N}_{\text {graft }}=24$. The interaction sets correspond to the plot letters as follows: lines on plot a correspond to interaction sets 1 (blue triangles) and 2 (black circle), lines on plot b correspond to interaction sets 3 (blue triangles) and 4 (black circle), lines on plot c correspond to interaction sets 5 (blue triangles) and 6 (black circle), lines on plot d correspond to interaction sets 7 (blue triangles) and 
8 (black circle), lines on plot e correspond to interaction sets 9 (blue triangles) and 10 (black circle), and lines on plot f correspond to interaction sets 11 (blue triangles) and 12 (black circle). The black lines with circle markers represent monomer-monomer attraction=1kT, while the blue triangles with triangle markers represent monomer-monomer attraction $=0.5 \mathrm{kT}$. All monomerparticle interactions are maintained as hard sphere interactions. 33

Figure 2.7: Average coordination number $\langle\mathrm{Z}\rangle$, average number of particles per cluster $\langle\mathrm{N}\rangle$, and relative shape anisotropy (RSA) as a function of monomer sequence for copolymer grafted nanoparticles of size $D=4 d$ and $D=12 d$ with six grafts of length $N_{\text {graft }}=24$. The lines on plot $a, d$, and g correspond to interaction sets 1 (triangles) and 2 (circles), lines on plot b, e, and h correspond to interaction sets 5 (triangles) and 6 (circles), lines on plot c, f, and i correspond to interaction sets 9 (triangles) and 10 (circles The circles represent monomer-monomer attraction=1kT, while the triangles represent monomer-monomer attraction $=0.5 \mathrm{kT}$. The filled symbols represent nanoparticles of size $D=4 d$ while the open symbols represent nanoparticles of size $D=12 d$. All monomer-particle interactions are maintained as hard sphere interactions . 35

Figure 2.8: Average height of A-monomers (top row) and B-monomers (bottom row) from the particle surface as a function of monomer sequences for copolymer grafted nanoparticles $D=12 \mathrm{~d}$ with six grafts of length $\mathrm{N}_{\text {graft }}=24$ for interaction sets with no particle monomer interactions (black diamonds), A-particle attraction=1kT (blue circles), and B-particle attraction=1kT (red squares). The interaction sets correspond to the plot letters as follows (with particle-monomer interactions indicated by line color and marker shape): lines on plot a and d correspond to interaction set 4 , lines on plot $\mathrm{b}$ and $\mathrm{e}$, correspond to interaction set 1 , lines on plot $\mathrm{c}$ and $\mathrm{f}$ correspond to interaction set 11 .

Figure 2.9: Average coordination number $\langle\mathrm{Z}\rangle$, average number of particles per cluster $\langle\mathrm{N}\rangle$, average radius of gyration of a cluster $\left\langle\mathrm{R}_{\mathrm{g}, \text { cluster }}\right\rangle^{0.5}$ and relative square anisotropy (RSA) as a function of monomer sequence for copolymer grafted nanoparticles $D=12 d$ with six grafts of length $\mathrm{N}_{\text {graft }}=24$ for systems with no particle monomer interactions (black diamonds), A-particle attraction=1kT (blue circles), and B-particle attraction=1kT (red squares). The interaction sets correspond to the plot letters as follows (with particle-monomer interactions indicated by line color and marker 
shape): lines on plot a, d, g, and $\mathrm{j}$ correspond to interaction set 4 , lines on plot $\mathrm{b}, \mathrm{e}, \mathrm{h}$, and $\mathrm{k}$ correspond to interaction set 1 , lines on plot $\mathrm{c}, \mathrm{f}, \mathrm{i}$, and 1 correspond to interaction set 11 .... 38

Figure 2.10: Schematic showing the three types of monomer contacts that could be made in these systems of copolymer grafted nanoparticles. 48

Figure 2.11: Average number of interparticle contacts as a function of monomer sequence for copolymer grafted nanoparticles of $D=4 d$ each with six grafts of length Ngraft=24 with no particle monomer interactions (black diamonds), A-monomer to particle attraction at -1kT (blue circles), and B-monomer to particle attraction at - $1 \mathrm{kT}$ (red squares). The system interactions correspond to the plot letters as in Table 2.2 (with particle-monomer interactions indicated by line color) ...... 50

Figure 2.12: Average number of interparticle contacts as a function of monomer sequence for copolymer grafted nanoparticles of $\mathrm{D}=12 \mathrm{~d}$ each with six grafts of length Ngraft=24 with no particle monomer interactions (black diamonds), A-monomer to particle attraction at -1kT (blue circles), and B-monomer to particle attraction at -1kT (red squares). The system interactions correspond to the plot letters as in Table 2.2 (with particle-monomer interactions indicated by line color) 50

Figure 2.13: Average number of inter-chain contacts, including inter and intra particle, as a function of monomer sequence for copolymer grafted nanoparticles of $D=4 d$ each with six grafts of length Ngraft=24 with no particle monomer interactions (black diamonds), A-monomer to particle attraction at -1kT (blue circles), and B-monomer to particle attraction at -1kT (red squares). The system interactions correspond to the plot letters as in Table 2.2 (with particle-monomer .. 51

Figure 2.14: Average number of inter-chain contacts, including inter and intra particle, as a function of monomer sequence for copolymer grafted nanoparticles of $D=12 \mathrm{~d}$ each with six grafts of length Ngraft=24 with no particle monomer interactions (black diamonds), A-monomer to particle attraction at -1kT (blue circles), and B-monomer to particle attraction at -1kT (red squares). The system interactions correspond to the plot letters as in Table 2.2 (with particle-monomer interactions indicated by line color). 51

Figure 2.15: Average number of inter-chain intra-particle contacts, including inter and intra particle, as a function of monomer sequence for copolymer grafted nanoparticles of $D=4 d$ each 
with six grafts of length Ngraft=24 with no particle monomer interactions (black diamonds), Amonomer to particle attraction at -1kT (blue circles), and B-monomer to particle attraction at -1kT (red squares). The system interactions correspond to the plot letters as in Table 2.2 (with particlemonomer interactions indicated by line color) 52

Figure 2.16: Average number of inter-chain intra-particle contacts, including inter and intra particle, as a function of monomer sequence for copolymer grafted nanoparticles of $D=12 d$ each with six grafts of length Ngraft=24 with no particle monomer interactions (black diamonds), Amonomer to particle attraction at -1kT (blue circles), and B-monomer to particle attraction at -1kT (red squares). The system interactions correspond to the plot letters as in Table 2.2 (with particlemonomer interactions indicated by line color)

Figure 2.17: Average number of A-particle contacts, including inter and intra particle, as a function of monomer sequence for copolymer grafted nanoparticles of $\mathrm{D}=4 \mathrm{~d}$ each with six grafts of length Ngraft=24 with no particle monomer interactions (black diamonds), A-monomer to particle attraction at $-1 \mathrm{kT}$ (blue circles), and B-monomer to particle attraction at -1kT (red squares). The system interactions correspond to the plot letters as in Table 2.2 (with particle-monomer interactions indicated by line color ). 53

Figure 2.18: Average number of A-particle contacts, including inter and intra particle, as a function of monomer sequence for copolymer grafted nanoparticles of $\mathrm{D}=12 \mathrm{~d}$ each with six grafts of length Ngraft=24 with no particle monomer interactions (black diamonds), A-monomer to particle attraction at $-1 \mathrm{kT}$ (blue circles), and B-monomer to particle attraction at $-1 \mathrm{kT}$ (red squares). The system interactions correspond to the plot letters as in Table 2.2 (with particle-monomer interactions indicated by line color). 53

Figure 2.19: Average number of B-particle contacts, including inter and intra particle, as a function of monomer sequence for copolymer grafted nanoparticles of $\mathrm{D}=4 \mathrm{~d}$ each with six grafts of length Ngraft=24 with no particle monomer interactions (black diamonds), A-monomer to particle attraction at -1kT (blue circles), and B-monomer to particle attraction at $-1 \mathrm{kT}$ (red squares). The system interactions correspond to the plot letters as in Table 2.2 (with particle-monomer interactions indicated by line color). 54 
Figure 2.20: Average number of B-particle contacts, including inter and intra particle, as a function of monomer sequence for copolymer grafted nanoparticles of $\mathrm{D}=12 \mathrm{~d}$ each with six grafts of length Ngraft=24 with no particle monomer interactions (black diamonds), A-monomer to particle attraction at -1kT (blue circles), and B-monomer to particle attraction at -1kT (red squares). The system interactions correspond to the plot letters as in Table 2.2 (with particle-monomer interactions indicated by line color). 55

Figure 2.21: Average height of an A-monomer as a function of monomer sequences for copolymer grafted nanoparticles of $\mathrm{D}=4 \mathrm{~d}$ each with six grafts of length $\mathrm{N}=24$ for systems with no particle monomer interactions (black diamonds), A-monomer to particle attraction at -1kT (blue circles), and B-monomer to particle attraction at -1kT (red squares). The system interactions correspond to the plot letters as in Table 2.2 (with particle-monomer interactions indicated by line color) ...... 55

Figure 2.22: Average height of an A-monomers as a function of monomer sequences for copolymer grafted nanoparticles of $\mathrm{D}=12 \mathrm{~d}$ each with six grafts of length $\mathrm{N}=24$ for systems with no particle monomer interactions (black diamonds), A-monomer to particle attraction at -1kT (blue circles), and B-monomer to particle attraction at -1kT (red squares). The system interactions correspond to the plot letters as in Table 2.2 (with particle-monomer interactions indicated by line color) ...... 56

Figure 2.23: Average height B-monomer as a function of monomer sequences for copolymer grafted nanoparticles of $\mathrm{D}=4 \mathrm{~d}$ each with six grafts of length $\mathrm{N}=24$ for systems with no particle monomer interactions (black diamonds), A-monomer to particle attraction at -1kT (blue circles), and B-monomer to particle attraction at -1kT (red squares). The system interactions correspond to the plot letters as in Table 2.2 (with particle-monomer interactions indicated by line color) ...... 56

Figure 2.24: Average height B-monomer as a function of monomer sequences for copolymer grafted nanoparticles of $\mathrm{D}=12 \mathrm{~d}$ each with six grafts of length $\mathrm{N}=24$ for systems with no particle monomer interactions (black diamonds), A-monomer to particle attraction at -1kT (blue circles), and B-monomer to particle attraction at $-1 \mathrm{kT}$ (red squares). The system interactions correspond to the plot letters as in Table 2.2 (with particle-monomer interactions indicated by line color) ...... 57

Figure 2.25: Simulation snapshots from Brownian dynamics of ungrafted copolymers of sequence $\left(\mathrm{A}_{1} \mathrm{~B}_{1}\right)_{10}$ (left) and $\left(\mathrm{A}_{2} \mathrm{~B}_{2}\right)_{6}$ (right) represented as iso-surfaces of density equal to 0.3 monomers $/ \mathrm{d}^{3}$. 
The top row shows both A and B iso-surfaces together while the middle and bottom show isosurfaces calculated from only A and only B monomers respectively. In both of these simulations, only the BB interactions are attractive and all others interactions are purely repulsive. The figures show that the $\left(\mathrm{A}_{2} \mathrm{~B}_{2}\right)_{5}$ sequence produces distinct, homogeneous domains of attractive $\mathrm{B}$ monomers (right column bottom figure), the $\left(\mathrm{A}_{1} \mathrm{~B}_{1}\right)_{10}$ sequence produces networked domains that span the simulation box (left column bottom figure). 58

Figure 2.26: Average coordination number $\langle\mathrm{Z}\rangle$, average number of particles per cluster $\langle\mathrm{N}\rangle$, and relative shape anisotropy (RSA) as a function of monomer sequences for copolymer grafted nanoparticles of $D=4 d$ and $D=12 d$ each with six grafts of length $N=24$. The lines on plot $a, d$, and g correspond to interaction sets 3 (blue and red triangles) and 4 (black and magenta circles), lines on plot b,e, and h correspond to interaction sets 7 (blue and red triangles) and 8 (black and magenta circles), lines on plot c, f, and i correspond to interaction sets 11 (blue and red triangles) and 12 (black and magenta circles). The circles represent attractive monomer-monomer interactions at a strength of $1 \mathrm{kT}$ while the triangles represent $0.5 \mathrm{kT}$. The filled symbols represent nanoparticles of size $D=4 d$ while open symbols represent interaction sets with nanoparticles of size $d=12 d$...... 65

Figure 2.27: (f-k) Plots showing the average number of A domains in a system for $D=12 d$. (l-q) Plots showing the average number of B domains in a system. The system interactions correspond to the plot letters as follows: The system interactions correspond to the plot letters as follows: lines on plot $\mathrm{f}$ and 1 correspond to interaction sets 1 (blue triangles) and 2 (black circles), lines on plot $\mathrm{g}$ and $\mathrm{m}$ correspond to interaction sets 3 (blue triangles) and 4 (black circles), lines on plot $\mathrm{h}$ and $\mathrm{n}$ correspond to interaction sets 5 (blue triangles) and 6 (black circles), lines on plot $\mathrm{i}$ and o correspond to interaction sets 7 (blue triangles) and 8 (black circles), lines on plot $\mathrm{j}$ and $\mathrm{p}$ correspond to interaction sets 9 (blue triangles) and 10 (black circle), and lines on plot $\mathrm{k}$ and $\mathrm{q}$ correspond to interaction sets 11 (blue triangles) and 12 (black circles). The black circles represent attractive monomer-monomer interactions at a strength of $1 \mathrm{kT}$ while the blue triangles represent ttractive monomer-monomer interactions at a strength of $0.5 \mathrm{kT}$

Figure 2.28: Plots showing the average height of an A-monomer (top row) and B-monomer (bottom row) as functions of monomer sequences for copolymer grafted nanoparticles of $\mathrm{D}=12 \mathrm{~d}$ each with six grafts of length $\mathrm{N}=24$. The system interactions correspond to the plot letters as 
follows: lines on plot a and g correspond to interaction sets 1 (blue triangles) and 2 (black circles), lines on plot $\mathrm{b}$ and $\mathrm{h}$ correspond to interaction sets 3 (blue triangles) and 4 (black circles), lines on plot $\mathrm{c}$ and i correspond to interaction sets 5 (blue triangles) and 6 (black circles), lines on plot d and $\mathrm{j}$ correspond to interaction sets 7 (blue triangles) and 8 (black circles), lines on plot e and $\mathrm{k}$ correspond to interaction sets 9 (blue triangles) and 10 (black circles), and lines on plot $\mathrm{f}$ and 1 correspond to interaction sets 11 (blue triangles) and 12 (black circles). The black circles represent attractive monomer-monomer interactions at a strength of $1 \mathrm{kT}$ while the blue triangles represent attractive monomer-monomer interactions at a strength of $0.5 \mathrm{kT}$ 67

Figure 2.29: Average coordination number $\langle\mathrm{Z}\rangle$ as a function of monomer sequences for copolymer grafted nanoparticles of $\mathrm{D}=4 \mathrm{~d}$ with six grafts of length $\mathrm{N}=24$ for systems with no particle monomer interactions (black diamonds), A-monomer to particle attraction at -1kT (blue circles), and Bmonomer to particle attraction at $-1 \mathrm{kT}$ (red squares). 70

Figure 2.30: Average coordination number $\langle Z\rangle$ as a function of monomer sequences for copolymer grafted nanoparticles of $\mathrm{D}=12 \mathrm{~d}$ with six grafts of length $\mathrm{N}=24$ for systems with no particle monomer interactions (black diamonds), A-monomer to particle attraction at -1kT (blue circles), and B-monomer to particle attraction at $-1 \mathrm{kT}$ (red squares).

Figure 2.31: Average number of particles per cluster $\langle\mathrm{N}\rangle$ as a function of monomer sequences for copolymer grafted nanoparticles of $\mathrm{D}=4 \mathrm{~d}$ with six grafts of length $\mathrm{N}=24$ for systems with no particle monomer interactions (black diamonds), A-monomer to particle attraction at -1kT (blue circles), and B-monomer to particle attraction at -1kT (red squares). 71

Figure 2.32: Average number of particles per cluster $\langle\mathrm{N}\rangle$ as a function of monomer sequences for copolymer grafted nanoparticles of $\mathrm{D}=12 \mathrm{~d}$ with six grafts of length $\mathrm{N}=24$ for systems with no particle monomer interactions (black diamonds), A-monomer to particle attraction at -1kT (blue circles), and B-monomer to particle attraction at -1kT (red squares). 71

Figure 2.33: Average radius of gyration of a cluster $\langle\mathrm{R} 2 \mathrm{~g}$,cluster $\rangle 0.5$ as a function of monomer sequences for copolymer grafted nanoparticles of $\mathrm{D}=4 \mathrm{~d}$ with six grafts of length $\mathrm{N}=24$ for systems with no particle monomer interactions (black diamonds), A-monomer to particle attraction at -1kT (blue circles), and B-monomer to particle attraction at -1kT (red squares). 72 
Figure 2.34: Average radius of gyration of a cluster $\langle\mathrm{R} 2 \mathrm{~g}$,cluster $\rangle 0.5$ as a function of monomer sequences for copolymer grafted nanoparticles of $D=12 \mathrm{~d}$ with six grafts of length $\mathrm{N}=24$ for systems with no particle monomer interactions (black diamonds), A-monomer to particle attraction at $-1 \mathrm{kT}$ (blue circles), and B-monomer to particle attraction at $-1 \mathrm{kT}$ (red squares). to interaction set 12 , and lines on plot $\mathrm{L}$ correspond to interaction set 11 72

Figure 2.35: Average relative square anisotropy (RSA) as a function of monomer sequences for copolymer grafted nanoparticles of $\mathrm{D}=4 \mathrm{~d}$ with six grafts of length $\mathrm{N}=24$ for systems with no particle monomer interactions (black diamonds), A-monomer to particle attraction at -1kT (blue circles), and B-monomer to particle attraction at - $1 \mathrm{kT}$ (red squares) 73

Figure 2.36: Average relative square anisotropy (RSA) as a function of monomer sequences for copolymer grafted nanoparticles of $\mathrm{D}=12 \mathrm{~d}$ with six grafts of length $\mathrm{N}=24$ for systems with no particle monomer interactions (black diamonds), A-monomer to particle attraction at $-1 \mathrm{kT}$ (blue circles), and B-monomer to particle attraction at $-1 \mathrm{kT}$ (red squares).

Figure 2.37: Average coordination number $(\langle\mathrm{Z}\rangle)$, average number of particles per cluster $(\langle\mathrm{N}\rangle)$, average cluster radius of gyration $\langle\mathrm{R} 2 \mathrm{~g}$,cluster $\rangle 0.5$ ), and relative square anisotropy (RSA) as a function of monomer sequences for copolymer grafted nanoparticles of $\mathrm{D}=4 \mathrm{~d}$ (left column) and $\mathrm{D}=12 \mathrm{~d}$ (right column) each with six grafts of length $\mathrm{N}=24$. The solid blue lines represent a system where A-A attraction is $1 \mathrm{kT}$ and A- particle attraction is $1 \mathrm{kT}$. The dashed blue lines represent a system where A-A attraction is $1 \mathrm{kT}$ and A-particle attraction is $16 \mathrm{kT}$. The solid red lines represent a system where B-B attraction is $1 \mathrm{kT}$ and B-particle attraction is $1 \mathrm{kT}$. The dashed red lines represent a system where B-B attraction is $1 \mathrm{kT}$ and B-particle attraction is $-16 \mathrm{kT}$. 74

Figure 3.1: PMF (in units of kT) versus inter-particle distance, r-D (in units of d), between grafted nanoparticles $(\mathrm{D}=5 \mathrm{~d})$ at $\sigma=0.1(\mathrm{a}), 0.25(\mathrm{~b})$, and 0.65 (c) chains $/ \mathrm{d}^{2}$ and $\mathrm{PDI}=1.0$ (circles), 1.5 (squares), 2.0 (upward facing triangles), and 2.5 (downward facing triangles) with $\mathrm{N}_{\mathrm{g}, \mathrm{avg}}=20$, in a dense solution $(\eta=0.1)$ of monodisperse homopolymer matrix chains with $\mathrm{N}_{\text {matrix }}=10$ (solid symbols) and $\mathrm{N}_{\text {matrix }}=40$ (open symbols). The insets have the same axes labels as the main plots. 
Figure 3.2: Penetration depth, $\lambda$ (in units of $d$ ), of matrix chains into grafted layer on nanoparticles ( $D=5 d$ ) grafted with polydisperse chains with $\mathrm{N}_{\mathrm{g}, \mathrm{avg}}=20$ at $\sigma=0.10$ chains $/ \mathrm{d}^{2}$ (solid lines) and $\sigma=0.25$ chains $/ \mathrm{d}^{2}$ (dashed lines) in a dense solution $(\eta=0.1)$ of monodisperse homopolymer matrix chains with $\mathrm{N}_{\text {matrix }}=10$ (filled symbols) and $\mathrm{N}_{\text {matrix }}=40$ (open symbols). 86

Figure 3.3: PMF (in units of $k T$ ) versus r-D (in units of $d$ ) between nanoparticles $(D=5 d)$ at $P D I=$ 1.0 (circles), PDI=1.5 (squares), PDI=2.0 (upward facing triangles), and PDI=2.5 (downward facing triangles) and $\mathrm{N}_{\mathrm{g}, \mathrm{avg}}=20$ at (a) $\sigma=0.1$ and (b) 0.65 chains $/ \mathrm{d}^{2}$ in a monodisperse melt-like matrix $\left(\eta=0.3\right.$ ) with $\mathrm{N}_{\text {matrix }}=10$ (solid symbol) and $\mathrm{N}_{\text {matrix }}=40$ (open symbol). The insets have the same axes labels as the main plots. 87

Figure 3.4: Potentials of mean force (in kT) versus inter-particle distances (in units of d) between nanoparticles of size $\mathrm{D}=5 \mathrm{~d}$ with monodisperse chains with $\mathrm{N}_{\mathrm{g}}=20$, at a grafting density of $\sigma=0.10$ chains $/ d^{2}$, in dense solution $(\eta=0.1$, subplot a) and melt $(\eta=0.3$, subplot $b)$ of monodisperse homopolymers of $\mathrm{N}_{\text {matrix }}=10$ (triangles), $\mathrm{N}_{\text {matrix }}=40$ (circles), $\mathrm{N}_{\text {matrix }}=80$ (squares).

Figure 3.5: Potentials of mean force, PMF (in units of kT), versus inter-particle distance, r-D (in units of $d$ ), between nanoparticles of size $D=5 d$, grafted with polydisperse chains at grafting densities $\sigma=0.65$ chains $/ \mathrm{d}^{2}$ with $\mathrm{PDI}=1.00$ (circles), $\mathrm{PDI}=1.05$ (upward facing triangles), $\mathrm{PDI}=1.10$ (squares), $\mathrm{PDI}=1.15$ (rightward facing triangles), $\mathrm{PDI}=1.20$ (diamonds), and PDI=1.40 (downward facing triangles) with $\mathrm{N}_{\mathrm{g}, \mathrm{avg}}=20$ in a dense solution $(\eta=0.1)$ of monodisperse homopolymer matrix chains with $\mathrm{N}_{\text {matrix }}=40$..... 94

Figure 3.6: Potentials of mean force (in units of kT) versus inter-particle distances (in units of d) between nanoparticles of size $\mathrm{D}=5 \mathrm{~d}$ with polydisperse chains of $\mathrm{PDI}=1.0$ (circles) and $\mathrm{PDI}=2.0$ (other symbols) with $\mathrm{N}_{\mathrm{g}, \mathrm{av}}=20$ and $\mathrm{N}_{\mathrm{g}, \mathrm{min}}=8$ at a grafting density of $\sigma=0.65$ chains $/ \mathrm{d}^{2}$, for five different log-normal chain length distributions (subplot $c$ ), in a dense solution $(\eta=0.1$, subplot a) and melt $\left(\eta=0.3\right.$, subplot b) of monodisperse homopolymers with $\mathrm{N}_{\text {matrix }}=40$. Each symbol, (besides circle) corresponds to a different log-normal chain distribution. 95

Figure 3.7: Potentials of mean force, PMF (in units of kT) versus inter-particle distance, r-D (in units of $d$ ), between nanoparticles of size $D=5 d$, grafted with polydisperse (filled symbols) or bidisperse (open symbols) chains at grafting densities $\sigma=0.65$ chains $/ \mathrm{d}^{2}$ (squares and upward 
triangle) and $\sigma=0.25$ chains $/ \mathrm{d}^{2}$ (circles and downward triangles) at PDI $=1.5$ with $\mathrm{N}_{\mathrm{g}, \mathrm{av}}=20$ in a dense solution $(\eta=0.1$, downward triangles and squares $)$ and melt-like $(\eta=0.3$, upward triangles and circles) matrix of monodisperse homopolymer matrix chains with Nmatrix $=40$.

Figure 3.8: Potentials of mean force, PMF (in units of kT) versus inter-particle distance, r-D (in units of $d$ ) between nanoparticles of size $D=8 d$ grafted with polydisperse chains at $\mathrm{PDI}=1.0$ (circles), PDI=1.5 (squares), PDI=2.0 (upward facing triangles), and PDI=2.5 (downward facing triangles) with $\mathrm{N}_{\mathrm{g}, \mathrm{av}}=20$ at a grafting density of $\sigma=0.1$ chains $/ \mathrm{d}^{2}$, in a dense solution $(\eta=0.1$, subplot a) and melt ( $\eta=0.3$, subplot $b$ ) of monodisperse homopolymers with $\mathrm{N}_{\text {matrix }}=40$. The insets have the same axes labels as the main plots. 97

Figure 4.1: PMF (in units of kT) versus inter-particle distance, r-D (in units of d), between grafted nanoparticles $(\mathrm{D}=5 \mathrm{~d})$ at $\sigma=0.65(\mathrm{a}), 0.25(\mathrm{~b})$, and 0.10 chains $/ \mathrm{d}^{2}$ and $\mathrm{PDI}=1.0$ (circles), 1.5 (squares), 2.0 (upward facing triangles), and 2.5 (downward facing triangles) with $\mathrm{N}_{\mathrm{g}, \mathrm{avg}}=20$, in a dense solution $(\eta=0.1)$ of monodisperse homopolymer matrix chains with $\mathrm{N}_{\text {matrix }}=10$ (solid symbols) and $\mathrm{N}_{\text {matrix }}=40$ (open symbols). The insets have the same axes labels as the main plots.

Figure 4.2: Second virial coefficient for polymer grafted nanoparticles in a polymer matrix as a function of graft polydispersity (PDI) at grafting densities of $\sigma=0.10$ chains $/ \mathrm{d}^{2}$ ( solid line), $\sigma=0.25$ chains $/ \mathrm{d}^{2}$ (dashed line), and $\sigma=0.65$ chains $/ \mathrm{d}^{2}($ dotted line $)$, and total system volume fraction of $\eta=0.1$. All data is for particle diameter $D=5 d$, average graft length $N_{g, a v g}=20$, and monodisperse matrix length $\mathrm{N}_{\text {matrix }}=40$. 115

Figure 4.3: Penetration depth (in units of $\mathrm{d}$ ) of the matrix chains into the grafted layer of nanoparticles $(\mathrm{D}=5 \mathrm{~d})$ with polydisperse chains $\left(\mathrm{N}_{\mathrm{g}, \mathrm{avg}}=20\right)$ at $\boldsymbol{\sigma}=\mathbf{0 . 1 0}$ chains $/ \mathbf{d}^{\mathbf{2}}$ (circles) and $\boldsymbol{\sigma}=$ $\mathbf{0 . 2 5}$ chains/ $\mathbf{d}^{\mathbf{2}}$ (triangles) in a dense solution $(\boldsymbol{\eta}=\mathbf{0 . 1})$ with $\mathbf{N}_{\text {matrix }}=\mathbf{1 0}$ (a) and $\mathbf{N}_{\text {matrix }}=\mathbf{4 0}$ (b) 117

Figure 4.4: PMF (in units of kT) versus inter-particle distance, r-D (in units of d), between grafted nanoparticles $(\mathrm{D}=5 \mathrm{~d})$ at $\sigma=0.65(\mathrm{a}), 0.25(\mathrm{~b})$, and 0.10 chains $/ \mathrm{d}^{2}$ and $\mathrm{PDI}=1.0$ (circles), 1.5 (squares), 2.0 (upward facing triangles), and 2.5 (downward facing triangles) with $\mathrm{N}_{\mathrm{g}, \mathrm{avg}}=20$, in a melt-like $(\eta=0.3)$ matrix of monodisperse homopolymer chains with $\mathrm{N}_{\text {matrix }}=10$ (solid symbols) and $\mathrm{N}_{\text {matrix }}=40$ (open symbols). The insets have the same axes labels as the main plots. 119 
Figure 4.5: Second virial coefficient for polymer grafted nanoparticles in a polymer matrix as a function of graft polydispersity (PDI) at grafting densities of $\sigma=0.10$ chains $/ \mathrm{d}^{2}$ ( (solid line), $\sigma=0.25$ chains $/ \mathrm{d}^{2}$ (dashed line), and $\sigma=0.65$ chains $/ \mathrm{d}^{2}$ (dotted line), and total system volume fraction of $\eta=0.3$. All data is for particle diameter $D=5 d$, average graft length $\mathrm{Ng}, \mathrm{avg}=20$, and monodisperse matrix length Nmatrix $=40$. 121

Figure 4.6: Potentials of mean force, PMF (in units of kT) versus inter-particle distance, r-D (in units of $\mathbf{d}$ ) between nanoparticles of size $\mathbf{D}=\mathbf{8 d}$ grafted with polydisperse chains at $\mathrm{PDI}=1.0$ (circles), PDI=1.5 (squares), PDI=2.0 (upward facing triangles), and PDI=2.5 (downward facing triangles) with $\mathrm{N}_{\mathrm{g}, \mathrm{av}}=20$ at a grafting density of $\sigma=0.1$ chains $/ \mathrm{d}^{2}$ (parts a and b) and 0.25 chains $/ \mathrm{d}^{2}$ (part c) in a dense solution $(\boldsymbol{\eta}=\mathbf{0 . 1}$, part a and c) and melt $(\boldsymbol{\eta}=\mathbf{0 . 3}$, part b) of monodisperse homopolymers with $\mathbf{N}_{\text {matrix }}=\mathbf{4 0}$. The insets have the same axes labels as the main plots. 122

Figure 4.7: Potentials of mean force, PMF (in units of kT) versus inter-particle distance, r-5 (in units of $\mathrm{d}$ ) between nanoparticles of size $\mathrm{D}=8 \mathrm{~d}$ (open symbol) and $\mathrm{D}=5 \mathrm{~d}$ (solid symbols) grafted with polydisperse chains at $\mathrm{PDI}=1.0$ (a), $\mathrm{PDI}=1.5$ (b), and $\mathrm{PDI}=2.0$ (c)with $\mathrm{N}_{\mathrm{g}, \mathrm{avg}}=20$ at a grafting density of $\boldsymbol{\sigma}=\mathbf{0 . 2 5}$ chains $/ \mathrm{d}^{2}$, in a dense solution $(\boldsymbol{\eta}=\mathbf{0 . 1})$ of monodisperse homopolymers with $\mathbf{N}_{\text {matrix }}=\mathbf{4 0}$. The insets have the same axes labels as the main plots.

Figure 4.8: Potentials of mean force between nanoparticles of size $\mathrm{D}=5 \mathrm{~d}$ with polydisperse chains of PDI=1.0 (circles) and PDI=2.0 (other symbols) with $\mathrm{N}_{\mathrm{g}, \mathrm{av}}=20$ and $\mathrm{N}_{\mathrm{g}, \mathrm{min}}=8$ at a grafting density of $\sigma=0.65 \mathrm{ch} / \mathrm{nm}^{2}$, for five different log-normal distributions, in a) a dense solution $(\eta=0.1)$ and b) melt $(\eta=0.3)$ of monodisperse homopolymers with $\mathrm{N}_{\text {matrix }}=40$. Each symbol (besides circle) corresponds to a different log-normal chain distribution. 126

Figure 4.9: PMF (in units of kT) versus inter-particle distance, r-D (in units of d), between grafted nanoparticles (D=5d) with monodisperse homopolymer grafts with $\mathrm{PDI}=1.0$ (solid line), polydisperse homopolymer grafts PDI=1.5 (solid circle), and bidisperse homopolymer grafts PDI=1.5 (open circle), all with $\mathrm{N}_{\mathrm{g}, \mathrm{avg}}=20$ and grafting density $\sigma=0.65$ (left), and 0.25 (right) chains $/ \mathrm{d}^{2}$ in a in a dense solution $(\eta=0.1)$ (top row) or melt-like $(\eta=0.3)$ (bottom row) monodisperse homopolymer matrix with $\mathbf{N}_{\text {matrix }}=\mathbf{4 0}$. The insets have the same axes labels as the main plots. 128 
Figure 4.10: Chain length distributions of polydisperse chains grafted on nanoparticles $(\mathrm{D}=5 \mathrm{~d})$ at $\sigma=0.1$ chains $/ \mathrm{d}^{2}(\mathrm{a}), \sigma=0.25$ chains $/ \mathrm{d}^{2}(\mathrm{~b}), \quad \sigma=0.65$ chains $/ \mathrm{d}^{2}(\mathrm{c})$, and $\mathrm{PDI}=1.5$ (squares), 2.0 (upward facing triangles), and 2.5 (downward facing triangles) with $\mathrm{N}_{\mathrm{g}, \mathrm{avg}}=20$ and $\mathrm{N}_{\mathrm{g}, \min }=8 \ldots 138$

Figure 4.11: Total monomer concentration profiles (in units of $\mathrm{d}^{-3}$ ) versus distance from particle surface, $r-(D+d) / 2$ (in units of $d$ ), between grafted (filled symbols) or matrix chains (open symbols) and grafted nanoparticles $(\mathrm{D}=5 \mathrm{~d})$ at $\sigma=0.65,0.25,0.10$ chains $/ \mathrm{d}^{2}$ and $\mathrm{PDI}=1.0$ (circles), 1.5 (squares), 2.0 (upward facing triangles), and 2.5 (downward facing triangles) with $\mathrm{N}_{\mathrm{g}, \mathrm{avg}}=20$, in a dense solution $(\eta=0.1)$ of monodisperse homopolymer matrix chains with $\mathrm{N}_{\text {matrix }}=40$ (top) and $\mathrm{N}_{\text {matrix }}=10$ (bottom). 139

Figure 4.12: Average radius of gyration of the monodisperse matrix chains as a function of distance of center of mass of matrix chain from particle surface, $r-(D+d) / 2$ (in units of d) for grafted monomers at varying polydispersity of grafted chains on nanoparticles have diameter $\mathrm{D}=5 \mathrm{~d}$ at grafting density $\sigma=0.65$ chains $/ \mathrm{d}^{2}$ in a dense solution $(\eta=0.1)$ of monodisperse homopolymer matrix chains with $\mathrm{N}_{\text {matrix }}=40$ (a) or $\mathrm{N}_{\text {matrix }}=10$ (b). For both plots, the symbols correspond to the polydispersity of the grafted chains as: PDI = 1.0 (circles), 1.5 (squares), 2.0 (upward triangles), and 2.5 (downward triangles). The solid symbols represent the mean value while the open symbols represent the upper and lower limits of the error bar. 140

Figure 4.13: End monomer concentration profiles (in units of $\mathrm{d}^{-3}$ ) versus distance from particle surface, $r-(D+d) / 2$ (in units of $d$ ), between grafted (filled symbols) or matrix chains (open symbols) and grafted nanoparticles $(\mathrm{D}=5 \mathrm{~d})$ at $\sigma=0.65,0.25,0.10$ chains $/ \mathrm{d}^{2}$ and $\mathrm{PDI}=1.0$ (circles), 1.5 (squares), 2.0 (upward facing triangles), and 2.5 (downward facing triangles) with $\mathrm{N}_{\mathrm{g}, \mathrm{avg}}=20$, in a dense solution $(\eta=0.1)$ of monodisperse homopolymer matrix chains with $N_{\text {matrix }}=40$ (top) and $\mathrm{N}_{\text {matrix }}=10$ (bottom).

Figure 4.14: PMF (in units of $k T$ ) versus inter-particle distance, $r-D$ (in units of $d$ ), between grafted nanoparticles $(D=5 d)$ at $\sigma=0.65$ chains $/ d^{2}$ and $P D I=1-1.5$ with $N_{g, a v g}=20$, in a dense solution $(\eta=0.1)$ of monodisperse homopolymer matrix chains with and $N_{\text {matrix }}=40$.

Figure 4.15: PMF (in units of kT) versus inter-particle distance, r-D (in units of d), between grafted nanoparticles $(D=5 d)$ at $\sigma=0.65$ chains $/ d^{2}$ in a polymer solution $(\eta=0.1)$ (top row) or melt-like 
$(\eta=0.3)$ (bottom row) matrix of monodisperse homopolymer chains. Parts a and $b$ are for monodisperse grafts of length 20 and monodisperse homopolymer matrix of $\mathrm{N}_{\text {matrix }}=10$ (triangles), $\mathrm{N}_{\text {matrix }}=40$ (circles), $\mathrm{N}_{\text {matrix }}=80$ (squares). Parts $\mathrm{c}$ and $\mathrm{d}$ are for polydisperse grafts with PDI=2.0 and monodisperse homopolymer matrix with $\mathrm{N}_{\text {matrix }}=10$ (solid circles), 40 (squares), 100 (upward triangles), 200 (downward triangles), 300 (open circles). The insets have the same axes labels as the main plots.

Figure 4.16: Penetration depth (in units of $\mathrm{d}$ ) of the matrix chains into the grafted layer of nanoparticles $(D=5 d)$ with polydisperse chains $\left(\mathrm{N}_{\mathrm{g}, \mathrm{avg}}=20\right)$ at $\sigma=0.10$ chains $/ \mathrm{d}^{2}$ (circles) and $\sigma=$ 0.25 chains $/ \mathrm{d}^{2}$ (triangles) in a dense solution $\left(\eta=0.3\right.$ ) with (a) $\mathrm{N}_{\text {matrix }}=10$ and (b) $\mathrm{N}_{\text {matrix }}=40 \ldots 146$

Figure 4.17: (left) PMF (in units of kT) versus inter-particle distance, r-D (in units of d) and graft monomer concentration profile (right) for grafted nanoparticles $(\mathbf{D = 5} \mathbf{d})$ with monodisperse homopolymer grafts with $\mathrm{PDI}=1.0$ (blue line), polydisperse homopolymer grafts PDI=1.5 (maroon line), and bidisperse homopolymer grafts PDI=1.5 (green line), all with $\mathrm{N}_{\mathrm{g}, \mathrm{avg}}=20$ and grafting density $\sigma=0.65$ chains $/ \mathrm{d}^{2}$ in a in a dense solution $(\eta=0.1)$ of monodisperse homopolymer matrix with $\mathbf{N}_{\text {matrix }}=\mathbf{4 0}$. The insets have the same axes labels as the main plots. 147

Figure 5.1: (a) Potentials of mean force (PMF), in units of $\mathrm{k}_{\mathrm{B}} \mathrm{T}$, versus interparticle distance, in units of $d$, and b) second virial coefficient, $B_{2}$, versus graft length PDI between nanoparticles of diameter $\mathrm{D}=5 \mathrm{~d}$ grafted with homopolymers with monodisperse or polydisperse log-normal length distribution $\left(\mathrm{N}_{\text {graft,avg }}=20\right)$ at $\sigma=0.65$ chains $/ \mathrm{d}^{2}$ in a homopolymer matrix $\left(\mathrm{N}_{\text {matrix }}=40\right)$. In subplot a, the symbols indicate the graft PDI=1.0 (solid) or 2.0 (open) and the particle-particle interactions $\varepsilon_{\mathrm{PP}}=0$ (black circles) or $\varepsilon_{\mathrm{PP}}=5.0 \mathrm{k}_{\mathrm{B}} \mathrm{T}$, (red triangles). The main figure shows the PMF at short interparticle distances, and inset shows the PMF at larger interparticle distances, and presence/absence of the mid-range attractive well. In subplot $\mathbf{b}$, symbols indicate $\varepsilon_{\mathrm{PP}}=0$ (black leftward triangles) and $\varepsilon_{\mathrm{PP}}=5.0 \mathrm{kB}$ (red squares). 155

Figure 5.2: (a) PMF, in units of $\mathrm{k}_{\mathrm{B}} \mathrm{T}$, versus interparticle distance, in units of $\mathrm{d}$, (b) total monomer concentration profile, $\mathrm{r}^{2} \mathrm{C}(\mathrm{r})$ versus bead-particle distance and (c) second virial coefficient, $\mathrm{B}_{2}$, versus PDI between nanoparticles of diameter $\mathrm{D}=5 \mathrm{~d}$ with particle-particle interactions at $\varepsilon_{\mathrm{PP}}=$ $5 \mathrm{k}_{\mathrm{B}} \mathrm{T}$ in a homopolymer matrix of length $\mathrm{N}_{\text {matrix }}=40$. The symbols in subplots a and $\mathbf{b}$ represent $\sigma=0.65$ chains $/ \mathrm{d}^{2}$ (1) log-normal length distributions with $\mathrm{PDI}=1.25, \mathrm{~N}_{\text {graft,avg }}=20$ (black solid 
circles) and (2) log-normal length distributions with $\mathrm{PDI}=1.33, \mathrm{~N}_{\text {graft,avg }}=14$ (black open circles), (3) bidisperse length distribution with $\sigma_{\mathrm{s}}=0.33, \sigma_{\mathrm{l}}=0.31, \beta=3, \mathrm{PDI}=1.25$, and $\mathrm{N}_{\text {graft,avg }}=20$ (blue solid triangles) and (4) bidisperse length distribution with $\sigma_{\mathrm{s}}=0.52, \sigma_{1}=0.12, \beta=3, \mathrm{PDI}=1.33$ and $\mathrm{N}_{\text {graft,avg }}=14$ (blue open triangles). The corresponding results at $\varepsilon_{\mathrm{PP}}=0 \mathrm{k}_{\mathrm{B}} \mathrm{T}$ are shown in the Supporting Information. In subplot $\mathbf{c}$ the open and closed squares represent the bidisperse distribution with $\sigma_{\mathrm{s}}>\sigma_{1}$ and $\sigma \approx \sigma_{\mathrm{s}}$ respectively while the open and closed circles represent $\log$ normal distributions, and both the open squares and circles have $\mathrm{N}_{\text {graft,avg }}=18,14,11$ from left to right. 158

Figure 5.3: PMF, in units of $\mathrm{k}_{\mathrm{B}} \mathrm{T}$, versus interparticle distance, in units of $\mathrm{d}$, between nanoparticles of diameter $\mathrm{D}=5 \mathrm{~d}$ with particle-particle interactions $\varepsilon_{\mathrm{PP}}=0$ (a) and $\varepsilon_{\mathrm{PP}}=5.0 \mathrm{k}_{\mathrm{B}} \mathrm{T}$ (b) for five different grafting systems: (1) bidisperse, $\mathrm{N}_{\text {graft,avg }}=20, \beta=3, \sigma_{\mathrm{l}}=0.31, \sigma_{\mathrm{s}}=0.33$ chains $/ \mathrm{d}^{2}$ (2) monodisperse, $\mathrm{N}_{\text {graft }}=10, \sigma=0.65$ chains $/ \mathrm{d}^{2}$ (3) monodisperse, $\mathrm{N}_{\text {graft }}=30, \sigma=0.65$ chains $/ \mathrm{d}^{2}$ (4) monodisperse, $\mathrm{N}_{\text {graft }}=10, \sigma=0.33$ chains $/ \mathrm{d}^{2}(5)$ monodisperse, $\mathrm{N}_{\text {graft }}=30, \sigma=0.31$ chains $/ \mathrm{d}^{2}$, in a homopolymer matrix of length $\mathrm{N}_{\text {matrix }}=40$. The main figure shows the PMF for short interparticle distances, while the inset shows the PMF at larger interparticle distances. 161

Figure 5.4: Monomer concentration profiles with the $\mathrm{r}^{2}$ prefactor, in units of $\mathrm{d}^{-1}$, versus beadparticle distance, in units of $\mathrm{d}$, for nanoparticles of diameter $\mathrm{D}=5 \mathrm{~d}$ with $\varepsilon_{\mathrm{PP}}=0$ (a,c,e) and $\varepsilon_{\mathrm{PP}}=5.0$ $\mathrm{k}_{\mathrm{B}} \mathrm{T}(\mathrm{b}, \mathrm{d}, \mathrm{f})$ particle-particle interactions grafted with homopolymers of $\mathrm{N}_{\text {graft,avg }}=20$, in a homopolymer matrix of length $\mathrm{N}_{\text {matrix }}=40$. The total system volume fraction is $\eta=0.1$ and the grafted nanoparticle filler fraction is kept at the dilute limit of $\varphi=0.001$. Subplots $\mathbf{a}$ and $\mathbf{b}$ show the total concentration profiles for all five systems. Subplots $\mathbf{c}$ and $\mathbf{d}$ show the concentration profiles of only the short chains $\left(\mathrm{N}_{\text {short }}=10\right)$ of system 1 (black circles), $1 / 2$ the concentration of all of the short chains of system 2 (red upward triangles), all of the (short) chains of system 4 (blue downward triangles). Subplots $\mathbf{e}$ and $\mathbf{f}$ show the concentration profiles for the long chains $\left(\mathrm{N}_{\text {long }}=30\right)$ of system 1 (black circles), $1 / 2$ the concentration of all of the long chains of system 3 (green leftward triangles), all of the (long) chains of system 5 (pink right triangles). 163

Figure 5.5: Colloid Lennard Jones potential, in units of $\mathrm{k}_{\mathrm{B}} \mathrm{T}$, versus interparticle distance, in units of $\mathrm{d}$, between particles of diameter $\mathrm{D}=5 \mathrm{~d}$ with well depths $\varepsilon_{\mathrm{PP}}=0.1$ (black circles), 1.0 (red upward 
triangles), 5.0 (green leftward triangles), 10.0 (blue downward triangles). The potential goes to infinity for $\mathrm{r}-\mathrm{D}<0$. See reference below for details on the Colloid Lennard Jones potential:... 171

Figure 5.6: Schematic of terminology used to describe various features of the potential of mean force (PMF) between homopolymer grafted nanoparticles with arbitrary particle-particle attraction in a chemically similar homopolymer matrix (not shown in diagrams). 172

Figure 5.7: (a) Potentials of mean force (PMF), in units of $\mathrm{k}_{\mathrm{B}} \mathrm{T}$, versus interparticle distance, in units of $\mathrm{d}$, and (b) root mean normalized square radius of gyration of grafted chains, $\left\langle 6 \mathrm{Rg}^{2} / \mathrm{N}\right\rangle^{0.5}$, in units of $d$, versus graft length PDI for nanoparticles of diameter $D=5 d$ grafted with homopolymers with log-normally distributed lengths at average length $\mathrm{N}_{\text {graft,avg }}=20$ at a grafting density of $\sigma=0.65$ chains $/ \mathrm{d}^{2}$ in a homopolymer matrix of length $\mathrm{N}_{\text {matrix }}=40$. The total system volume fraction is $\eta=0.1$ and the grafted nanoparticle filler fraction is kept at the dilute limit of $\varphi=0.001$. The error bars in subplot $\mathrm{b}$ represent standards error. The colors and symbols indicate the strength of particle-particle attraction as athermal $\varepsilon_{\mathrm{PP}}=0 \mathrm{k}_{\mathrm{B}} \mathrm{T}$ (black line, no symbols), $\varepsilon_{\mathrm{PP}}$ $=1 \mathrm{k}_{\mathrm{B}} \mathrm{T}$ (red circles), $\varepsilon_{\mathrm{PP}}=2 \mathrm{k}_{\mathrm{B}} \mathrm{T}$ (green upward triangles), $\varepsilon_{\mathrm{PP}}=3 \mathrm{k}_{\mathrm{B}} \mathrm{T}$ (blue leftward triangles), $\varepsilon_{\mathrm{PP}}$ $=4 \mathrm{k}_{\mathrm{B}} \mathrm{T}$ (pink downward triangles) and $\varepsilon_{\mathrm{PP}}=5 \mathrm{k}_{\mathrm{B}} \mathrm{T}$ (orange rightward triangles). 173

Figure 5.8: (a) PMF, in units of $k_{B} T$, versus interparticle distance, in units of $d$, and (b) second virial coefficient, $B_{2}$, versus PDI between nanoparticles of diameter $\mathrm{D}=5 \mathrm{~d}$ with particle-particle interactions at $\varepsilon_{\mathrm{PP}}=0 \mathrm{k}_{\mathrm{B}} \mathrm{T}$ in a homopolymer matrix of length $\mathrm{N}_{\text {matrix }}=40$. The symbols in subplot a represent at $\sigma=0.65$ chains $/ \mathrm{d}^{2}$ (1) log-normal length distributions with $\mathrm{PDI}=1.25, \mathrm{~N}_{\text {graft,avg }=20}$

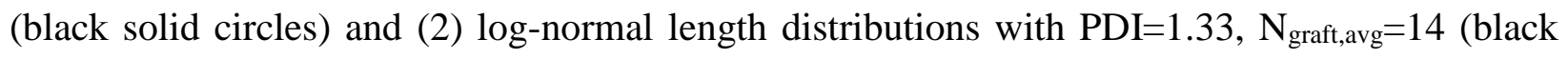
open circles), (3) bidisperse length distribution with $\sigma_{\mathrm{s}}=0.33, \sigma_{\mathrm{l}}=0.31, \beta=3, \mathrm{PDI}=1.25$, and $\mathrm{N}_{\text {graft,avg }}=20$ (blue solid triangles) and (4) bidisperse length distribution with $\sigma_{\mathrm{s}}=0.52, \sigma_{\mathrm{l}}=0.12, \beta=3$, $\mathrm{PDI}=1.33$ and $\mathrm{N}_{\text {graft,avg }}=14$ (blue closed triangles). The corresponding results at $\varepsilon_{\mathrm{PP}}=0 \mathrm{k}_{\mathrm{B}} \mathrm{T}$ are shown in the Supplementary Information. In subplot b the open and closed squares represent the bidisperse distribution with $\sigma_{s}>\sigma_{1}$ and $\sigma \approx \sigma_{s}$ respectively while the open and closed circles represents $\log$ normal distributions, and both the open squares and circles have $\mathrm{N}_{\text {graft,avg }}=18,14,11$ from left to right 174

Figure 5.9:Monomer concentration profiles both without (a, c units of $\left.\mathrm{d}^{-3}\right)$ and with $(\mathrm{b}, \mathrm{d}$ units of $\mathrm{d}^{-1}$ ) the $\mathrm{r}^{2}$ prefactor, versus bead-particle distance, in units of $\mathrm{d}$, for nanoparticles of diameter $\mathrm{D}=5 \mathrm{~d}$ 
with $\varepsilon_{\mathrm{PP}}=0(\mathrm{a}, \mathrm{b})$ and $\varepsilon_{\mathrm{PP}}=5.0 \mathrm{k}_{\mathrm{B}} \mathrm{T},(\mathrm{c}, \mathrm{d})$ particle-particle interactions grafted with homopolymers $\mathrm{N}_{\text {graft,avg }}=20$ at a total grafting density of $\sigma=0.65$ chains $/ \mathrm{d}^{2}$, in a homopolymer matrix of length $\mathrm{N}_{\text {matrix }}=40$ for four graft length distributions: 175

Figure 5.10: Potentials of mean force (PMF), in units of $\mathrm{k}_{\mathrm{B}} \mathrm{T}$, versus interparticle distance, in units of $\mathrm{d}$, between nanoparticles of diameter $\mathrm{D}=5 \mathrm{~d}$ with $\varepsilon_{\mathrm{PP}}=0$ (black circles) and $\varepsilon_{\mathrm{PP}}=5.0 \mathrm{k} \mathrm{T}$, (red triangles) particle-particle interactions grafted with homopolymers with a log-normal length distribution at average length $\mathrm{N}_{\text {graft,avg }}=20$ at a total grafting density of $\sigma=0.65$ chains $/ \mathrm{d}^{2}$ in a homopolymer matrix of length $\mathrm{N}_{\text {matrix }}=40$. The total system volume fraction is $\eta=0.1$ and the grafted nanoparticle filler fraction is kept at the dilute limit of $\varphi=0.001$. The closed symbols-solid lines represent a bidisperse system with $\beta=3$ and $N_{\text {graft,avg }}=20$ (system 1 ). The open symbols-dashed lines are the sum of the corresponding PMFs from systems 4 and 5. 176

Figure 6.1: A representative simulation snapshot of a polymer nanocomposite with polymer grafted particles of diameter $\mathrm{D}=5 \mathrm{~d}$ grafted with homopolymers (blue chains) with a monodisperse length distribution $\left(\mathrm{N}_{\text {graft }}=20\right)$ at grafting density 0.65 chains $/ \mathrm{d}^{2}$ and filler fraction $\phi=0.3$ in a bidisperse homopolymer matrix of lengths 20 (red chains) and 60 (green chains). The matrix chains are removed in the bottom image to show the grafted particles only. While the red, blue and green colors are used to distinguish the grafts, the short and long matrix chains in the snapshots, the grafts and all matrix polymers are of the same chemistry and modeled using athermal interactions.

Figure 6.2: Particle-monomer pair correlation functions $g_{i j}(r)$ versus particle-monomer distance, $r$ $(\mathrm{D}+\mathrm{d}) / 2$, in units of $\mathrm{d}$, for particles of diameter $\mathrm{D}=5 \mathrm{~d}$ grafted with homopolymers with a monodisperse length distribution $\left(\mathrm{N}_{\text {graft }}=20\right)$ at 0.65 chains $/ \mathrm{d}^{2}$ and filler fraction $\phi=0.3$ in a bidisperse or monodisperse homopolymer matrix. In subplots a and $b$ the symbols indicate the pair correlation between the particles and the monomers of a monodisperse matrix of $\mathrm{N}_{\text {matrix }}=30$, $\beta_{\text {matrix }}=1.0$ (solid black rightward triangles) or $\mathrm{N}_{\text {matrix }}=60, \beta_{\text {matrix }}=1.0$ (solid black leftward triangles), or the monomers belonging to the short (open red upward triangles, $\mathrm{N}_{\text {short }}=30$ ) or long (open green downward triangles, $\mathrm{N}_{\text {long }}=60$ ) chains of a bidisperse matrix with $\beta_{\text {matrix }}=3$ and $\mathrm{N}_{\text {matrix,avg }}=45$. In subplots $\mathrm{c}$ and $\mathrm{d}$, the symbols indicate the pair correlation between the particles and the monomers belonging to short (subplot c) or long (subplot d) chains of a bidisperse matrix 
with $\mathrm{N}_{\text {matrix,avg }}=40$ and $\beta_{\text {matrix }}=1.5$ (black squares), 3.0(blue leftward triangles), or 7.0 (pink rightward triangles).

Figure 6.3: Average per-monomer entropy of wetting versus $\beta_{\text {matrix }}$ for particles of diameter $D=5 d$ grafted with homopolymers with monodisperse length distribution $\left(\mathrm{N}_{\text {graft }}=20\right)$ at 0.65 chains $/ \mathrm{d}^{2}$ and filler fraction $\phi=0.3$ in a monodisperse or bidisperse matrix with $\mathrm{N}_{\text {matrix,avg }}=40$. The symbols correspond to the entropy contribution from the short (red upward triangles) or long (green downward triangles) matrix chains. The horizontal dashed lines correspond to the entropy of wetting calculated for monodisperse matrices. The numbers next to the symbols/lines indicate the matrix length for that datum.

Figure 6.4: Particle-particle (a) and particle-total matrix (b) pair correlation functions versus particle-particle and particle-monomer distance, in units of $d$ for nanoparticles of diameter $D=5 d$ grafted with homopolymers with a monodisperse or bidisperse graft length distribution at filler fraction $\phi=0.3$ in a bidisperse or monodisperse homopolymer matrix. The symbols correspond to: $\beta_{\text {graft }}=1.0, \beta_{\text {matrix }}=1.0, \mathrm{~N}_{\text {graft }}=20, \mathrm{~N}_{\text {matrix }}=40$ (black circles), $\beta_{\text {graft }}=1.0, \quad \beta_{\text {matrix }}=3.0, \mathrm{~N}_{\text {graft }}=20$, $\mathrm{N}_{\text {matrix,avg }}=40$ (red upward triangles), $\beta_{\text {graft }}=1.0, \beta_{\text {matrix }}=1.0, \mathrm{~N}_{\text {graft }}=20, \mathrm{~N}_{\text {matrix }}=60$ (green downward triangles), $\beta_{\text {graft }}=3.0, \beta_{\text {matrix }}=1.0, \mathrm{~N}_{\text {graft,avg }}=20, \mathrm{~N}_{\text {matrix }}=40$ (blue leftward triangles).

Figure 6.5: Mean-square internal distances versus number of connecting bonds, for a simulation for the long matrix chains a system of grafted nanoparticles of diameter $D=5 d$ grafted with homopolymers with a monodisperse length distribution $\left(\mathrm{N}_{\text {graft }}=20\right)$ at $\sigma=0.65$ chains $/ \mathrm{d}^{2}$ and filler fraction $\phi=0.3$ in a bidisperse matrix of $\mathrm{N}_{\text {matrix,short }}=20, \mathrm{~N}_{\text {matrix,long }}=60$. Details of this calculation can be found elsewhere[18] 198

Figure 6.6: Particle-short chain monomer (a) and Particle-long chain monomer (b) and particleparticle (c) pair correlation functions versus particle-monomer distance, in units of d, for nanoparticles of diameter $\mathrm{D}=5 \mathrm{~d}$ grafted with homopolymers with a monodisperse length distribution $\left(\mathrm{N}_{\text {graft }}=20\right)$ at $\sigma=0.65$ chains $/ \mathrm{d}^{2}$ and filler fraction of $\phi=0.3$ in a bidisperse homopolymer matrix with $\beta_{\text {matrix }}=3.0, \mathrm{~N}_{\text {matrix,avg }}=40$. The colors in the plot correspond to the five independent, randomly initialized trials in the simulation 199 
Figure 6.7: Simulation snapshots of PNCs with polymer grafted particles of diameter $\mathrm{D}=5 \mathrm{~d}$ grafted with homopolymers (blue chains) with a monodisperse length distribution $\left(\mathrm{N}_{\text {graft }}=20\right)$ at $\sigma=0.65$ chains $/ \mathrm{d}^{2}$ and filler fraction $\phi=0.1$ in a bidisperse matrix of lengths 20 (red chains) and 60 (green chains). While colors are used to distinguish the various chains in the snapshots, all interactions used in this study are athermal. Individually, the snapshots show all components of the simulation (a), only the short matrix chains (b), only the long matrix chains (c), only the grafted particles (d),

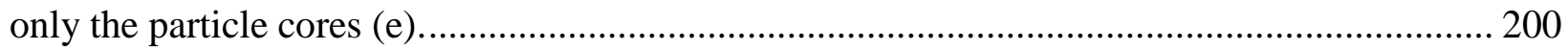

Figure 6.8: Simulation snapshots of polymer grafted particles of diameter $D=5 \mathrm{~d}$ grafted with homopolymers (blue chains) with a monodisperse and bidisperse graft length distribution $\left(\mathrm{N}_{\text {graft,avg }}=20\right)$ at $\sigma=0.65$ chains $/ \mathrm{d}^{2}$ and filler fraction $\phi=0.02$ in monodisperse (orange) or bidisperse (green,red) matrix. The snapshots correspond to $\mathrm{N}_{\text {graft }}=20, \mathrm{~N}_{\text {matrix }}=40, \beta_{\text {graft }}=1.0$, $\beta_{\text {matrix }}=1.0$ (a), $\mathrm{N}_{\text {graft }}=20, \mathrm{~N}_{\text {matrix }, \text { avg }}=40, \beta_{\text {graft }}=1.0, \beta_{\text {matrix }}=3.0(\mathrm{~b}), \mathrm{N}_{\text {graft,avg }}=20, \mathrm{~N}_{\text {matrix }}=40, \beta_{\text {graft }}=3.0$, $\beta_{\text {matrix }}=1.0$ (c), The snapshots show the top down view of cylindrical slice of matrix of depth $10 \sigma$ and diameter $30 \sigma$, centered around each grafted particle 201

Figure 6.9: Pair correlation functions (a-b,d-e) and ratios of pair correlation functions (c,f) versus monomer-monomer distance, in units of d (size of a Kuhn segment), for nanoparticles of diameter $\mathrm{D}=5 \mathrm{~d}$ grafted with homopolymers with a monodisperse length distribution $\left(\mathrm{N}_{\text {graft }}=20\right)$ at $\sigma=0.65$ chains $/ \mathrm{d}^{2}$ and filler fraction $\varphi=0.1$ in a bidisperse homopolymer matrix calculated from both liquid state theory (a-c) and molecular dynamics (d-f). For subplots a-b \& d-e, the upward triangles represent the graft bead - short matrix bead pair correlation functions, while the downward triangles are the graft bead - long matrix bead pair correlation functions for $\beta_{\text {matrix }}=1.5(\mathrm{a}, \mathrm{d})$ and $3.0(\mathrm{~b}, \mathrm{e})$. In subplot c,f, the ratio of the graft bead - short matrix bead to the graft bead - long matrix bead pair correlation functions are shown for $\beta_{\text {matrix }}=1.5$ (circles), 3.0, (upward triangles).

Figure 6.10: Particle-monomer pair correlation functions versus particle-monomer distance, in units of $\mathrm{d}$, for nanoparticles of diameter $\mathrm{D}=5 \mathrm{~d}$ grafted with homopolymers with a monodisperse length distribution $\left(\mathrm{N}_{\text {graft }}=20\right)$ at $\sigma=0.65$ chains $/ \mathrm{d}^{2}$ and filler fraction of $\phi=0.02(\mathrm{a}), 0.1$ (b), or 0.3(c) in a bidisperse or monodisperse homopolymer matrix. The symbols indicate the pair correlation between the particles and the monomers of a monodisperse matrix of $\mathrm{N}_{\text {matrix }}=40$, 
$\beta_{\text {matrix }}=1.0$ (black rightward triangles), or the monomers belonging to the short (red upward triangles, $\mathrm{N}_{\text {short }}=20$ ) or long (green downward triangles, $\mathrm{N}_{\text {long }}=60$ ) chains of a bidisperse matrix with $\beta_{\text {matrix }}=3$ and $\mathrm{N}_{\text {matrix,avg }}=40$.

Figure 6.11: Root-mean-square brush height, in units of d (size of Kuhn segment), versus $\beta_{\text {matrix }}$ for nanoparticles of diameter $\mathrm{D}=5 \mathrm{~d}$ grafted with homopolymers with monodisperse length distribution $\left(\mathrm{N}_{\text {graft }}=20\right)$ at $\sigma=0.65$ chains $/ \mathrm{d}^{2}$ and filler fractions $\varphi=0.02$ (square), 0.1 (leftward triangle), and 0.3 (rightward triangle) in a bidisperse matrix with $\mathrm{N}_{\text {matrix,avg }}=40$. The standard error is approximately 0.5 for all data in this plot. 204

Figure 6.12: Particle-particle pair correlation functions (a) and potentials of mean force, in units of $\mathrm{k}_{\mathrm{B}} \mathrm{T}$ (b) versus particle-particle distance, in units of $\mathrm{d}$, for nanoparticles of diameter $\mathrm{D}=5 \mathrm{~d}$ grafted with homopolymers with a monodisperse or bidisperse graft length distribution at filler fraction $\phi=0.05$ (a) and $\phi=0.001$ (b) in a bidisperse or monodisperse homopolymer matrix. The symbols correspond to: $\beta_{\text {graft }}=1.0, \beta_{\text {matrix }}=1.0 \mathrm{~N}_{\text {graft }}=20, \mathrm{~N}_{\text {matrix }}=40$, (circles), $\beta_{\text {graft }}=1.0, \beta_{\text {matrix }}=3.0$, $\mathrm{N}_{\text {graft }}=20, \quad \mathrm{~N}_{\text {matrix,avg }}=40 \quad$ (upward triangles), $\quad \beta_{\text {graft }}=3.0, \quad \beta_{\text {matrix }}=1.0, \mathrm{~N}_{\text {graft,avg }}=20, \quad \mathrm{~N}_{\text {matrix }}=40$ (downward triangles). All of these data come from liquid-state-theory derived calculations.... 206

Figure 6.13: Particle-particle pair correlation functions versus particle-particle distance, in units of $\mathrm{d}$ for nanoparticles of diameter $\mathrm{D}=5 \mathrm{~d}$ grafted with homopolymers with a monodisperse or bidisperse graft length distribution at filler fraction $\phi=0.3$ in a bidisperse or monodisperse homopolymer matrix. The symbols correspond to: $\beta_{\text {graft }}=1.0, \beta_{\text {matrix }}=1.0, \mathrm{~N}_{\text {graft }}=20, \mathrm{~N}_{\text {matrix }}=40$ (black circles), $\beta_{\text {graft }}=1.0, \beta_{\text {matrix }}=1.5, \mathrm{~N}_{\text {graft }}=20, \mathrm{~N}_{\text {matrix }, \text { avg }}=40$ (red upward triangles), $\beta_{\text {graft }}=1.0$, $\beta_{\text {matrix }}=3.0, \mathrm{~N}_{\text {graft }}=20, \mathrm{~N}_{\text {matrix,avg }}=40$ (green downward triangles), and $\beta_{\text {graft }}=1.0, \beta_{\text {matrix }}=7.0$, $\mathrm{N}_{\text {graft }}=20, \mathrm{~N}_{\text {matrix,avg }}=40$ (blue leftward triangles). 207

Figure 7.1: Graft (solid) and matrix (dashed) monomer concentration profiles for a single PGN with grafting density $=0.65$ chains $/ \sigma^{2}, \mathrm{~N}_{\text {graft }}=20$, and $\mathrm{N}_{\text {matrix }}=60$, with $\mathrm{K}=\mathrm{K}_{\text {graft }}=\mathrm{K}_{\text {matrix }}=0$ (black), 5(red) and 10 (blue) $\mathrm{k}_{\mathrm{B}} \mathrm{T} /$ radians $^{2}$. The brush heights are shown as vertical dash-dotted lines.. 216

Figure 7.2: Graft (solid) and matrix (dashed) monomer concentration profile for single PGN with grafting density $=0.65$ chains $/ \sigma^{2}, \mathrm{~N}_{\text {graft }}=20, \mathrm{~N}_{\text {matrix }}=60$, and $\mathrm{K}_{\text {graft }}=0$ and $\mathrm{K}_{\text {matrix }}=0$ (red), $\mathrm{K}_{\text {graft }}=0$ and $\mathrm{K}_{\text {matrix }}=5$ (green), $\mathrm{K}_{\text {graft }}=5$ and $\mathrm{K}_{\text {matrix }}=0$ (blue), and $\mathrm{K}_{\text {graft }}=5$ and $\mathrm{K}_{\text {matrix }}=5$ (black)...... 218 
Figure 7.3: Gain in mixing entropy, $\mathrm{T} \Delta \mathrm{S}_{\mathrm{mix}}$ (in $\mathrm{k}_{\mathrm{B}} \mathrm{T}$ ) as a function of graft and matrix flexibility calculated a) using simulation trajectory information and b) estimated approximately with minimal information from simulation.

Figure 7.4: a) Normalized change in the $\%$ wet matrix with increasing grafting density for $\mathrm{N}_{\text {graft }}=20$, b) normalized change in the $\%$ wet matrix with increasing graft length from 10 to 20 and 10 to 40 beads for flexible polymers $(\mathrm{K}=0)$ at high grafting density $\left(0.65\right.$ chains $\left./ \sigma^{2}\right)$, and $\left.\mathrm{c}\right)$ normalized change in the \% wet matrix with increasing graft length from 10 to 20 and 10 to 40 beads for semi-flexible polymers $(K=5)$ at high grafting density $\left(0.65\right.$ chains $\left./ \sigma^{2}\right)$, for a single PGN with $\mathrm{N}_{\text {matrix }}=60$. The normalized change in the $\%$ wet matrix is calculated as $(\mathrm{Y}-\mathrm{X}) / \mathrm{X}$ where $\mathrm{Y}$ is the $\%$ wet matrix at the higher grafting density or higher graft length and $\mathrm{X}$ is the $\%$ wet matrix at the lower grafting density or lower graft length.

221

Figure 7.5: a) Graft (solid) and matrix (dashed) monomer concentration profile and b) Particleparticle pair correlation function plotted versus the interparticle distance minus the brush height for 20 PGNs with grafting density $=0.65$ chains $/ \sigma^{2}$, and $\mathrm{N}_{\text {graft }}=20$ and $\mathrm{N}_{\text {matrix }}=100$ and increasing values of $\mathrm{K}=0$ (black) and $5(\mathrm{red})$. 223

Figure 7.6: Graft (solid) and matrix (dashed) monomer concentration profile for single polymer grafted particle with particle diameter $5 \mathrm{~nm}$ for $\mathrm{K}_{\text {graft }}=\mathrm{K}_{\text {matrix }}=0$ (left column) and $\mathrm{K}_{\text {graft }}=\mathrm{K}_{\text {matrix }}$ $=5$ (right column) for varying polymer grafting density $=0.25$ (black) and $0.65\left(\right.$ red) chains $/ \sigma^{2}$ with $\mathrm{N}_{\text {graft }}=20$ and $\mathrm{N}_{\text {matrix }}=60$ (in subplots $\mathrm{a}$ and b), varying matrix length $=20$ (black), 40 (blue) and 60 (red) with $\mathrm{N}_{\text {graft }}=20$ and grafting density 0.65 chains $/ \sigma^{2}$ (in subplots $\mathrm{c}$ and $\mathrm{d}$ ) and varying graft length $=10$ (black) and 20 (red) with $\mathrm{N}_{\text {matrix }}=60$ and grafting density 0.65 chains $/ \sigma^{2}$ (in subplots e and f). The brush heights, $\left\langle\mathrm{H}_{\mathrm{B}}{ }^{2}\right\rangle^{0.5}$, are shown with dotted lines. The insets in subplots $\mathrm{c}$ ) and $\mathrm{d}$ ) have the same axes as the main figure.

Figure 8.1: Schematic representation of the equilibration protocol used in the Brownian Dynamics simulations 239

Figure 8.2: (a) Dispersion-aggregation phase-diagram, (b) simulation snapshots, with bottom panel hiding the matrix polymer, and (c) low-q value of the matrix-matrix structure factor for particles of diameter $\mathrm{D}=5 d$ grafted with " $\mathrm{G}$ " homopolymer chains of length $\mathrm{N}_{\text {graft }}=10$ and grafting density 
$\Sigma=0.76$ chains $/ \mathrm{d}^{2}$ in a "M" homopolymer matrix of length $\mathrm{N}_{\text {matrix }}=50$ at a total volume fraction $\eta=0.35$ and with blend composition $\phi G=0.13$, and 0.20 . The total volume fraction, $\eta$ is defined as ratio of volume of all CG beads to the volume of the simulation box. The blend composition, $\phi_{\mathrm{G}}$, is defined as ratio of volume of graft CG beads to the volume of graft and matrix CG beads in the system. In part a, upward and downward triangles denote aggregated and dispersed states, respectively, in the simulations, and the circles denote the dispersion-aggregation transition. The snapshots in part b are for $\phi G=0.13$ blends. The error bars in part $\mathrm{c}$ are standard deviations calculated from 50 uncorrelated configurations for each system; the error bars when not visible are smaller than the size of the symbol 243

Figure 8.3: a) Graft monomer (dashed lines) and matrix monomer (solid lines) concentration ( in units of $d-3$ ) versus distance from the particle surface at $\phi G=0.13$. The graft concentration profile is calculated for each grafted particle independently and the average of these profiles is presented. In the calculation of each grafted particle's profile, we only include the contribution of grafts that are attached to the same particle, and do not include any intergrafted-particle contributions. b) Wet monomer fraction versus reduced temperature, $\mathrm{T}^{*}$. Also shown are the dispersion-aggregation transition temperature (vertical dot-dashed line) and the wet monomer fraction for the athermal $\phi G=0.13$ and 0.20 blends (horizontal dashed and dotted lines). The error bars in parts $\mathrm{a}$ and $\mathrm{b}$ are standard deviations calculated from 50 uncorrelated configurations for each system; the error bars when not visible are smaller than the size of the symbol. 245

Figure 8.4: a) Temperature dependence of Small Angle X-ray Scattering (SAXS) intensity data for blends of a 33k dPS-grafted silica (grafting density of 0.7 chains $/ \mathrm{nm}^{2}$ ) with a $226 \mathrm{k}$ PVME at a blend composition of $80 \%$ PVME by volume; data for the scattering intensity from a pure $33 \mathrm{k}$ dPS-grafted silica particles with no PVME matrix are shown for comparison. b) Temperature dependence of the Small Angle Neutron Scattering (SANS) intensity for the blend described in (a). c) Ornstein-Zernike representation of selected SANS data to estimate the intensity in the forward angle (I(0)) and correlation length $(\xi)$ as a function of temperature for the single phase blends. d) Temperature dependence of $\mathrm{I}^{-1}(0)$ from SANS and primary scattering peak position $\left(\mathrm{q}^{*}\right)$ from SAXS for the blend described in (a) and (b). TEM images of thin sections of unstained blends 
at (e) $25{ }^{\circ} \mathrm{C}$ showing individually-dispersed nanoparticles and after annealing at (f) $160{ }^{\circ} \mathrm{C}$ showing large-scale phase separation 248

Figure 8.5: a) Mixed-demixed phase-diagram comparing the grafted or free blend, the wet monomer fraction vs temperature $(b, c)$, graft radius of gyration $(\mathrm{d}, \mathrm{e})$, and matrix radius of gyration (f,g) for particles of diameter $\mathrm{D}=5 d$ grafted with chains of length $\mathrm{N}_{\text {graft }}=10$ and grafting density $\Sigma=0.76$ chains $/ \mathrm{d}^{2}$ in a matrix of length $\mathrm{N}_{\text {matrix }}=50(\mathrm{a}, \mathrm{b}, \mathrm{d}, \mathrm{f})$ or blends of $\mathrm{N}_{\text {graft }}=10$ and $\mathrm{N}_{\text {matrix }}=$ 50 chains (a,c,e,g) at a total volume fraction $\eta=0.35$ and filler fractions $\phi G=0.13$ (triangles), and 0.20 (diamonds). The error bars in parts b-g are standard errors calculated from 50 uncorrelated configurations for each system; the error bars when not visible are smaller than the size of the symbol. h) Change in experimentally measured radius of gyration of the outer shell (called $\mathrm{R}_{\mathrm{g}}{ }^{\text {corona }}$ ) with temperature from an excluded volume model for blends of a 33k dPS-grafted silica (grafting density of 0.7 chains $/ \mathrm{nm}^{2}$ ) with a 226k PVME at a blend composition of $80 \%$ PVME by volume. We note that $\mathrm{R}_{\mathrm{g}}{ }^{\text {corona }}$ does not represent the overall dimension of the polymer chains but only the outer shell of the grafted chains. 252

Figure 8.6: Particle-particle structure factors versus reduced wavenumber for particles of diameter $\mathrm{D}=5 d$ grafted with chains of length $\mathrm{N}_{\text {graft }}=10$ and grafting density $\Sigma=0.76$ chains $/ \mathrm{d}^{2}$ in a matrix of length $\mathrm{N}_{\text {matrix }}=50$ at a total volume fraction $\eta=0.35$ and filler fractions $\phi G=0.05$ (a), 0.13(b), and 0.20(c) at reduced temperatures as indicated by the colors and symbols defined in the legend. 262

Figure 8.7: Matrix-Matrix structure factors versus reduced wavenumber for the systems described in Figure S1, at reduced temperatures as indicated by the colors and symbols defined in the legend 263

Figure 8.8: Graft-Graft structure factors for the systems described in Figure 8.6 reduced temperatures as indicated by the colors and symbols defined in the legend. 264

Figure 8.9: Particle-graft (dashed lines) and particle-matrix (solid lines) monomer concentration $(d-3)$ versus particle-monomer distance $(\mathrm{d})$ at reduced temperatures as indicated by the colors and symbols defined in the legend. The data in these plots are for particles of diameter $\mathrm{D}=5 \mathrm{~d}$ grafted with chains of length $\mathrm{N}_{\text {graft }}=10$ and grafting density $\Sigma=0.76$ chains $/ \mathrm{d}^{2}$ in a matrix of 
length $\mathrm{N}_{\text {matrix }}=50$ at a total volume fraction $\eta=0.35$ and filler fractions $\phi G=0.05$ (a), 0.13(b), and $0.20(\mathrm{c})$ at reduced temperatures as indicated by the colors and symbols defined in the legend.

Figure 8.10: $\mathrm{SAXS}$ intensity profiles of pure $\mathrm{SiO}_{2}-\mathrm{dPS} 33 \mathrm{k}$ nanoparticles (no matrix) at various temperatures, showing no change in inter-particle distance or brush height. Sample was annealed at $140{ }^{\circ} \mathrm{C}$ overnight and cooled down to room temperature prior to measurement. 266

Figure 8.11: Zero-angle scattering and correlation length vs temperature. Calculation of spinodal temperature $\left(\mathrm{T}_{\mathrm{s}}\right)$ from the zero-angle scattering and correlation length data were obtained by linearly extrapolating ISANs $(0)^{-1} \mathrm{vs} \mathrm{T}^{-1}$ to the intercept value as well as by linearly extrapolating $\xi^{-}$ ${ }^{2} \mathrm{vs} \mathrm{T}^{-1}$ to the intercept value.

Figure 8.12: Matrix-Matrix structure factors as $\mathrm{q} \rightarrow 0$ versus reduced temperature for particles of diameter $\mathrm{D}=5 d$ grafted with chains of length $\mathrm{N}_{\text {graft }}=10$ and grafting density $\Sigma=0.76$ chains $/ \mathrm{d}^{2}$ in a matrix of length $\mathrm{N}_{\text {matrix }}=50$ (a) or blends of $\mathrm{N}_{\text {graft }}=10$ and $\mathrm{N}_{\text {matrix }}=50$ chains (b) at a total volume fraction $\eta=0.35$ and filler fractions $\phi G=0.05$ (circles), 0.13 (triangles), and 0.20 (diamonds). 268

Figure 8.13: (Left) Simulation snapshot of flat grafted surface being wetted by a free matrix. (right) Wet monomer fraction versus reduced temperature, $\mathrm{T}^{*}$ for equivalent flat and spherical grafted surfaces. For all data in the plot, there are 900 grafted chains of length $\mathrm{N}_{\text {graft }}=10$ and 1200 matrix chains of length $\mathrm{N}_{\text {matrix }}=50$ at composition of $\phi G=0.13$ and total volume fraction $\eta=0.35$. The simulation protocols for the flat cases are nearly identical to the spherical case (Figure 8.1), except for a slightly modified compression scheme that accounts for only compressing in the direction normal to the interface.

Figure 8.14: Synthesis procedure for the preparation of initiator-functionalized (a) and polymergrafted (b) silica nanoparticles.

Figure 8.15: IR spectra of the hybrid nanoparticle showing signature absorption bands of the different functional groups of the tethered brush. 276 
Figure 8.16: Thermogravimetric analysis plot of the dPS33k hybrid (solid line) and initiatorgrafted (dashed line) nanoparticle showing the weight loss due to the tethered brush and initiator, respectively.

Figure 8.17: Gel permeation chromatogram of cleaved deuterated polystyrene brush. 278

Figure 8.18: SAXS intensity profile of the pure dPS33k hybrid nanoparticle. 280

Figure 9.1: Schematic depiction of some of the graft and matrix chain compositions considered in this work. Note that the graft monomers are isotropically .grafted to the particle surface and that, in these images, some of the grafted chains are hidden for clarity. Grey beads represent athermal monomers, blue and green beads represent attractive monomers in graft and matrix chains respectively. This figure is best viewed in color. 287

Figure 9.2: Simulation snapshots, low-q value of the matrix-matrix structure factor, and wet monomer fraction. In all parts, the particle diameter is $\mathrm{D}=5 \mathrm{~d}$, the grafting density $=$ 0.76 chains $/ d 2$, the graft chain monomer volume fraction is $\phi G=0.13$ and the total volume fraction in the simulation box is $\eta=0.35$. Parts a, c and e are for composites with $f G=f M=$ 1.0 and parts $\mathrm{b}, \mathrm{d}$ and $\mathrm{f}$ are for composites with $f G=0.7, f M=0.1$. The graft length is $\mathrm{N}_{\text {graft }}=$ 10 in all parts, while a matrix of length $\mathrm{N}_{\text {matrix }}=10$ is denoted with red triangles and $\mathrm{N}_{\text {matrix }}=50$ is denoted with black circles. The horizontal dashed lines in parts c,d and e,f represent the low-q structure factor and wet monomer fraction (respectively) of the equivalent athermal ( $f G=f M=$ 0.0) composite. The vertical dashed lines in parts c-f mark the location of the dispersionaggregation transition. The error bars in part b-f are standard deviations calculated from 50 uncorrelated configurations for each system; the error bars when not visible are smaller than the size of the symbol.

Figure 9.3: Wet monomer fraction $a$ and $c$ and $S M M q \rightarrow 0$ (b and d) versus Flory-Huggins $\chi G M$ at Nmatrix $=50$ for varying symmetric ( $\mathrm{a}$ and $\mathrm{b}$ ) and asymmetric (c and d) attractive monomer graft and matrix chain compositions. Also shown are the dispersion-aggregation transition $\chi G M$ (vertical dashed lines) and the wet monomer fraction and $\operatorname{SMM}(q \rightarrow 0)$ for the equivalent athermal composite (horizontal dashed lines). The error bars are standard deviations 
calculated from 50 uncorrelated configurations for each system; the error bars when not visible are smaller than the size of the symbol. 296

Figure 9.4: Matrix chain radius of gyration, $R g 2$, distributions for varying $\chi G M$, at Nmatrix $=$ 50 , and for varying graft and matrix chain compositions $f G, f M$ (as labeled). 300

Figure 9.5: Graft chain radius of gyration, $R g 2$, distributions for varying $\chi G M$, at Nmatrix $=$ 50 , and for varying graft and matrix chain compositions $f G, f M$ (as labeled). 301

Figure 9.6: Matrix (a) and graft (b) chain radius of gyration, $R g 2$, distributions at the dispersionaggregation transition $\chi G M$ for each composite with varying graft and matrix chain compositions.

Figure 9.7: Simulated Flory-Huggins $\chi G M$ versus temperature for a blend of deuterated polystyrene (dPS) at NdPS $=465000$ and poly-vinyl-methyl-ether (PVME) at NPVME $=$ 10,000 . The data points were extracted from Figure 4 of [1] while the line is a linear fit intended as a guide to the eye. To convert from real experimental units to reduced simulation units, a reference volume of $1148 \mathrm{~cm} 3 / \mathrm{mol}$ (based on a PS Kuhn length of $1.24 \mathrm{~nm}$ [2]), and a coordination number of $\mathrm{z}=26$ was used.

Figure 9.8: Schematic diagram of the simulation protocol used in this study.

Figure 9.9: Overall average (solid), minimum and maximum (dashed) local volume fraction, $\eta$, of the simulated composites for Nmatrix $=50$ (black circles) and 10 (red triangles) and for graft and matrix chain composition $(f G, f M)$ as marked.

Figure 9.10: Low-q value of the graft-graft $(a, b)$ and particle-particle $(c, d)$ structure factors. In all parts, the particle diameter is $\mathrm{D}=5 \mathrm{~d}$, the grafting density $=0.76$ chains $/ d 2$, the graft chain monomer volume fraction is $\phi G=0.13$ and the total volume fraction is $\eta=0.35$. Parts a and $\mathrm{b}$ are for composites with $f G=f M=1.0$ and parts $\mathrm{c}$ and $\mathrm{d}$ are for composites with $f G=$ $0.7, f M=0.1$. The graft length is $\mathrm{N}_{\text {graft }}=10$ in all parts, while a matrix of length $\mathrm{N}_{\text {matrix }}=10$ is denoted with red triangles and $\mathrm{N}_{\text {matrix }}=50$ is denoted with black circles. The horizontal lines in represent the low-q structure factor of the equivalent athermal $(f G=f M=0.0)$ composite The 
error bars are standard deviations calculated from 50 uncorrelated configurations for each system; the error bars when not visible are smaller than the size of the symbol.

Figure 9.11: Graft-graft monomer structure factor $S G G q \rightarrow 0$ (a and c) and particle-particle structure factor $\operatorname{SPP}(q \rightarrow 0)$ (b and d) versus Flory-Huggins $\chi G M$ at Nmatrix $=50$ for varying symmetric ( $a$ and $b$ ) and asymmetric (c and d) graft and matrix chain compositions. Also shown are the dispersion-aggregation transition $\chi G M$ (vertical lines) and $S G G(q \rightarrow 0)$ or $\operatorname{SPP}(q \rightarrow 0)$ for the equivalent athermal composite (horizontal lines). The error bars are standard deviations calculated from 50 uncorrelated configurations for each system; the error bars when not visible are smaller than the size of the symbol

Figure 9.12: Average number of Graft-Graft (a,d) Graft-Matrix (b,e) and Matrix-Matrix (c,f) monomer contacts versus Flory-Huggins $\chi G M$ at Nmatrix $=50$ for varying symmetric (a, b, and c) and asymmetric (c, d, and f) graft and matrix chain compositions. Two monomers are in "contact" when they are within the cutoff distance for the attractive Lennard-Jones interactions (i.e. center to center distance $<2.5 \mathrm{~d}$ ).

Figure 9.13: Average $R g 2$ versus Flory-Huggins $\chi G M$ at Nmatrix $=50$ for graft (a and c) and matrix (b and d) chains at varying symmetric (a and b) and asymmetric (c and d) attractive monomer graft and matrix chain compositions as shown in the legend. 316 


\section{INTRODUCTION}

Polymer nanocomposites are a broad class of soft materials which, in general, consist of two types of components: a) synthetic or biological polymer which can be either free (matrix polymer) or grafted on filler surface (graft polymer) and b) a nanometer-sized filler material that modifies and improves the mechanical, thermal, optical, and/or electrical properties of the matrix polymer. While the chemical nature of the filler material and graft/matrix polymer are important in determining the macroscopic properties of a composite, the arrangement (i.e. morphology) of the filler and polymer are equally as important. In order to create materials with targeted macroscopic properties for specific applications, it is vital that control over the nanocomposite morphology is achieved. To this end, there has been much work in the soft materials community to come up with strategies for controlling nanocomposite morphology, either via the application of external fields (i.e. top-down control) or via the modification of the fundamental thermodynamic driving forces of the composite (i.e. bottom-up control). The latter is primarily achieved by grafting polymer chains to the surface of the filler material, such as spherical nanoparticles, thereby mediating the effective filler-filler interactions via the grafted polymer chains. The research described in this doctoral thesis is aimed at understanding how polymer-grafted nanoparticles should be designed to engineer nanocomposites with targeted morphologies and in turn targeted macroscopic properties.

In order to understand how the large number of physical parameters and chemical properties of polymer-grafted particle based nanocomposites affect their morphology, a robust and efficient set of techniques must be adopted. Molecular simulations and liquid state theory are tools that are set to handle the exploration of large materials parameters spaces more quickly than 
experiments, and, simultaneously, also provide molecular level insight into the thermodynamic driving forces that are harder to characterize in experiments. In this thesis, we will use Monte Carlo simulations, molecular dynamics simulations, and polymer reference interaction site model (PRISM) theory to understand and create new design rules for controlling nanocomposite morphology. Furthermore, wherever possible, we will highlight experimental studies conducted by other research groups that corroborate our computational and theoretical findings.

In Chapter 2, we use Monte Carlo simulations to study AB copolymer-grafted nanoparticles to elucidate the effect of blockiness (length of contiguous blocks of like-monomers) in the grafted monomer sequence on the shape, size, and structure of assembled nanoparticle clusters for a range of monomer-monomer and monomer-particle interactions. The graft sequence dictates the ease or difficulty of the grafted chains to form attractive like-monomer (A-A or B-B) contacts while minimizing repulsive unlike-monomer (A-B) contacts within an assembled cluster or dispersed state. When A-B repulsion is negligible, with increasing blockiness at constant graft length, the cluster size and average coordination number decrease in the presence of A-A or B-B attractions, and are approximately constant in the presence of A-A and B-B attractions. When AB repulsion is strong, the cluster size and average coordination number increase with increasing blockiness for small and large particles. For small particles, with strong B-B attraction and A-B repulsion, increasing blockiness leads to increasing anisotropy in cluster shape, while strong A-A attraction produces isotropic clusters regardless of graft sequence. The effect of graft sequence on cluster shape is reduced for large particles as compared to small particles, at constant graft length. Lastly, the extent to which monomer-particle attractive interactions change the above trends is highly dependent on the relative strength of monomer-particle to monomer-monomer interactions, in addition to the ratio of particle size to graft length, and the grafting density. This chapter 
illustrates how copolymer functionalization and tuning grafted copolymer sequence is an exciting new route for experimentalists to tailor the self-assembly of nanoparticles into selected nanostructures.

In Chapters 3-7, we shift our focus to systems of homopolymer grafted nanoparticles in a chemically identical free homopolymer matrix. In these systems, due to the chemical similarity of the graft and matrix chains, the driving forces that control the nanocomposite morphology are purely entropic in nature. In Chapters 3, 4, and 5, we explore the effect of graft-length polydispersity on nanocomposite morphology. Chapter 6 extends these results to include the effect of matrix-length bidispersity, and Chapter 7 focuses on how graft and matrix flexibility tunes morphology. In all of these cases, the phenomenon of interest is the mixing of the grafted and matrix chains i.e. wetting of the grafted layer by the matrix chains. In chemically-identical graftmatrix composites it is generally assumed that the wetting-dewetting and dispersion-aggregation transitions are simultaneous and identical transitions. Based on this concept, much of the focus of Chapters 3-7 will be on improving the wetting of the grafted layer which in turn stabilizes the dispersed morphology of the nanocomposite. Dispersed morphologies are desirable for applications where mechanical properties are important as they the maximize filler-matrix contact area and minimize the individual filler domain sizes.

In Chapter 3, we present an integrated theory and simulation study of polydisperse polymer grafted nanoparticles in a polymer matrix to demonstrate the effect of polydispersity in graft length on the potential of mean force (PMF) between the grafted nanoparticles. In dense polymer solutions, increasing polydispersity reduces the strength of repulsion at contact and weakens the attractive well at intermediate inter-particle distances, completely eliminating the latter at high polydispersity index (PDI). The reduction in contact repulsion is due to polydispersity relieving 
monomer crowding near the particle surface, especially at high grafting densities. The elimination of mid-range attractive well is due to the longer grafts in the polydisperse graft length distribution that introduce longer range steric repulsion, and alter the wetting of the grafted layer by matrix chains. Dispersion of the grafted particles is stabilized by increased penetration or wetting of the polydisperse grafted layer by the matrix chains. This study demonstrates that at high grafting densities, polydispersity in graft length stabilizes dispersions of grafted nanoparticles in a polymer matrix at conditions where monodisperse grafts would cause aggregation.

In Chapter 4, we extend these results presented in Chapter 3 to describe how the PMF between two grafted particles changes as a function of grafting density, ratio of matrix to graft length, and packing fraction of polymer matrix for polydisperse and bidisperse grafts. Comparison of a log-normal polydisperse distribution to a bidisperse distribution of graft chain lengths (with equal number of short and long chains) with same PDI and average length, shows that the polydisperse distribution better stabilize particle dispersions than the bidisperse distributions because of the longer chains in the polydisperse distribution. Additionally, in a bidisperse distribution, with all chains shorter than the matrix chain length, there is a reduction in the midrange attraction, thus confirming the role of reduced monomer crowding in the bidisperse grafted layer in increasing the grafted layer wetting by the matrix chains, and as a result, improving miscibility of grafted particles and matrix.

In Chapter 5, we investigate the roles of the short and long grafted chains in polydisperse polymer grafted nanoparticles in stabilizing particle dispersion in a chemically similar polymer matrix in the presence of particle-particle attractions. The effect of the short and long chains in a polydisperse or bidisperse graft length distribution on the PMF between the polymer grafted nanoparticles is coupled and distinct from their role in the corresponding deconstructed short and 
long monodisperse distributions. At high grafting density, the increased monomer crowding near the particle surface, from both short and long chains, maximizes shielding of particle-particle attraction, while the length and crowding of long chains away from the particle surface determines the location, range and strength of the steric repulsion and mid-range attraction. We find that to maximize grafted nanoparticle dispersion, it is best to synthesize grafted particles at high grafting density with polymer graft length distributions that maximize monomer crowding near the particle surface to shield particle-particle attraction, and minimize crowding at farther distances from the particle to increase wetting of the grafted layer by matrix chains. Polydisperse (log-normal) graft length distributions and bidisperse graft length distributions with few long chains among many short chains satisfy this criterion, and better disperse grafted particles in a chemically identical matrix than monodisperse grafts or bidisperse graft length distributions with equal number of short and long chains, with equivalent average graft length.

In Chapter 6, we present a simulation study showing the effect of bidispersity in matrix homopolymer length, for monodisperse graft lengths, on the wetting/dewetting of homopolymer grafted nanoparticles and the morphology of polymer nanocomposites where the graft and matrix polymer chemistries are identical. In a bidisperse matrix with equal number of short and long chains and average matrix length greater than the monodisperse graft length, the densely grafted polymer layer is preferentially wet by the short chains and relatively dewet by the long chains. This is driven by a larger gain in entropy of mixing between graft and matrix for short matrix chains than long matrix chains. Despite the preferential wetting of the short and dewetting of long chains, matrix length bidispersity does not significantly change the overall wetting of the grafted layer. Unlike graft length bidispersity which significantly improves particle dispersion, matrix length bidispersity slightly increases particle aggregation in the polymer matrix. 
In Chapter 7, we present a molecular dynamics simulation study of nanocomposites containing homopolymer grafted particles in a homopolymer matrix, where the graft and matrix chemistries are identical, to elucidate the effect of polymer flexibility on the wetting of the grafted layer by the matrix and the nanocomposite morphology. Decreasing flexibility of the graft and matrix causes increased wetting of the grafted layer by the matrix. This increased wetting of the grafted layer with decreasing flexibility is more significantly driven by decreasing the graft flexibility than by decreasing the matrix flexibility. This is due to a large increase in mixing entropy of the graft and matrix upon wetting rather than the reduction in conformational entropy loss of matrix upon wetting. Due to this improved wetting with decreasing flexibility of the graft and matrix, we observed increased particle dispersion in the polymer matrix.

In Chapters 8 and 9, we switch the focus away from composites with chemically-identical graft and matrix polymers to those with chemically-dissimilar graft and matrix. Composites where the graft and matrix chains are chemically dissimilar have competing enthalpic driving forces in addition to the entropic driving forces of the chemically identical composites discussed above. In these chapters, we will directly focus on the connection between the wetting-dewetting and dispersion-aggregations and furthermore how we can tune and control these transitions. We show that, unlike chemically-identical composites, chemically-distinct composites have a broad wettingdewetting transition that allows for the tuning of graft-matrix wetting/mixing. The ability to directly tune the graft-matrix mixing via temperature, solvent selection, or polymer sequence is an attractive route to creating composites with highly tunable mechanical and rheological properties.

In Chapter 8, we present simulations and experiments on mixtures containing polymer grafted nanoparticles in a chemically distinct polymer matrix, where the graft and matrix polymers exhibit attractive enthalpic interactions at low temperatures that become progressively repulsive 
as temperature is increased. Both coarse-grained molecular dynamics simulations, and x-ray scattering and neutron scattering experiments with deuterated polystyrene (dPS) grafted silica and poly(vinyl-methyl-ether) PVME matrix show that the sharp phase transition from (mixed) dispersed to (demixed) aggregated morphologies due to the increasingly repulsive effective interactions between the blend components is distinct from the continuous wetting-dewetting transition. Strikingly, this is unlike the extensively-studied chemically identical graft-matrix composites, where the two transitions have been considered to be synonymous, and is also unlike the free (ungrafted) blends of the same graft and matrix homopolymers, where the wettingdewetting is a sharp transition coinciding with the macrophase separation.

In Chapter 9, using coarse-grained molecular simulations we demonstrate that the extent of wetting of the grafted polymer layer and the particle dispersion-aggregation transition are tuned using the composition of graft and matrix polymers. Specifically, we study composites where the graft and matrix chains are random copolymers composed of attractive and athermal monomers. We vary the fraction of attractive monomers in the graft $\left(f_{G}\right)$ and matrix $\left(f_{M}\right)$ chains, graft-matrix chain composition ratio $\left(f_{G} / f_{M}\right)$, and the graft-matrix interaction strength, as characterized by the Flory Huggins interaction parameter between graft and matrix attractive monomers: $\chi_{G M}$. When $\chi_{G M}$ is negative, decreasing $f_{G}$ and/or $f_{M}$ decreases the extent of grafted layer wetting by matrix chains because the enthalpic driving force for wetting is reduced. As the $\chi_{G M}$ increases and becomes positive, the extent of wetting decreases gradually till it reaches the wetting of athermal graft-matrix composites. That value of $\chi_{G M}$ where the extent of wetting is the same as that of an analogous athermal graft-matrix polymer nanocomposite marks the onset of dispersionaggregation transition. For symmetric graft and matrix chain compositions $\left(f_{G}=f_{M}\right)$ the magnitude of $f_{G}$ and $f_{M}$ tunes the overall extent of wetting of the grafted particles in the dispersed 
state but not the dispersion-aggregation transition. Varying the asymmetry of the graft-matrix composition (i.e. $f_{G} / f_{M}$ ) tunes both the extent of wetting of the grafted layer and the dispersionaggregation transition.

The thesis ends with a chapter that presents a summary of these results and some future directions. Overall, this thesis presents several new and novel design criteria for controlling the morphology of different nanocomposite systems and should motivate the continued study of these systems by computational, theoretical, and experimental methods. 


\section{EFfect OF BlockinesS In GRAFted MONOMER SEQUENCES ON ASSEMBlY of Copolymer Grafted NANOPARTICLES: A Monte Carlo SimUlation STUDY}

Adapted from: Soft Matter 2011, 7 (13), 5952-5964.

\subsection{INTRODUCTION}

Controlled nanoparticle assembly is required to create nanostructured materials for applications such as metamaterials, photovoltaics, and microelectronics. One way to tailor nanoparticle assembly is via functionalization of the nanoparticle surface with ligands such as polymers,[1-31] DNA,[32-36] and proteins[37, 38]. Polymer functionalized nanoparticles, in particular, have been studied extensively in the recent years using theory,[1, 13, 15-17] simulation,[18-21] and experiments[15, 22, 23]. Experimental and computational studies of homopolymer grafted nanoparticles in polymer matrix, solvent, and pure bulk (no solvent or matrix) have demonstrated the ability to tune the assembly characteristics of these grafted nanoparticles via the grafting density, $[2,19,26]$ nanoparticle size, $[3,17,27]$ graft placement, $[8,28,29]$ graft and matrix molecular weight,[30] and nanoparticle shape.[20, 27, 31] Specifically, for homopolymer grafted nanoparticles, at high grafting density in a polymer matrix, it has been shown that when the grafted chains have a higher molecular weight than the matrix chains the nanoparticles disperse. It has also been shown that, at low grafting densities, the grafted chain layer is not dense and the interactions between the particles' cores becomes important, causing the lightly grafted polymer functionalized nanoparticles to assemble anisotropically.

Copolymer functionalization, as opposed to homopolymer functionalization, creates additional tuning parameters of graft sequence and monomer chemistry (or interactions) which provide further control over the assembly of polymer grafted nanoparticles. In the last few years, 
theory $[13,16,17]$ and simulations $[12,24,25]$ have been used to study copolymers grafted onto nanoparticles. Vorselaars et al.[16] have used (self-consistent field theory) SCFT to study dense layers of diblock copolymers grafted onto spherical nanoparticles. They found various domain shapes on particle surface depending on the composition of the copolymer, and discussed the stability of the various morphologies on these highly curved nanoparticle surface, in contrast to the case of zero curvature. Zhu et al.[17] have employed both SCFT and DFT (density fluctuation theory) to study a dense system of nanoparticles with a single diblock copolymer graft. When the particle surface was chemically neutral to the grafted chain, they observed typical block copolymer morphologies (i.e. cylinders and lamellae) determined by both the composition of the copolymer and the particle size. When the particle was repulsive to both blocks of the copolymer, they observed hierarchical morphologies, such as "lamellae with cylinders at interfaces" not typically observed with block copolymer melts. While the above studies focused on either a single copolymer grafted nanoparticle at high grafting density[16] or grafted nanoparticles with a single grafted chain[17], Jayaraman and coworkers[12, 13, 25] have conducted systematic studies of spherical copolymer grafted nanoparticles at low to intermediate grafting densities using a combination of theory and simulation techniques to better understand the effective interactions between the copolymer grafted particles and their assembly characteristics in both solvent and matrix. Using Monte Carlo simulations of a single copolymer grafted nanoparticle at low to intermediate grafting densities in an implicit solvent[25], they show that the grafted chain conformations change non-monotonically with monomer sequence, and depend on the monomer sequence and monomer interactions. In a following study[13], Nair and Jayaraman used an integrated theory and simulation approach (self-consistent PRISM-MC method) to study the effective interactions between two copolymer grafted nanoparticles in a homopolymer matrix as a 
function of grafted monomer sequence. They found that at the same composition, particle size and grafting density, using alternating $\mathrm{AB}$ copolymer grafts leads to a repulsive potential of mean force (PMF) between the grafted particles at contact in both A and B homopolymer matrix, while using diblock sequence $\mathrm{AB}$ copolymer grafts leads to an attractive PMF at contact and a repulsive PMF at larger inter-particle distance in B homopolymer matrix, and repulsive at contact and attractive at larger inter-particle distance in A homopolymer matrix. This was an interesting result because it demonstrated the isolated effect of varying the "arrangement" of monomers (monomer sequence) on the behavior of the grafted nanoparticles in a polymer matrix.

In recent work, we used lattice Monte Carlo simulations to study assembly of spherical nanoparticles grafted with $\mathrm{AB}$ alternating and diblock sequences[12] in an implicit solvent at varying monomer interactions, particle size, and graft length. We found that depending on the monomer interactions, the alternating sequence either favors dispersions, or formation of smaller, isotropic clusters, while the diblock sequence favors assembly producing larger, anisotropic clusters. In this paper, we use the same approach going beyond just alternating and diblock sequences, by focusing in detail on five different graft sequences with varying "blockiness" in sequence, where blockiness is defined as the length of contiguous blocks of like-monomers. Our goal here is to study how varying $\mathrm{AB}$ copolymer graft sequence blockiness affects the assembly of copolymer grafted nanoparticles at varying monomer-particle and monomer-monomer interactions. We find that the total number of attractive monomer contacts in the assembled nanoparticle cluster increases with increasing blockiness in graft sequence, with the alternating sequence being an outlier. As the graft sequence blockiness increases, attractive like monomers (A-A and/or B-B) aggregate more easily within an assembled nanoparticle cluster without having to constrain the grafted chains, thereby decreasing conformational entropic losses. The unique 
trend seen with the alternating sequence is explained by the alternating grafted chains forming "networks" of attractive monomer contacts within the cluster in contrast to the distinct domains of attractive monomers formed by other blockier sequences. In the case of like monomer (A-A and/or B-B) attraction in the absence of unlike (A-B) monomer repulsion, the assembled cluster size and average particle coordination number decreases with increasing blockiness. In the case of like monomer attraction in the presence of unlike monomer repulsion the clusters size and average particle coordination number within the cluster increases with increasing blockiness. For both small and large particle sizes, we observe that presence of B-B attraction with A-B repulsion produces the greatest range of anisotropy in clusters with varying blockiness in the grafted chains. All of the above trends are driven purely by how the monomer sequences enable/deter the grafted copolymer chains to bring together attractive monomer contacts for making inter-particle contacts. For the two particle sizes studied here, at constant graft length, how much the monomer-particle interactions affect the above trends are highly dependent on the particle size to graft length ratio, graft sequence, and the relative strengths of monomer-particle and monomer-monomer interactions.

Since copolymer-grafted nanoparticles are a relatively less-studied system as compared to homopolymer grafted nanoparticles in the polymer functionalized particles field, there is a need to predict how these copolymer functionalized nanoparticles assemble at a variety of parameters. Since the synthetic efforts to conduct a systematic parameter study are both time consuming and involve significant effort, we expect the results from this computational exploration to provide guidelines for synthetic chemists and other scientists attempting to create target morphologies through assembly of copolymer grafted nanoparticles. 
The paper is organized as follows. In Section 2.2 we provide details of our model, the simulation method, and analysis techniques. In Section 2.3 , we present the results showing the effect of varying monomer sequence, monomer-monomer, and monomer-particle interactions, and particle size on the shape, size and structure of assembled cluster of copolymer grafted nanoparticles. We conclude with a discussion on the observed general trends, limitations of this work, some future directions, and the impact of this computational work on experiments.

\subsection{APPROACH}

\subsubsection{Model}

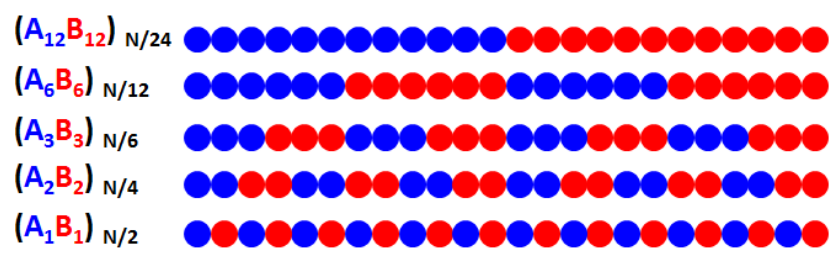

Figure 2.1: Schematic of the monomer sequences used in this study, in decreasing order of blockiness from top to bottom.

We model the system of AB copolymer grafted nanoparticles as hard sphere particles of diameter $D$ each with six grafted symmetric AB copolymers. Each grafted copolymer chain is modeled as a freely jointed chain on a cubic lattice with monomers of size $d$ of the order $1 \mathrm{~nm}$, and the first monomer is placed symmetrically $1 d$ away from the six poles of the spherical nanoparticle. The symmetric $\mathrm{AB}$ copolymers of length 24 monomers, have A and B monomers arranged in one of five sequences- $\left(A_{1} B_{1}\right)_{12},\left(A_{2} B_{2}\right)_{6},\left(A_{3} B_{3}\right)_{4},\left(A_{6} B_{6}\right)_{2},\left(A_{12} B_{12}\right)_{1}$-displayed in Figure 2.1. For all sequences the $\mathrm{AB}$ copolymer chains are grafted such that the first monomer attached to the particle surface is an A monomer. The identities of the second, third and higher monomers in each grafted chain depend on the chosen monomer sequence. 
The monomer-monomer interaction potential, $U_{i j}(r)$ between the $i^{\text {th }}$ and $j^{\text {th }}$ non-bonded grafted monomers is described by a square well potential shown in Equation (1)

$$
U_{i j}\left(r_{i j}\right)=\left\{\begin{array}{cc}
\infty & r_{i j}<d \\
\varepsilon_{i j} & d \leq r_{i j} \leq \sqrt{2} d \\
0 & \sqrt{2} d<r_{i j}
\end{array}\right.
$$

where $r_{i j}$ is the center-center distance between $i$ and $j$ monomers, $d$ is the diameter of the monomer (maintained the same for $\mathrm{A}$ and $\mathrm{B}$ monomer), $\sqrt{ } 2 d$ is the width of interaction (i.e interaction includes nearest neighbors and nearest diagonal neighbors), and $\varepsilon_{\mathrm{ij}}$ is the strength of interaction between the $i$ and $j$ monomers. Attractive interactions are characterized by a negative $\varepsilon_{\mathrm{ij}}$ and repulsive interactions are denoted by a positive $\varepsilon_{\mathrm{ij}}$. We have chosen $\varepsilon_{\mathrm{AA}}$, $\varepsilon_{\mathrm{BB}}$, and $\varepsilon_{\mathrm{AB}}$ to mimic varying chemistries as tabulated in Table 2.1 .

We model attractive interactions between monomer $i$ and the grafted particle $j$ using another square well potential:

$$
U_{i j}(r)=\left\{\begin{array}{cc}
\infty & r_{i j}<(d+D) / 2 \\
\varepsilon_{i j} & (d+D) / 2 \leq r_{i j} \leq D / 2+d \\
0 & D / 2+d<r_{i j}
\end{array}\right.
$$

where $D$ is the diameter of the particle. Particle-particle interactions are maintained as hard-sphere interactions.

Table 2.1: List of monomer-monomer and (in kT). Negative values of $\varepsilon$ values represent attractive interactions while positive values represent repulsive interactions, in the square well potential form. Each of these interactions is studied without monomer-particle interactions, with particle to A-particle attraction of $1 k T$, or with B-particle attraction $1 k T$.

\begin{tabular}{cccc}
\hline $\begin{array}{c}\text { Interaction } \\
\text { Set }\end{array}$ & $\varepsilon_{\mathrm{AB}} / \mathrm{kT}$ & $\varepsilon_{\mathrm{AA}} / \mathrm{kT}$ & $\varepsilon_{\mathrm{BB}} / \mathrm{kT}$ \\
\hline 1 & 0.0 & -0.5 & 0.0 \\
2 & 0.0 & -1.0 & 0.0 \\
3 & 0.0 & 0.0 & -0.5 \\
4 & 0.0 & 0.0 & -1.0
\end{tabular}




\begin{tabular}{cccc}
5 & 0.0 & -0.5 & -0.5 \\
6 & 0.0 & -1.0 & -1.0 \\
7 & 1.0 & -0.5 & 0.0 \\
8 & 1.0 & -1.0 & 0.0 \\
9 & 1.0 & 0.0 & -0.5 \\
10 & 1.0 & 0.0 & -1.0 \\
11 & 1.0 & -0.5 & -0.5 \\
12 & 1.0 & -1.0 & -1.0 \\
\hline
\end{tabular}

\subsubsection{Method}

We use Monte Carlo simulation on a cubic lattice to study the assembly of copolymer grafted nanoparticles. In this paper we are focused only on dilute concentration of $\mathrm{c} \sim 1 \times 10^{-5}$ particles per $\mathrm{nm}^{3}$, obtained by using 10 particles in a $100 \times 100 \times 100\left(\mathrm{~nm}^{3}\right)$ simulation box with periodic boundary conditions. In the first step of the simulation we grow the initial configuration by placing the spherical nanoparticles in random positions inside the simulation box. Then for each particle, we fix the first monomers of the six grafted chains at the predetermined symmetric sites on the sphere followed by placing the second monomer of each grafted chain in one of the five unoccupied lattice sites adjacent to the first monomer of that grafted chain. During this growth process if an $i^{\text {th }}$ monomer cannot be grown because all neighbouring sites of the $(i-1)^{\text {th }}$ monomer are occupied by other monomers, then all the monomers are subjected to local moves until a vacancy is created. This is repeated until all the grafted chains are grown to the desired chain length, $N_{\text {graft. }} N_{\text {graft }}$ is maintained as 24 in this study. This process is followed for all copolymer grafted particles within the simulation box while ensuring no overlaps. After the initial configuration is grown, the simulation proceeds to the initialization stage. The initialization stage helps us avoid any bias that might arise due to the nature of the initial configuration. In the initialization stage the chains are subjected to 100000 Monte Carlo (MC) steps with purely hardsphere interactions between all monomers in the simulation. An MC time step is defined either as 
$N_{\text {graft } \mathrm{x}} N_{g}$ random monomer moves, where $N_{\text {graft }}$ is the grafted copolymer chain length and $N_{g}$ is the number of grafts ( $N_{g}=6$ in this study), or one copolymer grafted nanoparticle translate or rotate move. In one monomer move, we randomly pick a monomer (with the exception of the first monomer that is fixed) on a randomly picked grafted chain of a randomly selected nanoparticle, and then move that monomer using a randomly chosen move_-"crankshaft", "kink" and "end" (end move for the last monomer only)[39]. In one single copolymer grafted nanoparticle move, one copolymer grafted nanoparticle is randomly chosen and the particle along with its constituent chains is translated or rotated. The moves are accepted or rejected based on the Metropolis algorithm[40] since interaction potentials are not turned on until the equilibration stage, all initialization-stage moves are made under athermal conditions and accepted as long as no overlaps occur during the move.

The initialization stage is followed by the equilibration stage. In the equilibration stage the chains goes through 20 million MC steps with a temperature annealing schedule going from dimensionless temperature $T_{\text {inital }}=3$ to $T_{\text {final }}=1$ with a temperature decrement of 0.9 at every $i^{\text {th }}$ stage ( $T_{i}=T_{i-1} \times 0.9$ ), and 3 million MC steps per temperature stage. This annealing schedule was chosen after rigorous testing to ensure equilibrium is reached at each temperature stage, and the resulting configurations at each temperature are independent of small variations in the annealing schedule (e.g. 3 million or 4 million MC steps per stage). The moves during the equilibration stage include the monomer moves (47.5\%) and grafted particle moves (47.5\%) described above and cluster moves (5\%). A cluster move is defined as a move of a collection of copolymer grafted nanoparticles, where every grafted particle is making at least one monomer contact with a monomer of another grafted particle in the cluster. During a cluster move we translate randomly picked clusters and accept the move only when the cluster move does not lead to an overlap or 
formation of a new cluster.[39] If there are no clusters in the configuration the chosen cluster move is rejected. Once the simulation has reached $T_{\text {final }}=1$ and our equilibrium condition has been satisfied - 40 consecutive block averages of energy are within $10 \%$ of each other-we collect the ensemble average of the block averages of the equilibrated simulation. We collect data on the thermodynamic property of interest over 10000 MC steps and calculate the block averages for every 100000 MC steps. For each parameter set, described below, we repeat 5 trials of simulation where each of the trials is initialized with different random number seeds. We obtain error bars for every data point presented in the Results section from the ensemble averages collected from 5 such trials.

\subsubsection{Analysis}

We employ a variety of metrics to assess the size and shape of the assembled cluster of the copolymer grafted particles, the monomer contacts within the cluster and the grafted chain configurations.

We calculate the average radius of gyration of the grafted chains using equation

$$
\left\langle R_{g, \text { chain }}^{2}\right\rangle^{0.5}=\left(\frac{1}{M * N_{\text {graft }}} \sum_{j=1}^{M} \sum_{i=1}^{N_{\text {graft }}}\left(r_{i j}-r_{c m, j}\right) \cdot\left(r_{i j}-r_{c m, j}\right)\right)^{0.5}
$$

where $r_{i j}$ is the position of the $i^{t h}$ monomer of the $j^{\text {th }}$ chain, $r_{c m, j}$ is the position of the center of mass of chain $j, N_{\text {graft }}$ is the number of monomers in grafted chain $j$ and $M$ is the total number of grafted chains in the simulation $\left(=N_{g} *\right.$ number of particles $)$.

We also calculate the mean height of each monomer type from the center of the particle it is grafted to. We first calculate the average concentration profile for each monomer type using

$$
C_{\alpha}(r)=\frac{1}{P} \sum_{i=1}^{P} \frac{N_{i, \alpha}(r)}{\frac{4}{3} \pi(r+1)^{3}-\frac{4}{3} \pi r^{3}}
$$


where $P$ is the total number of copolymer grafted nanoparticles particles in the system, $\alpha$ represents either A monomers or B monomers, $r$ is an integer radial distance from a particle center, and $N_{i, \alpha}(r)$ is the average number of monomers of type $\alpha$, grafted to particle $i$, that reside in the shell between $r$ and $r+1$. The second moment of the normalized $C_{\alpha}(r)$ is the mean squared height of monomer type $\alpha$ from the particle center as shown in Equation (5).

$$
H_{\alpha}=\left[\frac{\int_{0}^{R} C_{\alpha}(r) r^{2} d r}{\int_{0}^{R} c_{\alpha}(r) d r}\right]^{0.5}
$$

To quantify the shape of the assembled clusters of polymer grafted nanoparticles, we calculate relative shape anisotropy (RSA).[41] The RSA of a cluster varies between 0 and 1 , with 0 corresponding to perfectly isotropic (i.e. spherical symmetry) and 1 to perfectly anisotropic (i.e. rod-like) shape. Since the RSA calculation performs best when it's applied to many beads, we apply this analysis to the coordinates of the monomers of a cluster, as opposed to the coordinates of the particles of a cluster. We begin the calculation of RSA by first translating the center of mass of the monomer coordinates of a cluster to the origin and calculating the radius of gyration tensor for a cluster containing $\mathrm{N}$ monomers:

$$
S=\sum_{i=1}^{N} r_{i} r_{i}^{T}
$$

where $r_{i}$ is the translated coordinate vector of monomer $i$, and $r_{i}^{T}$ is the transpose of this coordinate vector. We then diagonalize $S$ in the following manner:

$$
S=V^{T} S V
$$

where $V$ is a $3 \times 3$ matrix with columns that correspond to the three eigenvectors of $S$. We then find the traceless part of $S$ :

$$
\widehat{\boldsymbol{S}}=\boldsymbol{S}-\frac{1}{3} \operatorname{tr}(\boldsymbol{S}) I
$$


where $I$ is the $3 \times 3$ identity matrix, and $\operatorname{tr}(S)$ is the trace of $\boldsymbol{S}$. Using the above quantities, we can calculate the relative shape anisotropy of the cluster of monomers:

$$
R S A=\frac{3}{2} \frac{\operatorname{tr}(\widehat{\boldsymbol{S}} \widehat{\boldsymbol{S}})}{\operatorname{tr}(\boldsymbol{S})^{2}}
$$

In the process of calculating $R S A$, we also can easily obtain the radius of gyration of a cluster which allows us to assess the size of clusters. This calculation is shown in Equation 10.

$$
\left[R_{g, \text { cluster }}^{2}\right]^{0.5}=\sqrt{\operatorname{tr}(\boldsymbol{S})}
$$

We also calculate the average number of particles per cluster, $\langle\mathrm{N}\rangle$, which serves as a separate metric of the size of clusters. To quantify the structure or particle-particle connectivity within a cluster we calculate the ensemble averaged coordination number, $\langle Z\rangle$, which is defined as the average number of "neighbors" a polymer grafted particle has. We define two polymer grafted nanoparticle as "neighbors" if they have at least one monomer-monomer contact.

In an assembly/dispersion each copolymer grafted nanoparticle exhibits an effective patchiness due to aggregation of monomers. In order to quantify this "patchiness" we perform analysis on the domains of like-monomers within the assembled cluster. We define a "domain" as a set of monomers of the same type that share at least one monomer contact with another monomer in the domain. Using this criterion, we can calculate various metrics such as the average number of domains in a cluster or the average radius of gyration of these domains. We also calculate the average total number of contacts between various pairs of monomers (e.g. A-A, A-B and B-B contacts) and between particle and monomers (e.g. A-P, B-P contacts) to show how the monomers aggregate in each cluster.

In order to verify that the data and trends that we present are significant, all of the shown trends have been subject to a statistical analysis. To verify that the data points within a specific trend are statistically significant from one another, each trend line is tested using a one-way 
analysis of variance with an acceptance criterion of 0.05 . A p-value below 0.05 indicates that the data in the trend are significantly different while a p-value above 0.05 indicates that the data are statistically the same (i.e. zero slope). To test data from different trends, for example weak monomer attraction vs. strong monomer attraction, we employ an unequal variance, two-tailed ttest with an acceptance criterion of 0.05 . All of the data in our presented figures have errors bars calculated using standard error; many of the error bars are smaller than the symbol representing the data.

\subsubsection{Driving Forces for Assembly of Grafted Nanoparticles}

In this section we discuss the forces that drive copolymer grafted nanoparticles to arrive at their equilibrium assembled/dispersed configuration. First, as stated earlier, the term "blockiness" is defined as the number of contiguously placed like monomers in a particular copolymer sequence. In other words, the longer the block of like monomers in a sequence is, the blockier the sequence. Therefore, in this study $\left(\mathrm{A}_{1} \mathrm{~B}_{1}\right)_{12}$ is the least blocky sequence (also called the alternating sequence), since it only has one like- monomer in each repeating block, while $\left(\mathrm{A}_{12} \mathrm{~B}_{12}\right)_{1}$ is the most blocky sequence as it has 12 like monomers contiguously placed in each block.

The equilibrium configuration of the copolymer grafted nanoparticles is achieved by maximizing the enthalpic gain through maximizing the number of attractive like monomer contacts, and minimizing the number of repulsive unlike-monomer contacts, while minimizing the entropic losses associated with making or avoiding these contacts. The conformational entropy of the grafted chains and translational entropy of nanoparticles is reduced upon assembly of the polymer grafted nanoparticles. While the translational entropy loss of the nanoparticles upon assembly should not vary much with the grafted monomer sequence, the conformational entropy loss of the grafted chains upon nanoparticle assembly is strongly dependent on the monomer 
sequence. This is because the assembly of the nanoparticles is driven by ability of the grafted chain to a) coil upon itself and make enthalpically favorable intra-chain contacts, and/or b) stretch out to make enthalpically favorable inter-chain intra-particle contacts, and/or c) stretch out towards another grafted nanoparticle and make enthalpically favorable inter-particle contacts. Supplementary Figure S1 shows schematically these different types of monomer contacts. The choice of one or more of these types of contacts is dictated by the strength of monomer attractions and repulsions, and by how the grafted chain sequence facilitates these contacts, while losing the least number of conformations or minimizing the entropic loss. For example, a highly blocky sequence, like $\left(\mathrm{A}_{12} \mathrm{~B}_{12}\right)_{1}$, can easily maximize the intra-chain $\mathrm{B}-\mathrm{B}$ and/or $\mathrm{A}$-A contacts while maintaining a high conformational entropy. The least blocky sequence, $\left(\mathrm{A}_{1} \mathrm{~B}_{1}\right)_{12}$, on the other hand, must seek highly compact conformations to form intra-chain contacts that lower the conformational entropy of the grafted chain. Thus, less blocky sequences could prefer to make "inter-chain intra-particle" or "inter-particle" contacts if the chain does not have to stretch too much to achieve those attractive contacts. Clearly, in addition to the sequence, the grafted chain length and the particle size also affect the above choice of types of contacts. For $e . g$., at constant chain length and constant number of grafts, a small nanoparticle can easily allow the formation of inter-chain intra-particle contacts as the chains are grafted closer together, but a larger nanoparticle will drive mostly inter-particle or intra-chain contacts due to the increased distance between the grafts.

In summary, the equilibrium configuration of copolymer grafted nanoparticles, assembled or dispersed, tightly clustered or loosely clustered, are all based on how the above factors - grafted chain length and sequence, monomer attractive and repulsive interaction strengths, graft chain 
length, and particle size -- balance the enthalpic gain and entropic losses. This balance is complex and non-intuitive, and therefore motivates this computational study.

\subsection{RESULTS}

\subsubsection{Effects of Monomer Sequence on Grafted Chain Conformations and Monomer Aggregation in the Cluster}

\subsubsection{Radius of Gyration and Monomer Contacts}

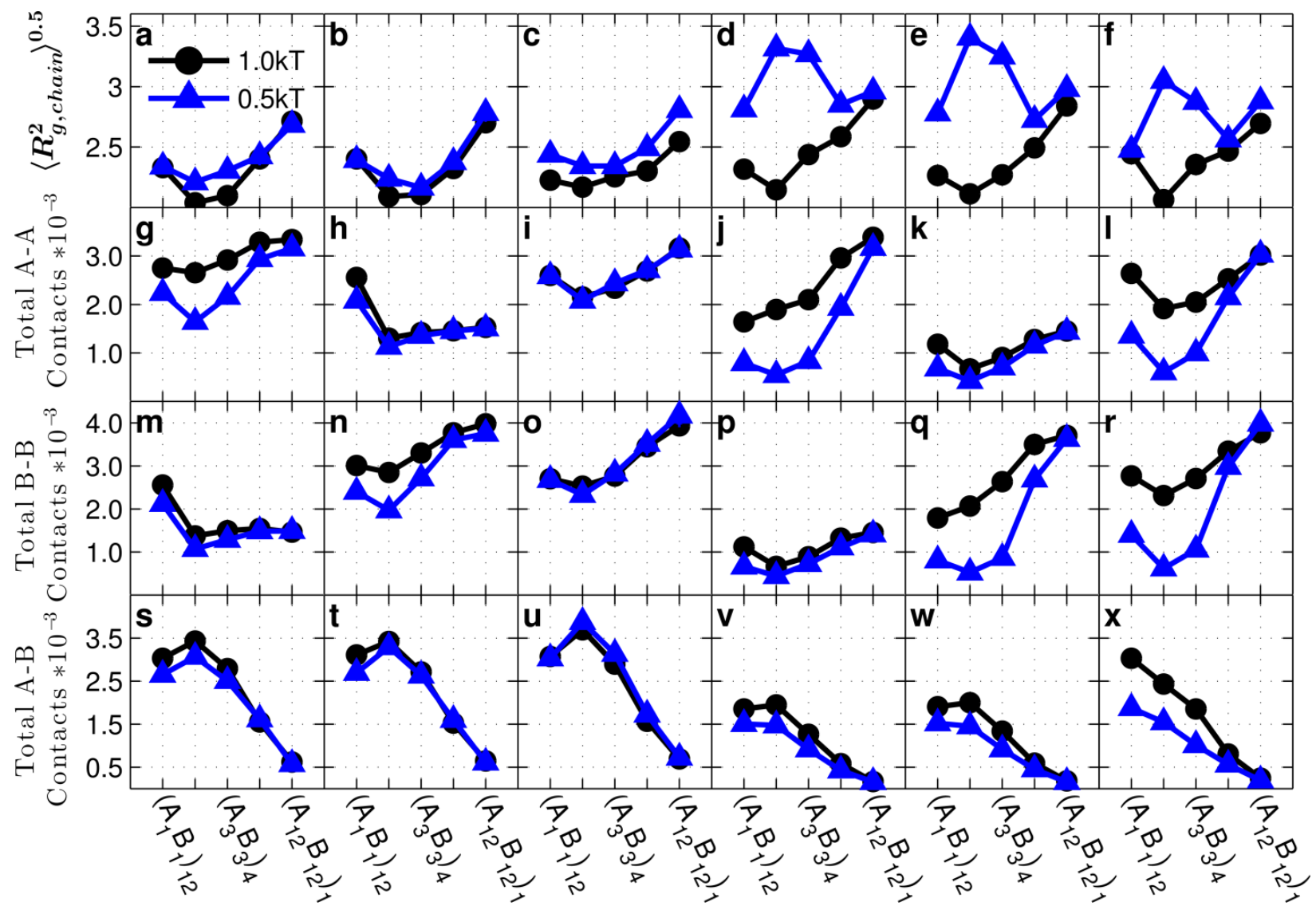

Figure 2.2: Average radius of gyration of a grafted chain $\left\langle R_{g \text {,chain }}\right\rangle^{0.5}$, average number of total $A A$ contacts, total BB contacts and total $A B$ contacts as a function of graft monomer sequence for copolymer grafted nanoparticles of size $D=4 d$ with six grafts of length $N_{\text {graft }}=24$. The $x$ - axis indices correspond to the graft sequence $\left(A_{1} B_{1}\right)_{12},\left(A_{2} B_{2}\right)_{6},\left(A_{3} B_{3}\right)_{4},\left(A_{6} B_{6}\right)_{2}$, and $\left(A_{12} B_{12}\right)_{1}$. The interaction sets correspond to the plot letters as follows: lines on plot $a, g, m$, and s correspond to interaction sets 1 (blue triangles) and 2 (black circles), lines on plot $b, h, n$, and $t$ correspond to 
interaction sets 3 (blue triangles) and 4 (black circles), lines on plot $c$, $i$, o, and $u$ correspond to interaction sets 5 (blue triangles) and 6 (black circles), lines on plot $d, j, p$, and v correspond to interaction sets 7 (blue triangles) and 8 (black circles), lines on plot $e, k, q$, and $w$ correspond to interaction sets 9 (blue triangles) and 10 (black circles), and lines on plot $f, l, r$, and $x$ correspond to interaction sets 11 (blue triangles) and 12 (black circles). The black lines with circle markers represent monomer-monomer attraction $=1 k T$, while the blue triangles with triangle markers represent monomer-monomer attraction=0.5kT. All monomer-particle interactions are maintained as hard sphere interactions.

First, we consider the effect of monomer sequence on grafted chain conformations in the assembled cluster/dispersed state for varying monomer-monomer interactions while maintaining monomer-particle interactions to be negligible (athermal). The effects of non-negligible monomerparticle interactions are discussed in Section 2.3.3. Figure 2.2a-f presents $\left\langle\mathrm{R}^{2} \mathrm{~g} \text {,chain }\right\rangle^{0.5}$ as a function of monomer sequence- $\left(\mathrm{A}_{1} \mathrm{~B}_{1}\right)_{12},\left(\mathrm{~A}_{2} \mathrm{~B}_{2}\right)_{6},\left(\mathrm{~A}_{3} \mathrm{~B}_{3}\right)_{4},\left(\mathrm{~A}_{6} \mathrm{~B}_{6}\right)_{2}$, and $\left(\mathrm{A}_{12} \mathrm{~B}_{12}\right)_{1}$-for interaction sets 1-12 in Table 2.1 with monomer-particle interaction maintained as hard-sphere for a system of 10 copolymer grafted nanoparticles of size $\mathrm{D}=4 \mathrm{~d}$ each with six grafts of length $\mathrm{N}_{\text {graft }}=24$.

In the absence of A-B repulsions, and in the presence of weak or strong A-A and/or B-B attractions (Figure 2.2a-c), with increasing blockiness from $\left(\mathrm{A}_{1} \mathrm{~B}_{1}\right)_{12}$ to $\left(\mathrm{A}_{12} \mathrm{~B}_{12}\right)_{1},\left\langle\mathrm{R}^{2} \text { g,chain }\right\rangle^{0.5}$ first decreases, reaches a minimum for sequences with intermediate blockiness, and then increases exhibiting the highest $\left\langle\mathrm{R}^{2} \text { g,chain }\right\rangle^{0.5}$ for the diblock sequence. The non-monotonic behavior with graft sequence can be explained by understanding how a grafted sequence can facilitate/deter attractive like-monomer contacts both within the same particle (intra-chain and intra-particle inter-chain) as well as with chains on another particle (inter-particle contacts). At higher blockiness, the grafted chains do not have to be too compact or too stretched to make these attractive contacts due to topologically favorable contiguous placement of like-monomers. In contrast, at low to intermediate blockiness, the grafted chains have to be either compact (low 
$\left\langle\mathrm{R}^{2} \text {,chain }\right\rangle^{0.5}$ ) for intra-chain attractive contacts or stretched (high $\left\langle\mathrm{R}^{2} \text { g,chain }\right\rangle^{0.5}$ ) for intra-particle inter-chain or inter-particle attractive contacts because the like-monomers are dispersed along the grafted chain. Compared to strong like-monomer attractions, the weak like-monomer attractions are inferior at driving the compaction of the grafted chains, especially at lower blockiness, because the enthalpic gain from weak attractive contacts cannot overcome the entropic loss of forming smaller chain conformations, leading to slightly higher $\left\langle\mathrm{R}^{2} \mathrm{~g} \text {,chain }\right\rangle^{0.5}$ at weak attractions than strong attractions.

In the presence of A-B repulsions, when the A-A and/or B-B attractions are strong enough to dominate over the A-B repulsions (black circles in Figure 2.2d-f), the results have the same trend as seen in the absence of A-B repulsion and our previous explanations for these trends hold. However, when A-A and/or B-B attractions are weak and A-B repulsions are strong (blue triangles in Figure 2.2d-e), the $\left\langle\mathrm{R}^{2} \text { g,chain }\right\rangle^{0.5}$ shows a different, non-monotonic trend. The three graft sequences with the lowest blockiness- $\left(\left(\mathrm{A}_{1} \mathrm{~B}_{1}\right)_{12},\left(\mathrm{~A}_{2} \mathrm{~B}_{2}\right)_{6},\left(\mathrm{~A}_{3} \mathrm{~B}_{3}\right)_{4}\right)$-lead to nanoparticles being dispersed (as discussed later in Figure 2.5j,k,1) and therefore all grafted chain conformations arise from maximizing intra-particle contacts. On the other hand, for the more blocky $\left(\left(\mathrm{A}_{6} \mathrm{~B}_{6}\right)_{2}\right.$ and $\left.\left(\mathrm{A}_{12} \mathrm{~B}_{12}\right)_{1}\right)$ sequences, the grafted nanoparticles assemble into clusters, where they are able to form inter-particle like-monomer contacts with lower stretching (i.e. lower $\left\langle\mathrm{R}^{2} \text { g,chain }\right\rangle^{0.5}$ ). Whether a sequence drives the copolymer grafted nanoparticles to form mostly inter-particle contacts or intra-particle contacts and how much the grafted chain has to stretch or coil up to make those contacts are dependent on the grafted sequence blockiness which governs how easy or hard it is to make attractive A-A or B-B contacts while avoiding repulsive A-B contacts.

We present the average number of total A-A, B-B, and A-B contacts in the second, third, and fourth row, respectively, in Figure 2.2 and the number of inter-particle, and inter-chain intra- 
particle contacts in Supplementary Section 2.7.2, as a function of monomer sequences at varying monomer-monomer interactions listed in Table 2.1.

For all interaction sets, leaving aside $\left(\mathrm{A}_{1} \mathrm{~B}_{1}\right)_{12}$ for now, as the grafted sequence blockiness increases from $\left(\mathrm{A}_{2} \mathrm{~B}_{2}\right)_{6}$ to $\left(\mathrm{A}_{12} \mathrm{~B}_{12}\right)_{1}$, the number of like monomer (A-A and $\left.\mathrm{B}-\mathrm{B}\right)$ contacts increases and the number of unlike monomer (A-B) contacts decreases. This is expected because, with increasing blockiness, the like-monomers are placed closer together thereby facilitating A-A and B-B aggregation and consequently minimizing A-B contacts. We will revisit the $\left(\mathrm{A}_{1} \mathrm{~B}_{1}\right)_{12}$ sequence shortly to discuss why it does not fit the trend shown by the other sequences. Interestingly, we note similar trends for the number of A-A contacts for grafted nanoparticles with A-A attraction (Figure 2.2g) and the number of B-B contacts for grafted nanoparticles with B-B attraction (Figure 2.2n) which allude to graft sequence and monomer-monomer interactions having a symmetric effect on chain conformations due to symmetric composition of the grafted copolymers. In other words, the A-A and B-B contact data are similar in pairs: Figure 2.2g and Figure 2.2n, Figure 2.2h and Figure 2.2m, Figure 2.2i and Figure 2.2o, Figure 2.2j and Figure 2.2q, Figure 2.2k and Figure 2.2p, Figure 2.21 and Figure 2.2r. Additionally, in Supplementary Section 2.7.2, the number of inter-particle, inter-chain, and intra-particle inter-chain contacts data further confirm these trends. They also show that, in the absence of A-B repulsion, with weak or strong A-A or B-B attraction, the total inter-particle and intra-particle inter-chain contacts decreases with increasing blockiness from $\left(\mathrm{A}_{2} \mathrm{~B}_{2}\right)_{6}$ to $\left(\mathrm{A}_{12} \mathrm{~B}_{12}\right)_{1}$. In the presence of A-B repulsion, with weak or strong A-A or B-B attraction the total inter-particle and intra-particle inter-chain contacts increases with increasing blockiness from $\left(\mathrm{A}_{2} \mathrm{~B}_{2}\right)_{6}$ to $\left(\mathrm{A}_{12} \mathrm{~B}_{12}\right)_{1}$. 


\subsubsection{Unique Behavior of $\left(A_{1} B_{1}\right)_{12}$}
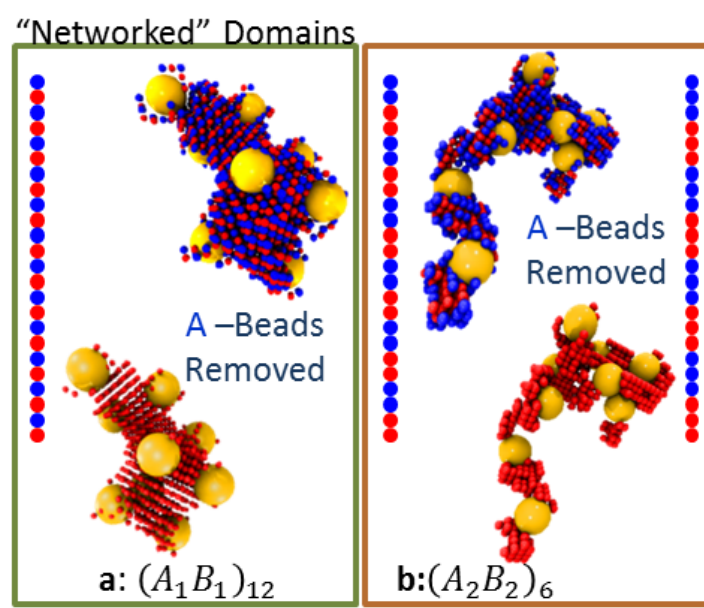

Distinct, Disconnected Domains
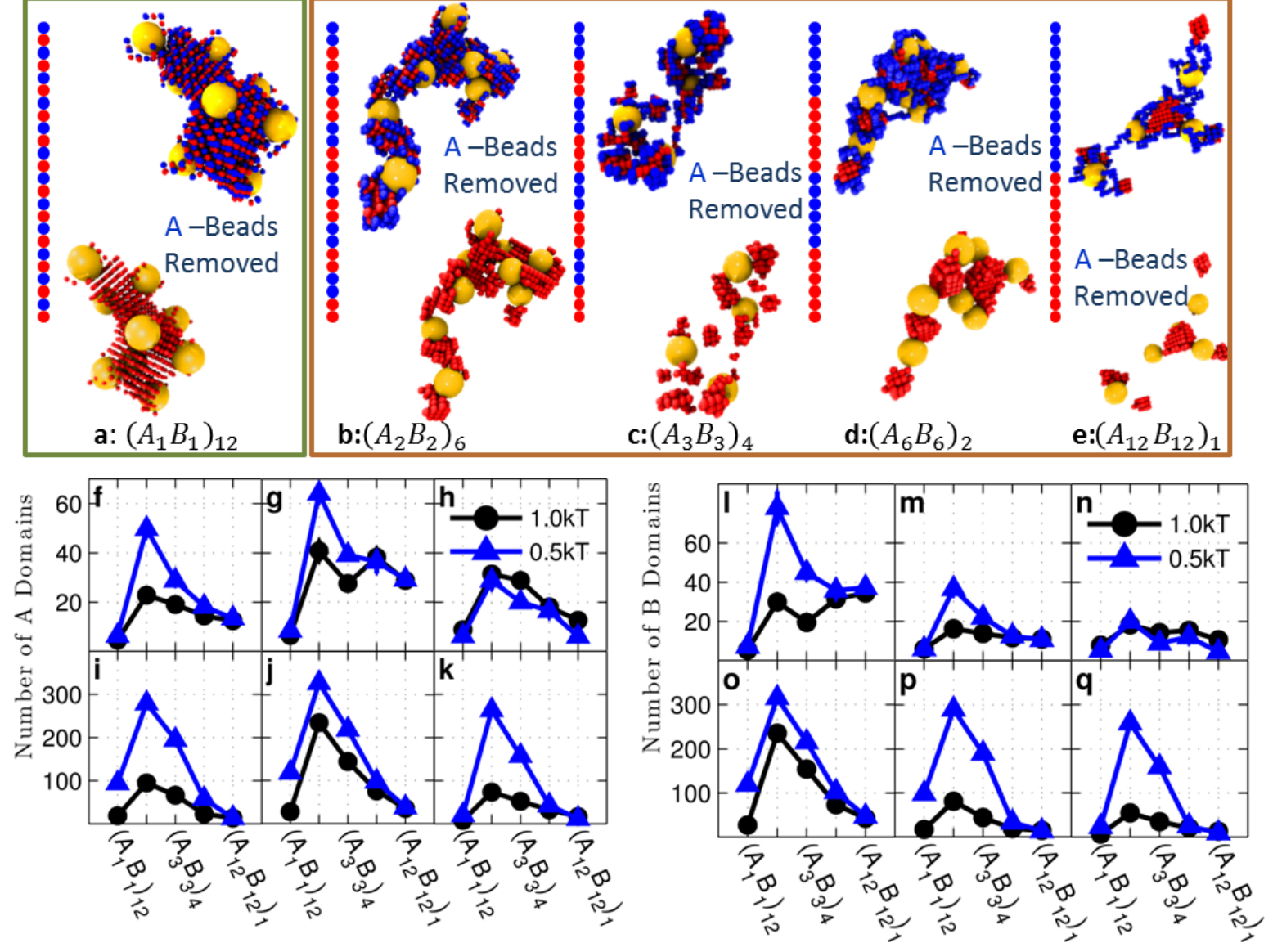

Figure 2.3: (a-e) Snapshots of clusters for systems with Interaction Set 4 and no monomer-particle attraction (best viewed in color). The plots show the average number of $A$ domains $(f-k)$ and $B$ domains (l-q) for copolymer grafted nanoparticles of size $D=4 d$ each with six grafts of length $N_{\text {graft }}=24$. The interaction sets correspond to the plot letters as follows: The interaction sets to the plot letters as follows: lines on plot f and l correspond to interaction sets 1 (blue triangles) and 2 (black circles), lines on plot $g$ and $m$ correspond to interaction sets 3 (blue triangles) and 4 (black circles), lines on plot h and n correspond to interaction sets 5 (blue triangles) and 6 (black circles), lines on plot $i$ and o correspond to interaction sets 7 (blue triangles) and 8 (black circles), lines on plot $j$ and p correspond to interaction sets 9 (blue triangles) and 10 (black circle), and lines on plot $k$ and $q$ correspond to interaction sets 11 (blue triangles) and 12 (black circles). The black circles represent monomer-monomer attraction $=1 \mathrm{kT}$, while the blue triangles represent monomermonomer attraction=0.5kT. All monomer-particle interactions are maintained as hard sphere interactions. 
The unique behavior of the alternating sequence, $\left(\mathrm{A}_{1} \mathrm{~B}_{1}\right)_{12}$, is observed across all of our analyses that describe chain conformations and monomer contacts. This behavior of $\left(A_{1} B_{1}\right)_{12}$ is connected to the patterns found within the aggregated monomer domains (Figure 2.3a) of the assembled clusters that are different from the patterns found within domains of assembled clusters formed by blockier sequences (Figure 2.3b-e). Figure 2.3a-e show simulation snapshots of particles grafted with copolymers in the presence of dominant B-B attraction (Table 2.1 interaction set 4). In the case of blockier sequences- $\left(\mathrm{A}_{2} \mathrm{~B}_{2}\right)_{6},\left(\mathrm{~A}_{3} \mathrm{~B}_{3}\right)_{4},\left(\mathrm{~A}_{6} \mathrm{~B}_{6}\right)_{2}$, and $\left(\mathrm{A}_{12} \mathrm{~B}_{12}\right)_{1}$-we find distinct domains of attractive like-monomers, where a domain is defined as a collection of like monomers that share mutual contacts. We can clearly see distinct domains of B monomers when the A monomers are hidden (bottom image of the snapshots). By making pure A and pure B domains, the grafted particles maximize attractive like-monomer contacts while reducing A-B contacts (if A-B repulsion is significant), while maintaining as high a conformational entropy as possible. In contrast to the distinct domains formed in blockier copolymers, in the case of $\left(A_{1} B_{1}\right)_{12}$ we see interpenetrated networks of like monomers (Figure 2.1) due to the frustrated-ABABsequence; in the presence A-B repulsion these domains are less ordered and tend to be fragmented into many "subdomains" (see supplementary movie online with Soft Matter 2011, 7 (13), 59525964.). The above domain patterns are also quantitatively represented via the average number of A (Figure 2.3f-k) and B (Figure 2.31-q) domains. Supplementary Section 2.7.3 confirms that while the bi-directional stripes are an artifact of the lattice model, the network structure is not biased due to the choice of a cubic lattice model, and is also seen in off-lattice models. For the $\left(\mathrm{A}_{1} \mathrm{~B}_{1}\right)_{12}$ sequences, the number of $\mathrm{A}$ and $\mathrm{B}$ domains are always low when compared to the intermediate blocky sequences, i.e. $\left(\mathrm{A}_{2} \mathrm{~B}_{2}\right)_{6},\left(\mathrm{~A}_{3} \mathrm{~B}_{3}\right)_{4}$, and $\left(\mathrm{A}_{6} \mathrm{~B}_{6}\right)_{2}$. For completeness, we have tabulated these data in Supplementary Section 2.7.4. For the blockier sequences, the number of distinct, 
homogeneous A or B domains decrease with increasing blockiness due to the increased likelihood of a neighboring monomer being a like-monomer. We believe these differences in the pattern of monomer aggregation between $\left(A_{1} B_{1}\right)_{12}$ and the other sequences helps explain why $\left(A_{1} B_{1}\right)_{12}$ does not follow the observed trends in chain conformation.

\subsubsection{Spatial Organization of Monomers}

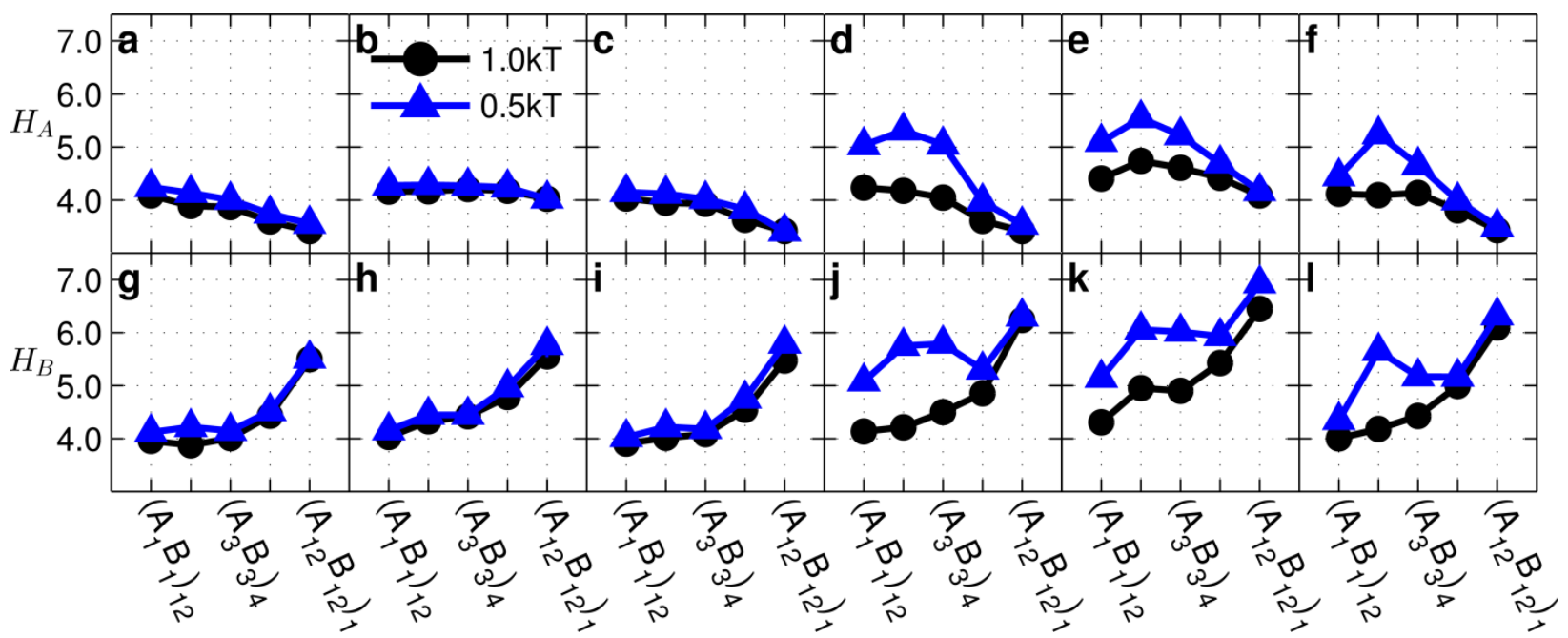

Figure 2.4: Average height of A-monomers (top row) and B-monomers (bottom row) from the particle centers as functions of monomer sequences for copolymer grafted nanoparticles of size $D=4 d$ with six grafts of length $N_{\text {graft }}=24$. The interaction sets correspond to the plot letters as follows: lines on plot a and g correspond to interaction sets 1 (blue triangles) and 2 (black circles), lines on plot b and h correspond to interaction sets 3 (blue triangles) and 4 (black circles), lines on plot c and $i$ correspond to interaction sets 5 (blue triangles) and 6 (black circles), lines on plot $d$ and $j$ correspond to interaction sets 7 (blue triangles) and 8 (black circles), lines on plot e and $k$ correspond to interaction sets 9 (blue triangles) and 10 (black circles), and lines on plot f and $l$ correspond to interaction sets 11 (blue triangles) and 12 (black circles). The black circles represent monomer-monomer attraction $=1 \mathrm{kT}$, while the blue triangles represent monomermonomer attraction=0.5kT. All monomer-particle interactions are maintained as hard sphere interactions.

While the discussion so far has been on the number of like (A-A or B-B) and unlike (A-B) monomer contacts and the nature of the aggregated A- or B-domains, it does not explain the spatial 
arrangement of these monomers around the particle surface. In Figure 2.4, we capture the spatial arrangement of the A and B monomers by plotting the average distance (or height) of A and B monomers from the center of the particle they are grafted to as a function of monomer sequence and monomer-monomer interactions.

When A-B repulsion is negligible and the A- and/or B- monomers are attractive, we observe that $\mathrm{H}_{\mathrm{A}}$ (Figure 2.4a-c) decreases slightly or is constant with increasing blockiness while $\mathrm{H}_{\mathrm{B}}$ (Figure 2.4g-i) increases with increasing blockiness. At low blockiness, both A and B monomers tend to be relatively close to the particle surface because the chains must be compact (Figure 2.2a-c) in order to make sufficient attractive like-monomer intrachain contacts. As blockiness increases, the monomers become more topologically separated, allowing attractive monomers to more easily aggregate either further from the surface (B-monomers) or closer to the particle surface (A-monomers). At higher blockiness, the B-monomers also tend to aggregate further from the particle surface in order to increase the likelihood of inter-particle contacts and therefore the number of possible B-B contacts, while A-monomers cannot access as many of these inter-particle contacts due to the steric hindrance from the B-block.

In the presence of A-B repulsion and strong A-A and/or B-B attraction, we observe similar trends in $\mathrm{H}_{\mathrm{A}}$ and $\mathrm{H}_{\mathrm{B}}$ as discussed above for the absence of $\mathrm{AB}$ repulsion. This is in line with our discussion of $\left\langle\left.\mathrm{R}_{\mathrm{g}, \text { chain }}^{2}\right|^{0.5}\right.$ which showed that, when $\varepsilon_{\mathrm{AB}}=\left(\varepsilon_{\mathrm{AA}}, \varepsilon_{\mathrm{BB}}\right)$, the effect of A-B repulsion is minimal on chain conformation. Cases with irregular trends in $\mathrm{H}_{\mathrm{A}}$ and $\mathrm{H}_{\mathrm{B}}$ (Figure 2.4jkl) are due to lower blockiness sequences favouring dispersions and higher blockiness sequences favouring assembly. 


\subsubsection{Effect of Monomer Sequence on Nanoparticle Arrangement in the Cluster}

\subsubsection{Size and Connectivity of Clusters}

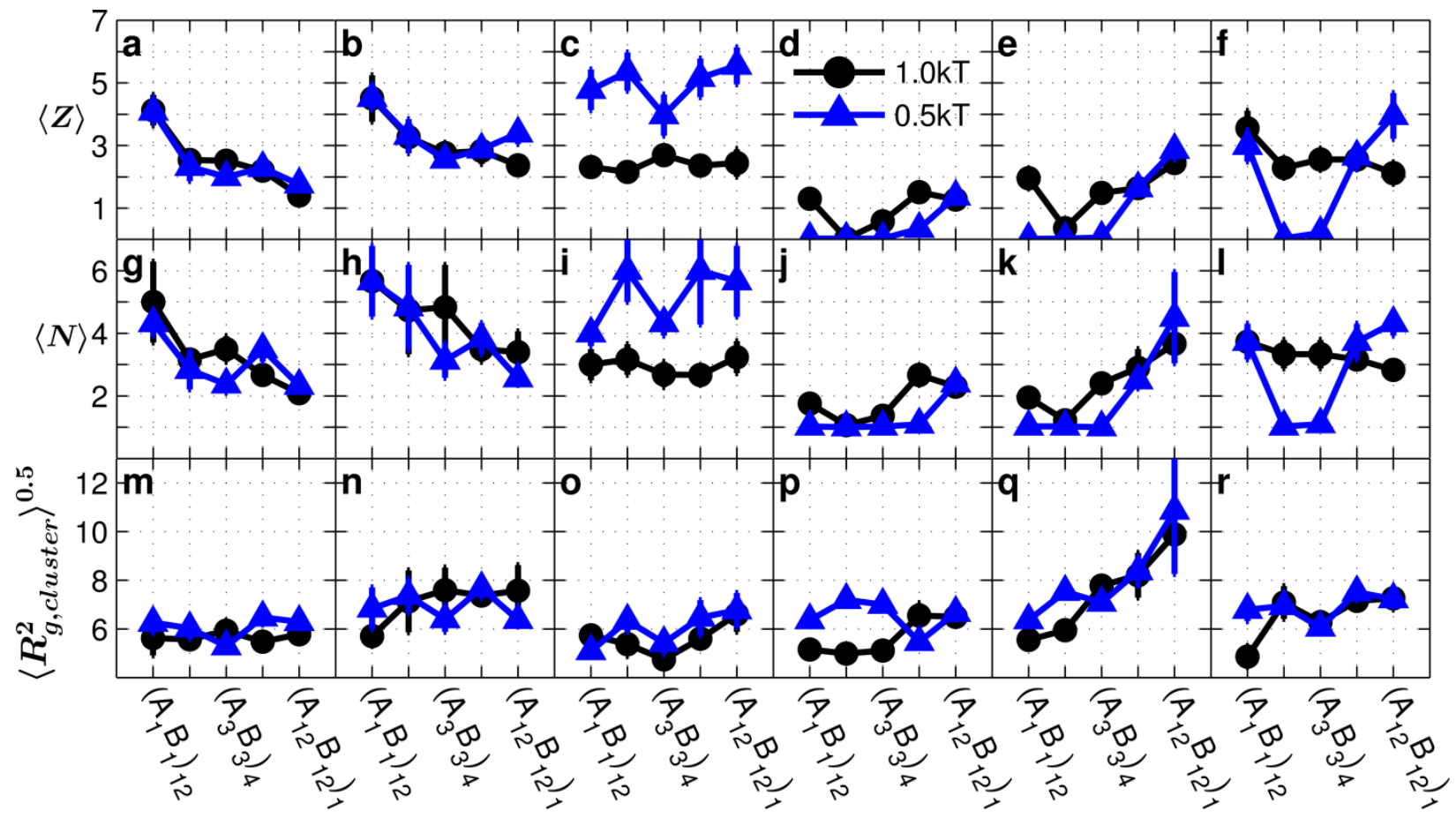

Figure 2.5: Average coordination number $(Z)$, average number of particles per cluster $(N)$, and average radius of gyration of a cluster $\left\langle R^{2}{ }_{g, c l u s t e r}\right\}^{0.5}$ as a function of monomer sequences for copolymer grafted nanoparticles of size $D=4 d$ with six grafts of length $N_{\text {graft }}=24$. The interaction sets correspond to the plot letters as follows: lines on plot $a, g$, and $m$ correspond to interaction sets 1 (blue triangles) and 2 (black circle), lines on plot $b, h$, and $n$ correspond to interaction sets 3 (blue triangles) and 4 (black circle), lines on plot $c$, $i$, and o correspond to interaction sets 5 (blue triangles) and 6 (black circle), lines on plot $d, j$, and p correspond to interaction sets 7 (blue triangles) and 8 (black circle), lines on plot $e, k$, and $q$ correspond to interaction sets 9 (blue triangles) and 10 (black circle), and lines on plot f, l, and r correspond to interaction sets 11 (blue triangles) and 12 (black circle). The black circles represent monomer-monomer attraction=1 $k T$, while the blue triangles represent monomer-monomer attraction $=0.5 \mathrm{kT}$. All monomer-particle interactions are maintained as hard sphere interactions. 
Next, we discuss the impact of the chain conformations and monomer aggregates presented above on the characteristics of the assembled cluster i.e. the relative sizes and overall structure within the cluster as a function of monomer sequence at various interactions.

In the absence of A-B repulsion when only A-A (Figure 2.5a,g) or B-B (Figure 2.5b,h) interactions are attractive, as blockiness increases, both the average number of neighbors each nanoparticle has in a cluster or the coordination number, $\langle Z\rangle$, and the average number of particles in a cluster, $\langle\mathrm{N}\rangle$, decrease. This is because, with increasing blockiness, the grafted chains are able to form a higher number of favorable intra-chain conformations without losing as much entropy, which in turn decreases the probability of forming inter-particle contacts, and hence the propensity for assembly is diminished. Using Supplementary Figure 2.13, which shows the average number inter-chain contacts, and the data in Figure 2.2, the approximate number of intra-chain contacts can be estimated. We also note the slightly higher $\langle\mathrm{Z}\rangle$ and $\langle\mathrm{N}\rangle$ for systems with B-B attraction when compared to systems with A-A attraction. This is due to the lower effective reach of the A monomers (Figure 2.4g) which inhibits grafted nanoparticles with A-A attractions from aggregating as compared to grafted nanoparticles with B-B attractions. Interestingly, while $\langle\mathrm{Z}\rangle$ and $\langle\mathrm{N}\rangle$ decrease with increasing blockiness, the $\left\langle\mathrm{R}_{\mathrm{g}, \mathrm{cluster}}^{2}\right\rangle^{0.5}$ (Figure $2.5 \mathrm{~m}$ and Figure $2.5 \mathrm{n}$ ) is either constant or increases slightly with increasing blockiness. This effect is a consequence of the grafts becoming less compact with increasing blockiness, as shown by $\left\langle\mathrm{R}_{\mathrm{g}, \mathrm{chain}}\right\rangle^{0.5}$ in Figure $2.2 \mathrm{a}$ and Figure 2.2b.

In the presence of both A-A and B-B attractions and absence of A-B repulsions (Figure 2.5c, Figure 2.5i, and Figure 2.5o), the copolymer chains seek to maximize both A-A and B-B contacts. For both strong (black circles) and weak (blue triangles) A-A and B-B attractions $\langle\mathrm{Z}\rangle$, $\langle\mathrm{N}\rangle$ and $\left\langle\mathrm{R}_{\mathrm{g}, \text { luster }}\right\rangle^{0.5}$ are statistically constant with monomersequence. In effect, the presence of 
both like-monomer attractions causes the grafted nanoparticles to achieve similar cluster sizes and internal connectivity regardless of monomer sequence. We also observe that the chain conformations with both A-A and B-B attraction and no A-B repulsion also show more constant trends with increasing blockiness than chains with only A-A or only B-B attraction, as evidenced by the data in Figure 2.2. Due to the fact that both A-A and B-B monomers are attractive, the probability of a chain making favorable monomer-monomer contact is greatly increased, also shown by the contact profiles in Figure 2.2. Due to the chains' reduced barriers to find favorable conformations, the effect of monomer sequence is diminished because monomer sequence mainly affects chain conformations by introducing barriers to favorable enthalpic contacts. Therefore, copolymer grafted nanoparticles with both A-A and B-B monomer attraction assemble into relatively consistent configurations regardless of graft sequence.

In the presence of A-B repulsion, when either A-A (Figure 2.5d,j,p), B-B (Figure 2.5e,k,q), or both are attractive (Figure 2.5f,j,r), $\langle\mathrm{Z}\rangle,\langle\mathrm{N}\rangle$, and $\left\langle\mathrm{R}^{2} \text { g,cluster }\right\rangle^{0.5}$ all increase with blockiness with the exception of $\left(\mathrm{A}_{1} \mathrm{~B}_{1}\right)_{12}$ which tends to deviate for strong A-A or B-B attraction strength (black circles). These trends are in contrast to the behavior seen in the absence of A-B repulsion, where increasing blockiness led to decreasing or constant $\langle\mathrm{N}\rangle$ and $\langle\mathrm{Z}\rangle$. This is because, at low blockiness, where A and B monomers are topologically not separated, the presence of A-B repulsion lowers the propensity to assemble and in turn reduces the $\langle\mathrm{N}\rangle$ and $\langle\mathrm{Z}\rangle$. As blockiness increases, the A-B repulsion plays a smaller role because the A and B monomers are segregated topologically, and therefore the values of $\langle\mathrm{Z}\rangle$ and $\langle\mathrm{N}\rangle$ remain similar to that seen in the absence of $A-B$ repulsion 


\subsubsection{Shape of Clusters}

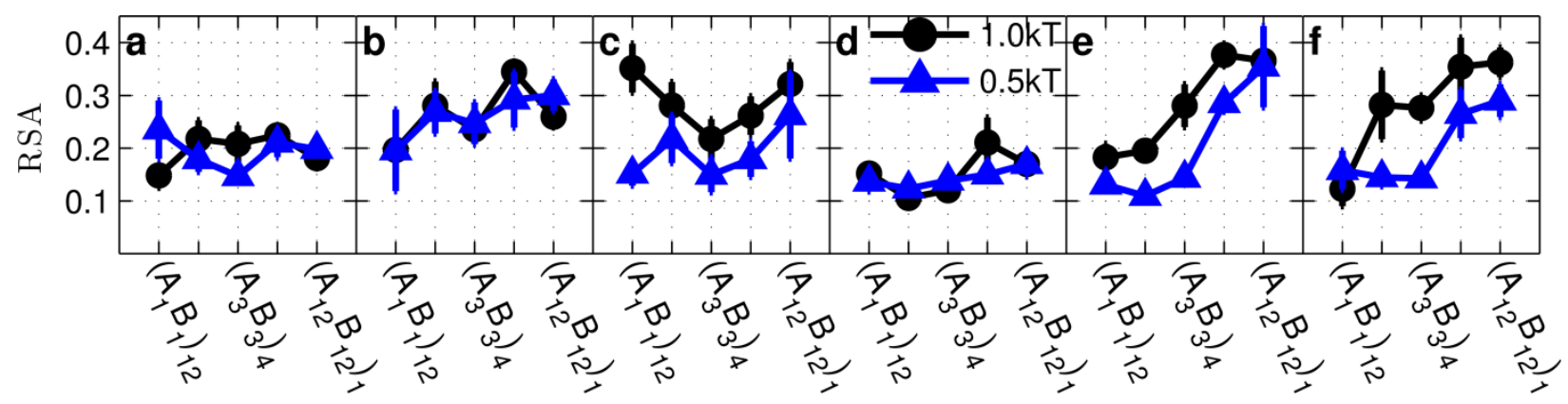

Figure 2.6: Relative shape anisotropy (RSA) as a function of monomer sequence on copolymer grafted nanoparticles for copolymer grafted nanoparticles of size $D=4 d$ with six grafts of length $N_{\text {graft }}=24$. The interaction sets correspond to the plot letters as follows: lines on plot a correspond to interaction sets 1 (blue triangles) and 2 (black circle), lines on plot b correspond to interaction sets 3 (blue triangles) and 4 (black circle), lines on plot c correspond to interaction sets 5 (blue triangles) and 6 (black circle), lines on plot d correspond to interaction sets 7 (blue triangles) and 8 (black circle), lines on plot e correspond to interaction sets 9 (blue triangles) and 10 (black circle), and lines on plotf correspond to interaction sets 11 (blue triangles) and 12 (black circle). The black lines with circle markers represent monomer-monomer attraction=1kT, while the blue triangles with triangle markers represent monomer-monomer attraction=0.5kT. All monomerparticle interactions are maintained as hard sphere interactions.

In order to quantify the shape of assembled clusters, we present relative shape anisotropy data (RSA) in Figure 2.6. The RSA data presented here characterizes the global anisotropy of a cluster rather than the local anisotropy about each polymer grafted particle in a cluster. For example, a large network formed by clustered copolymer grafted nanoparticles could have a low RSA because of global symmetry about the cluster's center of mass, even though there is significant anisotropy locally within the network of aggregated monomers.

In the absence of A-B repulsion and only A-A (Figure 2.6a) or B-B attractions (Figure 2.6b), we observe statistically constant RSA regardless of graft sequence while strong A-A and BB attraction (Figure 2.6c) show non-monotonic trends. In comparing simulations with only A-A 
attraction to simulations with only B-B attraction, we observe that statistically significant differences in RSA values only appear for $\left(\mathrm{A}_{6} \mathrm{~B}_{6}\right)_{2}$ and $\left(\mathrm{A}_{12} \mathrm{~B}_{12}\right)_{1}$. Since the differences between A-A and B-B attraction can be attributed to the differing locations of the A and B monomers along the chain, which becomes more pronounced with increasing blockiness as shown by $\mathrm{H}_{\mathrm{A}}$ and $\mathrm{H}_{\mathrm{B}}$ in Figure 2.4, it follows that the greatest difference in RSA between A-A and B-B attraction would be observed for the most blocky sequences. At high blockiness, when there are only A-A attractions, the attractive A-monomers aggregate close to the particle surface (Figure 2.4a) which forces other grafted nanoparticles to be close in order to make inter-particle A-A contacts. This means that particles need to pack neighbors into a smaller volume (closer to the surface) leading to a more isotropic distribution (low RSA). In contrast, when there is only B-B attractions at high blockiness, the attractive B monomers tend to aggregate farther from the particle surface into few patches (low $\langle Z\rangle$ and number of domains) leading to anisotropic cluster shapes.

In the presence of A-B repulsion and only A-A attractions (Figure 2.6d) we do not see a significant effect of sequence on RSA, since much of the cluster shape is driven by attractive Adomains being close to the surface forming isotropic clusters. Since presence of A-B repulsion affects the height of A-monomers (Figure 2.4a and Figure 2.4d) only for sequences with the lowest blockiness, where the sequence itself drives isotropic assembly, there is no effect of A-B repulsion on RSA. In contrast, for B-B attractions (either by themselves or with A-A attractions), the presence of A-B repulsions (Figure 2.6e and Figure 2.6f) seems to amplify the effect of increasing blockiness on RSA, making the clusters span a larger range of RSA. It is interesting that, unlike the contact data in Figure 2.2, there is no symmetry in the effect of A-A versus B-B attraction on cluster shape. Instead, we observe marked differences between the two types of monomer attractions. This lack of symmetry is again due to A-monomers being located much closer to the 
surface at high blockiness when compared to B-monomers. In contrast, for B-B attraction in the presence of A-B repulsion, $\mathrm{H}_{\mathrm{B}}$ in Figure 2.4k and Figure 2.41, and the number of $\mathrm{B}$ domains in Figure 2.3p and Figure 2.3q show that the B-monomers move further from particles' surfaces and become clustered into fewer domains which would cause clusters to become more "stretched" and therefore more anisotropic.

To summarize, systems with B-B monomer attraction in the presence of A-B repulsion show the greatest effect of graft sequence on cluster shape, while systems with A-A attraction produce relatively isotropic clusters regardless of graft sequence.

\subsubsection{Effect of Particle Size}

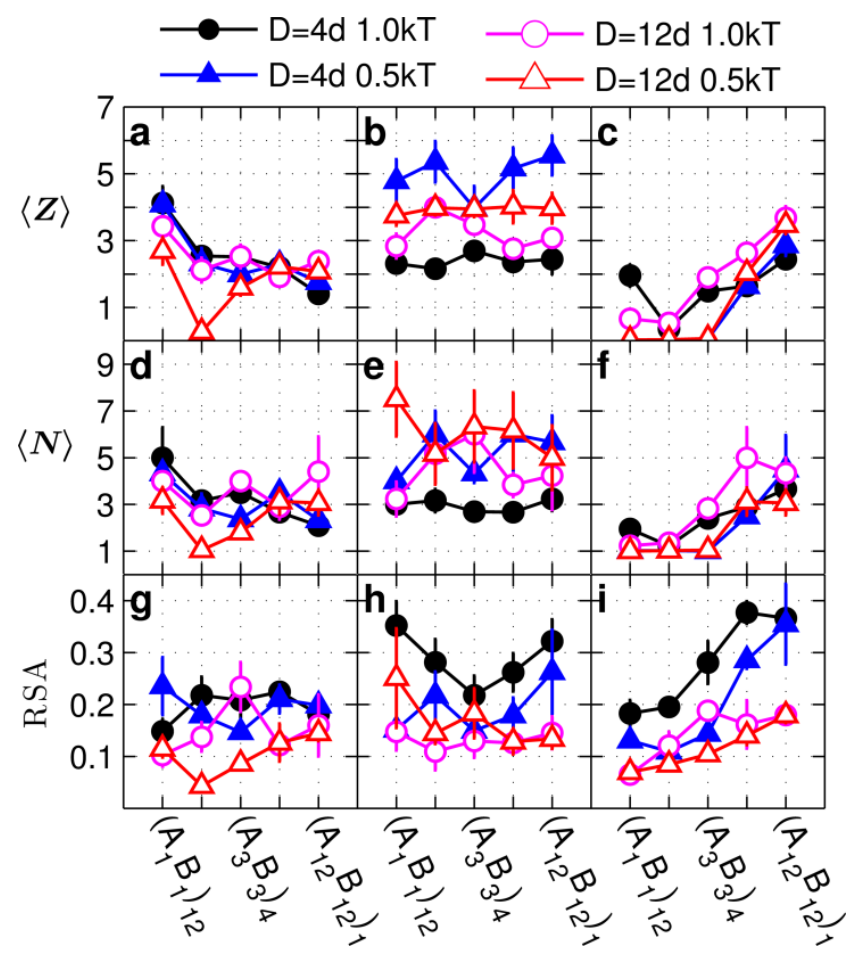

Figure 2.7: Average coordination number $(Z)$, average number of particles per cluster $(N)$, and relative shape anisotropy (RSA) as a function of monomer sequence for copolymer grafted nanoparticles of size $D=4 d$ and $D=12 d$ with six grafts of length $N_{\text {graft }}=24$. The lines on plot $a, d$, and $g$ correspond to interaction sets 1 (triangles) and 2 (circles), lines on plot $b$, $e$, and $h$ correspond to interaction sets 5 (triangles) and 6 (circles), lines on plot $c, f$, and $i$ correspond to 
interaction sets 9 (triangles) and 10 (circles The circles represent monomer-monomer attraction $=1 \mathrm{kT}$, while the triangles represent monomer-monomer attraction $=0.5 \mathrm{kT}$. The filled symbols represent nanoparticles of size $D=4 d$ while the open symbols represent nanoparticles of size $D=12 d$. All monomer-particle interactions are maintained as hard sphere interactions

To assess how the graft length to particle size ratio affects the trends presented so far, we present $\langle\mathrm{Z}\rangle,\langle\mathrm{N}\rangle$, and RSA for three selected interaction sets in Figure 2.7 for particles of size $\mathrm{D}=4 \mathrm{~d}$ and 12d. The remaining interaction sets are presented in Supplementary Figure 2.26, and show similar trends as the ones discussed in Figure 7.

In general, we expect grafted nanoparticles with higher particle-size to graft length (D/N $\mathrm{N}_{\text {graft }}$ ratios, at a constant number of grafts, to show lowered propensity for cluster formation because the grafted chains must adopt more extended and entropically costly conformations than lower $\mathrm{D} / \mathrm{N}_{\text {graft }}$ to form either intra-particle inter-chain or inter-particle contacts. In particular, the barrier to intra-particle inter-chain contacts, due to large $\mathrm{D}$ or small $\mathrm{N}_{\text {graft }}$, forces the chains to assume compact conformations to maximize intra-chain contacts, which in turn minimizes probability of forming inter-particle contacts due to reduced reach of the chain. The inter-particle and intra-particle inter-chain data in Supplementary Information Section 2.7.2 supports these conjectures. To summarize, increasing $\mathrm{D} / \mathrm{N}_{\text {graft }}$ introduces more entropic barriers to monomer aggregation by increasing the distance between intra-particle chains, and similarly reducing the number of accessible inter-particle contacts.

The differences in cluster connectivity, size and shape, between $D=4 d$ and $D=12 d$, are significant only for specific combinations of interaction set and interaction strength that complement the entropic barriers introduced by the increased (D/ $\left.\mathrm{N}_{\text {graft }}\right)$.

With only A-A attraction (Figure 2.7a,d), we see statistically indistinguishable trends in $\langle Z\rangle$ and $\langle N\rangle$ between $D=4 d$ and $D=12 d$ except for $\left(A_{2} B_{2}\right)_{6}$ with weak $A-A$ attraction. Like $D=4 d$, 
the trend in $\langle Z\rangle$ and $\langle N\rangle$ for $D=12 d$ is non-monotonic with graft sequence because the grafts of sequence $\left(A_{1} B_{1}\right)_{12}$ form spatially distributed networks of like monomers as opposed to the distinct disconnected domains seen with the other sequences (Figure 2.3 and Supplementary Figure 2.27). Also, the dip in $\langle Z\rangle$ at $\left(A_{2} B_{2}\right)_{6}$ for $D=12 d$ (open symbols) is exaggerated compared to the rest of the data because the nanoparticles are in a dispersed state.

In case of $\mathrm{A}-\mathrm{A}$ and $\mathrm{B}-\mathrm{B}$ attraction, in the absence of $\mathrm{A}-\mathrm{B}$ repulsion, the data (second column Figure 2.7 ) are mostly indistinguishable when comparing $D=4 d$ and $D=12 d$ for both weak and strong monomer attraction. As discussed above for $\mathrm{D}=4 \mathrm{~d}$, for $\mathrm{D}=12 \mathrm{~d}$ having both types of monomer attraction diminishes the effects of monomer sequence due to the increased number of possible favorable contacts, also reducing the effect of increasing $\mathrm{D} / \mathrm{N}_{\text {graft }}$.

\subsubsection{Effects of Monomer-Particle Interactions on Assembly}

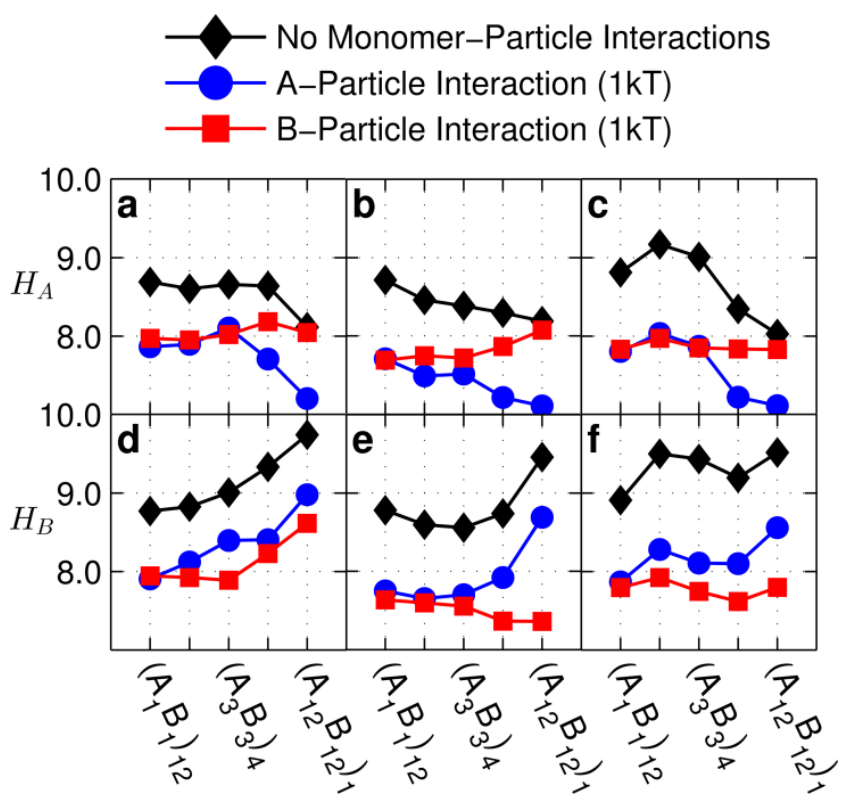

Figure 2.8: Average height of A-monomers (top row) and B-monomers (bottom row) from the particle surface as a function of monomer sequences for copolymer grafted nanoparticles $D=12 d$ with six grafts of length $N_{\text {graft }}=24$ for interaction sets with no particle monomer interactions (black diamonds), A-particle attraction=1kT (blue circles), and B-particle attraction=1 $\mathrm{kT}$ (red squares). The interaction sets correspond to the plot letters as follows (with particle-monomer interactions 
indicated by line color and marker shape): lines on plot a and d correspond to interaction set 4, lines on plot b and e, correspond to interaction set 1 , lines on plot $c$ and $f$ correspond to interaction set 11 .

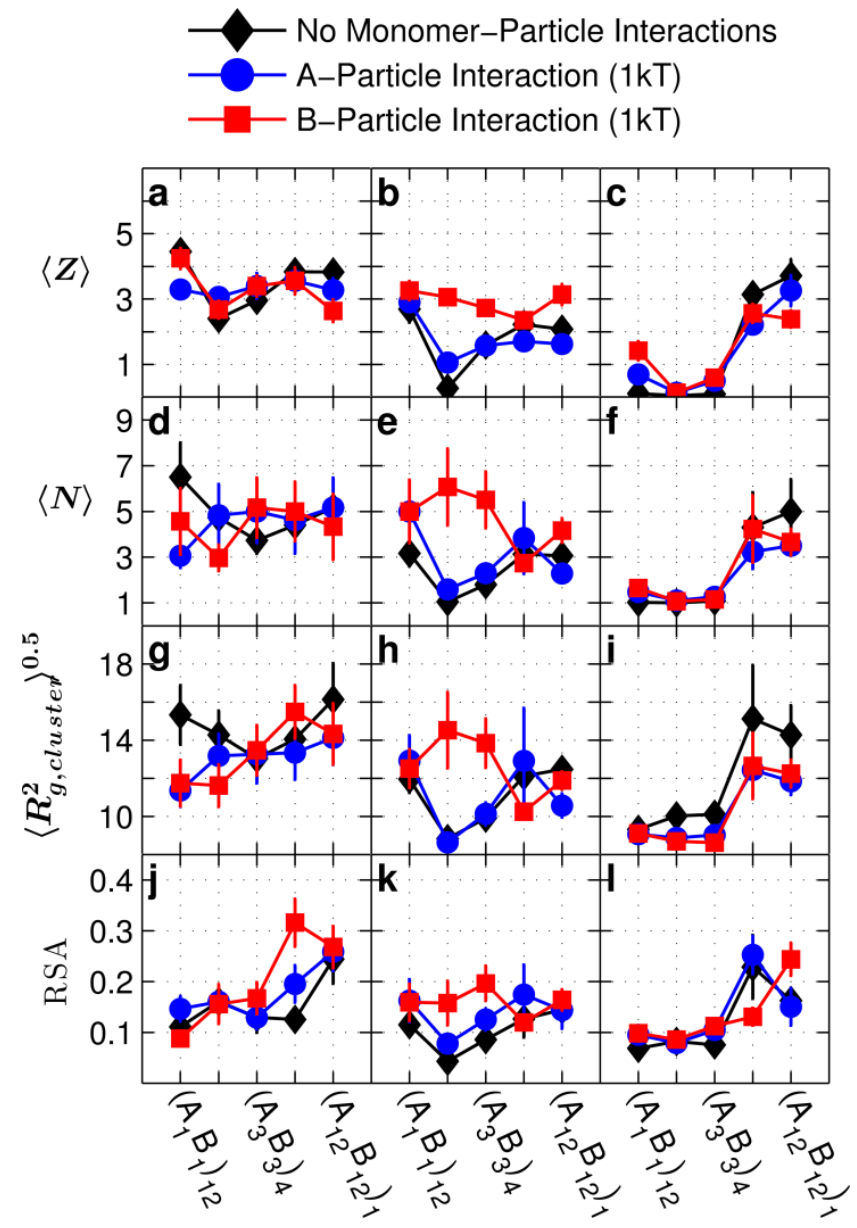

Figure 2.9: Average coordination number $(Z)$, average number of particles per cluster $\langle N\rangle$, average radius of gyration of a cluster $\left\langle R_{g, \text { cluster }}^{2}\right\rangle^{0.5}$ and relative square anisotropy (RSA) as a function of monomer sequence for copolymer grafted nanoparticles $D=12 d$ with six grafts of length $N_{\text {graft }}=24$ for systems with no particle monomer interactions (black diamonds), A-particle attraction $=1 \mathrm{kT}$ (blue circles), and B-particle attraction=1 $\mathrm{kT}$ (red squares). The interaction sets correspond to the plot letters as follows (with particle-monomer interactions indicated by line color and marker shape): lines on plot $a, d, g$, and $j$ correspond to interaction set 4 , lines on plot $b, e, h$, and $k$ correspond to interaction set 1 , lines on plot $c, f, i$, and $l$ correspond to interaction set 11 . 
The discussion of the effect of a grafted chain's monomer sequence on the assembled nanoparticle cluster size, structure, and shape presented so far has been in the absence of any monomer-particle interactions. For particle diameter $\mathrm{D}=4 \mathrm{~d}$, we find that while monomer-particle interactions (either A-particle or B-particle) affect the local ordering of monomers, these effects do not manifest into differences in coordination number, size, or shape of the assembled cluster. This is because, for a $\mathrm{D}=4 \mathrm{~d}$ particle with six grafts of length $\mathrm{N}_{\text {graft }}=24$ each, the small attractive surface area compared to the long grafts result in the number of monomer-monomer interactions outnumbering the possible monomer-particle interactions. See Supplementary Information Section 2.7.6 for a presentation of our full dataset on copolymer grafted nanoparticles in the presence of monomerparticle interactions. In Supplementary 2.7.7, we also provide a discussion of the differences between monomer-monomer interactions and monomer-particle interactions in our simulation.

For nanoparticles with $D=12 \mathrm{~d}$ with the same number of grafts and graft length as $D=4 \mathrm{~d}$, due to an increased surface area over $\mathrm{D}=4 \mathrm{~d}$, we observe a slightly increased effect of monomerparticle interactions on the chain conformations and particle assembly. In order to understand how these monomer-particle interactions affect the location of A and B monomers, we present the average height of an A monomer $\left(\mathrm{H}_{\mathrm{A}}\right)$ and $\mathrm{B}$ monomer $\left(\mathrm{H}_{\mathrm{B}}\right)$ from the particle surface in the presence of A-particle or B-particle attraction in Figure 2.8. We then connect this to the effect of monomer-particle interactions on assembly, via $\langle\mathrm{Z}\rangle,\langle\mathrm{N}\rangle,\left\langle\mathrm{R}_{\mathrm{g}, \mathrm{cluster}}{ }^{0.5}\right.$, and RSA data for three interaction sets of grafted particles of $\mathrm{D}=12 \mathrm{~d}$ in Figure 2.9. Our complete data set for $\mathrm{H}_{\mathrm{A}}, \mathrm{H}_{\mathrm{B}},\langle\mathrm{Z}\rangle$, $\langle\mathrm{N}\rangle,\left\langle\mathrm{R}_{\mathrm{g}, \mathrm{cluster}}\right\rangle^{0.5}$, and RSA in the presence of A-particle or B-particle interactions for both $\mathrm{D}=4 \mathrm{~d}$ and $\mathrm{D}=12 \mathrm{~d}$ is presented in the Supplementary Information Sections 2.7.2 and 2.7.6.

In Figure 2.8 for all interactions presented, we observe that A-particle attraction reduces $\mathrm{H}_{\mathrm{A}}$, and B-particle attraction reduces $\mathrm{H}_{\mathrm{B}}$ by approximately one unit for all sequences. We also see 
that $\mathrm{H}_{\mathrm{A}}$ decreases or is constant with increasing blockiness in the presence of A-particle attraction, while $\mathrm{H}_{\mathrm{A}}$ is relatively constant for all sequences in the presence of B-particle attraction. In contrast, $\mathrm{H}_{B}$ with B-particle attraction is increasing or constant with increasing blockiness while $\mathrm{H}_{\mathrm{B}}$ with A-particle attraction is always increasing. This is because, at low blockiness, the monomers attracted to the particle drags the unlike-monomers, while at higher blockiness, due to topological separation along the graft, the monomers not-attracted to the particle surface are not dragged.

Despite the significant effect of monomer-particle interactions on $\mathrm{H}_{\mathrm{A}}$ and $\mathrm{H}_{\mathrm{B}}$, in case of strong B-B attraction and no A-B repulsion, the monomer-particle interactions only slightly affect $\langle\mathrm{Z}\rangle$ (Figure 2.9a) and $\langle\mathrm{N}\rangle$ (Figure 2.9d) for two sequences- $\left(\mathrm{A}_{1} \mathrm{~B}_{1}\right)_{12}$ and $\left(\mathrm{A}_{12} \mathrm{~B}_{12}\right)_{1}$ and there are no statistically significant effects of monomer-particle interactions on the $\left\langle\mathrm{R}_{\mathrm{g} \text {,cluster }}\right\rangle^{0.5}$ for any of the sequences (Figure 2.9g). As for RSA, (Figure 2.9j) in the presence of B-particle attraction (red line), there seems to be a significant increase in RSA at $\left(\mathrm{A}_{6} \mathrm{~B}_{6}\right)_{2}$ as compared to no particlemonomer attraction (black diamonds) or with A-particle attraction (blue circles). However, this sharp rise in RSA does not seem to correspond with any change in the trend for monomer position in Figure 2.8a or Figure 2.8d or monomer contacts in Supplementary Figure 2.11-Figure 2.20.

In Figure 2.9b, Figure 2.9e, Figure 2.9h, and Figure 2.9k, with weak A-A attraction in the absence of A-B repulsion we see an increased effect of monomer-particle interactions on the assembled cluster as compared to the grafted nanoparticles with strong B-B attraction (Figure 2.9a, $\mathrm{d}, \mathrm{g}, \mathrm{j})$. In particular, we observe the greatest effect on the size $\left(\langle\mathrm{N}\rangle\right.$ and $\left.\left\langle\mathrm{R}_{\mathrm{g}, \mathrm{cluster}}^{2}\right\rangle^{0.5}\right)$ and connectivity $(\langle Z\rangle)$ of the clusters in the case of B-particle attraction, i.e. when the monomer-particle (B-particle) interactions do not compete with the monomer-monomer (A-A) interactions. When monomer-particle and monomer-monomer interactions do not compete, the monomers can easily aggregate to maximize favorable contacts both with the particle and other like monomers. 
Furthermore, leaving aside $\left(A_{1} B_{1}\right)_{12}$ for its uniqueness, the effect of monomer-particle attraction is greatest at low blockiness $\left(\left(\mathrm{A}_{2} \mathrm{~B}_{2}\right)_{6}\right.$ and $\left.\left(\mathrm{A}_{3} \mathrm{~B}_{3}\right)_{4}\right)$. Since the low blockiness sequences form tight, compact conformations (Figure 2.2a-f) that make inter-particle contacts difficult, it follows that for these sequences the monomer-particle interactions would encourage particle-monomer contacts more often than in blockier sequences. $\left(\mathrm{A}_{1} \mathrm{~B}_{1}\right)_{12}$ does not follow this trend due to its unique domain as discussed is Section 2.3.1.2. We observe that monomer-particle interactions do not have a significant effect on RSA (Figure 2.9k) for this interaction set.

In the case of weak A-A and B-B attraction in the presence of A-B repulsion (Figure 2.9c) either A-particle or B-particle attraction only slightly affects $\langle Z\rangle$ at low blockiness. We observe no significant effects of A-particle or B-particle attractions on $\langle\mathrm{N}\rangle$ (Figure 2.9f) or RSA (Figure 2.91), or $\left\langle\mathrm{R}_{\mathrm{g}, \mathrm{cluster}}{ }^{0.5}\right.$ (Figure 2.9i). The lack of an effect of particle-monomer interactions, suggests that the loss in conformational entropy by creating particle-monomer contacts cannot be overcome if the grafted chains also face enthalpic penalty due to repulsive A-B contacts made in the process of making favourable monomer-particle contacts.

Unlike the symmetry in monomer-monomer contacts seen in the absence of monomerparticle interactions (Figure 2.2), there is no symmetry in the effect of switching between Aparticle and B-particle interactions. With A-particle attractions, since the A-monomers are grouped closer to the surface of the nanoparticle with increasing blockiness (Figure 2.8), the A-monomers collapse onto the surface of the nanoparticle they are grafted on. This only slightly affects the reach of the attractive A-block resulting in clusters with negligible changes $\langle\mathrm{N}\rangle$ and $\left\langle\mathrm{R}_{\text {g,cluster }}^{2}{ }^{0.5}\right.$ (blue lines: Figure 2.9d, Figure 2.9e, Figure 2.9g, and Figure 2.9h). In contrast, B-particle contacts (either intra-particle or inter-particle) are harder to make than A-particle contacts, because B monomers are farther away from the surface and the A-monomers that are close to the particle 
surface sterically hinder B-particle contacts. Further compounding the complexity of monomerparticle interactions is the effect of competing or non-competing monomer-monomer interactions as was discussed above.

Overall, for particles of $\mathrm{D}=12 \mathrm{~d}$, the increased surface area of the larger particle enhances the effect of monomer-particle interaction, but the effect is still limited by the lower number of possible monomer-particle contacts as compared to the number of possible non-bonded monomermonomer contacts (see Supplementary Information Section 2.7.7 for a detailed discussion). One would expect that a significantly stronger monomer-particle attraction (>>1kT) would overwhelm the relatively weaker monomer-monomer attraction, and lead to significant effects on the assembly. We tested this hypothesis for alternating and diblock copolymer grafted particles, and observed a drastic effect of monomer-particle interaction for grafted nanoparticles with weak monomer-monomer $(\sim 0.5-1 \mathrm{kT})$ and strong monomer-particle interactions of $\sim 16 \mathrm{kT}$, as compared commensurate monomer-monomer and monomer-particle attractions (see Supplementary Figure 2.37). In general, when the monomer-particle interactions are greatly increased, we see that many of the trends going from $\left(\mathrm{A}_{1} \mathrm{~B}_{1}\right)_{12}$ to $\left(\mathrm{A}_{12} \mathrm{~B}_{12}\right)_{1}$ are flipped in comparison to the case where monomer-monomer and monomer-particle attractions are equal. Thus, the relative strength of monomer-particle interactions and monomer-monomer interactions heavily dictates the extent to which monomer-particle interactions can affect characteristics of the assembled cluster.

\subsection{CONCLUSIONS}

We have conducted Monte Carlo simulations to study copolymer grafted spherical nanoparticles placed in an implicit solvent to elucidate the effect of blockiness in the grafted copolymer sequence at varying monomer-monomer and monomer-particle interactions on size, shape and structure of assembled copolymer grafted nanoparticles. The grafted chain conformations are closely linked to 
how monomer sequence facilitates the chain's ability to form energetically favorable attractive monomer contacts while minimizing unfavorable repulsive monomer contacts. As the sequence blockiness increases, attractive monomer contacts are brought together more easily due to the favorable topological placement of like monomers along the chain. Within the assembled nanocluster, the alternating sequence, $\left(\mathrm{A}_{1} \mathrm{~B}_{1}\right)_{12}$, forms "networks" of attractive monomer contacts in contrast to the distinct domains of attractive monomers formed by blockier monomer sequences. In the case of A-A or B-B monomer attraction without A-B repulsion, we observed decreasing cluster size and decreasing connectivity within the cluster with increasing blockiness for particle sizes $\mathrm{D}=4 \mathrm{~d}$ and $\mathrm{D}=12 \mathrm{~d}$, with $\left(\mathrm{A}_{1} \mathrm{~B}_{1}\right)_{12}$ always being an outlier in these trends. With the addition of A-B repulsion to A-A or B-B monomer attraction, clusters of both particle size $\mathrm{D}=4 \mathrm{~d}$ and $12 \mathrm{~d}$ show increasing size and increasing connectivity with increasing blockiness. For $\mathrm{D}=4 \mathrm{~d}$ particles, with strong B-B attraction and A-B repulsion, increasing blockiness leads to increasing anisotropy in cluster shape, while strong A-A attraction produce isotropic clusters regardless of graft sequence. The effect of graft sequence on cluster shape is reduced for $D=12 d$ as compared to $D=4 d$ at constant graft length. Lastly, monomer-particle interactions only weakly affect the assembly of copolymer grafted nanoparticles when the particle size is small relative to the graft length, and the strength of particle-monomer interactions are commensurate with the monomer-monomer interactions. The effects of monomer-particle interactions on the cluster size, shape, and structure are enhanced with significantly strong particle-monomer interactions, and/or with substantially large particle sizes relative to graft lengths.

The studies presented in this paper are focused only on dilute concentration of $\mathrm{c} \sim 1 \mathrm{x} 10^{-5}$ particles per $\mathrm{nm}^{3}$, obtained by using 10 particles in a $100 \times 100 \times 100\left(\mathrm{~nm}^{3}\right)$ simulation box with periodic boundary conditions. Keeping in mind that an experimentalist working at these 
concentrations could observe clusters with greater than 10 nanoparticles we have been careful to emphasize qualitative trends in the data with varying parameters, and not discuss specific quantitative data (especially $\langle\mathrm{N}\rangle$ ). We have also carefully considered our choice of a lattice model and have tried to minimize lattice effects on our analysis, and focused on general qualitative trends that should hold true in off-lattice models as well. In some cases, we have conducted some offlattice tests to ensure unique behavior of alternating copolymer (e.g. network of A and B monomers) is not an artifact of the lattice model.

The trends presented here should guide experimentalists seeking to create target morphologies of copolymer grafted nanoparticles for various applications such as metamaterials, photonics, photovoltaics and electronics. For example, in materials design for photovoltaic application, the spatial organization of the constituent chemistries dictates how well charges separate and travel to corresponding electrodes, and in turn device efficiency[42]. This work shows how copolymer grafted nanoparticle assembly could be used and the sequence of grafted polymers chosen to control the number and structure of the monomers domains around the nanoparticles.

Some of the future directions of this work include investigating the dynamics during assembly of copolymer grafted particles. Also, understanding both equilibrium and dynamic behavior of these copolymer grafted nanoparticles in a polymer matrix would be useful to tailoring morphologies in polymer nanocomposites. Previous work using a self-consistent PRISM-MC scheme, has shown that the potential of mean force (PMF) between AB copolymer grafted nanoparticles in a A or B homopolymer matrix differs from that seen in homopolymer grafted nanoparticles in a homopolymer matrix (with graft and matrix chemistries being identical), and that the PMF is a function of copolymer sequence.[13] It would be interesting to study how at varying volume fractions of grafted particles, the presence of explicit polymer matrix, and matrix 
chain conformations affect the grafted chain conformations, and in turn the assembly/dispersion of the particles in the matrix.

\subsection{ACKNOWLEDGEMENTS}

This work was partially supported by National Science Foundation under grant number CBET0930940 and partially by National Science Foundation-Research Experience for Undergraduates (REU) for Functional Materials at University of Colorado.

\subsection{REFERENCES}

1. Camargo, M., S.A. Egorov, and C.N. Likos, Cluster formation in star-linear polymer mixtures: equilibrium and dynamical properties. Soft Matter, 2012.

2. Meng, D., et al., Effective interactions between grafted nanoparticles in a polymer matrix. Soft Matter, 2012. 8(18): p. 5002-5010.

3. Pryamtisyn, V., et al., Modeling the anisotropic self-assembly of spherical polymer-grafted nanoparticles. Journal of Chemical Physics, 2009. 131(22).

4. Witten, T.A. and P.A. Pincus, COLLOID STABILIZATION BY LONG GRAFTED POLYMERS. Macromolecules, 1986. 19(10): p. 2509-2513.

5. Clark, M.D. and R. Krishnamoorti, Dispersion of Functionalized Multiwalled Carbon Nanotubes. Journal of Physical Chemistry C, 2009. 113: p. 20861-20868.

6. Goel, V., et al., Structure of Polymer Tethered Highly Grafted Nanoparticles. Macromolecules, 2011. 44(20): p. 8129-8135.

7. Krishnamoorti, R., Strategies for Dispersing Nanoparticles in Polymers. MRS Bulletin, 2007. 32(4): p. 341-347.

8. Jayaraman, A. and K.S. Schweizer, Effect of the number and placement of polymer tethers on the structure of concentrated solutions and melts of hybrid nanoparticles. Langmuir, 2008. 24(19): p. 11119-11130.

9. Jayaraman, A. and K.S. Schweizer, Structure and Assembly of Dense Solutions and Melts of Single Tethered Nanoparticles. J. Chem. Phys., 2008. 128: p. 164904.

10. Jayaraman, A. and K.S. Schweizer, Effective Interactions, Structure, and Phase Behavior of Lightly Tethered Nanoparticles in Polymer Melts. Macromolecules, 2008. 41(23): p. 9430-9438.

11. Jayaraman, A. and K.S. Schweizer, Effective Interactions and Self-Assembly of Hybrid Polymer Grafted Nanoparticles in a Homopolymer Matrix. Macromolecules, 2009. 42 p. pp 8423-8434. 
12. Martin, T.B., A. Seifpour, and A. Jayaraman, Assembly of copolymer functionalized nanoparticles: a Monte Carlo simulation study. Soft Matter, 2011. 7(13): p. 5952-5964.

13. Nair, N. and A. Jayaraman, Self-Consistent PRISM Theory-Monte Carlo Simulation Studies of Copolymer Grafted Nanoparticles in a Homopolymer Matrix. Macromolecules, 2010. 43(19): p. 8251-8263.

14. Nair, N., N. Wentzel, and A. Jayaraman, Effect of bidispersity in grafted chain length on grafted chain conformations and potential of mean force between polymer grafted nanoparticles in a homopolymer matrix. Journal of Chemical Physics, 2011. 134: p. 194906.

15. Popov, K.I., et al., Interacting nanoparticles with functional surface groups. Journal of Polymer Science Part B-Polymer Physics, 2012. 50(12): p. 852-862.

16. Vorselaars, B., et al., Self-consistent field theory for diblock copolymers grafted to a sphere. Soft Matter, 2011. 7(11): p. 5128-5137.

17. Zhu, X., et al., Ordered Nanostructures Self-Assembled from Block Copolymer Tethered Nanoparticles. Acs Nano, 2010. 4(9): p. 4979-4988.

18. Dodd, P.M. and A. Jayaraman, Monte carlo simulations of polydisperse polymers grafted on spherical surfaces. Journal of Polymer Science Part B-Polymer Physics, 2012. 50(10): p. 694-705.

19. Ghanbari, A., et al., Interphase Structure in Silica-Polystyrene Nanocomposites: A CoarseGrained Molecular Dynamics Study. Macromolecules, 2012. 45(1): p. 572-584.

20. Hu, S.W., Y.J. Sheng, and H.K. Tsao, Self-Assembly of Organophilic Nanoparticles in a Polymer Matrix: Depletion Interactions. Journal of Physical Chemistry C, 2012. 116(2): p. 1789-1797.

21. Shen, J.X., et al., Revisiting the Dispersion Mechanism of Grafted Nanoparticles in Polymer Matrix: A Detailed Molecular Dynamics Simulation. Langmuir, 2011. 27(24): p. 15213-15222.

22. Akcora, P., et al., Anisotropic self-assembly of spherical polymer-grafted nanoparticles. Nature Materials, 2009. 8(4): p. 354-359.

23. Srivastava, S., P. Agarwal, and L.A. Archer, Tethered Nanoparticle-Polymer Composites: Phase Stability and Curvature. Langmuir, 2012. 28(15): p. 6276-6281.

24. Chan, E.R., L.C. Ho, and S.C. Glotzer, Computer simulations of block copolymer tethered nanoparticle self-assembly. Journal of Chemical Physics, 2006. 125(6): p. 9.

25. Seifpour, A., et al., Effect of monomer sequences on conformations of copolymers grafted on spherical nanoparticles: A Monte Carlo simulation study. Journal of Chemical Physics, 2010. 132(16).

26. Jiao, Y. and P. Akcora, Assembly of Polymer-Grafted Magnetic Nanoparticles in Polymer Melts. Macromolecules, 2012. 45(8): p. 3463-3470.

27. Lin, Y.L., et al., Self-Assembled Superstructures of Polymer-Grafted Nanoparticles: Effects of Particle Shape and Matrix Polymer. Journal of Physical Chemistry C, 2011. 115(13): p. 5566-5577. 
28. Iacovella, C.R. and S.C. Glotzer, Phase behavior of ditethered nanospheres. Soft Matter, 2009. 5(22): p. 4492-4498.

29. Glotzer, S.C., Nanotechnology Shape Matters. Nature, 2012. 481(7382): p. 450-452.

30. Lan, Q., L.F. Francis, and F.S. Bates, Silica nanoparticles dispersions in homopolymer versus block copolymer. Journal of Polymer Science Part B-Polymer Physics, 2007. 45: p. 2284-2299.

31. Glotzer, S.C. and J.A. Anderson, NANOPARTICLE ASSEMBLY Made to order. Nature Materials, 2010. 9(11): p. 885-887.

32. Knorowski, C. and A. Travesset, Materials design by DNA programmed self-assembly. Current Opinion in Solid State \& Materials Science, 2011. 15(6): p. 262-270.

33. van Anders, G. and S.C. Glotzer, DNA NANOTECHNOLOGY The world's smallest assembly line. Nature Chemistry, 2012. 4(2): p. 79-80.

34. Park, S.Y., et al., DNA programmable nanoparticle crystallization. Nature, 2008. 451: p. 553-556.

35. Jones, M.R., et al., DNA-nanoparticle superlattices formed from anisotropic building blocks. Nature Materials, 2010. 9(11): p. 913-917.

36. Crocker, J.C., Nanomaterials: Golden handshake. Nature, 2008. 451(7178): p. 528-529.

37. Kim, J., et al., Highly effective gold nanoparticle-enhanced biosensor array on the wettability controlled substrate by wiping. Journal of Applied Physics, 2011. 110(8): p. 7.

38. Keller, K., A. Amirian, and P. Akcora, Elastic Properties of a Protein-Polymer-Grafted Surface. Langmuir, 2012. 28(8): p. 3807-3813.

39. Frenkel, D. and B. Smit, Understanding Molecular Simulation: From Algorithms to Applications. 2nd ed. 2002, San Diego: Academic Press.

40. Metropolis, N., et al., EQUATION OF STATE CALCULATIONS BY FAST COMPUTING MACHINES. Journal of Chemical Physics, 1953. 21(6): p. 1087-1092.

41. Theodorou, D.N. and U.W. Suter, SHAPE OF UNPERTURBED LINEAR-POLYMERS POLYPROPYLENE. Macromolecules, 1985. 18(6): p. 1206-1214.

42. Slota, J.E., X. He, and W.T.S. Huck, Controlling nanoscale morphology in polymer photovoltaic devices. Nano Today, 2010. 5(3): p. 231-242. 


\subsection{SUPPLEMENTARY}

2.7.1 Types of Monomer Contacts in Clusters/Dispersions of Copolymer Grafted Nanoparticles

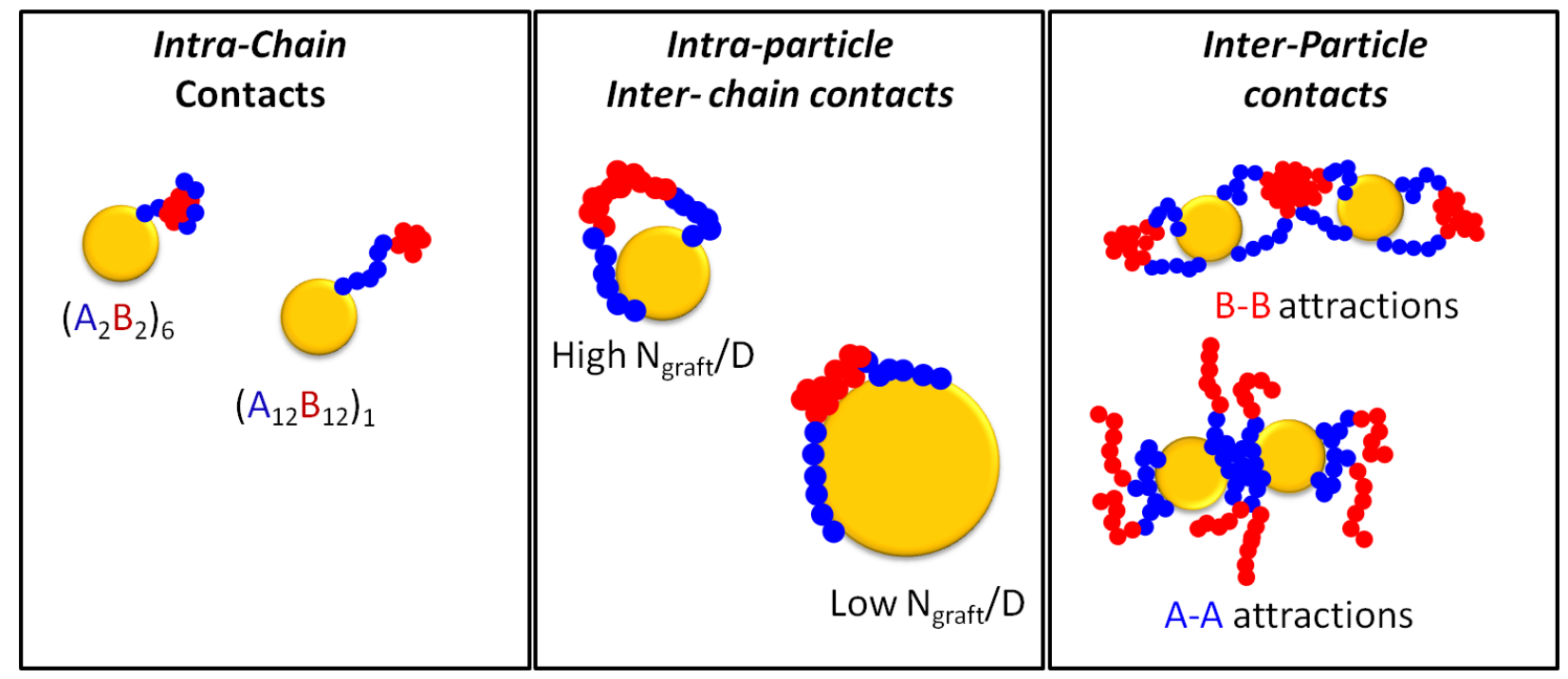

Figure 2.10: Schematic showing the three types of monomer contacts that could be made in these systems of copolymer grafted nanoparticles. 


\subsubsection{Effects of Monomer Sequence on Grafted Chain Conformations and Monomer Aggregation in the Cluster for $D=4 d$ and $D=12 d$ with and without Monomer-Particle Interactions}

The figures in this section consist of parts a-i that connect to the interaction sets in a way different from the main manuscript. The table below should help with connecting the figure parts to interaction sets. We present the data in this order to show some symmetry in A-A and B-B interaction effects, in presence and absence of A-B repulsion, and monomer-particle attractions.

Table 2.2: Interaction sets and figure parts

\begin{tabular}{cccccc}
$\begin{array}{c}\text { Interaction } \\
\text { Set }\end{array}$ & $\begin{array}{c}\text { Figure } \\
\text { part }\end{array}$ & $\boldsymbol{\varepsilon}_{\mathrm{AB}} / \mathbf{k} \mathbf{T}$ & $\boldsymbol{\varepsilon}_{\mathrm{AA}} / \mathbf{k} \mathbf{T}$ & $\mathbf{\varepsilon B B} / \mathbf{k T}$ & $\chi_{\mathrm{AB}} / \mathrm{kT}$ \\
\hline 1 & $\mathrm{~d}$ & 0.0 & -0.5 & 0.0 & 1 \\
2 & $\mathrm{~b}$ & 0.0 & -1.0 & 0.0 & 2 \\
3 & $\mathrm{c}$ & 0.0 & 0.0 & -0.5 & 1 \\
4 & $\mathrm{a}$ & 0.0 & 0.0 & -1.0 & 2 \\
5 & $\mathrm{j}$ & 0.0 & -0.5 & -0.5 & 2 \\
6 & $\mathrm{i}$ & 0.0 & -1.0 & -1.0 & 4 \\
7 & $\mathrm{~h}$ & 1.0 & -0.5 & 0.0 & 5 \\
8 & $\mathrm{f}$ & 1.0 & -1.0 & 0.0 & 6 \\
9 & $\mathrm{~g}$ & 1.0 & 0.0 & -0.5 & 5 \\
10 & $\mathrm{e}$ & 1.0 & 0.0 & -1.0 & 6 \\
11 & 1 & 1.0 & -0.5 & -0.5 & 6 \\
12 & $\mathrm{k}$ & 1.0 & -1.0 & -1.0 & 8 \\
\hline
\end{tabular}




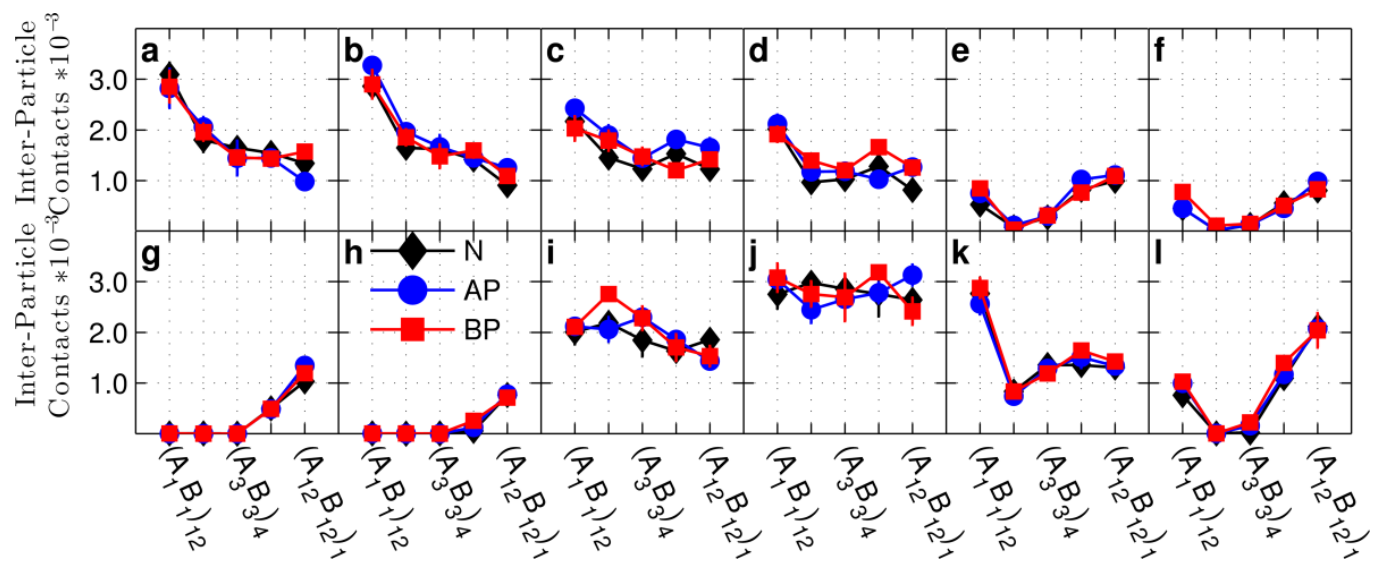

Figure 2.11: Average number of interparticle contacts as a function of monomer sequence for copolymer grafted nanoparticles of $D=4 d$ each with six grafts of length Ngraft $=24$ with no particle monomer interactions (black diamonds), A-monomer to particle attraction at -1kT (blue circles), and B-monomer to particle attraction at - $1 k T$ (red squares). The system interactions correspond to the plot letters as in Table 2.2 (with particle-monomer interactions indicated by line color)

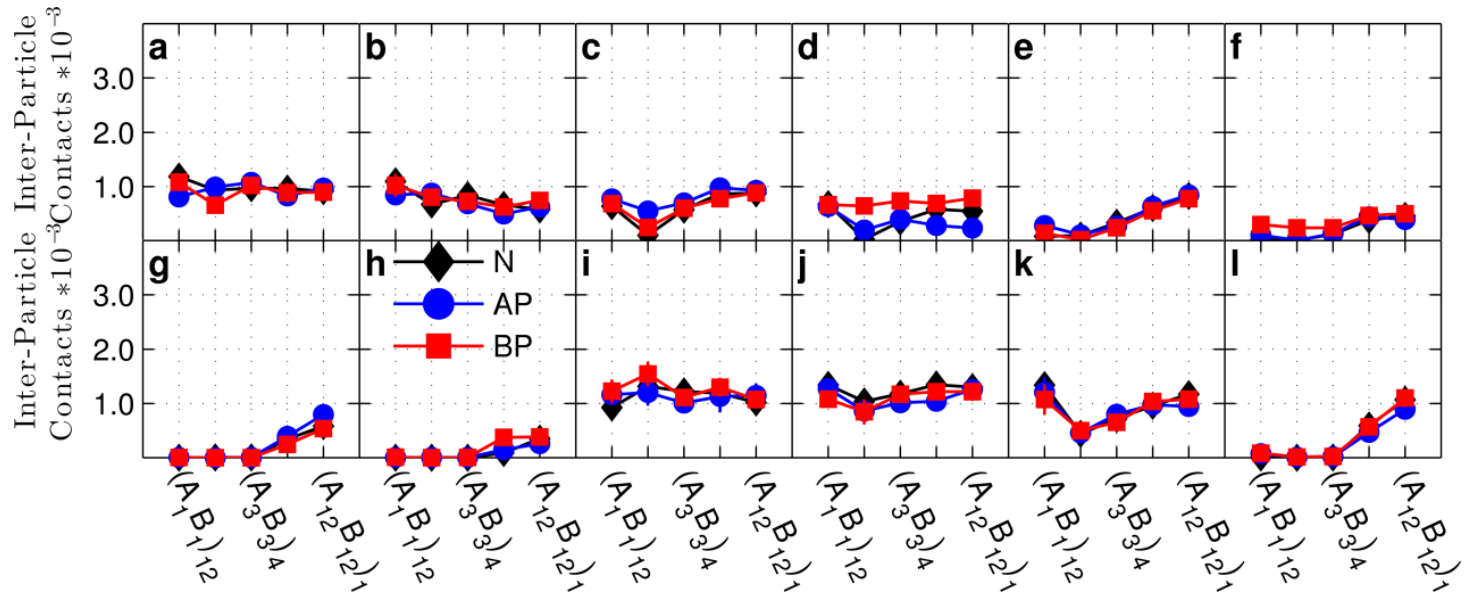

Figure 2.12: Average number of interparticle contacts as a function of monomer sequence for copolymer grafted nanoparticles of $D=12 d$ each with six grafts of length Ngraft=24 with no particle monomer interactions (black diamonds), A-monomer to particle attraction at -1kT (blue circles), and B-monomer to particle attraction at -1kT (red squares). The system interactions correspond to the plot letters as in Table 2.2 (with particle-monomer interactions indicated by line color) 


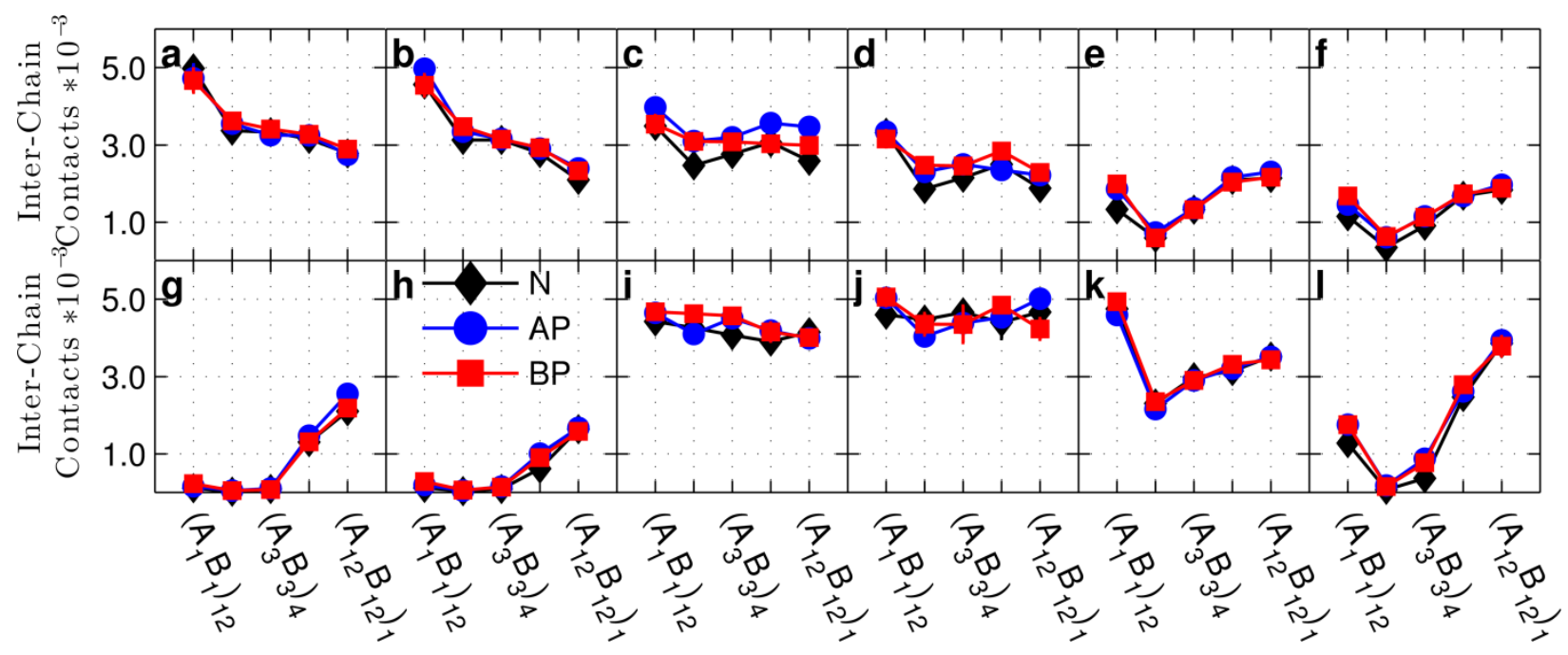

Figure 2.13: Average number of inter-chain contacts, including inter and intra particle, as a function of monomer sequence for copolymer grafted nanoparticles of $D=4 d$ each with six grafts of length Ngraft=24 with no particle monomer interactions (black diamonds), A-monomer to particle attraction at - $1 k T$ (blue circles), and B-monomer to particle attraction at - $1 k T$ (red squares). The system interactions correspond to the plot letters as in Table 2.2 (with particlemonomer

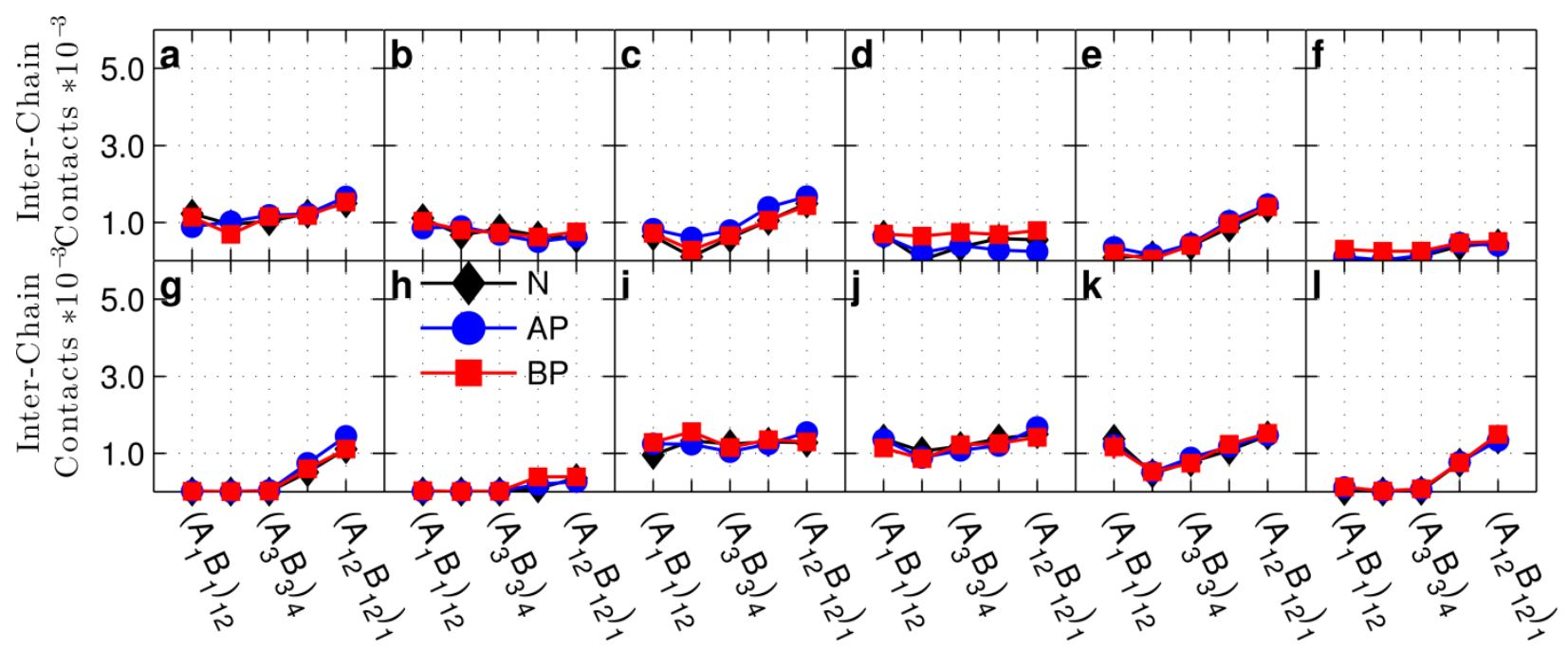

Figure 2.14: Average number of inter-chain contacts, including inter and intra particle, as a function of monomer sequence for copolymer grafted nanoparticles of $D=12 d$ each with six grafts of length Ngraft=24 with no particle monomer interactions (black diamonds), A-monomer to particle attraction at - $1 k T$ (blue circles), and B-monomer to particle attraction at - $1 k T$ (red 
squares). The system interactions correspond to the plot letters as in Table 2.2 (with particlemonomer interactions indicated by line color)

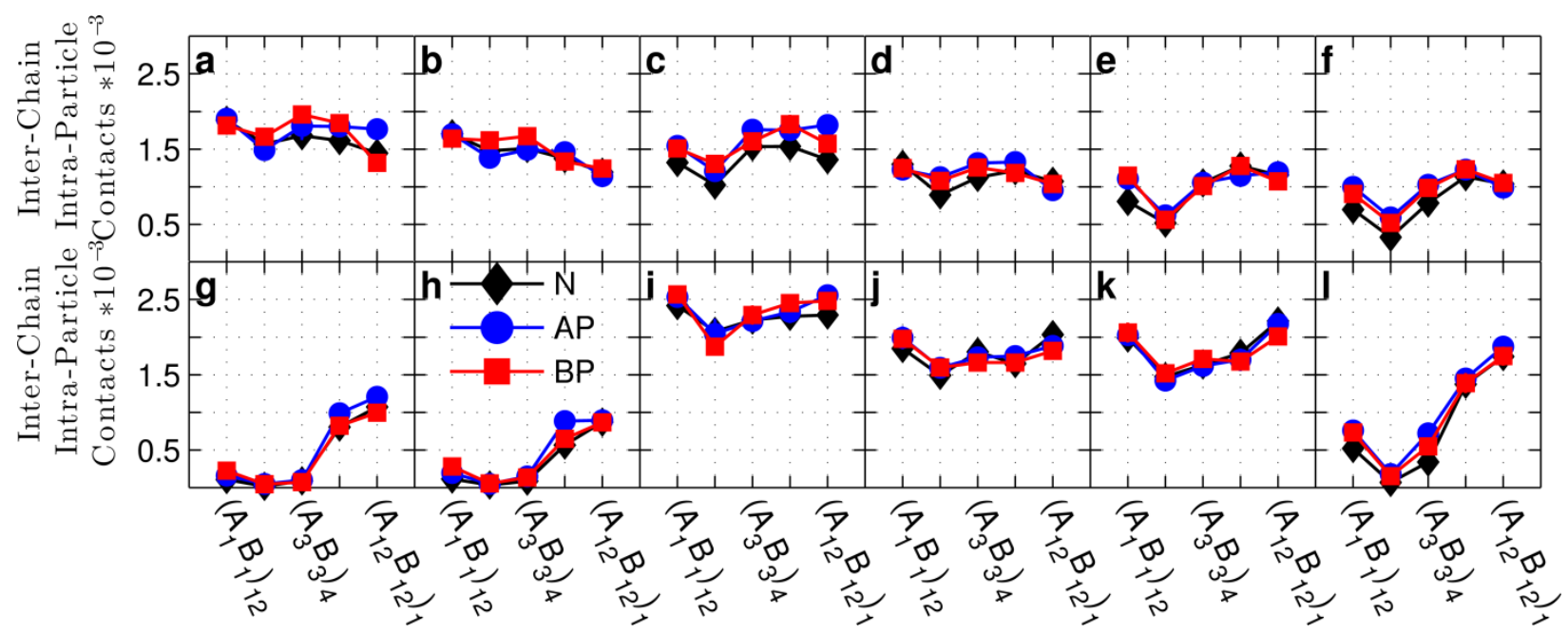

Figure 2.15: Average number of inter-chain intra-particle contacts, including inter and intra particle, as a function of monomer sequence for copolymer grafted nanoparticles of $D=4 d$ each with six grafts of length Ngraft=24 with no particle monomer interactions (black diamonds), Amonomer to particle attraction at -1kT (blue circles), and B-monomer to particle attraction at $1 k T$ (red squares). The system interactions correspond to the plot letters as in Table 2.2 (with particle-monomer interactions indicated by line color)

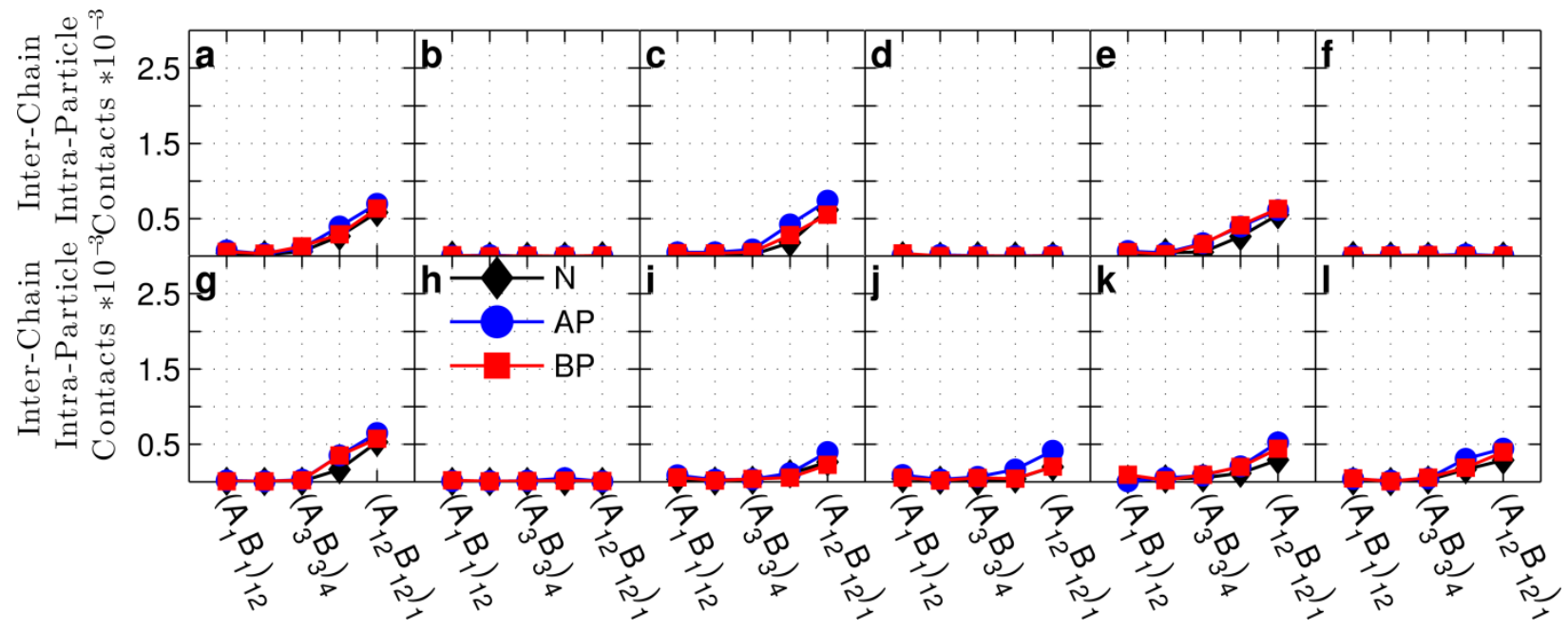

Figure 2.16: Average number of inter-chain intra-particle contacts, including inter and intra particle, as a function of monomer sequence for copolymer grafted nanoparticles of $D=12 \mathrm{~d}$ each with six grafts of length Ngraft=24 with no particle monomer interactions (black diamonds), A- 
monomer to particle attraction at - $1 k T$ (blue circles), and B-monomer to particle attraction at $1 k T$ (red squares). The system interactions correspond to the plot letters as in Table 2.2 (with particle-monomer interactions indicated by line color)

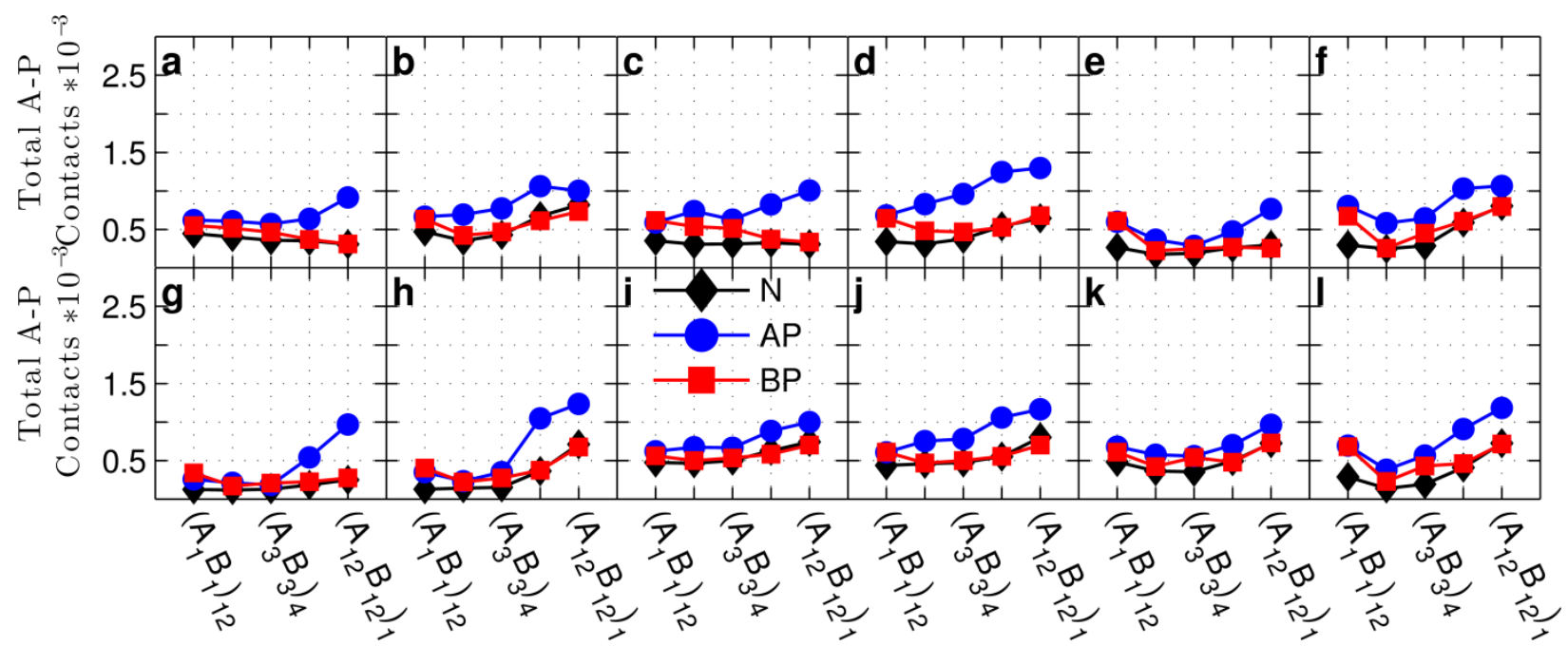

Figure 2.17: Average number of A-particle contacts, including inter and intra particle, as a function of monomer sequence for copolymer grafted nanoparticles of $D=4 d$ each with six grafts of length Ngraft=24 with no particle monomer interactions (black diamonds), A-monomer to particle attraction at - $1 k T$ (blue circles), and B-monomer to particle attraction at - $1 k T$ (red squares). The system interactions correspond to the plot letters as in Table 2.2 (with particlemonomer interactions indicated by line color ).

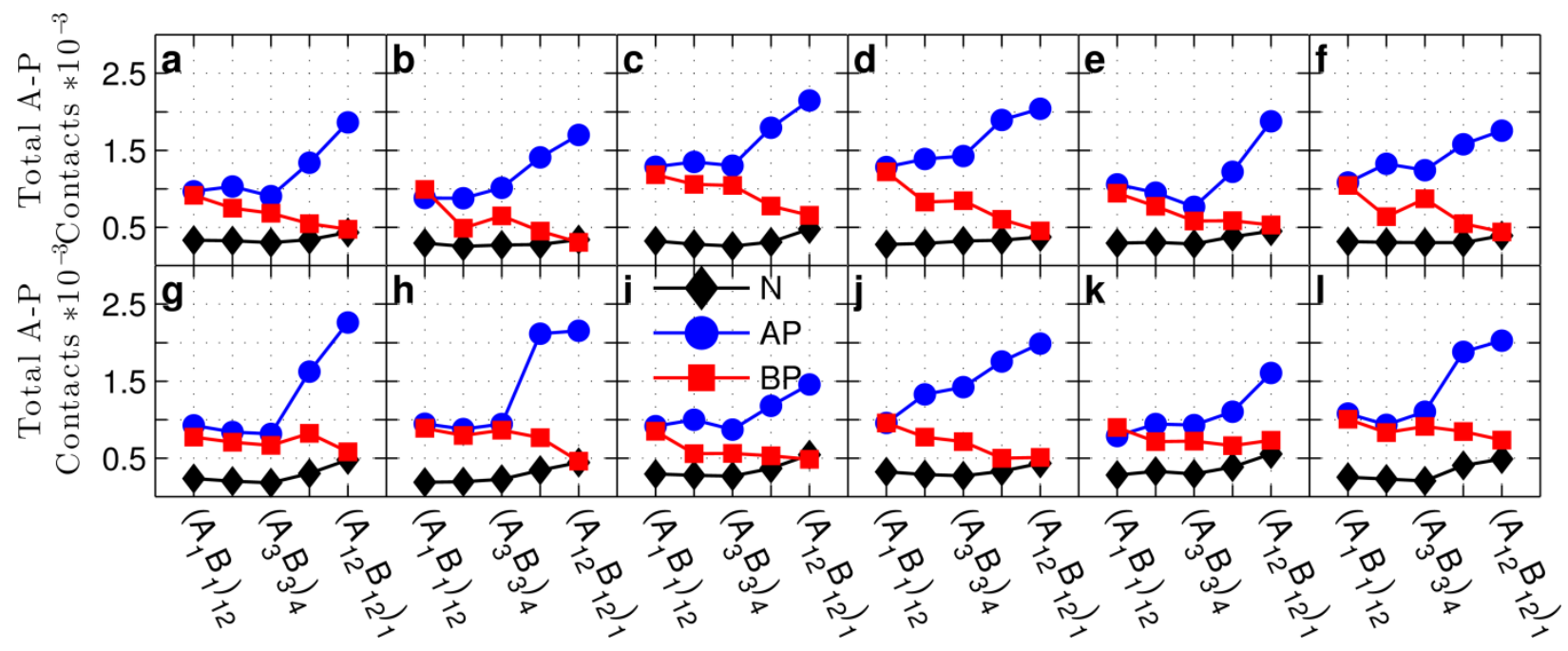

Figure 2.18: Average number of A-particle contacts, including inter and intra particle, as a function of monomer sequence for copolymer grafted nanoparticles of $D=12 \mathrm{~d}$ each with six grafts 
of length Ngraft=24 with no particle monomer interactions (black diamonds), A-monomer to particle attraction at -1kT (blue circles), and B-monomer to particle attraction at - $1 k T$ (red squares). The system interactions correspond to the plot letters as in Table 2.2 (with particlemonomer interactions indicated by line color).

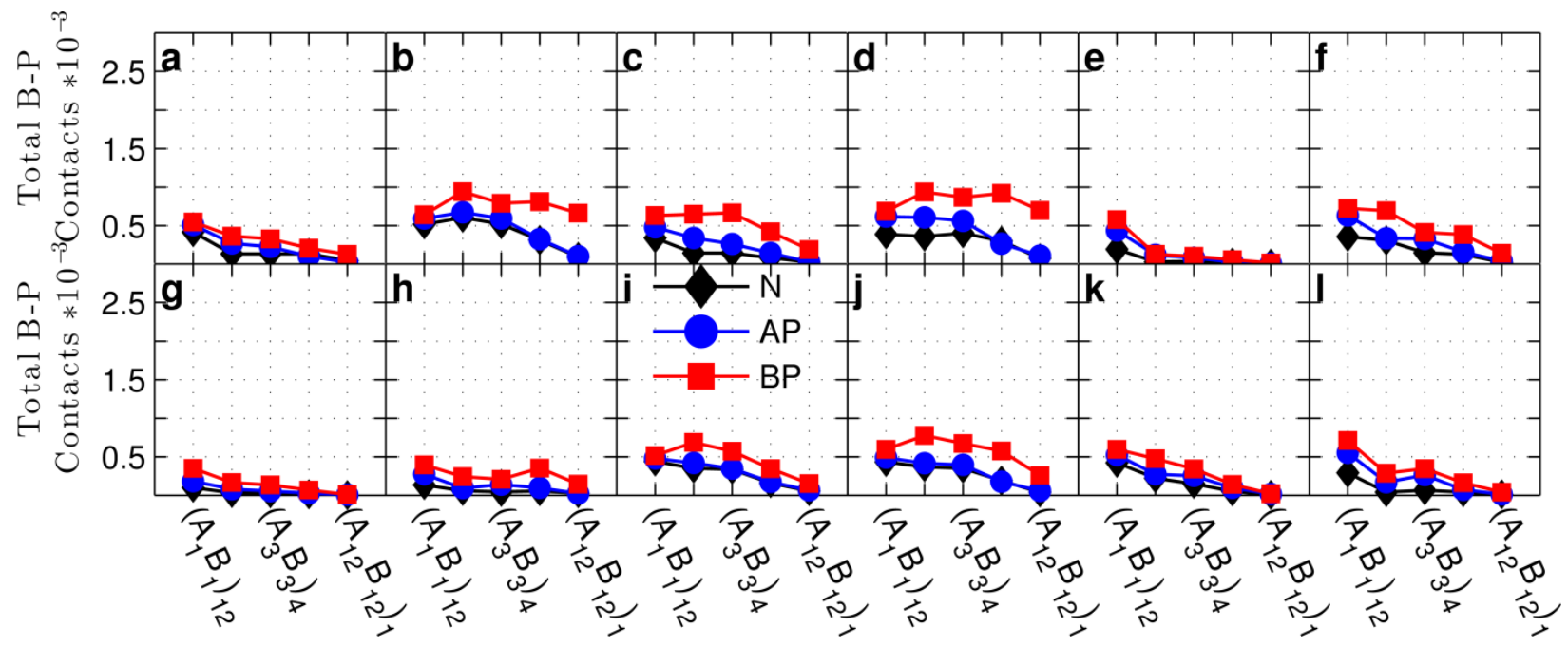

Figure 2.19: Average number of B-particle contacts, including inter and intra particle, as a function of monomer sequence for copolymer grafted nanoparticles of $D=4 d$ each with six grafts of length Ngraft=24 with no particle monomer interactions (black diamonds), A-monomer to particle attraction at - $1 k T$ (blue circles), and B-monomer to particle attraction at - $1 k T$ (red squares). The system interactions correspond to the plot letters as in Table 2.2 (with particlemonomer interactions indicated by line color) 


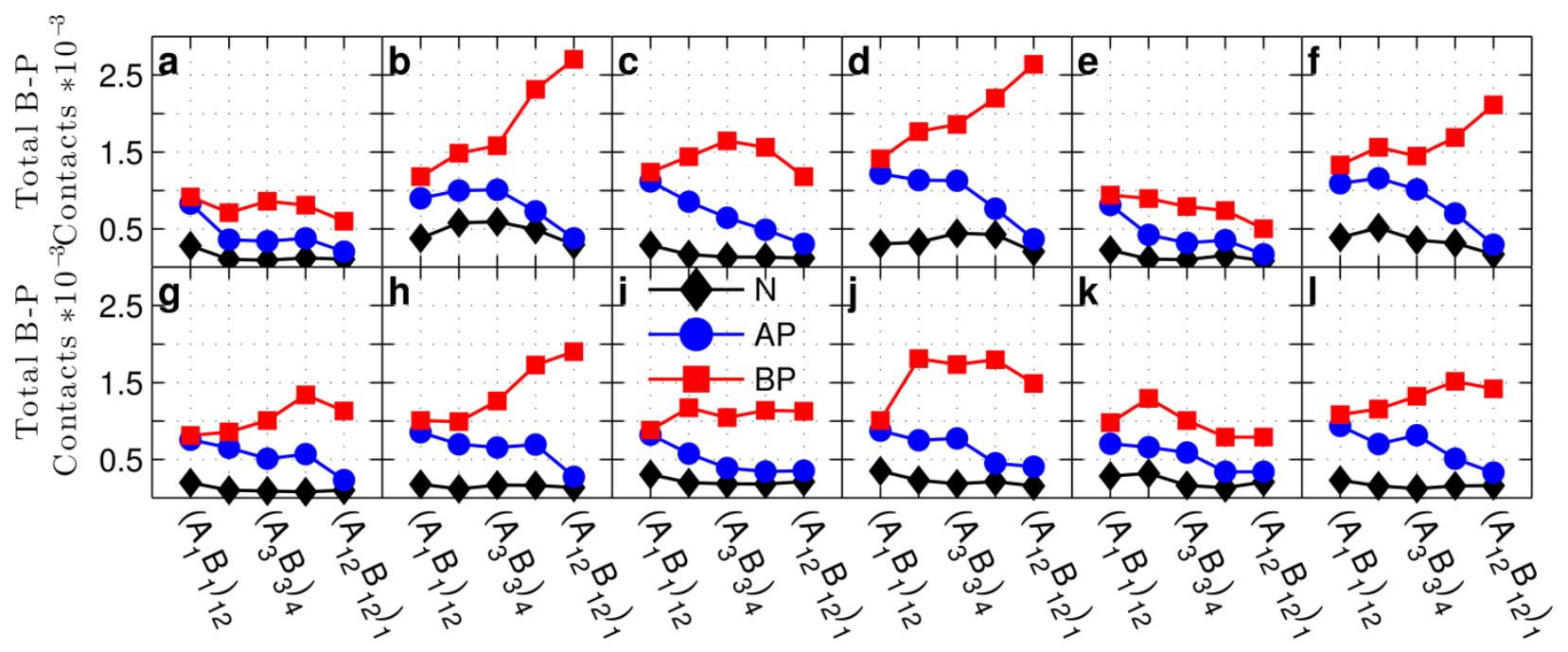

Figure 2.20: Average number of B-particle contacts, including inter and intra particle, as a function of monomer sequence for copolymer grafted nanoparticles of $D=12 \mathrm{~d}$ each with six grafts of length Ngraft=24 with no particle monomer interactions (black diamonds), A-monomer to particle attraction at - $1 k T$ (blue circles), and B-monomer to particle attraction at - $1 k T$ (red squares). The system interactions correspond to the plot letters as in Table 2.2 (with particlemonomer interactions indicated by line color)

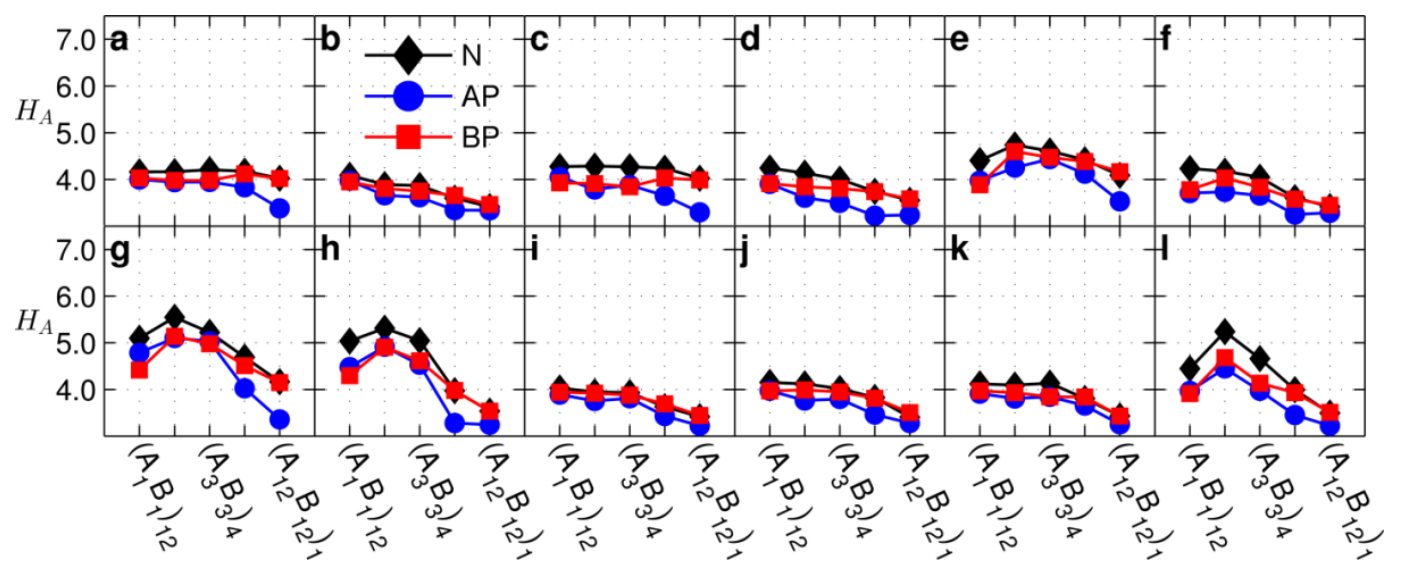

Figure 2.21: Average height of an A-monomer as a function of monomer sequences for copolymer grafted nanoparticles of $D=4 d$ each with six grafts of length $N=24$ for systems with no particle monomer interactions (black diamonds), A-monomer to particle attraction at -1kT (blue circles), and B-monomer to particle attraction at - $1 k T$ (red squares). The system interactions correspond to the plot letters as in Table 2.2 (with particle-monomer interactions indicated by line color) 


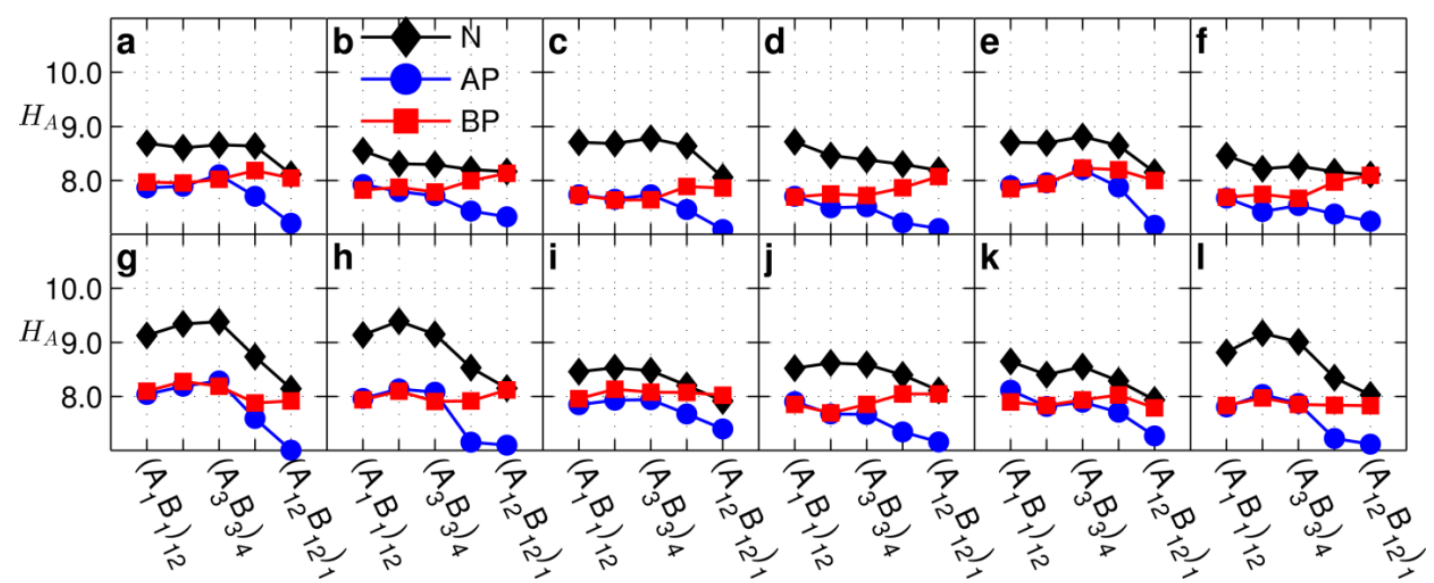

Figure 2.22: Average height of an A-monomers as a function of monomer sequences for copolymer grafted nanoparticles of $D=12 d$ each with six grafts of length $N=24$ for systems with no particle monomer interactions (black diamonds), A-monomer to particle attraction at -1kT (blue circles), and B-monomer to particle attraction at-1kT (red squares). The system interactions correspond to the plot letters as in Table 2.2 (with particle-monomer interactions indicated by line color)

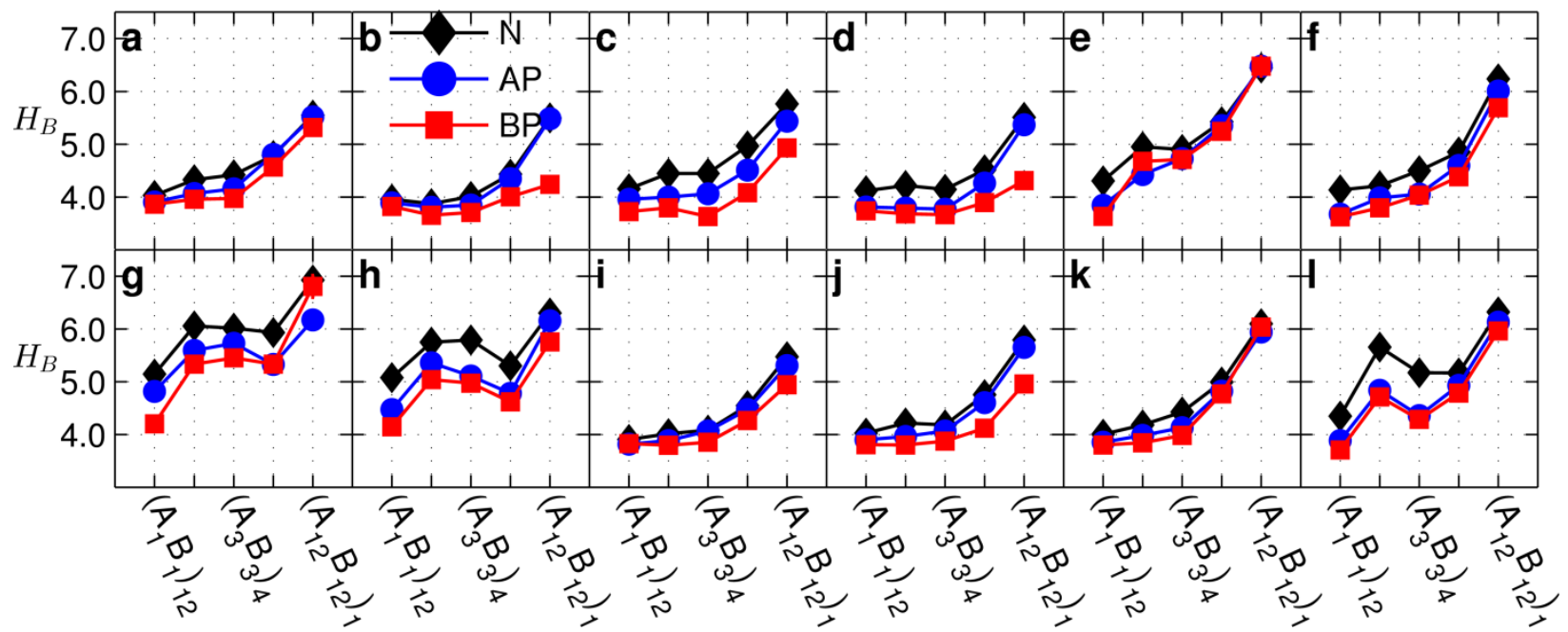

Figure 2.23: Average height B-monomer as a function of monomer sequences for copolymer grafted nanoparticles of $D=4 d$ each with six grafts of length $N=24$ for systems with no particle monomer interactions (black diamonds), A-monomer to particle attraction at-1kT (blue circles), and B-monomer to particle attraction at -1kT (red squares). The system interactions correspond to the plot letters as in Table 2.2 (with particle-monomer interactions indicated by line color) 


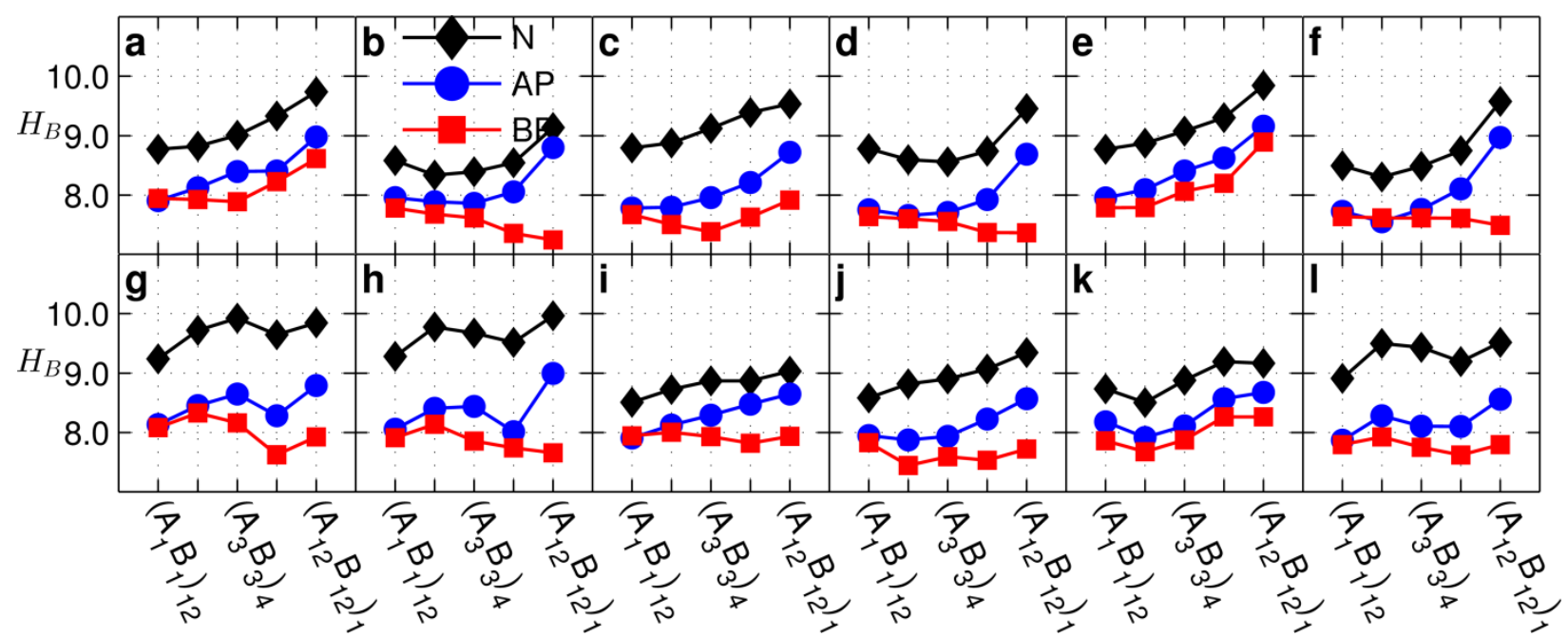

Figure 2.24: Average height B-monomer as a function of monomer sequences for copolymer grafted nanoparticles of $D=12 d$ each with six grafts of length $N=24$ for systems with no particle monomer interactions (black diamonds), A-monomer to particle attraction at -1kT (blue circles), and B-monomer to particle attraction at - $1 k T$ (red squares). The system interactions correspond to the plot letters as in Table 2.2 (with particle-monomer interactions indicated by line color) 


\subsubsection{Discussion of Lattice Effects and the Alternating Sequence}

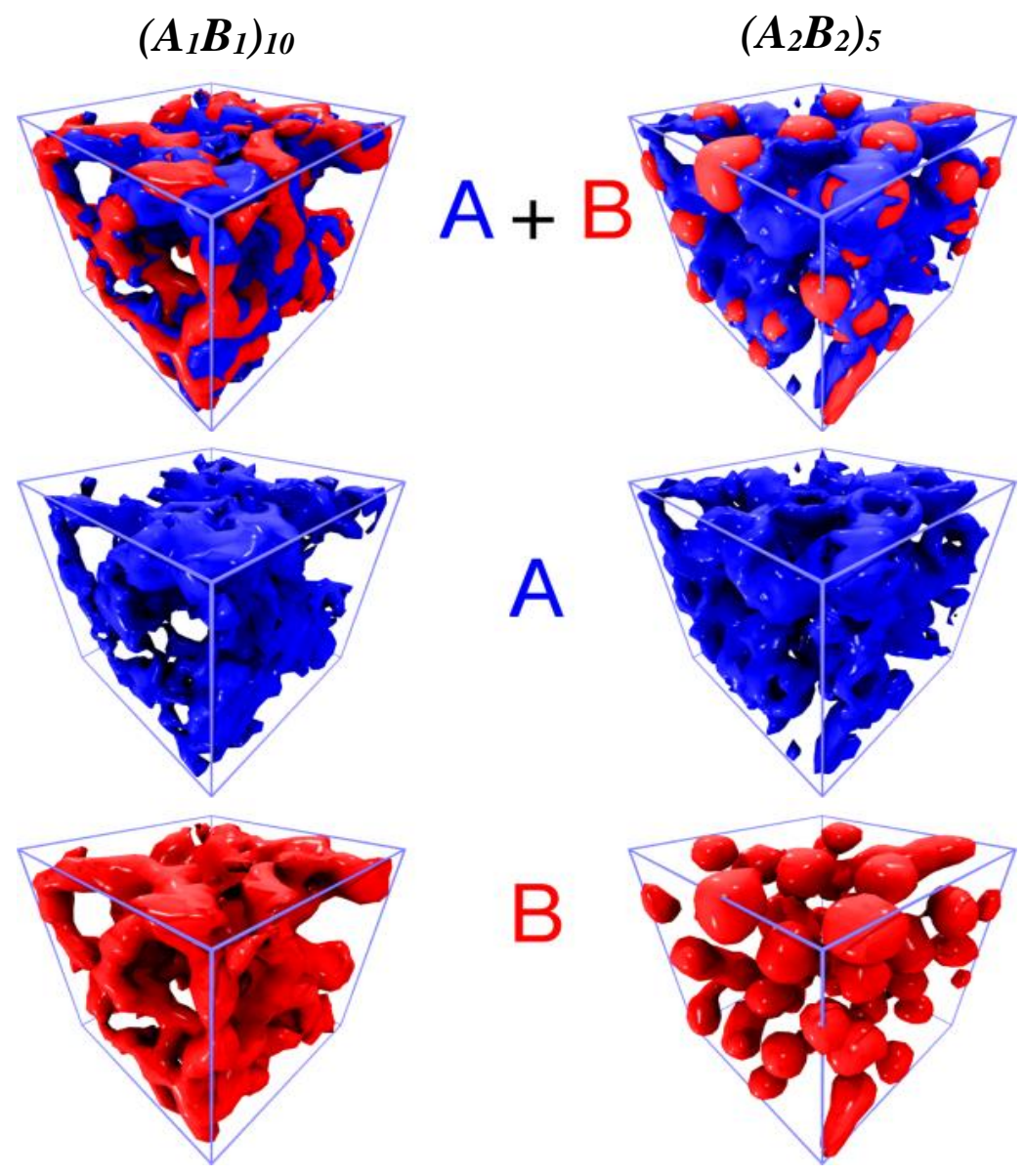

Figure 2.25: Simulation snapshots from Brownian dynamics of ungrafted copolymers of sequence $\left(A_{1} B_{1}\right)_{10}$ (left) and $\left(A_{2} B_{2}\right)_{6}$ (right) represented as iso-surfaces of density equal to 0.3 monomers/d $d^{3}$. The top row shows both $A$ and $B$ iso-surfaces together while the middle and bottom show isosurfaces calculated from only $A$ and only B monomers respectively. In both of these simulations, only the BB interactions are attractive and all others interactions are purely repulsive. The figures show that the $\left(A_{2} B_{2}\right)_{5}$ sequence produces distinct, homogeneous domains of attractive $B$ monomers (right column bottom figure), the $\left(A_{1} B_{1}\right)_{10}$ sequence produces networked domains that span the simulation box (left column bottom figure).

In order to alleviate concerns that the cubic lattice in our Monte Carlo (MC) simulations was biasing our results of monomer aggregation and domain shapes, specifically the unique behavior 
of $\left(A_{1} B_{1}\right)_{12}$, we have conducted short off-lattice Brownian Dynamics (BD) simulations of ungrafted copolymers. We have only done ungrafted copolymers here to focus the discussion on monomer-aggregation as a function of sequence, and not to mimic any nanoparticle assembly. We chose to run off-lattice BD simulations, as opposed to MC simulations, due to the availability of the open source HOOMD-blue platform ${ }^{1,2}$, which allowed us to quickly build our simulations and then rapidly simulate using GPU accelerated computing.

The simulations consisted of 350 ungrafted copolymer chains of length $N=20$ of either $\left(\mathrm{A}_{1} \mathrm{~B}_{1}\right)_{10}$ or $\left(\mathrm{A}_{2} \mathrm{~B}_{2}\right)_{5}$ sequence. The simulations progressed as follows. Initially, we modeled all interactions as purely repulsive Weeks-Chandler-Andersen (WCA) potentials. First, the system was integrated for $1 \mathrm{e} 6$ steps at $\mathrm{T}=5$ and a volume fraction of $\phi=0.029$ to remove any bias in the growth algorithm and "randomize" the system. The system was then compressed at $\mathrm{T}=5$ to a volume fraction of $\phi=0.23$ over a period of $2 \mathrm{e} 6$ time steps. At this point, the B-B interactions were switched to Lennard-Jones potentials with an attractive well depth of 3 while A-A and A-B interactions were maintained as WCA. After annealing the system using a linear gradient from $\mathrm{T}=5$ to $\mathrm{T}=1$ over $5 \mathrm{e} 6$ steps, we sampled our system and generated snapshots for $1 \mathrm{e} 5$ steps at $\mathrm{T}=1$. We note that the energy profiles of the simulations were relatively constant during the sampling period.

Figure 2.25 shows snapshots from simulations of $\left(\mathrm{A}_{1} \mathrm{~B}_{1}\right)_{10}$ (left) and $\left(\mathrm{A}_{2} \mathrm{~B}_{2}\right)_{5}$ (right) copolymers with the B and A monomers hidden for the second and third rows respectively. The isosurfaces represent a density value of 0.3 monomers $/ \mathrm{d}^{3}$. Similar to our results from MC simulations, we observe that while the $\left(A_{2} B_{2}\right)_{5}$ sequence produces distinct, homogeneous domains of attractive monomers, the $\left(A_{1} B_{1}\right)_{10}$ sequence produces networked domains that span the simulation box. These simulations support our conjecture that the alternating 
sequence is forced to assume networked morphologies due to the frustrating $-\mathrm{ABAB}-$ sequence both on and off lattice.

${ }^{1}$ HOOMD-blue web page: $h$ ttp://codeblue.umich.edu/hoomd-blue

2 J. A. Anderson, C. D. Lorenz, and A. Travesset. General purpose molecular dynamics simulations fully implemented on graphics processing units Journal of Computational Physics 227(10): 5342-5359, May 2008.

10.1016/j.jcp.2008.01.047 


\subsubsection{Tabulated Data for the number of $A$ and $B$ Domains for $D=4 d$ and $D=12 d$}

Table 2.3: Number of A Domains for copolymer grafted nanoparticle of varying sequences and monomer interactions as specified in Table 1 and $D=4 d, N g r a f t=24$, and $N g=6$. Note that all of these interaction sets are in the absence of particle monomer interactions.

\begin{tabular}{|c|c|c|c|c|c|c|c|c|c|c|c|c|}
\hline \multirow[b]{2}{*}{ Sequence } & \multicolumn{2}{|c|}{ Interaction Set 1} & \multicolumn{2}{|c|}{ Interaction Set 2} & \multicolumn{2}{|c|}{$\begin{array}{l}\text { Interaction Set } \\
3\end{array}$} & \multicolumn{2}{|c|}{ Interaction Set 4} & \multicolumn{2}{|c|}{ Interaction Set 5} & \multicolumn{2}{|c|}{ Interaction Set 6} \\
\hline & Avg & Err & Avg & Err & Avg & Err & Avg & Err & Avg & Err & Avg & Err \\
\hline (A1B1)12 & 4.60 & 0.93 & 6.40 & 0.75 & 6.40 & 1.29 & 8.40 & 0.24 & 8.80 & 1.66 & 6.20 & 0.66 \\
\hline$(\mathrm{A} 2 \mathrm{~B} 2) 6$ & 22.80 & 1.53 & 49.80 & 3.72 & 40.80 & 4.47 & 64.20 & 4.07 & 31.40 & 1.08 & 28.80 & 3.85 \\
\hline (А3B3)4 & 19.00 & 1.05 & 28.80 & 2.25 & 27.60 & 1.91 & 39.20 & 1.91 & 28.80 & 3.12 & 20.00 & 0.77 \\
\hline$(\mathrm{A} 6 \mathrm{~B} 6) 2$ & 14.40 & 1.36 & 18.20 & 1.20 & 38.20 & 3.99 & 36.40 & 4.68 & 18.00 & 2.05 & 16.40 & 3.09 \\
\hline \multirow[t]{2}{*}{ (A12B12)1 } & 12.40 & 1.29 & 13.40 & 0.81 & 28.80 & 2.46 & 29.20 & 1.24 & 12.60 & 0.68 & 6.00 & 0.95 \\
\hline & \multicolumn{2}{|c|}{ Interaction Set 7} & \multicolumn{2}{|c|}{ Interaction Set 8} & \multicolumn{2}{|c|}{$\begin{array}{l}\text { Interaction Set } \\
9\end{array}$} & \multicolumn{2}{|c|}{ Interaction Set 10} & \multicolumn{2}{|c|}{ Interaction Set 11} & \multicolumn{2}{|c|}{$\begin{array}{l}\text { Interaction Set } \\
12\end{array}$} \\
\hline Sequence & Avg & Err & Avg & Err & Avg & Err & Avg & Err & Avg & Err & Avg & Err \\
\hline (A1B1)12 & 18.40 & 1.96 & 94.40 & 2.62 & 28.00 & 2.30 & 118.60 & 4.06 & 7.80 & 0.97 & 20.00 & 2.00 \\
\hline$(\mathrm{A} 2 \mathrm{~B} 2) 6$ & 94.60 & 3.14 & 279.40 & 3.84 & 234.40 & 3.27 & 326.80 & 2.18 & 73.40 & 4.08 & 263.80 & 3.62 \\
\hline$(\mathrm{A} 3 \mathrm{~B} 3) 4$ & 66.20 & 2.08 & 194.60 & 2.09 & 144.20 & 3.15 & 218.80 & 1.24 & 52.20 & 2.35 & 157.60 & 4.51 \\
\hline$(\mathrm{A} 6 \mathrm{~B} 6) 2$ & 23.00 & 0.95 & 57.80 & 2.46 & 75.80 & 2.13 & 98.40 & 1.69 & 32.20 & 1.80 & 43.00 & 2.47 \\
\hline$(\mathrm{A} 12 \mathrm{~B} 12) 1$ & 13.00 & 0.45 & 13.00 & 0.32 & 35.00 & 1.92 & 38.00 & 1.14 & 16.60 & 0.40 & 11.40 & 0.75 \\
\hline
\end{tabular}


Table 2.4: Number of B Domains for copolymer grafted nanoparticle of varying sequences and monomer interactions as specified in Table 1 and $D=4 d, N g r a f t=24$, and $N g=6$. Note that all of these interaction sets are in the absence of particle monomer interactions.

\begin{tabular}{|c|c|c|c|c|c|c|c|c|c|c|c|c|}
\hline \multirow[b]{2}{*}{ Sequence } & \multicolumn{2}{|c|}{ Interaction Set 1} & \multicolumn{2}{|c|}{$\begin{array}{l}\text { Interaction Set } \\
2\end{array}$} & \multicolumn{2}{|c|}{ Interaction Set 3} & \multicolumn{2}{|c|}{ Interaction Set 4} & \multicolumn{2}{|c|}{ Interaction Set 5} & \multicolumn{2}{|c|}{ Interaction Set 6} \\
\hline & Avg & Err & Avg & Err & Avg & Err & Avg & Err & Avg & Err & Avg & Err \\
\hline (A1B1)12 & 5.40 & 1.03 & 7.20 & 0.20 & 5.80 & 0.86 & 5.80 & 0.66 & 7.80 & 0.80 & 5.20 & 0.73 \\
\hline (A2B2)6 & 29.80 & 2.40 & 78.00 & 8.42 & 16.20 & 1.50 & 36.60 & 3.66 & 18.20 & 2.37 & 20.00 & 2.68 \\
\hline$(\mathrm{A} 3 \mathrm{~B} 3) 4$ & 19.40 & 2.36 & 44.80 & 3.87 & 13.80 & 1.39 & 22.00 & 1.82 & 14.20 & 1.16 & 8.80 & 0.73 \\
\hline$(\mathrm{A} 6 \mathrm{~B} 6) 2$ & 31.40 & 2.80 & 35.80 & 4.93 & 11.60 & 1.40 & 12.40 & 1.33 & 15.40 & 1.94 & 12.00 & 3.54 \\
\hline \multirow[t]{2}{*}{ (A12B12)1 } & 34.40 & 2.32 & 37.20 & 1.93 & 11.00 & 0.55 & 10.60 & 0.51 & 10.60 & 0.81 & 4.40 & 0.68 \\
\hline & \multicolumn{2}{|c|}{ Interaction Set 7} & \multicolumn{2}{|c|}{$\begin{array}{l}\text { Interaction Set } \\
8\end{array}$} & \multicolumn{2}{|c|}{ Interaction Set 9} & \multicolumn{2}{|c|}{ Interaction Set 10} & \multicolumn{2}{|c|}{ Interaction Set 11} & \multicolumn{2}{|c|}{ Interaction Set 12} \\
\hline Sequence & Avg & Err & Avg & Err & Avg & Err & Avg & Err & Avg & Err & Avg & Err \\
\hline (A1B1)12 & 27.00 & 1.48 & 119.20 & 3.57 & 17.00 & 1.30 & 98.20 & 1.83 & 6.60 & 1.17 & 23.20 & 2.89 \\
\hline$(\mathrm{A} 2 \mathrm{~B} 2) 6$ & 235.20 & 4.16 & 315.60 & 2.01 & 81.20 & 4.04 & 289.80 & 3.34 & 55.00 & 3.99 & 258.60 & 4.38 \\
\hline (A3B3)4 & 154.00 & 1.82 & 215.40 & 1.25 & 44.20 & 0.58 & 189.80 & 2.65 & 34.80 & 0.97 & 159.20 & 2.91 \\
\hline$(\mathrm{A6B6}) 2$ & 73.40 & 2.27 & 102.60 & 1.86 & 19.20 & 1.32 & 33.40 & 1.29 & 21.20 & 1.59 & 25.20 & 1.32 \\
\hline (A12B12)1 & 42.80 & 0.80 & 47.00 & 1.05 & 14.80 & 1.16 & 13.60 & 0.87 & 13.40 & 1.03 & 9.20 & 0.37 \\
\hline
\end{tabular}


Table 2.5: Number of A Domains for copolymer grafted nanoparticle of varying sequences and monomer interactions as specified in Table 1 and $D=12 d, N g r a f t=24$, and $N g=6$. Note that all of these interaction sets are in the absence of particle monomer interactions.

\begin{tabular}{|c|c|c|c|c|c|c|c|c|c|c|c|c|}
\hline & \multicolumn{2}{|c|}{ Interaction Set 1} & \multicolumn{2}{|c|}{ Interaction Set 2} & \multicolumn{2}{|c|}{ Interaction Set 3} & \multicolumn{2}{|c|}{ Interaction Set 4} & \multicolumn{2}{|c|}{ Interaction Set 5} & \multicolumn{2}{|c|}{ Interaction Set 6} \\
\hline Sequence & Avg & Err & Avg & Err & Avg & Err & Avg & Err & Avg & Err & Avg & Err \\
\hline (A1B1)12 & 42.60 & 1.03 & 56.00 & 3.70 & 42.40 & 3.20 & 57.60 & 4.18 & 47.60 & 2.87 & 40.60 & 2.84 \\
\hline$(\mathrm{A} 2 \mathrm{~B} 2) 6$ & 54.00 & 2.63 & 117.20 & 5.17 & 141.60 & 7.17 & 185.40 & 3.60 & 69.00 & 5.05 & 76.60 & 4.86 \\
\hline (A3B3)4 & 46.60 & 1.03 & 78.80 & 3.97 & 101.20 & 2.78 & 127.00 & 9.45 & 58.40 & 1.63 & 65.40 & 4.30 \\
\hline$(\mathrm{A} 6 \mathrm{~B} 6) 2$ & 47.20 & 0.86 & 53.00 & 2.57 & 88.60 & 1.50 & 84.60 & 1.03 & 50.00 & 1.18 & 53.00 & 2.49 \\
\hline \multirow[t]{2}{*}{ (A12B12)1 } & 46.00 & 1.92 & 46.80 & 1.77 & 54.80 & 1.16 & 54.80 & 1.20 & 50.40 & 1.89 & 46.20 & 1.71 \\
\hline & \multicolumn{2}{|c|}{ Interaction Set 7} & \multicolumn{2}{|c|}{ Interaction Set 8} & \multicolumn{2}{|c|}{ Interaction Set 9} & \multicolumn{2}{|c|}{ Interaction Set 10} & \multicolumn{2}{|c|}{ Interaction Set 11} & \multicolumn{2}{|c|}{ Interaction Set 12} \\
\hline Sequence & Avg & Err & Avg & Err & Avg & Err & Avg & Err & Avg & Err & Avg & Err \\
\hline (A1B1)12 & 69.60 & 1.57 & 112.40 & 3.22 & 95.40 & 2.06 & 139.00 & 2.28 & 40.00 & 1.41 & 94.00 & 2.55 \\
\hline$(\mathrm{A} 2 \mathrm{~B} 2) 6$ & 103.20 & 4.43 & 292.80 & 2.82 & 274.20 & 3.71 & 316.00 & 2.59 & 119.20 & 3.29 & 279.00 & 3.96 \\
\hline (A3B3)4 & 80.00 & 1.14 & 203.80 & 2.06 & 194.20 & 3.32 & 225.80 & 1.28 & 106.80 & 4.57 & 196.00 & 1.92 \\
\hline$(\mathrm{A} 6 \mathrm{~B} 6) 2$ & 51.20 & 0.80 & 88.40 & 3.63 & 107.00 & 1.18 & 111.60 & 1.21 & 61.00 & 1.10 & 78.40 & 1.86 \\
\hline (A12B12)1 & 47.60 & 1.29 & 48.60 & 0.75 & 57.00 & 0.84 & 57.40 & 0.81 & 49.20 & 1.69 & 49.80 & 2.22 \\
\hline
\end{tabular}


Table 2.6: Number of B Domains for copolymer grafted nanoparticle of varying sequences and monomer interactions as specified in Table 1 and $D=12 d, N g r a f t=24$, and $N g=6$. Note that all of these interaction sets are in the absence of particle monomer interactions.

\begin{tabular}{|c|c|c|c|c|c|c|c|c|c|c|c|c|}
\hline \multirow[b]{2}{*}{ Sequence } & \multicolumn{2}{|c|}{ Interaction Set 1} & \multicolumn{2}{|c|}{$\begin{array}{l}\text { Interaction Set } \\
2\end{array}$} & \multicolumn{2}{|c|}{ Interaction Set 3} & \multicolumn{2}{|c|}{ Interaction Set 4} & \multicolumn{2}{|c|}{ Interaction Set 5} & \multicolumn{2}{|c|}{ Interaction Set 6} \\
\hline & Avg & Err & Avg & Err & Avg & Err & Avg & Err & Avg & Err & Avg & Err \\
\hline (A1B1)12 & 44.00 & 1.38 & 60.60 & 3.25 & 40.60 & 2.48 & 51.20 & 2.63 & 47.40 & 2.54 & 40.40 & 3.30 \\
\hline (A2B2)6 & 152.00 & 4.52 & 178.80 & 5.11 & 47.80 & 2.48 & 117.40 & 4.30 & 60.80 & 1.77 & 77.00 & 4.83 \\
\hline (A3B3)4 & 111.20 & 5.21 & 129.20 & 7.61 & 44.60 & 1.96 & 70.40 & 4.80 & 49.40 & 2.04 & 55.20 & 2.56 \\
\hline$(\mathrm{A} 6 \mathrm{~B} 6) 2$ & 91.20 & 1.80 & 91.40 & 2.20 & 36.80 & 1.59 & 41.00 & 1.34 & 42.40 & 1.72 & 42.00 & 2.88 \\
\hline \multirow[t]{2}{*}{ (A12B12)1 } & 55.60 & 1.25 & 54.80 & 0.97 & 26.20 & 1.77 & 24.20 & 1.83 & 35.80 & 2.76 & 33.20 & 1.83 \\
\hline & \multicolumn{2}{|c|}{ Interaction Set 7} & \multicolumn{2}{|c|}{$\begin{array}{l}\text { Interaction Set } \\
8\end{array}$} & \multicolumn{2}{|c|}{ Interaction Set 9} & \multicolumn{2}{|c|}{ Interaction Set 10} & \multicolumn{2}{|c|}{ Interaction Set 11} & \multicolumn{2}{|c|}{ Interaction Set 12} \\
\hline Sequence & Avg & Err & Avg & Err & Avg & Err & Avg & Err & Avg & Err & Avg & Err \\
\hline (A1B1)12 & 91.80 & 3.26 & 138.00 & 2.28 & 66.60 & 2.36 & 110.60 & 3.47 & 39.40 & 1.63 & 91.80 & 4.07 \\
\hline$(\mathrm{A} 2 \mathrm{~B} 2) 6$ & 271.40 & 5.19 & 320.40 & 1.50 & 96.20 & 4.93 & 284.20 & 2.58 & 108.60 & 2.32 & 273.40 & 1.12 \\
\hline (A3B3)4 & 204.20 & 1.53 & 224.40 & 1.60 & 64.60 & 0.60 & 201.40 & 1.54 & 78.40 & 2.54 & 188.40 & 2.62 \\
\hline$(\mathrm{A} 6 \mathrm{~B} 6) 2$ & 110.20 & 1.77 & 115.00 & 1.26 & 40.80 & 1.28 & 64.80 & 1.24 & 45.80 & 1.59 & 59.40 & 1.96 \\
\hline (A12B12)1 & 57.40 & 0.81 & 57.60 & 0.68 & 26.00 & 2.21 & 27.00 & 0.89 & 32.00 & 1.87 & 29.80 & 2.58 \\
\hline
\end{tabular}


2.7.5 Additional Results Showing Effect of Graft Length to Particle Size Ratio on Nanoparticle Assembly and Chain Conformations

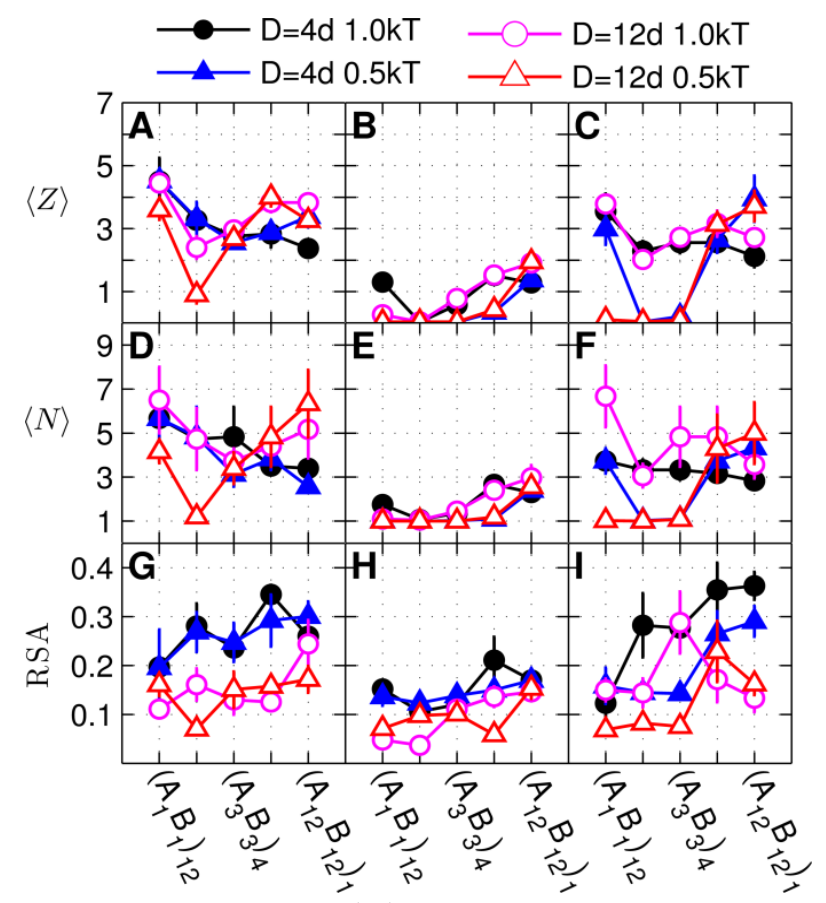

Figure 2.26: Average coordination number $(Z)$, average number of particles per cluster $(N)$, and relative shape anisotropy (RSA) as a function of monomer sequences for copolymer grafted nanoparticles of $D=4 d$ and $D=12 d$ each with six grafts of length $N=24$. The lines on plot $a, d$, and g correspond to interaction sets 3 (blue and red triangles) and 4 (black and magenta circles), lines on plot $b$, e, and h correspond to interaction sets 7 (blue and red triangles) and 8 (black and magenta circles), lines on plot $c, f$, and i correspond to interaction sets 11 (blue and red triangles) and 12 (black and magenta circles). The circles represent attractive monomer-monomer interactions at a strength of $1 \mathrm{kT}$ while the triangles represent $0.5 \mathrm{kT}$. The filled symbols represent nanoparticles of size $D=4 d$ while open symbols represent interaction sets with nanoparticles of size $d=12 d$.

Figure 2.7 in the main manuscript presented the data $\langle\mathrm{Z}\rangle,\langle\mathrm{N}\rangle$, and RSA for three selected sets of system interactions and both $\mathrm{D}=4 \mathrm{~d}$ and $12 \mathrm{~d}$. In Figure 2.26 we show the remaining interaction sets. For systems with B-B interactions in the absence of A-B repulsion (Figure 2.26a,d,g), we observe very similar trends to the data for the systems with only A-A attractions (Figure 2.7a,d,g). 
The largest difference between the two interaction sets is that the dip at $\left(\mathrm{A}_{2} \mathrm{~B}_{2}\right)_{6}$ for weak monomermonomer attraction (solid red line) seems to be much more pronounced in the case of B-B attraction. The data for A-A attraction in the presence of A-B repulsion (Figure 2.26b,e,h) shows that there is little effect from particle size on any of the assembly data. This is because the system is completely dispersed at low blockiness, where particle size has the greatest effect on assembly characteristics, and clusters only form at $\left(\mathrm{A}_{6} \mathrm{~B}_{6}\right)_{2}$ and $\left(\mathrm{A}_{12} \mathrm{~B}_{12}\right)_{1}$ where we have already shown that particle size has the least effect. The final column in Figure 2.26 represents the data for systems with both $\mathrm{A}-\mathrm{A}$ and $\mathrm{B}-\mathrm{B}$ attraction along with $\mathrm{A}-\mathrm{B}$ repulsion. Here we observe that particle size seems to only strongly affect $\langle\mathrm{Z}\rangle$ and $\langle\mathrm{N}\rangle$ at $\left(\mathrm{A}_{1} \mathrm{~B}_{1}\right)_{12}$ where the trend in the data for weak monomer-monomer attraction and $\mathrm{D}=4 \mathrm{~d}$ (solid blue line) deviates from the trend for weak monomer-monomer attraction and $\mathrm{D}=12 \mathrm{~d}$. This is mostly likely an artifact of the way that $\left(\mathrm{A}_{1} \mathrm{~B}_{1}\right)_{12}$ aggregates attractive monomers (distributed networks) and it only affects $D=4 d$ because $D=12 d$ cannot form enough intra-particle contacts to form these networks of like monomers. 

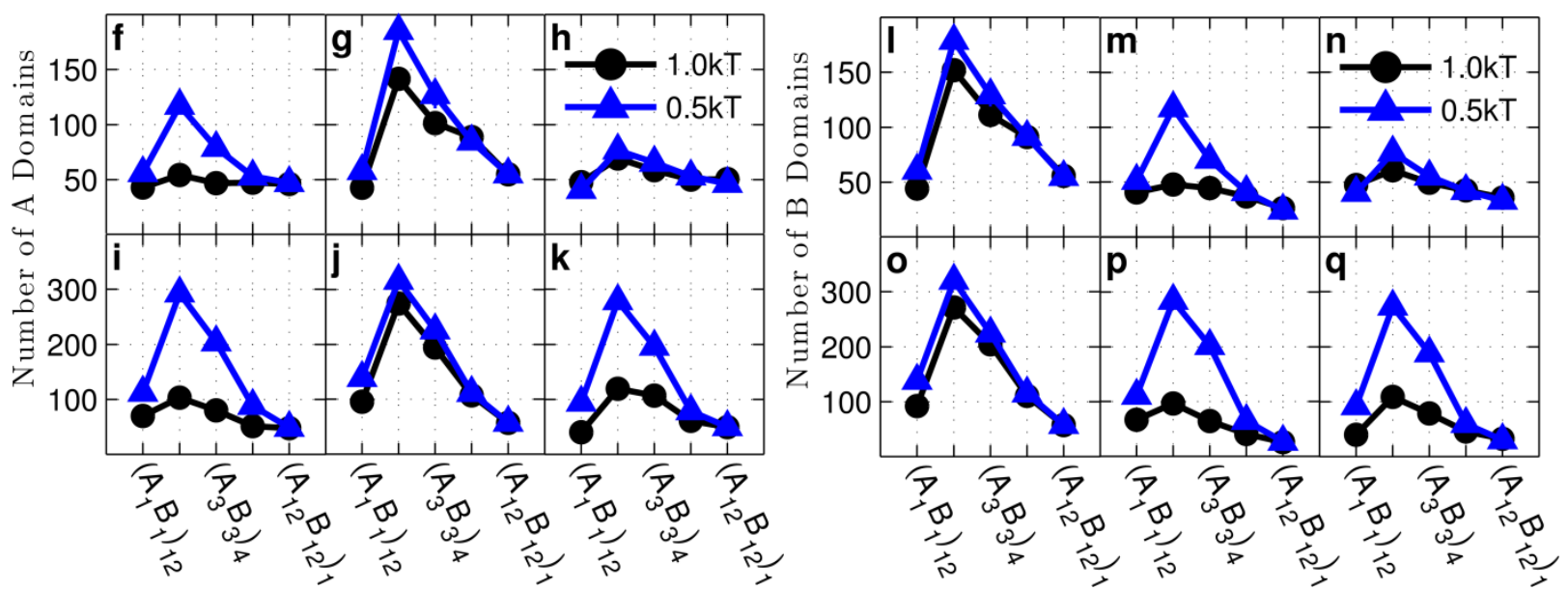

Figure 2.27: $(f-k)$ Plots showing the average number of $A$ domains in a system for $D=12 d$. $(l-q)$

Plots showing the average number of $B$ domains in a system. The system interactions correspond to the plot letters as follows: The system interactions correspond to the plot letters as follows: lines on plot f and l correspond to interaction sets 1 (blue triangles) and 2 (black circles), lines on plot $g$ and $m$ correspond to interaction sets 3 (blue triangles) and 4 (black circles), lines on plot h and $n$ correspond to interaction sets 5 (blue triangles) and 6 (black circles), lines on plot $i$ and o correspond to interaction sets 7 (blue triangles) and 8 (black circles), lines on plot $j$ and $p$ correspond to interaction sets 9 (blue triangles) and 10 (black circle), and lines on plot $k$ and $q$ correspond to interaction sets 11 (blue triangles) and 12 (black circles). The black circles represent attractive monomer-monomer interactions at a strength of $1 \mathrm{kT}$ while the blue triangles represent ttractive monomer-monomer interactions at a strength of $0.5 \mathrm{kT}$

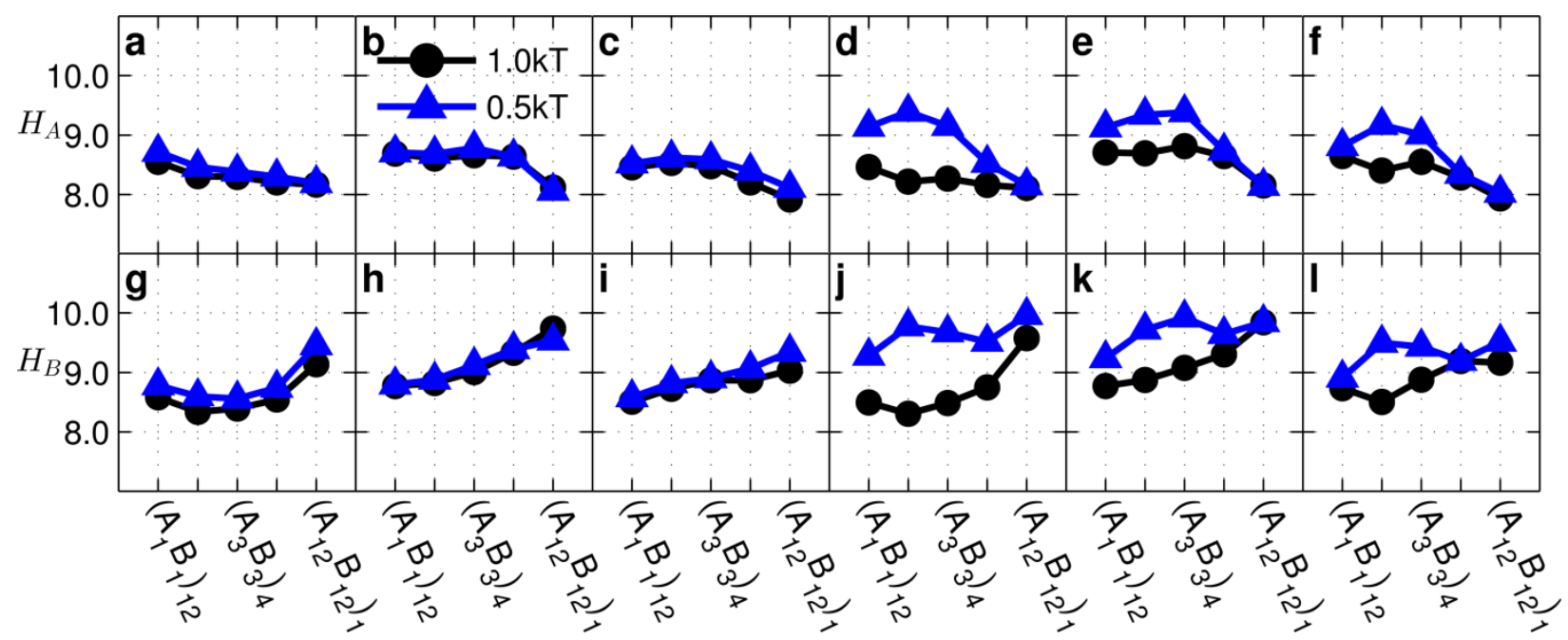

Figure 2.28: Plots showing the average height of an A-monomer (top row) and B-monomer (bottom row) as functions of monomer sequences for copolymer grafted nanoparticles of $D=12 d$ 
each with six grafts of length $N=24$. The system interactions correspond to the plot letters as follows: lines on plot a and g correspond to interaction sets 1 (blue triangles) and 2 (black circles), lines on plot $b$ and $h$ correspond to interaction sets 3 (blue triangles) and 4 (black circles), lines on plot $c$ and $i$ correspond to interaction sets 5 (blue triangles) and 6 (black circles), lines on plot $d$ and $j$ correspond to interaction sets 7 (blue triangles) and 8 (black circles), lines on plot e and $k$ correspond to interaction sets 9 (blue triangles) and 10 (black circles), and lines on plot $f$ and $l$ correspond to interaction sets 11 (blue triangles) and 12 (black circles). The black circles represent attractive monomer-monomer interactions at a strength of $1 \mathrm{kT}$ while the blue triangles represent attractive monomer-monomer interactions at a strength of $0.5 \mathrm{kT}$ 


\subsubsection{Effect of Monomer Sequence on Nanoparticle Arrangement in the Cluster for $D=4 d$ and}

$\mathrm{D}=12 \mathrm{~d}$ with and without Monomer-Particle Interactions

In the main manuscript in Section 2.3.3, we presented a detailed analysis of the effect of particlemonomer interactions on the assembly of copolymer grafted nanoparticles. We found that particle monomer interactions had limited effect on the assembly characteristics of the system due to limitations on available nanoparticle surface area, conformational restrictions of making particlemonomer contacts, and an overall inferiority of monomer-particle contacts to monomer-monomer contacts (Supplementary Section 2.7.7). In order to keep the analysis concise and avoid redundant analysis, we focused on three particular systems for $\mathrm{D}=12 \mathrm{~d}$ and discussed how these systems were affected by particle-monomer interactions. For posterity, on the following pages we present here in our complete data set of data on $\langle\mathrm{Z}\rangle,\langle\mathrm{N}\rangle,\left\langle\mathrm{R}_{\mathrm{g}, \text { cluster }}\right\rangle^{0.5}$, RSA for $\mathrm{D}=4 \mathrm{~d}$ and $\mathrm{D}=12 \mathrm{~d}$. These data support the conclusions discussed in the main article, therefore we will not provide any further analysis on them.

In the following figures the system interactions correspond to the plot letters as listed in Table 2.2 (with particle-monomer interactions indicated by line color). 
$\langle Z\rangle$

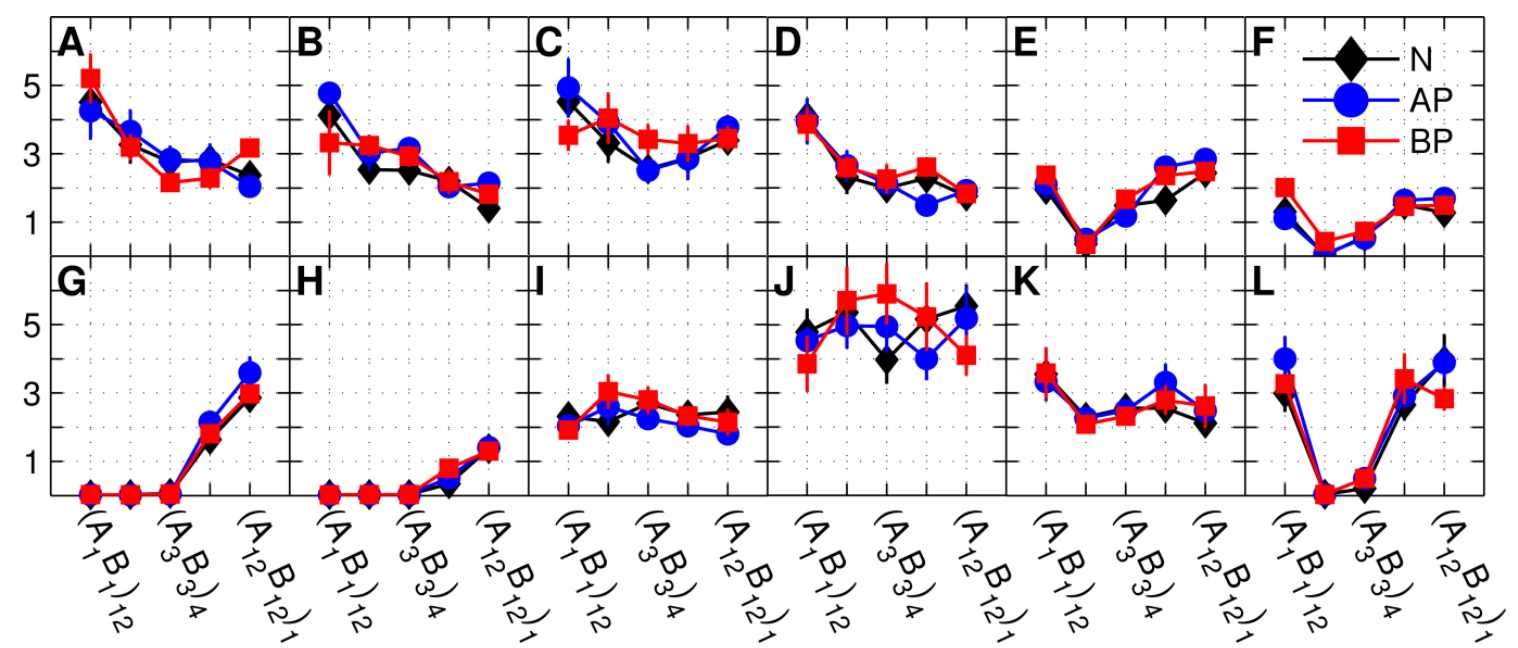

Figure 2.29: Average coordination number (Z) as a function of monomer sequences for copolymer grafted nanoparticles of $D=4 d$ with six grafts of length $N=24$ for systems with no particle monomer interactions (black diamonds), A-monomer to particle attraction at -1kT (blue circles), and Bmonomer to particle attraction at -1kT (red squares).

$\langle Z\rangle$

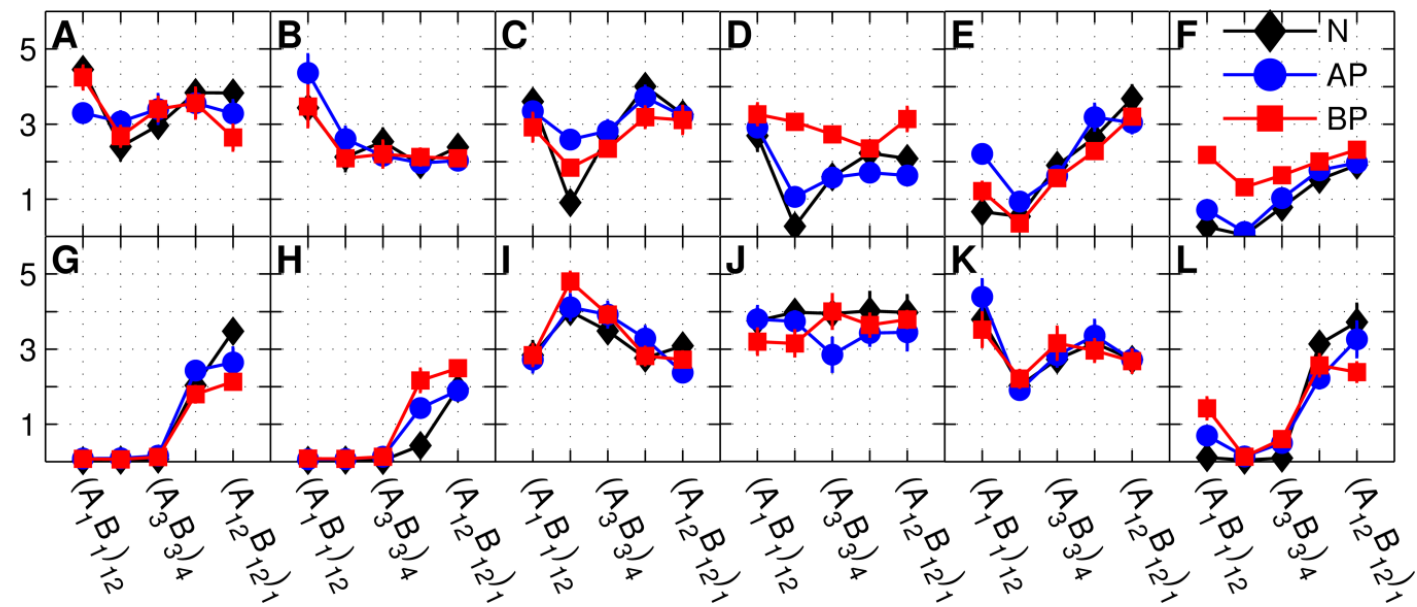

Figure 2.30: Average coordination number $(Z)$ as a function of monomer sequences for

copolymer grafted nanoparticles of $D=12 d$ with six grafts of length $N=24$ for systems with no particle monomer interactions (black diamonds), A-monomer to particle attraction at -1kT (blue circles), and B-monomer to particle attraction at $-1 k T$ (red squares). 


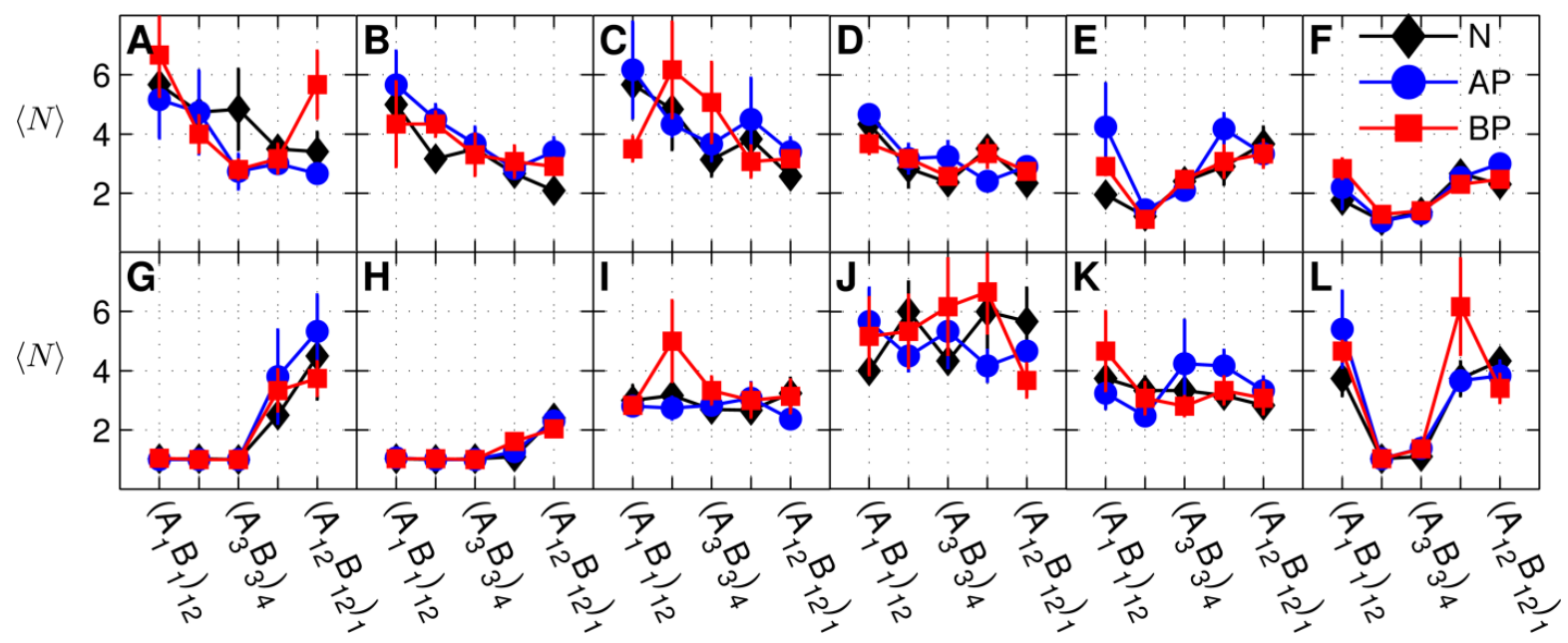

Figure 2.31: Average number of particles per cluster $(N)$ as a function of monomer sequences for copolymer grafted nanoparticles of $D=4 d$ with six grafts of length $N=24$ for systems with no particle monomer interactions (black diamonds), A-monomer to particle attraction at -1kT (blue circles), and B-monomer to particle attraction at -1kT (red squares).

$\langle N\rangle$

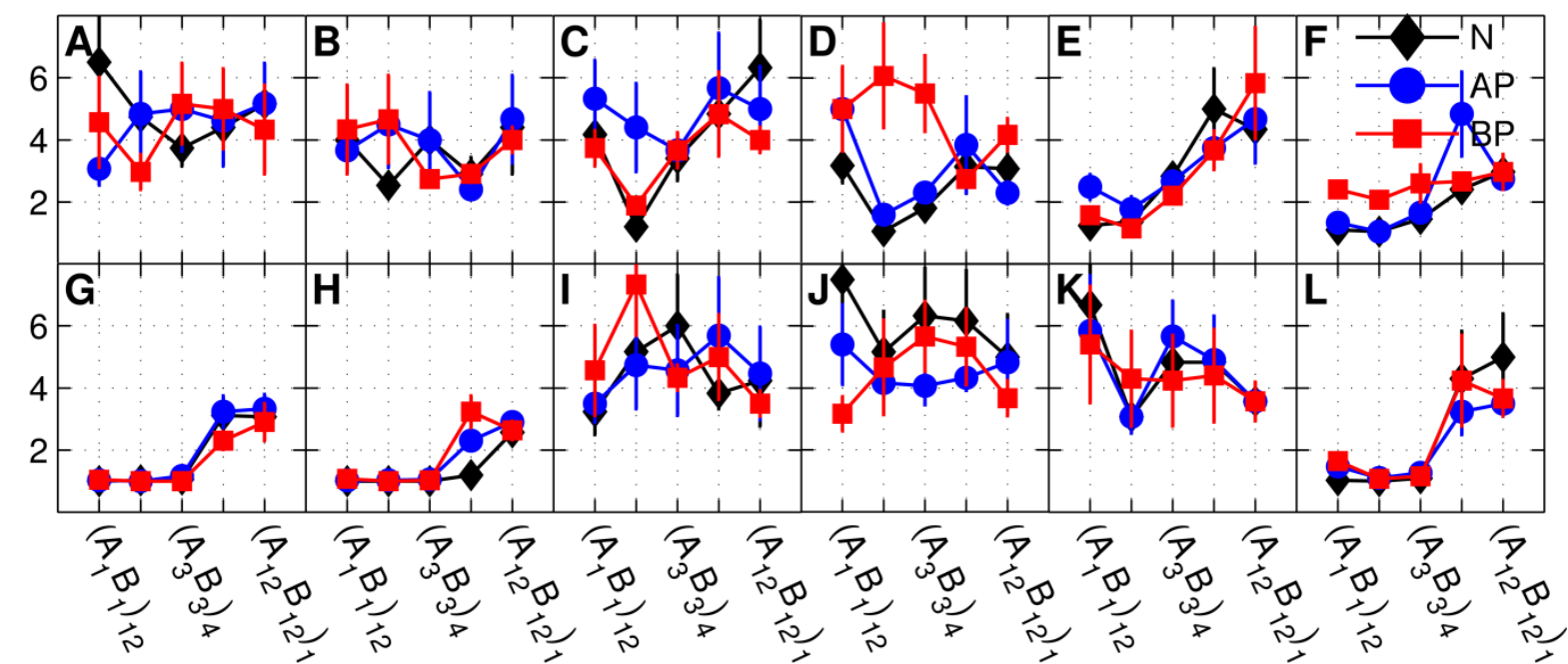

Figure 2.32: Average number of particles per cluster $(N)$ as a function of monomer sequences for copolymer grafted nanoparticles of $D=12 d$ with six grafts of length $N=24$ for systems with no particle monomer interactions (black diamonds), A-monomer to particle attraction at -1kT (blue circles), and B-monomer to particle attraction at -1kT (red squares). 


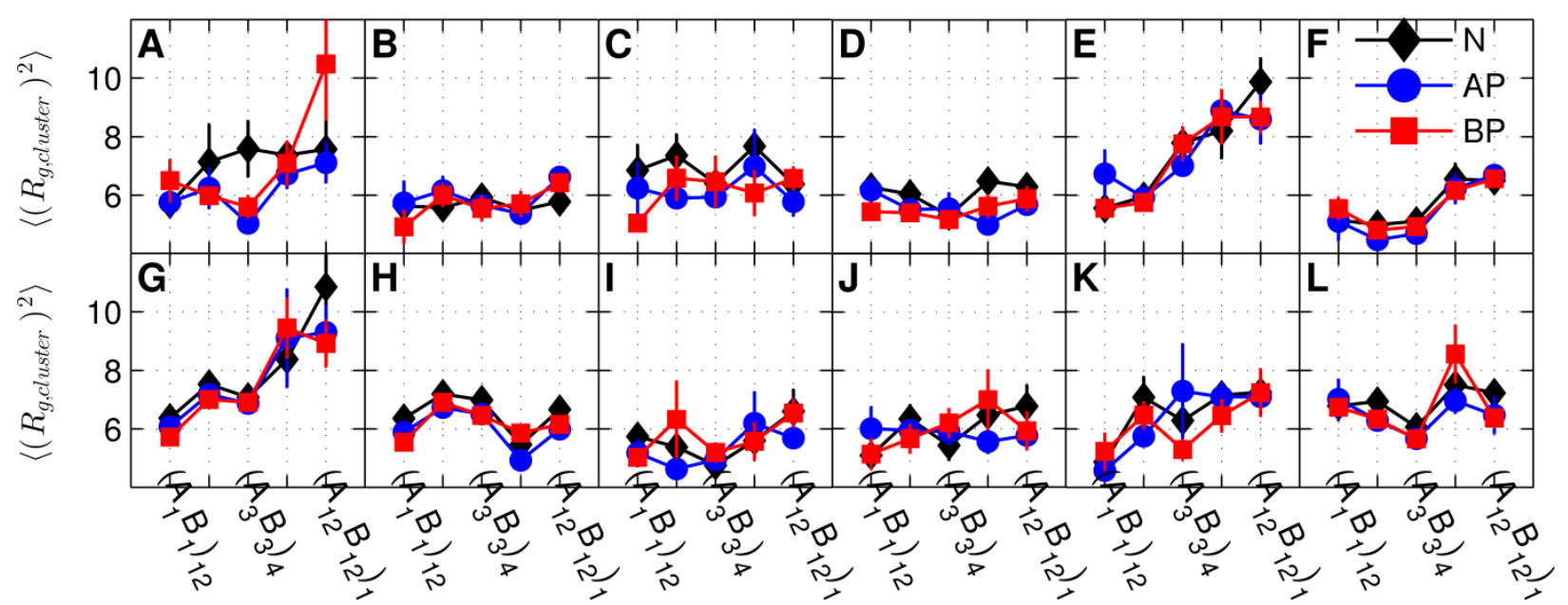

Figure 2.33: Average radius of gyration of a cluster (R2g,cluster $) 0.5$ as a function of monomer sequences for copolymer grafted nanoparticles of $D=4 d$ with six grafts of length $N=24$ for systems with no particle monomer interactions (black diamonds), A-monomer to particle attraction at -1kT (blue circles), and B-monomer to particle attraction at -1kT (red squares).

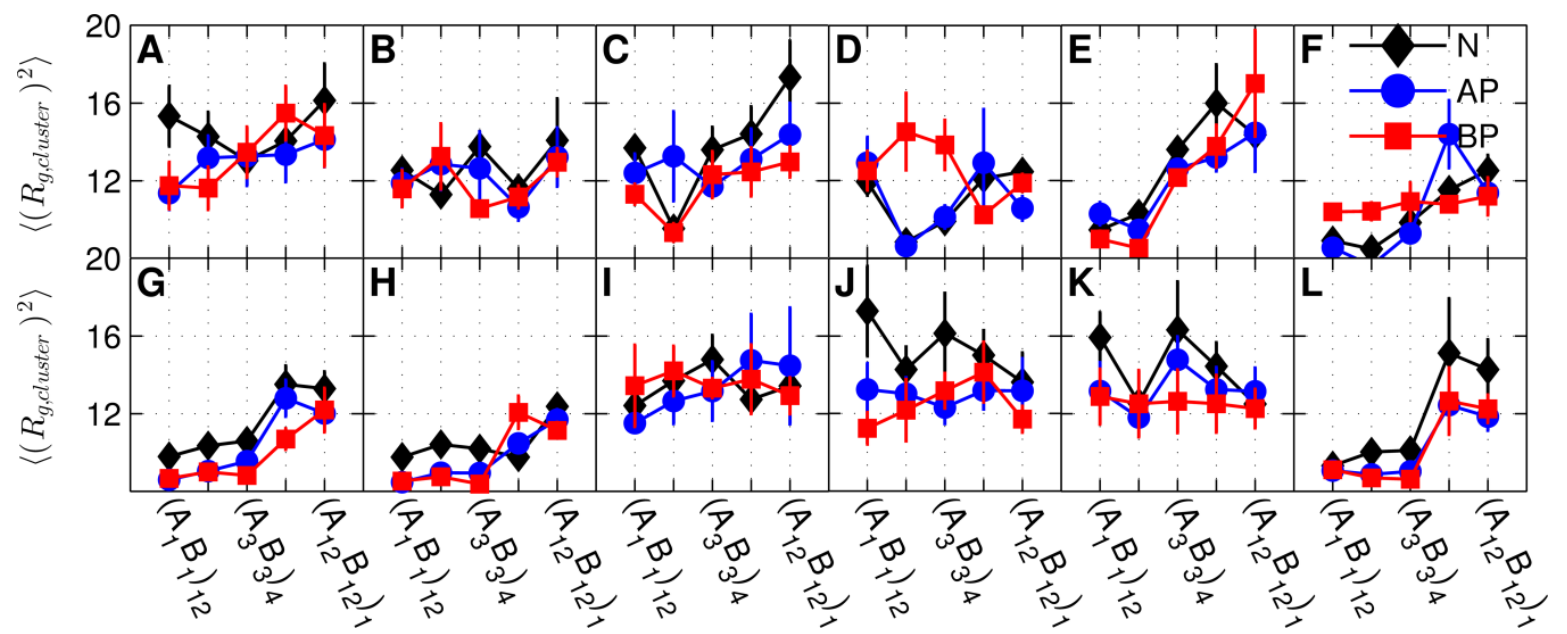

Figure 2.34: Average radius of gyration of a cluster (R2g,cluster ) 0.5 as a function of monomer sequences for copolymer grafted nanoparticles of $D=12 d$ with six grafts of length $N=24$ for systems with no particle monomer interactions (black diamonds), A-monomer to particle attraction at - $1 k T$ (blue circles), and B-monomer to particle attraction at - $1 k T$ (red squares). to interaction set 12, and lines on plot L correspond to interaction set 11 


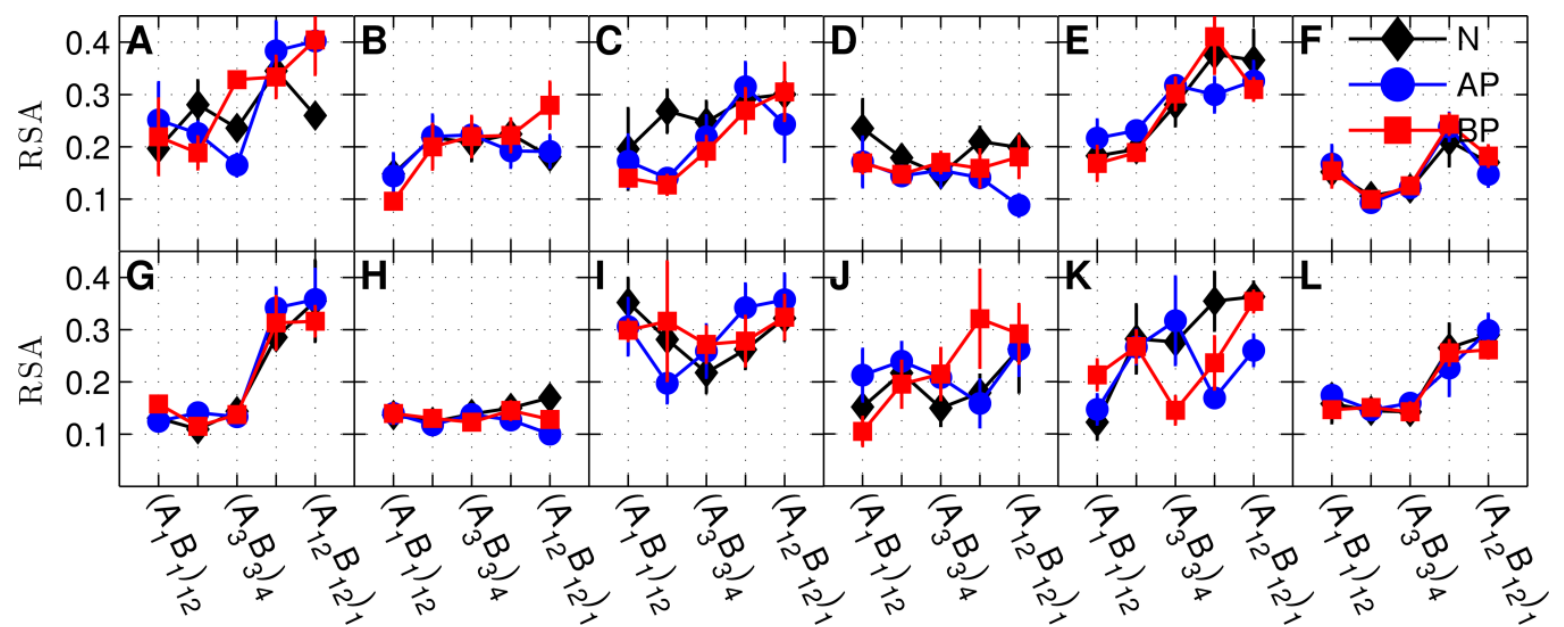

Figure 2.35: Average relative square anisotropy (RSA) as a function of monomer sequences for copolymer grafted nanoparticles of $D=4 d$ with six grafts of length $N=24$ for systems with no particle monomer interactions (black diamonds), A-monomer to particle attraction at -1kT (blue circles), and B-monomer to particle attraction at -1kT(red squares).

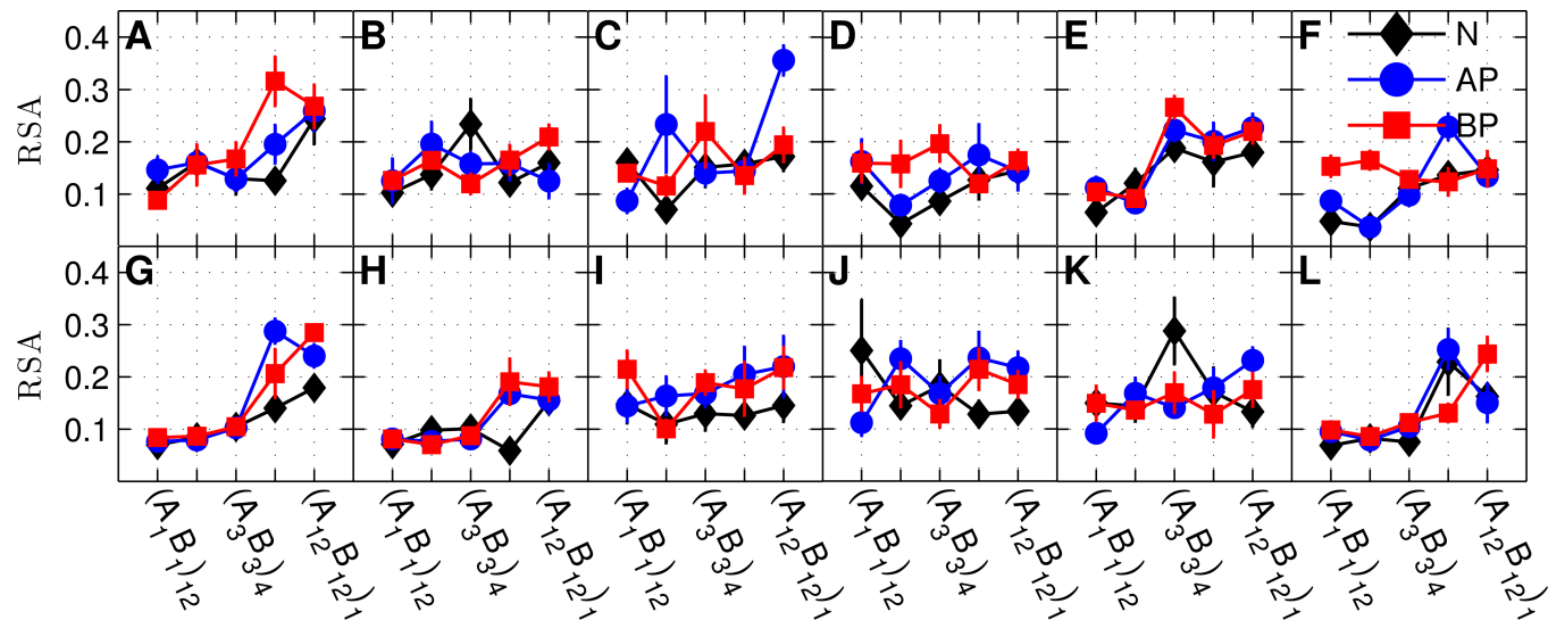

Figure 2.36: Average relative square anisotropy (RSA) as a function of monomer sequences for copolymer grafted nanoparticles of $D=12 d$ with six grafts of length $N=24$ for systems with no particle monomer interactions (black diamonds), A-monomer to particle attraction at -1kT (blue circles), and B-monomer to particle attraction at - $1 k T$ (red squares). 


\subsubsection{Relative Strength of Monomer-Particle and Monomer-Monomer Interactions for D-4d and $\mathrm{D}=12 \mathrm{~d}$}

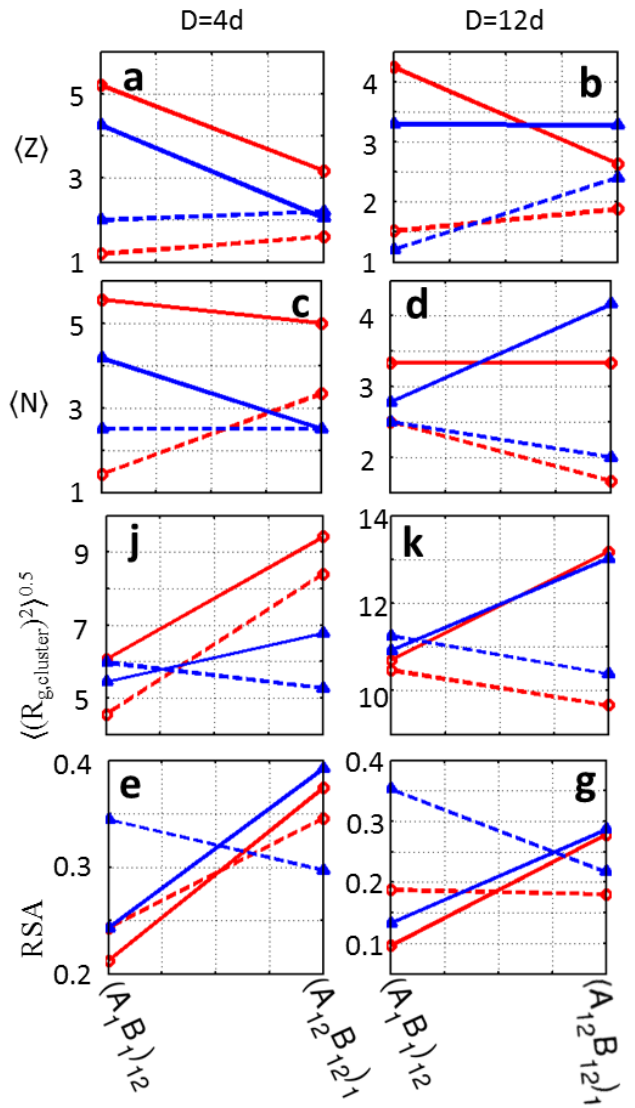

Figure 2.37: Average coordination number ( $(Z))$, average number of particles per cluster ( $(N\rangle)$, average cluster radius of gyration (R2g,cluster $\mathbf{0 . 5 )}$, and relative square anisotropy (RSA) as a function of monomer sequences for copolymer grafted nanoparticles of $D=4 d$ (left column) and $D=12 d$ (right column) each with six grafts of length $N=24$. The solid blue lines represent a system where A-A attraction is $1 k T$ and A-particle attraction is $1 k T$. The dashed blue lines represent a system where A-A attraction is $1 \mathrm{kT}$ and A-particle attraction is $16 \mathrm{kT}$. The solid red lines represent a system where B-B attraction is $1 k T$ and B-particle attraction is $1 k T$. The dashed red lines represent a system where $B-B$ attraction is $1 k T$ and $B$-particle attraction is $-16 k T$.

In the main manuscript in Section 2.3.3 we described the effect of particle-monomer interactions on the assembly of copolymer grafted nanoparticles. We concluded that the effect of these interactions on nanoparticle assembly is minimal due to the limited surface area on particles of size $\mathrm{D}=4 \mathrm{~d}$ and $12 \mathrm{~d}$, but there is also an effect from the 2-dimensional nature of particle-monomer 
contacts versus 3-dimensional nature of monomer-monomer contacts. In a polymer grafted particle, while a grafted monomer can have many monomer-monomer interacting neighbors within the square well width of $\sqrt{ } 2 \mathrm{~d}$ each monomer can only have one particle-monomer interaction within the square well depth of $(\mathrm{D}+\mathrm{d}) / 2$. When we compare systems with monomer-monomer interactions that are equal in magnitude to particle-monomer interactions (Figure 2.8a,b) with systems with monomer-monomer interactions that are half the magnitude of particle-monomer interactions (Figure $2.8 \mathrm{c}, \mathrm{d}$ ) we are effectively "doubling" the particle-monomer interaction strength. Because the number of possible monomer-monomer contacts is still significantly higher than particlemonomer contacts, we still do not observe an appreciable increase in the effect of particlemonomer interactions on characteristics of the assembled cluster.

In our lattice model, to make the particle-monomer contacts to significantly affect the assembly, the monomer-monomer epsilons $\left(\varepsilon_{A B}, \varepsilon_{A A}, \varepsilon_{B B}\right)$ would have to be approximately $1 / 16$ of the particle-monomer epsilons $\left(\varepsilon_{B P}, \varepsilon_{A P}\right)$. This large increase in interaction strength effectively makes one particle-monomer contact equal to 16 monomer-monomer contacts, therefore approximately equating their enthalpic potential. In Figure 2.37 we present data for $\langle\mathrm{Z}\rangle,\langle\mathrm{N}\rangle$, $\left\langle\mathrm{R}_{\mathrm{g}, \text { cluster }}\right\rangle^{0.5}$, and RSA at both $\mathrm{D}=4 \mathrm{~d}$ (left column) and $\mathrm{D}=12 \mathrm{~d}$ (right column) for both $\left(\mathrm{A}_{1} \mathrm{~B}_{1}\right)_{12}$ and $\left(\mathrm{A}_{12} \mathrm{~B}_{12}\right)_{1}$ that compares the effect of particle monomer interactions at a strength of $1 \mathrm{kT}$ (solid lines) and 16kT (dashed lines). We observe a drastic increase in the effect of particle monomer interactions for all of the data, especially for $\mathrm{D}=12 \mathrm{~d}$. In particular, nearly all of the trends in the data from $\left(A_{1} B_{1}\right)_{12}$ to $\left(A_{12} B_{12}\right)_{1}$ are reversed when the particle monomer-interactions are raised from $-1 \mathrm{kT}$ to $-16 \mathrm{kT}$. These data demonstrate that particle-monomer interactions have the potential to dramatically affect the assembly of copolymer grafted nanoparticles, but they must have extremely large interaction strengths in order to do so. 


\section{Polydispersity For Tuning the Potential of Mean Force Between

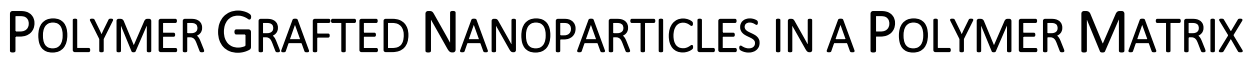

Adapted from: Phys. Rev. Lett. 2013, 110 (1), 018301

\subsection{INTRODUCTION}

Controlling the morphology of nanoscale additives in a polymer matrix is critical for tuning the macroscopic properties of the resulting polymer nanocomposite. One way to manipulate the morphology is by grafting the nanoparticle surface with polymers that are compatible with the matrix polymer, and, as a result, tuning the interactions between the grafted nanoparticles and the polymer matrix. A recent comprehensive review by Green [1] presents the extensive theoretical and experimental work that has shown that the molecular weights of the grafted and matrix polymer play a critical role in dictating the inter-particle interactions, both at high and low grafting density [References cited in Ref. 1]. At high grafting density, where the grafted chains are in the "strong brush" regime, nanoparticles disperse (aggregate) if the graft molecular weight is higher (lower) than matrix molecular weight with dispersion and aggregation being driven by wetting and dewetting of the grafted layer by matrix chains, respectively [1]. At low grafting density, larger graft molecular weight chains can better shield nanoparticles from direct particle-particle contacts and lead to dispersion of grafted particles in the polymer matrix[2]. Additionally, by tailoring the graft and matrix molecular weights along with the grafting density, one can further tune the shape of particle aggregation in the nanocomposite [3]. Despite the importance of graft molecular weight for controlling the morphology, experimental and theoretical studies on polymer grafted nanoparticles have not investigated how polydispersity in the grafted chains affects the morphology of the particles in a polymer matrix. 
Past studies have shown that polydispersity in chain lengths grafted on flat surfaces[4, 5] can alter chain conformations and the overall height of the grafted layer on these surfaces (with no curvature). Star polymers with polydisperse arms can be thought of as polydisperse polymers grafted on a nanoparticle with infinitely large curvature. The effective force, $F$, between polydisperse star polymers in a good solvent has been shown to have drastically different expression as compared to monodisperse star polymers [6] [7]. While these past studies justify further exploration of polydispersity effects, they do not predict the behavior of polydisperse polymers grafted on spherical hard nanoparticle surfaces with finite curvature in the presence of an explicit polymer matrix. Recently, using Monte Carlo (MC) simulations [8], Dodd and Jayaraman studied a single spherical polymer grafted nanoparticle with polydisperse grafted chains, in an implicit solvent, at a purely athermal limit, for varying polydispersity indices (PDI>12.5), particle diameter, and grafting density. Dodd and Jayaraman showed that the conformations of the grafted chains in a polydisperse system deviates significantly from the monodisperse counterpart, and approaches that of a single grafted chain on the same particle size because of polydispersity-induced relief in monomer crowding. Specifically, the radius of gyration of the short chains was lower at $\mathrm{PDI}>1$ than at $\mathrm{PDI}=1$ (monodisperse), and the long chains were less stretched at distances away from particle surface at PDI $>1$ than at PDI=1. These observations demonstrate that the chain conformations on hard nanoparticles with finite curvature are significantly affected by polydispersity in the grafted chain lengths. This leads to the question: Is the effect of polydispersity on grafted chain conformations large enough to alter how matrix chains wet/dewet/deplete the grafted layer? If yes, is this change in matrix wettability of the grafted layer predictable so that one could deliberately introduce polydispersity as a design knob to tailor interparticle interactions? In this letter, we answer these questions by exploring how polydispersity in 
polymer chains grafted on nanoparticles affects the potential of mean force (PMF) between the polymer grafted nanoparticles at varying grafting densities (e.g. low, intermediate, and high), in a dense solution of matrix polymers and melt-like polymer matrix at varying matrix lengths (e.g. less than and greater than average graft length) using a combined PRISM theory - Monte Carlo simulation approach. One of the key results is that, at high grafting density, polydispersity in the grafted polymers can stabilize dispersions in a monodisperse polymer matrix at conditions where corresponding monodisperse polymer grafted particles would exhibit aggregation.

\subsection{APPROACH}

We use a self-consistent Polymer Reference Interaction Site Model (PRISM) theory -Monte Carlo (MC) simulation approach to calculate the PMF for a system of a polymer matrix (dense solution and melt) with polymer grafted particles (filler) at infinitely dilute filler concentration. The details of the PRISM-MC method, including the choice of closures for the PRISM part and the limitations of the method are presented in Ref.[9], where this approach is reviewed in detail, and applied to study polymer grafted nanoparticles in polymer matrix. An overview of this method is presented below.

\subsubsection{Model}

We model the polymer grafted nanoparticles as a hard spherical nanoparticle of diameter $D$. Each particle has $\sigma$ number of freely jointed polymer chains grafted per particle surface area, with varying polydispersity in the grafted chain length. The grafted chains are permanently attached at random, non-overlapping points on the particle surface. We model the matrix chains as freely jointed polymer chains. The molecular weight of the polymer chains (grafted and matrix) is linked to the chain length of these coarse-grained polymers (grafted and matrix) by the number of coarsegrained beads $N_{\text {graft }}$ (where a bead mimics a Kuhn segment of the polymer). To achieve 
polydispersity, the grafted molecular weight (or chain lengths) are chosen from a log-normal distribution with the number average chain length $N_{\text {graft,avg }}$ equal to a target value. The matrix chain length is maintained to be monodisperse, equal to $N_{\text {matrix }}$. The chemical interactions between the grafted polymers and matrix polymers are maintained to be purely athermal, as the chemistries of the two polymers are identical. For high grafting densities $\left(>0.3 \mathrm{chains} / \mathrm{nm}^{2}\right)$, we are justified in using athermal interactions between the monomers and the particle as well, as the high monomer crowding on the surface reduces an effect of monomer-surface attraction.

\subsubsection{Method}

The full details of the PRISM-MC methodology are provided in Refs. [10, 11], and we present only a brief overview here. The PRISM portion of the self-consistent PRISM-MC approach consists of a matrix of Ornstein-Zernike-like integral equations, which in Fourier space is as follows:

$$
\mathrm{H}(k)=\Omega(k) \cdot C(k)(\Omega(k)+\mathrm{H}(k))
$$

where each of the terms in the above equation is an $\mathrm{NxN}$ matrix of terms, where $\mathrm{N}$ is the number of species in the system. The above equation relates the total site-site inter-molecular pair correlation function, $h_{i j}(r)$ where $h_{i j}(r)=g_{i j}(r)-1$, to the inter-molecular direct correlation function, $c_{i j}(r)$, and intra-molecular pair correlation function, $\omega_{i j}(r)$. In our system of homopolymer grafted particles in a homopolymer matrix there are three types of sites- graft monomers (A), particle (C), and matrix monomers (M). We note that, even though the matrix and graft monomer are of the same chemistry, we have to identify them as two different sites to distinguish them physically in the theory. The intra-molecular pair correlation function $\omega_{i j}(k)$ between sites $i$ and $j$ within a certain molecule in Fourier space provides the shape of each molecules (polymer grafted particle or matrix chain) and is served as one of the inputs to the theory. The $\omega_{i j}(k)$ of the polymer grafted 
nanoparticle is calculated as an ensemble average of the intramolecular pair correlation function calculated in a Monte Carlo (MC) simulation of a single polymer grafted nanoparticle with polydisperse grafted chain distribution placed in an effective solvation potential calculated by PRISM. Similarly the $\omega_{i j}(k)$ of the matrix chain is obtained from an MC simulation of a single matrix chain in an effective solvation potential calculated in PRISM. The PRISM-MC loop is executed in a self-consistent manner as $\omega_{i j}(k)$ and the solvation potential are inter-dependent. To solve the PRISM equations, we use closure relations connecting the direct pair correlation function $c_{i j}(r), h_{i j}(r)\left(=g_{i j}(r)-1\right)$ and interaction potentials $U_{i j}(r)$ (another input to the theory). Previous work on a polymer nanocomposites [12-20] has shown that the Percus-Yevick (PY) closure for polymerpolymer and polymer-particle and the hypernetted chain (HNC) closure for particle-particle work well, especially in case of athermal interactions. The non-linear coupled integral PRISM equations along with the closures are solved using the KINSOL algorithm [21] with the line search optimization strategy. With KINSOL, it has proven much easier to attain convergence for these complex nonlinear integral equations for a larger parameter set than is possible with Picard technique (the method used in much of the prior PRISM theory work).

Upon solving these equations, the output of the theory is the spatial organization of the system both in real space $g_{i j}(r)$ and Fourier space, $S_{i j}(k)$. The potential of mean force between two grafted particles in an explicit matrix (in case of infinitely dilute amount of grafted particles in an explicit matrix) is calculated from the inter-particle pair correlation function,

$$
P M F(r)=-k T \ln g(r)
$$

The advantage of the self-consistent PRISM-MC approach, over PRISM-only, is that PRISM-MC can tackle non-ideal conformations of the grafted and matrix polymers that have been neglected in PRISM-only studies of homopolymer-grafted nanoparticles, which assumes an ideal 
conformation in $\omega_{\mathrm{ij}}(\mathrm{k})[18-20,22]$. PRISM-MC is better than simulations of polymer grafted particles in implicit matrix, [23] because, in the latter, any spatial arrangements that could be occurring purely due to entropic reasons and excluded volume interactions (which is critical to wetting-dewetting phenomena) is completely ignored. In the self-consistent PRISM-MC approach even though the MC simulation does not account for the excluded volume interactions of the matrix chains, the PRISM part of the approach takes these excluded volume interactions into account when solving for the pair correlation functions. This PRISM-MC approach is also faster than either a CPU-based MC or molecular dynamics simulation with explicit matrix, thus allowing us to span the parameter space of varying polydispersity of the chains, $N_{\text {matrix }}$, particle size (D), and grafting density $(\sigma)$ in far less time than had we approached this problem with CPU-based simulations of the finite number of grafted particles in implicit or explicit matrix chains. The limitations with PRISM-MC approach: For some sets of parameters, e.g. large size disparity between sites (e.g. D>25d) or strong attractive interactions (or low $\mathrm{T}$ ) or high grafting densities $\left(\sigma>0.6\right.$ chains $\left./ \mathrm{nm}^{2}\right)$ self consistent PRISM-MC might not yield solutions due to numerical convergence issues. Additionally, the choice of closures is critical. In this study we used Percus Yevick and Hyper-netted Chain closures based on past success in predicting phase behavior in polymer nanocomposites. For other soft materials systems the most appropriate closures might be different, and have to be found based on direct comparisons with simulation results.

\subsubsection{Analysis}

Besides the various partial radial distribution functions and structure factors which are direct outputs of PRISM, we also calculate the matrix penetration depth. We begin by first truncating and shifting the partial pair distribution function between the nanoparticle and the matrix beads, $g_{P M}$, so that the domain of the function varies between the particle surface and the height of the 
grafted layer, $h_{g}$. We then calculate the square root of the normalized second moment of $g_{P M}$ in this domain to obtain the matrix penetration depth, $\lambda$.

$$
\lambda=\sqrt{\frac{\int_{0}^{h g} r^{2} g_{P M}(r) d r}{\int_{0}^{h g} g_{P M}(r) d r}}
$$

\subsubsection{Parameters}

In this work, polydispersity in the grafted chains is modeled using a log-normal distribution of chain lengths to achieve a target polydispersity index (PDI) while maintaining the average graft length, $\mathrm{N}_{\mathrm{g}, \mathrm{avg}}$, constant at 20 Kuhn segments and minimum graft length of 8 Kuhn segments (or "monomers"). The particle diameter (D) is maintained as either 5 or 8 times the monomer diameter (d). We vary the grafting density, $\sigma$, in the range $0.1-0.65$ chains $/ \mathrm{d}^{2}$, the matrix chain length $\mathrm{N}_{\text {matrix }}$ from 10 to 80 monomers, and total packing fraction, $\eta$, of the nanocomposite from 0.1 to mimic the matrix as a dense solution to 0.3 to mimic melt-like polymer matrix. We model all pair-wise interactions in the system to be hard sphere (athermal) interactions to capture the purely entropic effects of introducing polydispersity. These athermal interactions are also appropriate to mimic experimental systems where the graft and matrix monomers have similar chemistry, and particlemonomer interactions are negligible. 


\subsection{Results}

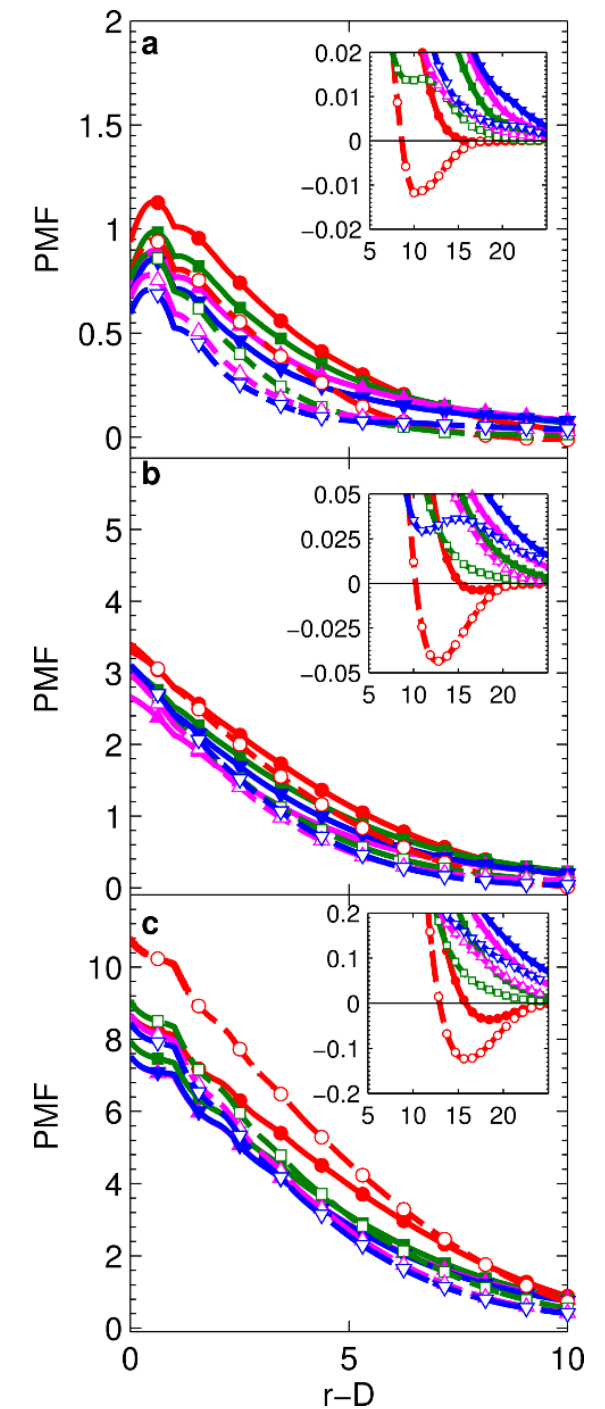

Figure 3.1: PMF (in units of kT) versus inter-particle distance, $r-D$ (in units of d), between grafted nanoparticles $(D=5 d)$ at $\sigma=0.1(a), 0.25$ (b), and 0.65 (c) chains $/ d^{2}$ and PDI= 1.0 (circles), 1.5 (squares), 2.0 (upward facing triangles), and 2.5 (downward facing triangles) with $N_{g, a v g}=20$, in a dense solution $(\eta=0.1)$ of monodisperse homopolymer matrix chains with $N_{\text {matrix }}=10$ (solid symbols) and $N_{\text {matrix }}=40$ (open symbols). The insets have the same axes labels as the main plots.

First, we present the PMF between polymer grafted nanoparticles, calculated as PMF= $\mathrm{k}_{\mathrm{B}} \mathrm{T} \ln \left[\mathrm{g}_{\text {particle-particle }}(\mathrm{r})\right]$, for particle size $\mathrm{D}=5 \mathrm{~d}$ at $\sigma=0.1$ chains $/ \mathrm{d}^{2}$ placed in a dense solution $(\eta=0.1)$ of monodisperse polymer matrix (Figure 3.1a). When $\mathrm{N}_{\text {matrix }}=10$ (solid symbols) and graft PDI=1.0 (monodisperse), the PMF exhibits repulsion at contact and no attractive well at intermediate inter- 
particle distances, which is in agreement with past experiments and theoretical studies for these lightly grafted systems (see review articles [1,24]). As the graft PDI increases, the PMF becomes slightly less repulsive at contact and slightly more repulsive at larger distances (Figure 3.1a inset). When $\mathrm{N}_{\text {matrix }}=40$ (open symbols) and graft PDI=1.0, the PMF is repulsive at contact and exhibits a weak attractive well at intermediate lengths. As graft PDI increases, the PMF loses the weak attractive well completely, and exhibits purely repulsive PMF (Figure 3.1a inset). Additionally, for both matrix lengths as PDI increases, the repulsive tail in the PMF increases in strength and extends to larger inter-particle distances. The decrease in repulsion at contact is driven by the polydisperse grafted polymers relieving some monomer crowding near particle surface by causing a change in grafted chain conformations to maximize overall conformational entropy upon introduction of polydispersity, as seen in recent study[8]. That study also showed that the effect of polydispersity on chain conformations is relatively minor at low grafting densities and more drastic at higher grafting densities where chain crowding is strong at the monodisperse limit. Therefore, one can expect that the attractive well at intermediate inter-particle distances, which was negligible at 0.1 chains $/ \mathrm{d}^{2}$ (Figure 3.1a), could be more significant at higher grafting densities and that the elimination of the attractive well could be more pronounced at higher grafting densities when polydispersity is introduced than seen at 0.1 chains $/ \mathrm{d}^{2}$.

At higher grafting densities of $\sigma=0.25$ (Figure 3.1b) and 0.65 chains $/ \mathrm{d}^{2}$ (Figure 3.1c), as expected from prior theoretical and experimental work on monodisperse grafts [1], we see that the repulsion at contact and attraction at intermediate distances increase in strength significantly compared to low grafting density, especially when $\mathrm{N}_{\text {matrix }}$ is larger than $\mathrm{N}_{\mathrm{g}, \text { avg }}$. While we only show $N_{\text {matrix }}=10$ and 40 here, we have confirmed as $N_{\text {matrix }}$ increases the attractive well at intermediate distances deepens (Supplementary Figure 3.4). We note that, for a few systems at the highest 
grafting density $\left(0.65\right.$ chains $\left./ \mathrm{d}^{2}\right)$ only, where matrix chains are expected to deplete/dewet large regions in the grafted layer near the particle surface, the choice of Percus-Yevick closure leads to negative values in $g_{\text {matrix-particle }}(\mathrm{r})$ at low $\mathrm{r}$ (where $\mathrm{g}_{\text {matrix-particle }}(\mathrm{r})$ should be 0 ), due to numerical issues. We also add that, for these specific systems, all other pair correlation functions are devoid of this issue and do not exhibit any negative values. Despite this issue, we note that PRISM-MC correctly predicts all known (qualitative and some quantitative) trends in monodisperse systems a) with increasing grafting density the mid-range attractive well deepens and shifts to higher interparticle distances (Figure 3.1); b) with increasing matrix chain length the attractive well depth deepens (Supplementary Figure 3.4); c) the value of the well depth seen at $0.65 \mathrm{chains} / \mathrm{d}^{2}$ is of the same order of magnitude ( 0.3-0.5kT in Supplementary Figure 3.4) as that seen for similar systems in recent simulation studies $[25,26]$ on systems with graft length of 10 monomers and matrix lengths of 10-70 monomers, and particle sizes approximately 10 times monomer size at high grafting density $\left(\sim 0.76\right.$ chains $\left./ \mathrm{nm}^{2}\right)$. The ability of PRISM-MC to predict the same qualitative trends as other theoretical methods[27], and, in certain cases quantitative agreement with prior simulations $[25,26]$ for monodisperse grafts suggests that this approach is capable of predicting correct qualitative trends for the polydisperse polymer grafted nanoparticles as well.

Continuing our discussion of higher grafting densities, as PDI increases the repulsion at contact is reduced more significantly at 0.65 chains $/ \mathrm{d}^{2}$ for $\mathrm{N}_{\text {matrix }}=40$ (Figure 3.1c) than at smaller grafting densities. This is because, at higher grafting densities, increasing polydispersity relieves the higher monomer crowding in the grafted layer at monodisperse limit. This is confirmed by the end-monomer concentration profiles which show larger values near the particle surface with increasing polydispersity, implying higher accessibility of the particle surface (Supplementary Table 3.1). The higher accessibility of the particle surface by end monomers is because of the 
shorter chains in the distribution as well as the changes in chain conformations due to reduced monomer crowding resulting from a wider grafted chain length distribution [8].

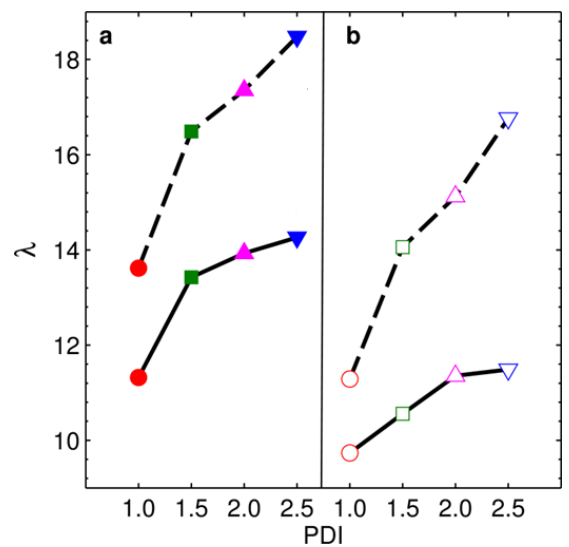

Figure 3.2: Penetration depth, $\lambda$ (in units of d), of matrix chains into grafted layer on nanoparticles $(D=5 d)$ grafted with polydisperse chains with $N_{g, a v g}=20$ at $\sigma=0.10$ chains $/ d^{2}$ (solid lines) and $\sigma=0.25$ chains $/ d^{2}$ (dashed lines) in a dense solution $(\eta=0.1)$ of monodisperse homopolymer matrix chains with $N_{\text {matrix }}=10$ (filled symbols) and $N_{\text {matrix }}=40$ (open symbols).

Most interestingly, at 0.65 chains $/ \mathrm{d}^{2}$ and $\mathrm{N}_{\text {matrix }}>\mathrm{N}_{\mathrm{g}, \text { avg }}$ (open symbols in Figure 3.1c) the attractive well of $\sim 0.1 \mathrm{kT}$ at intermediate distances seen in monodisperse systems is completely eliminated at PDI of 1.5 and above. Additional calculations at smaller PDI (1.05-1.4) (Supplementary Figure 3.5) found that there is a minimum PDI needed to eliminate the attractive well, and we expect this minimum PDI to be a function of grafting density, particle size and average graft length. The attractive well is eliminated at higher PDI because the longer chains in the polydisperse chain length distribution a) sterically repel the longer chains on the other grafted particle, and b) shift the entropic contributions more heavily towards the grafted chains than matrix chains, thus driving matrix chains to wet the grafted layer. The latter is confirmed from increasing penetration depth of the matrix chains into grafted layer, $\lambda$, (Figure 3.2), with increasing PDI. Since $\lambda$ is a measure of the average distance the matrix chains penetrate the grafted layer on the surface (Supplementary Table 3.1), Figure 3.2 implies increased matrix wetting of the grafted layer with increasing PDI. Since the mid-range attractive well has been shown to drive the nanoscale additives in polymer 
nanocomposites towards aggregation, eliminating the attractive well due to increased wetting of grafted layer suggests the exciting possibility of using polydispersity as a means to stabilize dispersions in systems where monodisperse grafts would drive aggregation.

To ensure that our choice of a specific discretized chain length distribution, that mimics a continuous log-normal distribution, does not bias the above results, we calculated the PMF for five different chain length distributions (all log-normal) for a select number of systems and found no significant change in PMF either in the repulsion at contact or attractive well (Supplementary Figure 3.6) in dense solutions. In contrast, when we compare PMFs from particles grafted with a log-normal chain length distribution, to those grafted with a bidisperse chain length distribution at the same PDI we observe some differences in both low-r repulsion and mid-range attraction (Supplementary Figure 3.7), and is the subject of our ongoing investigation.

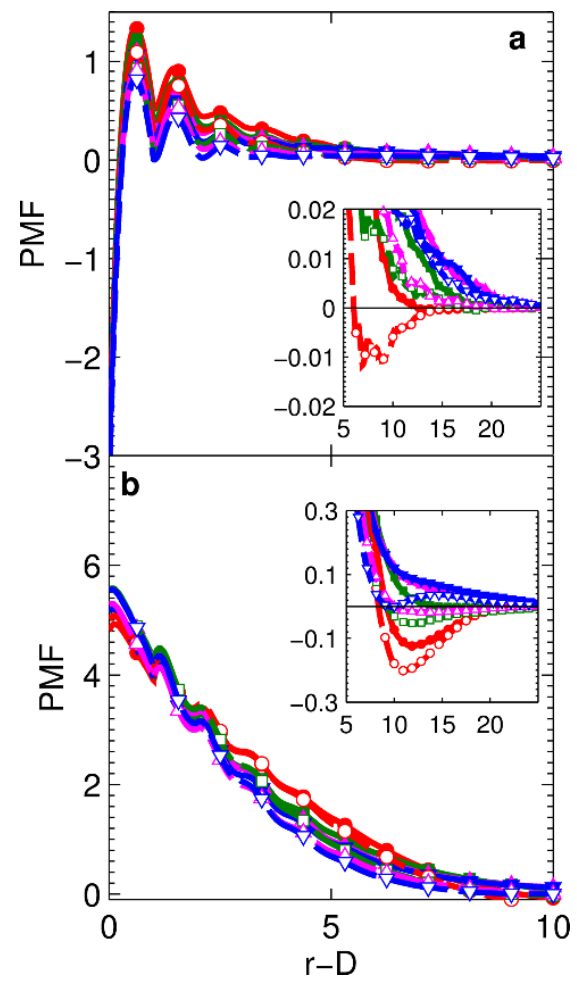

Figure 3.3: $P M F$ (in units of $k T$ ) versus $r-D$ (in units of $d$ ) between nanoparticles $(D=5 d)$ at $P D I=$ 1.0 (circles), $P D I=1.5$ (squares), PDI=2.0 (upward facing triangles), and PDI=2.5 (downward facing triangles) and $N_{g, a v g}=20$ at (a) $\sigma=0.1$ and (b) 0.65 chains $/ d^{2}$ in a monodisperse melt-like 
matrix ( $\eta=0.3)$ with $N_{\text {matrix }}=10$ (solid symbol) and $N_{\text {matrix }}=40$ (open symbol). The insets have the same axes labels as the main plots.

All the results presented so far are at a total packing fraction of $\eta=0.1$, which is characterized as a dense solution rather than a melt through calculations of the compressibility from the structure factor $S(k)$ as $k \rightarrow 0$ [28]. At a melt-like packing fraction of $\eta=0.3$, the matrix polymers have been shown to induce depletion like attractions between both bare and polymer grafted nanoparticles at infinitely dilute concentrations. This matrix-induced depletion-like attraction at low grafting densities shows up in the PMF as an attraction at contact (Figure 3.3a), and, at high grafting densities, significantly reduces the steric repulsion at contact (Figure 3.3b) and deepens the midrange attractive well (inset of Figure 3.3b versus Figure 3.1c). At low grafting density (a), the effects of polydispersity are reduced for $\eta=0.3$, as compared to $\eta=0.1$, as the values of attraction at contact $(\sim 3 \mathrm{kT})$ dominate at all PDI. Therefore in these conditions, polydispersity in grafted chains cannot overcome the matrix induced aggregation of the particles. At high grafting density (Figure 3.3b), comparing $\eta=0.3$ and $\eta=0.1$, the repulsion at contact is less sensitive to PDI for $\eta=0.3$, and a larger PDI is needed to eliminate the stronger attractive well at intermediate distances at $\eta=0.3$ (inset of Figure 3.3b). These results lead us to conclude that, for melt-like polymer matrices, one can stabilize dispersions using polydispersity only at high grafting densities, and the extent of polydispersity needed to stabilize dispersions is higher as compared to dense polymer solutions.

Past studies have shown that the relative graft length to particle diameter is an important parameter driving dispersion/aggregation in a polymer matrix [2, 29]. Higher D or lower curvature leads to increased monomer crowding, especially at high grafting densities. At PDI=1.0 and at ${ }_{\sigma}=0.1$ chains $/ \mathrm{d}^{2}$ the repulsion at contact and mid-range attraction is higher in the PMF for $\mathrm{D}=8 \mathrm{~d}$ 
than in the corresponding PMF for $\mathrm{D}=5 \mathrm{~d}$. This is in accordance with previous studies of monodisperse grafts [2, 29] that showed that decreasing curvature increases the monomer crowding near the particle surface, and decreases the propensity of matrix wetting the grafted layer. We see that at low grafting densities varying polydispersity of grafts on $D=8 \mathrm{~d}$ brings about the approximately the same effect as that seen for $D=5 d$ (Supplementary Figure 3.8). A detailed study of how curvature affects these polydispersity induced stabilization at a range of parameters is the subject of ongoing work.

\subsection{CONCLUSION}

In summary, this is one of the first studies demonstrating how increasing polydispersity in polymers grafted on spherical nanoparticles affects the PMF between homopolymer grafted nanoparticles in a homopolymer matrix. The effect of polydispersity is greatest at high grafting densities where polydispersity in grafted chain lengths has the largest effect on chain conformations [8] and the mid-range attraction is eliminated with increasing polydispersity. The implications that polydispersity can stabilize dispersions, even when the average graft molecular weight is lower than the matrix molecular weight, conditions that cause particle aggregation for monodisperse grafts, are exciting since much of the polymer synthesis community has been striving to achieve low polydispersity. This study motivates synthetic efforts to be directed towards obtaining controlled polydispersity in chain lengths as a design tool to program inter-particle interactions in a polymer matrix.

\subsection{ACKNOWLEDGEMENTS}

The authors acknowledge partial financial support by Department of Energy Early Career Award Program under Grant No. DE-SC0003912. 


\subsection{REFERENCES}

1. Green, P.F., The structure of chain end-grafted nanoparticle/homopolymer nanocomposites. Soft Matter, 2011. 7(18): p. 7914-7926.

2. Jayaraman, A. and K.S. Schweizer, Effective Interactions and Self-Assembly of Hybrid Polymer Grafted Nanoparticles in a Homopolymer Matrix. Macromolecules, 2009. 42(21): p. 8423-8434.

3. Akcora, P., et al., Anisotropic self-assembly of spherical polymer-grafted nanoparticles. Nature Materials, 2009. 8(4): p. 354-359.

4. de Vos, W.M. and F.A.M. Leermakers, Modeling the structure of a polydisperse polymer brush. Polymer, 2009. 50(1): p. 305-316.

5. Milner, S.T., T.A. Witten, and M.E. Cates, EFFECTS OF POLYDISPERSITY IN THE END-GRAFTED POLYMER BRUSH. Macromolecules, 1989. 22(2): p. 853-861.

6. Witten, T.A. and P.A. Pincus, COLLOID STABILIZATION BY LONG GRAFTED POLYMERS. Macromolecules, 1986. 19(10): p. 2509-2513.

7. Himmi, M., et al., Interaction force between colloidal particles clothed by end-grafted polydisperse polymer chains in solution. Journal of Molecular Liquids, 2003. 102(1-3): p. 347-363.

8. Dodd, P.M. and A. Jayaraman, Monte carlo simulations of polydisperse polymers grafted on spherical surfaces. Journal of Polymer Science Part B-Polymer Physics, 2012. 50(10): p. 694-705.

9. Agarwal, U. and F.A. Escobedo, Mesophase behaviour of polyhedral particles. Nature Materials, 2011. 10(3): p. 230-235.

10. Nair, N. and A. Jayaraman, Self-Consistent PRISM Theory-Monte Carlo Simulation Studies of Copolymer Grafted Nanoparticles in a Homopolymer Matrix. Macromolecules, 2010. 43(19): p. 8251-8263.

11. Jayaraman, A. and N. Nair, Integrating PRISM theory and Monte Carlo simulations for studying polymer functionalized particles and polymer nanocomposites. Molecular Simulations, 2012. in press(Special issue on New Developments in Molecular Simulations).

12. Hooper, J.B. and K.S. Schweizer, Contact aggregation, bridging, and steric stabilization in dense polymer-particle mixtures. Macromolecules, 2005. 38: p. 8858-8869.

13. Hooper, J.B. and K.S. Schweizer, Theory of phase separation in polymer nanocomposites. Macromolecules, 2006. 39: p. 5133-5142.

14. Hooper, J.B., et al., Structure, surface excess and effective interactions in polymer nanocomposite melts and concentrated solutions. Journal of Chemical Physics, 2004. 121(14): p. 6986-6997.

15. Hall, L.M., et al., Concentration Fluctuations, Local Order, and the Collective Structure of Polymer Nanocomposites. Macromolecules, 2009. 42(21): p. 8435-8442. 
16. Hall, L.M. and K.S. Schweizer, Many body effects on the phase separation and structure of dense polymer-particle melts. Journal of Chemical Physics, 2008. 128: p. 234901.

17. Hall, L.M. and K.S. Schweizer, Structure, scattering patterns and phase behavior of polymer nanocomposites with nonspherical fillers. Soft Matter, 2010. 6(5): p. 1015-1025.

18. Jayaraman, A. and K.S. Schweizer, Structure and Assembly of Dense Solutions and Melts of Single Tethered Nanoparticles. Journal of Chemical Physics, 2008. 128: p. 164904.

19. Jayaraman, A. and K.S. Schweizer, Effect of the number and placement of polymer tethers on the structure of dense solutions of hybrid nanoparticles. Langmuir, 2008. 24(19): p. 11119-11130.

20. Jayaraman, A. and K.S. Schweizer, Effective Interactions and Self-Assembly of Hybrid Polymer Grafted Nanoparticles in a Homopolymer Matrix. Macromolecules, 2009. 42 p. pp 8423-8434.

21. Hindmarsh, A.C., et al., SUNDIALS: Suite of nonlinear and differential/algebraic equation solvers. Acm Transactions on Mathematical Software, 2005. 31(3): p. 363-396.

22. Jayaraman, A. and K.S. Schweizer, Effective Interactions, Structure, and Phase Behavior of Lightly Tethered Nanoparticles in Polymer Melts. Macromolecules, 2008. 41(23): p. 9430-9438.

23. Akcora, P., et al., Anisotropic self-assembly of polymer-decorated spherical nanoparticles. Nature Materials, 2009. 8: p. 354-359.

24. Krishnamoorti, R., Strategies for Dispersing Nanoparticles in Polymers. MRS Bulletin, 2007. 32(4): p. 341-347.

25. Smith, G.D. and D. Bedrov, Dispersing Nanoparticles in a Polymer Matrix: Are Long, Dense Polymer Tethers Really Necessary? Langmuir, 2009. 25(19): p. 11239-11243.

26. Meng, D., et al., Effective interactions between grafted nanoparticles in a polymer matrix. Soft Matter, 2012. 8(18): p. 5002-5010.

27. Altintas, O., et al., Combining Modular Ligation and Supramolecular Self-Assembly for the Construction of Star-Shaped Macromolecules. Macromolecular Rapid Communications, 2012. 33(11): p. 977-983.

28. Jayaraman, A. and K.S. Schweizer, Structure and Assembly of Dense Solutions and Melts of Single Tethered Nanoparticles. J. Chem. Phys., 2008. 128: p. 164904.

29. Trombly, D.M. and V. Ganesan, Curvature effects upon interactions of polymer-grafted nanoparticles in chemically identical polymer matrices. Journal of Chemical Physics, 2010. $133(15)$. 


\subsection{SUPPLEMENTARY}

3.7.1 Effect of matrix length on the potential of mean force between monodisperse grafted nanoparticles

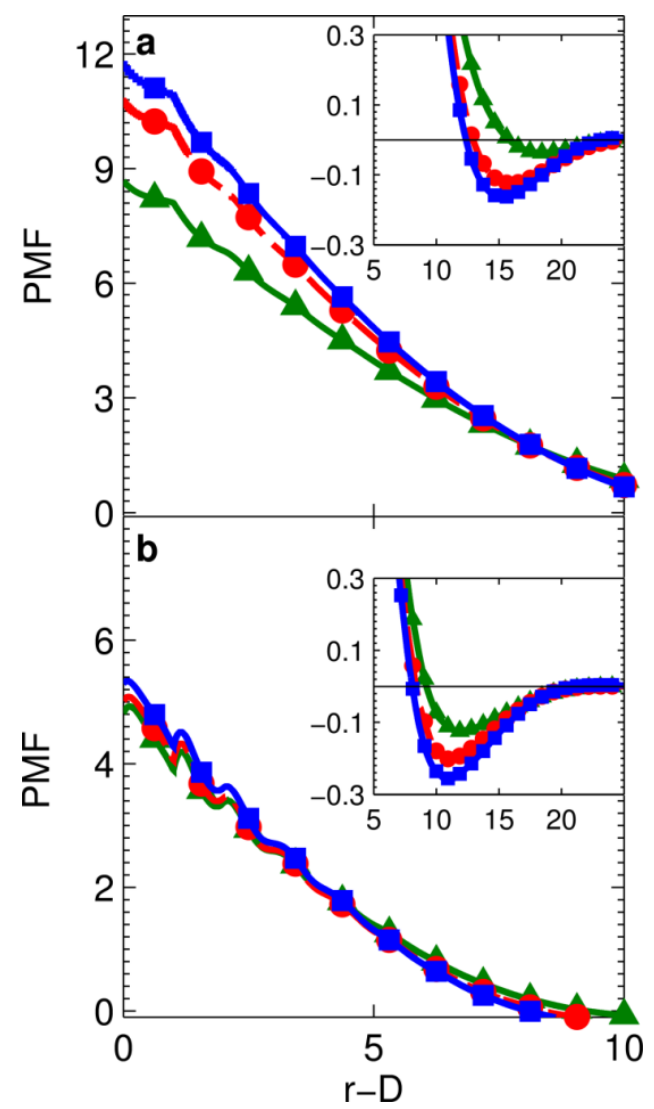

Figure 3.4: Potentials of mean force (in kT) versus inter-particle distances (in units of d) between nanoparticles of size $D=5 d$ with monodisperse chains with $N_{g}=20$, at a grafting density of $\sigma=0.10$ chains $/ d^{2}$, in dense solution ( $\eta=0.1$, subplot a) and melt $(\eta=0.3$, subplot b) of monodisperse homopolymers of $N_{\text {matrix }}=10$ (triangles), $N_{\text {matrix }}=40$ (circles), $N_{\text {matrix }}=80$ (squares). 
3.7.2 Dataset for concentration of end monomers at the particle surface and penetration depth of matrix into the grafted layer

Table 3.1: Tabulated data for the end monomer concentration at contact $\left(\varphi_{c}\right)$ and the penetration depth of the matrix into the grafted layer ( $\lambda$ ) in units of d, for varying PDI, matrix volume fraction $(\eta)$, grafting density $(\sigma)$, and matrix length ( $\left.N_{\text {matrix }}\right)$.

\begin{tabular}{|c|c|c|c|c|c|}
\hline & \multirow[b]{3}{*}{ PDI } & \multicolumn{4}{|c|}{$n=0.1$} \\
\hline & & \multicolumn{2}{|c|}{$\sigma=0.10$ chains $/ \mathrm{d}^{2}$} & \multicolumn{2}{|c|}{$\sigma=0.25$ chains $/ d^{2}$} \\
\hline & & $\phi_{c} \times 10^{3}$ & $\lambda$ & $\phi_{c} \times 10^{3}$ & $\lambda$ \\
\hline \multirow{4}{*}{ 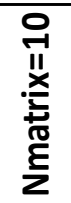 } & 1.0 & 0.0452 & 11.3 & 0.0377 & 13.6 \\
\hline & 1.5 & 0.0904 & 13.4 & 0.1356 & 16.5 \\
\hline & 2.0 & 0.1884 & 13.9 & 0.1733 & 17.4 \\
\hline & 2.5 & 0.1658 & 14.3 & 0.1206 & 18.5 \\
\hline \multirow{7}{*}{ 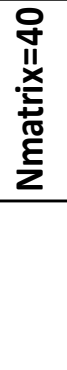 } & 1.0 & 0.0678 & 9.7 & 0.0527 & 11.3 \\
\hline & 1.5 & 0.0904 & 10.6 & 0.1959 & 14.1 \\
\hline & 2.0 & 0.098 & 11.4 & 0.2035 & 15.1 \\
\hline & 2.5 & 0.1959 & 11.5 & 0.1206 & 16.8 \\
\hline & & \multicolumn{4}{|c|}{$n=0.3$} \\
\hline & & \multicolumn{2}{|c|}{$\sigma=0.10$ chains $/ \mathrm{d}^{2}$} & \multicolumn{2}{|c|}{$\sigma=0.25$ chains $/ \mathrm{d}^{2}$} \\
\hline & PDI & $\phi_{\mathrm{c}} \times 10^{3}$ & $\lambda$ & $\phi_{c} \times 10^{3}$ & $\lambda$ \\
\hline \multirow{4}{*}{ 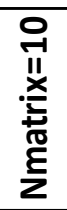 } & 1.0 & 0.1281 & 10.2 & 0.1236 & 10.7 \\
\hline & 1.5 & 0.1507 & 10.9 & 0.2336 & 12.6 \\
\hline & 2.0 & 0.2863 & 11.7 & 0.3517 & 14.1 \\
\hline & 2.5 & 0.1959 & 11.6 & 0.2321 & 14.8 \\
\hline \multirow{4}{*}{ 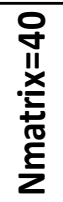 } & 1.0 & 0.1432 & 8.2 & 0.1507 & 8.6 \\
\hline & 1.5 & 0.1959 & 9.1 & 0.2035 & 10.2 \\
\hline & 2.0 & 0.2336 & 9.2 & 0.2863 & 12.2 \\
\hline & 2.5 & 0.2478 & 9.9 & 0.3918 & 12.3 \\
\hline
\end{tabular}


3.7.3 Effect of incremental increases in PDI on the intermediate-range attractive PMF well

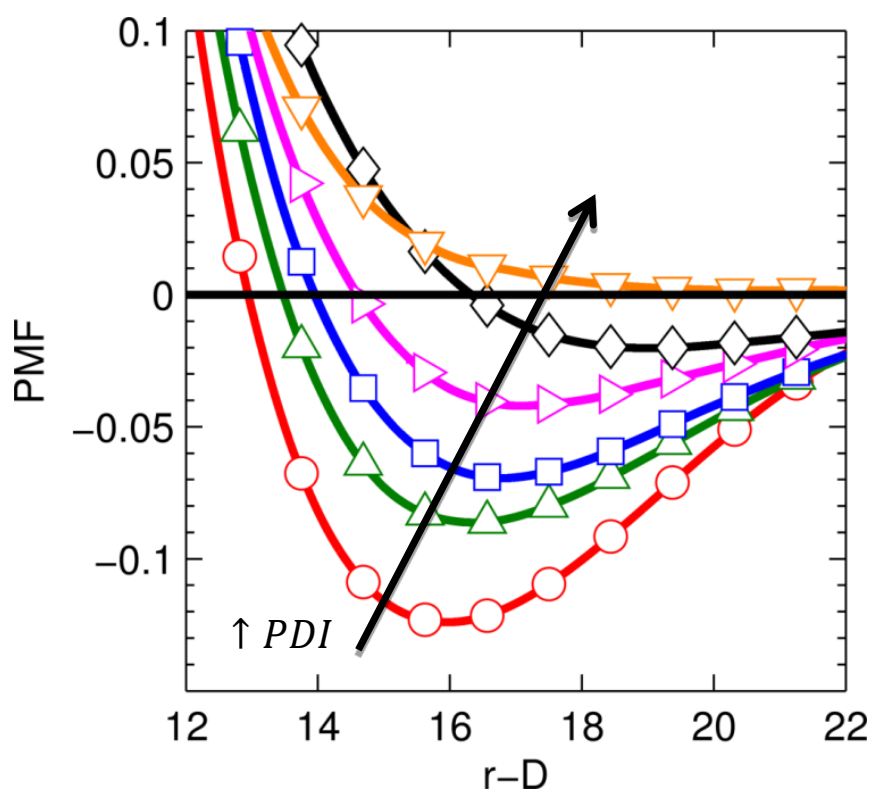

Figure 3.5: Potentials of mean force, $P M F$ (in units of $k T$ ), versus inter-particle distance, $r-D$ (in units of $d$ ), between nanoparticles of size $D=5 d$, grafted with polydisperse chains at grafting densities $\sigma=0.65$ chains $/ d^{2}$ with PDI= 1.00 (circles), PDI=1.05 (upward facing triangles), $P D I=1.10$ (squares), $P D I=1.15$ (rightward facing triangles), $P D I=1.20$ (diamonds), and $P D I=1.40$ (downward facing triangles) with $N_{g, a v g}=20$ in a dense solution $(\eta=0.1)$ of monodisperse homopolymer matrix chains with $N_{\text {matrix }}=40$ 
3.7.4 Effect of log-normal chain length distribution on the potential of mean force between polymer grafted nanoparticles
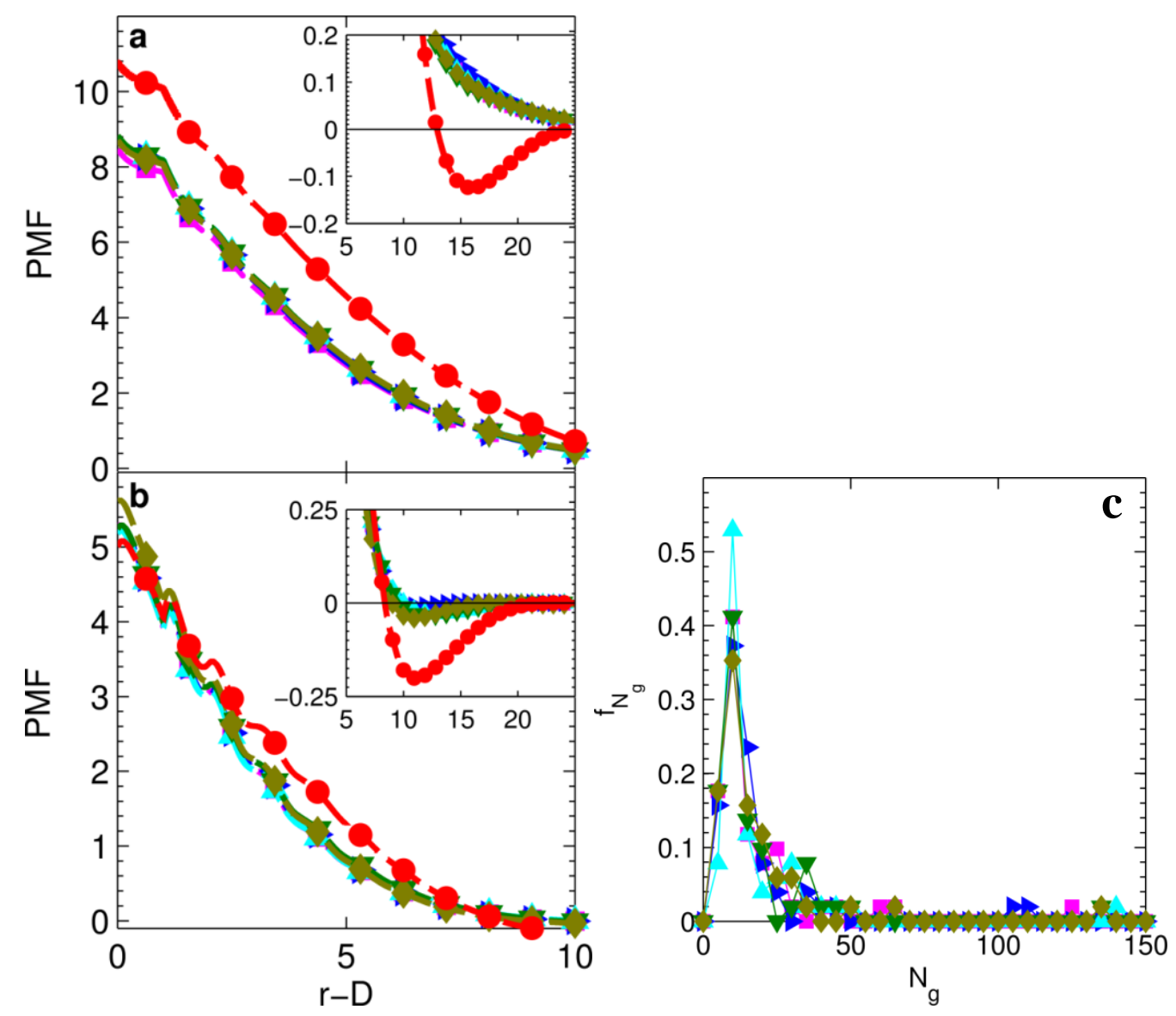

Figure 3.6: Potentials of mean force (in units of $k T$ ) versus inter-particle distances (in units of $d$ ) between nanoparticles of size $D=5 d$ with polydisperse chains of $P D I=1.0$ (circles) and PDI=2.0 (other symbols) with $N_{g, a v}=20$ and $N_{g, m i n}=8$ at a grafting density of $\sigma=0.65$ chains $/ d^{2}$, for five different log-normal chain length distributions (subplot $c$ ), in a dense solution ( $\eta=0.1$, subplot a) and melt ( $\eta=0.3$, subplot $b$ ) of monodisperse homopolymers with $N_{\text {matrix }}=40$. Each symbol, (besides circle) corresponds to a different log-normal chain distribution. 
3.7.5 Comparison of log-normal and bidisperse chain length distributions

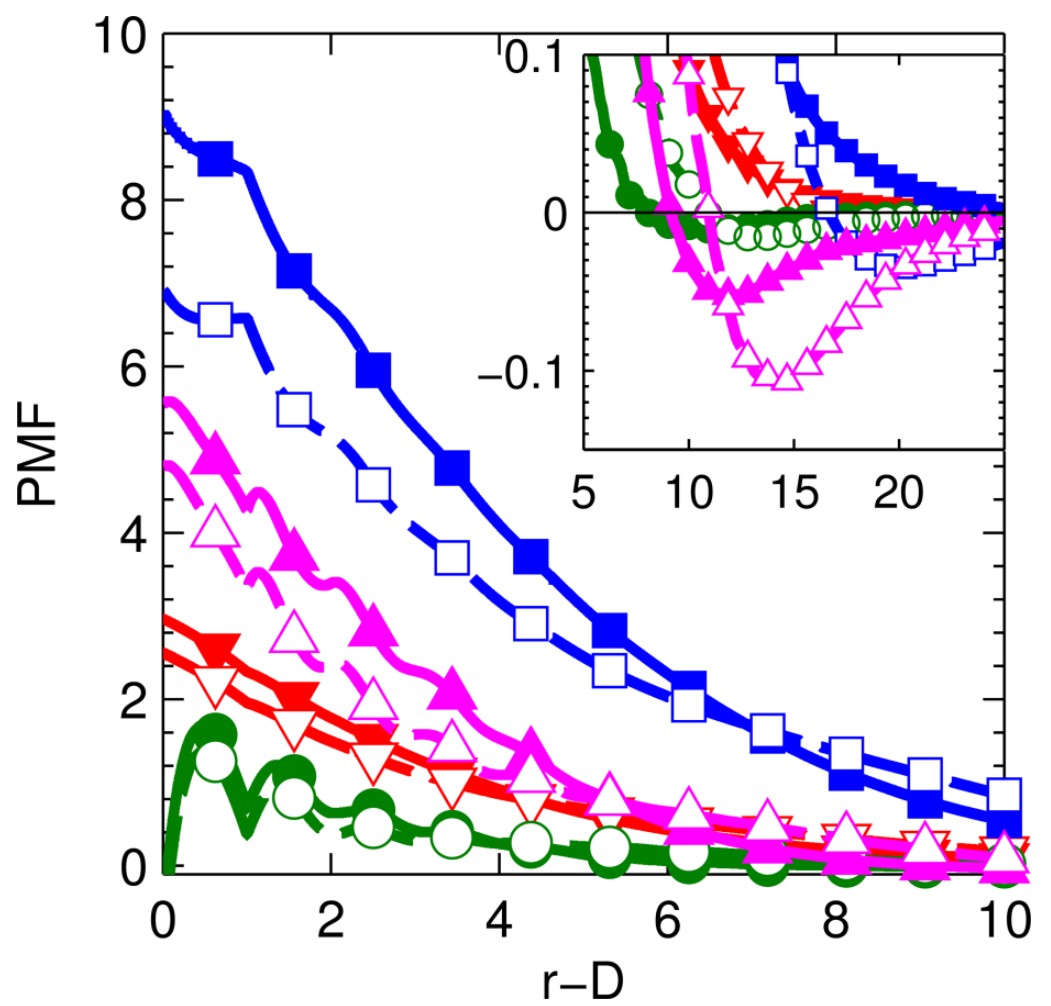

Figure 3.7: Potentials of mean force, PMF (in units of $k T$ ) versus inter-particle distance, $r-D$ (in units of $d$ ), between nanoparticles of size $D=5 d$, grafted with polydisperse (filled symbols) or bidisperse (open symbols) chains at grafting densities $\sigma=0.65$ chains/d ${ }^{2}$ (squares and upward triangle) and $\sigma=0.25$ chains $/ d^{2}$ (circles and downward triangles) at $P D I=1.5$ with $N_{g, a v}=20$ in a dense solution ( $\eta=0.1$, downward triangles and squares) and melt-like $(\eta=0.3$, upward triangles and circles) matrix of monodisperse homopolymer matrix chains with Nmatrix $=40$. 
3.7.6 Effect of lowered curvature on potential of mean force between polymer grafted nanoparticles

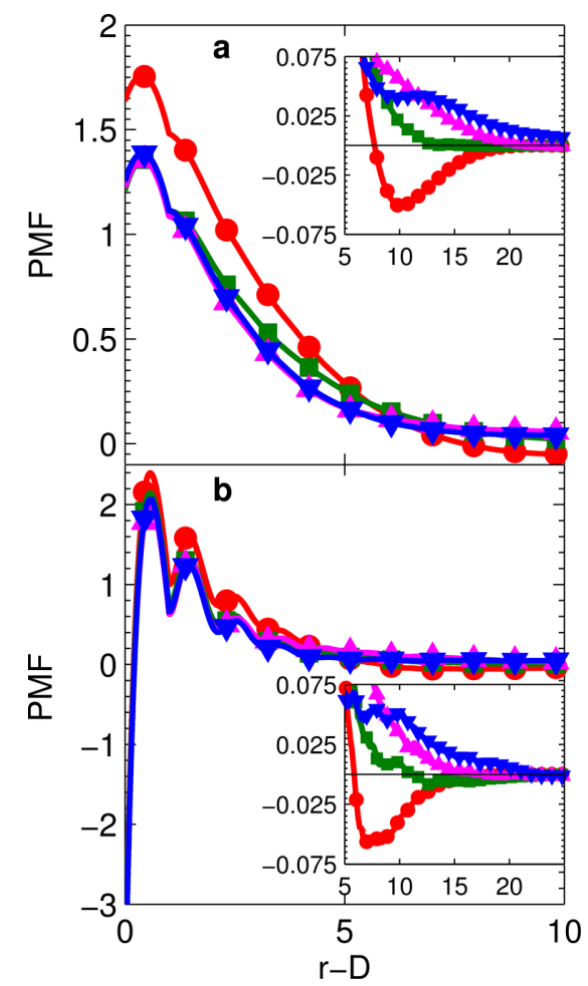

Figure 3.8: Potentials of mean force, PMF (in units of kT) versus inter-particle distance, r-D (in units of $\mathrm{d}$ ) between nanoparticles of size $\mathrm{D}=8 \mathrm{~d}$ grafted with polydisperse chains at $\mathrm{PDI}=1.0$ (circles), PDI=1.5 (squares), $\mathrm{PDI}=2.0$ (upward facing triangles), and $\mathrm{PDI}=2.5$ (downward facing triangles) with $\mathrm{N}_{\mathrm{g}, \mathrm{av}}=20$ at a grafting density of $\sigma=0.1$ chains $/ \mathrm{d}^{2}$, in a dense solution $(\eta=0.1$, subplot a) and melt ( $\eta=0.3$, subplot $b$ ) of monodisperse homopolymers with $N_{\text {matrix }}=40$. The insets have the same axes labels as the main plots. 


\section{Polydisperse Homopolymer GRAFTS Stabilize DisPERSIONS OF NANOPARTICLES IN A CHEMICALLY IDENTICAL HOMOPOLYMER MATRIX: AN INTEGRATED THEORY AND SIMULATION STUDY}

Adapted from: Soft Matter 9 (29), 6876-6889

\subsection{INTRODUCTION}

Controlling the morphology of nanoscale additives in a polymer matrix is critical for tuning the macroscopic properties of the resulting polymer nanocomposite. For example, superior mechanical properties of polymer nanocomposites can be achieved via good dispersion of nanoscale fillers in the polymer matrix. One approach to control the morphology of the nanocomposite is via functionalization of the nanoparticle surface with ligands, such as polymers and surfactants, that tune the effective interactions between the particles in the matrix leading to the target morphology. In particular, to achieve good dispersion of nanoparticles in a polymer matrix, the nanoparticle surface is grafted with polymers that are chemically identical to the matrix polymer. The chemical similarity between the graft and matrix improves the effective miscibility of the grafted particles in the matrix over that seen with bare (organic or inorganic) nanoparticles in the matrix. Extensive theoretical and experimental studies, both at high and low grafting density, for these chemically identical graft and matrix systems, have shown that the molecular weights of the grafted and matrix polymer play a critical role in dictating whether the grafted nanoparticles aggregate or disperse[110]. At high grafting density, where the grafted chains are stretched in the "brush" regime, nanoparticles disperse (aggregate) if the graft molecular weight is higher (lower) than matrix molecular weight. This is explained by the wetting and dewetting of the grafted layer by the matrix chains. In the case where the grafted polymer is the same chemistry as the matrix polymer the wetting/dewetting of the grafted chains by the matrix chains is driven purely by the entropy of the 
system. When the graft molecular weight is larger than the matrix molecular weight the conformational entropy of the grafted chain dominates, and the matrix chains penetrate (or wet) the grafted layer. When the graft molecular weight is smaller than the matrix molecular weight the conformational entropy of the matrix chain dominates, and the matrix chains deplete the grafted layer. The exact value of the matrix to graft chain molecular weight where this transition from wetting to dewetting occurs is a function of the grafting density and the curvature of the particle[1] In contrast to the high grafting density case, at low grafting density, dispersion/aggregation is dependent on the amount of exposed nanoparticle surface. Larger graft molecular weight chains can better shield nanoparticle surfaces from direct particle-particle contacts and lead to dispersion of grafted particles in the polymer matrix[11]. Despite the importance of graft molecular weight for controlling the morphology, there are very few experimental and theoretical studies on polymer grafted nanoparticles that have investigated the role of polydispersity in the grafted chains and its effect on miscibility of grafted chains with matrix chains.

While there have been less than a handful of studies on polydisperse polymers grafted on nanoparticle surfaces (with finite curvature), there have been many studies focused at the two extremes in the range of curvature - polymers grafted on flat surfaces (zero curvature) and star polymers (cores with infinite curvature). Polydispersity in chain lengths grafted on flat surfaces[12,13] have been shown to alter chain conformations and the overall height of the grafted layer on these surfaces (with no curvature). In case of star polymers with polydisperse arms the effective force, $F$, between polydisperse star polymers in a good solvent has been shown to have a drastically different expression as compared to monodisperse star polymers [14] [15]. To understand behavior of polydisperse grafted polymers on a surfaces of finite curvature Dodd and Jayaraman[16] studied using Monte Carlo simulations a single spherical polymer grafted nanoparticle with polydisperse grafted chains, in an implicit solvent, at a purely athermal limit, for varying polydispersity indices (PDI=1-2.5), particle diameter, and grafting density. Dodd and Jayaraman 
showed that the conformations of the polydisperse chains grafted chains on spherical nanoparticles $(5-8 \mathrm{~nm}$ in diameter) deviates from the monodisperse counterpart, and approach that of a single grafted chain on the same particle size because of polydispersity-induced relief in monomer crowding. Specifically, the radius of gyration of the short chains was lower at PDI $>1$ than at PDI=1 (monodisperse), and the long chains were less stretched at distances away from particle surface at PDI $>1$ than at PDI=1.

These past studies demonstrate that the grafted chain conformations are significantly affected by polydispersity in the grafted chain lengths. This leads to the question: In the presence of a polymer matrix, is the effect of polydispersity on grafted chain conformations large enough to alter how matrix chains wet/dewet/deplete the grafted layer? If yes, is this change in matrix wettability of the grafted layer predictable so that one could deliberately introduce polydispersity as a design knob to tailor inter-particle interactions? Past studies on flat surfaces have shown improved mixing of the grafted layer with the free chains in the presence of polydispersity[17-19]. It was observed that when graft-matrix interaction is repulsive, polydispersity does not affect the width of the interface between grafted brush and matrix [18].However, for attractive or athermal brush-matrix interaction there is increased stretching of the graft chains into the matrix as the polydispersity (measured by $\mathrm{PDI}=\mathrm{DP}_{\mathrm{w}} / \mathrm{DP}_{\mathrm{n}}$ ) increases from 1.0 to 3.0, indicating enhanced mixing between the matrix and highly polydisperse grafted chains with increasing polydispersity[18] when brush monomer-matrix monomer interactions are attractive or athermal. These results for flat brush cannot be extrapolated directly to the case of chains grafted on a high (convex) curvature nanoparticle surface. This is because the available volume per grafted chain on a flat surface is much lower than that on a convex surface, which changes the amount of crowding among the grafted chains, and results in significantly different conformations for the grafted chains.

To understand how polydispersity in grafted polymer affects miscibility of polymer grafted nanoparticles with finite convex curvature in a polymer matrix of same chemistry, in a recent letter Jayaraman and coworkers[20] explored how polydispersity in polymer chains grafted on nanoparticles affects the potential of mean force (PMF) between the polymer grafted nanoparticles at varying grafting densities (e.g. low, intermediate, and high), in a dense solution of matrix polymers and melt-like polymer 
matrix at varying matrix lengths (e.g. less than and greater than average graft length) using Polymer Reference Interaction Site Model (PRISM) - Monte Carlo (MC) approach. One of the key results was that at high grafting density, polydispersity in the grafted polymers removed the attractive well in the potential of mean force, suggesting that polydispersity could stabilize dispersions in a monodisperse polymer matrix at conditions where corresponding monodisperse polymer grafted particles would exhibit aggregation. In this paper we present detailed results from the above PRISM-MC study of infinitely dilute concentration of polydisperse polymer grafted nanoparticles in a monodisperse homopolymer matrix at varying grafting density, matrix packing fraction and particle diameter, along with some results from Brownian dynamics simulations of a single polydisperse polymer grafted nanoparticle in an explicit monodisperse homopolymer matrix. We find that, in the case of a polydisperse (log-normal) distribution of graft lengths and matrix lengths longer than graft lengths, the presence of the long chains and the improved wetting of the grafted layer by the matrix chains brought about by the reduced monomer crowding in the grafted layer, together remove the mid-range attraction seen at the corresponding monodisperse limit. Direct comparison of the effects of a statistically polydisperse distribution of graft lengths to a bidisperse distribution of graft lengths on the potential of mean force helps us understand how the chains lengths that are longer and shorter than the average chain length, and the reduced monomer crowding in the grafted layer contribute to the overall shape and features of the potential of mean force.

This paper is organized as follows. In the Section 4.2 we provide details of the model, theory and simulation, and analysis methods used in this work. In the Section 4.3 we present results from PRISM-MC approach that show the effect of polydispersity of graft lengths on potential of mean force (PMF) between two polymer grafted nanoparticles in a homopolymer matrix at varying grafting density, matrix chain length, matrix packing fraction, and particle diameter. We also present in certain cases the corresponding BD simulation results for concentration profiles and chain conformations for a single polymer grafted nanoparticles in a homopolymer matrix. We conclude the result section with a direct comparison of polydisperse grafted system with a 
bidisperse grafted system (Section 4.3.5). In the Section 4.4 we summarize this work and present the implications of the key results.

\subsection{APPROACH}

\subsubsection{Model}

We model homopolymer grafted spherical nanoparticle as a hard spherical nanoparticle of diameter $D$ with freely jointed chains permanently attached randomly on the particle surface. Through visual analysis we ensure that the chains are approximately equally spaced apart, and not clustered regionally on the particle. We model the matrix homopolymer chain as a freely jointed chain. Both the grafted and matrix homopolymer chains consist of monomer beads of diameter $d$ chosen to mimic a Kuhn segment of a linear synthetic polymer. While chains in the Monte Carlo (MC) simulations have a constant bond length of 1.4d, monomers in the Brownian Dynamics (BD) simulations are connected together with harmonic bonds with force constant $\mathrm{k}=30$ and bond rest length $\mathrm{r}_{0}=1.4 \mathrm{~d}$. The grafted homopolymer chains are monodisperse with chains of equal number of monomers $\left(\mathrm{N}_{\mathrm{graft}}=20\right)$, or polydisperse with log-normal distribution of chain lengths or bidisperse with a bimodal distribution consisting of (equal number of) short and long chains. We quantify the polydispersity via the polydispersity index (PDI). For systems with a polydisperse graft length distribution with PDI greater than 1 we fit the distribution of chain lengths to a lognormal distribution with minimum and number average graft lengths $\mathrm{N}_{\text {graft,min }}=8$ and $\mathrm{N}_{\text {graft,avg }}=20$. The details of the PDI calculation and the allocation of chain length distribution are given in the Supplemental Section Error! Reference source not found.. The matrix is maintained onodisperse throughout this study and its chain length is denoted by $N_{\text {matrix }}$. The total packing fraction $\eta$ is the volume fraction of the system occupied by the matrix chains and the polymergrafted nanoparticles (fillers). The volume fraction of $\eta$ occupied by the polymer-grafted 
nanoparticles is denoted by the filler fraction $\phi$. In this paper we choose $\phi=0.001$ to calculate potential of mean force between the grafted particles at the infinitely dilute (or 2-particle) filler limit. We maintain athermal interactions $\mathrm{U}_{\mathrm{ij}}(\mathrm{r})$ between all pairs of monomers (on grafted and matrix chains), particle and particle, and particle and monomers (on grafted and matrix chains). In the PRISM-MC calculations we model these athermal interactions using hard sphere potentials, while in the BD simulations, we use shifted-truncated LJ potentials (i.e. Weeks-ChandlerAndersen or WCA potential), defined as:

$$
V_{L J}(r)=\left\{\begin{array}{cc}
4 \varepsilon\left[\left(\frac{\sigma}{r}\right)^{12}-\left(\frac{\sigma}{r}\right)^{6}\right]+4 \varepsilon\left[\left(\frac{\sigma}{r_{\text {cut }}}\right)^{12}-\left(\frac{\sigma}{r_{\text {cut }}}\right)^{6}\right] & r<r_{\text {cut }} \\
0 & r \geq r_{\text {cut }}
\end{array}\right.
$$

where $\varepsilon$ is the interaction well depth, $\sigma$ is the bead diameter, and $\operatorname{rcut}\left(=2^{\frac{1}{6}} \sigma\right)$ is the cutoff distance for the potential. The choice of athermal interactions is appropriate to mimic experimental systems where the graft and matrix monomers have similar chemistry, and particle-monomer interactions are negligible. Additionally, the choice of athermal interactions ensures that we can capture the effective interactions resulting from entropic effects purely.

\subsubsection{PRISM-MC Method}

We use a self-consistent Polymer Reference Interaction Site Model theory and Monte Carlo simulation (PRISM-MC) approach recently developed by Nair and Jayaraman_ENREF_21 for studying polymer grafted particles[21], to calculate the potential of mean force between monodisperse and bidisperse polymer grafted particles in a homopolymer matrix. We use this integrated theory-simulation approach because it is computationally much faster than either pure Monte Carlo simulations or molecular dynamics simulations of polymer grafted particles in an explicit polymer matrix, thus allowing us to scan a large parameter space in a reasonable time. 
PRISM theory consists of a matrix of Ornstein-Zernike-like integral equations that relate the total site-site inter-molecular pair correlation function, $h_{i j}(r)$, to the inter-molecular direct correlation function, $c_{i j}(r)$, and intra-molecular pair correlation function, $\omega_{i j}(r)$. The PRISM equations in Fourier space are

$$
\begin{gathered}
H(q)=\Omega(q) C(q)[\Omega(q)+H(q)] \\
H_{i j}(q)=\rho_{i} \rho_{j} \hat{h}_{i j}(q) \\
\Omega_{i j}(q)=\rho \sum_{\alpha=1}^{N_{i}} \sum_{\beta=1}^{N_{j}} \Omega_{\alpha_{i} \beta_{j}}(q)
\end{gathered}
$$

where $H(q), C(q)$ and $\Omega(q)$ in this study are matrices of size $3 \times 3$ for the following 3 types of sites: graft monomers (A), particle (B), and matrix (M), with the matrix elements defined in equations 1b-c. We note that despite the chemistry of the graft and matrix being the same these sites are physically identified separately as graft or matrix. In the above equations, $N_{i}$ and $\rho_{i}$ are respectively the number and number density of site $i, \rho$ is the molecular number density, and $\Omega_{i j}(q)$ the intramolecular pair correlation function between sites $i$ and $j$ within a certain molecule in Fourier space. To solve equation 1 , we use closure relations connecting the real space $c_{i j}(r), h_{i j}(r)\left(=g_{i j}(r)-1\right)$ and interaction potentials $U_{i j}(r)$. The choice of closures depends on the system being studied. Previous work on a mixture of nanoparticles and polymers[22-31] shows that the Percus-Yevick (PY) closure for polymer-polymer and polymer-particle, and the hypernetted chain (HNC) closure for particle-particle work well. We have used the same combination of atomic closures, since this work also consists of polymers and nanoparticles. Given that $\sigma_{i j}$ is the distance of closest approach between sites $i$ and $j$, i.e. $\sigma_{i j}=d$ for monomer-monomer pairs and $\sigma_{i j}=\frac{D+d}{2}$ (as stated earlier, $d$ 
and $D$ are the monomer and particle diameters, respectively) and particle-monomer pairs, the impenetrability condition applies inside the hard core:

$$
g_{i j}(r)=0 \quad r<\sigma_{i j}
$$

Outside the hard core, the PY approximation describes the direct correlation function between all pairs of sites (except particle-particle):

$$
c_{i j}(r)=\left(1-e^{\beta U_{i j}(r)}\right) g_{i j}(r) \quad r>\sigma_{i j}
$$

and the HNC closure handles the particle-particle direct correlation function:

$$
c_{B B}(r)=h_{B B}(r)-\ln g_{B B}(r)-\beta U_{B B}(r) \quad r>D
$$

To efficiently solve this system of coupled nonlinear integral equations we employ the KINSOL algorithm[32] with the line search optimization strategy. Attaining convergence for complex nonlinear integral equations is much easier with KINSOL as compared to the Picard technique, the method used in prior PRISM theory work. The solution of the PRISM equations yields pair correlation functions, $g_{i j}(r)$, and the partial collective structure factors, $S_{i j}(q)$. We note that some sets of parameters, especially those involving larger particle sizes or longer polymer chains (graft or matrix) do not yield any solutions due to numerical issues.

We use a self-consistent approach linking PRISM theory and Monte Carlo simulations[21], where the intra-molecular pair correlation function $\Omega_{i j}$ that is input to PRISM are provided by MC simulations of a single polymer-grafted nanoparticle or a single matrix chain in an external medium-induced potential obtained from PRISM theory. The interdependence of the chain conformations $\left(\Omega_{i j}\right)$ and the medium-induced potential gives rise to the self-consistency in the approach. The advantage of this approach is that it can tackle non-ideal conformations along the grafted and matrix polymers that have been neglected in previous studies of homopolymer-grafted 
nanoparticles[28-31]. Furthermore, in contrast to previous self-consistent PRISM-MC studies on homopolymer melts alone[33-38] or on bare particles in a homopolymer melt[39, 40] where the self-consistent loop involved MC simulations of only a single matrix polymer chain, we use alternate self-consistent loops for a single polymer-grafted particle and a single matrix chain. This ensures that we account for non-idealities in both the grafted and matrix chain conformations.

Since the steps involved in this self-consistent approach are detailed in our previous papers $[21,41]$ where we describe this approach in detail and its application to study polymer grafted particles in polymer matrix we present here only a brief overview of this method. First, the pairwise-decomposed medium-induced solvation potential, $\Delta \psi_{i j}(r)$, is obtained from the PRISM equations; this describes the interaction between any two sites $i$ and $j$ as mediated by all the remaining sites in the system, i.e., including the matrix, grafts and particles themselves. The form of the solvation potential depends on the approximation used in its derivation[33-35, 42-44]and we use the PY-form

$$
\begin{gathered}
\Delta \psi_{i j}^{P Y}(r)=-k T \ln \left[1+c_{i k}(r) * s_{k k^{\prime \prime}}(r) * c_{k^{\prime} j}(r)\right] \\
S(q)=\Omega(q)+H(q)
\end{gathered}
$$

where '*' in equations 3 a denotes a convolution integral in spatial coordinates, $k$ is the Boltzmann constant, and $T$ is the temperature. $S(q)$ in equation $3 \mathrm{~b}$ is the structure factor in terms of intra- and inter-molecular pair correlation functions in Fourier space. The solvation potential $\Delta \psi_{i j}(r)$ is then fed to the MC simulation of a single polymer-grafted particle or a single matrix chain. In the MC simulation, the model of the polymer-grafted particle or the matrix chain is the same as that used in PRISM theory. The grafted chain length distribution (e.g. monodisperse, bidisperse or polydisperse) is assigned in the initial configuration of the polymer grafted nanoparticle in the MC simulation, and the effect of the polydisperse or bidisperse distribution of graft lengths is present 
in the PRISM section in the terms in the intra-molecular pair correlation function associated with the polymer grafted particle calculated in the MC simulation. In the simulation the total interaction between sites $i$ and $j$ separated by a distance $r, U_{i j}^{t o t}(r)(\mathrm{i}, \mathrm{j}=$ graft monomer, particle, or matrix), is the sum of $U_{i j}(r)$ and the solvation potential, $\Delta \psi_{i j}(r)$, obtained from the preceding PRISM step. We alternately simulate (a) a single polymer-grafted nanoparticle or (b) an isolated matrix chain with the set of solvation potentials from the most recent iteration of PRISM calculations. During the production stage of the MC simulation, the intra-molecular structure factors between site pairs are sampled every $5 \times 10^{5}$ steps and the ensemble average of the intra-molecular structure factors is calculated, which serves as the new input for the following iteration of PRISM calculations. The self-consistent PRISM-MC iterations are continued until convergence of $\Delta \psi_{i j}(r)$ between iterations.

\subsubsection{Brownian Dynamics Method}

Even though the system we study with PRISM-MC is polymer grafted nanoparticles in an explicit polymer matrix, in the MC simulation portion of PRISM-MC we alternately simulate a single polymer grafted nanoparticle and a single matrix chain in an effective solvation. This does not allow for simultaneous visualization of the polymer grafted nanoparticle in an explicit polymer matrix. To visualize and characterize certain physical aspects (e.g. matrix monomer concentration from the surface of the grafted particle) we simulate for a select few systems a single polymer grafted nanoparticle placed in an explicit monodisperse polymer matrix using NVT Brownian Dynamics simulations on the HOOMD-blue platform[45]. Using the HOOMD-blue code we are able to access faster simulation time on Graphical Processing Units (GPUs) than possible with traditional CPU-based codes. To initialize our simulations, we first build a grafted nanoparticle, with chains extending radially from the particle surface, in the absence of any matrix chains. A 
short simulation with strong Lennard-Jones monomer-monomer attraction is then run to compress the grafted chains. This compressed grafted nanoparticle, along with 1500 chains of length $\mathrm{N}_{\text {matrix }}$, are then placed in a large cubic box, which is then compressed to reach the target system packing fraction. There are 4 stages of initialization, compression and cooling before the final equilibration and production stage. The first stage is run for four million steps at a reduced temperature of $\mathrm{T}^{*}=5$, and box length of $\mathrm{L}=300$ in order to help eliminate any bias introduced in the system during the initialization. The system is then compressed over 1 million steps to a volume fraction of $\eta=0.1$ at $\mathrm{T}^{*}=5$. The third stage is another randomization stage at $\mathrm{T}^{*}=5$ for 5 million steps to allow the system to equilibrate at $\eta=0.1$. During stage 4 , the system is cooled linearly, from $T^{*}=5$ to $T^{*}=1$, over a period of five million steps. During the final stage, the system is sampled for 5 million steps at $\eta=0.1$ and $T^{*}=1$. The data collected in the final stage is verified to be equilibrated by ensuring the total energy of the system is constant. To be sure that we only use statistically independent samples in our averages, we calculate the correlation time of various data during the sampling period (final stage). We find that correlation times for our systems for all data range from 5,000 to 8,000 timesteps, so we sample every 10,000 timesteps.

\subsubsection{Analysis}

In the self-consistent PRISM-MC approach, at the end of the self-consistent loop we obtain the equilibrium inter-molecular pair correlation function, $g_{i j}(r)=h_{i j}(r)+1$, that characterizes the local structure of the grafted nanoparticles and the matrix polymer. The potential of mean force (PMF) between two nanoparticles, $W_{P P}(r)$, is calculated from the particle-particle pair correlation function, $\mathrm{g}_{\mathrm{PP}}(\mathrm{r})$ as follows:

$$
W_{P P}(r)=-k T \ln g_{P P}(r) .
$$


Using the $g(r)$, we can also calculate the second virial coefficient, B2, a measure of tendency for particle aggregation or dispersion.

$$
B_{2}=2 \pi \int r^{2}(1-g(r)) d r
$$

where $\mathrm{r}$ is a radial coordinate and $\mathrm{g}(\mathrm{r})$ is the value of the radial distribution function at radial coordinate $r$.

To calculate the matrix penetration depth we begin by first truncating and shifting the partial pair distribution function between the nanoparticle and the matrix beads, $g_{P M}$, obtained from PRISM-MC so that the domain of the function varies between the particle surface and the height of the grafted layer, $h_{g}$. We then calculate the square root of the normalized second moment of $g_{P M}$ in this domain to obtain the matrix penetration depth, $\lambda$.

$$
\lambda=\sqrt{\frac{\int_{0}^{h} r^{2} g_{P M}(r) d r}{\int_{0}^{h g} g_{P M}(r) d r}}
$$

We also calculate concentration profiles of the graft (in the MC simulations within the PRISM$\mathrm{MC}$ approach, and in the BD simulations) and matrix monomers (only in BD simulations) from the nanoparticle center or surface. The number of monomer beads of type $Y$ - grafted or matrix in concentric shells of width $\Delta r$ radiating outwards from the particle center is recorded. The monomer concentration $C_{Y}(r)$ at a distance $r$ from the nanoparticle surface is calculated by dividing the average population $n_{Y}(r)$ in a spherical shell by its volume:

$$
C_{Y}(r)=\frac{n_{Y}(r)}{4 \pi r^{2} \Delta r}
$$

\subsubsection{Parameters}

The system presented in this paper consists of homopolymer grafted spherical nanoparticles in an explicit homopolymer matrix where the grafted polymers are either monodisperse, polydisperse, 
or bidisperse, and the matrix polymers are monodisperse. The polydispersity of the grafted polymers is quantified by polydispersity index (PDI) is varied from 1 (monodisperse) to 2.5 . In the case of the polydisperse grafted polymers, the grafted polymer lengths are chosen from a log normal distribution of chain lengths representing a specific PDI, while maintaining the average grafted polymer length, $\mathrm{N}_{\mathrm{g}, \mathrm{avg}}$, to be $20 \mathrm{Kuhn}$ segments and minimum length to be 8 Kuhn segments. To study matrix polymer lengths lower and higher than the average graft polymer lengths, the matrix length is varied from 10 Kuhn segments to as high as 300 Kuhn segments, however much of the discussion in this paper focuses on 10 and 40 Kuhn segments. The spherical nanoparticle diameter is either $5 \mathrm{~d}$ (where $\mathrm{d}$ is the diameter of a Kuhn segment or "monomer") or $8 \mathrm{~d}$. The polymer grafting density, $\sigma$, on these particles is varied from high $\left(0.65\right.$ chains $\left./ \mathrm{d}^{2}\right)$ brushlike, to intermediate $\left(0.25\right.$ chains $\left./ \mathrm{d}^{2}\right)$ and low $\left(0.1\right.$ chain $\left./ \mathrm{d}^{2}\right)$ values. The total system packing fraction, $\eta$, is either 0.1 to mimic dense solution of matrix polymers or 0.3 to mimic melt-like polymer matrix. The volume fraction of the grafted particles, $\phi$, is maintained low $(\phi=0.001)$ to model an infinitely dilute concentration of the grafted particles. 


\subsection{RESULTS}

\subsubsection{Effect of Graft Length Polydispersity on Effective Interactions and Wetting}
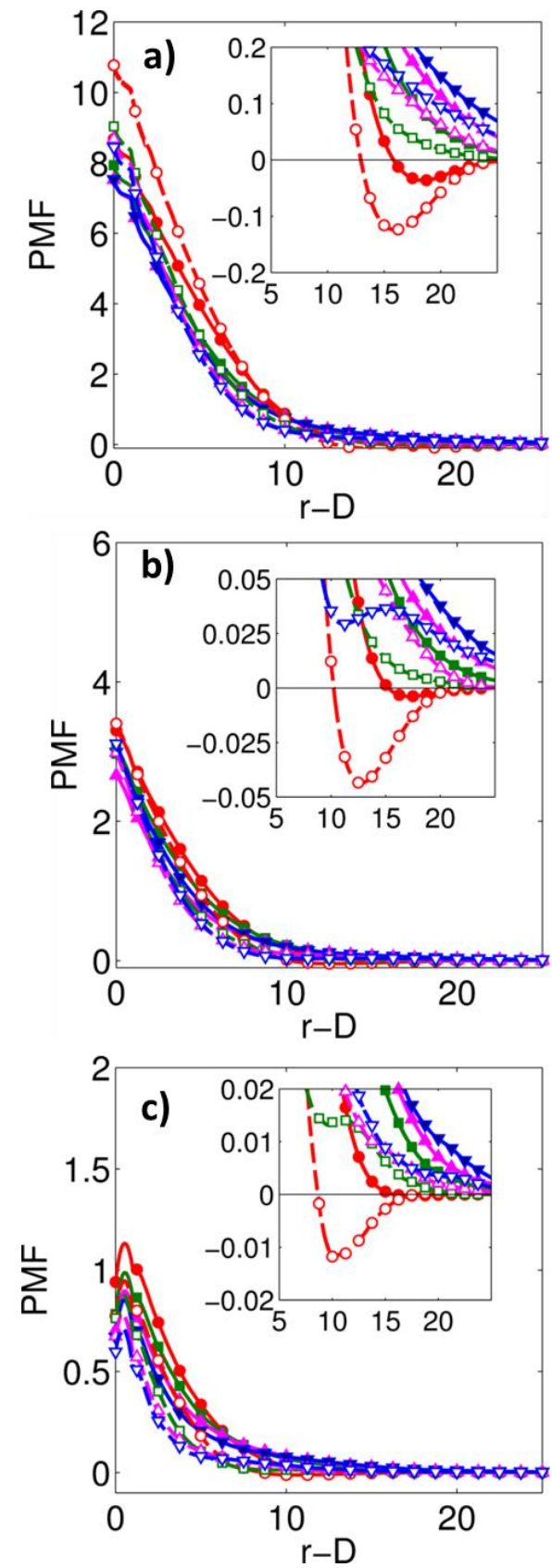

Figure 4.1: PMF (in units of kT) versus inter-particle distance, $r$-D (in units of d), between grafted nanoparticles $(D=5 d)$ at $\sigma=0.65(a), 0.25(b)$, and 0.10 chains $/ d^{2}$ and PDI= 1.0 (circles), 1.5 (squares), 2.0 (upward facing triangles), and 2.5 (downward facing triangles) with $N_{g, a v g}=20$, in a dense solution $(\eta=0.1)$ of monodisperse homopolymer matrix chains with $N_{\text {matrix }}=10$ (solid symbols) and $N_{\text {matrix }}=40$ (open symbols). The insets have the same axes labels as the main plots. 
In Figure 4.1, we present the PMF between polymer grafted nanoparticles with monodisperse $(\mathrm{PDI}=1)$ and polydisperse $(\mathrm{PDI}>1)$ homopolymer chains grafted on a spherical particle of size $\mathrm{D}=5 \mathrm{~d}$ at grafting densities of $0.65,0.25$ and 0.1 chains $/ \mathrm{d}^{2}$ and placed in a dense solution $(\eta=0.1)$ of monodisperse homopolymer matrix at athermal interactions between all species. At the higher grafting density of 0.65 chains/d $\mathrm{d}^{2}$ (Figure 4.1a), with monodisperse (PDI=1.0) grafts of $\mathrm{N}_{\mathrm{g}, \mathrm{avg}}=20$ and monodisperse matrix chains of $\mathrm{N}_{\text {matrix }}=10$ (red solid symbols), the PMF exhibits a repulsion at contact and weak attraction at intermediate distances. The corresponding PMF in matrix of $\mathrm{N}_{\text {matrix }}=40$ (red open symbols) also exhibits a repulsion at contact and an attractive well at intermediate distances (or mid-range attractive well) that is stronger than that seen at $\mathrm{N}_{\text {matrix }}=10$. The repulsion at contact is attributed to the grafted monomers on the particle sterically repelling the other polymer grafted particle as the particle surfaces approach each other, and is observed at both matrix lengths. The attractive well at intermediate distances is attributed to the overlap in grafted layers brought about by the dewetting of the monodisperse grafted layer by the monodisperse matrix chains, which is considerable when the matrix chain lengths are greater than the graft chain length. This behavior of increasing mid-range attraction strength with increasing ratio of matrix lengths to graft lengths seen in Figure 4.1a has been seen in prior theoretical and experimental work for polymer grafted nanoparticles at brush-like grafting densities at the monodisperse limits[1]. Here we see that, as polydispersity in grafts (PDI) increases from 1 to 2.5, the steric repulsion at contact weakens by 1-2 kT and the attractive well at contact is eliminated, for both matrix chain lengths. Additionally, as graft PDI increases, the repulsive tail in the PMF increases in strength and extends to larger inter-particle distances, for both matrix lengths. At the intermediate grafting density of 0.25 chains/ $\mathrm{d}^{2}$ (Figure $4.1 \mathrm{~b}$ ), the repulsion at contact and the attractive well at the monodisperse limit are weaker in strength than the corresponding 
monodisperse values at higher grafting density (Figure 4.1a). This reduction in contact repulsion and mid-range attraction with decreasing grafting density is also in agreement with prior theoretical and experimental work for polymer grafted nanoparticles with monodisperse grafts[1, $3,6,7]$. We see that the effect of increasing polydispersity at 0.25 chains $/ \mathrm{d}^{2}$ is qualitatively similar, but quantitatively weaker than that seen at 0.65 chains $/ \mathrm{d}^{2}$. At the low grafting density of 0.1 chains/ $\mathrm{d}^{2}$ (Figure 4.1c), the PMF exhibits repulsion at contact and no attractive well at intermediate inter-particle distances, which is also in agreement with past theoretical studies for these lightly grafted systems (see review articles $[1,46]$ ) where wetting/dewetting of the grafted layer by matrix chains does not occur due to absence of a grafted brush. At the lowest grafting density, as the graft PDI increases, the PMF becomes slightly less repulsive at contact and slightly more repulsive at larger distances (Figure 4.1c inset).

The decrease in repulsion at contact with increasing PDI is driven by the polydisperse grafted polymers relieving some monomer crowding in the grafted layer. The relief in crowding is caused by a change in grafted chain conformations to maximize the overall conformational entropy upon introduction of polydispersity, as seen in recent Monte Carlo simulation (MC) study in implicit matrix[16]. This MC study also showed that the effect of polydispersity on chain conformations is relatively minor at low grafting densities and more drastic at higher grafting densities where chain crowding in the grafted layer is strong at the monodisperse limit. In an explicit homopolymer matrix and high grafting density, $\left(0.65\right.$ chains $\left./ \mathrm{d}^{2}\right)$ Brownian dynamics simulations results (Supplemental Section 4.7.2) show that the grafted chain end-monomer concentration profiles show larger values near the particle surface with increasing polydispersity, implying higher accessibility of the particle surface. The higher accessibility of the particle surface by end monomers is due to the presence of shorter chains in the distribution, as well as the small 
changes in chain conformations due to reduced monomer crowding resulting from a wider grafted chain length distribution. The increasing relief in monomer crowding with increasing PDI, especially at higher grafting densities, manifests in the PMF as more significant reduction in repulsion at contact at 0.65 chains $/ \mathrm{d}^{2}$ for $\mathrm{N}_{\text {matrix }}=40$ (Figure $4.1 \mathrm{c}$ ) than at smaller grafting densities. Most interestingly, at 0.65 chains $/ \mathrm{d}^{2}$ and $\mathrm{N}_{\text {matrix }}>\mathrm{N}_{\text {g,avg }}$ (open symbols in Figure 4.1a) the attractive well of $\sim 0.1 \mathrm{kT}$ in the PMF at intermediate distances seen in monodisperse systems is completely eliminated at PDI of 1.5 and above. Additional calculations at smaller PDI (1.05-1.4) (Supplementary Figure 4.14) found that attractive well is not eliminated at all PDI>1, and that there is a minimum, or a critical, PDI needed to eliminate the attractive well. Since the strength of mid-range attraction is dependent on the grafting density, particle size and average graft and matrix length, one can expect the exact value of the minimum or critical PDI needed to eliminate this attractive well to also be a function of these parameters. The attractive well is eliminated at higher PDI because the longer chains in the polydisperse chain length distribution a) sterically repel the longer chains on the other grafted particle, and b) shift the entropic contributions more heavily towards the grafted chains than matrix chains, thus driving matrix chains to wet the grafted layer. This increased wetting is also captured via concentration profiles of the matrix monomers from the particle surface in the Brownian dynamics simulations (Supplementary Figure 4.11). The figure in Supplementary shows that as PDI increases the matrix monomer concentration profile goes further into the grafted layer (characterized by the grafted monomer concentration) as compared to monodisperse grafts.

Notably, many of the trends in how the effective interactions change with grafting density and ratio of matrix chain length to graft chain length for monodisperse grafts[1] remain the same even in the presence of polydispersity in grafts. 


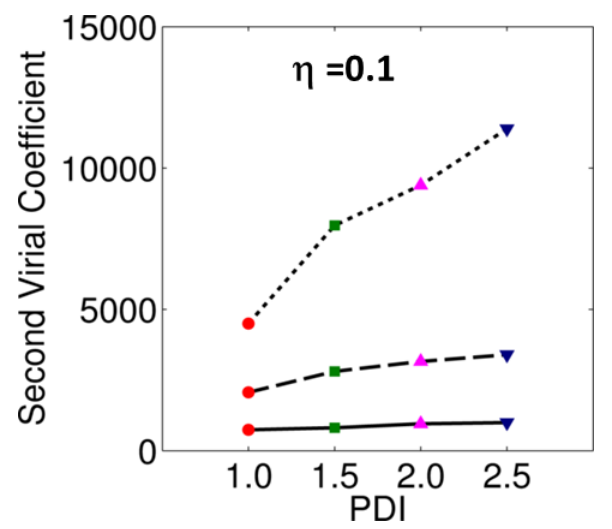

Figure 4.2: Second virial coefficient for polymer grafted nanoparticles in a polymer matrix as a function of graft polydispersity (PDI) at grafting densities of $\sigma=0.10$ chains $/ d^{2}$ (solid line), $\sigma=0.25$ chains/d2(dashed line), and $\sigma=0.65$ chains/d'dotted line), and total system volume fraction of $\eta=0.1$. All data is for particle diameter $D=5 d$, average graft length $N_{g, a v g}=20$, and monodisperse matrix length $N_{\text {matrix }}=40$.

The most exciting aspect of the above results is that, since the mid-range attractive well has been attributed to drive the nanoscale additives in polymer nanocomposites towards aggregation, eliminating the attractive well should stabilize dispersions of particles in systems where monodisperse grafts would drive aggregation. The second virial coefficient $\mathrm{B}_{2}$ (Figure 4.2), which characterizes the propensity of particles to assemble (negative $\mathrm{B}_{2}$ ) or disperse (positive $\mathrm{B}_{2}$ ) also shows that for all grafting densities as PDI increases the $\mathrm{B}_{2}$ value increases, with the net increase being largest at the highest grafting density. Our emphasis here is on the qualitative trend of increasing $\mathrm{B}_{2}$ value with increasing PDI, and not the value of $\mathrm{B}_{2}$ itself, as that would depend on the specific matrix and graft lengths, particle size, and the direct particle-particle attractive interactions as well (which are maintained as athermal here). Here the $\mathrm{B}_{2}$ value is positive even at the monodisperse limit, because the matrix chains are only 40 segments long, which is only twice that of the average graft length and the nanoparticle-nanoparticle interactions are athermal. Past studies have shown that for curved surfaces, when the ratio of matrix length to graft length is five to six, the particles aggregate at the monodisperse graft limit[1, 47]. While we only show results 
for $\mathrm{N}_{\text {matrix }}=10$ and 40 in this article, we have confirmed at the monodisperse graft limit that as $\mathrm{N}_{\text {matrix }}$ increases the attractive well at intermediate distances deepens (Supplementary Figure 4.15), thereby increasing tendency for particle aggregation, in agreement with past studies. Since the attractive well is stronger at intermediate distances one might also need larger PDI to eliminate that attraction. To test this hypothesis for a few cases, we conducted PRISM-MC calculations of polymer grafted particles with grafted chain polydispersity of $\mathrm{PDI}=2$ at the highest grafting density at large matrix molecular weights (Supplementary Figure 4.15). We observe that, at PDI=2, as the matrix chain length increases, the mid-range attractive well depth is not eliminated completely. This observation, along with stronger mid-range attraction at the monodisperse limit at high matrix chain lengths (Supplementary Figure 4.15), confirm our expectation that, at larger matrix chain lengths, larger PDI is needed to eliminate the attractive well. We could not conduct an extensive systematic calculation of PMF with increasing PDI at larger $\mathrm{N}_{\text {matrix }}$ because of lack of numerical convergence in PRISM-MC runs at long matrix lengths arising from numerical issues at these parameters. In this regard, we also note that, for a few systems, at the highest grafting density $(0.65$ chains $/ \mathrm{d}^{2}$ ) only, where matrix chains are expected to deplete/dewet large regions in the grafted layer near the particle surface, the choice of Percus-Yevick closure leads to negative values in gmatrix-particle $_{\text {( }) \text { ) at low } r}$ (where gmatrix-particle $(r)$ should be 0 ), due to numerical issues. We also add that, for these specific systems, all other pair correlation functions are devoid of this issue and do not exhibit any negative values. Despite this issue, the PRISM-MC results we show here correctly predicts all known (qualitative and some quantitative) trends in monodisperse systems - a) with increasing grafting density the mid-range attractive well deepens and shifts to higher inter-particle distances (Figure 4.1); b) with increasing matrix chain length the attractive well depth deepens (Supplementary Figure 4.15); c) the value of the well depth seen at 0.65 chains $/ \mathrm{d}^{2}$ is of the same 
order of magnitude ( $\sim 0.3-0.5 \mathrm{kT}$ in Supplementary Information) as that seen for similar systems in recent simulation studies $[3,48]$ on systems with graft length of 10 monomers and matrix lengths of 10-70 monomers, and particle sizes approximately 10 times monomer size at high grafting density ( $\sim 0.76$ chains $\left./ \mathrm{nm}^{2}\right)$. The ability of PRISM-MC to predict the same qualitative trends as prior monodisperse studies, and, in certain cases, show quantitative agreement with prior simulations for monodisperse grafts, suggests that this approach is capable of predicting correct qualitative trends for the polydisperse polymer grafted nanoparticles as well.
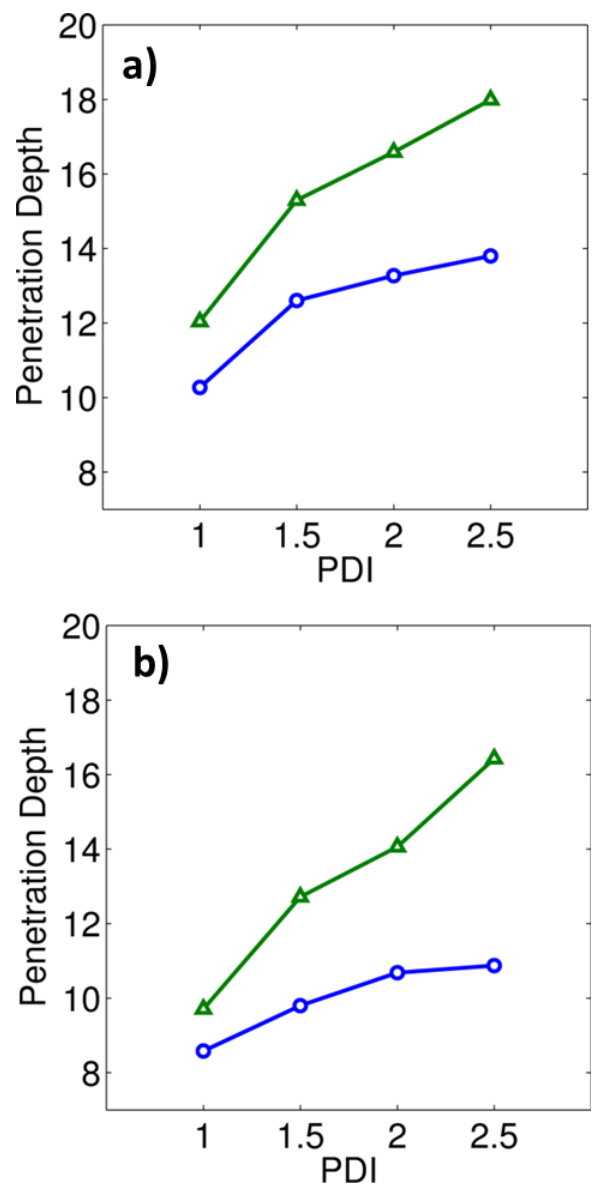

Figure 4.3: Penetration depth (in units of $d$ ) of the matrix chains into the grafted layer of nanoparticles $(D=5 d)$ with polydisperse chains $\left(N_{g, a v g}=20\right)$ at $\boldsymbol{\sigma}=\mathbf{0 . 1 0}$ chains $/ \boldsymbol{d}^{2}$ (circles) and $\sigma=$ 0.25 chains $/ d^{2}$ (triangles) in a dense solution $(\eta=0.1)$ with $N_{\text {matrix }}=10(a)$ and $N_{\text {matrix }}=40(b)$ 
Continuing with our discussion of reduction in attractive well depth with increasing polydispersity in grafts, while the graft and matrix concentration profiles in the Supplementary Section 4.7.2 show the increased region of overlap between the graft monomer concentration and matrix monomer concentration profile, to demonstrate the increased wetting of the polydisperse grafted layer by the matrix chains we present the penetration depth of the matrix chains into grafted layer, $\lambda$, (Figure 4.3). Figure 4.3 shows increasing $\lambda$ with increasing PDI, confirming increased wetting of the grafted layer by the matrix chains with increasing PDI for both matrix lengths. Also, there is a larger effect of polydispersity on improving wetting at higher grafting densities than lower grafting densities. The reason behind this is the same as mentioned earlier. Since the change in chain conformations due to increasing polydispersity is more drastic at the higher grafting densities than lower grafting densities, the resulting improved wetting of the grafted layer by the matrix is also more drastic at higher grafting density. 


\subsubsection{Effect of Increasing Matrix Packing Fraction}
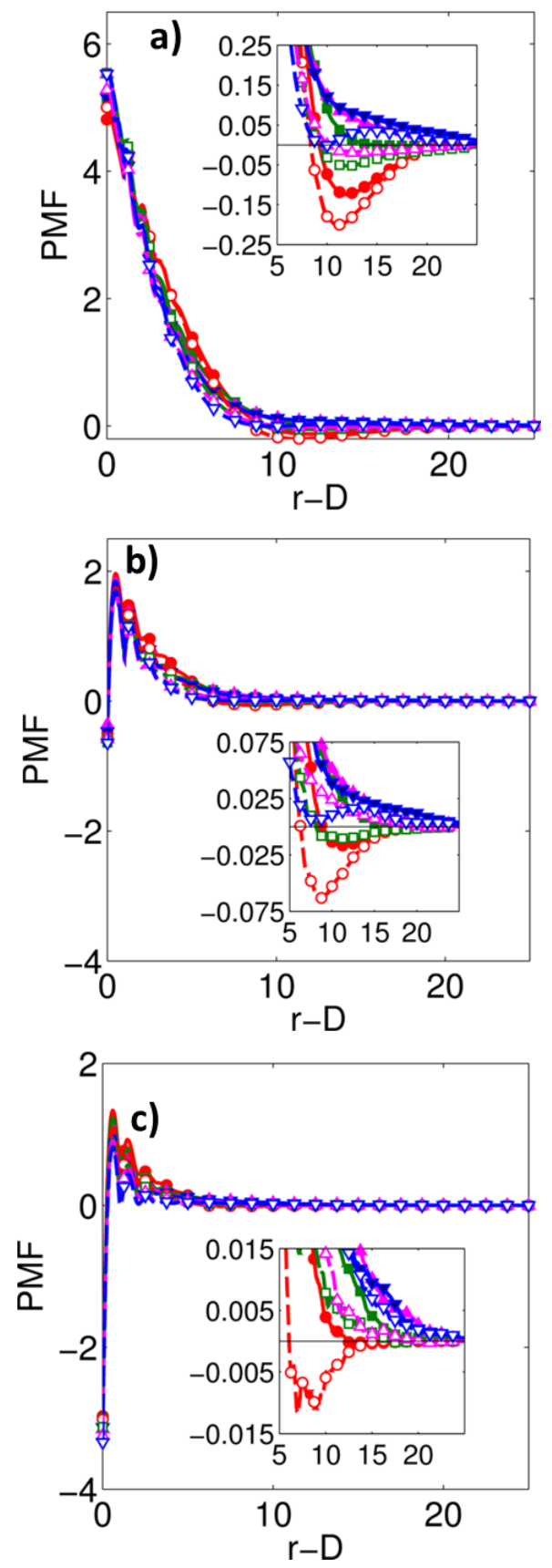

Figure 4.4: PMF (in units of kT) versus inter-particle distance, $r$-D (in units of d), between grafted nanoparticles $(D=5 d)$ at $\sigma=0.65(a), 0.25(b)$, and 0.10 chains $/ d^{2}$ and PDI= 1.0 (circles), 1.5 (squares), 2.0 (upward facing triangles), and 2.5 (downward facing triangles) with $N_{g, a v g}=20$, in a melt-like $(\eta=0.3)$ matrix of monodisperse homopolymer chains with $N_{\text {matrix }}=10$ (solid symbols) and $N_{\text {matrix }}=40$ (open symbols). The insets have the same axes labels as the main plots. 
All the results presented so far were at a total packing fraction of $\eta=0.1$, which we characterize as a dense polymer solution rather than a melt through calculations of the compressibility from the structure factor $\mathrm{S}(\mathrm{k})$ as $\mathrm{k} \rightarrow 0$ [28]. At a melt-like packing fraction of $\eta=0.3$, the matrix polymers have been shown to induce depletion like attractions between both bare and monodisperse polymer grafted nanoparticles at infinitely dilute concentrations[30, 49, 50]. At high grafting densities, this matrix-induced depletion-like attraction significantly reduces the steric repulsion at contact and deepens the mid-range attractive well (inset of Figure 4.4a versus Figure 4.1a). At low grafting densities, the matrix-induced depletion-like attraction manifests itself in the PMF as attraction at contact (Figure $4.4 \mathrm{c}$ versus Figure $4.1 \mathrm{c}$ ). Comparing $\eta=0.3$ and $\eta=0.1$ at high grafting density (of Figure 4.4a versus Figure 4.1a), the repulsion at contact is less sensitive to PDI at $\eta=0.3$, and a larger PDI is needed to eliminate the stronger attractive well at intermediate distances at $\eta=0.3$ (inset of Figure 4.4a). At low grafting density (Figure 4.4c), the effects of polydispersity are reduced at $\eta=0.3$ as compared to $\eta=0.1$, as the values of attraction at contact $(\sim 3 \mathrm{kT})$ dominate at all PDI. This implies that in melt-like polymer matrices one can stabilize dispersions using polydispersity only at high grafting densities, and the extent of polydispersity needed to stabilize dispersions is higher as compared to the graft polydispersity needed to stabilize dispersions in dense polymer solutions. In a melt-like polymer matrix at low grafting densities, any effect of polydispersity in grafts will be overcome by the dominant matrix-induced depletion attraction that will enhance the tendency for aggregation of particles. 


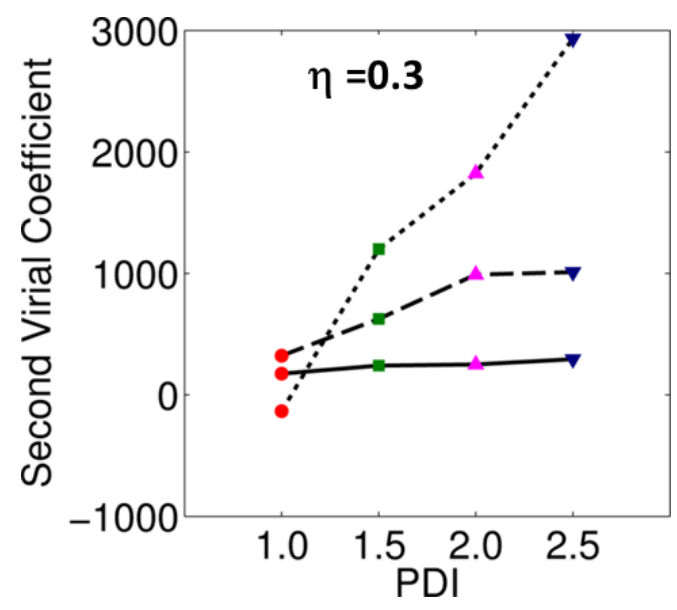

Figure 4.5: Second virial coefficient for polymer grafted nanoparticles in a polymer matrix as a function of graft polydispersity (PDI) at grafting densities of $\sigma=0.10$ chains $/ d^{2}$ (solid line), $\sigma=0.25$ chains $/ d^{2}$ (dashed line), and $\sigma=0.65$ chains/d $d^{2}$ (dotted line), and total system volume fraction of $\eta=0.3$. All data is for particle diameter $D=5$ d, average graft length $N g$, avg $=20$, and monodisperse matrix length Nmatrix $=40$.

This is further confirmed by comparing the second virial coefficient at $\eta=0.3$ (Figure 4.5) as compared to $\eta=0.1$ (Figure 4.2). At high grafting densities (dotted lines in Figure 4.5 and Figure 4.2) at each PDI, the $B_{2}$ is much smaller for $\eta=0.3$ as compared to $\eta=0.1$. Similar behavior is seen at intermediate grafting density (dashed line in in Figure 4.5 and Figure 4.2). At low grafting densities the value of $\mathrm{B}_{2}$ is weakly positive, and does not change with increasing PDI as seen at $\eta=0.1$. Comparison of penetration depth at $\eta=0.3$ (Supplementary Figure 4.16) and $\eta=0.1$ (Figure 4.3) shows that the matrix penetrates a lot less at high matrix packing fraction than in dense solutions. This lower penetration depth can be explained partly by the reduction in the thickness of the grafted layer at higher matrix packing fraction due to compression of the grafted monomers by the melt-like matrix chains[51]. Overall, the net effect of polydispersity is lowered at high meltlike matrix packing fraction due to matrix-induced depletion interactions dominating the physics in these systems. 


\subsubsection{Effect of Particle Diameter or Curvature}
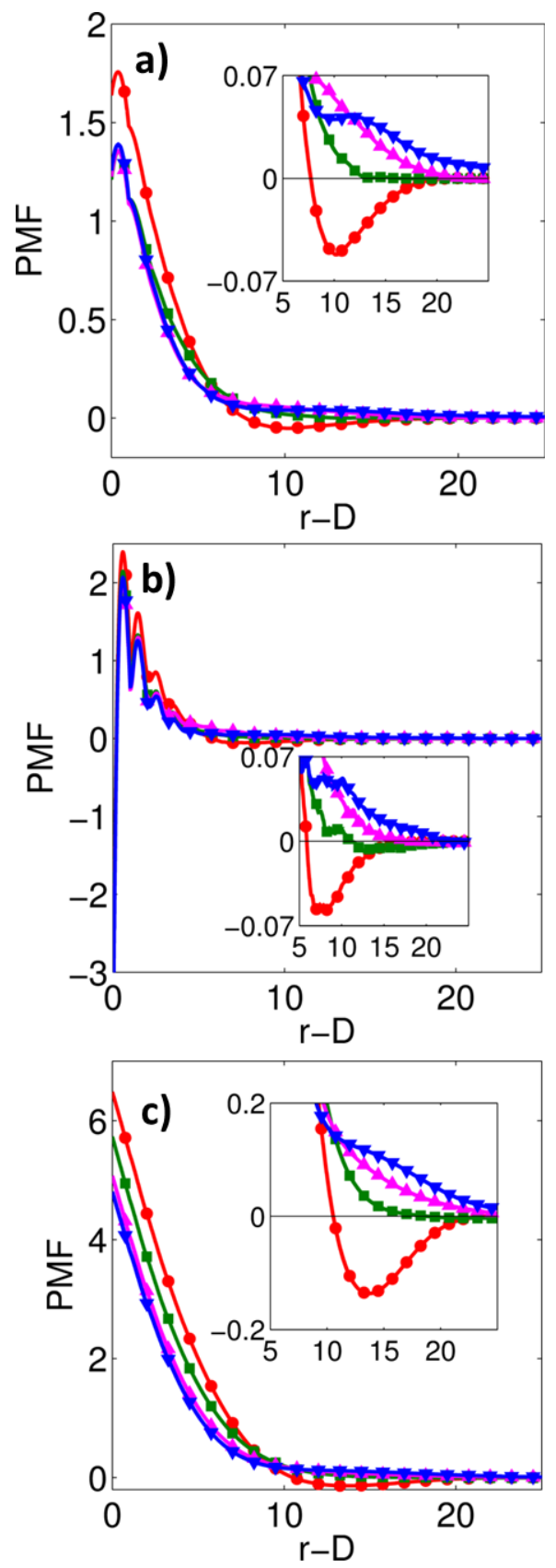

Figure 4.6: Potentials of mean force, PMF (in units of $k T$ ) versus inter-particle distance, $r$-D (in units of $d$ ) between nanoparticles of size $\boldsymbol{D}=8 \boldsymbol{d}$ grafted with polydisperse chains at PDI= 1.0 (circles), PDI=1.5 (squares), PDI=2.0 (upward facing triangles), and PDI=2.5 (downward facing triangles) with $N_{g, a v}=20$ at a grafting density of $\sigma=0.1$ chains $/ d^{2}$ (parts $a$ and $b$ ) and 0.25 chains $/ d^{2}$ (part c) in a dense solution $(\boldsymbol{\eta}=\mathbf{0 . 1}$, part $a$ and $c)$ and melt $(\boldsymbol{\eta}=\mathbf{0 . 3}$, part b) of 
monodisperse homopolymers with $\boldsymbol{N}_{\text {matrix }}=\mathbf{4 0}$. The insets have the same axes labels as the main plots.

So far we have observed that the effect of polydispersity is enhanced at conditions where there is large monomer crowding at the monodisperse limit (e.g. higher grafting density) and therefore the relief to that crowding brought about by polydispersity is more significant. Based on that observation, one could expect that at constant grafting density, since the monomer crowding is larger on surfaces with lower curvature, the effect of polydispersity induced relief in crowding would also be larger on surfaces with lower curvature. To test this, we calculated the PMFs for grafted particles with diameter $\mathrm{D}=8 \mathrm{~d}$ with polydisperse grafts at 0.1 and 0.25 chains $/ \mathrm{d}^{2}$ in a dense homopolymer matrix (Figure 4.6a, (Figure 4.6c) and at 0.1 chains $/ \mathrm{d}^{2}$ in a melt-like homopolymer matrix ((Figure 4.6b) with $\mathrm{N}_{\text {matrix }}=40$. In a dense solution, upon comparing the PMF for $D=8 \mathrm{~d}$ (Figure 4.6a and (Figure 4.6c) to the corresponding PMF for D=5d (open symbols in Figure 4.1c and Figure 4.1b) we observe the following. At the monodisperse graft limit the repulsion at contact and mid-range attractive well are stronger for $D=8 d$ as compared to $D=5 d$ when $N_{\text {matrix }}=40$ for both grafting densities. 

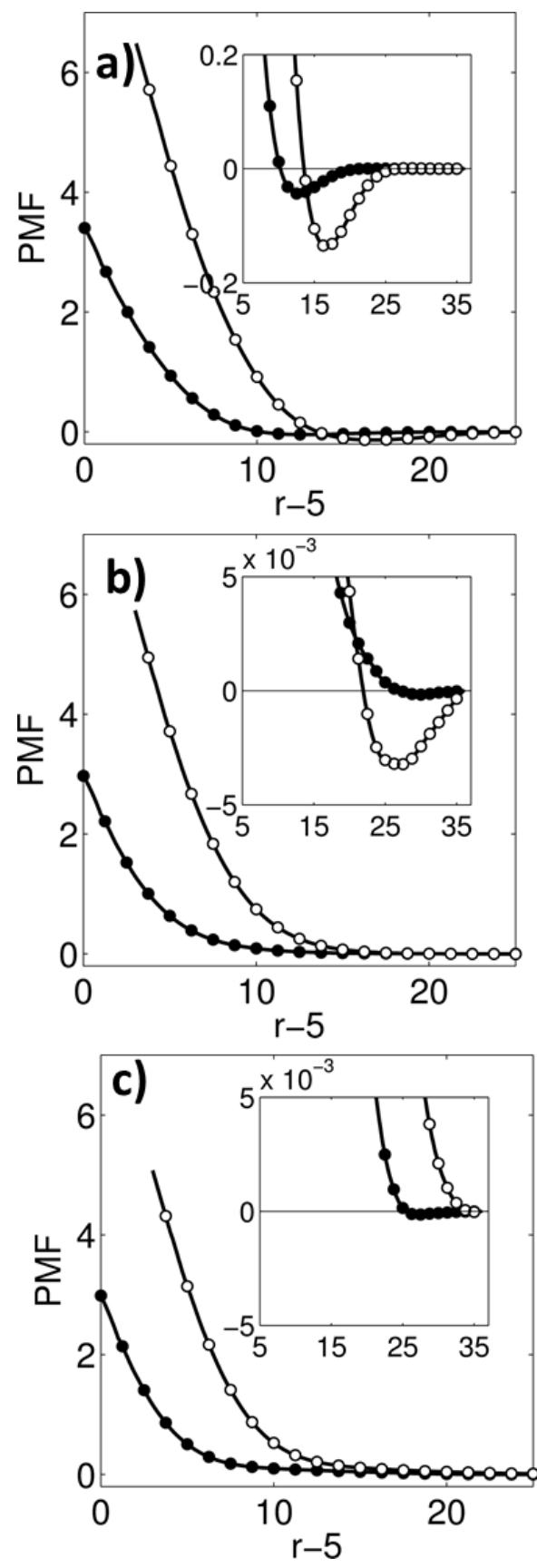

Figure 4.7: Potentials of mean force, PMF (in units of kT) versus inter-particle distance, $r-5$ (in units of $d$ ) between nanoparticles of size $D=8 d$ (open symbol) and $D=5 d$ (solid symbols) grafted with polydisperse chains at $P D I=1.0(a), P D I=1.5(b)$, and $P D I=2.0$ (c)with $N_{g, a v g}=20$ at a grafting density of $\boldsymbol{\sigma}=\mathbf{0 . 2 5}$ chains $/ d^{2}$, in a dense solution $(\boldsymbol{\eta}=\mathbf{0 . 1})$ of monodisperse homopolymers with $\boldsymbol{N}_{\text {matrix }}=40$. The insets have the same axes labels as the main plots. 
Figure 4.7 a presents a direct comparison of the monodisperse grafts on $D=5 d$ to $D=8 d$ at 0.25 chains $/ \mathrm{d}^{2}$ grafting density in a dense solution of matrix of length $\mathrm{N}_{\text {matrix }}=40$ and confirms the above trend of higher repulsion at contact and stronger mid-range attraction in $D=8 \mathrm{~d}$ as compared $\mathrm{D}=5 \mathrm{~d}$. Figure $4.7 \mathrm{a}$ also shows the slight shift of the mid-range attraction to larger inter-particle distances. These trends are in accord with previous studies of monodisperse grafts $[6,11]$ that showed that decreasing curvature increases the monomer crowding near the particle surface, increases the grafted layer thicknesses, and decreases the propensity of matrix to wet the grafted layer. As graft polydispersity increases, the repulsion at contact is reduced and the attractive well at intermediate distances is replaced by a long repulsive tail for $D=8 d$, similar to $D=5 d$.

Table 4.1: Minimum values for the potentials of mean force, PMF (in units of kT), versus PDI between nanoparticles of size $D=8 d$ and $D=5 d$ ( grafted with polydisperse chains with $N_{g, a v g}=20$ at a grafting density of $\boldsymbol{\sigma}=\mathbf{0 . 2 5}$ chains $/ d^{2}$, in a dense solution $(\boldsymbol{\eta}=\mathbf{0 . 1})$ of monodisperse homopolymers with $\boldsymbol{N}_{\text {matrix }}=\mathbf{4 0}$.

\begin{tabular}{ccc}
\hline PDI & $D=5 d$ & $D=8 d$ \\
\hline 1 & -0.0436 & -0.1363 \\
1.5 & -0.0002 & -0.0032 \\
2 & -0.0001420 & -0.0000230 \\
\hline
\end{tabular}

Table 1 presents a direct comparison of the attractive well depth for $D=8 d$ and $D=5 d$ at PDI $=1.5$ and 2, grafting density of 0.25 chains $/ \mathrm{d}^{2}$ and $\mathrm{N}_{\text {matrix }}=40$ which demonstrates that, at $\mathrm{PDI}=1.5$ in case of $\mathrm{D}=8 \mathrm{~d}$, we can reduce a much larger attractive well seen at $\mathrm{PDI}=1$ as compared to the relatively smaller attractive well of $\mathrm{D}=5 \mathrm{~d}$. At 0.1 chains $/ \mathrm{d}^{2}$ and $\eta=0.3$ (Figure $4.6 \mathrm{~b}$ for $\mathrm{D}=8 \mathrm{~d}$, Figure $4.4 \mathrm{c}$ for $\mathrm{D}=5 \mathrm{~d}$ ) we do not see any significant qualitative differences in polydispersity effects for $D=8 d$ and $D=5 d$, as expected since the crowding is minimal at that low grafting density and 
the matrix induced depletion-like attraction starts to dominate. The trends seen so far suggest that with increasing diameter or decreasing curvature, we can expect polydispersity in grafts to play a larger role in reducing the tendency of grafted particle aggregation.. For low curvatures, as the well-depth deepens significantly in the monodisperse limit with increasing matrix length beyond those presented here, we might also need larger polydispersity to eliminate the relatively stronger mid-range attraction.

\subsubsection{Effect of Distribution of Chain Lengths}
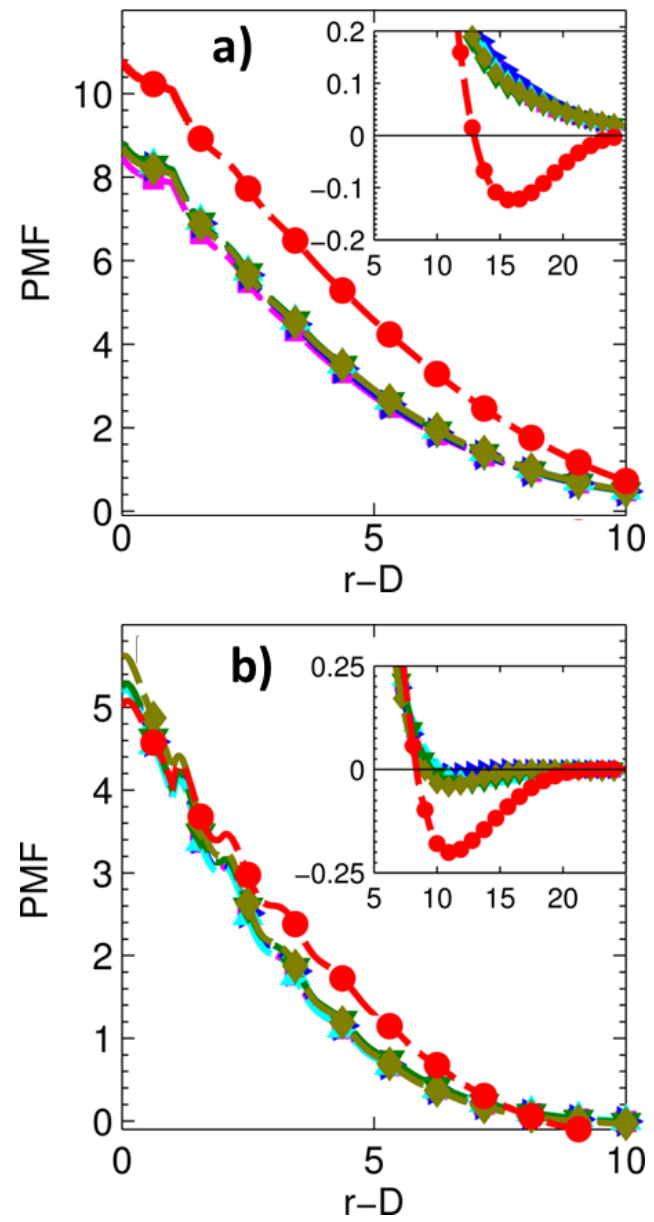

Figure 4.8: Potentials of mean force between nanoparticles of size $D=5 d$ with polydisperse chains of PDI $=1.0$ (circles) and PDI $=2.0$ (other symbols) with $N_{g, a v}=20$ and $N_{g, \min }=8$ at a grafting density of $\sigma=0.65 \mathrm{ch} / \mathrm{nm}^{2}$, for five different log-normal distributions, in a) a dense solution $(\eta=0.1)$ and 
b) melt $(\eta=0.3)$ of monodisperse homopolymers with $N_{\text {matrix }}=40$. Each symbol (besides circle) corresponds to a different log-normal chain distribution.

The key result in this paper is that when polydisperse homopolymer chains with a broad chain length distribution are grafted on nanoparticle surfaces they eliminate or reduce the mid-range attractive well in the PMF that has been shown to cause aggregation in monodisperse limits. To ensure that our choice of a specific discretized chain length distribution, that mimics a continuous log-normal distribution, does not bias the above results, we calculated the PMF for five different chain length distributions (all log-normal) for a select few systems. In Figure 4.8 we present PMF between nanoparticles of size $\mathrm{D}=5 \mathrm{~d}$ with polydisperse chains of $\mathrm{PDI}=1.0$ (circles) and five different $\log$-normal distributions with $\mathrm{PDI}=2.0$ (other symbols) with $\mathrm{N}_{\mathrm{g}, \mathrm{avg}}=20$ at a grafting density of $\sigma=0.65$ chains $/ \mathrm{d}^{2}$ and in a dense solution or $\eta=0.1$ (Figure 8a) and melt-like matrix $\eta=0.3$ (Figure $4.8 \mathrm{~b}$ ) of monodisperse homopolymers with $\mathrm{N}_{\text {matrix }}=40$. Clearly, for both $\eta=0.1$ and 0.3, the PMF between the particles with polydisperse chain length distribution are quantitatively similar for the five distributions, and quantitatively distinct from the corresponding PMF between particles with monodisperse grafts. We also expect that the choice of another form of distribution (different from log normal) would not change the effect of polydispersity removing the mid-range attraction, because the distribution we have chosen is a discretized version of a continuous lognormal distribution and another discretized broad distribution of chain lengths should bring about the same effect. This then begs the question- how will the PMF obtained for particles with a bimodal distribution of graft lengths or bidisperse graft length distribution compare with that of the PMFs seen so far with a statistically polydisperse distribution. 


\subsubsection{Polydisperse versus Bidisperse.}
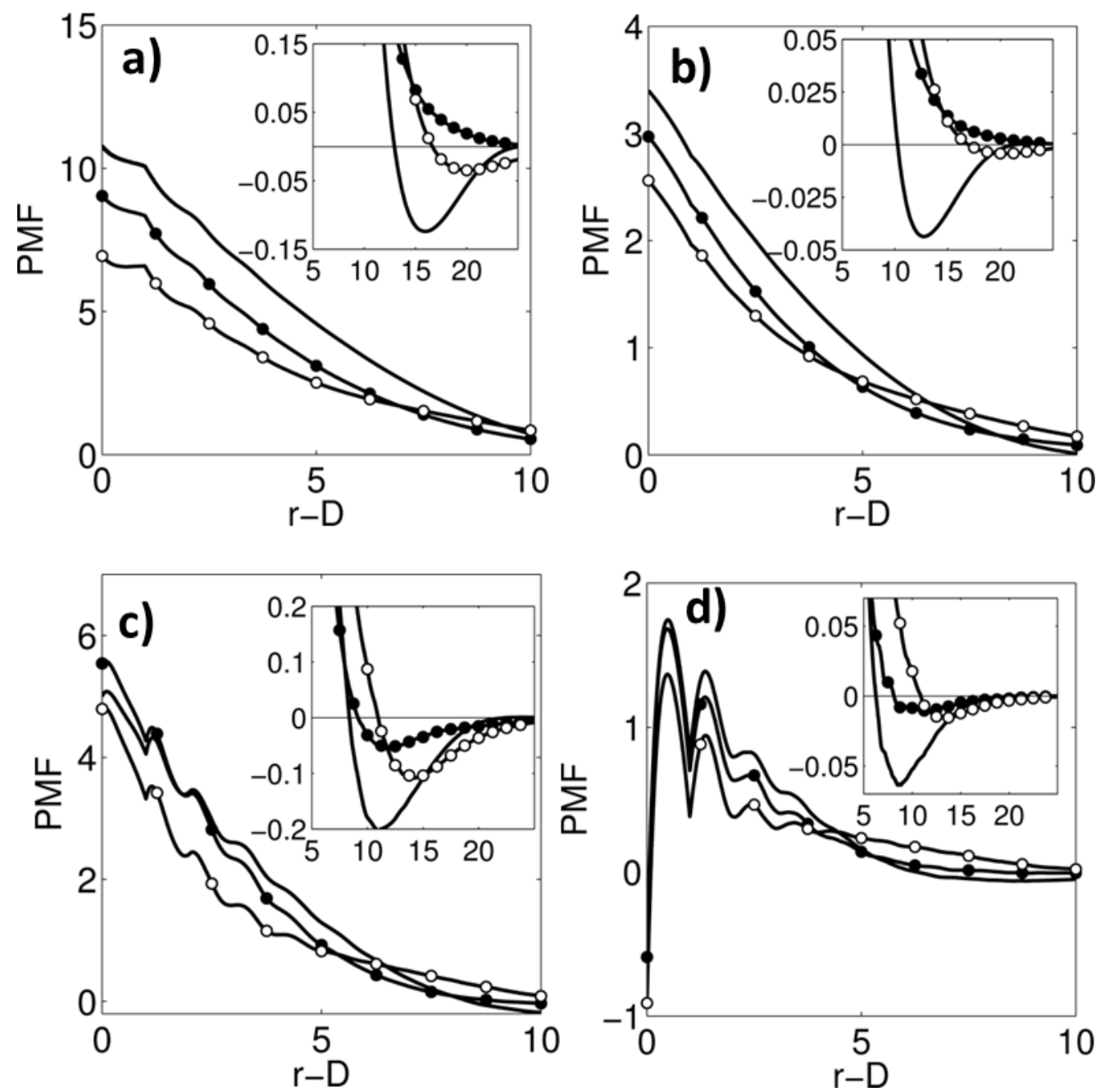

Figure 4.9: PMF (in units of kT) versus inter-particle distance, $r$-D (in units of d), between grafted nanoparticles $(\boldsymbol{D}=\mathbf{5 d})$ with monodisperse homopolymer grafts with PDI=1.0 (solid line), polydisperse homopolymer grafts $P D I=1.5$ (solid circle), and bidisperse homopolymer grafts $P D I=1.5$ (open circle), all with $N_{g, a v g}=20$ and grafting density $\sigma=0.65$ (left), and 0.25 (right) chains/d ${ }^{2}$ in a in a dense solution $(\eta=0.1)$ (top row) or melt-like $(\eta=0.3)$ (bottom row) monodisperse homopolymer matrix with $\boldsymbol{N}_{\text {matrix }}=40$. The insets have the same axes labels as the main plots.

In Figure 4.9, we compare PMFs from particles grafted with a log-normal chain length distribution (solid circles) to those grafted with a bidisperse chain length distribution (open circles) at the same $\mathrm{PDI}=1.5$, and the corresponding monodisperse grafts with same average graft length (no markers). 
These bidisperse chain length distributions have equal number of monodisperse short $(=6$ segments) and monodisperse long ( $=36$ segments) chains with $\mathrm{N}_{\text {graft,avg }}=20$ and PDI $=1.5$. At both 0.65 and 0.25 chains $/ \mathrm{d}^{2}$ and both $\eta=0.1$ and 0.3 , we observe distinct behavior between the monodisperse, polydisperse (log-normal) and bidisperse PMFs both at contact and at intermediate

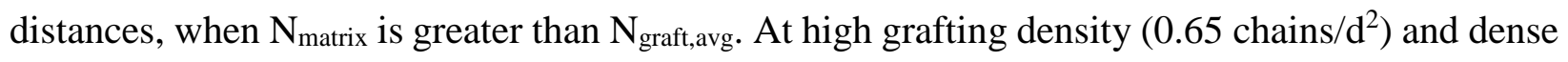
solution matrix (Figure 4.9a), the repulsion at contact is most reduced by a bidisperse distribution while the attractive well at intermediate distances is most reduced by the polydisperse distribution. The former is because half the chains in the bidisperse distribution are short (6 monomers), while there are only six chains in the polydisperse distribution $(\mathrm{PDI}=1.5)$ that are less than 10 monomers long. The latter is because in the polydisperse distributions the long chains are significantly longer than the long chains in the bidisperse distribution ( $\mathrm{N}_{\text {graft,long }}=36$ monomers). We have attributed the elimination of mid-range attraction in the PMF to i) the presence of these significantly long chains sterically hindering other long chains and ii) increased graft chains mixing with the matrix chains due to reduced grafted layer crowding in the polydisperse system. The fact that the long chains in the bidisperse distribution are shorter than the $\mathrm{N}_{\text {matrix }}$ (systems where aggregation is observed) and yet the bidisperse distribution is able to reduce the mid-range attraction emphasizes the role of lowered monomer crowding (brought about having short and long chains) in reducing the mid-range attraction.

In the melt-like matrix (Figure 4.9b) we also see a shift in the mid-range attraction to the lower distances in the case of polydisperse distribution as compared to bidisperse distribution. In the melt-like matrices the matrix induced depletion attraction pushes the grafted particles together. The inter-particle distance where the two grafted layers start to overlap due to this depletion-like attraction corresponds to the position of the mid-range attraction in the PMF. This overlap between 
the grafted layers occurs at the outer-most region of the grafted layer in the monodisperse case, and therefore corresponds to the distance where the graft concentration profile drops close to zero. The graft concentration profile is slightly different in the the polydisperse case when compared to the bidisperse case (Supplementary Figure 4.17). Close to the particle surface, the polydisperse and bidisperse graft monomer concentrations profiles have similar values. At intermediate distances from the particle surface, the bidisperse has the lowest concentration; the region where the long chains of the bidisperse distribution have a "stem-like" conformation[51] and polydisperse chains have a distribution of chain conformations. At larger distances from the particle surface (outer region of the grafted layer) the bidisperse concentration is higher than polydisperse and monodisperse because of the "crown" region of the long chains in the bidisperse region[51]. Due to these features in the grafted layer monomer distribution, the overlap of two grafted layers and the corresponding mid-range attractive well occurs at slightly different interparticle distances for polydisperse, bidisperse, and monodisperse graft distributions. The above trends between bidisperse and polydisperse distributions on the mid-range attraction are also observed at 0.25 chains/d ${ }^{2}$ (Figure $4.9 \mathrm{~b}$ and Figure $4.9 \mathrm{~d}$ ).

These results in Figure 4.9 suggest that for this specific type of bidisperse distribution (equal number of short and long chains), with the same average graft length and PDI as polydisperse distribution, the differences of monomer arrangement within the grafted layer between polydisperse and bidisperse grafts leads to polydisperse grafts being able to stabilize particle dispersion better than bidisperse grafts, in dense polymer matrix. However, we note that if we relaxed some of the conditions chosen in Figure 4.9 (e.g. equal number of short and long chains) or change the average graft length in the bidisperse distribution, there could be cases where the bidisperse or bimodal distributions provide better dispersion of particles as compared to a 
polydisperse distribution, In melt-like polymer matrix, the effect of bidispersity or polydispersity is lower than the corresponding dense polymer matrix; the attractive well in the PMF is not removed in melt-like matrix at the PDI where the corresponding dense solution matrix has been eliminated by polydispersity/bidispersity. The different positions for the mid-range attraction in the PMF between grafted particles with bidisperse and polydisperse grafted chains suggests that the choice of graft length distribution can be used as a way to tune the structure (e.g. inter-particle spacing) within the aggregates of these grafted particles.

\subsection{CONCLUSION}

In summary, this article presents a theory and simulation study that demonstrates how polydispersity in polymers grafted on spherical nanoparticles affects the effective inter-particle interactions between homopolymer grafted nanoparticles in a chemically identical homopolymer matrix. Graft polydispersity reduces the strength of repulsion at contact and weakens the attractive well at intermediate inter-particle distances in the potential of mean force (PMF) between grafted particles. This effect is attributed to polydispersity in grafted chain lengths reducing the grafted layer monomer crowding seen at monodisperse limits, which in turn increases wettability of the grafted layer by the matrix chains. The reduction/elimination of the attractive well suggests that graft polydispersity can reduce the tendency for particle aggregation, even stabilizing dispersion in some cases where the monodisperse grafts would cause aggregation. As the grafting density decreases from a brush-like grafting density, the effect of polydisperse grafts on the potential of mean force reduces because the relief in monomer crowding brought about by polydisperse grafts is insignificant at low grafting density. As the matrix packing fraction increases, the matrixinduced depletion-like attraction between the grafted particles becomes dominant, reducing the above graft polydispersity effects. At high grafting density, this matrix induced depletion-like 
attractions results in a need for higher polydispersity to eliminate the mid-range attraction completely, while at low grafting density the effect of polydispersity is completely removed. At the monodisperse graft limit the larger particles (lower curvature) have increased monomer crowding, especially at high grafting densities, compared to smaller (highly curved) particles, and as a result the effect of polydispersity is enhanced at lower curvature surfaces.

Comparison of bidisperse and polydisperse distributions of graft lengths elucidate the role of long chains in removing the attractive well by essentially adding a repulsive tail to the PMF through long grafts sterically repelling the grafts on the other particle. At the same PDI and average graft length, the presence of longer "long" chains in the polydisperse distribution eliminates the attractive well in the PMF better than the shorter "long" chains in the bidisperse distribution. In a bidisperse distribution with both short and long chains that are shorter than the matrix chains, we see reduction in mid-range attraction attributed to the lowered monomer crowding in the bidisperse grafted layer, that in turn increases the grafted layer wetting by the matrix chains. In a melt-like matrix, where the matrix-induced depletion attraction starts to dominate over the polydispersity effects, the different conformations of the grafted chains in the bidisperse and polydisperse distribution, captured through the graft monomer concentration profiles, change the location of the attractive well in the potential of mean force.

In conclusion, one of the key findings in this paper is that polydispersity can stabilize dispersions even when the average graft polymer molecular weight is lower than matrix polymer molecular weight, conditions that would cause particle aggregations for monodisperse grafts. This study motivates synthetic efforts to be directed towards obtaining controlled polydispersity in chain lengths as a design tool to program inter-particle interactions in a polymer matrix, and in turn the morphology of the polymer nanocomposite. 


\subsection{ACKNOWLEDGEMENTS}

The authors acknowledge financial support by Department of Energy Early Career Award Program under Grant No. DE-SC0003912

\subsection{REFERENCES}

1. Green, P.F., The structure of chain end-grafted nanoparticle/homopolymer nanocomposites. Soft Matter, 2011. 7(18): p. 7914-7926.

2. Sunday, D., J. Ilavsky, and D.L. Green, A Phase Diagram for Polymer-Grafted Nanoparticles in Homopolymer Matrices. Macromolecules, 2012. 45(9): p. 4007-4011.

3. Meng, D., et al., Effective interactions between grafted nanoparticles in a polymer matrix. Soft Matter, 2012. 8(18): p. 5002-5010.

4. Lin, Y.L., et al., Self-Assembled Superstructures of Polymer-Grafted Nanoparticles: Effects of Particle Shape and Matrix Polymer. Journal of Physical Chemistry C, 2011. 115(13): p. 5566-5577.

5. Chevigny, C., et al., Polymer-Grafted-Nanoparticles Nanocomposites: Dispersion, Grafted Chain Conformation, and Rheological Behavior. Macromolecules, 2011. 44(1): p. 122133.

6. Trombly, D.M. and V. Ganesan, Curvature effects upon interactions of polymer-grafted nanoparticles in chemically identical polymer matrices. Journal of Chemical Physics, 2010. 133(15).

7. Kalb, J., et al., End grafted polymer nanoparticles in a polymeric matrix: Effect of coverage and curvature. Soft Matter. 7(4): p. 1418-1425.

8. Akcora, P., et al., Anisotropic self-assembly of spherical polymer-grafted nanoparticles. Nature Materials, 2009. 8(4): p. 354-359.

9. Harton, S.E. and S.K. Kumar, Mean-field theoretical analysis of brush-coated nanoparticle dispersion in polymer matrices. Journal of Polymer Science Part B-Polymer Physics, 2008. 46(4): p. 351-358.

10. Xu, J., et al., Morphology and interactions of polymer brush-coated spheres in a polymer matrix. Journal of Polymer Science Part B-Polymer Physics, 2006. 44(19): p. 2811-2820.

11. Jayaraman, A. and K.S. Schweizer, Effective Interactions and Self-Assembly of Hybrid Polymer Grafted Nanoparticles in a Homopolymer Matrix. Macromolecules, 2009. 42(21): p. 8423-8434.

12. de Vos, W.M. and F.A.M. Leermakers, Modeling the structure of a polydisperse polymer brush. Polymer, 2009. 50(1): p. 305-316.

13. Milner, S.T., T.A. Witten, and M.E. Cates, EFFECTS OF POLYDISPERSITY IN THE END-GRAFTED POLYMER BRUSH. Macromolecules, 1989. 22(2): p. 853-861. 
14. Witten, T.A. and P.A. Pincus, COLLOID STABILIZATION BY LONG GRAFTED POLYMERS. Macromolecules, 1986. 19(10): p. 2509-2513.

15. Himmi, M., et al., Interaction force between colloidal particles clothed by end-grafted polydisperse polymer chains in solution. Journal of Molecular Liquids, 2003. 102(1-3): p. 347-363.

16. Dodd, P.M. and A. Jayaraman, Monte carlo simulations of polydisperse polymers grafted on spherical surfaces. Journal of Polymer Science Part B-Polymer Physics, 2012. 50(10): p. 694-705.

17. Shull, K.R., Wetting behavior of polymer melts on polydisperse grafted polymer layers. Macromolecules, 1996. 29(26): p. 8487-8491.

18. Laub, C.F. and J.T. Koberstein, EFFECT OF BRUSH POLYDISPERSITY ON THE INTERPHASE BETWEEN END-GRAFTED BRUSHES AND POLYMERIC MATRICES. Macromolecules, 1994. 27(18): p. 5016-5023.

19. Chremos, A., et al., Structure of solvent-free grafted nanoparticles: Molecular dynamics and density-functional theory. Journal of Chemical Physics, 2011. 135(11).

20. Akcora, P., et al., Segmental Dynamics in PMMA-Grafted Nanoparticle Composites (vol 43, pg 8275, 2010). Macromolecules, 2011. 44(2): p. 416-416.

21. Nair, N. and A. Jayaraman, Self-Consistent PRISM Theory-Monte Carlo Simulation Studies of Copolymer Grafted Nanoparticles in a Homopolymer Matrix. Macromolecules, 2010. 43(19): p. 8251-8263.

22. Hooper, J.B. and K.S. Schweizer, Contact aggregation, bridging, and steric stabilization in dense polymer-particle mixtures. Macromolecules, 2005. 38: p. 8858-8869.

23. Hooper, J.B. and K.S. Schweizer, Theory of phase separation in polymer nanocomposites. Macromolecules, 2006. 39: p. 5133-5142.

24. Hooper, J.B., et al., Structure, surface excess and effective interactions in polymer nanocomposite melts and concentrated solutions. Journal of Chemical Physics, 2004. 121(14): p. 6986-6997.

25. Hall, L.M., et al., Concentration Fluctuations, Local Order, and the Collective Structure of Polymer Nanocomposites. Macromolecules, 2009. 42(21): p. 8435-8442.

26. Hall, L.M. and K.S. Schweizer, Many body effects on the phase separation and structure of dense polymer-particle melts. Journal of Chemical Physics, 2008. 128: p. 234901.

27. Hall, L.M. and K.S. Schweizer, Structure, scattering patterns and phase behavior of polymer nanocomposites with nonspherical fillers. Soft Matter, 2010. 6(5): p. 1015-1025.

28. Jayaraman, A. and K.S. Schweizer, Structure and Assembly of Dense Solutions and Melts of Single Tethered Nanoparticles. J. Chem. Phys., 2008. 128: p. 164904.

29. Jayaraman, A. and K.S. Schweizer, Effect of the number and placement of polymer tethers on the structure of dense solutions of hybrid nanoparticles. Langmuir, 2008. 24(19): p. 11119-11130. 
30. Jayaraman, A. and K.S. Schweizer, Effective Interactions, Structure, and Phase Behavior of Lightly Tethered Nanoparticles in Polymer Melts. Macromolecules, 2008. 41(23): p. 9430-9438.

31. Jayaraman, A. and K.S. Schweizer, Effective Interactions and Self-Assembly of Hybrid Polymer Grafted Nanoparticles in a Homopolymer Matrix. Macromolecules, 2009. 42 p. pp 8423-8434.

32. Hindmarsh, A.C., et al., SUNDIALS: Suite of nonlinear and differential/algebraic equation solvers. Acm Transactions on Mathematical Software, 2005. 31(3): p. 363-396.

33. Grayce, C.J., A. Yethiraj, and K.S. Schweizer, Liquid state theory of the density dependent conformation of nonpolar linear polymers. Journal of Chemical Physics, 1994. 100: p. 6857-6872.

34. Melenkevitz, J., K.S. Schweizer, and J.G. Curro, Self consistent integral eqn theory for the eqbm properties of polymer solns. Macromolecules, 1993. 26: p. 6190-6196.

35. Khalatur, P.G. and A.R. Khokhlov, Hybrid MC/RISM technique for simulation of polymer solutions: MC+RISM integral eqns. Molecular Physics, 1998. 93: p. 555-572.

36. Mendez, S., et al., An integral equation theory for polymer solutions: explicit inclusion of the solvent molecules. Journal of Chemical Physics, 2001. 115: p. 5669-5678.

37. Putz, M., J.G. Curro, and G.S. Grest, Self-consistent integral equation theory for polyolefins: comparison to molecular dynamics simulations and x-ray scattering. Journal of Chemical Physics, 2001. 114: p. 2847-2860.

38. Yethiraj, A. and C.K. Hall, Monte Carlo simulations and integral equation theory for microscopic correlations in polymeric fluids. Journal of Chemical Physics, 1991. 96: p. 797-807.

39. Frischknecht, A.L., E.S. McGarrity, and M.E. Mackay, Expanded chain dimensions in polymer melts with nanoparticle fillers. Journal of Chemical Physics, 2010. 132: p. 204901.

40. Khalatur, P.G., L.V. Zherenkova, and A.R. Khokhlov, Entropy-driven polymer collapse: Application of the hybrid MC/RISM method to the study of conformational transitions in macromolecules interacting with hard colloidal particles. European Physical Journal B, 1998. 5(4): p. 881-897.

41. Agarwal, U. and F.A. Escobedo, Mesophase behaviour of polyhedral particles. Nature Materials, 2011. 10(3): p. 230-235.

42. Schweizer, K.S. and J.G. Curro, Integral equation theory of polymer melts: intramolecular structurem local order, and the correlation hole. Macromolecules, 1988. 21: p. 3070-3081.

43. Grayce, C.J. and K.S. Schweizer, Solvn potentials for macromolecules. Journal of Chemical Physics, 1994. 100: p. 6846-6856.

44. Schweizer, K.S. and J.G. Curro, PRISM Theory of the Structure, Thermodynamics, and Phase-Transitions of Polymer Liquids and Alloys. Advances in Polymer Science, 1994. 116: p. 319-377.

45. HOOMD-blue. http://codeblue.umich.edu/hoomd-blue. 
46. Krishnamoorti, R., Strategies for Dispersing Nanoparticles in Polymers. MRS Bulletin, 2007. 32(4): p. 341-347.

47. Egorov, S.A. and K. Binder, Effect of solvent quality on the dispersibility of polymergrafted spherical nanoparticles in polymer solutions. Journal of Chemical Physics, 2012. 137(9).

48. Smith, G.D. and D. Bedrov, Dispersing Nanoparticles in a Polymer Matrix: Are Long, Dense Polymer Tethers Really Necessary? Langmuir, 2009. 25(19): p. 11239-11243.

49. Hall, L.M., A. Jayaraman, and K.S. Schweizer, Molecular theories of polymer nanocomposites. Current Opinion in Solid State \& Materials Science, 2010. 14(2): p. 3848.

50. Jayaraman, A. and K.S. Schweizer, Liquid state theory of the structure and phase behaviour of polymer-tethered nanoparticles in dense suspensions, melts and nanocomposites. Molecular Simulation, 2009. 35(10-11): p. 835-848.

51. Nair, N., N. Wentzel, and A. Jayaraman, Effect of bidispersity in grafted chain length on grafted chain conformations and potential of mean force between polymer grafted nanoparticles in a homopolymer matrix. Journal of Chemical Physics, 2011. 134(19). 


\subsection{SUPPLEMENTARY}

\subsubsection{Additional Model Details}

We model polymer grafted spherical nanoparticle as a hard spherical nanoparticle of diameter $D$ with $N_{\text {graft }}$ freely jointed homopolymer chains permanently attached symmetrically on the particle surface, and model the matrix chain as a freely jointed homopolymer chain. The polymer chains (grafted and matrix) consist of monomer beads of diameter $d$ chosen to mimic a Kuhn segment of a linear synthetic polymer. While chains in the Monte Carlo (MC) simulations have a constant bond length, monomers in the Brownian Dynamics (BD) simulations are connected together with harmonic bonds with force constant $\mathrm{k}=30$ and bond rest length $\mathrm{r}_{0}=1.4 \mathrm{~d}$ :

$$
V_{H}(r)=\frac{1}{2} k\left(r-r_{0}\right)
$$

where $\mathrm{k}$ is the force constant, $\mathrm{r}$ is the center to center distance between the bonded beads, and $\mathrm{r}_{0}$ is the bond rest length. The grafted chains are either monodisperse, with chains of equal length (or number of monomers), or polydisperse with a log normal distribution or bidisperse with equal number of short and long chains. In the case of polydisperse grafted chains, we quantify the polydispersity via the polydispersity index (PDI):

$$
P D I=\frac{E\left[\left(N_{\text {graft }}\right)^{2}\right]}{E\left[N_{\text {graft }}\right]^{2}}=\frac{\bar{N}_{\text {graft }, w}}{\bar{N}_{\text {graft }, \text { avg }}}
$$

where $\mathrm{E}\left[\left(\mathrm{N}_{\text {graft }}\right)^{\mathrm{n}}\right]$ is the nth moment of the distribution of $\mathrm{N}_{\text {graft }}, \overline{\mathrm{N}}_{\mathrm{graft}, \mathrm{w}}$ is the weight average molecular weight of the graft length distribution, and $\overline{\mathrm{N}}_{\text {graft,avg }}$ is the number average molecular weight. For systems with graft PDI>1, we fit the chain lengths to a log-normal distribution with minimum and number average graft lengths $\mathrm{N}_{\text {graft,min }}=8$ and $\mathrm{N}_{\text {graft,avg }}=20$. The probability density function for the log-normal distribution is:

$$
P\left(N_{\text {graft }}\right)=\frac{1}{N_{\text {graft }} \sigma \sqrt{2 \pi}} \exp \left(-\frac{\left(\ln N_{\text {graft }}-\mu\right)^{2}}{2 \sigma^{2}}\right)
$$


where $\mu$ and $\sigma$ are the mean and standard deviation of $\ln \left(\mathrm{N}_{\text {graft }}\right)$. We generate our desired chain distribution by first solving the following linear system for the log-normal distribution parameters using PDI, $\mathrm{N}_{\text {graft,avg, }}$ and $\mathrm{N}_{\text {graft,min }}$ as inputs.

$$
\begin{gathered}
\mu+\frac{1}{2} \sigma=\ln \left(\bar{N}_{\text {graft }, \text { avg }}-N_{\text {graft }, \text { min }}\right) \\
\mu+\sigma=\frac{1}{2} \ln \left[P D I\left(\bar{N}_{\text {graft }, \text { avg }}\right)^{2}-2\left(N_{\text {graft }, \text { min }}\right)\left(\bar{N}_{\text {graft }, \text { avg }}\right)+\left(N_{\text {graft }, \text { min }}\right)^{2}\right]
\end{gathered}
$$

The $\mu$ and $\sigma$ are then used with the above equation to generate a distribution of chain lengths with the desired properties.

Supplementary Figure 4.10 shows the distribution of chain lengths for the system whose results are presented in Figure 4.1 in main manuscript.
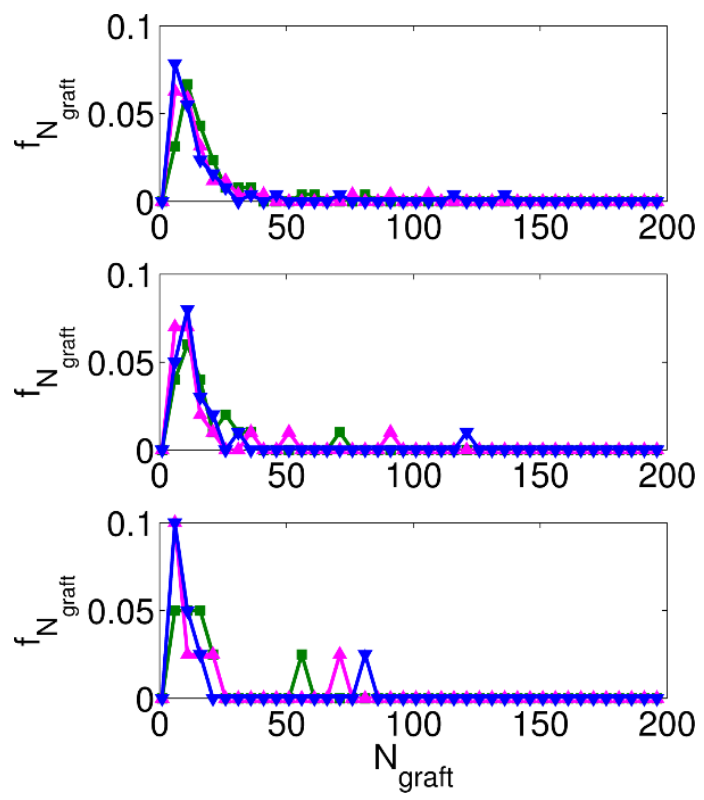

Figure 4.10: Chain length distributions of polydisperse chains grafted on nanoparticles $(D=5 d)$ at $\sigma=0.1$ chains $/ d^{2}(a), \sigma=0.25$ chains $/ d^{2}(b), \sigma=0.65$ chains $/ d^{2}(c)$, and $P D I=1.5$ (squares), 2.0 (upward facing triangles), and 2.5 (downward facing triangles) with $N_{g, a v g}=20$ and $N_{g, \min }=8$ 


\subsubsection{Brownian Dynamics Simulation Results}

In Supplementary Figure 4.11 - Figure 4.13 we present the total monomer concentration profiles for grafted chains and matrix chains, distribution of average radius of gyration of matrix chains, end-monomer concentration profiles, respectively.
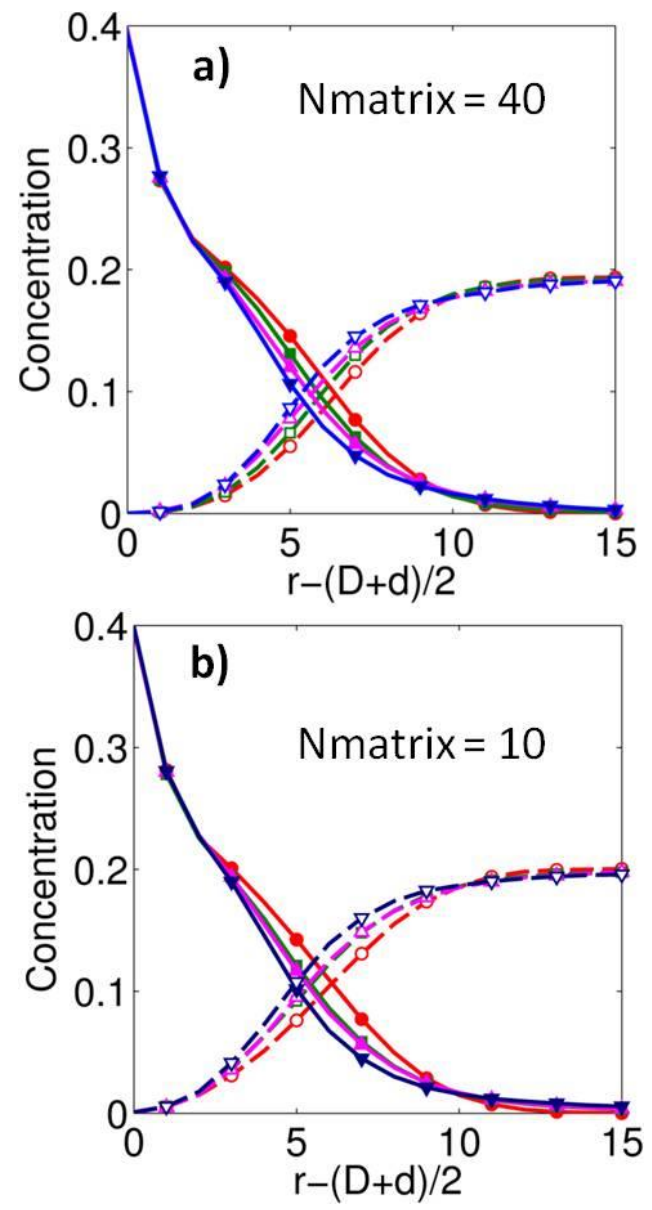

Figure 4.11: Total monomer concentration profiles (in units of $d^{-3}$ ) versus distance from particle surface, $r-(D+d) / 2$ (in units of $d$ ), between grafted (filled symbols) or matrix chains (open symbols) and grafted nanoparticles $(D=5 d)$ at $\sigma=0.65,0.25,0.10$ chains $/ d^{2}$ and $P D I=1.0$ (circles), 1.5 (squares), 2.0 (upward facing triangles), and 2.5 (downward facing triangles) with $N_{g, a v g}=20$, in a dense solution $(\eta=0.1)$ of monodisperse homopolymer matrix chains with $N_{\text {matrix }}=40$ (top) and $N_{\text {matrix }}=10$ (bottom). 
This figure shows that with increasing polydispersity in the grafted chains we see increasing penetration of the grafted layer by the matrix chains. The grafted chain concentration profile also shows changes with increasing polydispersity. We do not see differences near the particle surface. We see lower graft monomer concentration at intermediate distances from the particle surface for higher PDI attributed to the reduced monomer crowding brought about by polydispersity. At farther distances from particle surface we see higher graft monomer concentration at high PDI due to the presence of longer chains in the highly polydisperse distribution.
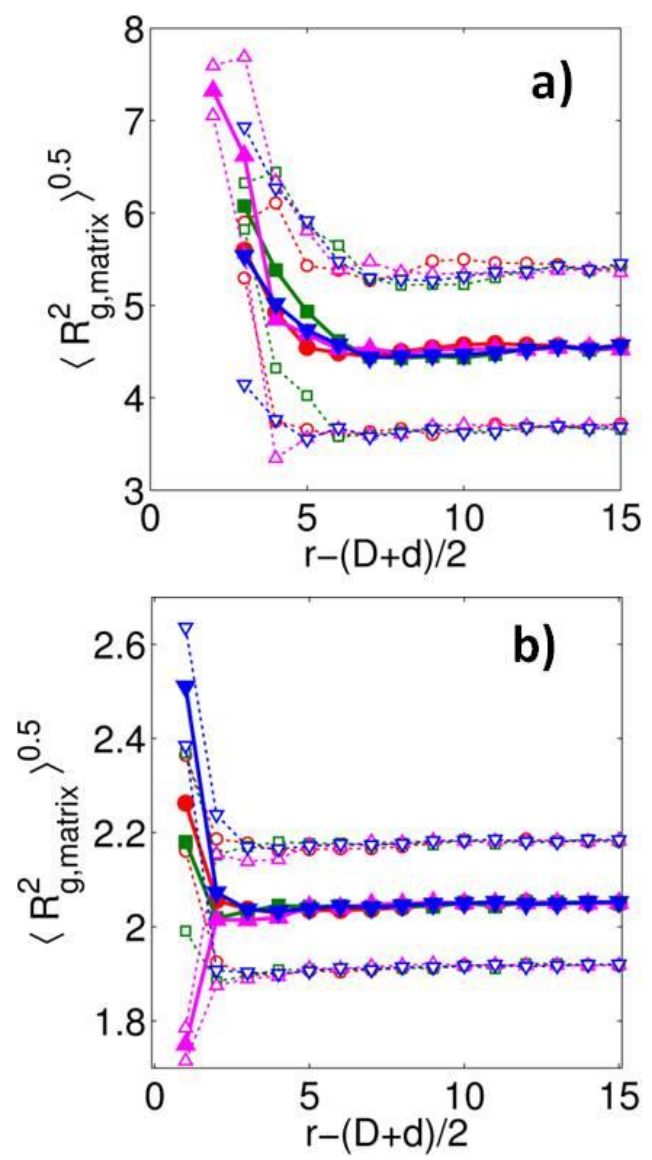

Figure 4.12: Average radius of gyration of the monodisperse matrix chains as a function of distance of center of mass of matrix chain from particle surface, $r-(D+d) / 2($ in units of $d)$ for grafted monomers at varying polydispersity of grafted chains on nanoparticles have diameter $D=5 d$ at grafting density $\sigma=0.65$ chains $/ d^{2}$ in a dense solution $(\eta=0.1)$ of monodisperse 
homopolymer matrix chains with $N_{\text {matrix }}=40$ (a) or $N_{\text {matrix }}=10(\mathrm{~b})$. For both plots, the symbols correspond to the polydispersity of the grafted chains as: PDI = 1.0 (circles), 1.5 (squares), 2.0 (upward triangles), and 2.5 (downward triangles). The solid symbols represent the mean value while the open symbols represent the upper and lower limits of the error bar.

This figure characterizes the matrix conformations as a function of distance of matrix chain from the particle surface. In both cases of matrix chain lengths equal to 10 and 40 segments the error bars are large enough that we do not expect there to be a significant differences in matrix conformations as a function of distance. However we do note the slight increase in the mean matrix chain radius of gyration as the matrix chains approach the particle surface. This could be expected as the matrix chains might have to stretch while penetrating a densely grafted polymer layer.
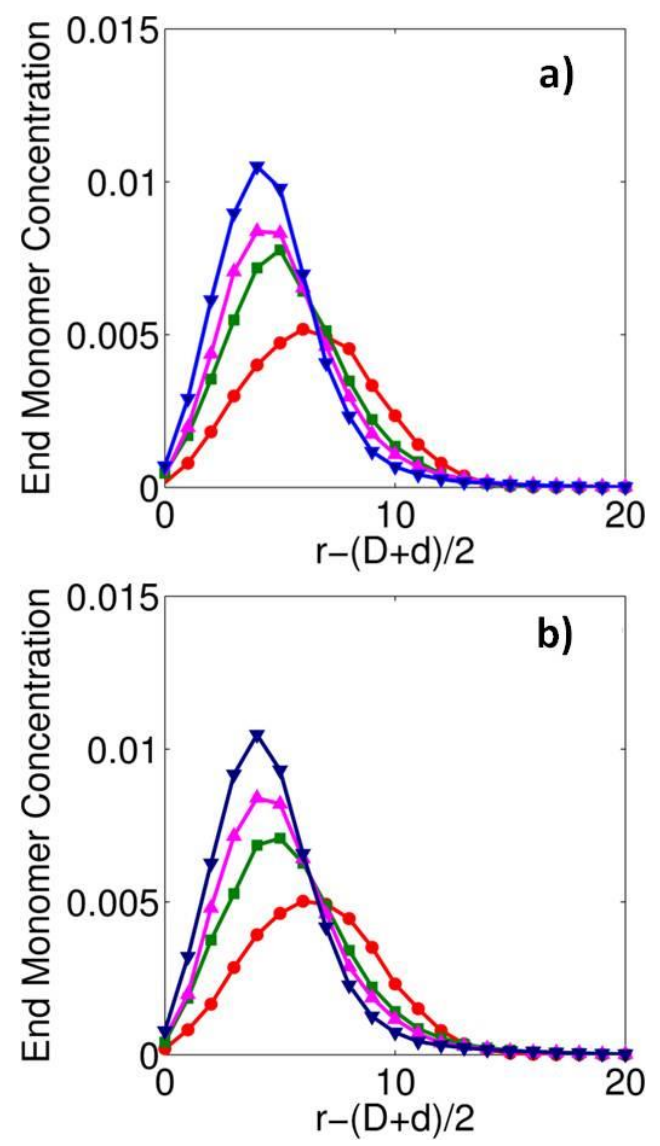

Figure 4.13: End monomer concentration profiles (in units of $d^{-3}$ ) versus distance from particle surface, $r-(D+d) / 2$ (in units of $d$ ), between grafted (filled symbols) or matrix chains (open symbols) 
and grafted nanoparticles $(D=5 d)$ at $\sigma=0.65,0.25,0.10$ chains $/ d^{2}$ and PDI= 1.0 (circles), 1.5 (squares), 2.0 (upward facing triangles), and 2.5 (downward facing triangles) with $N_{g, a v g}=20$, in a dense solution $(\eta=0.1)$ of monodisperse homopolymer matrix chains with $N_{\text {matrix }}=40$ (top) and $N_{\text {matrix }}=10$ (bottom).

This figure characterizes the distribution of the end monomers of the grafted chains within the grafted layer as a function of polydispersity index. As seen in prior work by Dodd and Jayaraman where the polymer grafted nanoparticles were in an implicit matrix, we see a shift of the end monomer distribution closer to the surface and becomes narrower with increasing PDI. 


\subsubsection{Additional PRISM-MC Results}

\subsubsection{PMF for small changes in PDI}

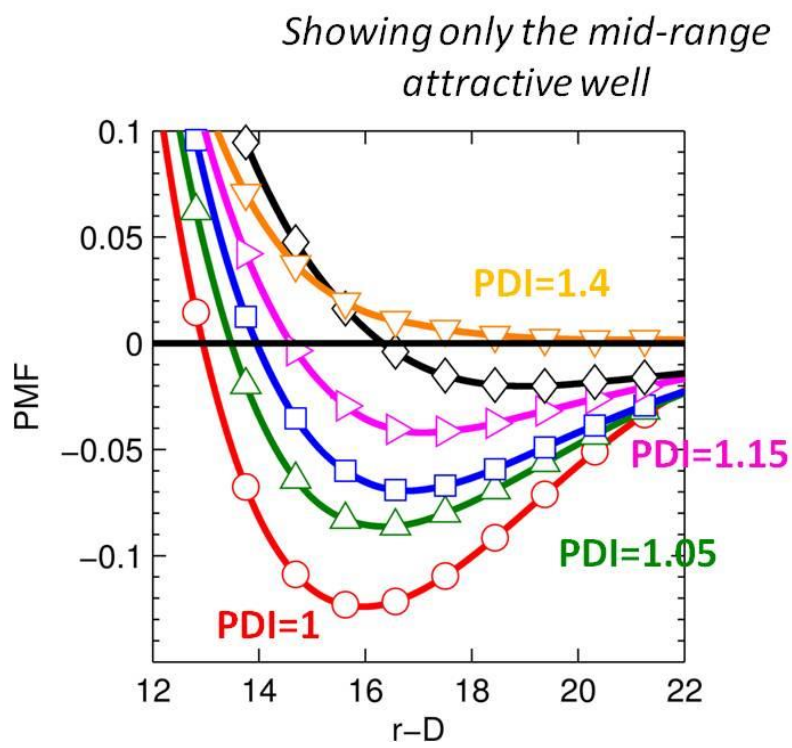

Figure 4.14: PMF (in units of kT) versus inter-particle distance, $r$ - $D$ (in units of $d$ ), between grafted nanoparticles $(D=5 d)$ at $\sigma=0.65$ chains $/ d^{2}$ and $P D I=1-1.5$ with $N_{g, a v g}=20$, in a dense solution $(\eta=0.1)$ of monodisperse homopolymer matrix chains with and $N_{\text {matrix }}=40$.

In the main paper at 0.65 chains/ $\mathrm{d}^{2}$ and $\mathrm{N}_{\text {matrix }}>\mathrm{N}_{\mathrm{g}, \text { avg }}$ (open symbols in Figure $4.1 \mathrm{a}$ ) the attractive well of $\sim 0.1 \mathrm{kT}$ at intermediate distances seen in monodisperse systems is completely eliminated at PDI of 1.5 and above. The above figures shows additional calculations at smaller PDI (1.05-1.4) demonstrating that there is a minimum PDI needed to eliminate the attractive well. Since this minimum PDI needed to remove this attraction is dependent on the strength of the midrange attraction (which is a function of grafting density, particle size and average graft and matrix lengths) we expect this minimum PDI to be a function of grafting density, particle size and average graft and matrix length. 


\subsubsection{PMF for long matrix lengths}

While we only show results for $\mathrm{N}_{\text {matrix }}=10$ and 40 in the main manuscript we have confirmed at the monodisperse graft limit that as $\mathrm{N}_{\text {matrix }}$ increases the attractive well at intermediate distances deepens thereby increasing particle aggregation (parts a and b), in agreement with past studies.
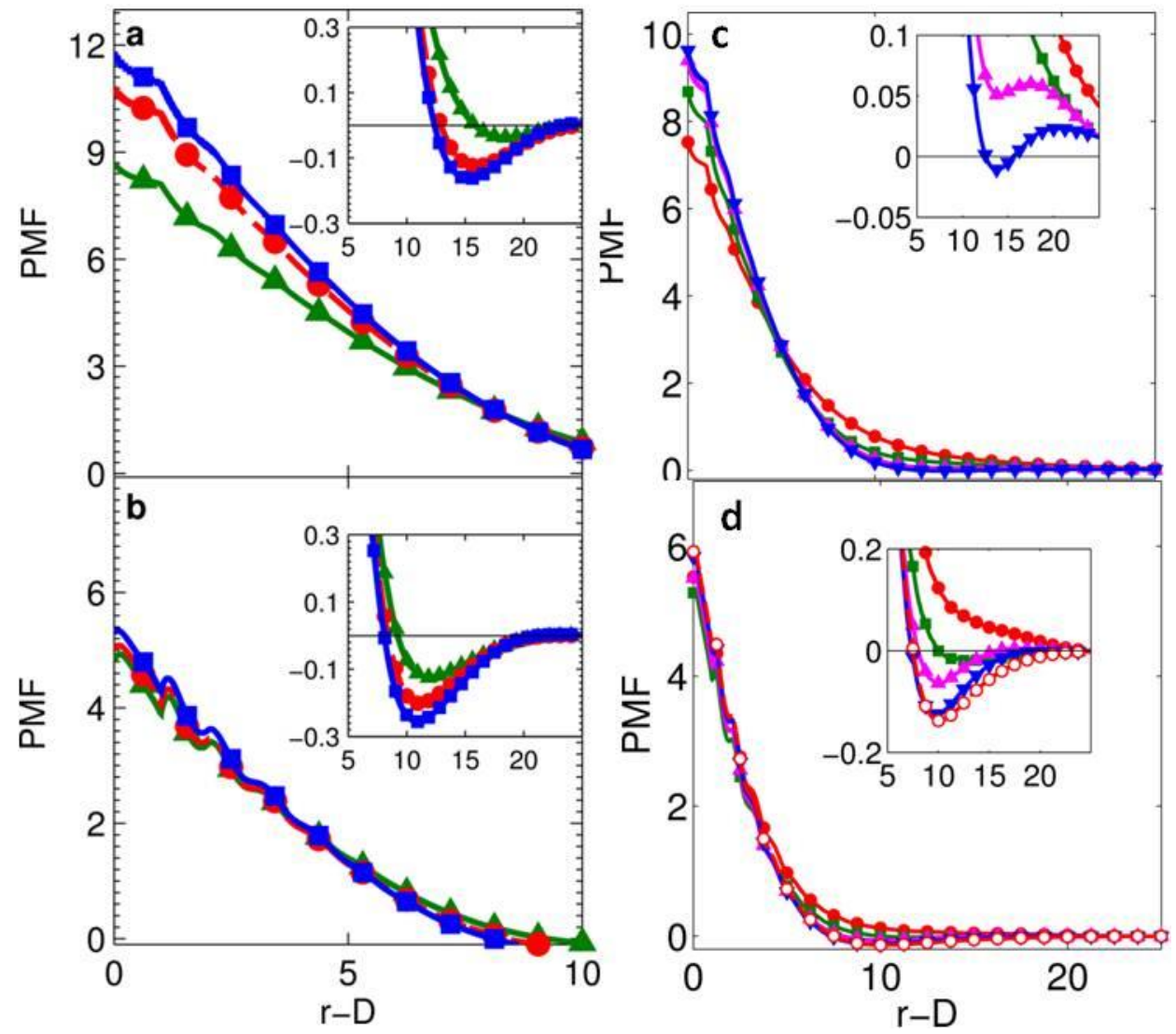

Figure 4.15: PMF (in units of $k T$ ) versus inter-particle distance, $r$ - $D$ (in units of $d$ ), between grafted nanoparticles $(D=5 d)$ at $\sigma=0.65$ chains $/ d^{2}$ in a polymer solution $(\eta=0.1)$ (top row) or meltlike $(\eta=0.3)$ (bottom row) matrix of monodisperse homopolymer chains. Parts $a$ and $b$ are for monodisperse grafts of length 20 and monodisperse homopolymer matrix of $N_{\text {matrix }}=10$ (triangles), $N_{\text {matrix }}=40$ (circles), $N_{\text {matrix }}=80$ (squares). Parts $c$ and $d$ are for polydisperse grafts with PDI=2.0 and monodisperse homopolymer matrix with $N_{\text {matrix }}=10$ (solid circles), 40 (squares), 100 (upward triangles), 200 (downward triangles), 300 (open circles). The insets have the same axes labels as the main plots. 
Parts $\mathrm{c}$ and $\mathrm{d}$ show that with grafts of polydispersity $\mathrm{PDI}=2$ the mid-range attraction is weakened for all matrix lengths, and completely removed for lower matrix lengths where the mid-range attraction at the monodisperse limit ( $a$ and $b$ ) are weaker than the longer matrix lengths. This figure supports the comment in the main manuscript that as the matrix length increases we would need larger PDI to remove the tendency of the grafted particles to aggregate. In other words, larger PDI is needed to induced wetting of the grafted layer by longer matrix chains. 
4.7.3.3 Effect of increasing matrix packing fraction on penetration depth
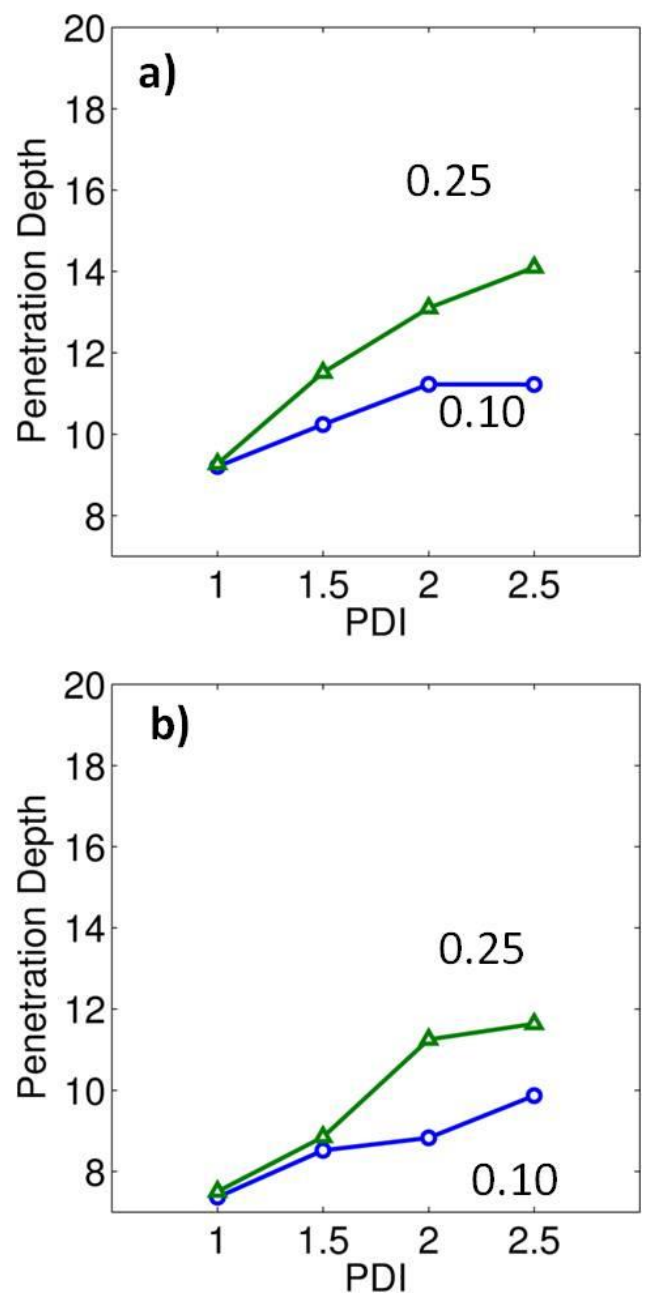

Figure 4.16: Penetration depth (in units of $d$ ) of the matrix chains into the grafted layer of nanoparticles $(D=5 d)$ with polydisperse chains $\left(N_{g, a v g}=20\right)$ at $\sigma=0.10$ chains $/ d^{2}$ (circles) and $\sigma=$ 0.25 chains $/ d^{2}$ (triangles) in a dense solution $(\eta=0.3)$ with (a) $N_{\text {matrix }}=10$ and (b) $N_{\text {matrix }}=40$. 
4.7.3.4 PMF for Polydisperse distribution versus Bidisperse distribution
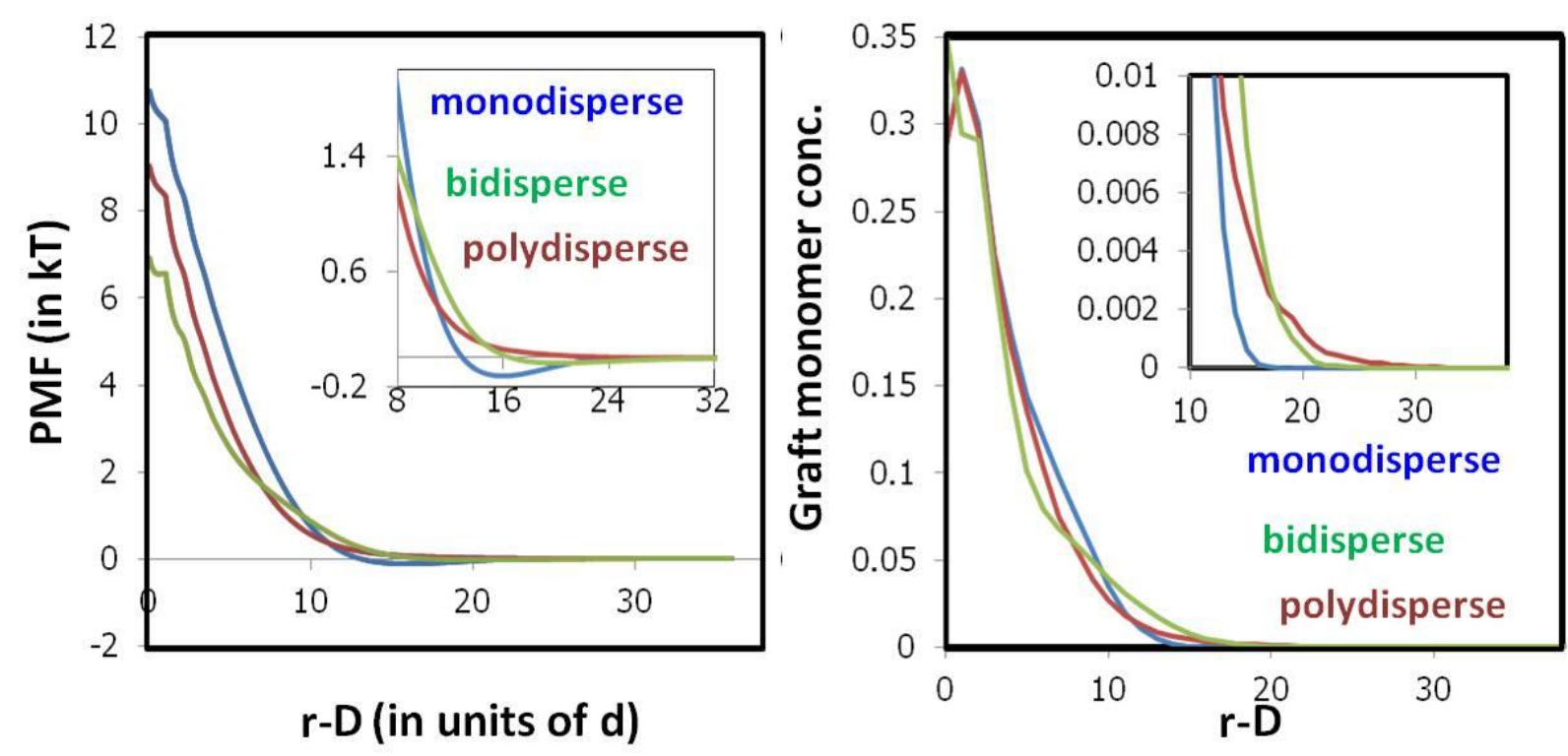

Figure 4.17: (left) PMF (in units of $k T$ ) versus inter-particle distance, $r$-D (in units of d) and graft monomer concentration profile (right) for grafted nanoparticles $(\boldsymbol{D}=\mathbf{5 d})$ with monodisperse homopolymer grafts with PDI=1.0 (blue line), polydisperse homopolymer grafts PDI=1.5 (maroon line), and bidisperse homopolymer grafts $P D I=1.5$ (green line), all with $N_{g, a v g}=20$ and grafting density $\sigma=0.65$ chains $/ d^{2}$ in a in a dense solution ( $\left.\eta=0.1\right)$ of monodisperse homopolymer matrix with $\boldsymbol{N}_{\text {matrix }}=40$. The insets have the same axes labels as the main plots. 


\section{IDENTIFYING THE IDEAL CHARACTERISTICS OF THE GRAFTED POLYMER CHAIN LENGTH DISTRIBUTION FOR MAXIMIZING DISPERSION OF POLYMER GRAFTED NANOPARTICLES IN A POLYMER MATRIX}

Adapted from: Macromolecules 46 (22), 9144-9150

\subsection{INTRODUCTION}

Linking the morphology of nanoparticles in a dense polymer matrix to the underlying molecular features of the nanoparticles and polymer matrix is important for the rational design of polymer nanocomposite materials for various applications in the automobile, electronics, optics, and photonics industries. [1,2] Furthermore, since the macroscopic properties of these polymer nanocomposites are strongly correlated with their microscopic morphology, the goal of such a rational design is to controllably create target morphologies. The morphologies in polymer nanocomposites fall broadly into two categories: dispersed morphologies, where the nanoparticles seek to maximize interparticle distance within the matrix, and aggregated morphologies, where the nanoparticles can assemble into a variety of isotropic or anisotropic structures within the matrix. Dispersed morphologies are generally needed for applications where the mechanical properties of the nanocomposite are important, while ordered aggregates of nanoparticles are desired in optics and electronics applications.[2,3] One way to control the morphology of the nanoparticles in a polymer matrix is by functionalizing the surface of the nanoparticles with polymers that are chemically similar or dissimilar to the polymer matrix in order to tune the effective particle-particle and particle-matrix interactions.[4-16] When the grafted polymers are the same chemistry as the matrix polymers, and are functionalized at high grafting density, the ratio of graft to matrix molecular weight $\left(\mathrm{N}_{\text {graft }} / \mathrm{N}_{\text {matrix }}\right)$ dictates whether the grafted nanoparticles will aggregate or disperse in the polymer matrix, with the grafted particles aggregating when 
$N_{\text {graft }} / N_{\text {matrix }}<1$, and dispersing when $N_{\text {graft }} / N_{\text {matrix }}>1 .[5,8]$ In these densely grafted systems, since the graft and matrix polymers are the same chemistry, the morphology at these $\mathrm{N}_{\text {graft }} / \mathrm{N}_{\text {matrix }}$ conditions is driven primarily by the entropy of the graft and matrix polymers.[17, 18] When $\mathrm{N}_{\text {graft }} / \mathrm{N}_{\text {matrix }}$ is small, the matrix chains penetrate, or wet, the grafted polymer layer. When $\mathrm{N}_{\text {graft }} / \mathrm{N}_{\text {matrix }}$ is large, the matrix chains deplete, or dewet, the grafted layer. Promoting wetting (dewetting) of the grafted polymer layer by the matrix leads to dispersed (aggregated) morphologies. The exact value of $\mathrm{N}_{\text {graft }} / \mathrm{N}_{\text {matrix }}$ where the systems crosses over from dispersed to aggregated morphologies depends on the value of particle/substrate curvature and the grafting density. For example, at high particle curvature and high grafting densities past studies have shown that $\mathrm{N}_{\text {graft }} / \mathrm{N}_{\text {matrix }}>4-5$ causes dispersion of the polymer grafted particles in a chemically identical polymer matrix. $[5,8,19]$ And, as the curvature of the grafted surface decreases towards zero (approaching a flat surface) the value of $\mathrm{N}_{\text {graft }} / \mathrm{N}_{\text {matrix }}$ where the wetting to dewetting transition happens decreases, due to reduced volume available within the grafted layer at low curvatures.

In recent theoretical work, we have shown that polydispersity in grafted homopolymer chain lengths stabilizes dispersions of grafted nanoparticles (high curvature) in a monodisperse polymer matrix with the same chemistry as the grafted polymer, even when the average $\mathrm{N}_{\text {average_graft }} / \mathrm{N}_{\text {matrix }}<1$, conditions where the corresponding monodisperse case would cause aggregation.[20,21] Polydisperse grafts stabilize nanoparticle dispersion a) by introducing steric repulsion due to the long chains in the polydisperse graft length distribution, which are not present in the monodisperse system with same average graft length, and b) by reducing the monomer crowding in the grafted layer, allowing the matrix chains to more easily wet the grafted layer. Similar dispersion stabilizing effects are also seen with bidisperse grafted polymer chain length distributions. [20, 22, 23] In agreement with our theoretical prediction, recent experimental work 
by Schadler, Benicewicz and coworkers shows that a bimodal grafted chain length distribution greatly improves the dispersion of grafted nanoparticles in a polymer matrix over that observed with a monomodal graft length distribution.[24-26] These experiments suggest that the improved dispersion comes from a combination of the short grafted chains shielding the particle-particle attractions, and the sparsely grafted long chains improving the wetting of the grafted layer by the matrix chains. It is important to note that while our previous theoretical predictions [20, 21] were obtained using athermal interactions to capture purely the entropic effect of wetting/dewetting in the presence of polydisperse grafts, these experiments [24-26] were conducted in the presence of strong particle-particle attractions. Therefore, in this paper using theory and simulations, first, we elucidate how the dispersion stabilizing effect of polydisperse grafts seen previously at athermal conditions is altered in the presence of significant particle-particle attraction for bidisperse and polydisperse graft distributions. Second, we extract the role of the short and long grafted chains in shielding nanoparticle-nanoparticle attractions and improving wetting of the grafted layer, so as to identify the ideal features of a densely grafted polymer length distribution that can maximize the dispersion of the polymer grafted nanoparticles in a chemically identical matrix polymer.

In this paper we find that for densely grafted systems, particle-particle attraction has little effect on the ability of graft length polydispersity to stabilize dispersions of polymer grafted nanoparticles in a polymer matrix. At high grafting density increasing particle-particle attraction leads only to minor changes in the potential of mean force (PMF) between two polymer grafted particles in a chemically identical matrix as compared to that at purely athermal interactions, and does not significantly alter the graft and matrix monomer concentration profiles. In the presence of particle-particle attraction strength of 5kT, the PMF between two polymer grafted particles with bidisperse graft length distributions with equal number of short and long chains has a stronger 
attraction at contact and reduced close range repulsion as compared to particles with log-normal polydisperse graft length distributions, at same polydispersity index and average graft length. This is because, in the presence of particle-particle attraction, the bidisperse distribution with equal number of short and long chains is worse at shielding particle-particle attraction than the lognormal distribution at the same polydispersity index and average graft length, due to reduced monomer concentration (for bidisperse case) near the particle surface which allows the nanoparticles to more easily approach one another to within the range of the attractive particleparticle potential. In contrast, we find similar monomer concentration profiles and consequently similar steric repulsion and mid-range attraction in the PMF for particles with bidisperse graft length distribution with many short chains and few long chains and particles with polydisperse log-normal graft length distribution with the same average graft length and polydispersity index, These results suggest that to find an optimal graft distribution that best disperses the polymer grafted particles it is important to understand the individual role of the short and long chains on the resulting PMF between the particles in the polymer matrix. We find that the screening of the particle-particle attraction and the resulting value of PMF close to contact is driven not by the short chains alone, but primarily by the number and arrangement of the monomers near the surface irrespective of the chain (short or long) the monomers belong to. The length and crowding of the long chains determine the location and strength of the steric repulsion and mid-range attraction in the PMF. We also find that the PMF between particles with a bidisperse distribution cannot be predicted by the simple addition of the PMF between particles with only the short chains and PMF between particles with only the long chains, highlighting the coupled roles of the short and long chains on the potential of mean force. Based on these comparisons, we find that the ideal graft length distribution that maximizes dispersion of a polymer grafted particle in a chemically identical 
matrix polymer, is one that provides a) maximum monomer crowding near the surface of the nanoparticle, in order to shield short-range particle-particle attraction and sterically repelling other approaching grafted nanoparticles, and b) minimal crowding near the edge of the grafted layer in order to maximize wetting of the grafted layer by the matrix chains. Polydisperse (log-normal) graft length distributions and bidisperse graft length distributions with few long chains among many short chains satisfy this criterion, and better disperse grafted particles in a chemically identical matrix than monodisperse grafts or bidisperse graft length distributions with equal number of short and long chains, with equivalent average graft length.

\subsection{APPROACH}

\subsubsection{Model}

We model a homopolymer grafted spherical nanoparticle as a hard spherical nanoparticle of diameter $D=5 d$, where $d$ is the size of 1 Kuhn segment of a generic homopolymer, with polymer chains permanently grafted randomly on the particle surface at total grafting density $\sigma=0.65$ chains $/ \mathrm{d}^{2}$ (brush-like grafting density). Through visual analysis we confirm that the grafting points of the chains are approximately equally spaced, ensuring uniform grafting density on the particle surface. We model the matrix and graft homopolymer chains as freely jointed chains, with monomer beads of diameter $d$ and constant bond length equal to $1.4 d$. The matrix chain lengths are monodisperse throughout this study and denoted by $\mathrm{N}_{\text {matrix. Three types of grafted }}$ homopolymer chain length distributions are considered: i) monodisperse with all chains of length $\mathrm{N}_{\text {graft }}=20$, ii) polydisperse log-normal distribution with minimum graft length $\mathrm{N}_{\text {graft,min }}=8$ and average graft lengths $\mathrm{N}_{\text {graft,avg }}=14$ and 20; the polydispersity of the grafted chains is characterized by a polydispersity index (PDI) [20, 21], and iii) bidisperse distribution with short and long chains at equal grafting densities $\left(\sigma_{\mathrm{s}} \approx \sigma_{\mathrm{l}} \approx 0.32\right.$ chains $\left./ \mathrm{d}^{2}\right)$ and $\mathrm{N}_{\text {graft,avg }}=20$, or bidisperse distribution with 
unequal grafting densities $\left(\sigma_{\mathrm{s}}>\sigma_{1}\right.$ chains $\left./ \mathrm{d}^{2}\right)$ and varying $\mathrm{N}_{\text {graft,avg }}$; the bidispersity is characterized both using PDI as well as graft length ratio, $\beta=\mathrm{N}_{\text {long }} / \mathrm{N}_{\text {short. }}$. Since the focus here is to find distributions that stabilize dispersion we choose monodisperse matrix chains of length $\mathrm{N}_{\text {matrix }}=40$ Kuhn segments to maintain $\mathrm{N}_{\text {graft,avg }} / \mathrm{N}_{\text {matrix }}<1$, condition where aggregation is expected at the monodisperse graft limit.

\subsubsection{Method}

We use self-consistent Polymer Reference Interaction Site Model (PRISM)-Monte Carlo (MC) simulation method described in detail in Refs [27, 28] where we solve non-linear integral equations that relate the total site-site inter-molecular pair correlation function, $h_{i j}(r)$, to the inter-molecular direct correlation function, $c_{i j}(r)$, and intra-molecular pair correlation function, $\omega_{i j}(r)$, between all $i$ and $j$ sites in the system (Equation 1), along with atomic closures.[29, 30]

$$
\begin{gathered}
H(q)=\Omega(q) C(q)[\Omega(q)+H(q)] \\
H_{i j}(q)=\rho_{i} \rho_{j} \hat{h}_{i j}(q) \\
\Omega_{i j}(q)=\rho \sum_{\alpha=1}^{N_{i}} \sum_{\beta=1}^{N_{j}} \Omega_{\alpha_{i} \beta_{j}}(q)
\end{gathered}
$$

We use Percus Yevick (PY) closure for all pairwise interactions except particle-particle which is represented with hypernetted chain (HNC) closure, as shown in Equation 2.

$$
\begin{gathered}
g_{i j}(r)=0 \quad r<\sigma_{i j} \\
P Y: c_{i j}(r)=\left(1-e^{\beta U_{j}(r)}\right) g_{i j}(r) \quad r>\sigma_{i j} \\
H N C: c_{B B}(r)=h_{B B}(r)-\ln g_{B B}(r)-\beta U_{B B}(r) \quad r>D
\end{gathered}
$$

We choose to keep this section brief as all the details of this PRISM-MC approach, the selfconsistent loop, and the choice of closures are presented in a recent review by Jayaraman about this method and its application to study polymer grafted particles. [27] As the numerical solution 
of the self-consistent PRISM-MC equations we obtain the equilibrium inter-molecular pair correlation function, $g_{i j}(r)=h_{i j}(r)+1$, that characterizes the local structure of the grafted nanoparticles and the matrix polymer. The effective interactions or potential of mean force (PMF) between two nanoparticles is calculated from the particle-particle pair correlation function, $\mathrm{g}_{\mathrm{PP}}(\mathrm{r})$ as $P M F(r)=-k_{B} T \ln g_{P P}(r)$. See Supporting Information Figure 5.6 for a schematic of the PMF and the information gleaned from the various features of the PMF. Using $g_{P P}(r)$ we also calculate the second virial coefficient, $\mathrm{B}_{2}$, a measure of the tendency for particle aggregation or dispersion as $B_{2}=2 \pi \int r^{2}\left(1-g_{P P}(r)\right) d r$. We also calculate the total or partial monomer concentration profiles $C(r)$ versus distance $r$ from the nanoparticle surface from the MC portion of calculation. $C(r)$ at a distance $r$ from the nanoparticle surface is calculated by dividing the average population $n(r)$ in a spherical shell by its volume: ${ }_{C}(r)=\frac{n(r)}{4 \pi r^{2} \Delta r}$. We plot $r^{2} C(r)$ versus distance from the particle surface to clearly distinguish two or more concentration profiles, especially near the particle surface.

\subsubsection{Parameters}

The total packing fraction $(\eta=0.1)$ is the volume fraction of the system occupied by the matrix chains and the polymer-grafted nanoparticles (fillers). The fraction of $\eta$ that is occupied by the fillers is denoted by $\phi$, and chosen to be $\phi=0.001$ here to calculate potential of mean force (PMF) between the grafted particles at the infinitely dilute filler limit. We vary the particle-particle interaction from an athermal (hard sphere) potential (denoted by $\varepsilon_{\mathrm{PP}}=0$ ) to attractive potentials modeled with colloid Lennard Jones (cLJ) potential shown in Supporting Information Figure 5.5, with varying well depths, $\varepsilon_{\mathrm{PP}}=0.1$ to $5 \mathrm{k}_{\mathrm{B}} \mathrm{T}$. We maintain athermal (hard sphere) interactions between all monomer pairs (both graft and matrix) and all particle-monomer pairs (graft and matrix). The choice of athermal interactions between monomers and particle and monomers is 
appropriate to mimic experimental systems where the graft and matrix monomers have similar chemistry, and particle-monomer interactions are negligible.

\subsection{RESULTS}

\subsubsection{Effect of particle-particle attraction on polydispersity- and bidispersity- stabilized dispersion:}
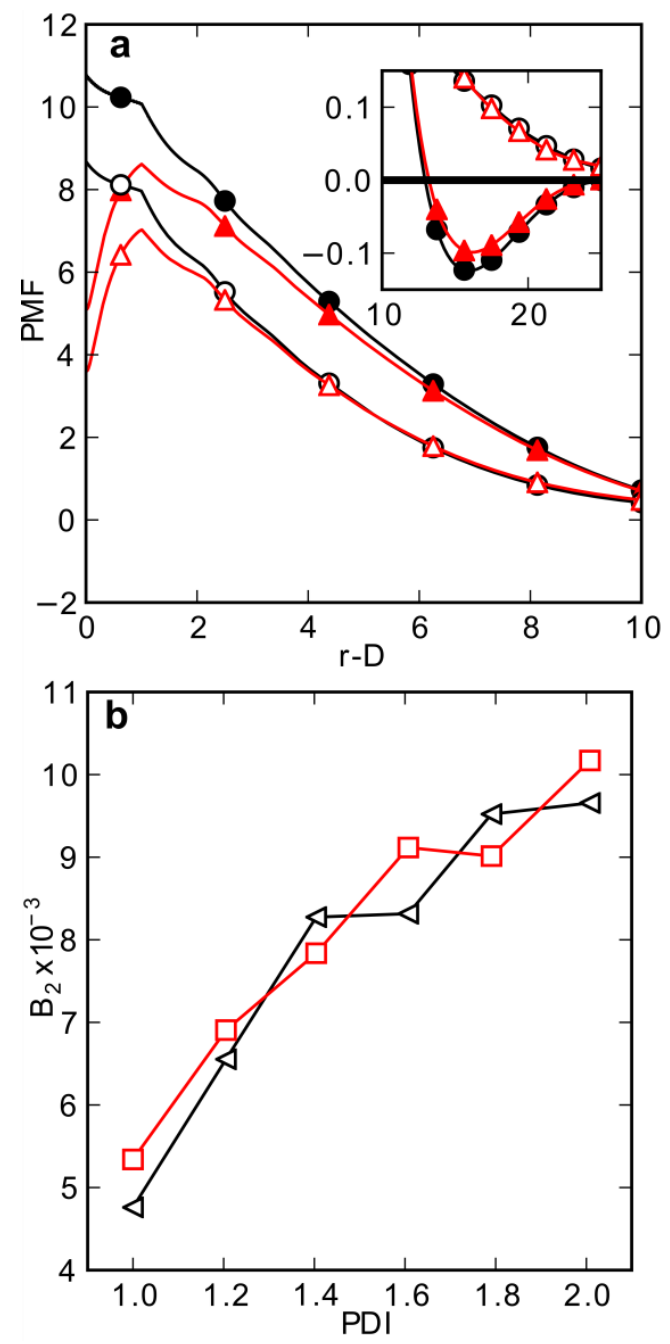

Figure 5.1: (a) Potentials of mean force (PMF), in units of $k_{B} T$, versus interparticle distance, in units of $d$, and $\left.\boldsymbol{b}\right)$ second virial coefficient, $B_{2}$, versus graft length PDI between nanoparticles of diameter D=5d grafted with homopolymers with monodisperse or polydisperse log-normal length distribution $\left(N_{\text {graft,avg }}=20\right)$ at $\sigma=0.65$ chains $/ d^{2}$ in a homopolymer matrix $\left(N_{\text {matrix }}=40\right)$. In $\underline{\text { subplot a, }}$, the symbols indicate the graft PDI=1.0 (solid) or 2.0 (open) and the particle-particle interactions $\varepsilon_{P P}=0$ (black circles) or $E P P=5.0 k_{B} T$, (red triangles). The main figure shows the PMF at short interparticle distances, and inset shows the PMF at larger interparticle distances, and presencelabsence of the mid-range attractive well. In subplot b, symbols indicate $\varepsilon_{P P}=0($ black leftward triangles) and $\varepsilon P P=5.0 \mathrm{kB}$ (red squares). 
Figure 5.1 shows how increasing the strength of particle-particle attraction impacts the dispersion stabilizing effect of a polydisperse log-normal graft length distribution. When the nanoparticles grafts have a polydisperse graft length distribution, we find that increasing particle-particle attraction leads to only minor changes in the PMF (Figure 5.1a) and chain conformations (Supporting Information Figure 5.7) as compared to the purely athermal interactions, and does not alter the overall observations that polydispersity in grafts stabilizes particle dispersion (Figure 5.1b). Specifically, increasing the particle-particle attraction strength introduces a close range contact attraction in the PMF between grafted nanoparticles, but this contact attraction is too short ranged to have a large effect on the overall tendency for grafted nanoparticle dispersion, as seen in $\mathrm{B}_{2}$ values (Figure 5.1b). Even at attraction strengths as high as $5 \mathrm{k}_{\mathrm{B}} \mathrm{T}$, the short range vdW particle-particle attraction is fully shielded by the densely grafted polymer chains. Overall, our findings indicate that, for densely grafted systems, particle-particle attraction has little effect on the ability of graft length polydispersity to stabilize dispersions of polymer grafted nanoparticles in a polymer matrix. .

Next, we compare how particle-particle attraction impacts the PMF between bidisperse polymer grafted nanoparticles. We have previously shown, for athermal particle-particle interaction when $\sigma_{\mathrm{s}} \approx \sigma_{1}$ (equal number of, and thus equal grafting densities, of short and long chains), the bidisperse grafted chain length distribution is not as good as the polydisperse lognormal distribution at removing the mid-range attractive well at the same polydispersity index (PDI) and average graft length $\left(\mathrm{N}_{\text {graft,avg }}\right)$.[20] At a particle-particle attraction strength of $\varepsilon_{\mathrm{PP}}=$ $5 \mathrm{k}_{\mathrm{B}} \mathrm{T}$, the PMF of the bidisperse distribution with $\sigma_{\mathrm{s}} \approx \sigma_{1}$ in Figure 5.2a (solid triangles) has a stronger attraction at contact and reduced close range repulsion as compared to log-normal 
distributions at same PDI 1.25 and $\mathrm{N}_{\text {graft,avg }}=20$ (solid circles). The bidisperse distribution with $\sigma_{\mathrm{s}} \approx \sigma_{1}$ is worse than the log-normal distribution at shielding particle-particle attraction because, at

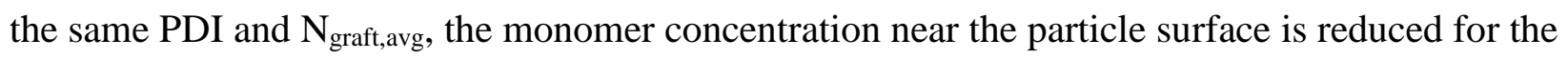
bidisperse $\sigma_{\mathrm{s}} \approx \sigma_{1}$ case (Figure 5.2b) which means nanoparticles can more easily approach one another to within the range of the attractive particle-particle potential. Additionally, when compared to the log-normal distribution at the same $\mathrm{N}_{\text {graft,avg }}$ and PDI, there are more long chains in the bidisperse distributions with $\sigma_{\mathrm{s}} \approx \sigma_{1}$ (see Table 5.1 to Table 5.3 of chain length distributions in the Supporting Information) that are not as easily wet by the matrix as evidenced from a deeper mid-range attraction for the bidisperse case with $\sigma_{\mathrm{s}} \approx \sigma_{1}$ (solid triangles in Figure 5.2a inset). 

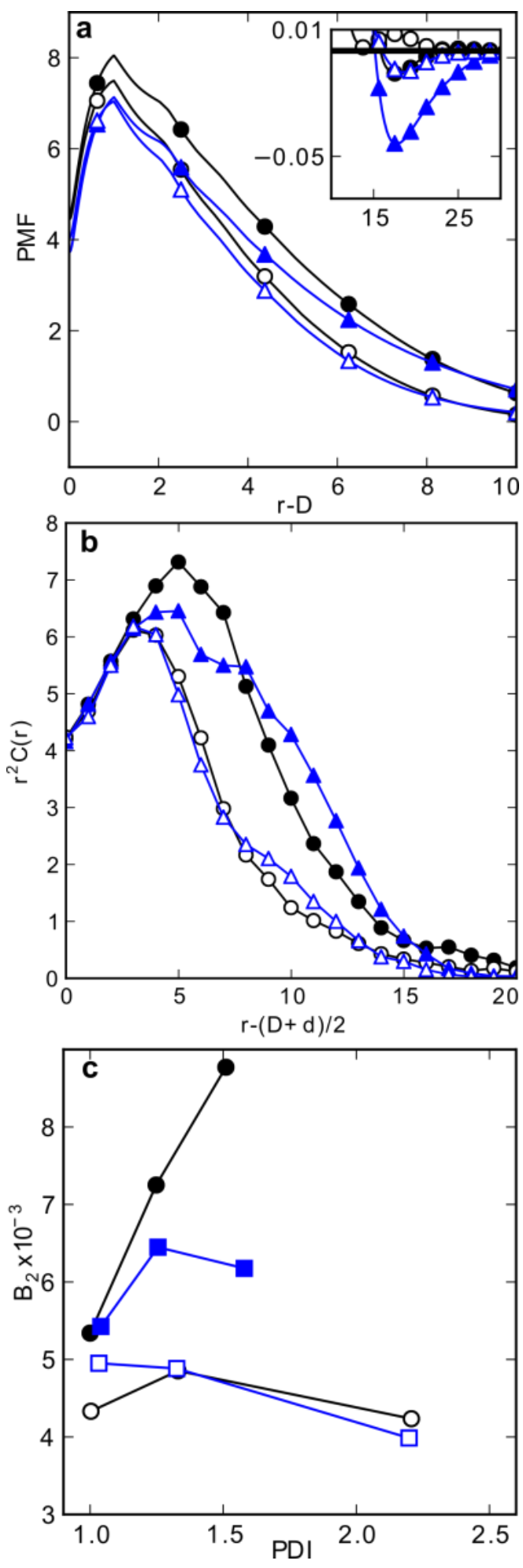

Figure 5.2: (a) PMF, in units of $k_{B} T$, versus interparticle distance, in units of $d$, (b) total monomer concentration profile, $r^{2} C(r)$ versus bead-particle distance and (c) second virial coefficient, $B_{2}$, versus PDI between nanoparticles of diameter $D=5 d$ with particle-particle interactions at $\varepsilon_{P P}=$ 
$5 k_{B} T$ in a homopolymer matrix of length $N_{\text {matrix }}=40$. The symbols in subplots $\boldsymbol{a}$ and $\boldsymbol{b}$ represent $\sigma=0.65$ chains $/ d^{2}$ (1) log-normal length distributions with $P D I=1.25, N_{\text {graft, avg }}=20$ (black solid circles) and (2) log-normal length distributions with $P D I=1.33, N_{\text {graft,avg }}=14$ (black open circles), (3) bidisperse length distribution with $\sigma_{s}=0.33, \sigma_{l}=0.31, \beta=3, P D I=1.25$, and $N_{\text {graft, avg }}=20$ (blue solid triangles) and (4) bidisperse length distribution with $\sigma_{s}=0.52, \sigma_{l}=0.12, \beta=3, P D I=1.33$ and $N_{\text {graft,avg }}=14$ (blue open triangles). The corresponding results at $\varepsilon_{P P}=0 k_{B} T$ are shown in the Supporting Information. In subplot c the open and closed squares represent the bidisperse distribution with $\sigma_{s}>\sigma_{l}$ and $\sigma \approx \sigma_{s}$ respectively while the open and closed circles represent log normal distributions, and both the open squares and circles have $N_{\text {graff,avg }}=18,14,11$ from left to right.

Next, we compare the bidisperse graft length distribution with $\sigma_{\mathrm{s}} \approx \sigma_{1}$ (solid triangles in Figure 5.2a) to one with $\sigma_{\mathrm{s}}>\sigma_{1}$ i.e. few long chains among many short chains (open triangles in Figure 5.2a). Our new consideration of the bidisperse system with $\sigma_{\mathrm{s}}>\sigma_{1}$ is partly driven by the fact that it is a closer analogue to the polydisperse log-normal distribution than bidispserse with $\sigma_{\mathrm{s}} \approx \sigma_{.1}$ We see that the effectiveness of the bidisperse distribution at removing the mid-range attractive well in the PMF improves in case of $\sigma_{\mathrm{s}}>\sigma_{1}$ (open triangle in Figure 5.2a) despite it having the lower average graft length $\left(\mathrm{N}_{\text {graft,avg }}=14\right.$ versus $\left.\mathrm{N}_{\text {graft,avg }}=20\right)$ and a lower $\mathrm{N}_{\text {graft,avg }} / \mathrm{N}_{\text {matrix. }}$ However, the lowered $\mathrm{N}_{\text {graft,avg }}$ leads to lowered steric repulsion from the grafted layer for the bidisperse $\sigma_{\mathrm{s}}>\sigma_{1}$ system, due to the reduced number of total monomers in the grafted layer. Comparing the bidisperse graft length distribution with $\sigma_{\mathrm{s}}>\sigma_{1}$ (open triangles in Figure 5.2a) to a polydisperse lognormal graft length distribution with the same average graft length and PDI $\left(\mathrm{N}_{\text {graft,avg }}=14\right.$, $\mathrm{PDI} \approx 1.33$ ), we see similar steric repulsion and mid-range attraction for both systems. This can be explained by the similar total monomer concentration profiles for the bidisperse graft length distribution with $\sigma_{\mathrm{s}}>\sigma_{1}$ and polydisperse log-normal graft length distribution with the same average graft length and PDI in Figure 5.2b. The effect of these trends in PMF on the overall $\mathrm{B}_{2}$ is discussed next. 
By comparing the values of $\mathrm{B}_{2}$ (Figure 5.2c) at the same PDI, the overall tendency for dispersion of the graft distributions is higher for the polydisperse log-normal length distribution with $\mathrm{N}_{\text {graft,avg }}=20$ (black solid circles) than a bidisperse length distribution with $\sigma_{\mathrm{s}}=0.33, \sigma_{\mathrm{l}}=0.31$ and $\mathrm{N}_{\text {graft,avg }}=20$ (blue solid triangles). Interestingly, the tendency to disperse grafted particles is similar for bidisperse length distribution with $\sigma_{\mathrm{s}}=0.52>\sigma_{\mathrm{l}}=0.12$ (blue open triangles) and polydisperse log-normal with same PDI and $\mathrm{N}_{\text {graft,avg. }}$ These results show that while the bidisperse graft length distribution (with $\sigma_{s} \approx \sigma_{1}$ ) is worse than the polydisperse log-normal distribution at stabilizing dispersions, increasing $\sigma_{s} / \sigma_{1}$ causes the bidisperse distribution to have similar dispersive tendencies to its polydisperse log-normal analogues. As expected, at similar PDI, the systems with higher $\mathrm{N}_{\text {graft,avg }}$ (solid symbols, $\mathrm{N}_{\text {graft,avg }}=20$ ) exhibits higher values of $\mathrm{B}_{2}$ than a polydisperse $\log$ normal distribution with lower average graft length (open symbols, $\mathrm{N}_{\text {graft,avg }}=18,14$, and 11). Overall, the choice of the number and length of short and long chains in the distribution impacts the ability to stabilize dispersion of the polymer grafted particles. This suggests that to find an optimal graft distribution that best disperses the polymer grafted particles we have to understand the individual role of the short and long chains on the effective interactions between the particles in the polymer matrix.

\subsubsection{Role of Short and Long Chains on the Potential of Mean Force}

To extract the role of the short and long chains in these distributions on the effective interactions between the grafted particles in a polymer matrix, we deconstruct the bidisperse system with $\sigma_{s} \approx \sigma_{1}$ (system 1 in Figure 5.3) into four monodisperse cases (systems 2-5 in Figure 5.3) so as to elucidate the individual effects of the short and long grafted chains on the different features of the PMF, at constant matrix chain length. 

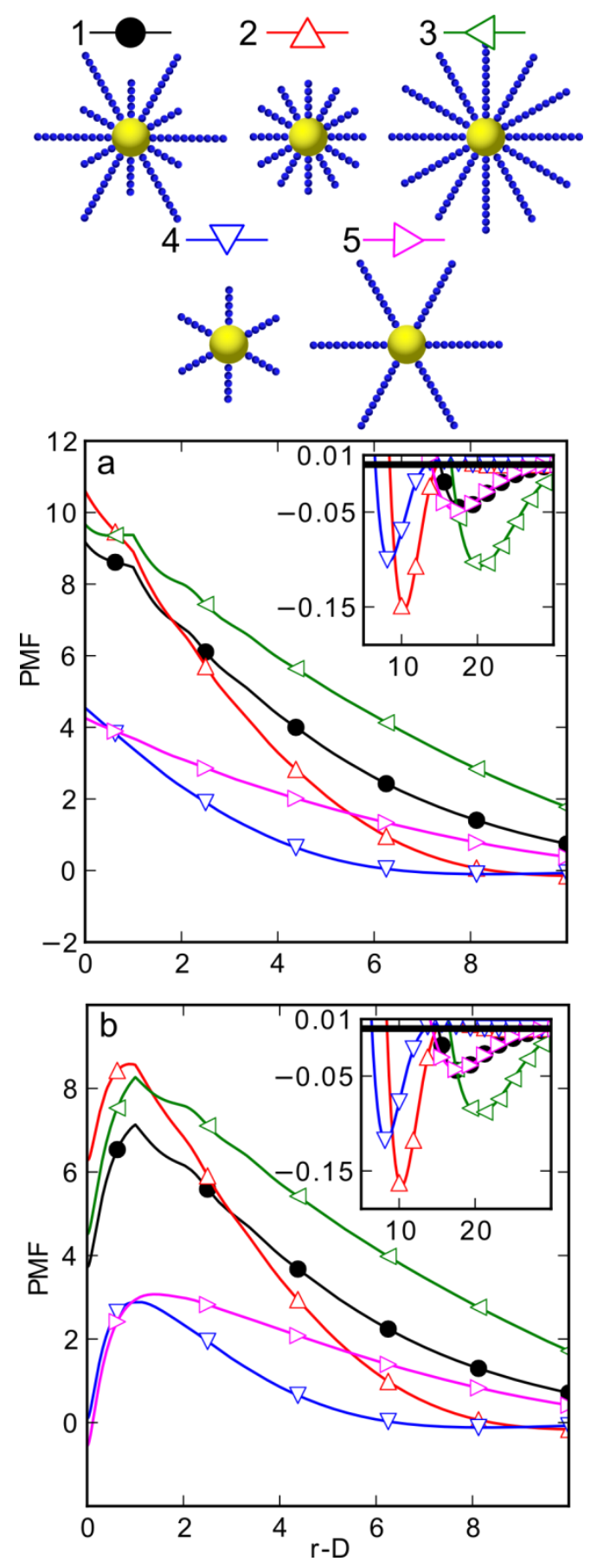

Figure 5.3: PMF, in units of $k_{B} T$, versus interparticle distance, in units of $d$, between nanoparticles of diameter $D=5 d$ with particle-particle interactions $\varepsilon_{P P}=0 \quad$ (a) and $\varepsilon_{P P}=5.0 k_{B} T$ (b) for five different grafting systems: (1) bidisperse, $N_{\text {graft,avg }}=20, \beta=3, \sigma_{l}=0.31, \sigma_{s}=0.33$ chains $/ d^{2}$ (2) monodisperse, $N_{\text {graft }}=10, \sigma=0.65$ chains $/ d^{2}$ (3) monodisperse, $N_{\text {graft }}=30, \sigma=0.65$ chains $/ d^{2}$ (4) monodisperse, $N_{\text {graft }}=10, \sigma=0.33$ chains $/ d^{2}(5)$ monodisperse, $N_{\text {graft }}=30, \sigma=0.31$ chains $/ d^{2}$, in a 
homopolymer matrix of length $N_{\text {matrix }}=40$. The main figure shows the PMF for short interparticle distances, while the inset shows the PMF at larger interparticle distances.

At $\varepsilon_{\mathrm{PP}}=0 \mathrm{k}_{\mathrm{B}} \mathrm{T}$ (Figure 5.3a) systems 1-3 have similar contact PMF due to equal total grafting densities, and similar (not equal) total monomer crowding near the particle surface as shown in Figure 4a. But since the average graft length increases from system 2 to 1 to 3 , a) the range of the steric repulsion increases to larger inter-particle distances, and b) the mid-range attractive well shifts to larger distances, as expected from past work[31]. The mid-range attractive well is the weakest for the bidisperse grafts (system 1) when compared to the monodisperse short (system 2) and long (system 3) grafts, all of which have $\mathrm{N}_{\text {graft,avg }}<\mathrm{N}_{\text {matrix }}$, showing what has already been shown, that bidispersity improves wetting of the grafted layer better than the monodisperse systems. Systems 4 and 5 show the behavior of the short and the long grafts in the absence of the other, and thus have approximately half the grafting density of systems 1-3. Due to reduced monomer crowding, the contact repulsion in the PMF is smaller and mid-range attraction is weaker for systems 4 and 5 as compared 2 and 3 (approximately equal for systems 4 and 3). At $\varepsilon_{\mathrm{PP}}=5 \mathrm{k}_{\mathrm{B}} \mathrm{T}$ (Figure 5.3b) we observe similar trends between these five systems; the only notable change with increasing particle-particle attraction is the decreasing value at contact PMF for all five systems. 


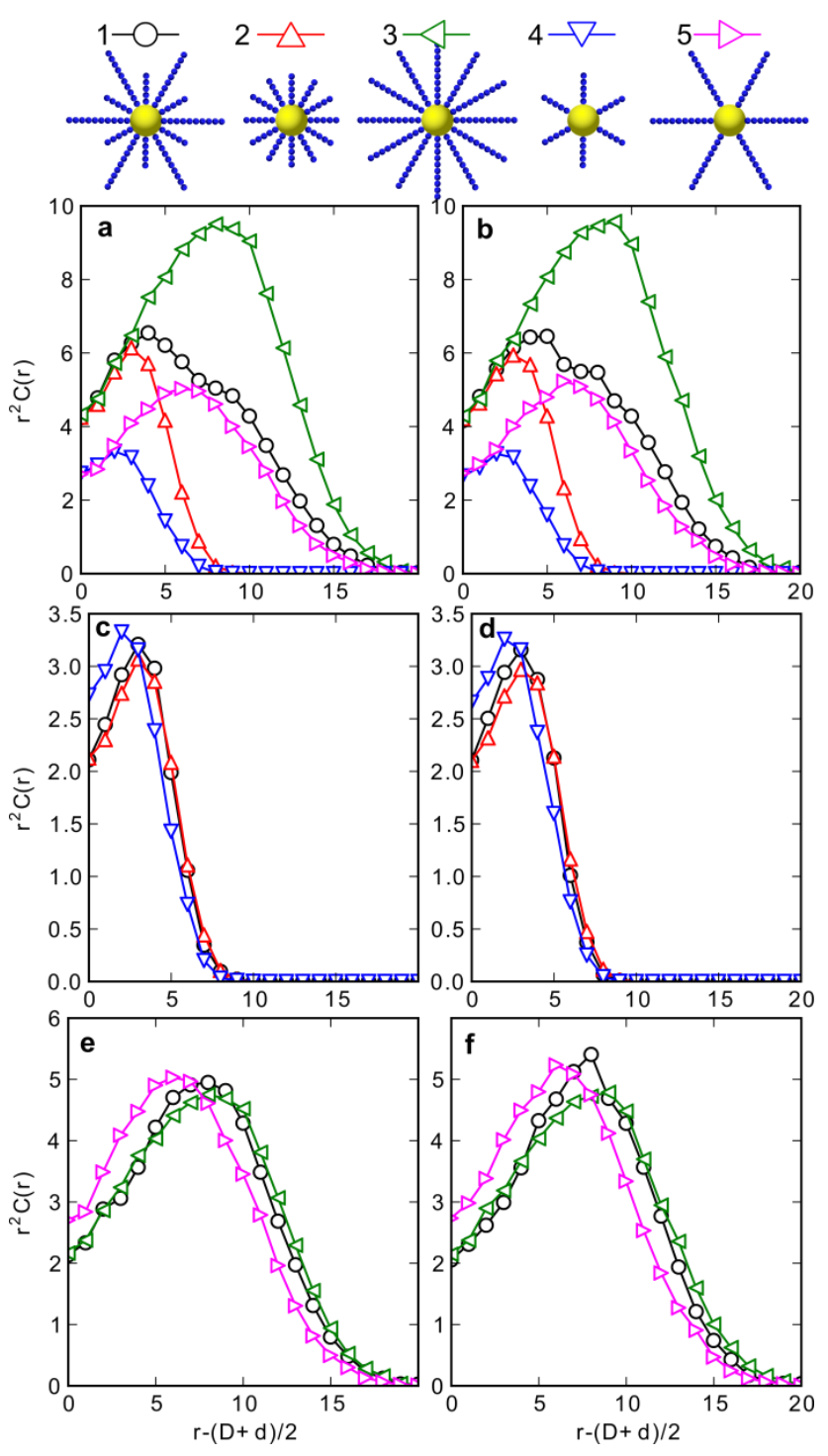

Figure 5.4: Monomer concentration profiles with the $r^{2}$ prefactor, in units of $d^{-1}$, versus beadparticle distance, in units of $d$, for nanoparticles of diameter $D=5 d$ with $\varepsilon_{P P}=0(a, c, e)$ and $\varepsilon_{P P}=5.0$ $k_{B} T(b, d, f)$ particle-particle interactions grafted with homopolymers of $N_{\text {graft,avg }}=20$, in a homopolymer matrix of length $N_{\text {matrix }}=40$. The total system volume fraction is $\eta=0.1$ and the grafted nanoparticle filler fraction is kept at the dilute limit of $\varphi=0.001$. Subplots $\boldsymbol{a}$ and $\boldsymbol{b}$ show the total concentration profiles for all five systems. Subplots $\boldsymbol{c}$ and $\boldsymbol{d}$ show the concentration profiles of only the short chains $\left(N_{\text {short }}=10\right)$ of system 1 (black circles), $1 / 2$ the concentration of all of the short chains of system 2 (red upward triangles), all of the (short) chains of system 4 (blue downward triangles). Subplots $\boldsymbol{e}$ and $\boldsymbol{f}$ show the concentration profiles for the long chains $\left(N_{\text {long }}=30\right)$ of system 1 (black circles), 1/2 the concentration of all of the long chains of system 3 (green leftward triangles), all of the (long) chains of system 5 (pink right triangles). 
Comparing systems 1 (bidisperse) and 2 (all short) which are at equal total grafting density, the bidisperse system leads to a lower contact PMF than monodisperse short, both at $\varepsilon_{\mathrm{PP}}=0 \mathrm{k}_{\mathrm{B}} \mathrm{T}$ and $5 \mathrm{k}_{\mathrm{B}} \mathrm{T}$. Additionally, as expected, the range of the steric repulsion from the grafted layer is shorter and mid-range attraction is stronger for system 2 than system 1, purely arising from average graft chain length being smaller for system 2 than 1 . The small differences in the short chain conformations of system 1 (bidisperse) and 2 (all short) in Figure 5.4c $\left(0 \mathrm{k}_{\mathrm{B}} \mathrm{T}\right)$ and Figure $5.4 \mathrm{~d}$ $\left(5 \mathrm{k}_{\mathrm{B}} \mathrm{T}\right)$, at equal grafting density, are significant enough to impact the screening of particle-particle attraction by the short chains. It is important to note that one might expect the near surface monomer arrangement to be the same for system 1 and 2 as the grafting density is the same, and yet the short chain conformations are different, purely because of the presence of long chains in system 1 (that are absent in system 2). System 4, which has the same number of short chains as the bidisperse case (system 1), has a weaker contact repulsion than system 1, due to system 4 having half the total grafting density of system 1. System 3, consisting of all long chains at same total grafting density as 1 and 2, has a contact value of the PMF that is in-between that of systems 1 and 2, suggesting system 3 (with no short chains) can screen particle-particle attractions as well as systems 1 and 2. Thus, the screening of the particle-particle attraction and the resulting value of PMF close to contact is driven not by the short chains alone, as hypothesized by some experiments, but primarily by the number and arrangement of the monomers near the surface irrespective of the chain the monomers belong to. The number and arrangement of monomers is dictated by grafting density and choice of chain length distribution e.g. monodisperse, bidisperse or polydisperse.

The equal strength of the mid-range attractive well in the PMFs of systems 1 and 5 confirm that the longer chains in the bidisperse distribution dictate whether or not the grafted layer is wet/dewet by the matrix. The deeper mid-range attraction of system 3, with the same graft length 
as system 5 and same grafting density as system 1 shows that reducing the crowding among long chains (either by replacing some long chains with short chains or reducing overall grafting density) improves wetting of the long chains. The magnitude and range of the steric repulsion in the PMF arising from the grafted layer are dictated by the crowding among and the length of the long chains in the distribution.

By comparing the bidisperse distribution to its deconstructed monodisperse counterparts we have shown that one cannot completely decouple the effect of the short and long chains on the PMF of polymer grafted nanoparticles with polydisperse/bidisperse graft length distributions. Contrary to the hypothesis that it is primarily the short chains in the bidisperse environment that screen the particle-particle attraction, we find that it is the monomers near the surface, irrespective of whether they belong to the short or long chains, that provide this shielding effect. It is the length and crowding of the long chains that determine the location and strength of the steric repulsion and mid-range attraction. The inseparable nature of the contributions of the short and long chains to the bidisperse PMF is further highlighted by the fact that the bidisperse PMF cannot be predicted by the simple addition of the PMFs of systems 4 and 5. [Supporting Information Figure 5.10]. This non-additive nature of the PMF stems from the fact that the chain conformations of the short and long chains in presence of each other (system 1) are not the same as when they are apart (systems 4 and 5), as seen in the concentration profiles in Figure 5.4.

\subsubsection{Features of an Ideal Graft Length Distribution for Maximizing Particle Dispersion:}

This understanding of the effect of short and long chains in a polydisperse/bidisperse graft distribution is useful in identifying the 'ideal' graft length distribution for dispersing polymer grafted nanoparticles in a chemically similar polymer matrix. Our results suggest that the ideal graft length distribution is one that provides a) maximum monomer crowding near the surface of 
the nanoparticle, in order to shield short-range particle-particle attraction and sterically repelling other approaching grafted nanoparticles, and b) minimal crowding near the edge of the grafted layer in order to maximize wetting of the grafted layer by the matrix chains. Using this criteria, it is not surprising that the polydisperse log-normal graft length distribution and bidisperse graft length distribution with few long chains among many short chains showed a higher tendency for dispersion (Figure 5.2c) over ones with the bidisperse graft length distribution with equal number of short and long chains $\left(\sigma_{\mathrm{s}} \approx \sigma_{1}\right)$.The polydisperse log-normal distribution at high grafting density has a range of chain lengths which contribute monomers near the particle surface to increase closerange repulsion, and has few chains with length greater than $\mathrm{N}_{\text {graft,avg }}$ (see Supporting Information Table 5.1 to Table 5.3) that cause reduced long-chain crowding and increased wetting of the grafted layer by the matrix. As its definition suggests, the bidisperse graft length distribution with few long chains and many short chains also displays these optimal features of the polymer graft distribution that will provide good particle dispersion.

\subsection{CONCLUSION}

We have conducted self-consistent PRISM-MC studies of polymer grafted nanoparticles with three types of graft length distribution at high grafting density-i) polydisperse (log-normal) graft length distribution, and bidisperse graft length distributions with ii) equal number of short and long chains $\left(\sigma_{s} \approx \sigma_{1}\right)$, and iii) mostly short chains with a few long chains $\left(\sigma_{\mathrm{s}}>\sigma_{1}\right)$ - placed in a monodisperse polymer matrix that is chemically identical to the grafted polymer, at varying particle-particle attractions. By comparing the potential of mean force, monomer concentration profiles, and second virial coefficients between systems of equal average graft length and polydispersity index, at varying particle-particle attraction strengths, we find that particles with polydisperse log-normal graft length distribution showed a higher tendency for dispersion over particles with a bidisperse 
graft length distribution with $\sigma_{\mathrm{s}} \approx \sigma_{\mathrm{l}}$, and a similar improved dispersion as ones with the bidisperse distribution with $\sigma_{\mathrm{s}}>\sigma_{1}$ (Figure 5.2c). By deconstructing the bidisperse distribution into its component short and long chains we elucidate the role of the short and long chains on the various features of the potential of mean force. We find that the increased crowding of monomers from both short and long chains near the particle surface shields the particle-particle attraction, and maximizes steric repulsion in the potential of mean force between two polymer grafted particles in a chemically identical polymer matrix. The length and crowding of long chains in the grafted chain length distribution determines the location and strength of both the mid-range steric repulsion and attraction.

We conclude that to maximize grafted nanoparticle dispersion, it is best to synthesize polymer grafted particles with polymer graft length distributions that maximize monomer crowding near the particle surface to shield particle-particle attraction (e.g. high grafting density), and minimize crowding at farther distances from the particle to increase wetting of the grafted layer by matrix chains (e.g. few long chains amidst many short chains). Polydisperse (log-normal) graft length distributions and bidisperse graft length distributions with few long chains among many short chains satisfy this criterion, and better disperse grafted particles in a chemically identical matrix than monodisperse or bidisperse graft length distributions with equal number of short and long chains, with same average graft length.

In summary, this paper provides a valuable fundamental understanding on the effect of different features of graft length distributions on both shielding particle-particle (enthalpic) interactions and reducing (entropic) depletion-like attraction between polymer grafted nanoparticles in a polymer matrix. This knowledge can be leveraged by material scientists and 
synthetic chemists to tailor grafted nanoparticles to have a stable dispersed phase in chemically identical polymer matrices.

\subsection{ACKNOWLEDGEMENTS}

The authors acknowledge partial financial support by Department of Energy Early Career Award Program under Grant No. DE-SC0003912, National Science Foundation GRFP Award Number DGE 1144083, and Teets Family Endowed Fellowship to T. Martin.

\subsection{REFERENCES}

1. Winey, K.I. and R.A. Vaia, Polymer nanocomposites. Mrs Bulletin, 2007. 32(4): p. 314319.

2. Krishnamoorti, R. and R.A. Vaia, Polymer nanocomposites. Journal of Polymer Science Part B-Polymer Physics, 2007. 45(24): p. 3252-3256.

3. Vaia, R.A. and J.F. Maguire, Polymer nanocomposites with prescribed morphology: Going beyond nanoparticle-filled polymers. Chemistry of Materials, 2007. 19(11): p. 2736-2751.

4. Goel, V., et al., Structure of Polymer Tethered Highly Grafted Nanoparticles. Macromolecules, 2011. 44(20): p. 8129-8135.

5. Krishnamoorti, R., Strategies for Dispersing Nanoparticles in Polymers. MRS Bulletin, 2007. 32(4): p. 341-347.

6. Leibler, L., Theory of Microphase Separation in Block Copolymers. Macromolecules, 1980. 13(6): p. 1602-1617.

7. Maillard, D., et al., Polymer-Grafted-Nanoparticle Surfactants. Nano Letters, 2011. 11(11): p. 4569-4573.

8. Green, P.F., The structure of chain end-grafted nanoparticle/homopolymer nanocomposites. Soft Matter, 2011. 7(18): p. 7914-7926.

9. Hong, B.B. and A.Z. Panagiotopoulos, Molecular Dynamics Simulations of Silica Nanoparticles Grafted with Poly(ethylene oxide) Oligomer Chains. Journal of Physical Chemistry B, 2012. 116(8): p. 2385-2395.

10. Goyal, S. and F.A. Escobedo, Structure and transport properties of polymer grafted nanoparticles. Journal of Chemical Physics, 2011. 135(18).

11. Sunday, D., J. Ilavsky, and D.L. Green, A Phase Diagram for Polymer-Grafted Nanoparticles in Homopolymer Matrices. Macromolecules, 2012. 45(9): p. 4007-4011.

12. Jiao, Y. and P. Akcora, Assembly of Polymer-Grafted Magnetic Nanoparticles in Polymer Melts. Macromolecules, 2012. 45(8): p. 3463-3470. 
13. Ndoro, T.V.M., et al., Interface of Grafted and Ungrafted Silica Nanoparticles with a Polystyrene Matrix: Atomistic Molecular Dynamics Simulations. Macromolecules, 2011. 44(7): p. 2316-2327.

14. Chevigny, C., et al., Polymer-Grafted-Nanoparticles Nanocomposites: Dispersion, Grafted Chain Conformation, and Rheological Behavior. Macromolecules, 2011. 44(1): p. 122-133.

15. Kalb, J., et al., End grafted polymer nanoparticles in a polymeric matrix: Effect of coverage and curvature. Soft Matter. 7(4): p. 1418-1425.

16. Min, K., et al., One-Pot Synthesis of Hairy Nanoparticles by Emulsion ATRP. Macromolecules, 2009. 42(5): p. 1597-1603.

17. Matsen, M.W., Scaling behavior of a brush-homopolymer interface in the limit of high grafting density. Journal of Chemical Physics, 2005. 122(14).

18. Ferreira, P.G., A. Ajdari, and L. Leibler, Scaling law for entropic effects at interfaces between grafted layers and polymer melts. Macromolecules, 1998. 31(12): p. 3994-4003.

19. Trombly, D.M. and V. Ganesan, Curvature effects upon interactions of polymer-grafted nanoparticles in chemically identical polymer matrices. Journal of Chemical Physics, 2010. 133(15).

20. Sen, S., et al., Chain conformations and bound-layer correlations in polymer nanocomposites. Physical Review Letters, 2007. 98(12).

21. Cui, S.T., P.T. Cummings, and H.D. Cochran, The calculation of the viscosity from the autocorrelation function using molecular and atomic stress tensors. Molecular Physics, 1996. 88(6): p. 1657-1664.

22. Nair, N., N. Wentzel, and A. Jayaraman, Effect of bidispersity in grafted chain length on grafted chain conformations and potential of mean force between polymer grafted nanoparticles in a homopolymer matrix. Journal of Chemical Physics, 2011. 134(19).

23. Chremos, A., et al., Structure of solvent-free grafted nanoparticles: Molecular dynamics and density-functional theory. Journal of Chemical Physics, 2011. 135(11).

24. Tuteja, A., P.M. Duxbury, and M.E. Mackay, Polymer chain swelling induced by dispersed nanoparticles. Physical Review Letters, 2008. 100(7).

25. Bates, F.S., et al., Multiblock Polymers: Panacea or Pandora's Box? Science, 2012. 336(6080): p. 434-440.

26. Padmanabhan, V., Effect of grafting on nanoparticle segregation in polymer/nanoparticle blends near a substrate. Journal of Chemical Physics, 2012. 137(9).

27. Agarwal, U. and F.A. Escobedo, Mesophase behaviour of polyhedral particles. Nature Materials, 2011. 10(3): p. 230-235.

28. Jayaraman, A. and K.S. Schweizer, Liquid state theory of the structure and phase behaviour of polymer-tethered nanoparticles in dense suspensions, melts and nanocomposites. Molecular Simulation, 2009. 35(10-11): p. 835-848. 
29. Nair, N. and A. Jayaraman, Self-Consistent PRISM Theory-Monte Carlo Simulation Studies of Copolymer Grafted Nanoparticles in a Homopolymer Matrix. Macromolecules, 2010. 43(19): p. 8251-8263.

30. Schweizer, K.S. and A. Yethiraj, POLYMER REFERENCE INTERACTION SITE MODEL-THEORY - NEW MOLECULAR CLOSURES FOR PHASE-SEPARATING FLUIDS AND ALLOYS. Journal of Chemical Physics, 1993. 98(11): p. 9053-9079.

31. Smith, G.D. and D. Bedrov, Dispersing Nanoparticles in a Polymer Matrix: Are Long, Dense Polymer Tethers Really Necessary? Langmuir, 2009. 25(19): p. 11239-11243. 


\subsection{SUPPLEMENTARY}

\subsubsection{Colloid Lennard Jones Potential}

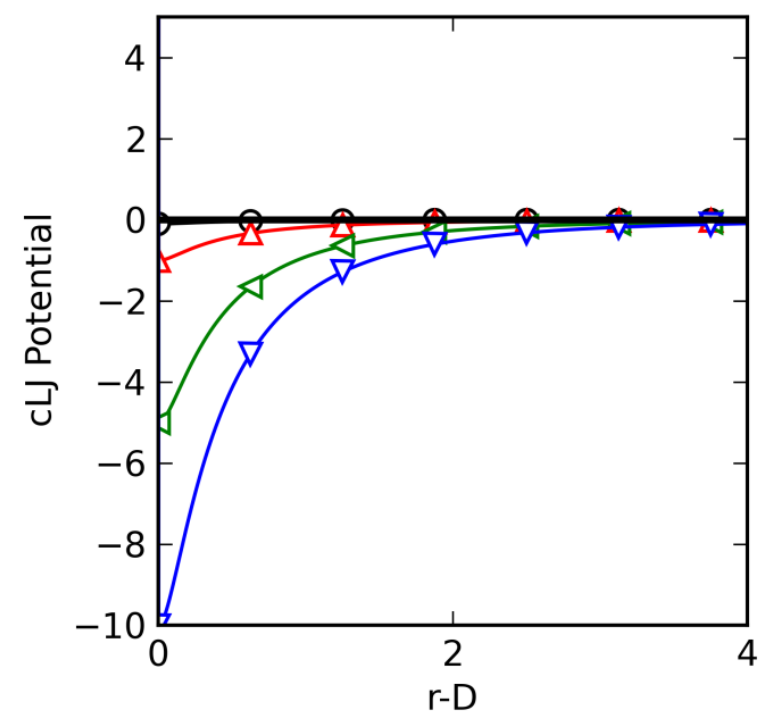

Figure 5.5: Colloid Lennard Jones potential, in units of $k_{B} T$, versus interparticle distance, in units of $d$, between particles of diameter $D=5 d$ with well depths $\varepsilon_{P P}=0.1$ (black circles), 1.0 (red upward triangles), 5.0 (green leftward triangles), 10.0 (blue downward triangles). The potential goes to infinity for $r-D<0$. See reference below for details on the Colloid Lennard Jones potential:

Henderson, D.; Duh, D. M.; Chu, X. L.; Wasan, D., An expression for the dispersion force between colloidal particles. J. Colloid Interface Sci. 1997, 185 (1), 265-268. 
5.7.2 Schematic of terminology used to describe the potential of mean force

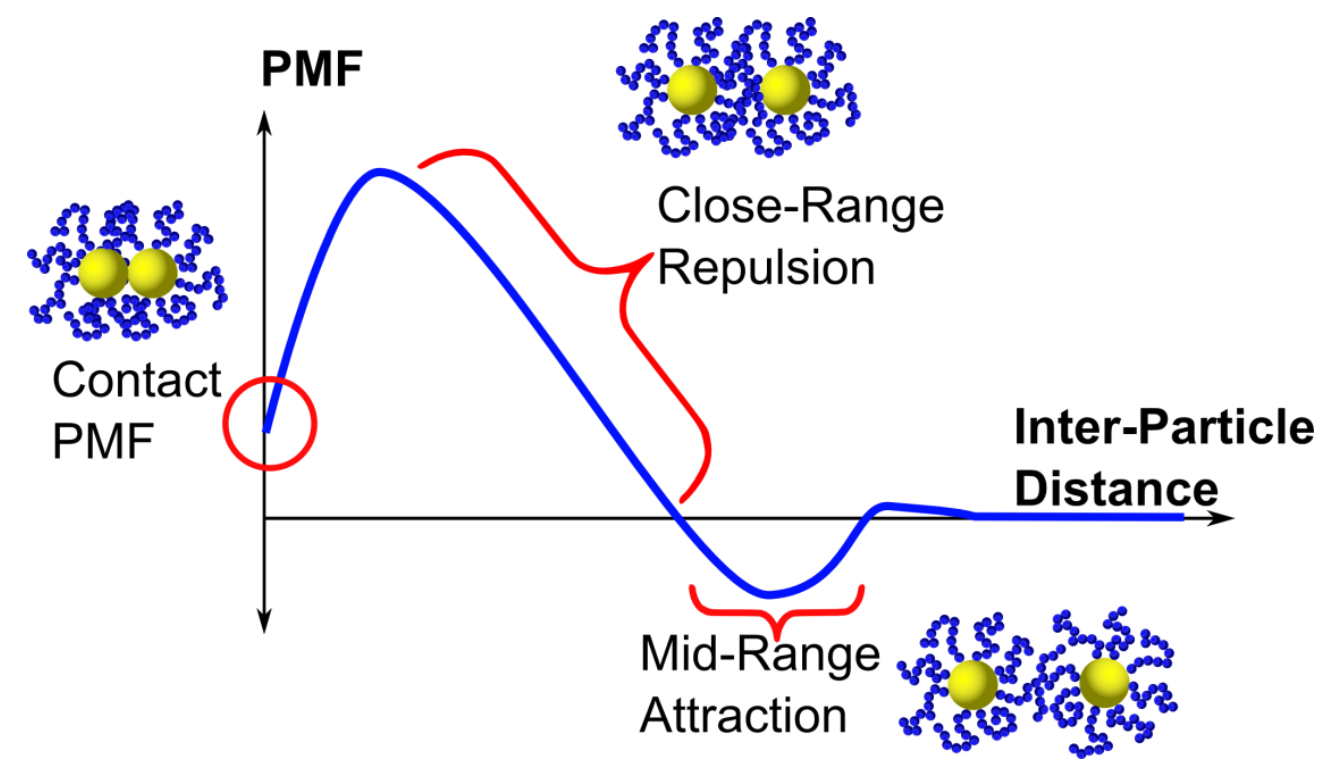

Figure 5.6: Schematic of terminology used to describe various features of the potential of mean force $(P M F)$ between homopolymer grafted nanoparticles with arbitrary particle-particle attraction in a chemically similar homopolymer matrix (not shown in diagrams). 


\subsubsection{Effect of Particle-Particle attraction on the Potential of Mean Force and Grafted Chain Conformations}
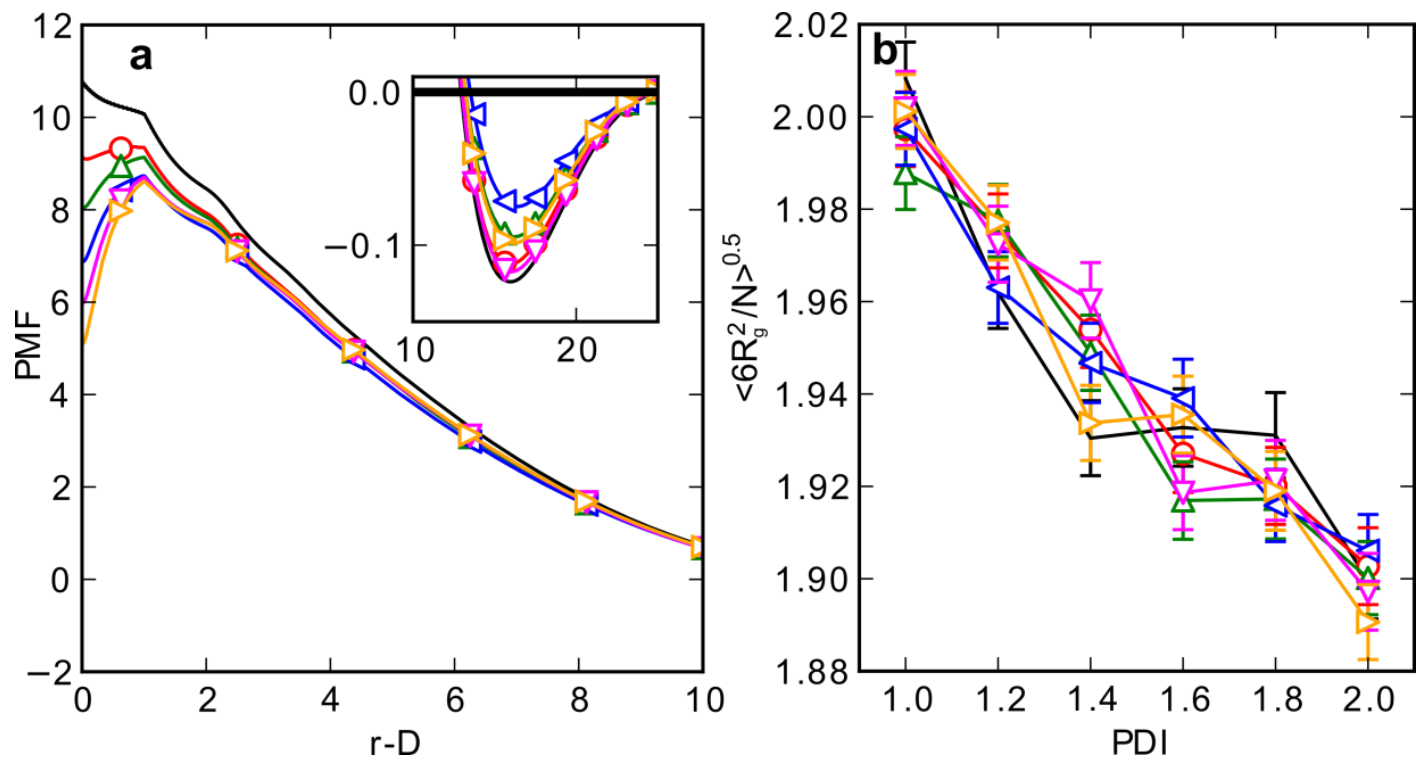

Figure 5.7: (a) Potentials of mean force (PMF), in units of $k_{B} T$, versus interparticle distance, in units of $d$, and (b) root mean normalized square radius of gyration of grafted chains, $\left\langle 6 R g^{2} / N\right\rangle^{0.5}$, in units of $d$, versus graft length PDI for nanoparticles of diameter $D=5 d$ grafted with homopolymers with log-normally distributed lengths at average length $N_{\text {graft,avg }}=20$ at a grafting density of $\sigma=0.65$ chains $/ d^{2}$ in a homopolymer matrix of length $N_{\text {matrix }}=40$. The total system volume fraction is $\eta=0.1$ and the grafted nanoparticle filler fraction is kept at the dilute limit of $\varphi=0.001$. The error bars in subplot $b$ represent standards error. The colors and symbols indicate the strength of particle-particle attraction as athermal $\varepsilon_{P P}=0 k_{B} T$ (black line, no symbols), $\varepsilon_{P P}$ $=1 k_{B} T$ (red circles), $\varepsilon_{P P}=2 k_{B} T$ (green upward triangles), $\varepsilon_{P P}=3 k_{B} T$ (blue leftward triangles), $\varepsilon_{P P}$ $=4 k_{B} T$ (pink downward triangles) and $\varepsilon_{P P}=5 k_{B} T$ (orange rightward triangles). 


\subsubsection{PMF and $B_{2}$ corresponding to Fig. 1 with $\varepsilon_{P P}=0 k_{B} T$}
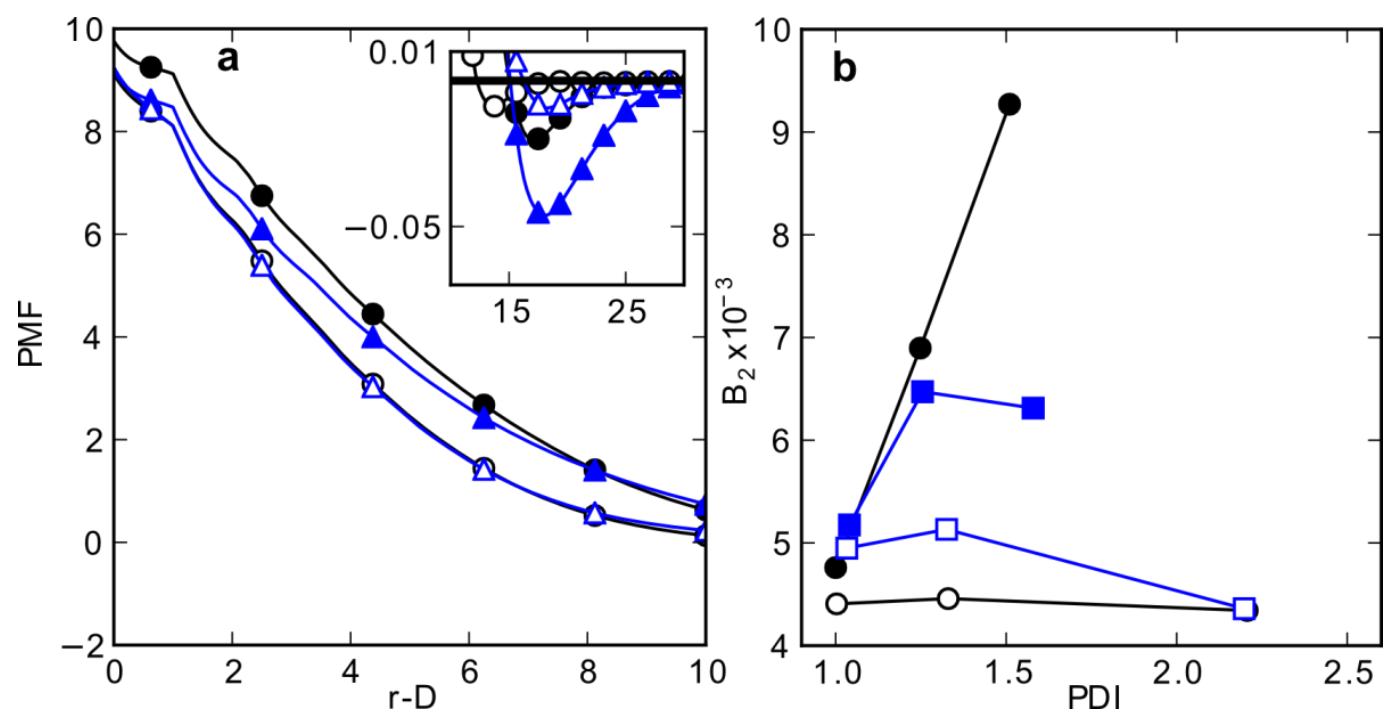

Figure 5.8: (a) PMF, in units of $k_{B} T$, versus interparticle distance, in units of $d$, and (b) second virial coefficient, $B_{2}$, versus PDI between nanoparticles of diameter $D=5 d$ with particle-particle interactions at $\varepsilon_{P P}=0 k_{B} T$ in a homopolymer matrix of length $N_{\text {matrix }}=40$. The symbols in subplot a represent at $\sigma=0.65$ chains/d ${ }^{2}$ (1) log-normal length distributions with $P D I=1.25, N_{\text {graft,avg }}=20$ (black solid circles) and (2) log-normal length distributions with PDI=1.33, $N_{\text {graft,avg }}=14$ (black open circles), (3) bidisperse length distribution with $\sigma_{s}=0.33, \sigma_{l}=0.31, \beta=3, P D I=1.25$, and $N_{\text {graft, }, \text { vvg }}=20$ (blue solid triangles) and (4) bidisperse length distribution with $\sigma_{s}=0.52, \sigma_{l}=0.12$, $\beta=3, P D I=1.33$ and $N_{\text {graft,avg }}=14$ (blue closed triangles). The corresponding results at $\varepsilon_{P P}=0 k_{B} T$ are shown in the Supplementary Information. In subplot b the open and closed squares represent the bidisperse distribution with $\sigma_{s}>\sigma_{l}$ and $\sigma \approx \sigma_{s}$ respectively while the open and closed circles represents log normal distributions, and both the open squares and circles have $N_{\text {graft,avg }}=18,14$, 11 from left to right. 


\subsubsection{Log-Normal versus Bidisperse Monomer Concentration Profiles}
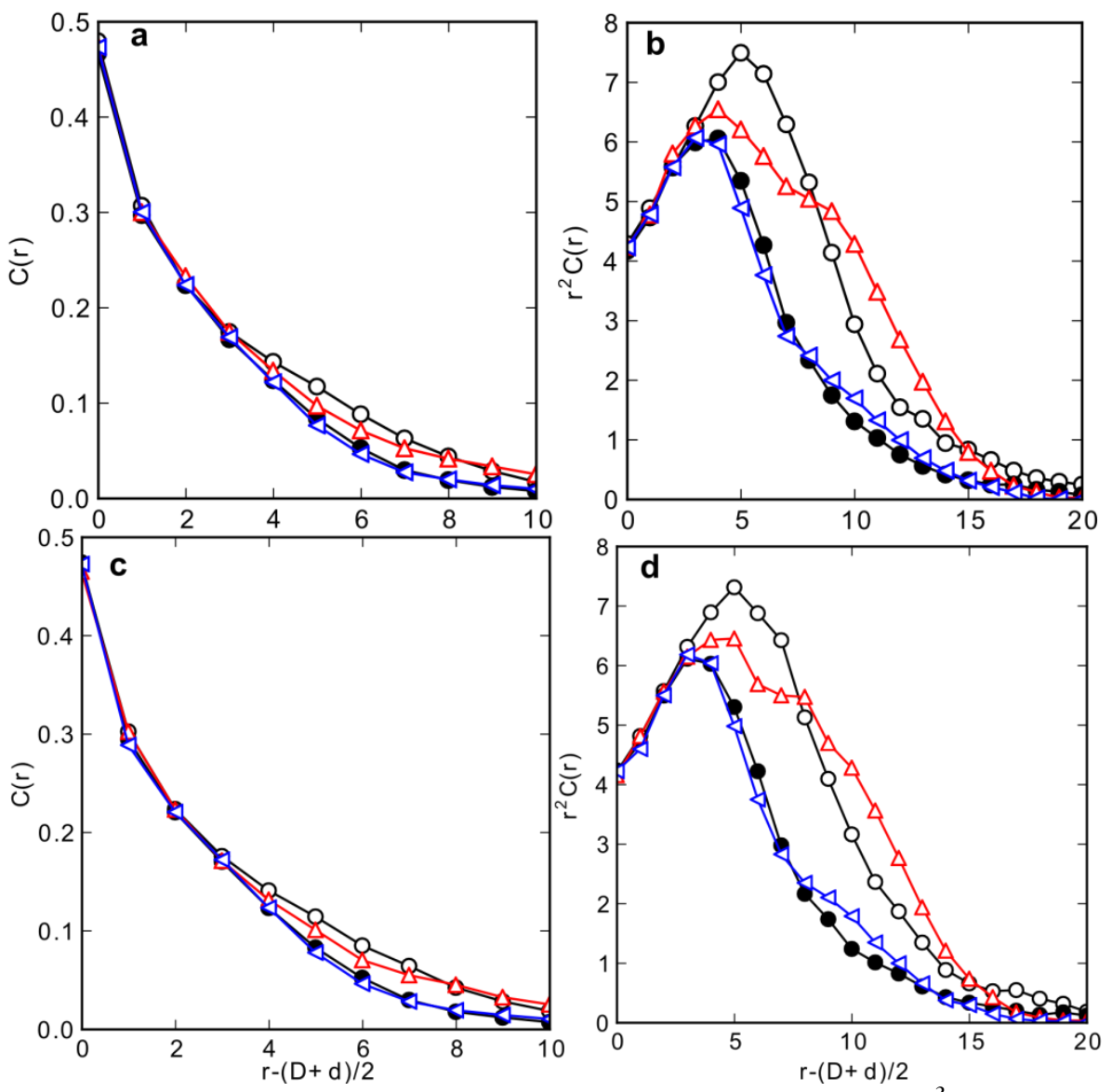

Figure 5.9:Monomer concentration profiles both without ( $a, c$ units of $\left.d^{-3}\right)$ and with $(b, d$ units of $\left.d^{-1}\right)$ the $r^{2}$ prefactor, versus bead-particle distance, in units of $d$, for nanoparticles of diameter $D=5 d$ with $\varepsilon_{P P}=0(a, b)$ and $\varepsilon_{P P}=5.0 k_{B} T,(c, d)$ particle-particle interactions grafted with homopolymers $N_{\text {graft,avg }}=20$ at a total grafting density of $\sigma=0.65$ chains $/ d^{2}$, in a homopolymer matrix of length $N_{\text {matrix }}=40$ for four graft length distributions:

(black open circles) polydisperse, $N_{\text {graft,avg }}=20, P D I=1.25$;

(black closed circles) polydisperse, $N_{\text {graft,avg }}=14, P D I=1.33$;

(red upward triangles) bidisperse, $N_{\text {graft,avg }}=20, \beta=3$ or $P D I=1.25, \sigma_{s}=0.33, \sigma_{l}=0.31$ chains $/ d^{2}$; (blue leftward triangles) bidisperse, $N_{\text {graff,avg }}=14, \beta=3$ or $P D I=1.33, \sigma_{s}=0.52, \sigma_{l}=0.12$ chains $/ d^{2}$. The total system volume fraction is $\eta=0.1$ and the grafted nanoparticle filler fraction is kept at the dilute limit of $\varphi=0.001$. 


\subsubsection{Comparison of Bidisperse PMF to Direct Addition of PMFs for Systems 4 and 5}

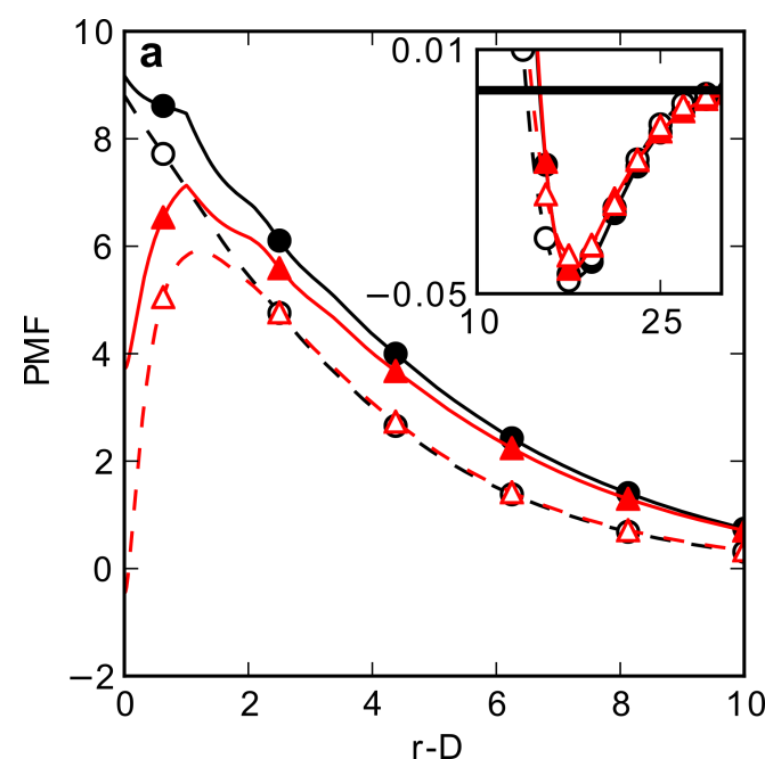

Figure 5.10: Potentials of mean force $(P M F)$, in units of $k_{B} T$, versus interparticle distance, in units of $d$, between nanoparticles of diameter $D=5 d$ with $\varepsilon_{P P}=0$ (black circles) and $\varepsilon_{P P}=5.0 k_{B} T$, (red triangles) particle-particle interactions grafted with homopolymers with a log-normal length distribution at average length $N_{\text {graft, avg }}=20$ at a total grafting density of $\sigma=0.65$ chains $/ d^{2}$ in a homopolymer matrix of length $N_{\text {matrix }}=40$. The total system volume fraction is $\eta=0.1$ and the grafted nanoparticle filler fraction is kept at the dilute limit of $\varphi=0.001$. The closed symbols-solid lines represent a bidisperse system with $\beta=3$ and $N_{\text {graft,avg }}=20$ (system 1). The open symbols-dashed lines are the sum of the corresponding PMFs from systems 4 and 5. 


\subsubsection{Tables of Number of Long Chains for Bidisperse and Log-Normal Distributions}

Table 5.1: Number of Chains $w / N>N_{\text {graft,avg }}$ for Bidisperse Distributions at $\sigma=0.65$ chains $/ d^{2}$

\begin{tabular}{|c|c|c|}
\hline & \multicolumn{2}{|c|}{ Number of Chains $w / N>N_{\text {graft,avg }}$} \\
\hline Beta & Bidisperse $w / \sigma_{\mathrm{s}} \approx \sigma_{\mathrm{I}}$ & Bidisperse $\mathrm{w} / \sigma_{\mathrm{s}} \neq \sigma_{\mathrm{I}}$ \\
\hline 1.5 & 25 & 10 \\
\hline 3 & 25 & 10 \\
\hline 7 & 25 & 10 \\
\hline
\end{tabular}

Table 5.2: Number of Chains $w / \quad N>N_{\text {graft,avg }}$ for Log Normal Distributions at $\sigma=0.65$ chains $/ d^{2}$ and $N_{\text {graft,avg }}=20$

\begin{tabular}{|c|c|}
\hline PDI & Number of Chains $\mathrm{w} / \mathrm{N}>\mathrm{N}_{\text {graft,avg }}$ \\
\hline 1.2 & 18 \\
\hline 1.3 & 16 \\
\hline 1.4 & 14 \\
\hline 1.5 & 15 \\
\hline 1.6 & 17 \\
\hline 1.7 & 17 \\
\hline 1.8 & 14 \\
\hline 1.9 & 12 \\
\hline 2.0 & 11 \\
\hline
\end{tabular}

Table 5.3: Number of Chains w/ $N>N_{\text {graft,avg }}$ for Log Normal Distributions at $\sigma=0.65$ chains $/ d^{2}$ and $N_{\text {graft,avg }}=14$

\begin{tabular}{|c|c|}
\hline PDI & Number of Chains $w / N>N_{\text {graft,avg }}$ \\
\hline 1.03 & 32 \\
\hline 1.33 & 14 \\
\hline 2.2 & 11 \\
\hline
\end{tabular}




\section{Effect of Matrix Bidispersity on the Morphology of Polymer

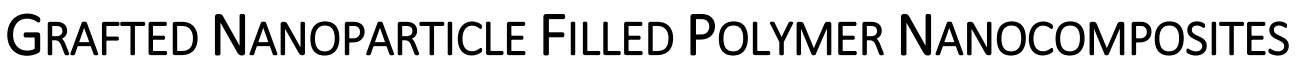

Adapted from: Journal of Polymer Science Part B: Polymer Physics 52 (24), 1661-1668

\subsection{INTRODUCTION}

In order to engineer polymer nanocomposites with desirable macroscopic properties, the ability to control the microscopic arrangement, or morphology, of the matrix and fillers (additives) is important.[1, 2] Polymer nanocomposite morphologies fall into two broad classes: aggregated morphologies where the fillers assemble into a variety of isotropic and anisotropic structures[3, 4], and dispersed morphologies where the average filler-filler distance is maximized.[2, 5-8] By grafting ligands to the surface of the fillers (e.g. nanoparticles), the filler-filler and filler-matrix interactions, and thereby the morphology of the polymer nanocomposites can be tuned. For a homopolymer matrix filled with homopolymer grafted nanoparticles, where the matrix and graft chemistry are identical and the grafting density is high, it has been shown that the ratio of the graft to matrix molecular weight $\left(\mathrm{N}_{\text {graft }} / \mathrm{N}_{\text {matrix }}\right)$ dictates whether grafted particles stay dispersed or aggregated.[6] Specifically, for spherical nanoparticles where the radius of the particle is similar to the radius of gyration of the grafted chains, one observes particle dispersion when $\mathrm{N}_{\text {graft }} / \mathrm{N}_{\text {matrix }}$

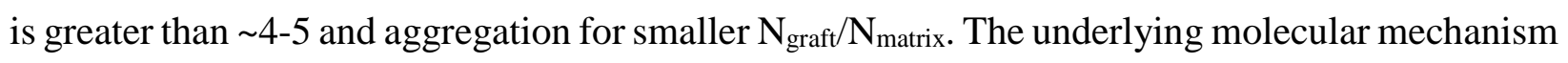
for particle dispersion and aggregation has been suggested to be a transition from a wet brush to dry brush.[6]

Exploiting this idea of wetting/dewetting driven dispersion/aggregation, we recently investigated how polydispersity in grafted chain lengths affects the wetting of grafted nanoparticles by a monodisperse polymer matrix. We predicted using theory that in the regimes where particle aggregation is expected, graft length polydispersity stabilized the dispersed phase of the grafted 
particles by promoting wetting of the densely grafted polymer layer by the matrix. Due to increased wetting of the grafted layer, the mid-range attraction in the particle-particle potential of mean force is eliminated, thereby removing the tendency for aggregation of the particles. $[9,10][11]$ We also found that the ideal graft length distribution for promoting particle dispersion is one that provides a) maximum monomer crowding near the surface of the nanoparticle, in order to shield short-range particle-particle attraction and sterically repel other approaching grafted nanoparticles, and b) minimal crowding near the edge of the grafted layer in order to maximize wetting of the grafted layer by the matrix chains.[12] In agreement with our theoretical predictions of graft polydispersity stabilized particle dispersion, experimental work by Schadler and coworkers shows that a bimodal grafted chain length distribution greatly improves the dispersion of grafted nanoparticles in a polymer matrix over that observed with a monodisperse graft length distribution.[13, 14]

While the above theoretical and experimental studies have focused on the effect of graft length polydispersity on the morphology of polymer nanocomposites, to the best of our knowledge, the effect of matrix length polydispersity on the morphology of homopolymer grafted particle filled polymer nanocomposites has not been investigated. One past study worth noting is that by Broseta et al. who studied the effect of polydispersity on morphology of strongly segregating homopolymer blends. They found that the polydispersity reduced the interfacial tension of the homopolymers, with the shorter polymer chains segregating to the interface.[15] Despite the significance of their results, since their study focused on entropically driven segregation of short chains to flat interfaces formed by two strongly (enthalpically) segregating polymers, one cannot extend their prediction to a blend of polymer grafted particles and polymer matrix where the graftmatrix polymer chemistries are the same (no enthalpically driven segregation), and the graft-matrix interfaces are curved. In this article we present a computational study of the effect of matrix length 
polydispersity on the wetting/dewetting phenomena and the morphology of homopolymer grafted nanoparticle filled polymer nanocomposites, which to the best of our knowledge has not been studied before.

\subsection{APPROACH}

We simulate the polymer nanocomposites (PNCs) in this study using Brownian dynamics (BD) ${ }^{[16]}$ with coarse grained models of grafted and matrix chains. The details of the computational approach - model, simulation and parameters varied- are as follows.

\subsubsection{Model}

We model the grafted and matrix chains as freely jointed chains of beads of diameter 1d, where $d$ is the size of a Kuhn segment in the polymers (graft or matrix). The polymer beads which represent Kuhn segments, are connected by harmonic bonds:

$$
U_{\text {bond }}(r)=k\left(r-r_{0}\right)^{2}
$$

where $\mathrm{k}$ is the bond strength, and $\mathrm{r}_{0}$ is the resting distance of the bond. We model the nanoparticles as rigid bodies[10] of overlapping spheres of diameter 1d. These spheres of the nanoparticle serve as both the surface of the particle and as grafting sites. We ensure that both the surface beads and the grafting sites are evenly distributed across the particle for desired arbitrary diameters (D) and grafting densities (chains/ $\mathrm{d}^{2}$ ).

We maintain athermal interactions between all entities to mimic PNCs with identical graft and matrix chemistry, and with effective shielding of particle-particle attraction at high grafting density. Our past work has shown that the steric repulsion due to the polymers at high grafting density effectively shields the particle-particle attraction[12]. To model these athermal interactions all pairs of non-bonded beads in the system interact via purely-repulsive WeekChandler-Andersen potentials[17]: 


$$
U_{W C A}(r)=\left\{\begin{array}{c}
4 \varepsilon\left[\left(\frac{\sigma}{r}\right)^{12}-\left(\frac{\sigma}{r}\right)^{6}\right]+\varepsilon, r \leq 2^{\frac{1}{6}} \sigma \\
0.0, r>2^{\frac{1}{6}} \sigma
\end{array}\right.
$$

where $r$ is the bead-bead distance, $\varepsilon$ is the steepness of the repulsive potential, and $\sigma$ is the contact distance for the two beads.

\subsubsection{Method}

We conduct Brownian Dynamics (BD) simulations using the HOOMD-blue platform[16]. We initialize our systems as follows: we first build a grafted nanoparticle, with grafted chains extending radially from the particle surface, in the absence of any matrix chains. A short simulation with strong Lennard-Jones monomer-monomer and monomer-particle attraction is then run purely to make the grafted chains take up a compact conformation. Note that this is the only time we use attractive non-bonded interactions in our simulation. Copies of this compact grafted nanoparticle, along with the desired number of short and long matrix chains, are then randomly placed in a large cubic box. The randomly placed molecules are integrated using a Brownian dynamics integrator for $0.5 \mathrm{e} 6$ time steps to both mix and relax the grafted and matrix chains. The box is then compressed to the desired volume fraction over $0.5 \mathrm{e} 6$ steps, and then mixed again for $0.5 \mathrm{e} 6$ steps at the compressed state. Finally, the production simulation runs for 100-200 million time steps where snapshots of the system are saved every $0.5 \mathrm{e} 6$ time steps. The analysis is conducted using these snapshots and ensemble average results obtained from five independent simulations are reported.

One of the simulation challenges in such densely packed systems is ensuring that the ensemble average results are indeed representing equilibrium states. In the Supplementary Material, we show the evolution of the average mean square internal distances of our matrix chains to demonstrate that the polymer chains relax on a timescale that is faster than our sampling period 
(Supplementary Figure 6.5).[18] Additionally, in Supplementary Figure 6.6 we show paircorrelation functions for five independent simulation trials with randomly initialized structures of the same composite, and demonstrate that we are are reaching statistically similar equilibrium results. We also use liquid state theory based calculations[7, 19, 20] (described next), which are devoid of these equilibration issues, and find qualitative agreement between the theoretical and simulation results, further confirming that we are indeed reporting equilibrium behavior in the simulations.

We use a variant of the self-consistent Polymer Reference Interaction Site Model theory and Monte Carlo simulation (PRISM-MC)[20, 21], in which we use Brownian Dynamics (BD) rather than Monte Carlo to calculate the intramolecular structure factor. PRISM theory consists of a matrix of Ornstein-Zernike-like integral equations that relate the total site-site inter-molecular pair correlation function, $h_{i j}(r)$, to the inter-molecular direct correlation function, $c_{i j}(r)$, and intramolecular pair correlation function, $\omega_{i j}(r)$. The PRISM equations in Fourier space are

$$
\begin{gathered}
H(q)=\Omega(q) C(q)[\Omega(q)+H(q)] \\
H_{i j}(q)=\rho_{i} \rho_{j} \hat{h}_{i j}(q) \\
\Omega_{i j}(q)=\rho \sum_{\alpha=1}^{N_{i}} \sum_{\beta=1}^{N_{j}} \Omega_{\alpha_{i} \beta_{j}}
\end{gathered}
$$

where $H(q), C(q)$ and $\Omega(q)$ in this study are matrices of size $4 \mathrm{x} 4$ for the following 4 types of sites: graft monomers (A), particle (B), short matrix monomers (C), and long matrix monomers (D), with the matrix elements defined in equations 4 and 5 . We note that despite the chemistry of the graft and matrix being the same, these sites are physically identified separately as graft or matrix. In the above equations, $N_{i}$ and $\rho_{i}$ are respectively the number and number density of site $i, \rho$ is the molecular number density, and $\Omega_{i j}(q)$ the intra-molecular pair correlation function between sites $i$ and $j$ within a certain molecule in Fourier space. To solve equation 3, we use closure relations 
connecting the real space $c_{i j}(r), h_{i j}(r)\left(=g_{i j}(r)-1\right)$ and interaction potentials $U_{i j}(r)$. Previous work on a mixture of nanoparticles and polymers[22-31] shows that the Percus-Yevick (PY) closure for polymer-polymer and polymer-particle, and the hypernetted chain (HNC) closure for particleparticle provides good agreement between theory and simulations. Thus, we have used the same combination of atomic closures, since this work also consists of polymers and nanoparticles. Given that $\sigma_{i j}$ is the distance of closest approach between sites $i$ and $j$, i.e. $\sigma_{i j}=d$ for monomer-monomer pairs and $\sigma_{i j}=(\mathrm{D}+\mathrm{d}) / 2$ (as stated earlier, $d$ and $D$ are the monomer and particle diameters, respectively) and particle-monomer pairs, the impenetrability condition applies inside the hard core:

$$
g_{i j}(r)=0, r<\sigma_{i j}
$$

Outside the hard core, the PY approximation describes the direct correlation function between all pairs of sites (except particle-particle):

$$
c_{i j}(r)=\left(1-e^{\beta U_{i j}(r)}\right) g_{i j}(r), r>\sigma_{i j}
$$

and the $\mathrm{HNC}$ closure handles the particle-particle direct correlation function:

$$
c_{B B}=h_{B B}(r)-\ln \left(g_{B B}(r)\right)-\beta U_{B B}(r), r>D
$$

To efficiently solve this system of coupled nonlinear integral equations we employ the KINSOL algorithm[32] with the line search optimization strategy. Attaining convergence for complex nonlinear integral equations is much easier with KINSOL as compared to the Picard technique, the method used in prior PRISM theory work. The solution of the PRISM equations yields pair correlation functions, $g_{i j}(r)$, and the partial collective structure factors, $S_{i j}(q)$. We note that some sets of parameters, especially those involving larger particle sizes or longer polymer chains (graft or matrix) do not yield any solutions due to numerical issues. 
We use a self-consistent approach linking PRISM theory and Brownian Dynamics simulations (BD), where the chain conformations (via the intra-molecular pair correlation function $\left.\Omega_{i j}\right)$ input to PRISM are provided by BD simulations of a single polymer-grafted nanoparticle or a single matrix chain in an external medium-induced potential obtained from PRISM theory. The interdependence of the chain conformations and the medium-induced potential gives rise to the self-consistency. The self-consistent loop starts with three different BD simulations of i) a single grafted particle (in the absence of any matrix chains), ii) a single long matrix chain (in the absence of grafted particle), and iii) a single short matrix chain (in the absence of grafted particle). The model and interactions used in these BD simulations is identical to the one described before. The grafted and matrix chains are integrated for 1e6 time steps with snapshots being collected every $0.25 \mathrm{e} 5$ time steps. Using these snapshots, the intramolecular structure factors: $\omega_{\mathrm{AA}}$ (graft-graft), $\omega_{\mathrm{AB}}$ (graft-particle), $\omega_{\mathrm{CC}}$ (short matrix-short matrix), $\omega_{\mathrm{DD}}$ (long matrix-long matrix)- are calculated. Next, the PRISM equations are solved with the $\omega_{\mathrm{ij}}$ and intermolecular potentials $\mathrm{U}_{\mathrm{ij}}$ as input. Using the results from the PRISM calculation, the pairwise-decomposed medium-induced solvation potential, $\Delta \psi_{i j}(r)$ is calculated; this describes the interaction between any two sites $i$ and $j$ as mediated by all the remaining sites in the system, i.e., including the matrix, grafts and particles themselves. The form of the solvation potential depends on the approximation used in its derivation[33-38] and we use the PY-form:

$$
\Delta \psi_{i j}^{P Y}(r)=-k T \ln \left(1+c_{i k}(r) * s_{k k^{\prime}}(r) * c_{k^{\prime} j}(r)\right)
$$

where ' $*$ ' in equation 9 denotes a convolution integral in spatial coordinates, $k$ is the Boltzmann constant, and $T$ is the temperature. The solvation potential $\Delta \psi_{i j}(r)$ is then used in all following BD simulations of a single polymer-grafted particle, a single short matrix chain, or a single long matrix chain, completing the self-consistent loop. In all BD simulations after the first self-consistent loop, 
the non-bonded interactions between sites are a sum of the WCA potential and the solvation potential from the previous PRISM calculation:

$$
U_{i j}^{t o t}(r)=U_{W C A, i j}(r)+\Delta \psi_{i j}(r)
$$

Note that, while the WCA forces are calculated internally in HOOMD-blue, a table of forces must be created for the solvation potential force using finite-difference approximations. The selfconsistent PRISM-BD iterations are continued until convergence of $\Delta \psi_{i j}(r)$ is achieved. To define the convergence criteria, we first specify 'error' of loop $\mathrm{n}$ as:

$$
E_{n}=\sum_{i, j} \sum_{r}\left[\Delta \psi_{i j}^{n+1}(r)-\Delta \psi_{i j}^{n}(r)\right]^{2}
$$

where the summation of $\mathrm{i}$ and $\mathrm{j}$ is over the site-pairs that are relevant to the BD simulations: AA $\mathrm{AB}, \mathrm{CC}, \mathrm{DD}$. The self-consistent loop converges on when $\mathrm{E}_{\mathrm{n}} / \mathrm{E}_{0}<0.01$ for three consecutive loops.

\subsubsection{Parameters}

The system presented in this paper consists of homopolymer grafted spherical nanoparticles in an explicit homopolymer matrix where the grafted and matrix polymers are either monodisperse or bidisperse. The bidispersity ratio $\beta_{X}=N_{X}$,long $/ N_{X \text {,short }}$ (where $X=$ graft or matrix) and average matrix $\left(\mathrm{N}_{\text {matrix,avg }}\right)$ and graft $\left(\mathrm{N}_{\text {graft,avg }}\right)$ lengths are varied to test the effect of varying extents of bidispersity. The spherical nanoparticle has diameter $D=5 d$ (where $d$ is the diameter of a Kuhn segment or "monomer") and the polymer grafting density is kept in the brush-like regime at 0.65 chains $/ \mathrm{d}^{2}$. The total system packing fraction $\eta$ is 0.1 to mimic a dense solution of matrix polymers. The filler fraction of the grafted particles, $\phi$, defined as the fraction of the total occupied volume that is occupied by particles or grafted monomers, is varied from low $\phi(\phi=0.02)$ to high $\phi(\phi=$ 0.3) during the study, even though we only present results at filler fraction of 0.3 as the qualitative trends described in the results section is seen both at dilute filler fraction and high filler fraction. 
In Figure 6.1 we show simulation snapshots of the system at $\phi=0.3$. Simulation snapshots for $\phi$ $=0.1$ are provided in Supplementary Figure 6.7.

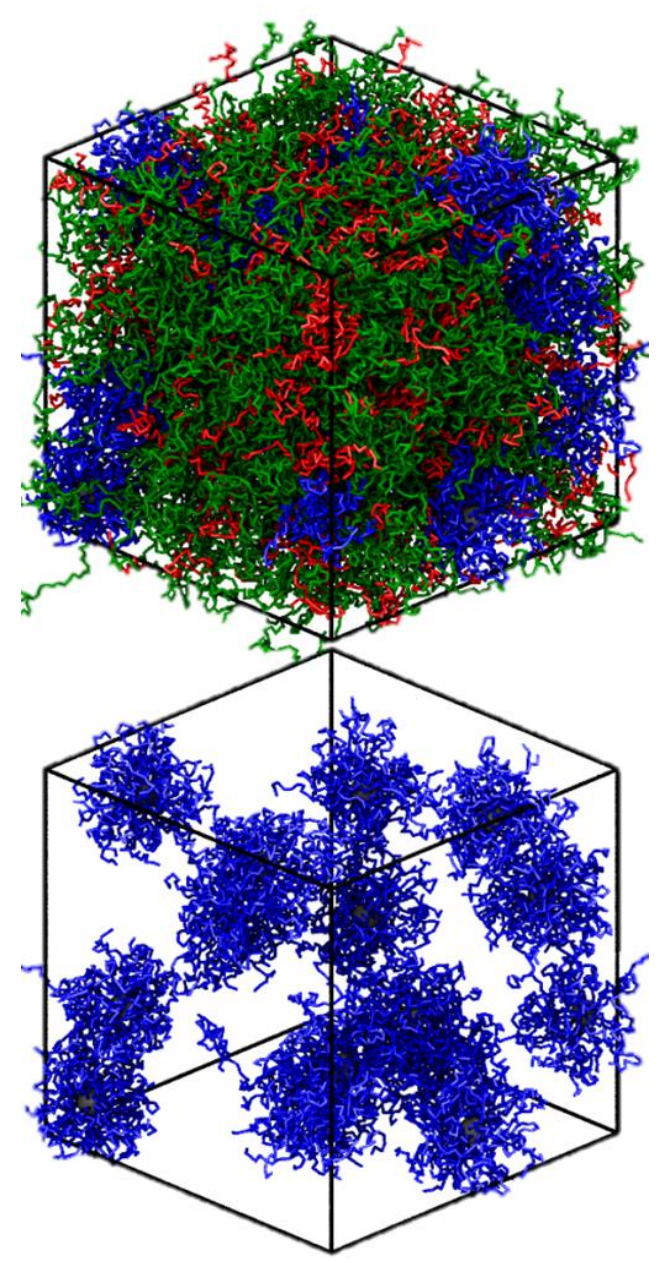

Figure 6.1: A representative simulation snapshot of a polymer nanocomposite with polymer grafted particles of diameter $D=5 d$ grafted with homopolymers (blue chains) with a monodisperse length distribution $\left(N_{\text {graft }}=20\right)$ at grafting density 0.65 chains $/ d^{2}$ and filler fraction $\phi=0.3$ in a bidisperse homopolymer matrix of lengths 20 (red chains) and 60 (green chains). The matrix chains are removed in the bottom image to show the grafted particles only. While the red, blue and green colors are used to distinguish the grafts, the short and long matrix chains in the snapshots, the grafts and all matrix polymers are of the same chemistry and modeled using athermal interactions. 


\subsection{RESULTS}
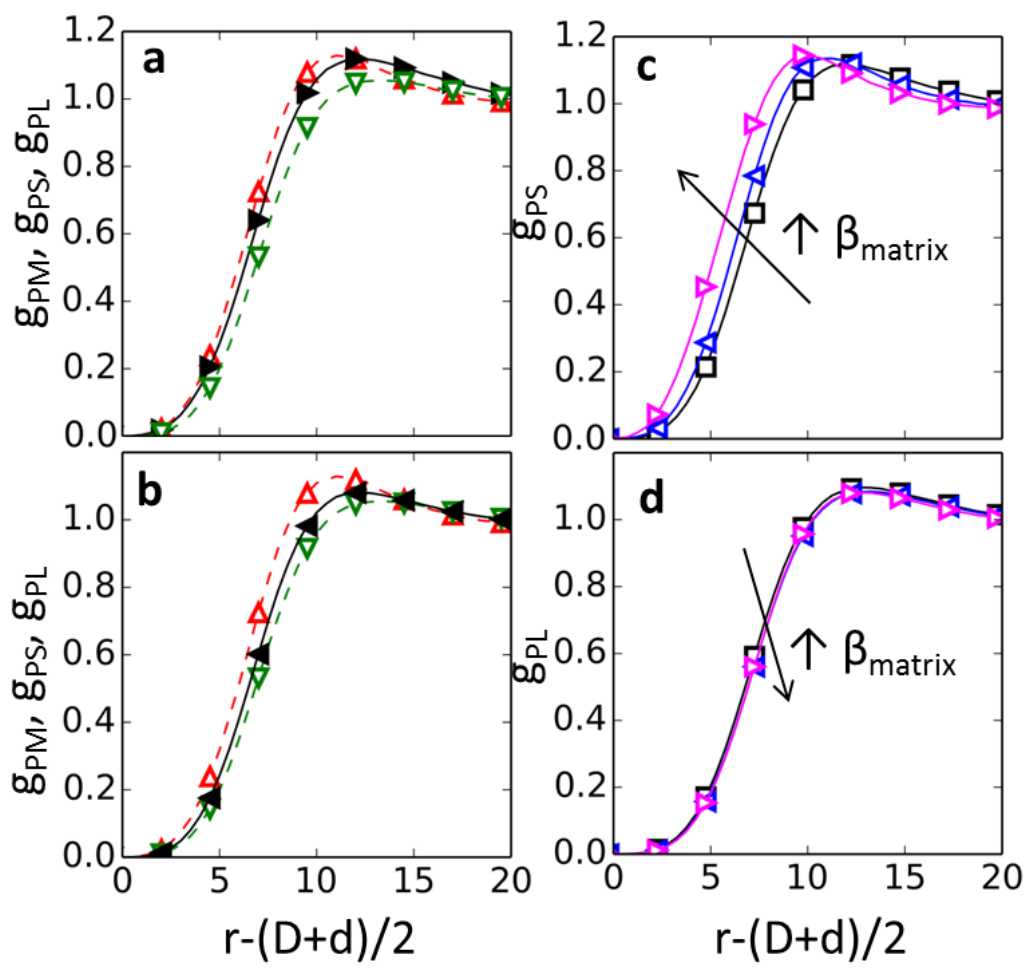

Figure 6.2: Particle-monomer pair correlation functions $g_{i j}(r)$ versus particle-monomer distance, $r-(D+d) / 2$, in units of $d$, for particles of diameter $D=5 d$ grafted with homopolymers with a monodisperse length distribution $\left(N_{\text {graft }}=20\right)$ at 0.65 chains $/ d^{2}$ and filler fraction $\phi=0.3$ in a bidisperse or monodisperse homopolymer matrix. In subplots a and b the symbols indicate the pair correlation between the particles and the monomers of a monodisperse matrix of $N_{\text {matrix }}=30$, $\beta_{\text {matrix }}=1.0$ (solid black rightward triangles) or $N_{\text {matrix }}=60, \beta_{\text {matrix }}=1.0$ (solid black leftward triangles), or the monomers belonging to the short (open red upward triangles, $N_{\text {short }}=30$ ) or long (open green downward triangles, $N_{\text {long }}=60$ ) chains of a bidisperse matrix with $\beta_{\text {matrix }}=3$ and $N_{\text {matrix,avg }}=45$. In subplots $c$ and $d$, the symbols indicate the pair correlation between the particles and the monomers belonging to short (subplot c) or long (subplot d) chains of a bidisperse matrix with $N_{\text {matrix,avg }}=40$ and $\beta_{\text {matrix }}=1.5$ (black squares), 3.0(blue leftward triangles), or 7.0 (pink rightward triangles).

In Figure 6.2a, we show the pair correlation functions between the particle centers and monodisperse matrix monomers ( $\mathrm{g}_{\mathrm{PM}}$, solid black rightward triangles, $\mathrm{N}_{\text {matrix }}=30$ ), short matrix 
chain monomers (gPs, red upward triangles, $\mathrm{N}_{\text {matrix,short }}=30$ ), and long matrix chain monomers $\left(\mathrm{g}_{\mathrm{PL}}\right.$, green downward triangles, $\mathrm{N}_{\text {matrix,short }}=60$ ) of a bidisperse matrix.

In the bidisperse matrix, the short chains show a higher correlation with the particle centers at short distances than the long chains in the same system. This means that the short matrix chains preferentially wet the grafted layer over the long chains. In the monodisperse matrix, it is well understood that, at constant $\mathrm{N}_{\text {graft }}$, wetting of the grafted layer should decrease with increasing matrix chain length.[6, 9,39$]$ Since both $\mathrm{N}_{\text {matrix,short }}$ and $\mathrm{N}_{\text {matrix,long }}$ are greater than $\mathrm{N}_{\text {graft, }}$, the matrix chains should dewet the grafted layer. Furthermore, since $N_{\text {matrix,long }}>N_{\text {matrix,short }}$ it might seem unsurprising that the short chains preferentially wet the grafted layer more than the long chains. However, in Figure 6.2a if we now compare the short chains of the bidisperse pair (open red upward triangles) to a monodisperse matrix of the same length as the short chains (solid rightward triangles), the short chains in the bidisperse environment show a higher correlation with the particles at short distances than in a monodisperse environment. This demonstrates that it is the matrix length bidispersity (simultaneous presence of short and long chains) that is driving the preferential wetting of the grafted layer by the short chains. In Figure $6.2 \mathrm{~b}$ we compare the same bidisperse system to a monodisperse matrix (solid black leftward triangles, $\mathrm{N}_{\text {matrix }}=60$ ) of the same length as the long bidisperse chains. The long chains in the bidisperse environment dewet the grafted layers more than the monodisperse matrix of the same length as the long chains, showing that matrix bidispersity drives both increased wetting of the grafted layer by short matrix chains, and decreased wetting by long matrix chains. See Supplementary Figure 6.8 for additional simulation snapshots that (to some extent) visually depict the extent of wetting of the grafted layer by the matrix chains. 
To see how the behavior in Figure 6.2a and Figure 6.2b changes with increasing matrix bidispersity, in Figure 6.2c and Figure 6.2d we plot the particle - short matrix pair correlation functions ( $g_{P S}$ in Figure 6.2c) and the particle - long matrix correlation functions ( $g_{P L}$ in Figure 6.2d) for three bidispersity ratios $\beta_{\text {matrix }}=\mathrm{N}_{\text {matrix,long }} / \mathrm{N}_{\text {matrix,short }}=1.5,3.0,7.0$ at constant $\mathrm{N}_{\text {matrix,avg }}=40$. With increasing $\beta_{\text {matrix }}$, the preferential wetting of the grafted layer by the short matrix chains becomes more pronounced, while the dewetting of the grafted layer by the long chains is relatively less affected. To address any questions that may arise regarding efficient sampling and equilibration, in Supplementary Figure 6.9 we present a comparison of the graft short matrix pair correlation functions $\left(\mathrm{g}_{\mathrm{GS}}\right)$ and the graft - long matrix correlation functions $\left(\mathrm{g}_{\mathrm{GL}}\right)$ from both simulations and liquid-state theory. From both methods, we observe the grafted layer being preferential wet by the short chains. As liquid-state theory is an equilibrium theory, these results confirm that our simulation results are indicative of the equilibrium behavior of the system. Also to confirm that our results are not strongly dependent on chosen filler fraction (defined as the fraction of the total occupied volume that is occupied by particles or grafted monomers), we also studied $\phi$ ranging from 0.02 to 0.3 and found the preferential wetting of the grafted layer by the short-matrix is present at all $\phi$ within this range (Supplementary Figure 6.10.)

Since we maintain athermal interactions using Weeks-Chandler-Andersen (WCA) pairwise potentials between all monomers, the driving forces that cause the short-matrix chains to preferentially wet the grafted layer are purely entropic in nature. We show the average entropy change of wetting $\left(\Delta \mathrm{S}_{\text {wetting }}\right)$ for a monomer belonging to either a short or long chain in a bidisperse matrix or any chain in a monodisperse matrix. We estimate these values via Flory-Huggins mixing theory[40]:

$$
\left(\frac{1}{k_{B}}\right) \Delta S_{\text {wetting }}=-\frac{1}{N_{X}} \ln \frac{\rho_{X, w e t}}{\rho_{X, \text { unwet }}}
$$


where $\mathrm{X}$ is either short, long, or monodisperse; $\mathrm{N}_{\mathrm{X}}$ is the length of the $\mathrm{X}$ matrix component, $\rho_{\mathrm{X} \text {,wet }}$ is the ratio of volume $\mathrm{X}$ monomers that have wet the grafted layer to the volume of all (graft, short and long matrix) monomers in the grafted layer, $\rho_{X}$,unwet is the ratio of volume of $\mathrm{X}$ monomers that are outside the grafted layer to the volume of all monomers outside the grafted layer, and $\mathrm{k}_{\mathrm{B}}$ is the Boltzmann constant. All the volume fractions used in Equation 12 are calculated from our BD simulations. We define a monomer as having 'wet' the grafted layer when it is within the average root-mean-square brush height (Supplementary Figure 6.11) for the system. $\Delta \mathrm{S}_{\text {wetting }}$ represents the gain in mixing entropy upon a matrix monomer leaving the bulk matrix and entering (wetting) the grafted layer.

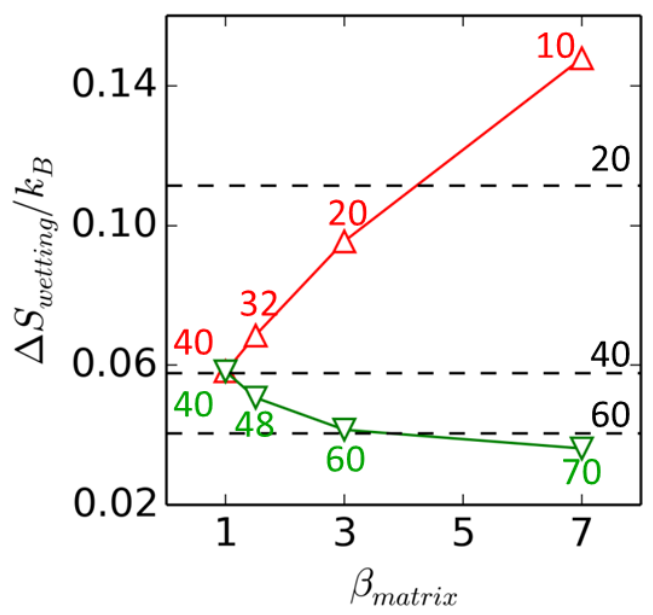

Figure 6.3: Average per-monomer entropy of wetting versus $\beta_{\text {matrix }}$ for particles of diameter $D=5 d$ grafted with homopolymers with monodisperse length distribution $\left(N_{\text {graft }}=20\right)$ at 0.65 chains $/ d^{2}$ and filler fraction $\phi=0.3$ in a monodisperse or bidisperse matrix with $N_{\text {matrix, avg }}=40$. The symbols correspond to the entropy contribution from the short (red upward triangles) or long (green downward triangles) matrix chains. The horizontal dashed lines correspond to the entropy of wetting calculated for monodisperse matrices. The numbers next to the symbols/lines indicate the matrix length for that datum. 


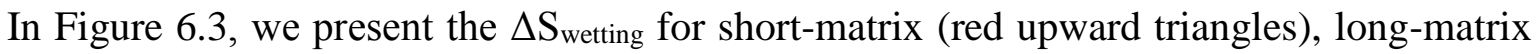
(green downward triangles) and monodisperse matrix (dashed lines), with the length of the matrix component shown next to the corresponding symbol/line. With increasing $\beta_{\text {matrix }}$, the $\Delta \mathrm{S}_{\text {wetting }}$ for

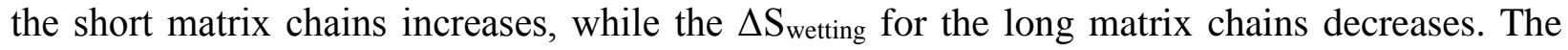
$\Delta \Delta \mathrm{S}_{\text {wetting }}$ between the short and long chains is the effective driving force for preferential wetting of the grafted layer by the short chains and, this driving force increases with increasing $\beta_{\text {matrix. }}$ This is consistent with our observation that with increasing $\beta_{\text {matrix }}$, there is increasing wetting of the grafted layer by short matrix chains and (slightly) decreasing wetting by long matrix chains. Interestingly, the monodisperse matrix with $\mathrm{N}_{\text {matrix }}=20$ has a higher $\Delta \mathrm{S}_{\text {wetting }}$ than the bidisperse short chains of the same length $\left(\mathrm{N}_{\text {matrix,short }}=20, \beta_{\text {matrix }}=3.0\right.$ ). This is because the $\rho_{\text {unwet }}$ for the monodisperse system is much greater than $\rho_{\text {unwet }}$ for the bidisperse system, due to the presence of long matrix chains in the bulk matrix for the bidisperse case (Supplementary Table 6.2). The higher $\rho_{\text {unwet }}$ means that the short chains in the monodisperse environment gain more entropy by wetting the grafted layer than their bidisperse counterparts, and therefore they have a higher $\Delta \mathrm{S}_{\text {wetting. Note }}$ that it does not contradict our findings of preferential wetting of the grafted layer by short matrix chains, as it is the $\Delta \Delta \mathrm{S}_{\text {wetting }}$ between the short and long chains that drives the preferential wetting and not the individual $\Delta \mathrm{S}_{\text {wetting }}$ of the short or the long matrix chains.

When compared to a monodisperse matrix of either short or long chains, the bidisperse matrix has increased the wetting of the grafted layer by short matrix chains, but also slightly decreased the wetting by the long matrix chains. With increased and decreased wetting of the grafted layer, by different components of the bidisperse matrix, how does the bidispersity affect the tendency for aggregation of the grafted nanoparticles? 

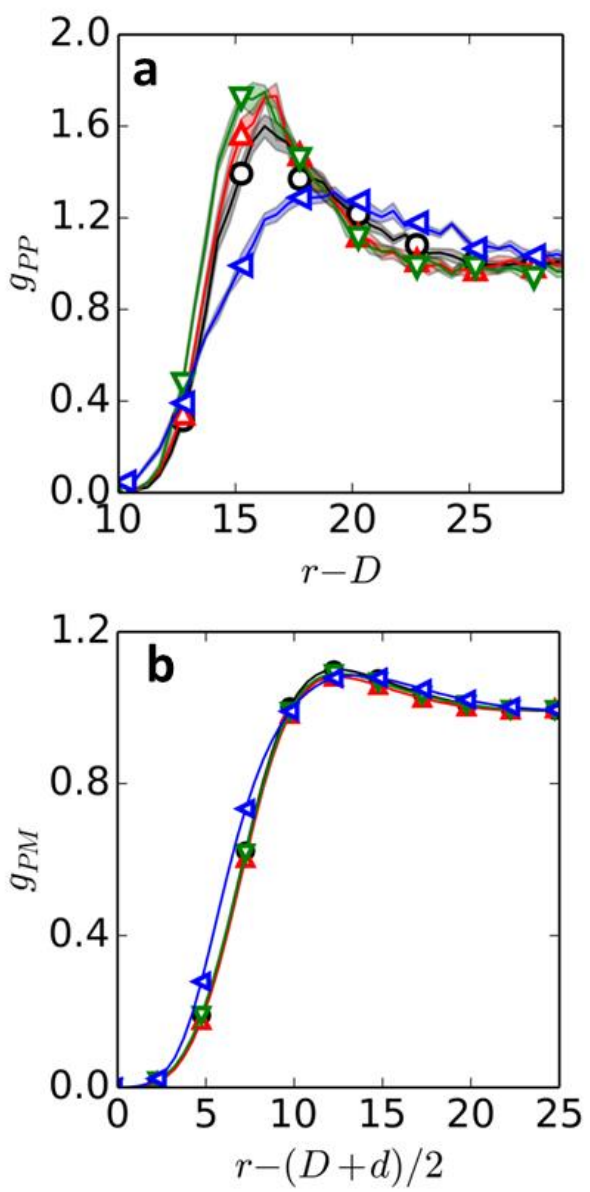

Figure 6.4: Particle-particle (a) and particle-total matrix (b) pair correlation functions versus particle-particle and particle-monomer distance, in units of $d$ for nanoparticles of diameter $D=5 d$ grafted with homopolymers with a monodisperse or bidisperse graft length distribution at filler fraction $\phi=0.3$ in a bidisperse or monodisperse homopolymer matrix. The symbols correspond to: $\beta_{\text {graft }}=1.0, \beta_{\text {matrix }}=1.0, N_{\text {graft }}=20, N_{\text {matrix }}=40$ (black circles), $\beta_{\text {graft }}=1.0, \beta_{\text {matrix }}=3.0, N_{\text {graft }}=20$, $N_{\text {matrix,avg }}=40$ (red upward triangles), $\quad \beta_{\text {graft }}=1.0, \quad \beta_{\text {matrix }}=1.0, N_{\text {graft }}=20, N_{\text {matrix }}=60$ (green downward triangles), $\beta_{\text {graft }}=3.0, \beta_{\text {matrix }}=1.0, N_{\text {graft, avg }}=20, N_{\text {matrix }}=40$ (blue leftward triangles).

In Figure 6.4a, we show the pair-correlation functions between the particles, $\mathrm{g}_{\mathrm{PP}}$, for monodisperse or bidisperse matrix systems. The peak in $\mathrm{g}_{\mathrm{PP}}$ for $\beta_{\text {matrix }}=3.0, \mathrm{~N}_{\text {matrix,avg }}=40$ (red upward triangles) is slightly higher than the peak for $\beta_{\text {matrix }}=1.0$ (monodisperse) $\mathrm{N}_{\text {matrix }}=40$ (black circles) indicating that the bidisperse matrix increases the tendency for aggregation, when 
compared to a monodisperse system with the same $\mathrm{N}_{\text {matrix,avg. Furthermore, at constant }}$ $\mathrm{N}_{\text {matrix,avg }}=40$ as $\beta_{\text {matrix }}$ increases we observe increasing tendency for aggregation (Supplementary Figure 6.12), likely due to the length of the longer matrix chain increasing. In Figure 4a if we compare the $\beta_{\text {matrix }}=3.0$ and $\mathrm{N}_{\text {matrix,long }}=60$ (red upward triangles), to a monodisperse matrix with $\mathrm{N}_{\text {matrix }}=60$ (green downward triangles), the peak in $\mathrm{g}_{\mathrm{PP}}$ is smaller for the bidisperse matrix case, indicating that the bidisperse matrix increases tendency for dispersion (slightly) more than a monodisperse matrix with $\mathrm{N}_{\text {matrix }}=\mathrm{N}_{\text {matrix,long. }}$ To understand the effect of matrix bidispersity on particle aggregation/dispersion we look at the total wetting of the grafted layer via the total particle-matrix pair-correlation function ( $\mathrm{g}_{\mathrm{PM}}$, Figure 6.4b), where the correlation of the short and long chains in the bidisperse matrix are combined. The overall wetting of the grafted layer shows only small variations between the monodisperse (circles, downward triangles) and bidisperse (upward triangles) matrix systems. While matrix bidispersity does increase the wetting of the grafted layer by short matrix chains, it does not increase the overall wetting of the grafted layer. In contrast to the bidisperse matrix, a system consisting of bidisperse grafts with $\beta_{\text {graft }}=3.0$, $\mathrm{N}_{\text {graft,avg }}=20$ in a monodisperse matrix (leftward triangles) exhibits a significantly reduced peak in gP when compared to monodisperse grafted particles in monodisperse/bidisperse matrix. This trend is also consistent with what we observe from liquid-state theory calculations (Supplementary Figure 6.13) where the mid-range attraction in the potential of mean force is eliminated completely for bidisperse grafts, but remains for bidisperse matrix. In Figure 4b, we can see that the wetting of the grafted layer is increased for the system with bidisperse grafts as compared to any of the other systems with monodisperse grafts. Unlike bidispersity in the matrix chain lengths, bidispersity in the grafted chain lengths is able to increase the wetting of the grafted layer by the matrix chains, which in turn improves dispersion of the polymer grafted particles. 


\subsection{CONCLUSION}

This article presents a coarse-grained computational study of polymer nanocomposites consisting of polymer grafted nanoparticles in a bidisperse polymer matrix, with athermal polymer - polymer, polymer - particle and particle - particle interactions. This study focuses on the effects of varying matrix bidispersity, average matrix length and filler volume fraction, while maintaining high grafting density and keeping the particle diameter and graft length constant. Furthermore, in most cases, the matrix chain lengths are chosen to be larger than the graft chain lengths to explore the effect of matrix bidispersity on systems where de-wetting of the grafted layer by the matrix chains has been observed in previous studies with monodisperse systems.

We find that the short-matrix chains show a higher correlation with the particle than either the long matrix chains or monodisperse matrix chains of the same length as the short chains. In other words the short matrix chains preferentially wet the grafted layer on the particles in the presence of matrix bidispersity. Due to athermal interactions, this preferential wetting of the grafted layer by the short chains is largely driven by the disparity in mixing entropies between the grafted layer and the short or long matrix chains. Although the matrix bidispersity increases wetting of the grafted layer by short matrix chains, the overall wetting of the grafted layer is largely unaffected, as long chains dewet the grafted layer more than their monodisperse counterpart. As a

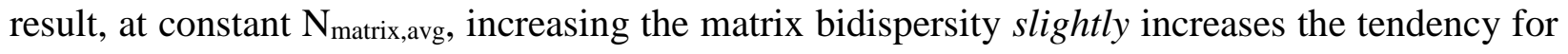
aggregation of the grafted particles, in contrast to the bidispersity in the grafted layer which stabilizes dispersion of the grafted particles.

\subsection{ACKNOWLEDGEMENTS}

This work acknowledges financial support by Department of Energy under Grant DE-SC0003912 (AJ) and National Science Foundation GRFP Award Number DGE 1144083 (TBM). We are 
grateful for supercomputing time on Janus supercomputer, which was used for some of the work, and is supported by the National Science Foundation (award number CNS-0821794) and the University of Colorado Boulder. The Janus supercomputer is a joint effort of the University of Colorado Boulder, the University of Colorado Denver and the National Center for Atmospheric Research. We are also grateful for resources of the National Energy Research Scientific Computing Center, which was used for some of the work, and is supported by the Office of Science of the U.S. Department of Energy under Contract DE-AC02-05CH11231.

\subsection{REFERENCES}

1. Ganesan, V. and A. Jayaraman, Theory and simulation studies of effective interactions, phase behavior and morphology in polymer nanocomposites. Soft Matter, 2014. 10(1): p. 13-38.

2. Krishnamoorti, R., Strategies for Dispersing Nanoparticles in Polymers. MRS Bulletin, 2007. 32(4): p. 341-347.

3. Akcora, P., et al., Anisotropic self-assembly of spherical polymer-grafted nanoparticles. Nature Materials, 2009. 8(4): p. 354-359.

4. Jiao, Y. and P. Akcora, Assembly of Polymer-Grafted Magnetic Nanoparticles in Polymer Melts. Macromolecules, 2012. 45(8): p. 3463-3470.

5. Chevigny, C., et al., Polymer-Grafted-Nanoparticles Nanocomposites: Dispersion, Grafted Chain Conformation, and Rheological Behavior. Macromolecules, 2011. 44(1): p. 122133.

6. Green, P.F., The structure of chain end-grafted nanoparticle/homopolymer nanocomposites. Soft Matter, 2011. 7(18): p. 7914-7926.

7. Hall, L.M., A. Jayaraman, and K.S. Schweizer, Molecular theories of polymer nanocomposites. Current Opinion in Solid State \& Materials Science, 2010. 14(2): p. 3848.

8. Hasegawa, R., Y. Aoki, and M. Doi, Optimum graft density for dispersing particles in polymer melts. Macromolecules, 1996. 29(20): p. 6656-6662.

9. Martin, T.B., P.M. Dodd, and A. Jayaraman, Polydispersity for Tuning the Potential of Mean Force between Polymer Grafted Nanoparticles in a Polymer Matrix. Physical Review Letters, 2013. 110(1): p. 018301.

10. Martin, T.B. and A. Jayaraman, Polydisperse homopolymer grafts stabilize dispersions of nanoparticles in a chemically identical homopolymer matrix: an integrated theory and simulation study. Soft Matter, 2013. 
11. Nair, N., N. Wentzel, and A. Jayaraman, Effect of bidispersity in grafted chain length on grafted chain conformations and potential of mean force between polymer grafted nanoparticles in a homopolymer matrix. Journal of Chemical Physics, 2011. 134(19).

12. Martin, T.B. and A. Jayaraman, Identifying the Ideal Characteristics of the Grafted Polymer Chain Length Distribution for Maximizing Dispersion of Polymer Grafted Nanoparticles in a Polymer Matrix. Macromolecules, 2013. 46(22): p. 9144-9150.

13. Li, Y., et al., Ligand Engineering of Polymer Nanocomposites: From the Simple to the Complex. Acs Applied Materials \& Interfaces, 2014.

14. Tao, P., et al., Transparent luminescent silicone nanocomposites filled with bimodal PDMS-brush-grafted CdSe quantum dots. Journal of Materials Chemistry C, 2013. 1(1): p. 86-94.

15. Broseta, D., et al., MOLECULAR-WEIGHT AND POLYDISPERSITY EFFECTS AT POLYMER POLYMER INTERFACES. Macromolecules, 1990. 23(1): p. 132-139.

16. Anderson, J.A., C.D. Lorenz, and A. Travesset, General purpose molecular dynamics simulations fully implemented on graphics processing units. Journal of Computational Physics, 2008. 227(10): p. 5342-5359.

17. Weeks, J.D., D. Chandler, and H.C. Andersen, ROLE OF REPULSIVE FORCES IN DETERMINING EQUILIBRIUM STRUCTURE OF SIMPLE LIQUIDS. Journal of Chemical Physics, 1971. 54(12): p. 5237-+.

18. Auhl, R., et al., Equilibration of long chain polymer melts in computer simulations. Journal of Chemical Physics, 2003. 119(24): p. 12718-12728.

19. Schweizer, K.S. and J.G. Curro, INTEGRAL-EQUATION THEORY OF THE STRUCTURE OF POLYMER MELTS. Physical Review Letters, 1987. 58(3): p. 246-249.

20. Agarwal, U. and F.A. Escobedo, Mesophase behaviour of polyhedral particles. Nature Materials, 2011. 10(3): p. 230-235.

21. Nair, N. and A. Jayaraman, Self-Consistent PRISM Theory-Monte Carlo Simulation Studies of Copolymer Grafted Nanoparticles in a Homopolymer Matrix. Macromolecules, 2010. 43(19): p. 8251-8263.

22. Hooper, J.B. and K.S. Schweizer, Contact aggregation, bridging, and steric stabilization in dense polymer-particle mixtures. Macromolecules, 2005. 38: p. 8858-8869.

23. Hooper, J.B. and K.S. Schweizer, Theory of phase separation in polymer nanocomposites. Macromolecules, 2006. 39: p. 5133-5142.

24. Hooper, J.B., et al., Structure, surface excess and effective interactions in polymer nanocomposite melts and concentrated solutions. Journal of Chemical Physics, 2004. 121(14): p. 6986-6997.

25. Hall, L.M., et al., Concentration Fluctuations, Local Order, and the Collective Structure of Polymer Nanocomposites. Macromolecules, 2009. 42(21): p. 8435-8442.

26. Hall, L.M. and K.S. Schweizer, Many body effects on the phase separation and structure of dense polymer-particle melts. Journal of Chemical Physics, 2008. 128: p. 234901. 
27. Hall, L.M. and K.S. Schweizer, Structure, scattering patterns and phase behavior of polymer nanocomposites with nonspherical fillers. Soft Matter, 2010. 6(5): p. 1015-1025.

28. Jayaraman, A. and K.S. Schweizer, Structure and Assembly of Dense Solutions and Melts of Single Tethered Nanoparticles. J. Chem. Phys., 2008. 128: p. 164904.

29. Jayaraman, A. and K.S. Schweizer, Effect of the number and placement of polymer tethers on the structure of dense solutions of hybrid nanoparticles. Langmuir, 2008. 24(19): p. $11119-11130$.

30. Jayaraman, A. and K.S. Schweizer, Effective Interactions, Structure, and Phase Behavior of Lightly Tethered Nanoparticles in Polymer Melts. Macromolecules, 2008. 41(23): p. 9430-9438.

31. Jayaraman, A. and K.S. Schweizer, Effective Interactions and Self-Assembly of Hybrid Polymer Grafted Nanoparticles in a Homopolymer Matrix. Macromolecules, 2009. 42 p. pp 8423-8434.

32. Hindmarsh, A.C., et al., SUNDIALS: Suite of nonlinear and differential/algebraic equation solvers. Acm Transactions on Mathematical Software, 2005. 31(3): p. 363-396.

33. Schweizer, K.S. and J.G. Curro, Integral equation theory of polymer melts: intramolecular structurem local order, and the correlation hole. Macromolecules, 1988. 21: p. 3070-3081.

34. Melenkevitz, J., K.S. Schweizer, and J.G. Curro, Self consistent integral eqn theory for the eqbm properties of polymer solns. Macromolecules, 1993. 26: p. 6190-6196.

35. Grayce, C.J. and K.S. Schweizer, Solvn potentials for macromolecules. Journal of Chemical Physics, 1994. 100: p. 6846-6856.

36. Grayce, C.J., A. Yethiraj, and K.S. Schweizer, Liquid state theory of the density dependent conformation of nonpolar linear polymers. Journal of Chemical Physics, 1994. 100: p. 6857-6872.

37. Khalatur, P.G. and A.R. Khokhlov, Hybrid MC/RISM technique for simulation of polymer solutions: MC+RISM integral eqns. Molecular Physics, 1998. 93: p. 555-572.

38. Schweizer, K.S. and J.G. Curro, PRISM Theory of the Structure, Thermodynamics, and Phase-Transitions of Polymer Liquids and Alloys. Advances in Polymer Science, 1994. 116: p. 319-377.

39. Trombly, D.M. and V. Ganesan, Curvature effects upon interactions of polymer-grafted nanoparticles in chemically identical polymer matrices. Journal of Chemical Physics, 2010. 133(15).

40. Flory, P.J., THE CONFIGURATION OF REAL POLYMER CHAINS. Journal of Chemical Physics, 1949. 17(3): p. 303-310. 


\subsection{SUPPLEMENTARY}

\subsubsection{Internal Distances Calculation Showing Relaxation of Chains}

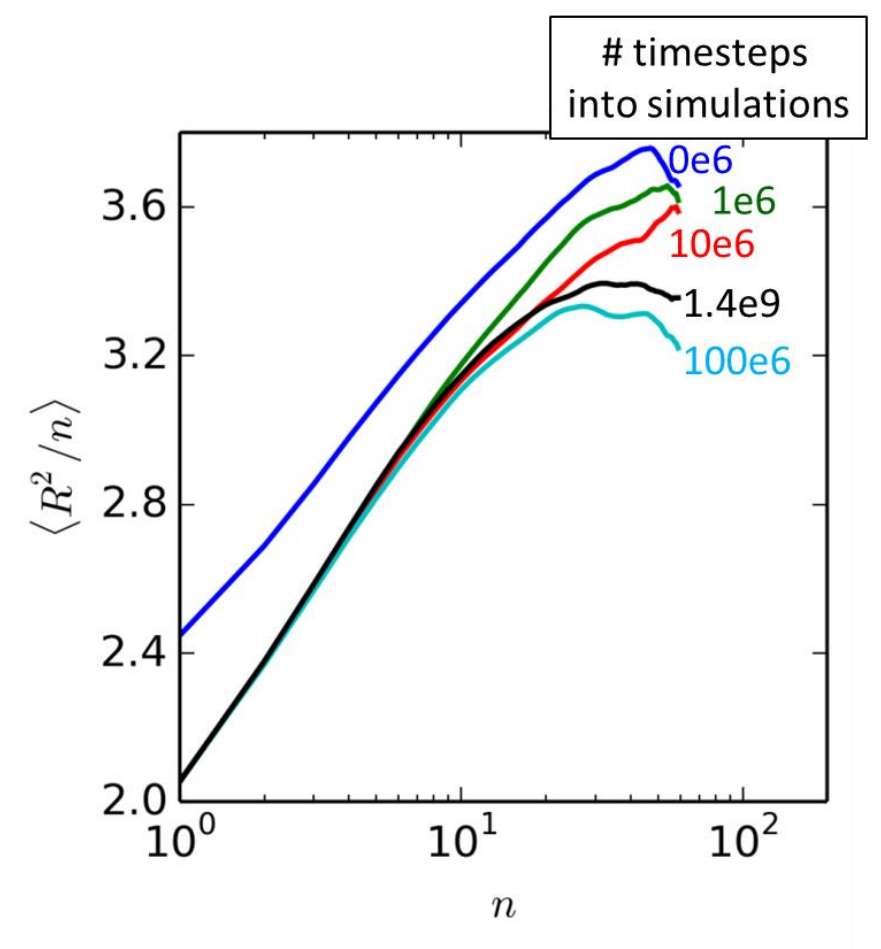

Figure 6.5: Mean-square internal distances versus number of connecting bonds, for a simulation for the long matrix chains a system of grafted nanoparticles of diameter $D=5 d$ grafted with homopolymers with a monodisperse length distribution $\left(N_{\text {graft }}=20\right)$ at $\sigma=0.65$ chains $/ d^{2}$ and filler fraction $\phi=0.3$ in a bidisperse matrix of $N_{\text {matrix }, \text { short }}=20, N_{\text {matrix,long }}=60$. Details of this calculation can be found elsewhere[18]

After the first 1e6 steps of the simulation, the matrix chains relax and show oscillatory behavior at large $\mathrm{n}$, indicative of the chains properly sampling end-to-end distances. 
6.7.2 Pair correlation function data showing variation in individual trials
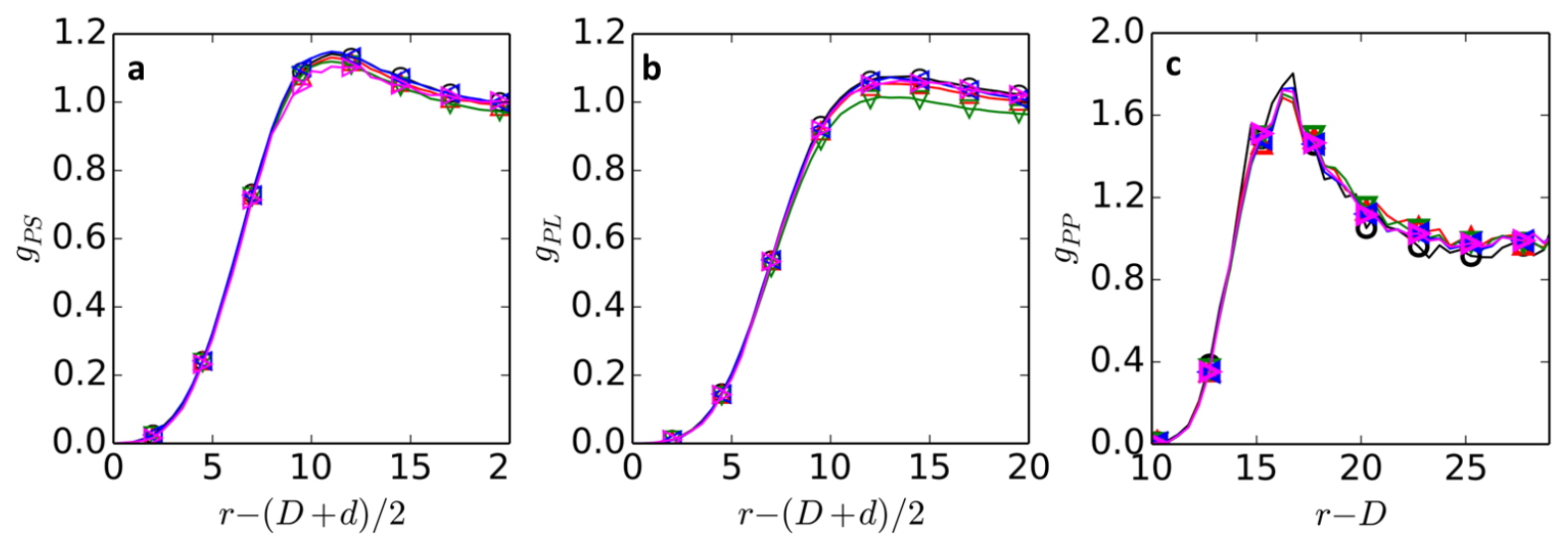

Figure 6.6: Particle-short chain monomer (a) and Particle-long chain monomer (b) and particleparticle (c) pair correlation functions versus particle-monomer distance, in units of $d$, for nanoparticles of diameter $D=5 d$ grafted with homopolymers with a monodisperse length distribution $\left(N_{\text {graft }}=20\right)$ at $\sigma=0.65$ chains $/ d^{2}$ and filler fraction of $\phi=0.3$ in a bidisperse homopolymer matrix with $\beta_{\text {matrix }}=3.0, N_{\text {matrix,avg }}=40$. The colors in the plot correspond to the five independent, randomly initialized trials in the simulation.

These data indicate that our independent trials are reaching statistically similar equilibrium states. 


\subsubsection{Simulation Snaphots}

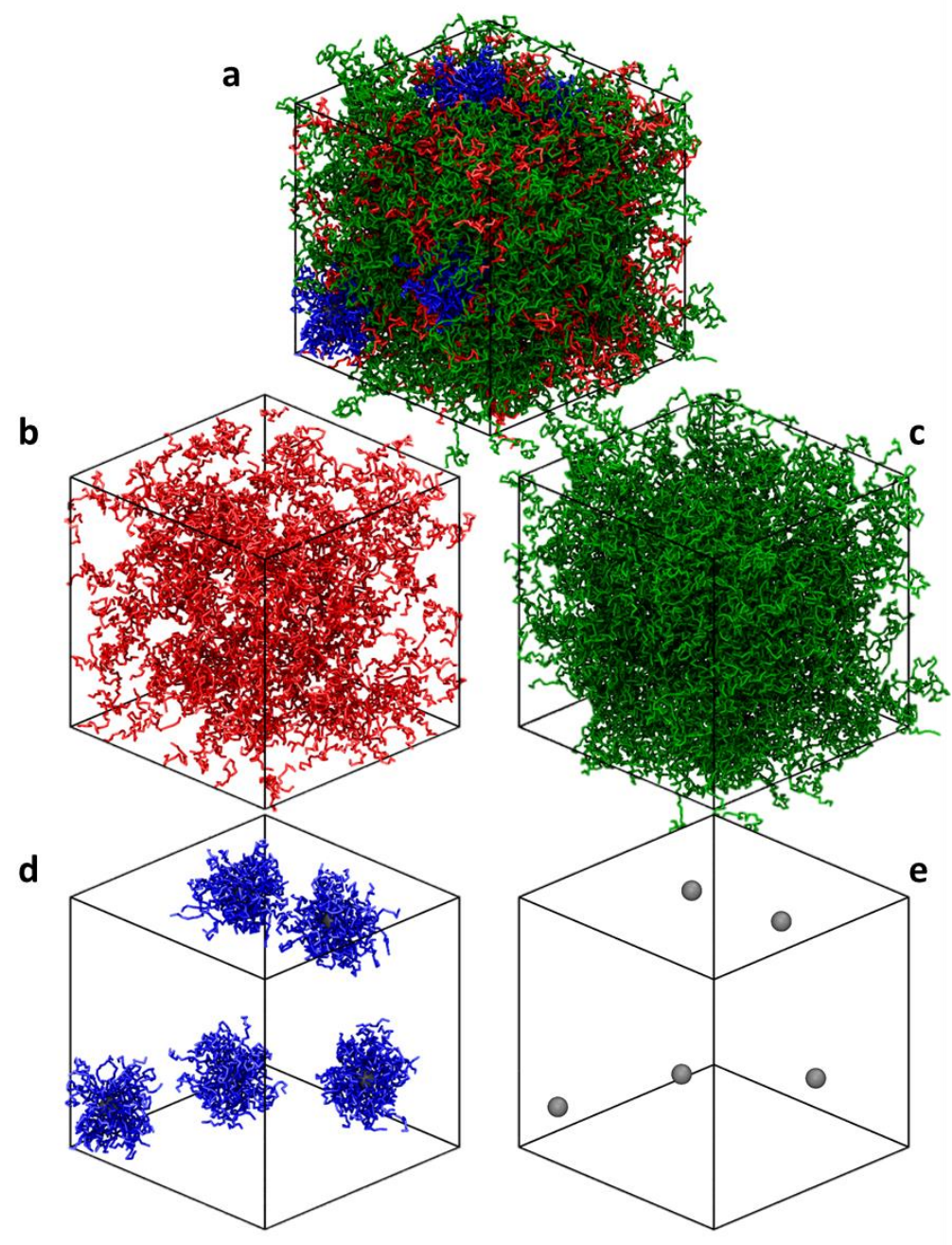

Figure 6.7: Simulation snapshots of PNCs with polymer grafted particles of diameter D=5d grafted with homopolymers (blue chains) with a monodisperse length distribution $\left(N_{\text {graft }}=20\right)$ at $\sigma$ $=0.65$ chains $/ d^{2}$ and filler fraction $\phi=0.1$ in a bidisperse matrix of lengths 20 (red chains) and 60 (green chains). While colors are used to distinguish the various chains in the snapshots, all interactions used in this study are athermal. Individually, the snapshots show all components of the simulation (a), only the short matrix chains (b), only the long matrix chains (c), only the grafted particles $(d)$, only the particle cores (e). 


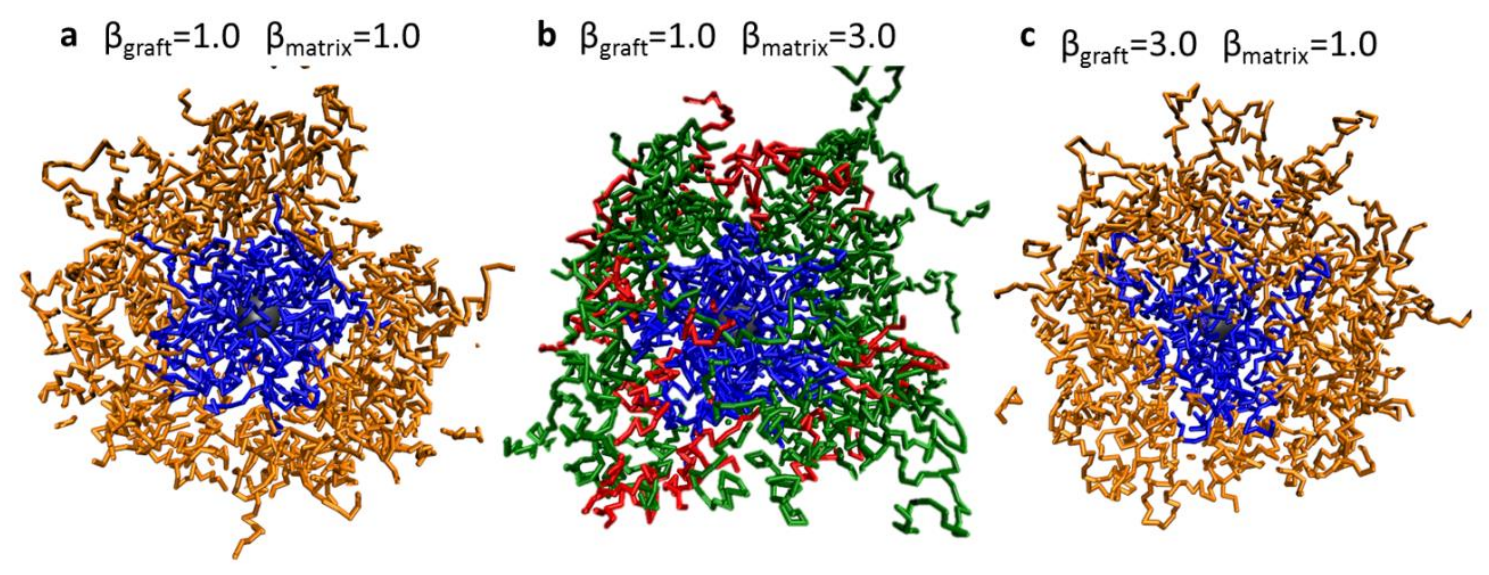

Figure 6.8: Simulation snapshots of polymer grafted particles of diameter D=5d grafted with homopolymers (blue chains) with a monodisperse and bidisperse graft length distribution $\left(N_{\text {graft, avg }}=20\right)$ at $\sigma=0.65$ chains $/ d^{2}$ and filler fraction $\phi=0.02$ in monodisperse (orange) or bidisperse (green,red) matrix. The snapshots correspond to $N_{\text {graft }}=20, N_{\text {matrix }}=40, \beta_{\text {graft }}=1.0$, $\beta_{\text {matrix }}=1.0$ (a), $\quad N_{\text {graft }}=20, \quad N_{\text {matrix }, \text { avg }}=40, \quad \beta_{\text {graft }}=1.0, \beta_{\text {matrix }}=3.0(b), \quad N_{\text {graft }, \text { avg }}=20, \quad N_{\text {matrix }}=40$, $\beta_{\text {graft }}=3.0, \beta_{\text {matrix }}=1.0$ (c), The snapshots show the top down view of cylindrical slice of matrix of depth $10 \sigma$ and diameter $30 \sigma$, centered around each grafted particle 


\subsubsection{Comparison of Brownian Dynamics Simulations and Liquid-State Theory Calculations}
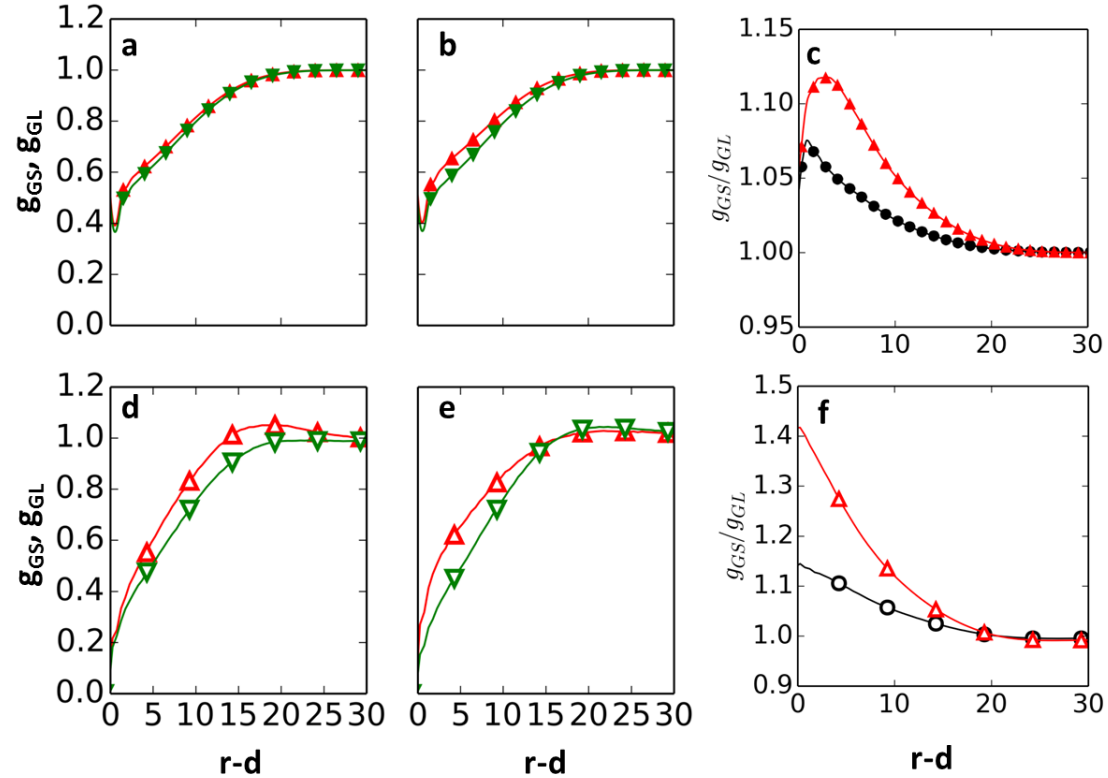

Figure 6.9: Pair correlation functions $(a-b, d-e)$ and ratios of pair correlation functions $(c, f)$ versus monomer-monomer distance, in units of d (size of a Kuhn segment), for nanoparticles of diameter $D=5 d$ grafted with homopolymers with a monodisperse length distribution $\left(N_{\text {graft }}=20\right)$ at $\sigma=0.65$ chains $/ d^{2}$ and filler fraction $\varphi=0.1$ in a bidisperse homopolymer matrix calculated from both liquid state theory $(a-c)$ and molecular dynamics (d-f). For subplots $a-b \& d$-e, the upward triangles represent the graft bead - short matrix bead pair correlation functions, while the downward triangles are the graft bead - long matrix bead pair correlation functions for $\beta_{\text {matrix }}=$ $1.5(a, d)$ and $3.0(b, e)$. In subplot c,f, the ratio of the graft bead - short matrix bead to the graft bead - long matrix bead pair correlation functions are shown for $\beta_{\text {matrix }}=1.5$ (circles), 3.0, (upward triangles).

These data show that the results from our equilibrium liquid-state theory (a-c) calculations match up qualitatively with our results from Brownian dynamics simulations (d-f), in that both methods show that the short-matrix chains preferentially wet the grafted layer over the long matrix chains. Because liquid-state theory results are devoid of equilibration issues, the above qualitative agreement indicates that we are sampling the equilibrium ensemble with our Brownian dynamics simulations. 


\subsubsection{Variation in Short-Matrix Preferential Wetting with Varying Filler-Fraction}
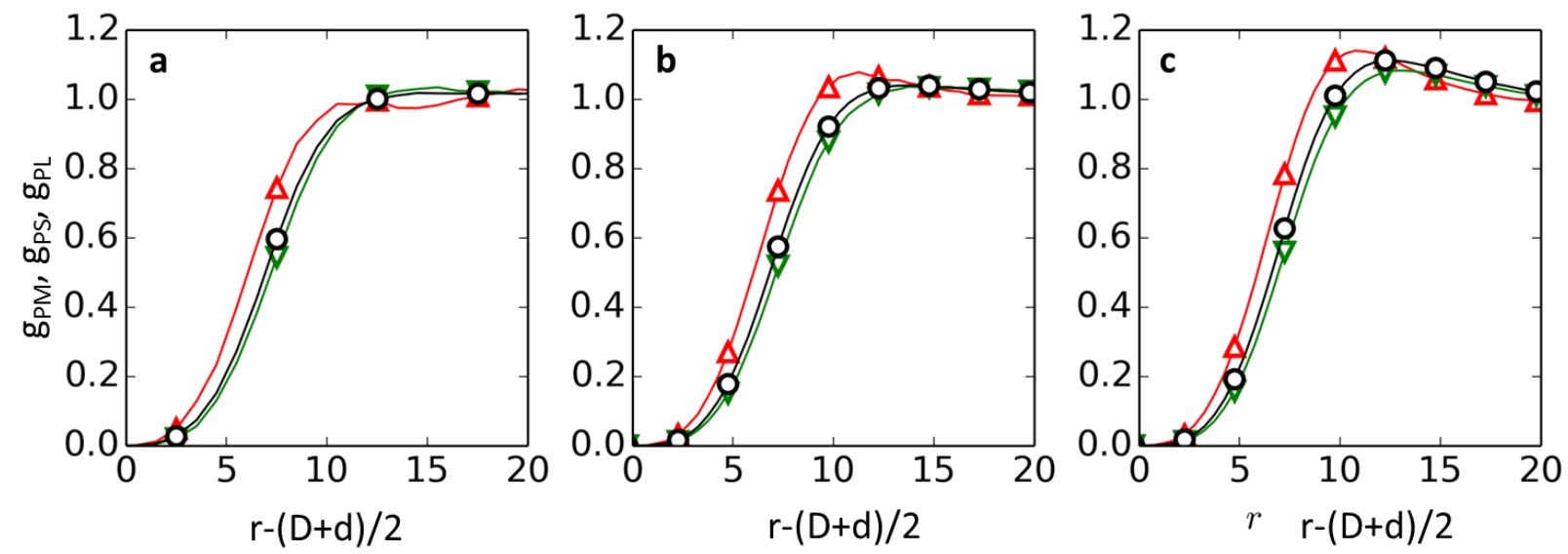

Figure 6.10: Particle-monomer pair correlation functions versus particle-monomer distance, in units of $d$, for nanoparticles of diameter $D=5 d$ grafted with homopolymers with a monodisperse length distribution $\left(N_{\text {graft }}=20\right)$ at $\sigma=0.65$ chains $/ d^{2}$ and filler fraction of $\phi=0.02(a), 0.1(b)$, or 0.3(c) in a bidisperse or monodisperse homopolymer matrix. The symbols indicate the pair correlation between the particles and the monomers of a monodisperse matrix of $N_{\text {matrix }}=40$, $\beta_{\text {matrix }}=1.0$ (black rightward triangles), or the monomers belonging to the short (red upward triangles, $N_{\text {short }}=20$ ) or long (green downward triangles, $N_{\text {long }}=60$ ) chains of a bidisperse matrix with $\beta_{\text {matrix }}=3$ and $N_{\text {matrix }, \text { avg }}=40$.

These data indicate that preferential wetting of the grafted layer by short matrix chains is apparent at a range of filler fractions. 


\subsubsection{Effect of matrix bidispersity on the average brush height of the grafted particles}

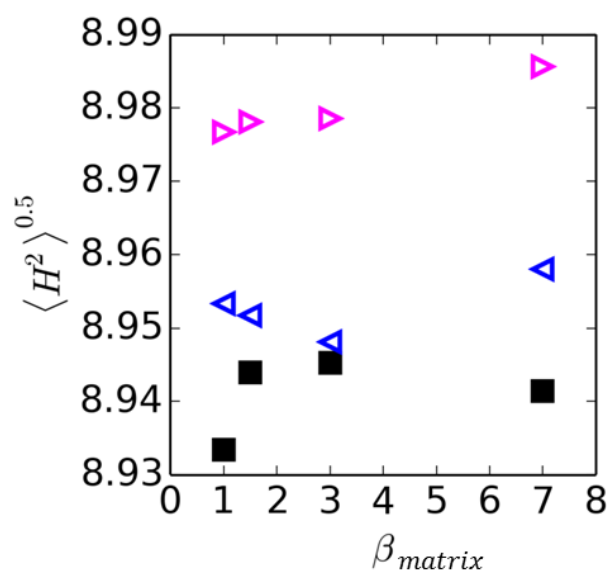

Figure 6.11: Root-mean-square brush height, in units of d (size of Kuhn segment), versus $\beta_{\text {matrix }}$ for nanoparticles of diameter $D=5 d$ grafted with homopolymers with monodisperse length distribution $\left(N_{\text {graft }}=20\right)$ at $\sigma=0.65$ chains $/ d^{2}$ and filler fractions $\varphi=0.02$ (square), 0.1 (leftward triangle), and 0.3 (rightward triangle) in a bidisperse matrix with $N_{\text {matrix,avg }}=40$. The standard error is approximately 0.5 for all data in this plot.

These are calculated as the root-mean-square distance of a grafted monomer from the center of grafted particle. We observe that there is no signifiant change in the brush height with varying $\beta_{\text {matrix }}$, despite the variation in the wetting of the grafted layer. Although there appears to be a trend in these data with $\varphi$, we point out that the variation in these datasets is $1 / 100^{\text {th }}$ the diameter of a single grafted monomer, and thus not to be treated as significantly different. 


\subsubsection{Data tables for $\Delta \mathrm{S}_{\text {wetting }}$ Calculation}

Table 6.1: Wetted and Dewetted Monomer Counts used for $\Delta S_{\text {wetting }}$ Calculation

\begin{tabular}{|c|c|c|c|c|c|c|c|c|c|c|}
\hline \multicolumn{2}{|c|}{} & \multicolumn{3}{c|}{ \# short monomers } & \multicolumn{3}{c|}{ \# long monomers } & \multicolumn{3}{c|}{ \# grafted monomers } \\
\hline$\beta_{\text {matrix }}$ & $N_{\text {matrix,avg }}$ & wet & dewet & Total & wet & dewet & total & wet & dewet & total \\
\hline 1.0 & 20 & 34 & 12465 & 12500 & 34 & 12465 & 12500 & 574 & 445 & 1020 \\
\hline 1.0 & 40 & 31 & 24968 & 25000 & 31 & 24968 & 25000 & 574 & 445 & 1020 \\
\hline 1.0 & 60 & 27 & 24932 & 24959 & 27 & 24932 & 24959 & 573 & 446 & 1020 \\
\hline 1.5 & 40 & 28 & 19971 & 20000 & 33 & 29966 & 29999 & 574 & 445 & 1020 \\
\hline 3.0 & 40 & 23 & 12476 & 12500 & 39 & 37460 & 37500 & 574 & 445 & 1020 \\
\hline 7.0 & 40 & 17 & 6232 & 6249 & 43 & 43706 & 43750 & 575 & 444 & 1020 \\
\hline
\end{tabular}

Table 6.2: Example $\Delta S_{\text {wetting }}$ Calculation for Chains of in Monodisperse and Bidisperse Matrices

\begin{tabular}{|c|c|c|c|c|}
\hline & $\rho_{\text {wet }}$ & Punwet & $\rho_{\text {wet }} / \rho_{\text {unwet }}$ & $-1 / \mathrm{N} \ln \left(\rho_{\text {wet }} / \rho_{\text {unwet }}\right)$ \\
\hline $\begin{array}{l}\beta_{\text {matrix }}=1.0 \\
N_{\text {matrix }}=20\end{array}$ & 0.106 & 0.982 & 0.108 & 0.111 \\
\hline $\begin{array}{c}\beta_{\text {matrix }}=3.0, \\
\mathrm{~N}_{\text {matrix,short }}=20\end{array}$ & 0.037 & 0.248 & 0.148 & 0.095 \\
\hline $\begin{array}{l}\beta_{\text {matrix }}=1.0 \\
\mathrm{~N}_{\text {matrix }}=60\end{array}$ & 0.088 & 0.992 & 0.089 & 0.040 \\
\hline $\begin{array}{c}\beta_{\text {matrix }}=3.0, \\
N_{\text {matrix,long }}=60\end{array}$ & 0.061 & 0.744 & 0.083 & 0.042 \\
\hline
\end{tabular}

We present the numbers in the table as the raw data used in the $\Delta \mathrm{S}_{\text {wetting }}$ calculations. We define a monomer as 'wetting' the grafted layer when it is within the average root-mean-square brush height (Fig S2) for the system. We note that, as these data are averages over multiple frames and multiple trials, there seem to be some discrepancies in the data (e.g. fo Table S1 $\beta_{\text {matrix }}=1.0$, $\mathrm{N}_{\text {matrix,avg }}=60$ the total monomer count seems to be missing a single bead), but these are just due to error in the averages. 


\subsubsection{Liquid-State Theory Results for the Particle-Particle Correlation Function and Potential of}

Mean Force between Grafted Particles
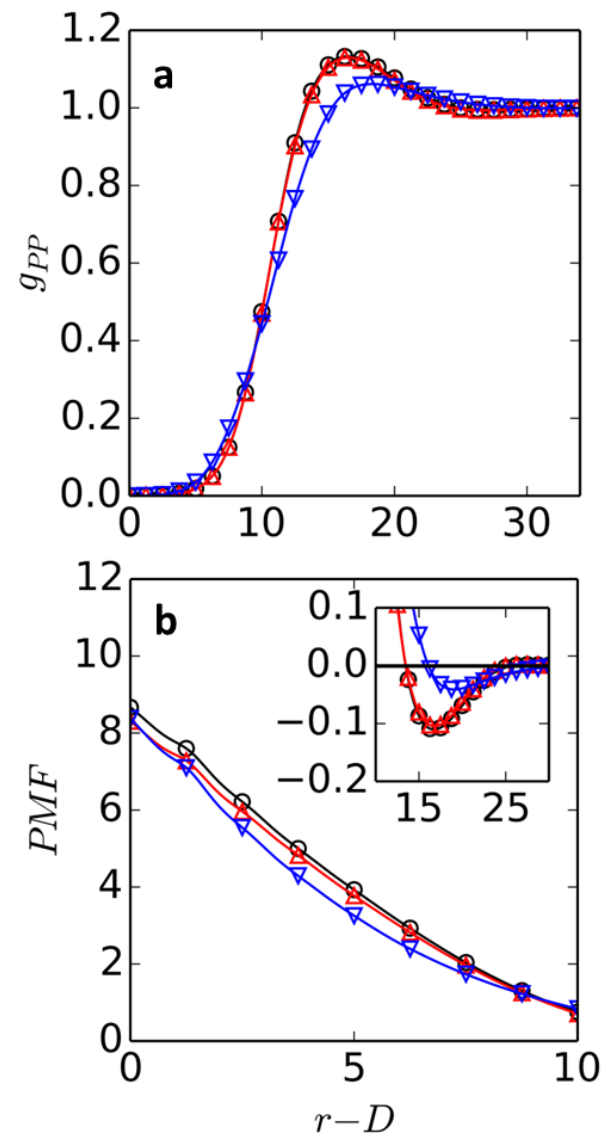

Figure 6.12: Particle-particle pair correlation functions (a) and potentials of mean force, in units of $k_{B} T(b)$ versus particle-particle distance, in units of $d$, for nanoparticles of diameter $D=5 d$ grafted with homopolymers with a monodisperse or bidisperse graft length distribution at filler fraction $\phi=0.05$ (a) and $\phi=0.001$ (b) in a bidisperse or monodisperse homopolymer matrix. The symbols correspond to: $\beta_{\text {graft }}=1.0, \beta_{\text {matrix }}=1.0 N_{\text {graft }}=20, N_{\text {matrix }}=40$, (circles), $\beta_{\text {graft }}=1.0, \beta_{\text {matrix }}=3.0, N_{\text {graft }}=20, N_{\text {matrix }, \text { avg }}=40$ (upward triangles), $\beta_{\text {graft }}=3.0, \beta_{\text {matrix }}=1.0, N_{\text {graft, avg }}=20, N_{\text {matrix }}=40$ (downward triangles). All of these data come from liquid-state-theory derived calculations.

These data are in qualitative aggrement with the simulation results in the main article. While bidispersity in matrix slightly increases the tendency for particle aggreagtion, bidisperse in the grafted chain length significantly decreases the tendency for particle aggregation. 


\subsubsection{Particle-Particle Pair Correlation Function for Varying $\beta_{\text {matrix }}$}

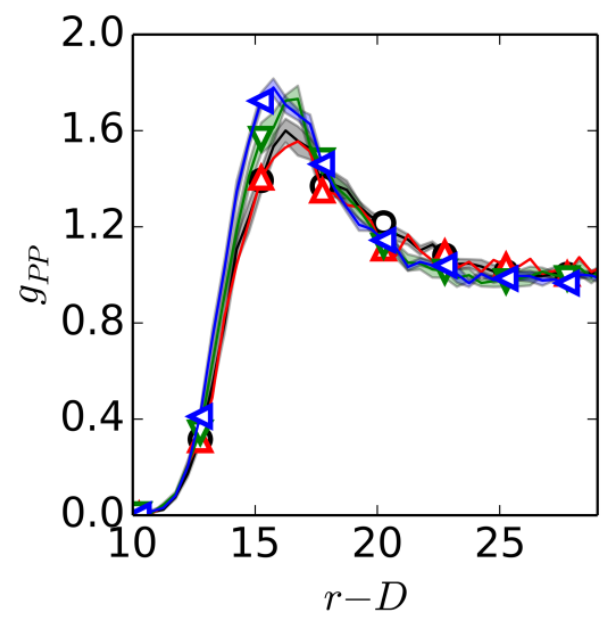

Figure 6.13: Particle-particle pair correlation functions versus particle-particle distance, in units of $d$ for nanoparticles of diameter $D=5 d$ grafted with homopolymers with a monodisperse or bidisperse graft length distribution at filler fraction $\phi=0.3$ in a bidisperse or monodisperse homopolymer matrix. The symbols correspond to: $\beta_{\text {graft }}=1.0, \beta_{\text {matrix }}=1.0, N_{\text {graft }}=20, N_{\text {matrix }}=40$ (black circles), $\beta_{\text {graft }}=1.0, \beta_{\text {matrix }}=1.5, N_{\text {graft }}=20, N_{\text {matrix }, \text { avg }}=40$ (red upward triangles), $\beta_{\text {graft }}=1.0$, $\beta_{\text {matrix }}=3.0, N_{\text {graft }}=20, N_{\text {matrix } \text { avg }}=40$ (green downward triangles), and $\beta_{\text {graft }}=1.0, \beta_{\text {matrix }}=7.0$, $N_{\text {graft }}=20, N_{\text {matrix }, \text { avg }}=40$ (blue leftward triangles).

These data show that, the tendency for aggregation of the particles increases slightly with increasing $\beta_{\text {matrix. }}$ 


\section{Decreasing Polymer Flexibility Improves Wetting and Dispersion of Polymer Grafted Particles in a Chemically Identical Polymer MATRIX}

Adapted from: ACS Macro Letters 3 (7), 628-632

\subsection{INTRODUCTION}

Past studies have elucidated the various parameters that govern dispersion/assembly in polymer nanocomposites (PNCs) containing polymer grafted nanoparticles (PGNs).[1-8] They have mostly focused on PGNs with flexible homopolymers that are chemically identical to the matrix homopolymers, and shown that polymer grafting density[3, 9], particle curvature [10], molecular weights and polydispersity of the graft and matrix[11-15] affect PGN aggregation or dispersion. At high grafting density, on curved surfaces, aggregation/dispersion is driven largely by dewetting/wetting of the PGN grafted layer by the matrix. When the molecular weight of the matrix is less than that of the graft, the grafted layer is wet by the matrix, which leads to increased PGN dispersion. At low grafting density, large molecular weight of grafts shield the highly curved nanoparticle surface from interparticle attractive interactions, and promote particle dispersion.[16] Decreasing surface curvature decreases the wetting of the grafted layer by the matrix due to increased graft crowding near the surface of the particle. Polydispersity in the graft molecular weight has been shown to eliminate the mid-range attractive well in the potential of mean force between PGNs and to stabilize particle dispersion.[11-15] In all of these studies, the impact of polymer flexibility on wetting/dewetting and dispersion/aggregation of PGNs in PNCs has largely been unexplored.

Several studies have focused on semi-flexible polymers (in the absence of nanoparticles) [17-32], near surfaces and interfaces [32-37], as well as on composites of semi-flexible polymers 
and bare particles.[38-41] For example, the effect of semi-flexibility on coil to globule transition of the polymer [20,42], the formation of spherical or toroidal globules [20, 28], and the isotropicnematic liquid crystalline transitions have been explained. [18, 21, 22, 31] In a composite of semiflexible polymers and bare nanoparticles near substrates, decreasing polymer flexibility causes an increase in polymer density near the surface, resulting in lower nanoparticle density near the substrate compared to flexible polymers.[39] Polymer semi-flexibility also impacts the depletion attraction in systems of particles and polymers, with the relative ratio of correlation length and persistence length dictating the depletion thickness and the effects of particle curvature on depletion attraction[38]. These studies point to the importance of changing polymer flexibility on the polymer conformations and effective interactions between the bare particle/surfaces and polymers, and motivate our work here on the role of flexibility in PNCs containing PGNs.

We present a molecular simulation study of PGNs in a chemically identical homopolymer matrix to elucidate the effect of decreasing flexibility in grafts and matrix polymers on wetting of the grafted layer and PNC morphology. Decreasing graft and matrix flexibility leads to increased wetting of the PGNs by the matrix, and improved dispersion of particles with semi-flexible grafts. Changing the flexibility of the grafts has a more significant effect on improving wetting of the grafted layer than changing the flexibility of the matrix does. We also quantify the effect of decreasing flexibility on the known trends of varying graft and matrix length and grafting density on wetting of the grafted layer by the matrix.

\subsection{APPROACH}

\subsubsection{Model}

We model polymer grafted spherical nanoparticles in a polymer matrix using a generic coarsegrained model, where the nanoparticles are modeled as a rigid-body of several $d=1 \sigma$ beads $(\sigma \approx$ 
$1 \mathrm{~nm})$, and the polymers as bead-spring chains. The nanoparticle consists of surface beads to preserve the excluded volume of the particle and grafting sites to anchor the grafted chains. The surface and grafting site beads overlap in the rigid body of the particle, with the grafting site beads isotropically located in the spherical particle surface. Each grafted or matrix polymer is modeled as a bead-spring chain[43], with each bead of size $d=1 \sigma$ representing a group of monomers on the polymer chain, and harmonic springs linking the beads having a force constant of $\mathrm{k}_{\mathrm{bond}}=50 \mathrm{k} \mathrm{B} / \sigma^{2}$ and a bond rest length of $\mathrm{r}_{0}=1 \sigma$, mathematically represented as

$$
U_{\text {bond }}(r)=\frac{1}{2} k_{\text {bond }}\left(r-r_{0}\right)^{2}
$$

where $r$ is the center to center distance between the bonded beads.

We model decreasing flexibility in the graft and matrix polymers through a harmonic angle potential with varying force constant of $\mathrm{K}=0-10 \mathrm{k} \mathrm{B} /$ radians $^{2}$, and a rest angle of $\theta_{0}=\pi$ radians, mathematically represented as[44]

$$
U_{\text {angle }}(r)=\frac{1}{2} K_{X}\left(\theta-\theta_{0}\right)^{2}
$$

where $\mathrm{X}$ is graft or matrix, and $\theta$ is the angle between the two bond vectors that define the potential. Polymer chains with the values of K studied here would have persistence lengths[45] as shown in the Table 7.1 using three different calculation methods[45]:

$$
\left\{\begin{array}{c}
L_{p 1}=\frac{\left\langle\vec{R}_{e e} \cdot \vec{b}_{1}\right\rangle}{\langle b\rangle} \\
\frac{\left\langle\vec{b}_{i} \cdot \vec{b}_{1}\right\rangle}{\langle b\rangle^{2}}=\exp \left(-\frac{i}{L_{p 2}}\right) \\
\left\langle R_{e e}^{2}\right\rangle=2 L_{p 3} N_{X}-2 L_{p 3}^{2}\left(1-\exp \left(-\frac{N_{X}}{L_{p 3}}\right)\right)
\end{array}\right.
$$

where $L_{p n}$ is the persistence length calculated using method $n, \vec{R}_{\text {ee }}$ is a chain end-to-end vector, $\vec{b}_{1}$ is the bond vector for the first bond (from bead 0 to 1 ) of a chain, $\langle b\rangle$ is the average bond length 
where \langle\rangle denotes ensemble averages over 200 independent snapshots and all grafted or matrix chains, $\vec{b}_{i}$ is the $\mathrm{i}^{\text {th }}$ bond vector (from bead $\mathrm{i}-1$ to i) of a chain , $\left\langle\mathrm{R}_{\mathrm{ee}}{ }^{2}\right\rangle$ is the mean-square end-toend distance (calculation described below), and $\mathrm{N}_{\mathrm{X}}(\mathrm{X}=$ graft or matrix) is the chain length. While Equation $3 a$ can be solved directly for $\mathrm{L}_{\mathrm{p} 1}$, in Equation $3 b \mathrm{~L}_{\mathrm{p} 2}$ is solved for by linearly interpolating to find when the LHS is equal to $\mathrm{e}^{-1}$ (i.e. when $\mathrm{L}_{\mathrm{p} 2}$ is equal to $\mathrm{i}$ ), and Equation $3 \mathrm{c}$ must be iteratively solved for $\mathrm{L}_{\mathrm{p} 3}$. Using three estimates for $\mathrm{L}_{\mathrm{p}}$ allows us to assess the effects of the specific assumptions in Equation 3a $(\mathrm{N} \rightarrow \infty)$, Equation $3 \mathrm{~b}$ (fixed bond angle), and Equation 3c (worm-like chain model).

Table 7.1: Expected persistence lengths $L_{p}$, (in units of $\sigma$ ), of graft and matrix chains at given grafting density and chain lengths calculated using three methods

\begin{tabular}{|c|c|c|c|c|c|c|c|c|c|c|}
\hline \multirow[b]{2}{*}{$\mathrm{K}_{\text {graft }}$} & \multirow[b]{2}{*}{$\mathrm{K}_{\text {matrix }}$} & Grafting & \multirow[b]{2}{*}{$\begin{array}{l}\mathrm{N}_{\text {graft }} \\
\left.{ }^{2}\right)\end{array}$} & \multirow[b]{2}{*}{$\mathrm{N}_{\text {matrix }}$} & \multicolumn{3}{|c|}{ Grafts (units of $\sigma$ ) } & \multicolumn{3}{|c|}{ Matrix (units of $\sigma$ ) } \\
\hline & & $\begin{array}{l}\text { Density } \\
\text { (chains } / \sigma^{2}\end{array}$ & & & $\mathrm{~L}_{\mathrm{p} 1}$ & $\mathrm{~L}_{\mathrm{p} 2}$ & $\mathrm{~L}_{\mathrm{p} 3}$ & $\mathrm{~L}_{\mathrm{p} 1}$ & $\mathrm{~L}_{\mathrm{p} 2}$ & $\mathrm{~L}_{\mathrm{p} 3}$ \\
\hline 5 & 5 & 0.65 & 20 & 60 & 7.1 & 6.1 & 5.7 & 5.8 & 4.1 & 5.3 \\
\hline 10 & 10 & 0.65 & 20 & 60 & 9.9 & 10.1 & 10.3 & 10.9 & 9.1 & 9.4 \\
\hline
\end{tabular}

We model a purely athermal system where all pairs of coarse-grained beads, including grafted, matrix, and surface beads, interact via the Weeks-Chandler-Andersen[46] (WCA) potential.

$$
\begin{cases}U_{W C A}(r)=4 \varepsilon\left[\left(\frac{\sigma}{r}\right)^{12}-\left(\frac{\sigma}{r}\right)^{6}\right]+\varepsilon & r<r_{c u t} \\ U_{W C A}(r)=0 & r>r_{c u t}\end{cases}
$$


where $\varepsilon=1$ (in units of $\mathrm{k}_{\mathrm{B}} \mathrm{T}$ ), $\sigma=1$ and $\mathrm{r}_{\mathrm{cut}}=\sigma * 2^{1 / 6}$

\subsubsection{Simulation Method}

Using the model described above, we conduct Brownian dynamics (BD) simulations in the canonical ensemble using the graphical processing unit based HOOMD-blue package.[47, 48] We first create an initial configuration in the following manner: We generate a particle of a desired diameter with isotropically distributed graft points, with the chains extending radially from these graft points embedded on the particle surface. In order to make it easier to insert the grafted particle into the simulation box, a short simulation with strong Lennard-Jones monomer-monomer and monomer-particle attraction is then run to compress the grafted chains from these extended conformations. We note that this is the only time we use attractive non-bonded interactions in our simulation, as the study is focused on a system with athermal interactions. Copies of this one compressed grafted nanoparticle are then randomly placed in a large cubic box to achieve the desired number of particles along with the desired number of matrix chains. This initial configuration is then integrated using a Brownian dynamics integrator for $0.5 \mathrm{e} 6$ time steps to both mix and relax the grafted and matrix chains. The box is then compressed to the desired volume fraction over $0.5 \mathrm{e} 6$ steps, and then mixed again for $0.5 \mathrm{e} 6$ steps at the compressed state. Using this relaxed initial configuration at the appropriate packing fraction at reduced temperature $T^{*}=1$, we finally conduct the production simulation runs for at least 40 million time steps where snapshots of the system are saved every $0.1 \mathrm{e} 6$ time steps.

\subsubsection{Analysis}

We calculate a number of structural features (e.g. monomer concentration profiles, radii of gyration, graft and matrix end-end distances) and thermodynamic information (e.g. mixing 
entropy. Data is calculated from 200 independent uncorrelated snapshots, with the error bars calculated as the standard error between these 200 data points.

We quantify monomer concentration profiles of the grafted and matrix chains from the particle surface as follows:

$$
C_{X}(r)=\frac{\left\langle n_{X}(r)\right\rangle}{4 \pi r^{2} \Delta r}
$$

where $\mathrm{C}_{\mathrm{x}}(\mathrm{r})\left(\mathrm{X}=\mathrm{graft}\right.$ or matrix) is the monomer concentration profile, in units of $\sigma^{(-3)}$, as a function of $\mathrm{r}$, the distance between the particle surface and the monomer bead, and $\left\langle\mathrm{n}_{\mathrm{X}}(\mathrm{r})\right\rangle$ is the average number of beads of type $\mathrm{X}$ that are within a shell of thickness $\Delta \mathrm{r}$ at distance $\mathrm{r}$.

The brush height defines the effective thickness of the grafted layer of the grafted particle and is calculated as the root mean square of the distance of the grafted beads from the surface of the particle.

$$
\left\langle H_{B}^{2}\right\rangle^{0.5}=\sqrt{\frac{1}{\left(n_{P} * n_{G}\right)} \sum_{i=1}^{n_{G}} r_{i}^{2}}
$$

where $\left\langle\mathrm{H}_{\mathrm{B}}{ }^{2}\right\rangle^{0.5}$ is the brush height in units of $\sigma, \mathrm{r}_{\mathrm{i}}$ is the distance of the $\mathrm{i}^{\text {th }}$ graft bead from the surface of the particle the graft belongs to, $n_{P}$ is the total number of grafted particles, and $n_{G}$ is the total number of graft beads in the system (across all grafted particles).

The average end-to-end distance of the polymer chain is calculated by averaging the distance between the first and last bead of each matrix chain over the number of matrix chains in the system:

$$
\left\langle R_{e e}^{2}\right\rangle=\frac{1}{n_{M C}} \sum_{i=1}^{n_{M C}}\left(r_{i, n}-r_{i, 1}\right)^{2}
$$


where $\left\langle\mathrm{R}_{\mathrm{ee}}^{2}\right\rangle$ is the average squared end to end distance of the matrix chains, in units of $\sigma^{2}, \mathrm{n}_{\mathrm{MC}}$ is the number of matrix chains, and $r_{i, n}-r_{i, 1}$ is the distance between the first and last beads of the $i^{\text {th }}$ chain.

The average radius of gyration of the grafted chains quantifies the size of the grafted chain conformations averaged over all of the grafted chains in the system.

$$
\left\langle R_{g}^{2}\right\rangle=\left(\frac{1}{n_{G C} * N_{G}}\right) \sum_{i=1}^{n_{G C}} \sum_{j=1}^{N_{G}}\left(r_{i, j}-r_{i, c o m}\right)^{2}
$$

where $\left\langle\mathrm{R}_{\mathrm{g}}{ }^{2}\right\rangle$ is the average squared radius of gyration, in units of $\sigma^{2}, \mathrm{n}_{\mathrm{GC}}$ is the number of grafted chains in the system(across all particles), $\mathrm{N}_{\mathrm{G}}$ is the length of the grafted chains, $\mathrm{r}_{\mathrm{i}, \mathrm{j}}$ is the position of bead $\mathrm{j}$ on chain $\mathrm{i}$, and $\mathrm{r}_{\mathrm{i}, \mathrm{com}}$ is the center of mass of chain $\mathrm{i}$.

The wet matrix bead percentage quantifies the degree of wetting of the grafted layer by matrix beads, and is calculated as:

$$
\text { wet matrix } \%=\frac{n_{M, w e t}}{n_{M}}(100 \%)
$$

where $n_{M}$ is the total number of matrix beads in the system and $n_{M \text {,wet }}$ is the number of matrix beads that are within the brush height $\left(\left\langle\mathrm{H}_{\mathrm{B}}{ }^{2}\right\rangle^{0.5}\right)$ i.e. the number of matrix beads that have wet the grafted layer of any particle.

We also calculate the particle-particle pair correlation function, $g_{P P}(r)$, which describes the extent of aggregation/dispersion of the grafted particles in the polymer matrix, by quantifying the correlation between the particle centers[49]. This is calculated only for the systems with multiple grafted particles.

We also estimate the total gain in mixing entropy upon the matrix chains wetting the grafted layer, $\Delta \mathrm{S}_{\mathrm{wet}}$, as shown below in Equation 10 . We calculate this quantity using 


$$
\begin{gathered}
\Delta S_{\text {wet }}=-\frac{k_{B}}{N_{M}} \sum_{n_{M, w e t}=1}^{\left\langle n_{M, w e t}\right\rangle} \ln \frac{\phi_{M, w e t}}{\phi_{M, \text { unwet }}} \\
\phi_{M, w e t}=\frac{n_{M, w e t}}{n_{M, w e t}+n_{G, w e t}}, \quad \phi_{M, \text { unwet }}=\frac{n_{M}-n_{M, w e t}}{n_{M}+n_{G}-\left(n_{M, w e t}+n_{G, w e t}\right)}
\end{gathered}
$$

where $\Delta S_{\text {wet }}$ is the total gain in mixing entropy, $k_{B}$ is Boltzmann's constant, $N_{M}$ is the length of the matrix chains, $\phi_{\mathrm{M} \text {,wet }}$ is the volume fraction of matrix beads that have wet the grafted layer, and $\phi_{M \text {,unwet }}$ is the volume fraction of matrix beads that are outside the grafted layer. Since the volumes of the individual matrix and grafted beads are equal, the volume fractions can be calculated as number fractions, where $\mathrm{n}_{\mathrm{M}}$ is the total number of matrix beads in the system, $\mathrm{n}_{\mathrm{M} \text {,wet }}$ is the number of wet matrix beads, $\mathrm{n}_{\mathrm{G}}$ is the total number of graft beads in the system, and $\mathrm{n}_{\mathrm{G} \text {,wet }}$ is the number of wet graft beads. We calculate the number fractions in two separate ways: (a) explicitly counting graft and matrix beads in the simulation that are within the brush height and averaging that over snapshots and independent trials or (b) assuming that $\left\langle n_{G, w e t}\right\rangle$ is approximately $0.6 \mathrm{n}_{\mathrm{G}}$, based on our observations for number of grafted beads within grafted layer, and calculating $\left\langle n_{M, w e t}\right\rangle$ by assuming the density of the matrix beads in the grafted layer is equal to the bulk density:

$$
\left\langle n_{M, w e t}\right\rangle=\frac{4}{3} \pi\left[\left(\left\langle H_{B}^{2}\right\rangle^{0.5}+\frac{D}{2}\right)^{3}-\left(\frac{D}{2}\right)^{3}\right] \rho_{M, \text { bulk }}
$$

where $\rho_{\mathrm{M}, \text { bulk }}$ is the bulk density of matrix beads in the simulation, $\left\langle\mathrm{H}_{\mathrm{B}}{ }^{2}\right\rangle^{0.5}$ is the brush height or grafted layer thickness, and D is the diameter of the particle. Method (b) is intended to be a crude check for trends found with method (a) as it involves less information from the simulation, and allows us to decouple the entropy estimations from the simulation data more than method (a)

\subsubsection{Parameters}

In this study the nanoparticle size is maintained at $5 \sigma$, grafting density is varied from 0.25 to 0.65 chains $/ \sigma^{2}$, the matrix polymer length is varied from $20-100$ coarse-grained beads, the graft 
polymer length is varied from 10-40 beads, and the angle potential force constant is varied from 0 to $10 \mathrm{k}_{\mathrm{B}} \mathrm{T} /$ radians $^{2}$, with the majority of the results shown for 0 and $5 \mathrm{k}_{\mathrm{B}} \mathrm{T} /$ radians $^{2}$. For single particle simulations, we use 60,000 matrix beads and for multi particle simulations we use 120,000 matrix beads. The simulation box volume for single particle simulations is about $68 \times 68 \times 68 \sigma^{3}$, and for multi particle simulations is about $91 \times 91 \times 91 \sigma^{3}$. The total occupied volume fraction in the simulation box is maintained to be 0.1 for all systems and the number of grafted particles is varied from 1 to 20 particles.

\subsection{RESULTS}

\subsubsection{Effect of polymer flexibility on wetting of the grafted layer by matrix}

Figure 7.1 shows that as the graft and matrix flexibility decreases the grafts adopt extended conformations, thereby increasing the brush height from the particle surface. Most importantly, with decreasing polymer flexibility, the matrix concentration profile extends further into the grafted layer, implying increasing wetting of the grafted layer by the matrix chains.
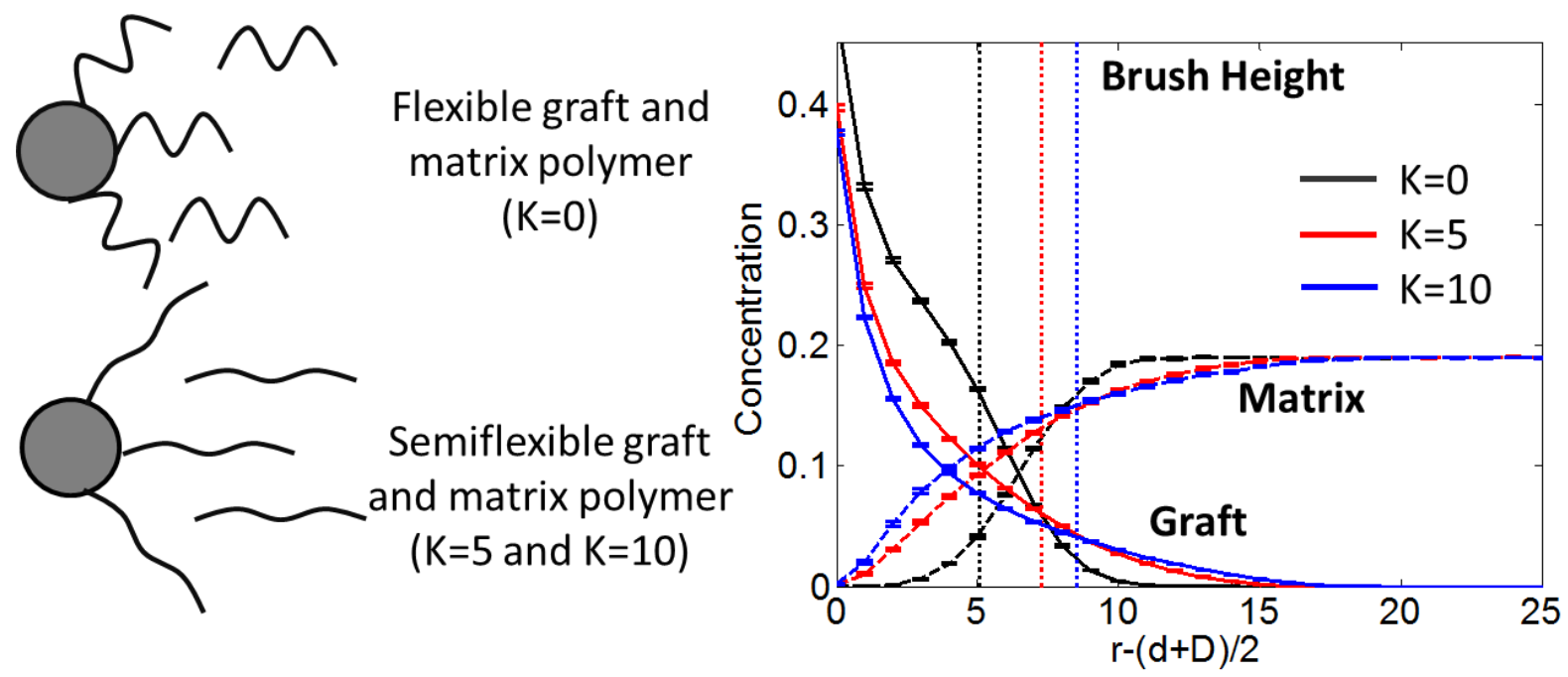

Figure 7.1: Graft (solid) and matrix (dashed) monomer concentration profiles for a single PGN with grafting density $=0.65$ chains $/ \sigma^{2}, N_{\text {graft }}=20$, and $N_{\text {matrix }}=60$, with $K=K_{\text {graft }}=K_{\text {matrix }}=0$ (black), 5 (red) and 10 (blue) $k_{B} T /$ radians $^{2}$. The brush heights are shown as vertical dash-dotted lines. 
Since we maintain athermal interactions, the wetting/dewetting of the grafted layer by matrix is driven by the balance of gain in mixing entropy and losses in conformational entropy of the graft and matrix upon wetting. Decreasing flexibility of the matrix is expected to decrease its conformational entropy in the bulk, and as a result, decrease the conformational entropy loss the matrix would face upon entering/wetting the crowded grafted layer. As expected, as matrix flexibility decreases, the average end-end distance of the matrix increases (Supplementary Table 7.3); the average and the distribution of end-end distances is a signature of the conformational entropy of the matrix chains. While decreasing graft flexibility also reduces the conformational entropy loss of the grafts upon being wet, it is relatively negligible when compared to the matrix due to the grafts being constrained to the particle surface and crowded by other grafts. More importantly, decreasing graft flexibility increases the brush height, which increases the grafted layer volume and likely the mixing entropy gain upon wetting. By decreasing the flexibility of the graft and matrix, the conformational entropy losses upon wetting of the grafted layer are reduced, while the gains in mixing entropy are increased, driving the increased wetting of the grafted layer. Next, to understand which of the above factors more significantly drives the increased wetting of the grafted layer by matrix chains with decreasing flexibility, we tune the graft and matrix flexibility individually. 


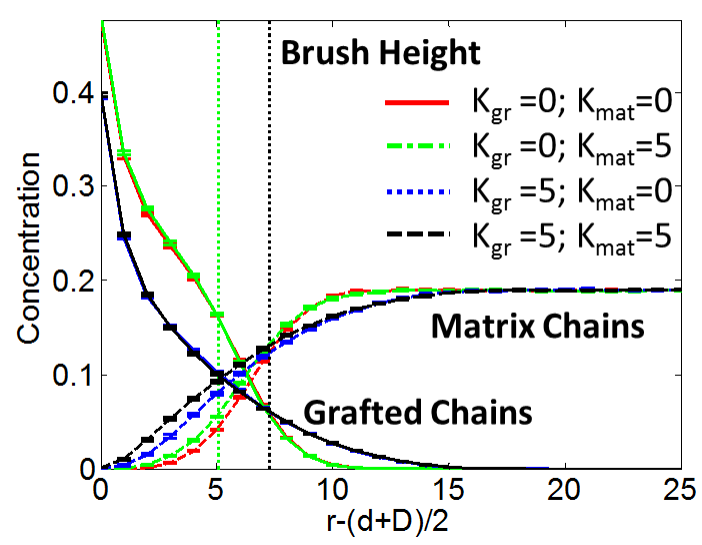

Figure 7.2: Graft (solid) and matrix (dashed) monomer concentration profile for single PGN with grafting density $=0.65$ chains $/ \sigma^{2}, N_{\text {graft }}=20, N_{\text {matrix }}=60$, and $K_{\text {graft }}=0$ and $K_{\text {matrix }}=0($ red $), K_{\text {graft }}=0$ and $K_{\text {matrix }}=5$ (green), $K_{\text {graft }}=5$ and $K_{\text {matrix }}=0$ (blue), and $K_{\text {graft }}=5$ and $K_{\text {matrix }}=5$ (black).

In Figure 7.2 the matrix concentration at short distances from the particle surface is higher and thus the grafted layer wetting is larger for decreased graft flexibility $\left(\mathrm{K}_{\text {graft }}>0, \mathrm{~K}_{\text {matrix }}=0\right)$ than for decreased matrix flexibility $\left(\mathrm{K}_{\mathrm{graft}}=0, \mathrm{~K}_{\text {matrix }}>0\right)$. Similarly, the $\%$ matrix beads that have wet the grafted layer (denoted as "\% Wet Matrix" in Table 7.2), calculated as the percent of matrix beads that are within the brush height of any grafted particle is significantly more for the case of $\mathrm{K}_{\text {graft }}>0, \mathrm{~K}_{\text {matrix }}=0$ than that for the $\mathrm{K}_{\text {graft }}=0, \mathrm{~K}_{\text {matrix }}>0$ case, indicating that decreasing graft flexibility improves wetting more than decreasing matrix flexibility. At constant graft flexibility, decreasing the matrix flexibility has little effect on the brush height, and therefore little effect on the grafted layer volume. This means that when the graft is flexible and matrix is semi-flexible, any changes in wetting come solely from the matrix chains losing less conformational entropy upon wetting the grafted layer. Since the distribution of end-end distances of matrix upon wetting (Supplementary Table 7.3) is not significantly different for flexible and semi-flexible matrix, the reduced loss in conformational entropy of matrix with decreasing matrix flexibility is likely small. Conversely, at constant matrix flexibility, decreasing the graft flexibility increases the brush height 
dramatically and results in significantly higher grafted layer volume, which increases the entropy of mixing of the graft and matrix chains without altering the conformational entropy loss of the matrix. This increased gain in entropy of mixing of the graft and matrix chains is driving the improved wetting behavior, as quantified next.

Table 7.2: Average percentage of matrix beads that wet the grafted layer (\% Wet Matrix) as a function of graft and matrix lengths, grafting density and flexibility.

\begin{tabular}{cccccccc}
\hline $\begin{array}{c}\text { \# of } \\
\text { nanoparticles }\end{array}$ & $\mathrm{K}_{\text {graft }}$ & $\mathrm{K}_{\text {matrix }}$ & $\begin{array}{c}\text { Grafting } \\
\text { Density } \\
\left.\text { (chains/ } \sigma^{2}\right)\end{array}$ & $\mathrm{N}_{\text {graft }}$ & $\mathrm{N}_{\text {matrix }}$ & $\begin{array}{c}\text { \% Wet } \\
\text { Matrix }\end{array}$ & Error \\
\hline 1 & 0 & 0 & 0.65 & 20 & 60 & $0.03 \%$ & 0.002 \\
1 & 0 & 5 & 0.65 & 20 & 60 & $0.05 \%$ & 0.002 \\
1 & 5 & 0 & 0.65 & 20 & 60 & $0.49 \%$ & 0.006 \\
1 & 5 & 5 & 0.65 & 20 & 60 & $0.58 \%$ & 0.006 \\
1 & 10 & 10 & 0.65 & 20 & 60 & $1.18 \%$ & 0.007 \\
1 & 0 & 0 & 0.25 & 20 & 60 & $0.10 \%$ & 0.003 \\
1 & 5 & 5 & 0.25 & 20 & 60 & $0.87 \%$ & 0.005 \\
1 & 0 & 0 & 0.65 & 20 & 20 & $0.05 \%$ & 0.002 \\
1 & 5 & 5 & 0.65 & 20 & 20 & $0.63 \%$ & 0.006 \\
1 & 0 & 0 & 0.65 & 10 & 60 & $0.003 \%$ & 0.0004 \\
1 & 5 & 5 & 0.65 & 10 & 60 & $0.08 \%$ & 0.002 \\
1 & 0 & 0 & 0.65 & 40 & 60 & $0.23 \%$ & 0.005 \\
1 & 5 & 5 & 0.65 & 40 & 60 & $2.81 \%$ & 0.012 \\
1 & 0 & 0 & 0.65 & 20 & 40 & $0.03 \%$ & 0.002 \\
1 & 5 & 5 & 0.65 & 20 & 40 & $0.59 \%$ & 0.006 \\
1 & 0 & 0 & 0.65 & 20 & 100 & $0.03 \%$ & 0.002 \\
1 & 5 & 5 & 0.65 & 20 & 100 & $0.57 \%$ & 0.006 \\
20 & 0 & 0 & 0.65 & 20 & 100 & $0.01 \%$ & 0.0002 \\
20 & 5 & 5 & 0.65 & 20 & 100 & $0.26 \%$ & 0.0007 \\
\hline & & & & & & & \\
\hline
\end{tabular}



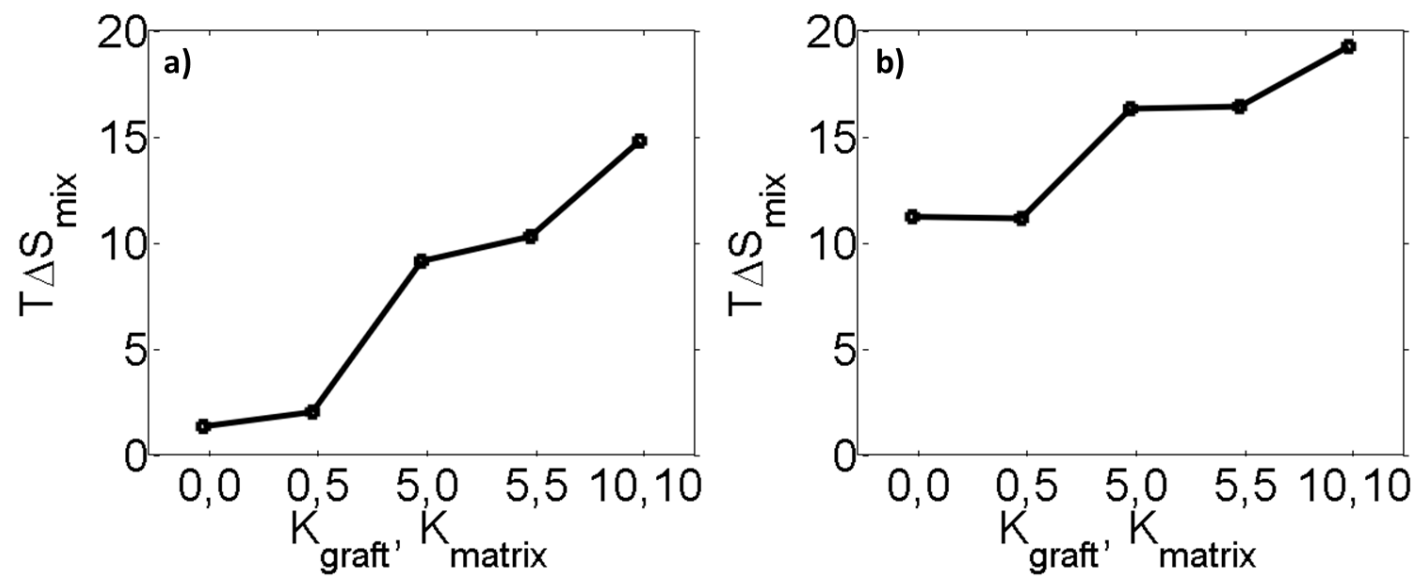

Figure 7.3: Gain in mixing entropy, $T \Delta S_{\text {mix }}$ (in $k_{B} T$ ) as a function of graft and matrix flexibility calculated a) using simulation trajectory information and b) estimated approximately with minimal information from simulation.

In Figure 7.3 we show the gain in mixing entropy of wetting the grafted layer by matrix chains $\mathrm{T} \Delta \mathrm{S}_{\mathrm{mix}}$ versus decreasing flexibility for graft and matrix. In Figure 7.3a, where $\mathrm{T} \Delta \mathrm{S}_{\mathrm{mix}}$ is calculated from the simulation trajectories by explicitly counting the number of matrix beads that wet the grafted layer, the $\mathrm{T} \Delta \mathrm{S}_{\text {mix }}$ is much larger when the graft flexibility is decreased than when the matrix flexibility is decreased. Furthermore, when the flexibility of both graft and matrix is decreased, the increase in $\mathrm{T} \Delta \mathrm{S}_{\mathrm{mix}}$ is largest. To minimize the bias introduced by using simulation data in the calculation of $\mathrm{T} \Delta \mathrm{S}_{\mathrm{mix}}$, we recalculated the $\mathrm{T} \Delta \mathrm{S}_{\text {mix }}$ using only the average brush height from each simulation (see Section 7.2.3). In Figure 7.3b, we find identical qualitative trends as seen in Figure 7.3a, with the magnitude of the $\mathrm{T} \Delta \mathrm{S}_{\mathrm{mix}}$ being greater with the approximate method (Figure $7.3 \mathrm{~b}$ ). Despite the over-estimation of $\mathrm{T} \Delta \mathrm{S}_{\mathrm{mix}}$ in Figure $7.3 \mathrm{~b}$ (due to the approximation that the density of the matrix beads in the grafted layer is equal to the bulk matrix density) both methods agree that decreasing flexibility increases the $\mathrm{T} \Delta \mathrm{S}_{\mathrm{mix}}$. We can conclusively say that the mixing entropy of the 
grafted and matrix chains is a primary driving force for wetting/dewetting in this system and that flexibility of the graft and matrix directly tunes this driving force.

\subsubsection{Effect of polymer flexibility on trends of varying grafting density, graft and matrix length on wetting/dewetting}
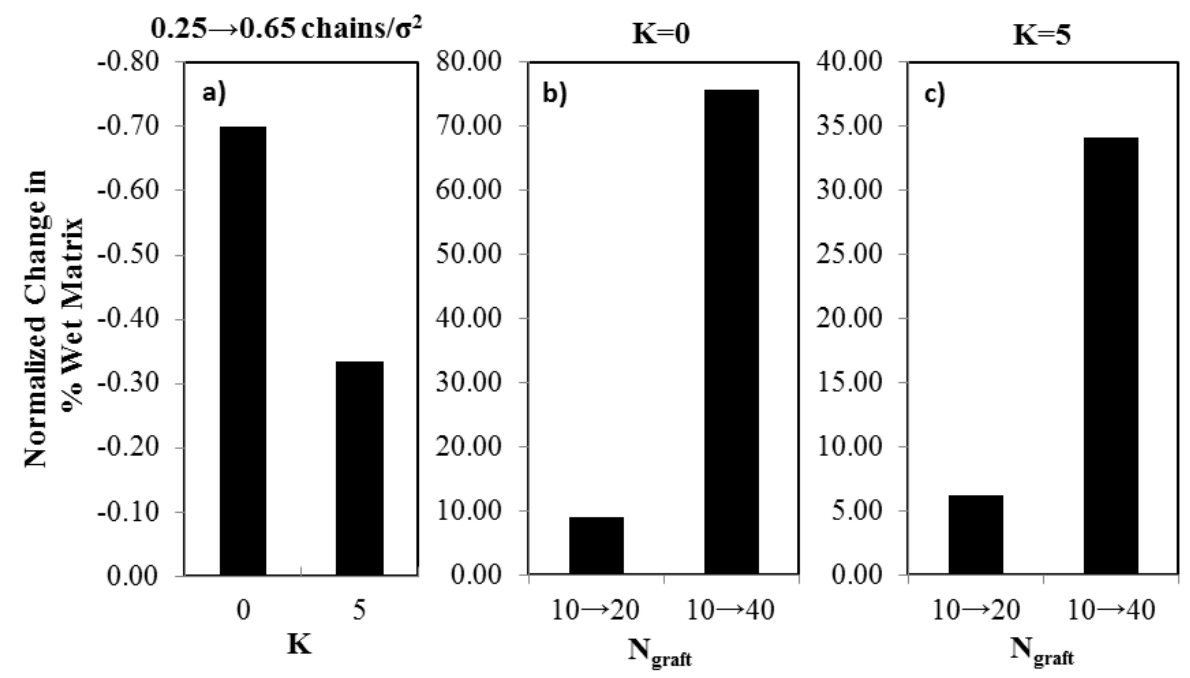

Figure 7.4: a) Normalized change in the \% wet matrix with increasing grafting density for $\left.N_{\text {graft }}=20, b\right)$ normalized change in the \% wet matrix with increasing graft length from 10 to 20 and 10 to 40 beads for flexible polymers $(K=0)$ at high grafting density $\left(0.65\right.$ chains $\left./ \sigma^{2}\right)$, and $\left.c\right)$ normalized change in the \% wet matrix with increasing graft length from 10 to 20 and 10 to 40 beads for semi-flexible polymers $(K=5)$ at high grafting density $\left(0.65\right.$ chains $\left./ \sigma^{2}\right)$, for a single PGN with $N_{\text {matrix }}=60$. The normalized change in the \% wet matrix is calculated as $(Y-X) / X$ where $Y$ is the $\%$ wet matrix at the higher grafting density or higher graft length and $X$ is the \% wet matrix at the lower grafting density or lower graft length.

For flexible graft and matrix, where the $\mathrm{N}_{\text {matrix }}>\mathrm{N}_{\text {graft, }}$ as grafting density increases, based on the $\%$ wet matrix data in Table 7.2, wetting of the grafted layer decreases, due to increased crowding in the grafted layer[50]. With increasing grafting density, the percentage change in the number of matrix beads that have wet the grafted layer, normalized by the lower grafting density, shows a drop of 0.70 for $K=0$ and only a drop of 0.33 for $K=5$ (Figure $7.4 a$ ). This suggests that the effect 
of increasing grafting density on wetting of the grafted layer is reduced for semi-flexible graft and matrix. This is because, as the grafting density increases, the change in brush height for semiflexible grafts is lower than that for flexible grafts (Supplementary Figure 7.6), also confirmed by the change in average radius of gyration of grafts with increasing grafting density being smaller for semi-flexible polymers than flexible polymers (Supplementary Table 7.4). In short, decreasing the flexibility reduces the effect of grafting density on the graft conformations, and in turn wetting behavior.

As the $N_{\text {matrix }}$ increases at constant $\mathrm{N}_{\text {graft }}$, in the case of flexible polymers, the wetting of the grafted layer by the matrix decreases. Decreasing flexibility does not alter how the wetting of the grafted layer by the matrix changes with increasing $\mathrm{N}_{\text {matrix }}$. With increasing $\mathrm{N}_{\text {matrix }}$ monomer concentration profiles in Supplementary Figure 7.6 show decreasing penetration of the matrix chains for both $\mathrm{K}=0$ and $\mathrm{K}=5$, and $\%$ wet matrix data in Table 7.2 decreases for both $\mathrm{K}=0$ and $\mathrm{K}=5$. As the $N_{\text {graft }}$ increases at constant $\mathrm{N}_{\text {matrix }}$, in the case of flexible polymers, it is known that the wetting of the grafted layer by matrix increases. With decreasing flexibility of graft and matrix, the effect of increasing $\mathrm{N}_{\text {graft }}$ on increasing wetting of the grafted layer is reduced. For flexible polymers and $\mathrm{N}_{\text {matrix }}=60$, going from graft length of 10 to 20 , the normalized change (increase) in $\%$ wet matrix is $\sim 10$ and going from 10 to 40 is $\sim 76$ (Figure $7.4 \mathrm{~b}$ ). In contrast, for semi-flexible polymers and $\mathrm{N}_{\text {matrix }}=60$, going from graft length of 10 to 20 , the normalized change (increase) in $\%$ wet matrix is $\sim 7$ and going from graft length of 10 to 40 is $\sim 35$ (Figure $7.4 \mathrm{c}$ ) 


\subsubsection{Effect of polymer flexibility on particle assembly/dispersion at finite filler fraction:}
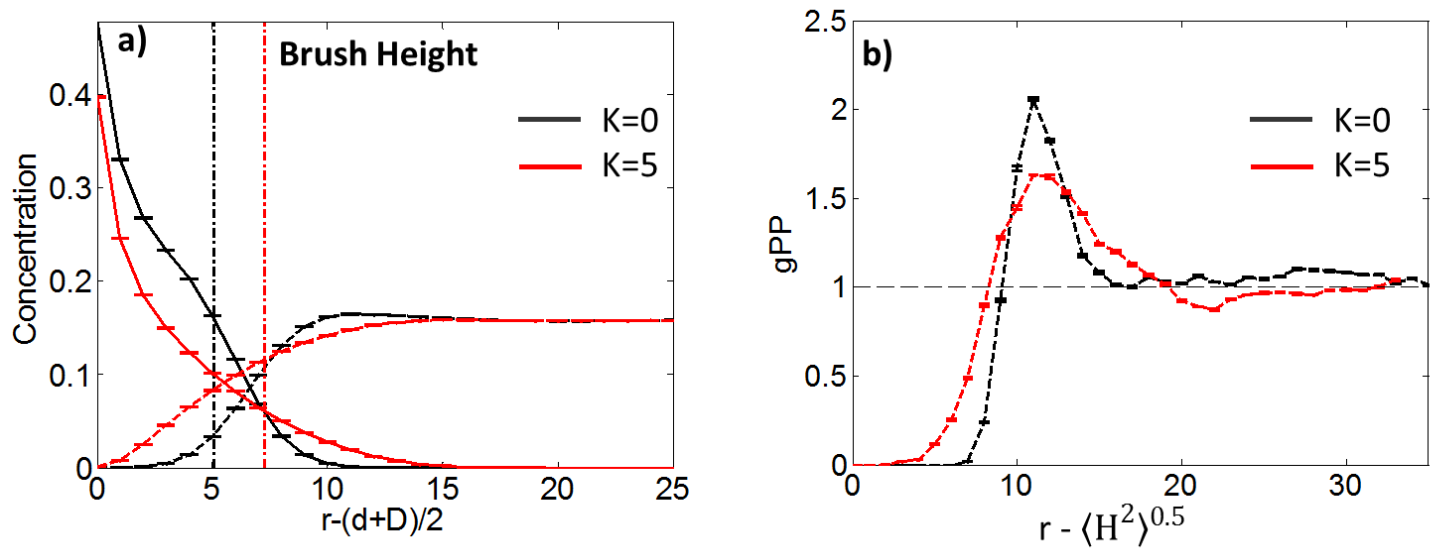

Figure 7.5: a) Graft (solid) and matrix (dashed) monomer concentration profile and b) Particleparticle pair correlation function plotted versus the interparticle distance minus the brush height for $20 P G N$ s with grafting density $=0.65$ chains $/ \sigma^{2}$, and $N_{\text {graft }}=20$ and $N_{\text {matrix }}=100$ and increasing values of $K=0$ (black) and $5(\mathrm{red})$

Figure 7.5a shows that at finite filler fractions, we continue to see the improved wetting with decreasing polymer flexibility that is seen at the dilute filler fraction or single PGN limit. Table 7.2, Supplementary Table 7.3, and Supplementary Table 7.4 also show that increasing filler fraction does not alter the graft radius of gyration and matrix end-end distance seen for single PGN for both flexible and semi-flexible systems. Since increasing wetting of the grafted layer has been connected to increased dispersion for flexible PGNs in past studies [12-14], we compare the particle-particle pair correlation for the flexible and semi-flexible graft and matrix cases. For semiflexible graft and matrix case, we see a reduced correlation at the contact peak, with the contact peak shifting to larger distances (Figure 7.5b) compared to the flexible case. This confirms that reducing flexibility in graft and matrix improves dispersion of PGNs in a chemically identical polymer matrix, due to increased wetting of the grafted layer by matrix chains. 


\subsection{CONCLUSION}

In summary, the wetting of PGNs by chemically identical matrix is strongly dependent on the flexibility of the polymer chains. Decreasing flexibility of the grafts more significantly improves the wetting of the grafted layer than decreasing flexibility of the matrix. Finite filler fraction simulations show that decreasing flexibility improves dispersion due to increasing wetting of the grafted layer. These results suggest that in PGN filled PNCs with larger persistence length polymers (graft and matrix), one would see a larger window in the phase space where the particles would be dispersed.

\subsection{ACKNOWLEDGEMENTS}

This work acknowledges financial support by Department of Energy under Grant DE-SC0003912 (AJ) and National Science Foundation GRFP Award Number DGE 1144083 (TBM), and supercomputing resources of the NERSC, supported by the Office of Science of the U.S. Department of Energy under Contract DE-AC02-05CH11231.

\subsection{REFERENCES}

1. Hall, L.M., A. Jayaraman, and K.S. Schweizer, Molecular theories of polymer nanocomposites. Current Opinion in Solid State \& Materials Science, 2010. 14(2): p. 3848.

2. Jayaraman, A. and N. Nair, Integrating PRISM theory and Monte Carlo simulation to study polymer-functionalised particles and polymer nanocomposites. Molecular Simulation, 2012. 38(8-9): p. 751-761.

3. Jayaraman, A., Polymer Grafted Nanoparticles: Effect of Chemical and Physical Heterogeneity in Polymer Grafts on Particle Assembly and Dispersion. Journal of Polymer Science Part B-Polymer Physics, 2013. 51(7): p. 524-534.

4. Ganesan, V. and A. Jayaraman, Theory and simulation studies of effective interactions, phase behavior and morphology in polymer nanocomposites. Soft Matter, 2014. 10(1): p. 13-38.

5. Krishnamoorti, R., Strategies for Dispersing Nanoparticles in Polymers. MRS Bulletin, 2007. 32(4): p. 341-347. 
6. Zhang, R. and K.S. Schweizer, Theory of nonlinear elasticity, stress-induced relaxation, and dynamic yielding in dense fluids of hard nonspherical colloids. Journal of Chemical Physics, 2012. 136(15).

7. Green, P.F., The structure of chain end-grafted nanoparticle/homopolymer nanocomposites. Soft Matter, 2011. 7(18): p. 7914-7926.

8. Goyal, S. and F.A. Escobedo, Structure and transport properties of polymer grafted nanoparticles. Journal of Chemical Physics, 2011. 135(18).

9. Akcora, P., et al., Anisotropic self-assembly of spherical polymer-grafted nanoparticles. Nature Materials, 2009. 8(4): p. 354-359.

10. Trombly, D.M. and V. Ganesan, Curvature effects upon interactions of polymer-grafted nanoparticles in chemically identical polymer matrices. Journal of Chemical Physics, 2010. 133(15).

11. Nair, N., N. Wentzel, and A. Jayaraman, Effect of bidispersity in grafted chain length on grafted chain conformations and potential of mean force between polymer grafted nanoparticles in a homopolymer matrix. Journal of Chemical Physics, 2011. 134(19).

12. Martin, T.B., P.M. Dodd, and A. Jayaraman, Polydispersity for Tuning the Potential of Mean Force between Polymer Grafted Nanoparticles in a Polymer Matrix. Physical Review Letters, 2013. 110(1).

13. Martin, T.B. and A. Jayaraman, Identifying the Ideal Characteristics of the Grafted Polymer Chain Length Distribution for Maximizing Dispersion of Polymer Grafted Nanoparticles in a Polymer Matrix. Macromolecules, 2013. 46(22): p. 9144-9150.

14. Martin, T.B. and A. Jayaraman, Polydisperse homopolymer grafts stabilize dispersions of nanoparticles in a chemically identical homopolymer matrix: an integrated theory and simulation study. Soft Matter, 2013. 9(29): p. 6876-6889.

15. Bates, F.S., et al., Multiblock Polymers: Panacea or Pandora's Box? Science, 2012. 336(6080): p. 434-440.

16. Jayaraman, A. and K.S. Schweizer, Effective Interactions and Self-Assembly of Hybrid Polymer Grafted Nanoparticles in a Homopolymer Matrix. Macromolecules, 2009. 42(21): p. 8423-8434.

17. Dijkstra, M. and D. Frenkel, SIMULATION STUDY OF THE ISOTROPIC-TO-NEMATIC TRANSITIONS OF SEMIFLEXIBLE POLYMERS. Physical Review E, 1995. 51(6): p. 5891-5898.

18. Dupre, D.B. and S.J. Yang, LIQUID-CRYSTALLINE PROPERTIES OF SOLUTIONS OF PERSISTENT POLYMER-CHAINS. Journal of Chemical Physics, 1991. 94(11): p. 74667477.

19. Fynewever, H. and A. Yethiraj, Phase behavior of semiflexible tangent hard sphere chains. Journal of Chemical Physics, 1998. 108(4): p. 1636-1644.

20. Ivanov, V.A., et al., Structures of stiff macromolecules of finite chain length near the coilglobule transition: A Monte Carlo simulation. Macromolecular Theory and Simulations, 2000. 9(8): p. 488-499. 
21. Jaffer, K.M., et al., The nematic-isotropic phase transition in semiflexible fused hardsphere chain fluids. Journal of Chemical Physics, 2001. 114(7): p. 3314-3324.

22. Kindt, J.T. and W.M. Gelbart, Chain self-assembly and phase transitions in semiflexible polymer systems. Journal of Chemical Physics, 2001. 114(3): p. 1432-1439.

23. Kong, M., et al., Systematic Coarse-Graining of the Dynamics of Self-Attractive Semiflexible Polymers. Macromolecules, 2014. 47(4): p. 1494-1502.

24. Milchev, A., PHASE-TRANSITIONS IN POLYDISPERSE POLYMER MELTS. Polymer, 1993. 34(2): p. 362-368.

25. Pedersen, J.S., M. Laso, and P. Schurtenberger, Monte Carlo study of excluded volume effects in wormlike micelles and semiflexible polymers. Physical Review E, 1996. 54(6): p. R5917-R5920.

26. Schnurr, B., F. Gittes, and F.C. MacKintosh, Metastable intermediates in the condensation of semiflexible polymers. Physical Review E, 2002. 65(6).

27. Steinhauser, M.O., Static and dynamic scaling of semiflexible polymer chains-a molecular dynamics simulation study of single chains and melts. Mechanics of Time-Dependent Materials, 2008. 12(4): p. 291-312.

28. Stukan, M.R., et al., Chain length dependence of the state diagram of a single stiff-chain macromolecule: Theory and Monte Carlo simulation. Journal of Chemical Physics, 2003. 118(7): p. 3392-3400.

29. Wiggins, P.A. and P.C. Nelson, Generalized theory of semiflexible polymers. Physical Review E, 2006. 73(3).

30. Wilhelm, J. and E. Frey, Radial distribution function of semiflexible polymers. Physical Review Letters, 1996. 77(12): p. 2581-2584.

31. Yethiraj, A. and H. Fynewever, Isotropic to nematic transition in semiflexible polymer melts. Molecular Physics, 1998. 93(5): p. 693-701.

32. Yethiraj, A. and K.S. Schweizer, INTEGRAL-EQUATION THEORY OF POLYMER BLENDS - NUMERICAL INVESTIGATION OF MOLECULAR CLOSURE APPROXIMATIONS. Journal of Chemical Physics, 1993. 98(11): p. 9080-9093.

33. Ivanov, V.A., et al., Conformational Properties of Semiflexible Chains at Nematic Ordering Transitions in Thin Films: A Monte Carlo Simulation. Macromolecules, 2014. 47(3): p. 1206-1220.

34. Morse, D.C. and G.H. Fredrickson, SEMIFLEXIBLE POLYMERS NEAR INTERFACES. Physical Review Letters, 1994. 73(24): p. 3235-3238.

35. Muralidhar, A., et al., Interplay between chain stiffness and excluded volume of semiflexible polymers confined in nanochannels. Journal of Chemical Physics, 2014. 140(8).

36. Sintes, T., K. Sumithra, and E. Straube, Adsorption of semiflexible polymers on flat, homogeneous surfaces. Macromolecules, 2001. 34(5): p. 1352-1357. 
37. Tallury, S.S. and M.A. Pasquinelli, Molecular Dynamics Simulations of Polymers with Stiff Backbones Interacting with Single-Walled Carbon Nanotubes. Journal of Physical Chemistry B, 2010. 114(29): p. 9349-9355.

38. Ganesan, V., L. Khounlavong, and V. Pryamitsyn, Equilibrium characteristics of semiflexible polymer solutions near probe particles. Physical Review E, 2008. 78(5).

39. Padmanabhan, V., A.L. Frischknecht, and M.E. Mackay, Effect of Chain Stiffness on Nanoparticle Segregation in Polymer/Nanoparticle Blends Near a Substrate. Macromolecular Theory and Simulations, 2012. 21(2): p. 98-105.

40. Xu, M., et al., Integral Equation Prediction of Surface-Induced Layering Transition of Polymer Nanocomposites. Journal of Physical Chemistry C, 2013. 117(38): p. 1940919418.

41. Zhang, D., et al., Phase separation and crystallization of binary nanoparticles induced by polymer brushes. Soft Matter, 2013. 9(6): p. 1789-1797.

42. Schnurr, B., F.C. MacKintosh, and D.R.M. Williams, Dynamical intermediates in the collapse of semiflexible polymers in poor solvents. Europhysics Letters, 2000. 51(3): p. 279-285.

43. Ceperley, D., M.H. Kalos, and J.L. Lebowitz, Computer Simulation of the Dynamics of a Single Polymer Chain. Physical Review Letters, 1978. 41(5): p. 313-316.

44. Winkler, R.G., P. Reineker, and L. Harnau, Models and equilibrium properties of stiff molecular chains. The Journal of Chemical Physics, 1994. 101(9): p. 8119-8129.

45. Cifra, P., Differences and limits in estimates of persistence length for semi-flexible macromolecules. Polymer, 2004. 45(17): p. 5995-6002.

46. Weeks, J.D., D. Chandler, and H.C. Andersen, Role of Repulsive Forces in Determining the Equilibrium Structure of Simple Liquids. The Journal of Chemical Physics, 1971. 54(12): p. 5237-5247.

47. Kalb, J., et al., End grafted polymer nanoparticles in a polymeric matrix: Effect of coverage and curvature. Soft Matter, 2011. 7(4): p. 1418-1425.

48. http://codeblue.umich.edu/hoomd-blue/. HOOMD-Blue webpage. Available from: http://codeblue.umich.edu/hoomd-blue/ (2010).

49. Hansen, J.-P. and I.R. McDonald, Theory of Simple Liquids

3rd ed, ed. Elsevier. 2006.

50. Frischknecht, A.L. and A. Yethiraj, Two- and three-body interactions among nanoparticles in a polymer melt. The Journal of Chemical Physics, 2011. 134(17): p. 174901-11. 


\subsection{SUPPLEMENTARY}

\subsubsection{Effect of polymer flexibility on wetting of the grafted polymer layer by matrix polymers:}

Table 7.3: Average end-end distance of the matrix polymers for varying matrix and graft lengths, grafting densities, and flexibilities. The graft and matrix end-to-end distances are defined as the average over all of the graft and matrix chains respectively. The "wet matrix" end-to-end distance is defined as the average over any chain that has any bead within the grafted layer "brush" height, $\left\langle H_{B}{ }^{2}\right\rangle^{0.5}$, and the "unwet matrix" end-to-end distance is the average over the remainder of the chains. The standard deviation of the independent snapshots is shown.

\begin{tabular}{|c|c|c|c|c|c|c|c|c|c|c|c|c|c|}
\hline $\mathrm{n}_{\mathrm{p}}$ & $K_{\text {graf }}$ & $\mathrm{K}_{\text {matrix }}$ & $\begin{array}{l}\text { Grafting } \\
\text { Density } \\
\left(\text { chains } / \sigma^{2}\right)\end{array}$ & $\mathrm{N}_{\text {graf }}$ & $\mathrm{N}_{\text {matrix }}$ & $\begin{array}{l}\text { Graft } \\
<\mathrm{R}_{\text {end-end }}^{2}>^{0.5} \\
(\text { units of } \sigma \text { ) }\end{array}$ & $\begin{array}{c}\text { Stdev Graft } \\
<\mathrm{R}_{\text {end-end }}{ }^{2}>0.5 \\
\text { (units of } \sigma \text { ) }\end{array}$ & $\begin{array}{c}\text { Matrix } \\
<\mathrm{R}_{\text {end-end }}^{2}>^{0.5} \\
(\text { units of } \sigma)\end{array}$ & $\begin{array}{l}\text { Stdev Matrix } \\
<\mathrm{R}_{\text {end-end }}{ }^{2}>^{0.5} \\
\text { (units of } \sigma \text { ) }\end{array}$ & $\begin{array}{l}\text { Wet Matrix } \\
<\mathrm{R}_{\text {end-end }}{ }^{2}>0.5 \\
(\text { units of } \sigma \text { ) }\end{array}$ & $\begin{array}{c}\text { Stdev Wet } \\
\text { Matrix } \\
<\mathrm{R}_{\text {end-end }}^{2}>^{0.5} \\
\text { (units of } \sigma \text { ) }\end{array}$ & $\begin{array}{c}\text { Unwet } \\
\text { Matrix } \\
<\mathrm{R}_{\text {end-end }}^{2}>^{0.5} \\
\text { (units of } \sigma \text { ) }\end{array}$ & $\begin{array}{c}\text { Stdev Unwet } \\
\text { Matrix } \\
5<\mathrm{R}_{\text {end-end }}^{2}>^{0.5} \\
\text { (units of } \sigma \text { ) }\end{array}$ \\
\hline 1 & 0 & 0 & 0.65 & 20 & 60 & 8.3 & 1.9 & 12.6 & 4.5 & 12.9 & 4.3 & 12.6 & 4.5 \\
\hline 1 & 0 & 5 & 0.65 & 20 & 60 & 8.2 & 1.9 & 24.3 & 7.9 & 24.5 & 8 & 24.3 & 8.3 \\
\hline 1 & 5 & 0 & 0.65 & 20 & 60 & 12.8 & 2.7 & 12.6 & 4.5 & 13 & 4.7 & 12.6 & 4.5 \\
\hline 1 & 5 & 5 & 0.65 & 20 & 60 & 12.9 & 2.6 & 24.3 & 7.9 & 24.9 & 8.1 & 24.2 & 8.3 \\
\hline 1 & 10 & 10 & 0.65 & 20 & 60 & 15.2 & 2.2 & 32.5 & 7.6 & 32.8 & 9.1 & 32.4 & 9.2 \\
\hline 1 & 0 & 0 & 0.25 & 20 & 60 & 7.3 & 2 & 12.6 & 4.5 & 12.9 & 4.7 & 12.6 & 4.5 \\
\hline 1 & 5 & 5 & 0.25 & 20 & 60 & 12.6 & 2.8 & 24.2 & 7.9 & 24.9 & 8.2 & 24.2 & 8.3 \\
\hline 1 & 0 & 0 & 0.65 & 20 & 20 & 8.3 & 1.9 & 6.7 & 2.1 & 6.9 & 2.1 & 6.7 & 2.1 \\
\hline
\end{tabular}




\begin{tabular}{|c|c|c|c|c|c|c|c|c|c|c|c|c|c|}
\hline 1 & 5 & 5 & 0.65 & 20 & 20 & 12.9 & 2.7 & 12.4 & 2.9 & 12.5 & 2.8 & 12.4 & 2.9 \\
\hline 1 & 0 & 0 & 0.65 & 10 & 60 & 5.1 & 1.1 & 12.6 & 4.5 & 13 & 4.5 & 12.6 & 4.5 \\
\hline 1 & 5 & 5 & 0.65 & 10 & 60 & 7.4 & 1 & 24.2 & 7.9 & 24.8 & 8.4 & 24.2 & 8.3 \\
\hline 1 & 0 & 0 & 0.65 & 40 & 60 & 12.6 & 3.2 & 12.6 & 4.5 & 13 & 4.6 & 12.6 & 4.5 \\
\hline 1 & 5 & 5 & 0.65 & 40 & 60 & 20.3 & 5.6 & 24.2 & 7.9 & 24.9 & 8.2 & 24.1 & 8.3 \\
\hline 1 & 0 & 0 & 0.65 & 20 & 40 & 8.3 & 1.9 & 10 & 3.5 & 10.2 & 3.4 & 10 & 3.5 \\
\hline 1 & 5 & 5 & 0.65 & 20 & 40 & 12.9 & 2.7 & 19.2 & 6 & 19.7 & 5.9 & 19.2 & 6 \\
\hline 1 & 0 & 0 & 0.65 & 20 & 100 & 8.2 & 1.9 & 16.7 & 6.1 & 17.2 & 6 & 16.7 & 6.1 \\
\hline 1 & 5 & 5 & 0.65 & 20 & 100 & 12.8 & 2.7 & 32 & 8.2 & 32.4 & 11.8 & 32 & 11.8 \\
\hline 20 & 0 & 0 & 0.65 & 20 & 100 & 8.3 & 1.9 & 16.6 & 6.1 & 17.1 & 6.2 & 16.6 & 6.1 \\
\hline 20 & 5 & 5 & 0.65 & 20 & 100 & 12.9 & 2.7 & 31.9 & 11.8 & 32.6 & 11.8 & 31.3 & 11.7 \\
\hline
\end{tabular}


Table 7.4: Average radii of gyration of the graft chains. The standard deviation for all values is $<0.1 \sigma$

\begin{tabular}{lllllll}
\hline $\mathrm{n}_{\mathrm{p}}$ & $\mathrm{K}_{\text {graft }}$ & $\mathrm{K}_{\text {matrix }}$ & $\begin{array}{l}\text { Grafting } \\
\text { Density } \\
\text { (chains/ } \sigma^{2} \text { ) }\end{array}$ & $\mathrm{N}_{\text {graft }}$ & $\mathrm{N}_{\text {matrix }}$ & $\begin{array}{l}\left\langle\mathrm{Rg}^{2}\right\rangle^{1 / 2} \\
(\sigma)\end{array}$ \\
\hline 1 & 0 & 0 & 0.65 & 20 & 60 & 3.0 \\
1 & 0 & 5 & 0.65 & 20 & 60 & 2.9 \\
1 & 5 & 0 & 0.65 & 20 & 60 & 4.5 \\
1 & 5 & 5 & 0.65 & 20 & 60 & 4.5 \\
1 & 10 & 10 & 0.65 & 20 & 60 & 5.0 \\
1 & 0 & 0 & 0.25 & 20 & 60 & 2.8 \\
1 & 5 & 5 & 0.25 & 20 & 60 & 4.4 \\
1 & 0 & 0 & 0.65 & 20 & 20 & 3.0 \\
1 & 5 & 5 & 0.65 & 20 & 20 & 4.5 \\
1 & 0 & 0 & 0.65 & 10 & 60 & 1.9 \\
1 & 5 & 5 & 0.65 & 10 & 60 & 2.6 \\
1 & 0 & 0 & 0.65 & 40 & 60 & 4.4 \\
1 & 5 & 5 & 0.65 & 40 & 60 & 7.4 \\
1 & 0 & 0 & 0.65 & 20 & 40 & 3.0 \\
1 & 5 & 5 & 0.65 & 20 & 40 & 4.5 \\
1 & 0 & 0 & 0.65 & 20 & 100 & 2.9 \\
1 & 5 & 5 & 0.65 & 20 & 100 & 4.5 \\
20 & 0 & 0 & 0.65 & 20 & 100 & 3.0 \\
20 & 5 & 5 & 0.65 & 20 & 100 & 4.5 \\
\hline & & & & & &
\end{tabular}


7.7.2 Effect of polymer flexibility on trends of varying grafting density and varying matrix and graft polymer length on wetting/dewetting:
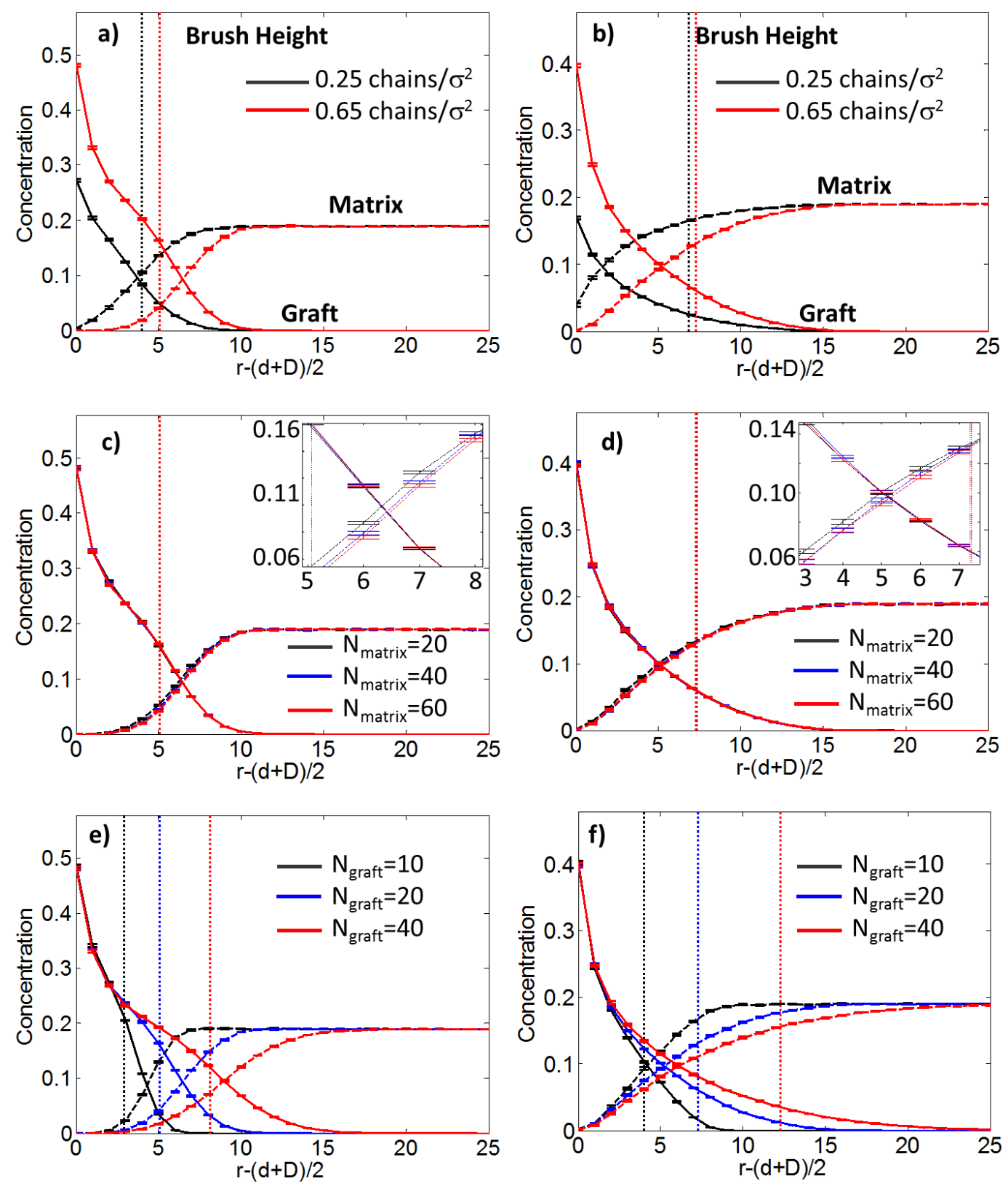

Figure 7.6: Graft (solid) and matrix (dashed) monomer concentration profile for single polymer grafted particle with particle diameter $5 \mathrm{~nm}$ for $K_{\text {graft }}=K_{\text {matrix }}=0$ (left column) and $K_{\text {graft }}=K_{\text {matrix }}$ $=5$ (right column) for varying polymer grafting density $=0.25$ (black) and 0.65 (red) chains $/ \sigma^{2}$ with $N_{\text {graft }}=20$ and $N_{\text {matrix }}=60$ (in subplots $a$ and $b$ ), varying matrix length $=20$ (black), 40 (blue) and 60 (red) with $N_{\text {graft }}=20$ and grafting density 0.65 chains $/ \sigma^{2}$ (in subplots $c$ and $d$ ) and varying graft length $=10$ (black) and 20 (red) with $N_{\text {matrix }}=60$ and grafting density 0.65 chains $/ \sigma^{2}$ (in subplots e 
and f). The brush heights, $\left\langle H_{B}{ }^{2}\right\rangle^{0.5}$, are shown with dotted lines. The insets in subplots $\left.c\right)$ and d) have the same axes as the main figure.

For flexible graft and matrix polymers, where the matrix length is greater than graft length, as grafting density increases, wetting of the grafted layer decreases due to increased crowding in the grafted layer[50]. As the flexibility of graft and matrix polymer chains is reduced, we see that the effect of changing the grafting density on grafted layer wetting seems qualitatively unaltered, as seen in Figure 7.6a and Figure 7.6b. However, the percent change in the number of matrix beads that have wet the grafted layer with increasing grafting density, normalized by the lower grafting density, shows a $70 \%$ drop for $\mathrm{K}=0$ and a $33 \%$ drop for $\mathrm{K}=5$. This suggests that the effect of increasing grafting density on wetting of the grafted layer is reduced with decreasing flexibility. We justify this trend as follows: For flexible grafted polymers at low grafting densities, the brush height is small because the grafted chains adopt mushroom conformations on the particle. As the grafting density increases, the grafted chains adopt extended conformations and the brush height increases. In contrast, for grafted polymers with reduced flexibility, at low grafting densities, the grafted chain conformations are extended to some degree, resulting in a larger brush height than the corresponding flexible grafted polymer, and therefore significantly improved wetting. As the grafting density increases, the change in brush height for grafted chains with reduced flexibility (Figure 7.6b) is lower than that for flexible grafted chains (Figure 7.6a). This is also confirmed by the change in average radius of gyration of grafted polymer chains with increasing grafting density being smaller for semiflexible polymers than flexible polymers (Supplementary Table 7.4). In short, decreasing the flexibility reduces the effect of grafting density on the graft conformations, brush height, and wetting behavior.

In the case of flexible polymers, it is known that as the matrix polymer chain length increases at constant graft length, the wetting of the grafted layer by the matrix chains decreases. 
In Figure 7.6c and Figure 7.6d, despite the different shapes of the graft and matrix monomer concentration profiles with decreasing flexibility, we see that decreasing flexibility does not alter how the wetting of the grafted layer by the matrix chains changes with increasing matrix chain length, both visually as well as quantitatively (using data in Table 7.2 of main manuscript). Our reasoning for this is as follow. In the flexible polymers case, the loss in conformational entropy of the matrix chain upon wetting the grafted layer is expected to increase slightly as the matrix polymer chain length increases (at constant graft length). Even though we know that as the flexibility decreases the matrix polymer conformational entropy decreases, both in the unwet and wet states, the trend of increasing loss in conformational entropy of the matrix chain upon wetting with increasing matrix length is likely the same as that in the completely flexible case.

In the case of flexible polymers, it is known that as the graft polymer length increases, at constant matrix length, the wetting of the grafted layer by matrix chains increases. While this trend is true for semi-flexible polymers as well (Figure 7.6e and Figure 7.6f) we find quantitative differences between flexible and semi-flexible cases. Using the data in Table 7.2 of main manuscript, for semi-flexible polymers and $\mathrm{N}_{\text {matrix }}=60$, going from graft length of 10 to 20 , the wetting increases approximately 7 times and going from graft length of 10 to 40 the wetting increases about 35 times. In contrast for flexible polymers, going from graft length of 10 to 20 , the wetting increases 10 times and going from 10 to 40 the wetting increases 77 times. This suggests that with decreasing flexibility of graft and matrix polymers, the effect of increased graft length on wetting of the grafted layer is reduced. This is likely because the wetting of the grafted layer by the matrix chain is large even at short graft length, for semi-flexible polymers (esp. as we approach chains length close to persistence lengths), that increasing the graft length does not change the wetting as much as it does for flexible polymers. 


\section{Wetting-DeWetting AND DISPERSION-AggREgation TRANSITIONS ARE Distinct FOR POlYMER GRAFTEd NANOPARTICleS IN Chemically DISSIMILAR POLYMER MATRIX}

Adapted from: Journal of the American Chemical Society 137 (33), 10624-10631

\subsection{INTRODUCTION}

Driven by the need to develop functionally superior materials, significant effort has been directed towards the understanding of structure and thermodynamics of polymer blends [1-5] and polymernanoparticle mixtures/blends [6-11]. Fundamentally, the delicate balance of enthalpic and entropic driving forces, arising from the interplay of polymer and particle chemistry, polymer molecular weight, architecture, particle size, and the blend composition dictates the phase transition from mixed (dispersed) to demixed (aggregated) states. A large body of scientific work [10, 12-22] has focused on the development of polymer nanocomposites wherein the particles are grafted with polymers in order to maximize the particle dispersability in a chemically similar matrix polymer. Through these numerous studies that are described in the review articles [12, 23-26], it is now well understood that the extent of matrix polymer penetration (exclusion) into the grafted polymer layer, also termed as "wetting" ("dewetting") dictates extent of dispersion (aggregation) of polymer grafted particles in the free matrix polymer. Experiments, theory, and simulations have shown ways to tune this extent of wetting by varying grafting density (mushroom to brush regimes), ratio of matrix to graft molecular weights, and particle size or curvature. Especially in the dense grafting regime, where the direct particle-particle interactions are screened [27], the chemical similarity of the graft and matrix reduces the thermodynamic driving forces for wettingdewetting to being purely entropic. While the gain in entropy of mixing drives wetting of the grafted polymer by the matrix chains, the conformational entropy loss due to matrix penetrating a 
dense grafted layer drives dewetting. Decreasing ratio of matrix to graft molecular weights [28], increasing graft molecular weight dispersity [27, 29-32], and decreasing polymer flexibility[33] have been shown to increase the wetting and, in turn, stabilize particle dispersion.

While the wetting-dewetting and dispersion-aggregation phase behavior of chemically identical dense brush graft-matrix blends is largely governed by these entropic driving forces, there is a non-trivial competition between the enthalpic and entropic driving forces for chemically dissimilar graft-matrix composites. Theoretical studies of flat surfaces or colloids grafted with a dense polymer brush placed in a chemically dissimilar polymer matrix have predicted how the choice of chemically dissimilar graft and matrix polymers with negative or positive Flory-Huggins $\chi$ parameter (quantifying the enthalpic interactions between the blend components) impacts the graft conformations and the wetting-dewetting. [34-38] Using highly-controlled synthesis and characterization techniques, a few experiments have also shown that particle dispersion and aggregation is tuned by enthalpic interactions between grafted and matrix chains. [38-41] Especially in the case where there are attractive enthalpic interactions between the graft and matrix chains, the mixture is driven to a dispersed phase even with grafts that are shorter than matrix chains, a case where chemically identical systems would exhibit dewetting and particle aggregation.[39] This ability to disperse particles due to graft-matrix miscibility allows for higher filler loadings than athermal chemically identical graft-matrix composites.[39] Choosing graftmatrix polymers with specific H-bonding also enables thermoreversibility to particle dispersionaggregation. [41]

In the above studies, the wetting-dewetting transition and dispersion-aggregation transition have been assumed to occur simultaneously, and are treated synonymously. [9, 10, 12, 20, 22, 2426, 42-44]. In contrast, in this paper, using simulations and experiments, we show that in 
chemically dissimilar graft-matrix polymer nanocomposites, the wetting-dewetting transition occurs gradually with increasing temperature and is distinct from the sharp dispersion-aggregation (phase separation) transition. The coarse-grained molecular dynamics (CGMD) simulations focus on generic chemically dissimilar graft-matrix polymer pairs that exhibit a lower critical solution temperature (LCST) phase behavior with increasing temperature. The experiments focus on deuterated polystyrene (dPS) grafted silica particles in a poly(vinyl-methyl-ether) (PVME) matrix as dPS-PVME blends have been shown to exhibit miscibility at room temperature, and a LCST phase behavior with increasing temperatures. [45] Through a comprehensive characterization of the composite using $\mathrm{x}$-ray scattering and neutron scattering experiments, along with morphology and chain conformations from CGMD simulations, we provide the molecular mechanism underlying the gradual wetting to dewetting transition being separate from the sharp dispersionto-aggregation transition.

\subsection{COMPUtATIONAL APPROACH}

\subsubsection{Model}

We model polymer grafted spherical nanoparticles in a polymer matrix using a generic coarsegrained model, where the nanoparticles (denoted as $\mathrm{P}$ ) are modeled as a rigid-body of several $\mathrm{D}=$ 1d beads $(\mathrm{d} \approx 1 \mathrm{~nm})$, and the polymers as bead-spring chains. The nanoparticle consists of surface beads to preserve the excluded volume of the particle and grafting sites to anchor the grafted chains. The surface and grafting site beads overlap in the rigid body of the particle, with the grafting site beads isotropically located in the spherical particle surface. Each grafted $(\mathrm{G})$ or matrix

(M) polymer is modeled as a fully-flexible bead-spring chain[46], with each bead of size d representing a group of monomers on the polymer chain, and harmonic springs linking the beads 
having a force constant of $\mathrm{k}=50 \mathrm{k} \mathrm{B} / \mathrm{d}^{2}$ and a bond rest length of $\mathrm{r}_{0}=1 \mathrm{~d}$, mathematically represented as

$$
U_{\text {bond }}(r)=\frac{1}{2} k\left(r-r_{0}\right)^{2}
$$

where $r$ is the center to center distance between the bonded beads.

The monomer-monomer interactions are modeled using a Lennard-Jones potential:

$$
U_{L J}(r)=4 \varepsilon\left[\left(\frac{\sigma}{r}\right)^{12}-\left(\frac{\sigma}{r}\right)^{6}\right]
$$

where $\varepsilon$ is the well depth, $\sigma$ is the contact distance, and $\mathrm{r}$ is the center-center distance of the interacting beads. All particle-particle and particle-monomer interactions are athermal via a purely-repulsive Weeks-Chandler-Andersen potential with $\varepsilon=1.0 k_{B} T$ :

$$
U_{W C A}(r)=\left\{\begin{array}{c}
4 \varepsilon\left[\left(\frac{\sigma}{r}\right)^{12}-\left(\frac{\sigma}{r}\right)^{6}\right]+\varepsilon, \quad r<2^{\frac{1}{6} \sigma} \\
0, \quad r \geq 2^{\frac{1}{6} \sigma}
\end{array}\right.
$$

In this study the nanoparticle size is maintained at $\mathrm{D}=5 \mathrm{~d}$, grafting density at $\Sigma=0.76$ chains $/ \mathrm{d}^{2}$, and the graft and matrix lengths are kept at $\mathrm{N}_{\text {graft }}=10$ and $\mathrm{N}_{\text {matrix }}=50$ respectively. The filler fraction is varied over $\phi_{G}=0.05-0.20$ (main paper focuses on 0.13 and 0.20 ), where $\phi_{1}$ is defined as

$$
\phi_{G}=\frac{V_{\text {graft }}}{V_{\text {graft }}+V_{\text {matrix }}}
$$

where $V_{X}$ is the total volume occupied by component $\mathrm{X}$ in the system. The form of the filler fraction is chosen to be able to easily compare simulations of grafted particle in a matrix to simulations of blends of free "graft" and matrix polymers. For all simulations, the total occupied volume fraction in the simulation box is maintained to be $\eta=0.35$, and we maintain 60,000 matrix beads to enforce a minimum box size of $\approx 44 \mathrm{~d} x 44 \mathrm{~d} \times 44 \mathrm{~d}$ regardless of our chosen filler fraction. 
The graft-graft and matrix-matrix interactions are maintained constant with $\varepsilon_{G G}=\varepsilon_{M M}=$ $0.5 k_{B} T$, while the graft-matrix interactions are varied to simulate varying reduced temperature $T^{*}=1 / \varepsilon_{G M}^{*}=1.25-3.33$.

\subsubsection{Method}

Using the model described above, we conduct Brownian dynamics (BD) simulations in the canonical ensemble using the graphical processing unit based HOOMD-blue package [47, 48]. We first create an initial configuration in the following manner: We generate a particle of a desired diameter with isotropically distributed graft points, with the chains extending radially from these graft points embedded on the particle surface. In order to make it easier to insert the grafted particle into the simulation box, a short simulation with strong Lennard-Jones monomer-monomer and monomer-particle attraction is then run to compress the grafted chains from these extended conformations. Copies of this one compressed grafted nanoparticle are then randomly placed in a large cubic box to achieve the desired filler fraction along with the desired number of matrix chains. At an integrator temperature of $\mathrm{T}_{\text {inital }}=5.0$, this initial configuration is then integrated using a Brownian dynamics integrator for $5 \mathrm{e} 6$ time steps to both mix and relax the grafted and matrix chains. The box is then compressed to the desired volume fraction over $10 \mathrm{e} 6$ steps, and then mixed again for $15 \mathrm{e} 6$ steps at the compressed state. We then vary the integrator temperature $\mathrm{T}$ from $\mathrm{T}_{\text {inital }}$ $=5.0$ to $\mathrm{T}_{\text {final }}=1.0 \mathrm{using}$ ten, geometrically sized quenches over a period of $50 \mathrm{e} 6$ time steps. After the annealing is completed, we run the simulations for an additional 50e6 time steps where we sample the equilibrium configurations of the system every $0.5 \mathrm{e} 6$ time steps to calculate the ensemble averages of various thermodynamic and structural properties. The starting temperature, ending temperature, number of quenches, quench temperatures, and number of time steps of each stage of the protocol are chosen after rigorously testing many protocols, and finding that this 
protocol is the most computationally efficient that produced statistically similar equilibrium states from different initial conditions. Our simulation protocol is visually depicted in Figure 8.1.

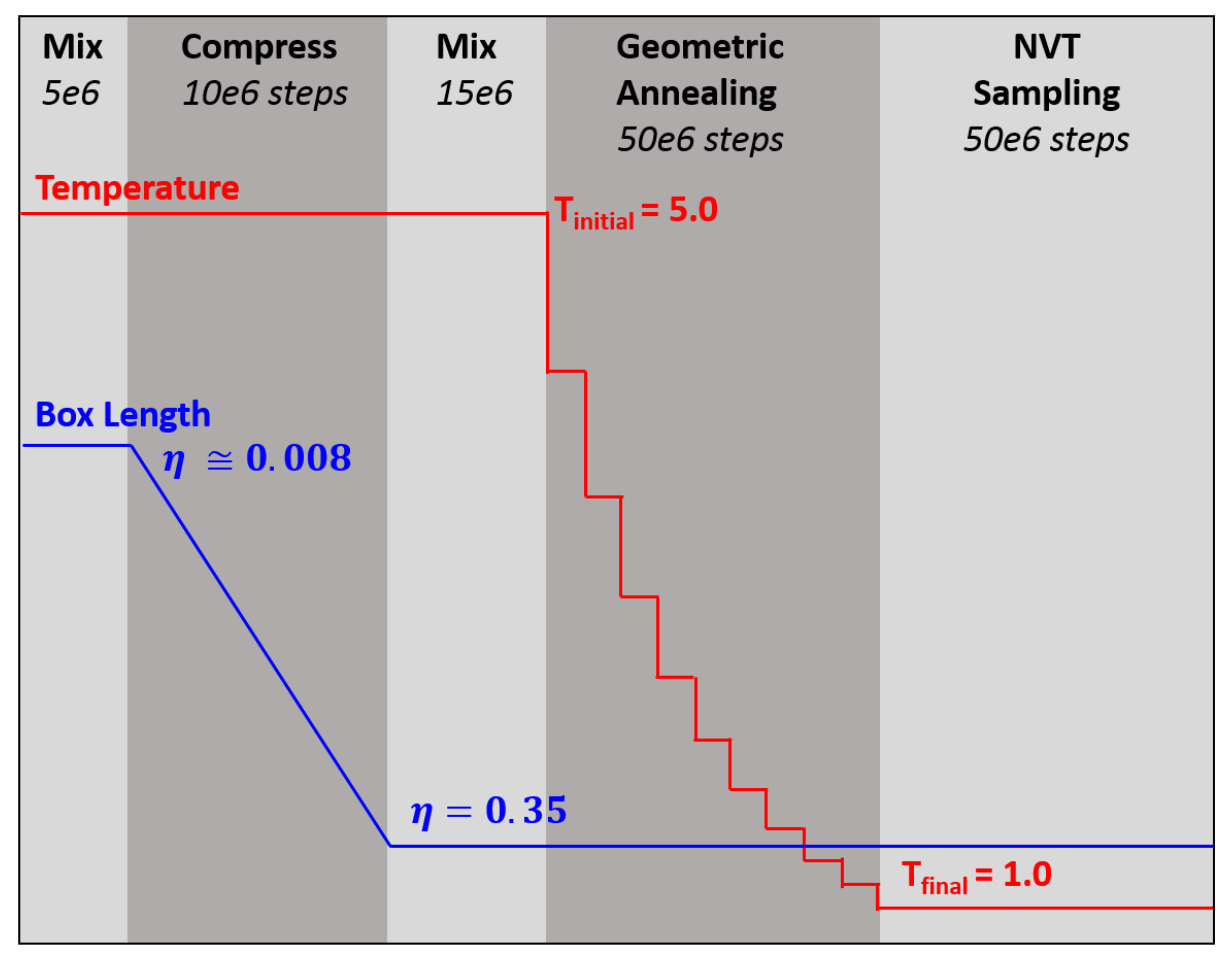

Simulation Time

Figure 8.1: Schematic representation of the equilibration protocol used in the Brownian Dynamics simulations

\subsubsection{Analysis}

We quantify monomer concentration profiles of the graft and matrix chains from the particle surface as follows:

$$
C_{P X}(r)=\frac{\left\langle n_{X}(r)\right\rangle}{4 \pi r^{2} \Delta r}
$$

where $\mathrm{C}_{\mathrm{PX}}(\mathrm{r})(\mathrm{X}=\mathrm{G}$ or $\mathrm{M})$ is the particle-graft or particle-matrix monomer concentration profile, in units of $\mathrm{d}^{(-3)}$, as a function of $\mathrm{r}$, the distance between the particle surface and the monomer, and $\left\langle\mathrm{n}_{\mathrm{X}}(\mathrm{r})\right\rangle$ is the average number of monomers of type $\mathrm{X}$ that are within a shell of thickness $\Delta \mathrm{r}$ at distance $r$. 
The average radius of gyration of the graft or matrix chains quantifies the size of the chain conformations averaged over all of the graft or matrix chains in the system.

$$
\left\langle R_{g}^{2}\right\rangle=\left(\frac{1}{n_{X C} * N_{X}}\right) \sum_{i=1}^{n_{X C}} \sum_{j=1}^{N_{X}}\left(r_{i, j}-r_{i, c o m}\right)^{2}
$$

where $\left\langle\mathrm{R}_{\mathrm{g}}{ }^{2}\right\rangle$ is the average squared radius of gyration, in units of $\mathrm{d}^{2}, \mathrm{n}_{\mathrm{XC}}$ is the number of graft or matrix chains in the system(across all particles), $\mathrm{N}_{\mathrm{X}}$ is the length of the graft or matrix chains, $\mathrm{r}_{\mathrm{i}, \mathrm{j}}$ is the position of monomer $\mathrm{j}$ on chain $\mathrm{i}$, and $\mathrm{r}_{\mathrm{i}, \mathrm{com}}$ is the center of mass of chain $\mathrm{i}$.

The wet matrix fraction quantifies the degree of wetting of the grafted layer by matrix monomers:

$$
\text { wet matrix fraction }=\frac{n_{M, w e t}}{n_{M}}
$$

where $n_{M}$ is the total number of matrix monomers in the system and $n_{M}$,wet is the number of matrix monomers that are wetting a grafted layer. We find $\mathrm{n}_{\mathrm{M}}$, wet by first calculating the grafted-monomer count field throughout the simulation box, then finding the spatial locations of the isosurfaces corresponding to a monomer count of 0.01 , and then counting the number of matrix monomers that are within this closed isosurface. For this study, we found that an isosurface corresponding to an average monomer count of 0.01 best captures the outer edge of the grafted layer of isolated particles or clusters of particles.

We calculate the particle-particle, graft-graft, and matrix-matrix pair correlation functions, $\left(g_{P P}(r) g_{G G}(r)\right.$, and $\left.g_{M M}(r)\right)$, to quantify the spatial correlation between these various species[49]. In order to better assess the degree of phase separation between the components of our simulation, we also invert the pair correlation functions to structure factors via:

$$
S_{X X}(k)=1+\frac{4 \pi \rho_{X}}{k} \int r \cdot\left(g_{X X}(r)-1\right) \cdot \sin (k r) d r
$$


where $\mathrm{S}_{\mathrm{XX}}(\mathrm{k})$ is the value of the structure factor for pair XX (PP, MM, or GG) at wavenumber k, $\rho_{X}$ is the number density of beads of type $\mathrm{X}$, and $\mathrm{gXX}_{\mathrm{XX}}(\mathrm{r})$ is the pair correlation function for pair $\mathrm{XX}$ at a real-space distance $\mathrm{r}$.

\subsection{EXPERIMENTAL APPROACH}

\subsubsection{Experimental preparation of polymer-grafted nanoparticles}

Initiator-functionalized silica nanoparticles $\left(\mathrm{SiO}_{2}\right.$-initiator) were prepared according to the procedure in Ref. [50] (see details in Supplementary Section 8.8.2). Deuterated polystyrenegrafted $\mathrm{SiO}_{2}$ nanoparticles $\left(\mathrm{SiO}_{2}-\mathrm{dPS}\right)$ were then synthesized using a modified AGET ATRP reaction [51] where briefly, the $\mathrm{Cu}(\mathrm{II}) \mathrm{Br}$ catalyst and dNbpy ligand were combined with d-styrene in a flask and bubbled with nitrogen for 30 minutes. In another flask, the $\mathrm{Sn}(\mathrm{EH})_{2}$ reducing agent and $\mathrm{SiO}_{2}$-initiator were dissolved in toluene, purged with nitrogen and transferred to the first flask. The reaction mixture was then placed in an oil bath at $90^{\circ} \mathrm{C}$ and allowed to proceed for $\sim 40$ hours. After polymerization, the reaction mixture was further diluted with THF, filtered through a column of neutral aluminum oxide to remove the catalyst, concentrated in vacuo and precipitated in excess amount of cold methanol. The recovered polymer-grafted nanoparticles were further purified through a mixed solvent precipitation method using toluene and methanol. This step ensured the removal of unfunctionalized silica nanoparticles as well as free ungrafted chains from the grafted hybrid sample (see Supplementary Section 8.8.2). Prior to gel permeation chromatography (GPC) experiments to determine the polymer brush molecular weight, the grafted polymers were cleaved from the $\mathrm{SiO}_{2}$ surface by first dissolving the hybrid nanoparticles in $2 \mathrm{ml}$ THF and then adding 2 $\mathrm{ml}$ of a $2 \%(\mathrm{v} / \mathrm{v})$ solution of aqueous HF. After stirring the solution overnight, the polymer was precipitated in excess amount of methanol and dried under vacuum for at least 24 hours. GPC measurements gave a brush molecular weight $\left(\mathrm{M}_{\mathrm{w}}\right)$ of $33,000 \mathrm{~g} / \mathrm{mol}$ and polydispersity index 
$\left(\mathrm{M}_{\mathrm{w}} / \mathrm{M}_{\mathrm{n}}\right)$ of 1.27 . The grafted nanoparticles $\left(\mathrm{SiO}_{2}-\mathrm{dPS} 33 \mathrm{k}\right)$ were also subjected to thermogravimetric analysis to calculate the polymer grafting density, determined to be $\approx 0.7 \mathrm{chain} / \mathrm{nm}^{2}$.

\subsubsection{Experimental preparation of nanocomposites}

The nanocomposite was prepared following a simple solution mixing procedure. Initially, poly(vinyl methyl ether) (PVME) $\left(\mathrm{M}_{\mathrm{w}}=226,000 \mathrm{~g} / \mathrm{mol}, \mathrm{PVME} 226 \mathrm{k}\right)$ was dried at $70^{\circ} \mathrm{C}$ under vacuum overnight and cooled down to room temperature prior to use. After which, a 20:80 (by weight) blend composition of $\mathrm{SiO}_{2}-\mathrm{dPS} 33 \mathrm{k} / \mathrm{PVME} 226 \mathrm{k}$ was prepared by co-dissolving predetermined amounts of $\mathrm{SiO}_{2}-\mathrm{dPS} 33 \mathrm{k}$ and PVME226k in toluene and mixing at room temperature for at least 24 hours. The polymer blend was obtained by precipitating the solution in a large excess of hexane and collecting the solid sample by vacuum filtration. The resulting composite was allowed to dry in air for 2 days and annealed at $60^{\circ} \mathrm{C}$ under vacuum for at least 24 hours before the scattering measurements were performed. The samples were heated from room temperature to elevated temperature for the scattering measurements. In some cases the samples were also studied in cooling and we found reproducible data between heating and cooling as long as the temperature did not exceed $145{ }^{\circ} \mathrm{C}$. Additional details of the experimental methods can be found in the Supplementary Section 8.8.2.

\subsection{RESULTS AND DISCUSSION}

Coarse-grained (CG) molecular dynamics (MD) simulations of homopolymer grafted particles in a chemically distinct homopolymer matrix were conducted for a blend with $\mathrm{N}_{\text {matrix }}=50 \mathrm{Kuhn}$ segments (or effective "monomers"), $\mathrm{N}_{\text {graft }}=10$ Kuhn segments, particle size $\mathrm{D}=5 \mathrm{~d}$, where $\mathrm{d}$ is the size of the Kuhn segment, at grafting density of 0.76 chains $/ \mathrm{d}^{2}$ for two different blend/mixture compositions. 
a)

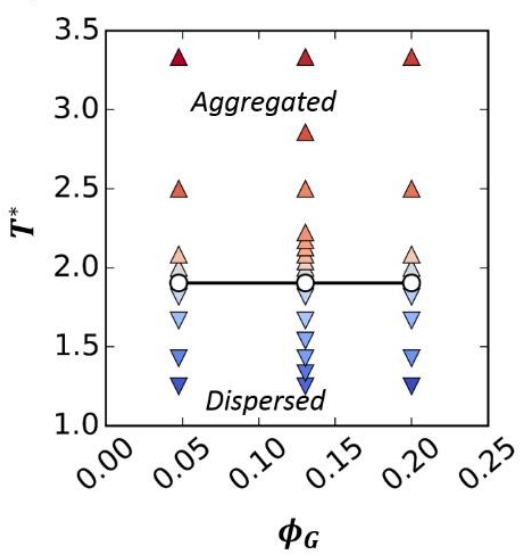

b)

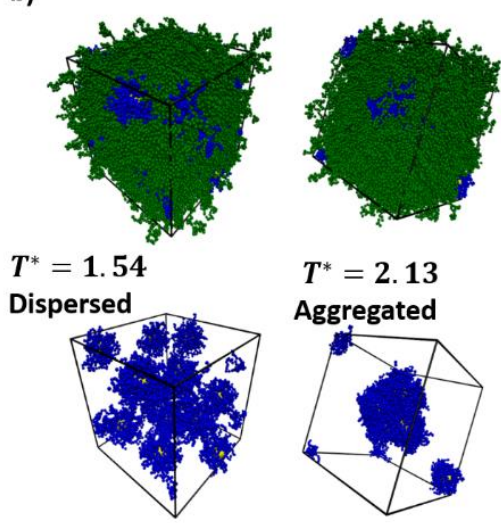

c)

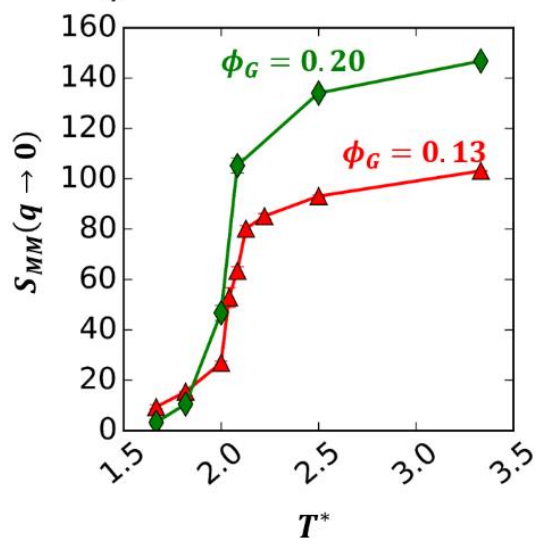

Figure 8.2: (a) Dispersion-aggregation phase-diagram, (b) simulation snapshots, with bottom panel hiding the matrix polymer, and (c) low-q value of the matrix-matrix structure factor for particles of diameter $D=5 d$ grafted with " $G$ " homopolymer chains of length $N_{\text {graft }}=10$ and grafting density $\Sigma=0.76$ chains $/ d^{2}$ in a " $M$ " homopolymer matrix of length $N_{\text {matrix }}=50$ at a total volume fraction $\eta=0.35$ and with blend composition $\phi_{G}=0.13$, and 0.20. The total volume fraction, $\eta$ is defined as ratio of volume of all CG beads to the volume of the simulation box. The blend composition, $\phi_{G}$, is defined as ratio of volume of graft $C G$ beads to the volume of graft and matrix CG beads in the system. In part a, upward and downward triangles denote aggregated and dispersed states, respectively, in the simulations, and the circles denote the dispersion-aggregation transition. The snapshots in part b are for $\phi_{G}=0.13$ blends. The error bars in part c are standard deviations calculated from 50 uncorrelated configurations for each system; the error bars when not visible are smaller than the size of the symbol.

Figure 8.2a shows that there is a transition from particle dispersion to particle aggregation with increasing (reduced) temperature, $\mathrm{T}^{*}$. The evidence of dispersion at this high matrix to graft molecular weight ratio is in contrast to the chemically similar graft-matrix blends, where aggregation is observed at high matrix to graft molecular weight purely due to entropic driving forces. The reason we see dispersion at low $\mathrm{T}^{*}$ for this high matrix to graft molecular weight ratio in this chemically dissimilar graft $(\mathrm{G})$-matrix $(\mathrm{M})$ polymer pair system is because the attractive graft-matrix enthalpic interactions (negative $\chi$ ) drive graft-matrix contacts at low $\mathrm{T}^{*}$. This 
favorable enthalpic driving force for wetting or mixing overcomes the net entropic driving forces that favor graft-matrix dewetting or demixing, and result in well-dispersed blends. As the temperature is increased, the enthalpic forces become repulsive (positive $\chi$ ) and along with the entropic driving forces favor demixing of grafted and matrix chains, and result in aggregated blends. The dispersion to aggregation transition is seen both in simulation snapshots rendered with visual molecular dynamics (VMD) [52] (e.g. Figure 8.2b), as well as partial structure factors (MM, PP and GG) (Supplementary Figure 8.6-Figure 8.8) which show an upturn with increasing temperature as $q \rightarrow 0$, indicative of the onset of particle aggregation. These partial structure factors are calculated via Fourier transform of MM, PP and GG radial distribution functions, as described in the supplementary information, and an upturn at low q indicates increasing aggregation of like-pairs, MM, PP or GG. Since the simulations are run at discrete temperatures, the dispersion-aggregation phase transition (circles in Figure 8.2a) is marked as the temperature where we see the onset of the low-q upturn in these partial structures. The $\mathrm{S}_{\mathrm{MM}}(\mathrm{q} \rightarrow 0)$ versus $\mathrm{T}^{*}$ data for the $\phi_{\mathrm{G}}=0.13$ and 0.20 compositions (Figure 8.2c) show a sharp transition over a small $\mathrm{T}^{*}$ range, indicating a first-order dispersion-aggregation transition. We are unable to resolve differences in the dispersion-aggregation $\mathrm{T}^{*}$ of $\phi_{\mathrm{G}}=0.13$ and 0.20 due to the discrete steps in $\mathrm{T}^{*}$ at which simulations are run. 

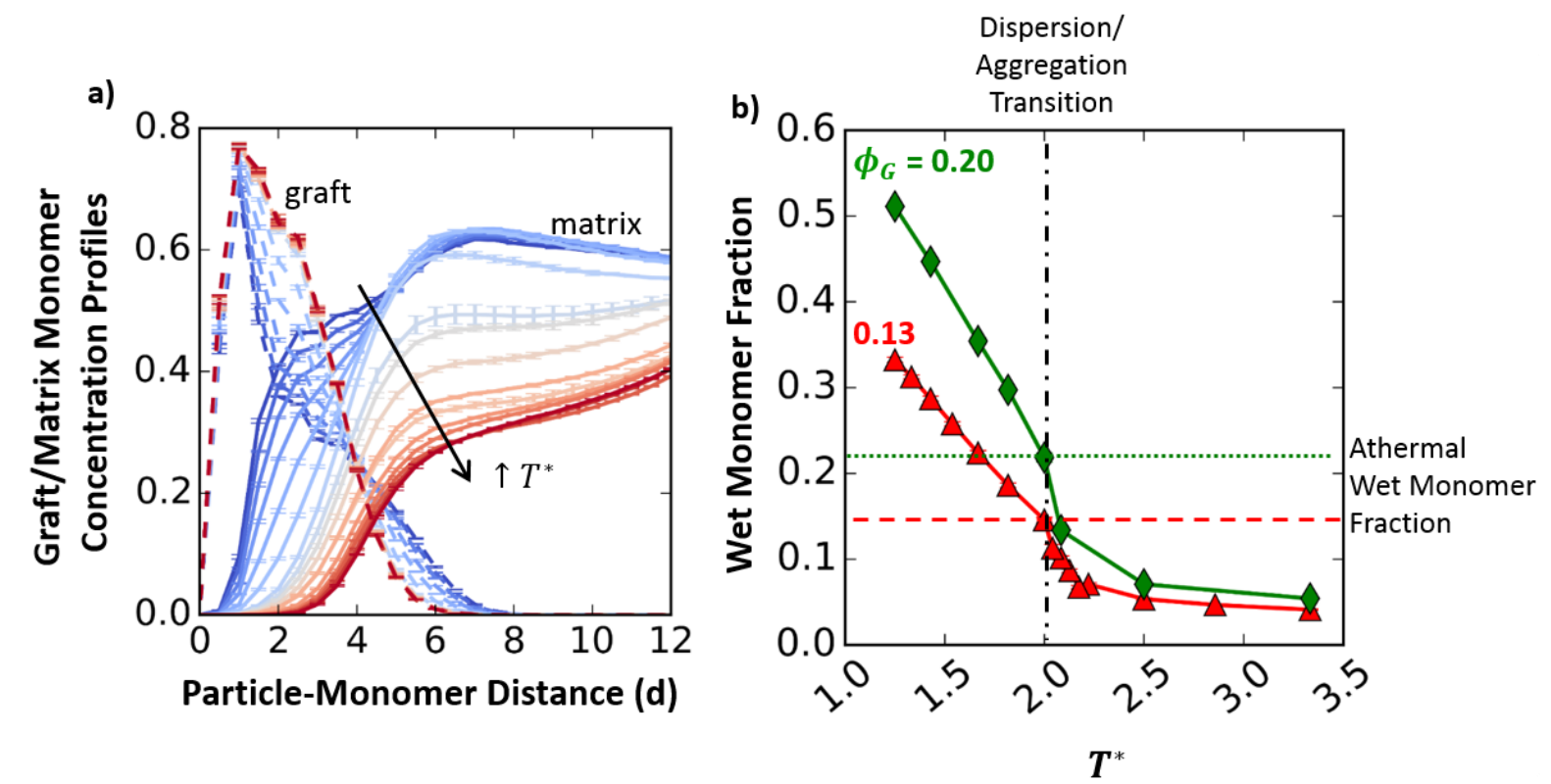

Figure 8.3: a) Graft monomer (dashed lines) and matrix monomer (solid lines) concentration (in units of $d^{-3}$ ) versus distance from the particle surface at $\phi_{G}=0.13$. The graft concentration profile is calculated for each grafted particle independently and the average of these profiles is presented. In the calculation of each grafted particle's profile, we only include the contribution of grafts that are attached to the same particle, and do not include any inter-grafted-particle contributions. b) Wet monomer fraction versus reduced temperature, $T^{*}$. Also shown are the dispersion-aggregation transition temperature (vertical dot-dashed line) and the wet monomer fraction for the athermal $\phi_{G}=0.13$ and 0.20 blends (horizontal dashed and dotted lines). The error bars in parts $a$ and $b$ are standard deviations calculated from 50 uncorrelated configurations for each system; the error bars when not visible are smaller than the size of the symbol.

Molecular simulations are also a powerful means to characterize the wetting to dewetting transition associated with the penetration of the grafted polymer chains by the chemically dissimilar polymers. Using CGMD simulations we calculated the extent of wetting in the dispersed and aggregated states using two different methods. In the first method, the graft and matrix Kuhn segment (or effective "monomer") concentration profiles (Figure 8.3a for $\phi_{\mathrm{G}}=0.13$, and Supplementary Figure 8.9 for all $\phi_{\mathrm{G}}$ ) confirm that, with increasing temperature, there is a gradual decrease in overlap between graft and matrix monomer concentration profiles. In the second 
method, which results in a single number that characterizes the extent of wetting, we identify the spatial location of the anisotropic, closed isosurface corresponding to the edges of the aggregates (at high $\mathrm{T}^{*}$ ) or isolated grafted particles (at low $\mathrm{T}^{*}$ ), and then calculate the fraction of matrix monomers that are inside (wet) or outside (dewet) of this boundary as a function of temperature (Figure 8.3b). At any temperature, the absolute value of the wet monomer fraction increases with increasing amount of grafted polymer chains in the blends, as would be anticipated based on a simple volumetric scaling of the number of particles. On the other hand, when a normalized wet monomer fraction (normalized by the surface area of the aggregates) is calculated from these simulations, the data collapses onto a single curve irrespective of blend composition (not shown). Remarkably, we observe no discontinuous or abrupt change in the wetting fraction shown in Figure 8.3 and instead observe a continuous transition.

Another important feature of the gradual wetting to dewetting transition (Figure 8.3) and the sharp dispersion to aggregation transition (Figure 8.2) is that the onset of dewetting occurs at a temperature well below the dispersion-aggregation transition temperature, and that the dewetting continues at temperatures higher than the dispersion-aggregation transition. These observations imply that the wetting-dewetting and dispersion-aggregation are two distinct transitions. This is in contrast to past literature, especially for chemically similar graft-matrix systems, where the two transitions are treated synonymously. [9, 10, 12, 20, 22, 24-26, 42-44]

Interestingly, for a $\phi_{\mathrm{G}}=0.13$ blend with all WCA excluded volume only (athermal) interactions, that would mimic a chemically similar $\chi=0$ graft $(\mathrm{G})$-matrix $(\mathrm{M})$ polymer pair with the same grafting density, particle size, matrix and graft chain lengths, the wet monomer fraction (horizontal dashed line in Figure 8.3b) coincides with the intersection of the wet-monomer fraction profile and dispersion-aggregation transition of the $\phi_{\mathrm{G}}=0.13$ chemically dissimilar composite. 
This means that when the extent of wetting for the chemically dissimilar graft (G)-matrix (M) polymer system reaches the (threshold or critical) wetting seen in chemically similar athermal system, the chemically dissimilar graft-matrix system goes through the dispersion-aggregation transition. 

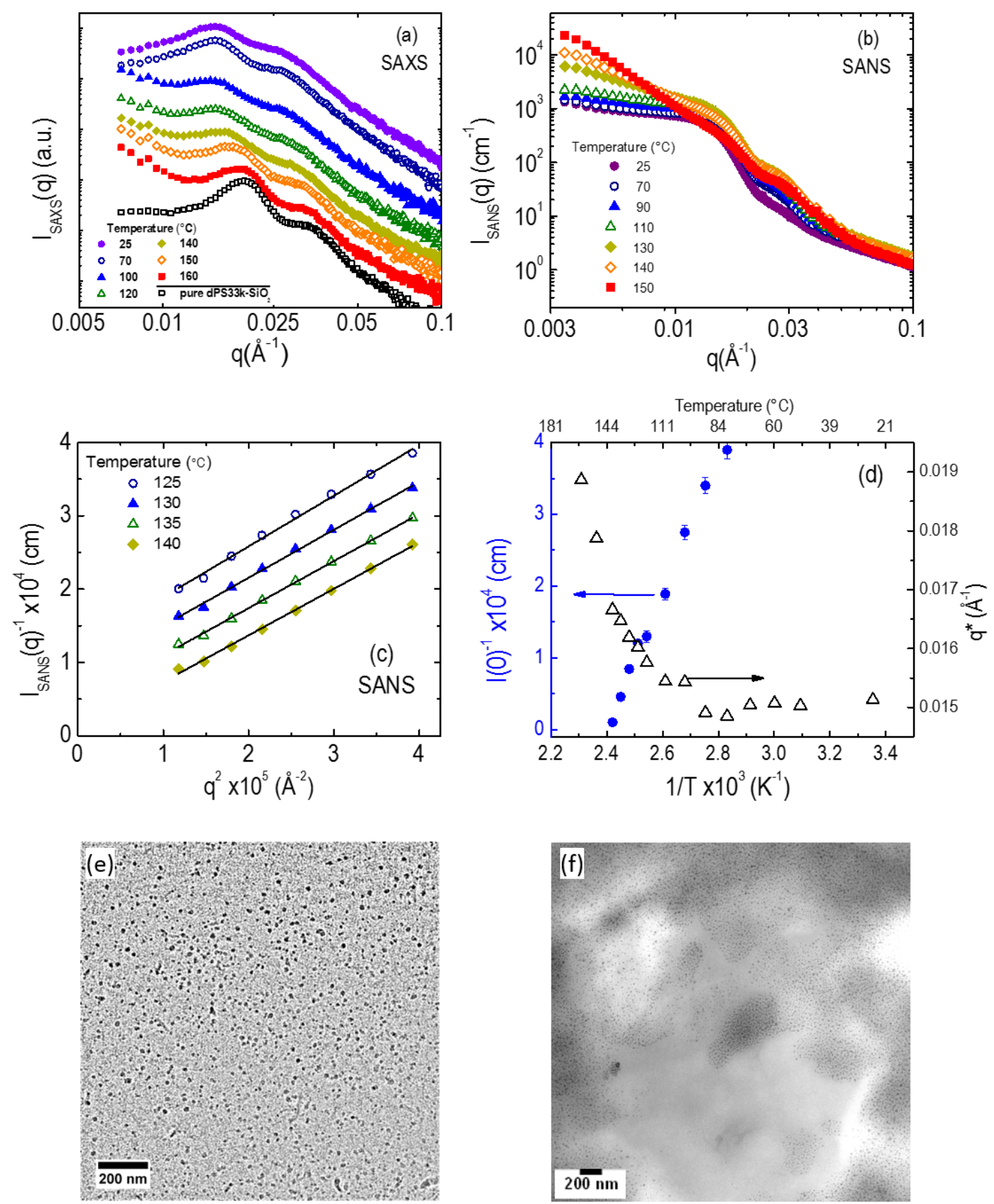

Figure 8.4: a) Temperature dependence of Small Angle X-ray Scattering (SAXS) intensity data for blends of a 33k dPS-grafted silica (grafting density of 0.7 chains $/ \mathrm{nm}^{2}$ ) with a $226 \mathrm{k} P V M E$ at a blend composition of $80 \%$ PVME by volume; data for the scattering intensity from a pure 33k dPSgrafted silica particles with no PVME matrix are shown for comparison. b) Temperature dependence of the Small Angle Neutron Scattering (SANS) intensity for the blend described in (a). 
c) Ornstein-Zernike representation of selected SANS data to estimate the intensity in the forward angle $(\mathrm{I}(0))$ and correlation length $(\xi)$ as a function of temperature for the single phase blends. $d)$ Temperature dependence of $I^{-1}(0)$ from SANS and primary scattering peak position $\left(q^{*}\right)$ from SAXS for the blend described in (a) and (b). TEM images of thin sections of unstained blends at (e) 25 ${ }^{\circ} \mathrm{C}$ showing individually-dispersed nanoparticles and after annealing at $(f) 160{ }^{\circ} \mathrm{C}$ showing largescale phase separation

We conducted Small Angle X-ray and Neutron scattering (SAXS and SANS) studies as a function of temperature on blends of 226k PVME (80 vol \%) with 33k dPS-grafted silica particles (grafting density $=0.7$ chains $/ \mathrm{nm}^{2}$ ). These scattering techniques provide structural information that covers a broad range of length scales, and can be compared to the simulation trends presented. Since the x-ray contrast between the polymers is small and their contrast with the silica particles dominate the SAXS data, we use this method and the observed peaks in the SAXS data to track the correlations between silica particles and therefore monitor the changes in wetting of the d-PS brushes on the silica particles. Separately, since the largest contrast for neutrons are between dPS and PVME (see Supplementary Section 8.8.2) and because of the small amount of silica particles in the scattering volume, we monitor the bulk phase behavior (and aggregation) of the dPS-grafted silica in PVME using the low q behavior in SANS.

It is well understood that sufficiently concentrated nanoparticles will exhibit a liquid like ordering characterized by a peak in the pair correlation function. This translates into a peak in the scattered intensity which is essentially the Fourier transform of the real space distribution of material. Thus the first order peak in the scattered $\mathrm{x}$-ray intensity, denoted $\mathrm{q}^{*}$ in Figure $8.4 \mathrm{a}$, is a direct measure of the distance between silica particles and, in the case of no matrix or solvent, is a measure of the height of the brush as established by Goel et al. [53] The peak in the x-ray scattering from the blend of dPS grafted $\mathrm{SiO}_{2}$ and PVME at room temperature in Figure 8.4a indicates the 
silica particles are fairly homogenously dispersed. This is further confirmed through a direct space transmission electron micrograph shown in Figure 8.4e.

With increasing temperature, the primary scattering peak shifts to higher q indicating the silica particles are moving closer together. Since the concentration of particles does not change this is a clear indication of a depletion of the matrix polymers between particles, and thus strongly suggests that the PVME matrix chains are dewetting the dPS grafted layer. Parenthetically, we note that the dPS starts to dewet the portions of the grafted brush structure closest to the silica particle (and densest) first and progressively dewets portions farther away from the silica core with increasing temperature. Finally at the highest temperature the peak location almost coincides with that of the pure dPS-grafted $\mathrm{SiO}_{2}$ nanoparticles in its melt state, indicating no matrix polymer is left between the nanoparticles, suggesting a complete dewetting and the formation of dense nanoparticle aggregates. The formation of nanoparticle aggregates is further confirmed by TEM of a sample held at $160{ }^{\circ} \mathrm{C}$ and is shown in Figure 8.4f. The peak position for the pure $\mathrm{SiO}_{2}-$ dPS33k nanoparticles (i.e., with no PVME added) gives the particle to particle distance in its space filling state, and thus the grafted brush height of $\sim 9 \mathrm{~nm}$ (Supplementary Figure 8.10). The gradual shift of the peak position with temperature from evenly dispersed to "dry" grafted nanoparticle clusters suggests a gradual dewetting in agreement with the gradual decrease in wet monomer fraction seen in the simulations (Figure 8.3b).

The increase in low q intensity as a function of temperature in both the SAXS and SANS data indicates an increasing contribution of large scale inhomogeneities. SAXS is only sensitive to silica density inhomogeneities within the polymer matrix whereas the SANS data is sensitive to all three components, and in particular, the large neutron contrast between dPS and PVME make SANS especially sensitive to large scale concentration fluctuations between dPS and PVME which 
would be characteristic of graft polymer-matrix polymer phase separation. We note that while the silica (especially as it aggregates) does contribute to the scattering in this q-regime, the magnitude of such scattering is small because of the small volume fraction of silica used in these samples and therefore we adopt a pseudo-binary approach to analyze the SANS data. Indeed, the increase in the low q SANS intensity data (Figure 8.4b) is consistent with a lower critical solution temperature (LCST) system where the concentration fluctuations increase with increasing temperature. For a binary blend, the scattering at low $\mathrm{q}$ in such a system can be described by the Ornstein- Zernike equation:

$$
I_{S A N S, C o h}(q)=\frac{I(0)}{1+\xi^{2} q^{2}}
$$

where $\xi$ is the correlation length. The scattered intensity in the forward direction, $\mathrm{I}(0)$, is obtained by extrapolating the $1 / \mathrm{I}(\mathrm{q})$ vs. $\mathrm{q}^{2}$ data to $\mathrm{q}=0$ (Figure $8.4 \mathrm{c}$ ). Both the zero-angle scattering intensity, $\mathrm{I}(0)$, and correlation length $\xi$ diverge as the spinodal temperature is approached (Supplementary Figure 8.11). Plotting $1 / \mathrm{I}(0)$ and $1 / \xi^{2}$ against $1 / \mathrm{T}$ (Figure 8.4c and Supplementary Figure 8.11) allows a linear extrapolation to $q=0$, and leads to the identification of the spinodal temperature, $\mathrm{T}_{\mathrm{s}} \sim 143 \pm 2{ }^{\circ} \mathrm{C}$. The behavior of the intensity and correlation length with temperature bears remarkable similarity to that observed for polymer blends and star-PS / PVME mixtures. $[54,55]$

These dPS (graft) - PVME (matrix) blend experiments are thus in remarkable agreement with the simulations using a generic LCST graft-matrix pair, suggesting that a) the wetting to dewetting transition is a gradual process with increasing temperature while the dispersionaggregation or macrophase separation transition is first order, b) the onset of wetting to dewetting occurs at temperatures lower than the dispersion to aggregation transition, and c) dewetting continues at temperatures above the spinodal temperature in the aggregated state. 

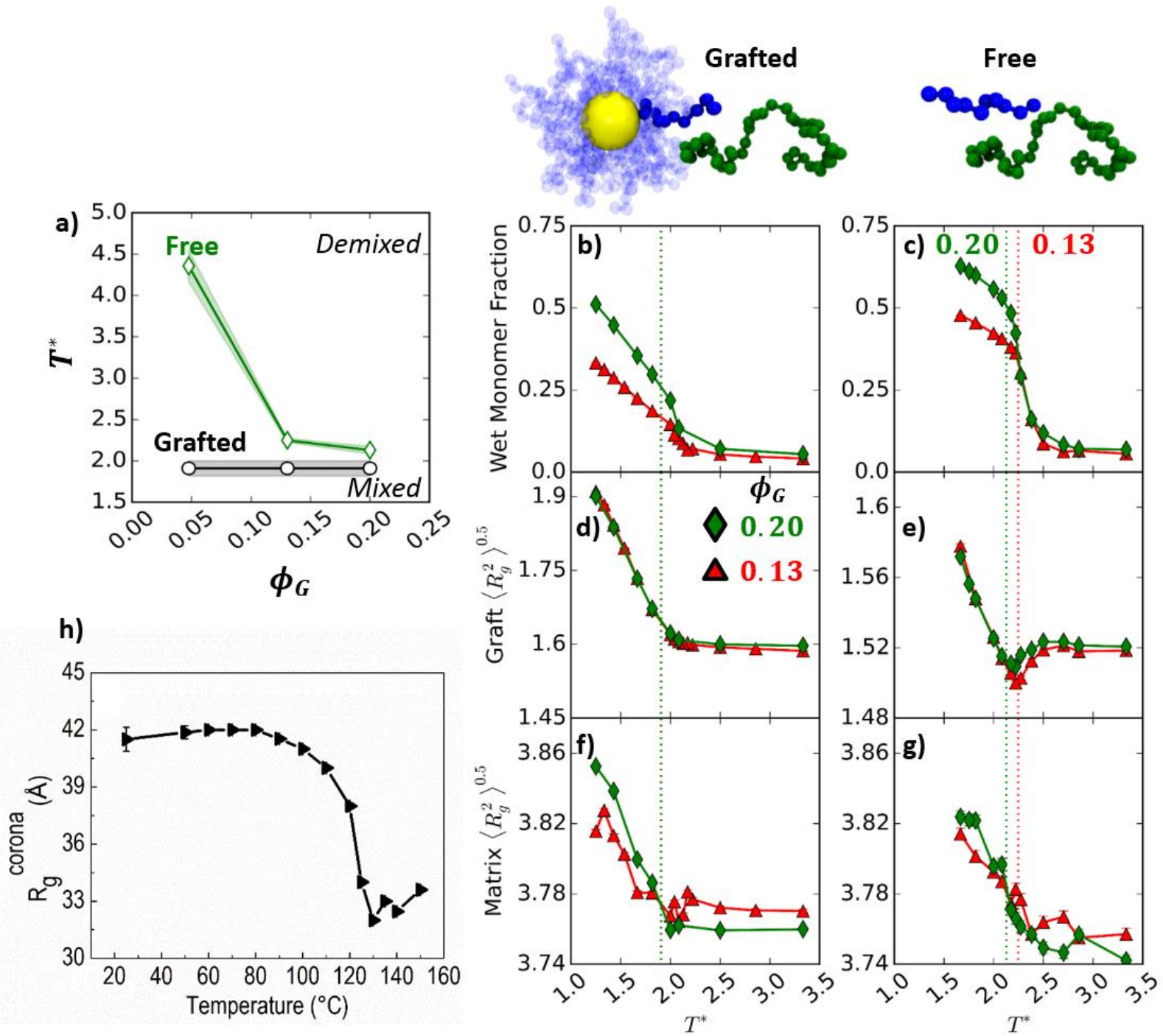

Figure 8.5: a) Mixed-demixed phase-diagram comparing the grafted or free blend, the wet monomer fraction vs temperature $(b, c)$, graft radius of gyration (d,e), and matrix radius of gyration $(f, g)$ for particles of diameter $D=5 d$ grafted with chains of length $N_{\text {graft }}=10$ and grafting density $\Sigma=0.76$ chains $/ d^{2}$ in a matrix of length $N_{\text {matrix }}=50(a, b, d, f)$ or blends of $N_{\text {graft }}=10$ and $N_{\text {matrix }}=50$ chains $(a, c, e, g)$ at a total volume fraction $\eta=0.35$ and filler fractions $\phi_{G}=0.13$ (triangles), and 0.20 (diamonds). The error bars in parts b-g are standard errors calculated from 50 uncorrelated configurations for each system; the error bars when not visible are smaller than the size of the symbol. $h$ ) Change in experimentally measured radius of gyration of the outer shell (called $R_{g}{ }^{{ }^{c o r o n a}}$ ) with temperature from an excluded volume model for blends of a 33k dPS-grafted 
silica (grafting density of 0.7 chains $/ \mathrm{nm}^{2}$ ) with a 226k PVME at a blend composition of $80 \%$ PVME by volume. We note that $R_{g}{ }^{\text {corona }}$ does not represent the overall dimension of the polymer chains but only the outer shell of the grafted chains.

To better understand the chain conformations that are linked to the above wettingdewetting behavior, we unravel the effect of grafting one of the polymers in the LCST graft $(\mathrm{G})$ matrix (M) polymer pair, on the enthalpic and entropic driving forces of the phase behavior. To do this we also simulate using CGMD a blend of ungrafted (G) polymer and ungrafted (M) polymer, termed the free blend, with the same number of $\mathrm{G}$ and $\mathrm{M}$ chains and chain lengths as the grafted blend, for the blend compositions discussed so far. Figure 8.5a shows that, for the free blend, the transition from mixed to demixed states occurs at higher temperatures and has a stronger dependence on the blend composition than the grafted blend. We explain this difference between the free and grafted blends through the enthalpic and entropic contributions to the change in free energy to go from mixed (dispersed) to demixed (aggregated) states, $\Delta \mathrm{A}$, defined as

$$
\Delta A_{\text {mixed } \rightarrow \text { demixed }}=\Delta U-T \Delta S
$$

where $\Delta \mathrm{U}$ is the change in internal energy and $\Delta \mathrm{S}$ the change in entropy going from mixed (dispersed) to demixed (aggregated) states.

If we assume that the G-M attractive interactions are the dominant energetic driving force for G-M mixing, we can define $\Delta \mathrm{U} \sim \Delta \mathrm{n} \varepsilon_{\mathrm{GM}}$, where $\Delta \mathrm{n}$ is the change in number of contacts between $\mathrm{G}$ and $\mathrm{M}$ monomers going from mixed (dispersed) to demixed (aggregated) states, and $\varepsilon_{\mathrm{GM}}$ the attractive interaction strength between $\mathrm{G}$ and $\mathrm{M}$ monomers. Since both free and grafted blends have the same number of $\mathrm{G}$ and $\mathrm{M}$ monomers, the total number of possible pair G-M monomer contacts should be the same for the grafted and free blends. However, in the grafted blend, the dense grafting shields some of the $\mathrm{G}$ monomers from making contacts with the $\mathrm{M}$ monomers in the mixed (dispersed) state, as seen in the monomer concentration profiles in Figure 8.3a. It is fair 
to assume that in the demixed (aggregated) state, the number of G-M contacts is minimal and negligibly different between the grafted and free blend. Thus, the (negative) $\Delta \mathrm{n}$ and (positive) $\Delta \mathrm{U}$ going from mixed (dispersed) to demixed (aggregated) states is smaller for the grafted blend than the free blend.

The major contributions to the change in entropy going from mixed (dispersed) to demixed (aggregated) states is the conformational entropy gain of matrix chains, $\Delta \mathrm{S}_{\text {conf, }}$ and mixing entropy loss, $\Delta \mathrm{S}_{\mathrm{mix}}$. Even though these two contributions to entropy cannot be fully decoupled, it is useful to see individually how these impact the total entropy change.

$\Delta \mathrm{S}_{\mathrm{conf}}$, the conformational entropy gain of the matrix chains going from mixed (dispersed) to demixed (aggregated) state is larger for grafted blend than free blend. This is because the M matrix chains that penetrate the grafted layer in the dispersed state have fewer conformations in the crowded grafted $G$ layer than they do outside the grafted layer in the aggregated state. In contrast, we expect that the free blend has relatively negligible differences in matrix conformations in the mixed and demixed state. $\Delta \mathrm{S}_{\mathrm{mix}}$, the mixing entropy loss going from mixed (dispersed) to demixed (aggregated) state is smaller for the grafted blend than free blend.[56] This is because in the mixed state, due to the $\mathrm{G}$ chains being end-grafted to the particle surface in the grafted case, the volume available for $\mathrm{G}$ and $\mathrm{M}$ monomer mixing is significantly lower in the grafted blends compared to the free blends. In the demixed state the grafted and free blends should have similar low mixing entropy.

Thus, with a larger energetic and entropic driving force to stay mixed, the free blends have a higher mixed-to-demixed transition temperature than the grafted blends. The grafted vs. free blend phase behavior is, thus, not surprising due to the above intuitive thermodynamic driving forces. 
Surprisingly, in contrast to the gradual continuous transition of wet monomer fraction with increasing temperature for the grafted blends, for free blends the wet monomer fractions, which characterizes the extent of G-M monomer mixing, shows a sharp transition with increasing temperature. Furthermore, for the $\phi_{\mathrm{G}}=0.13$ and 0.20 free blends this mixing-demixing transition coincides with the macrophase separation transition (dotted lines), in contrast to the grafted blends at those compositions. We conjecture that the gradual wetting to dewetting transition for the grafted blend/composite in contrast to the sharp transition for the free blend is due to the permanent tethering of the grafted chains. Tethering of graft chains to the particle creates regions in the grafted layer with variable entropic driving forces for graft-matrix mixing. With increasing distance from the particle surface, each successive layer of grafted monomers has increased free volume available for mixing with matrix monomers. This creates a gradient in graft-matrix mixing entropy gain and conformational entropy loss upon wetting. For example, the grafted region closest to the particle center has a higher conformational entropy loss and lower mixing entropy gain from graft-matrix wetting than the outer region in the grafted layer. Thus, as the temperature increases likely various regions of the grafted layers are dewet in a step by step fashion rather than sharply as in the case of free blends. While curvature of the grafted surface affects the available volume for mixing within the grafted layer, the gradual wetting to dewetting transition is also seen in zerocurvature flat surface limit (Supplementary Figure 8.13). This further strengthens the argument that the grafting/tethering of one of the polymers in the blend leads to the gradual continuous wetting to dewetting transition.

To elucidate the single chain conformations within the blends during this sharp vs. continuous wetting-dewetting phenomena for free blends vs. grafted blends, respectively, we calculate the ensemble average radius of gyration for the $\mathrm{G}$ and $\mathrm{M}$ chains in the grafted and free 
blends. There are clear differences between the behavior of the $G$ homopolymer chains when grafted (Figure 8.5d) and free (Figure 8.5e). First, due to dense grafting-induced chain extension, the size of the G chains in the grafted systems is larger than those in the free blends. Second, as temperature increases, coinciding with the wetting-dewetting transition, the grafted $\mathrm{G}$ chains' size decreases gradually till they plateau to a value when the system has completed the wetting to dewetting transition. In contrast, the free $\mathrm{G}$ polymer chains go through a coil-globule-coil transition, coinciding with the mixing-demixing transition in the free blend. In this case it has been argued that as the temperature increases, the (minority component) $\mathrm{G}$ chains have an increasing drive to shrink or collapse to a globule because they are amidst increasingly unfavorable M matrix chains. Once macrophase separation occurs, the $\mathrm{G}$ chains are in a domain of other $\mathrm{G}$ chains and are able to relax their entropically-unfavorable globule configurations and expand modestly. In the grafted blend, the segregation of the G chains in the grafted layer decreases the need for the $G$ chains to collapse to the same extent as free $\mathrm{G}$ chains, as the grafted architecture prevents single $\mathrm{G}$ chains from ever being totally surrounded by $\mathrm{M}$ chains. Additionally, the dense grafting also prevents the $\mathrm{G}$ chains from going through the drastic changes in chain conformations that they do in the free blends. Interestingly, applying an excluded volume model [57] to quantitatively model the SANS data for the dPS-grafted $\mathrm{SiO}_{2}$ and PVME blends, allows for the extraction of a radius of gyration for outer layer of the grafted chains (Figure 8.5h). The decreasing radius of gyration of the outer layer of the grafted chains in experiments (Figure 8.5h) and decreasing graft radius of gyration in molecular simulations (Figure $8.5 \mathrm{~d}$ ) are remarkably similar. The matrix $\mathrm{M}$ chains in the grafted and free blends adopt similar chain conformations at all $\phi_{G}$, with the effect of temperature on the matrix chain conformations decreasing with decreasing $\phi_{G}$ for both free and grafted blends. 


\subsection{CONCLUSION}

In summary, through a combination of coarse-grained simulations and $\mathrm{x}$-ray and neutron scattering, we demonstrate that, unlike chemically identical graft-matrix systems, a sharp dispersion to aggregation transition is distinct from the gradual continuous wetting to dewetting transition in a polymer nanocomposite with a chemically dissimilar graft-matrix polymer pair that exhibits LCST behavior. We also show that the dispersion to aggregation transition occurs when the extent of wetting in the attractive graft-matrix polymer pair approaches that of the analogous chemically similar graft-matrix polymer composite.

\subsection{ACKNOWLEDGEMENTS}

AJ acknowledges financial support from U.S. Department of Energy under Grant DE-SC0003912 and TBM acknowledges financial support from NSF graduate fellowship (award number DGE 1144083). RK and KM acknowledge financial support from ExxonMobil Chemical Company. We acknowledge B. Natarajan for assistance with the TEM images. SAXS data were obtained using an instrument obtained on a National Science Foundation (NSF DMR 1040446) funded instrument. This work used NERSC supercomputing supported by the U.S. Department of Energy (DE-AC02- 05CH11231), Farber supercomputer at University of Delaware, and Opuntia supercomputer at University of Houston. This work utilized the NIST Center for Neutron Research (NCNR) supported in part by the National Science Foundation under Grant \#DMR-0944772.

\subsection{REFERENCES}

1. McMaster, L.P., ASPECTS OF POLYMER-POLYMER THERMODYNAMICS. Macromolecules, 1973. 6(5): p. 760-773.

2. Bates, F.S., POLYMER-POLYMER PHASE-BEHAVIOR. Science, 1991. 251(4996): p. 898-905.

3. Paul, D.R. and C.B. Bucknall, Polymer Blends: Formulation and Performance (Two volume set). 2000: Wiley. 
4. Ryan, A.J., Polymer science: Designer polymer blends. Nat Mater, 2002. 1(1): p. 8-10.

5. Eitouni, H. and N. Balsara, Thermodynamics of Polymer Blends, in Physical Properties of Polymers Handbook, J. Mark, Editor. 2007, Springer New York. p. 339-356.

6. $\quad$ Mackay, M.E., et al., General Strategies for Nanoparticle Dispersion. Science, 2006. 311(5768): p. 1740-1743.

7. Balazs, A.C., T. Emrick, and T.P. Russell, Nanoparticle polymer composites: Where two small worlds meet. Science, 2006. 314(5802): p. 1107-1110.

8. McGarrity, E.S., et al., Surface-induced first-order transition in athermal polymernanoparticle blends. Physical Review Letters, 2007. 99(23).

9. Sen, S., et al., Chain conformations and bound-layer correlations in polymer nanocomposites. Physical Review Letters, 2007. 98(12).

10. Akcora, P., et al., Anisotropic self-assembly of spherical polymer-grafted nanoparticles. Nature Materials, 2009. 8(4): p. 354-359.

11. Crawford, M.K., et al., Chain Conformation in Polymer Nanocomposites with Uniformly Dispersed Nanoparticles. Physical Review Letters, 2013. 110(19): p. 5.

12. Green, P.F., The structure of chain end-grafted nanoparticle/homopolymer nanocomposites. Soft Matter, 2011. 7(18): p. 7914-7926.

13. Corbierre, M.K., et al., Gold nanoparticlelpolymer nanocomposites: Dispersion of nanoparticles as a function of capping agent molecular weight and grafting density. Langmuir, 2005. 21: p. 6063-6072.

14. Goel, V., et al., Viscoelastic properties of silica-grafted poly(styrene-acrylonitrile) nanocomposites. Journal of Polymer Science Part B-Polymer Physics, 2006. 44(14): p. 2014-2023.

15. Tsubokawa, N., Surface grafting of polymers onto nanoparticles in a solvent-free drysystem and applications of polymer-grafted nanoparticles as novel functional hybrid materials. Polymer Journal, 2007. 39(10): p. 983-1000.

16. Kim, B.J., et al., Effect of areal chain density on the location of polymer-modified gold nanoparticles in a block copolymer template. Macromolecules, 2006. 39(12): p. 41084114.

17. Lan, Q., L.F. Francis, and F.S. Bates, Silica nanoparticles dispersions in homopolymer versus block copolymer. Journal of Polymer Science Part B-Polymer Physics, 2007. 45: p. 2284-2299.

18. Shull, K.R., THEORY OF END-ADSORBED POLYMER BRUSHES IN POLYMERIC MATRICES. Journal of Chemical Physics, 1991. 94(8): p. 5723-5738.

19. Harton, S.E. and S.K. Kumar, Mean-field theoretical analysis of brush-coated nanoparticle dispersion in polymer matrices. Journal of Polymer Science Part B-Polymer Physics, 2008. 46: p. 351-358.

20. Trombly, D.M. and V. Ganesan, Curvature effects upon interactions of polymer-grafted nanoparticles in chemically identical polymer matrices. Journal of Chemical Physics, 2010. 133(15). 
21. $\mathrm{Xu}, \mathrm{C}$., et al., Dispersion of polymer-grafted magnetic nanoparticles in homopolymers and block copolymers. Polymer, 2008. 49(16): p. 3568-3577.

22. Leibler, L., Theory of Microphase Separation in Block Copolymers. Macromolecules, 1980. 13(6): p. 1602-1617.

23. Krishnamoorti, R. and R.A. Vaia, Polymer nanocomposites. Journal of Polymer Science Part B-Polymer Physics, 2007. 45(24): p. 3252-3256.

24. Krishnamoorti, R., Strategies for Dispersing Nanoparticles in Polymers. MRS Bulletin, 2007. 32(4): p. 341-347.

25. Chevigny, C., et al., Polymer-Grafted-Nanoparticles Nanocomposites: Dispersion, Grafted Chain Conformation, and Rheological Behavior. Macromolecules, 2011. 44(1): p. 122-133.

26. Frischknecht, A.L. and A. Yethiraj, Two- and three-body interactions among nanoparticles in a polymer melt. The Journal of Chemical Physics, 2011. 134(17): p. 174901-11.

27. Martin, T.B. and A. Jayaraman, Identifying the Ideal Characteristics of the Grafted Polymer Chain Length Distribution for Maximizing Dispersion of Polymer Grafted Nanoparticles in a Polymer Matrix. Macromolecules, 2013. 46(22): p. 9144-9150.

28. Kumar, S.K., et al., Nanocomposites with Polymer Grafted Nanoparticles. Macromolecules, 2013. 46(9): p. 3199-3214.

29. Nair, N., N. Wentzel, and A. Jayaraman, Effect of bidispersity in grafted chain length on grafted chain conformations and potential of mean force between polymer grafted nanoparticles in a homopolymer matrix. Journal of Chemical Physics, 2011. 134(19).

30. Bates, F.S., et al., Multiblock Polymers: Panacea or Pandora's Box? Science, 2012. 336(6080): p. 434-440.

31. Martin, T.B., P.M. Dodd, and A. Jayaraman, Polydispersity for Tuning the Potential of Mean Force between Polymer Grafted Nanoparticles in a Polymer Matrix. Physical Review Letters, 2013. 110(1): p. 018301.

32. Padmanabhan, V., Effect of grafting on nanoparticle segregation in polymer/nanoparticle blends near a substrate. Journal of Chemical Physics, 2012. 137(9).

33. Barata, T., et al., Computational design principles for bioactive dendrimer based constructs as antagonists of the TLR4-MD-2-LPS complex. Biomaterials, 2011. 32(33): $\mathrm{p}$. 8702-8711.

34. Borukhov, I. and L. Leibler, Enthalpic stabilization of brush-coated particles in a polymer melt. Macromolecules, 2002. 35(13): p. 5171-5182.

35. Borukhov, I. and L. Leibler, Stabilizing grafted colloids in a polymer melt: Favorable enthalpic interactions. Physical Review E, 2000. 62(1): p. R41-R44.

36. Laub, C.F. and J.T. Koberstein, EFFECT OF BRUSH POLYDISPERSITY ON THE INTERPHASE BETWEEN END-GRAFTED BRUSHES AND POLYMERIC MATRICES. Macromolecules, 1994. 27(18): p. 5016-5023.

37. Muller, M. and L.G. MacDowell, Wetting of a short chain liquid on a brush: First-order and critical wetting transitions. Europhysics Letters, 2001. 55: p. 221. 
38. Kim, B., et al., Dewetting of PMMA on PS-Brush Substrates. Macromolecules, 2009. 42(20): p. 7919-7923.

39. Ojha, S., et al., Strategies for the Synthesis of Thermoplastic Polymer Nanocomposite Materials with High Inorganic Filling Fraction. Langmuir, 2013. 29(28): p. 8989-8996.

40. Brown, H.R., K. Char, and V.R. Deline, Enthalpy-driven swelling of a polymer brush. Macromolecules, 1990. 23(13): p. 3383-3385.

41. Cui, L., et al., A facile "graft from" method to prepare molecular-level dispersed graphenepolymer composites. Journal of Polymer Science Part a-Polymer Chemistry, 2012. 50(21): p. 4423-4432.

42. Kalb, J., et al., End grafted polymer nanoparticles in a polymeric matrix: Effect of coverage and curvature. Soft Matter. 7(4): p. 1418-1425.

43. Jiao, Y. and P. Akcora, Assembly of Polymer-Grafted Magnetic Nanoparticles in Polymer Melts. Macromolecules, 2012. 45(8): p. 3463-3470.

44. Sunday, D., J. Ilavsky, and D.L. Green, A Phase Diagram for Polymer-Grafted Nanoparticles in Homopolymer Matrices. Macromolecules, 2012. 45(9): p. 4007-4011.

45. de Espinosa, L.M., et al., Side-Chain Modification and "Grafting Onto" via Olefin CrossMetathesis. Macromolecular Rapid Communications, 2012. 33(23): p. 2023-2028.

46. Ceperley, D., M.H. Kalos, and J.L. Lebowitz, Computer Simulation of the Dynamics of a Single Polymer Chain. Physical Review Letters, 1978. 41(5): p. 313-316.

47. Kalb, J., et al., End grafted polymer nanoparticles in a polymeric matrix: Effect of coverage and curvature. Soft Matter, 2011. 7(4): p. 1418-1425.

48. http://codeblue.umich.edu/hoomd-blue/. HOOMD-Blue webpage. Available from: http://codeblue.umich.edu/hoomd-blue/ (2010).

49. Hansen, J.-P. and I.R. McDonald, Theory of Simple Liquids

3rd ed, ed. Elsevier. 2006.

50. Pyun, J., et al., Synthesis and Characterization of Organic/Inorganic Hybrid Nanoparticles: Kinetics of Surface-Initiated Atom Transfer Radical Polymerization and Morphology of Hybrid Nanoparticle Ultrathin Films. Macromolecules, 2003. 36(14): p. 5094-5104.

51. Jakubowski, W. and K. Matyjaszewski, Activator Generated by Electron Transfer for Atom Transfer Radical Polymerization. Macromolecules, 2005. 38(10): p. 4139-4146.

52. Humphrey, W., A. Dalke, and K. Schulten, VMD - Visual Molecular Dynamics. J. Molec. Graphics, 1996. 14: p. 33-38.

53. Goel, V., et al., Structure of Polymer Tethered Highly Grafted Nanoparticles. Macromolecules, 2011. 44(20): p. 8129-8135.

54. Faust, A.B., et al., Influence of star-core exclusion on polymer-polymer miscibility. Macromolecules, 1989. 22(3): p. 1250-1254.

55. Russell, T.P., et al., Concentration fluctuations in mixtures of linear and star-shaped polymers. Macromolecules, 1990. 23(2): p. 654-659. 
56. Daoud, M., ADSORPTION FROM A BIDISPERSE POLYMER MIXTURE. Macromolecules, 1991. 24(25): p. 6748-6753.

57. Hammouda, B., SANS from homogeneous polymer mixtures: A unified overview, in Polymer Characteristics. 1993, Springer Berlin Heidelberg. p. 87-133.

58. Natarajan, B., et al., The Evolution of Carbon Nanotube Network Structure in Unidirectional Nanocomposites Resolved by Quantitative Electron Tomography. ACS Nano, 2015. 9(6): p. 6050-6058. 


\subsection{SUPPLEMENTARY}

\subsubsection{Additional Results}

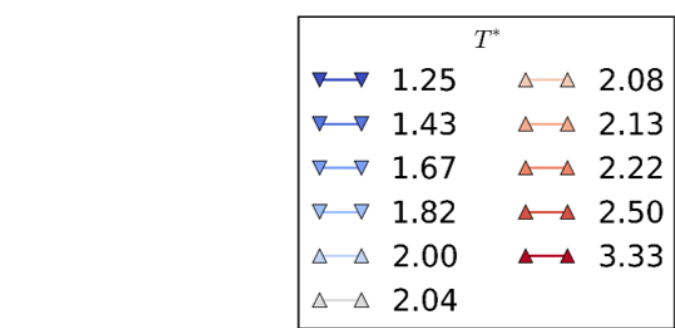
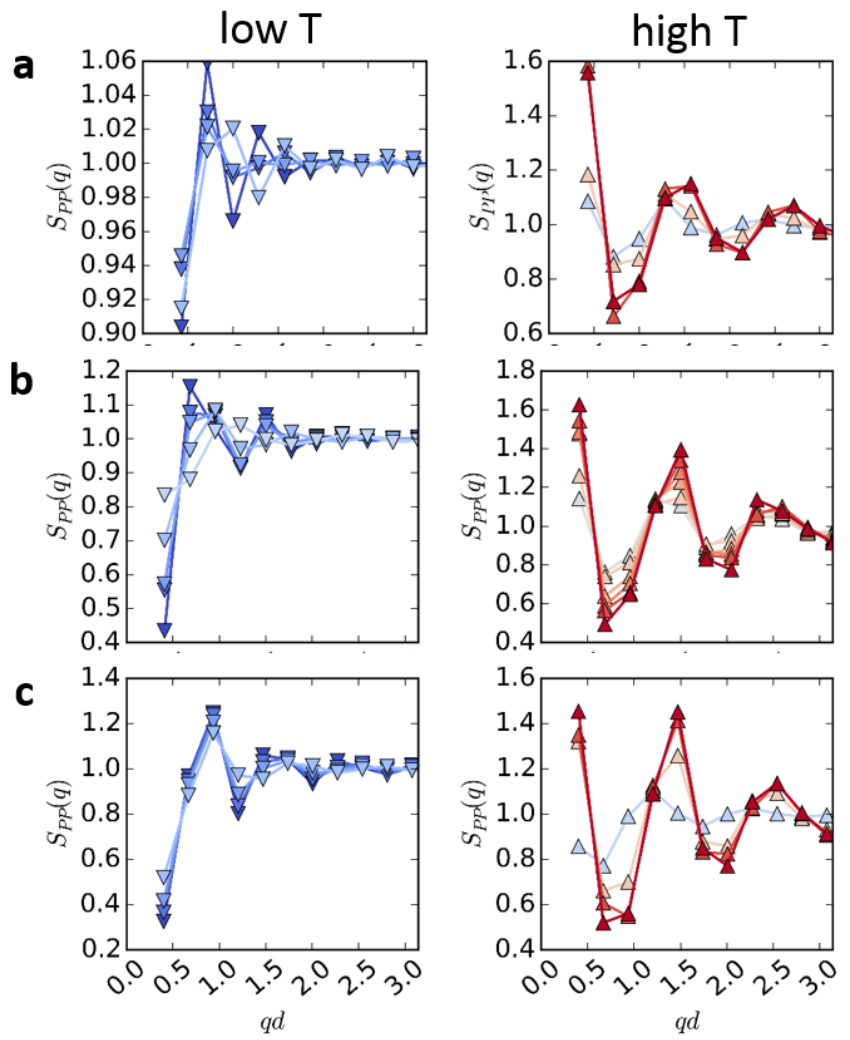

Figure 8.6: Particle-particle structure factors versus reduced wavenumber for particles of diameter $D=5 d$ grafted with chains of length $N_{\text {graft }}=10$ and grafting density $\Sigma=0.76$ chains $/ d^{2}$ in a matrix of length $N_{\text {matrix }}=50$ at a total volume fraction $\eta=0.35$ and filler fractions $\phi_{G}=0.05$ (a), 0.13(b), and 0.20(c) at reduced temperatures as indicated by the colors and symbols defined in the legend. 

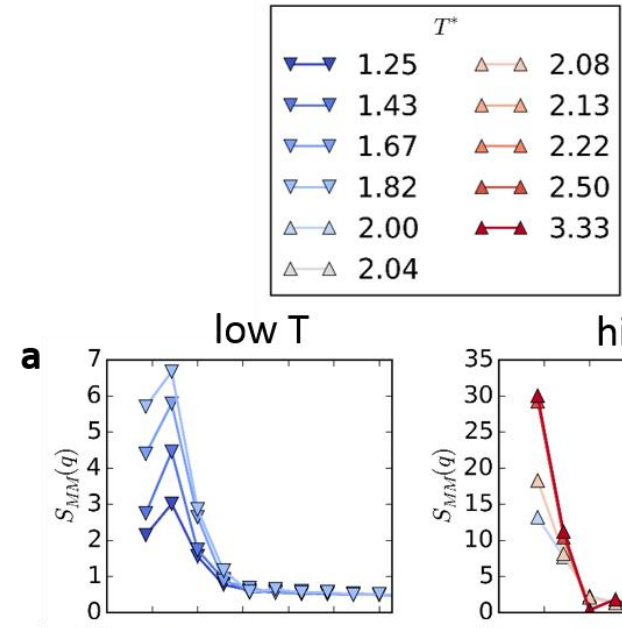

b

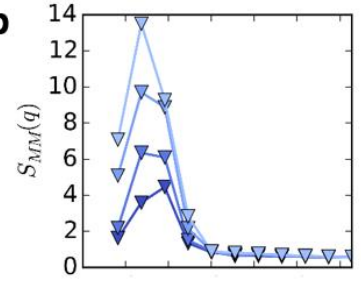

C

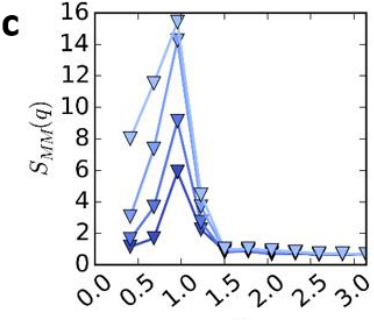

$q d$ high $\mathrm{T}$
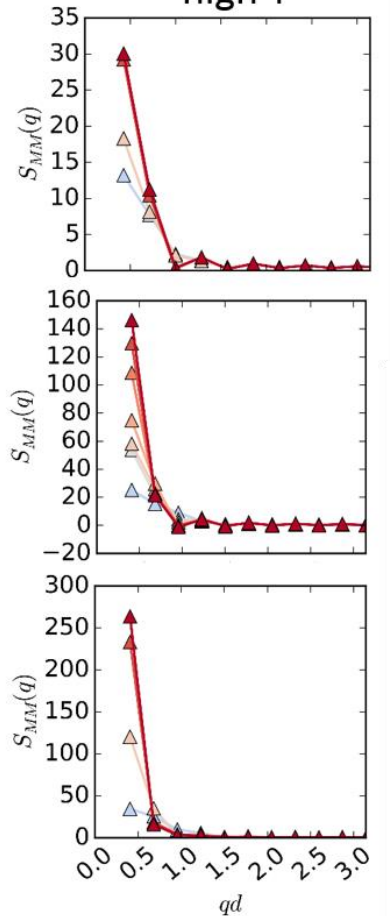

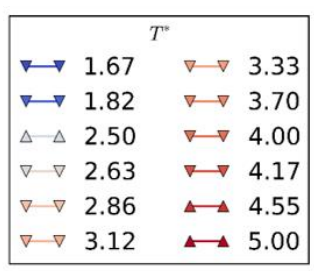

low $T$

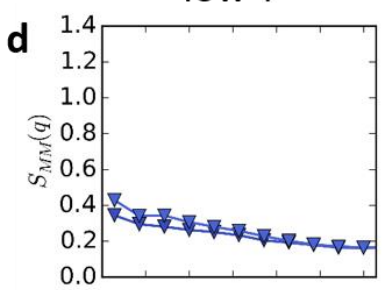

e $\quad 3.5$
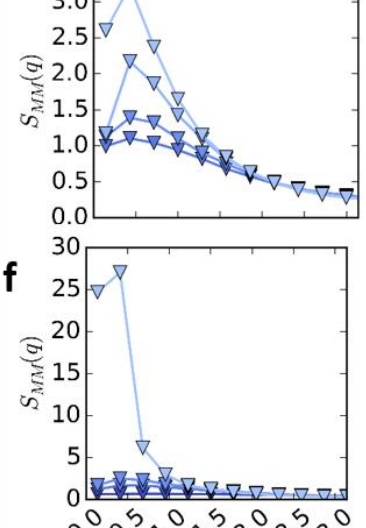

0.0 .21 .2 .5 .2 .3 .0

qd high $\mathrm{T}$
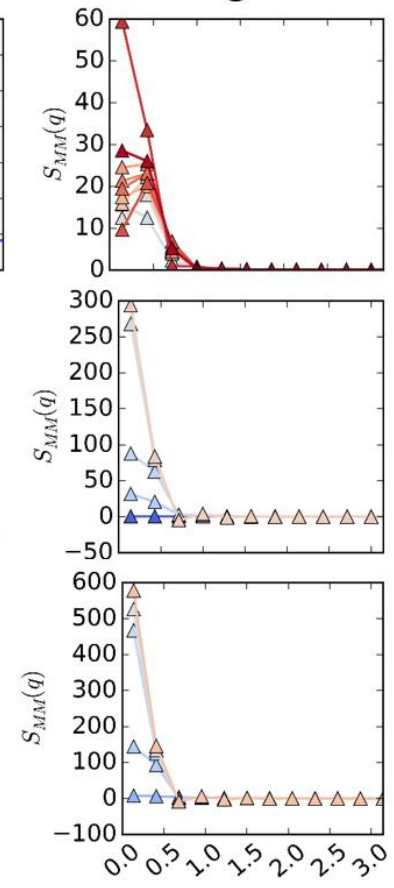

$q d$

Figure 8.7: Matrix-Matrix structure factors versus reduced wavenumber for the systems described in Figure S1, at reduced temperatures as indicated by the colors and symbols defined in the legend 


\begin{tabular}{|c|c|c|c|}
\hline \multicolumn{4}{|c|}{$T^{*}$} \\
\hline$\nabla \longrightarrow$ & 1.25 & $\Delta \Delta$ & 2.08 \\
\hline$\nabla \longrightarrow$ & 1.43 & $\Delta \Delta$ & 2.13 \\
\hline$\nabla \nabla$ & 1.67 & $\Delta \Delta$ & 2.22 \\
\hline$\nabla \nabla$ & 1.82 & $\Delta \Delta$ & 2.50 \\
\hline$\Delta \Delta$ & 2.00 & $\longleftrightarrow$ & 3.33 \\
\hline$\Delta \Delta$ & 2.04 & & \\
\hline
\end{tabular}

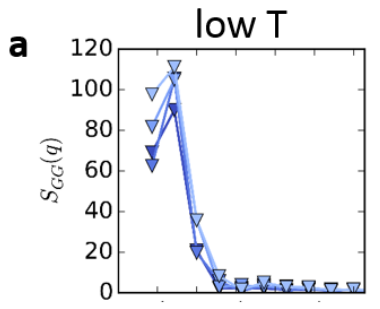

b

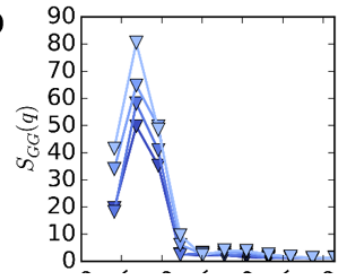

C

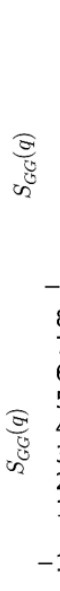
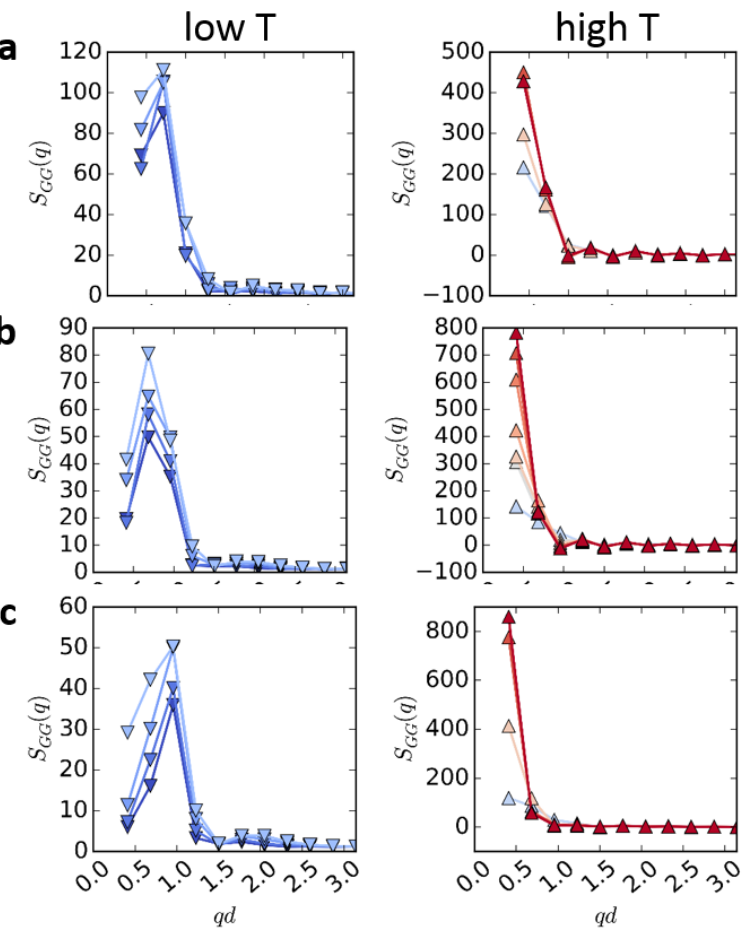

\begin{tabular}{|c|c|c|c|}
\hline \multicolumn{4}{|c|}{$T^{*}$} \\
\hline$\vec{\nabla}$ & 1.67 & $\nabla \nabla$ & 3.33 \\
\hline$\nabla \longrightarrow$ & 1.82 & $\nabla-\nabla$ & 3.70 \\
\hline$\Delta \Delta$ & 2.50 & $\nabla \longrightarrow \nabla$ & 4.00 \\
\hline$\nabla \quad \nabla$ & 2.63 & $\nabla \longrightarrow$ & 4.17 \\
\hline$\nabla \quad \nabla$ & 2.86 & $\Delta \Delta$ & 4.55 \\
\hline$\nabla \nabla$ & 3.12 & 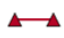 & 5.00 \\
\hline
\end{tabular}
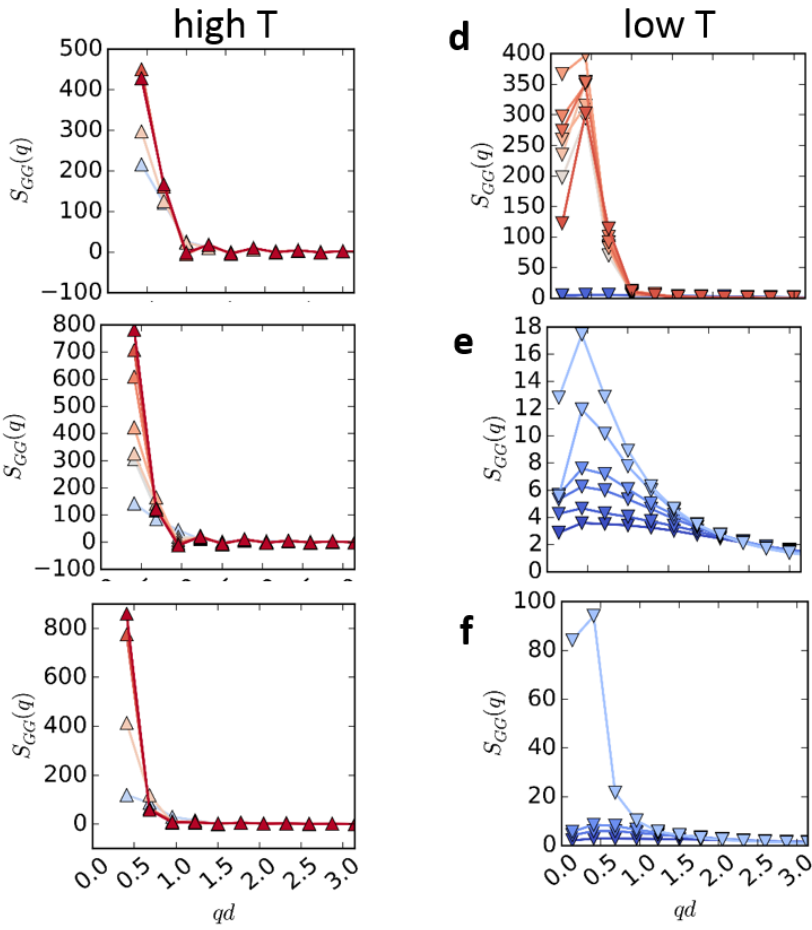

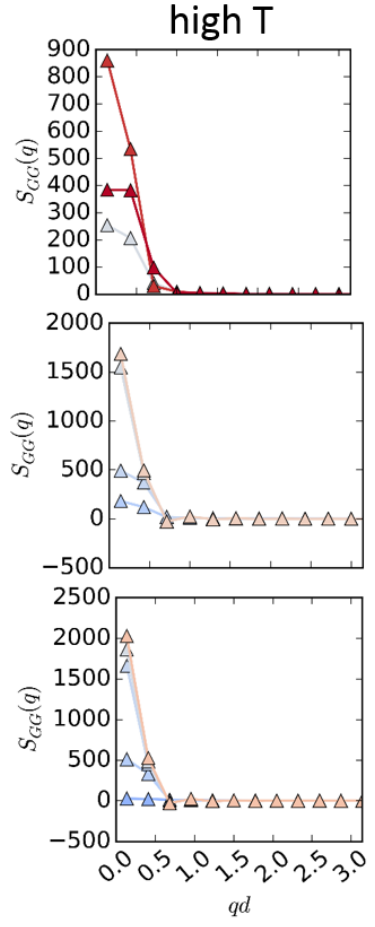

Figure 8.8: Graft-Graft structure factors for the systems described in Figure 8.6 reduced temperatures as indicated by the colors and symbols defined in the legend. 

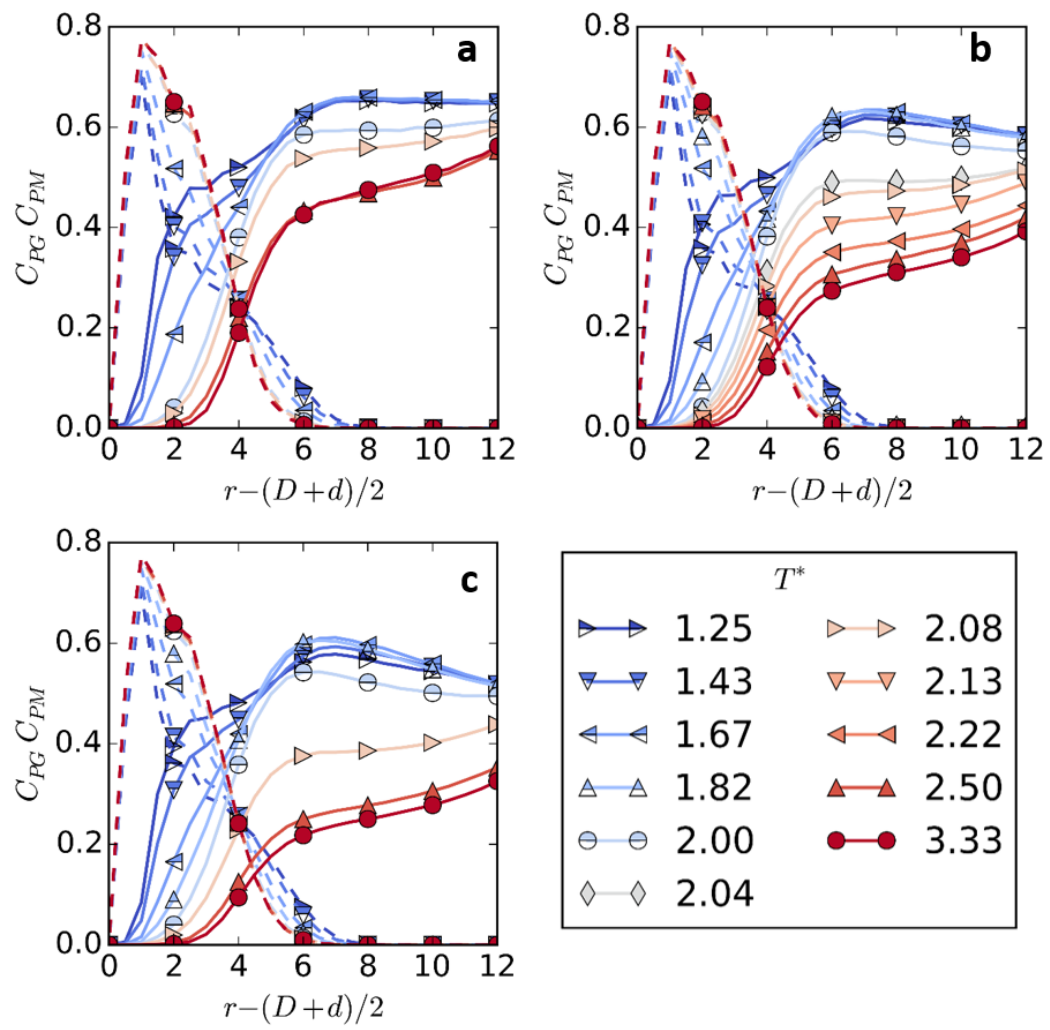

Figure 8.9: Particle-graft (dashed lines) and particle-matrix (solid lines) monomer concentration $\left(d^{-3}\right)$ versus particle-monomer distance $(d)$ at reduced temperatures as indicated by the colors and symbols defined in the legend. The data in these plots are for particles of diameter $D=5 d$ grafted with chains of length $N_{\text {graft }}=10$ and grafting density $\Sigma=0.76$ chains $/ d^{2}$ in a matrix of length $N_{\text {matrix }}=50$ at a total volume fraction $\eta=0.35$ and filler fractions $\phi_{G}=0.05(a), 0.13(b)$, and $0.20(c)$ at reduced temperatures as indicated by the colors and symbols defined in the legend. 


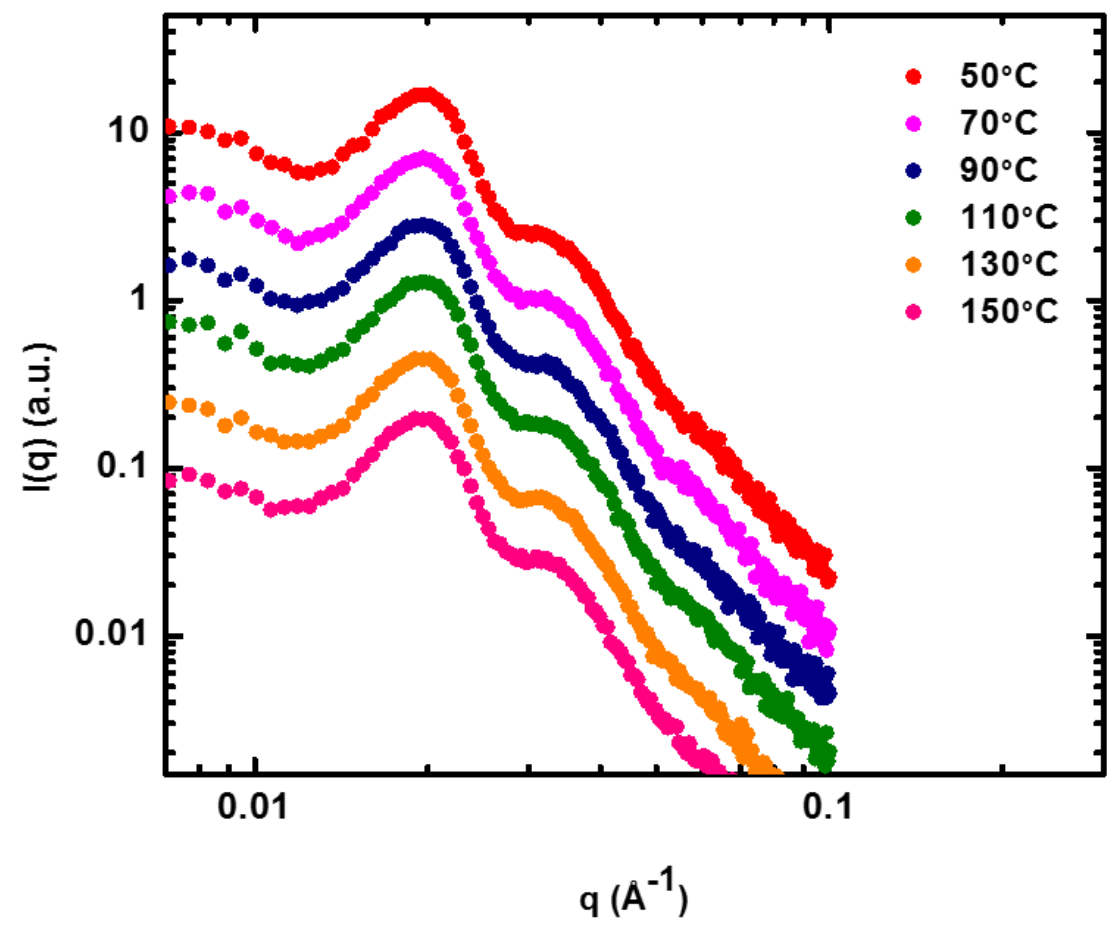

Figure 8.10: $\mathrm{SAXS}$ intensity profiles of pure $\mathrm{SiO}_{2}-d P S 33 k$ nanoparticles (no matrix) at various temperatures, showing no change in inter-particle distance or brush height. Sample was annealed at $140{ }^{\circ} \mathrm{C}$ overnight and cooled down to room temperature prior to measurement. 


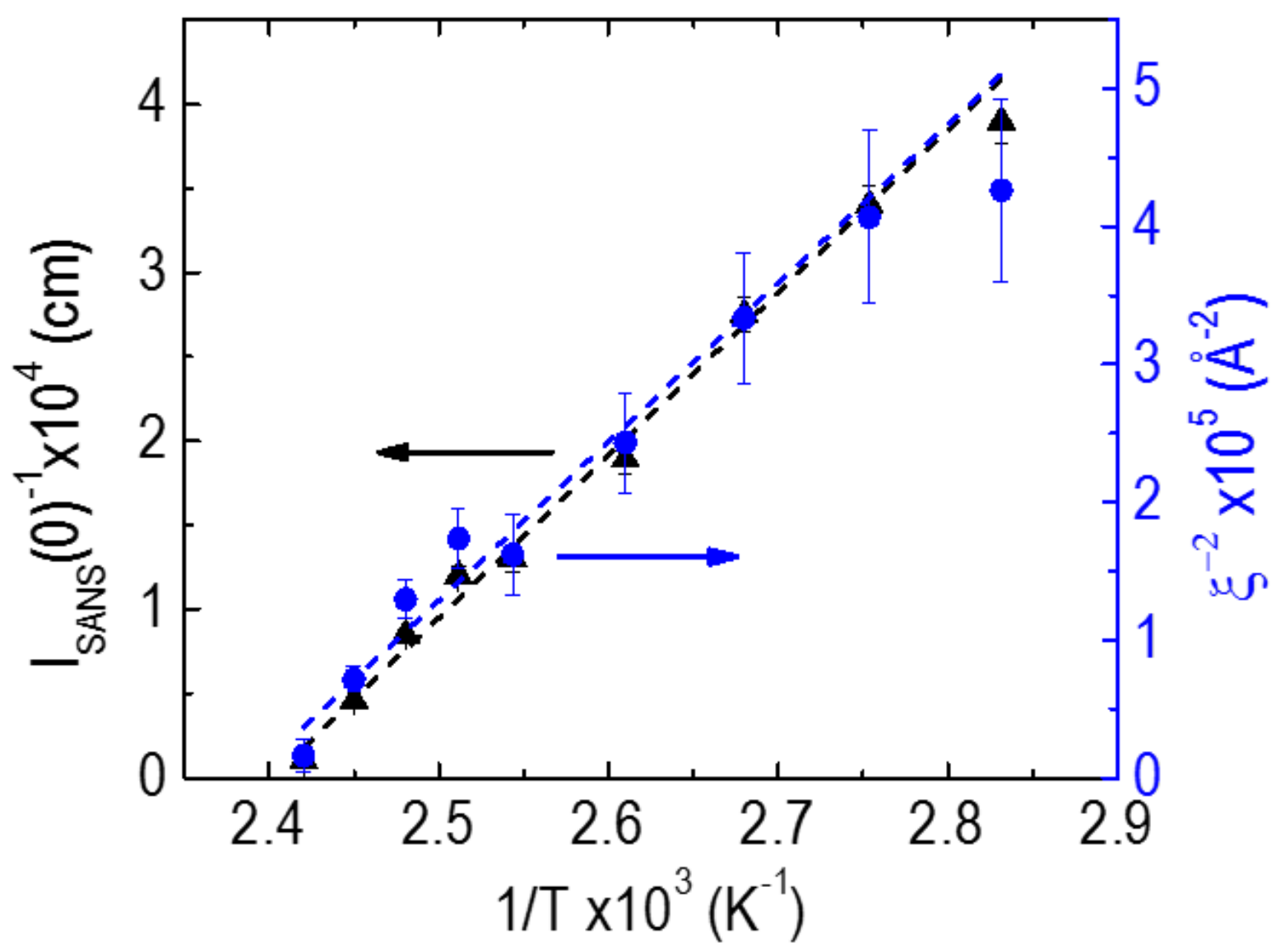

Figure 8.11: Zero-angle scattering and correlation length vs temperature. Calculation of spinodal temperature $\left(T_{s}\right)$ from the zero-angle scattering and correlation length data were obtained by linearly extrapolating $I_{S A N S}(0)^{-1}$ vs $T^{-1}$ to the intercept value as well as by linearly extrapolating $\xi$ ${ }^{2}$ vs $T^{-1}$ to the intercept value. 

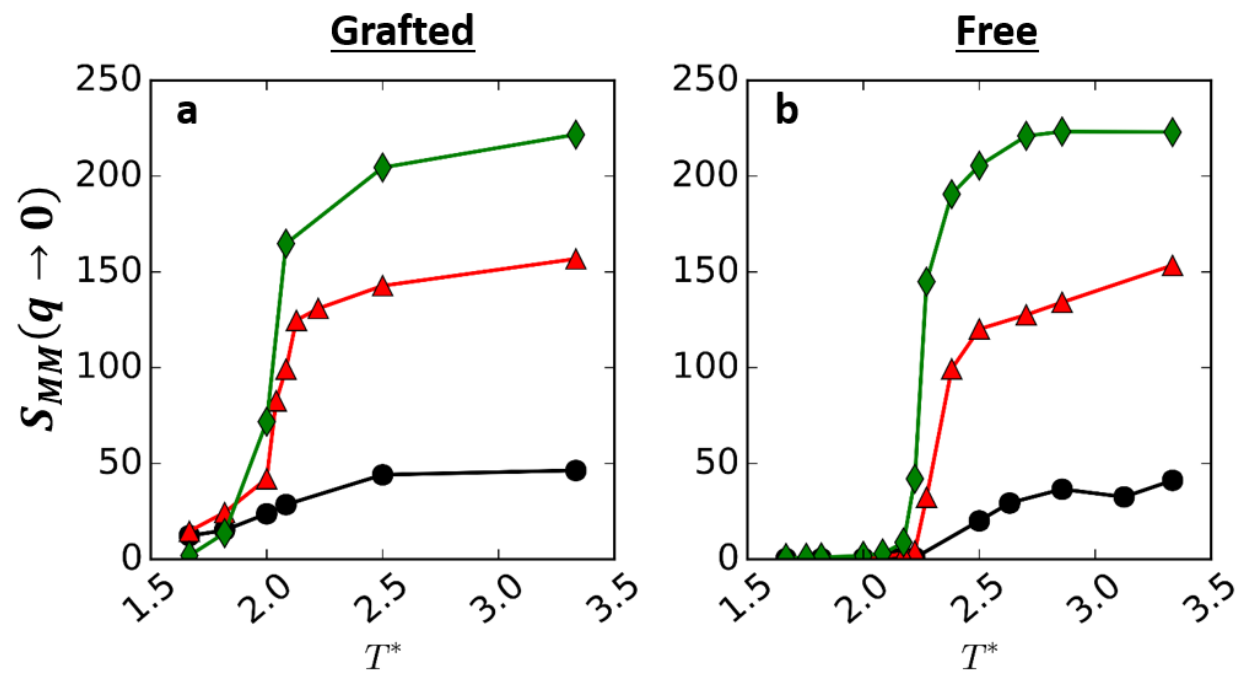

Figure 8.12: Matrix-Matrix structure factors as $q \rightarrow 0$ versus reduced temperature for particles of diameter $D=5 d$ grafted with chains of length $N_{\text {graft }}=10$ and grafting density $\Sigma=0.76$ chains $/ d^{2}$ in a matrix of length $N_{\text {matrix }}=50(a)$ or blends of $N_{\text {graft }}=10$ and $N_{\text {matrix }}=50$ chains $(b)$ at a total volume fraction $\eta=0.35$ and filler fractions $\phi_{G}=0.05$ (circles), 0.13 (triangles), and 0.20 (diamonds). 

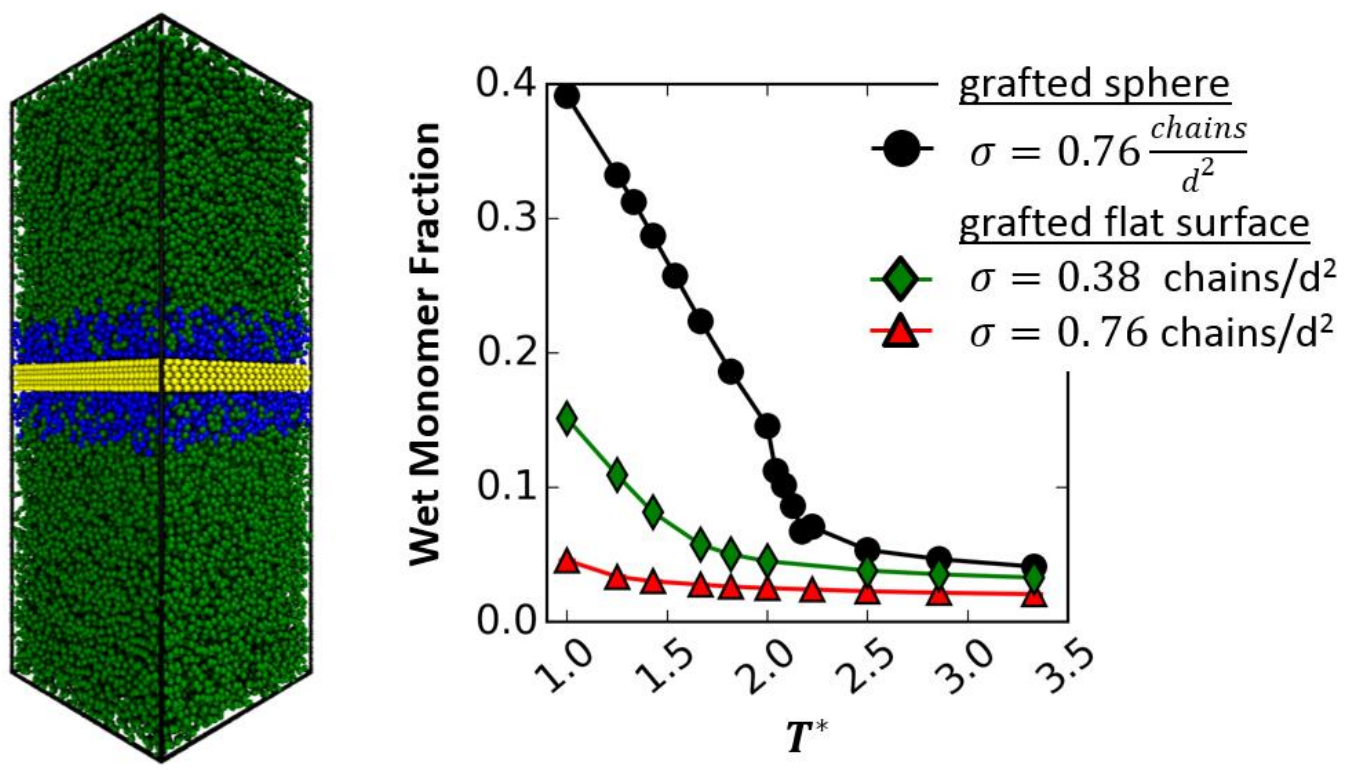

Figure 8.13: (Left) Simulation snapshot of flat grafted surface being wetted by a free matrix. (right) Wet monomer fraction versus reduced temperature, $T^{*}$ for equivalent flat and spherical grafted surfaces. For all data in the plot, there are 900 grafted chains of length $N_{\text {graft }}=10$ and 1200 matrix chains of length $N_{\text {matrix }}=50$ at composition of $\phi_{G}=0.13$ and total volume fraction $\eta=0.35$. The simulation protocols for the flat cases are nearly identical to the spherical case (Figure 8.1), except for a slightly modified compression scheme that accounts for only compressing in the direction normal to the interface.

In an attempt to explain the molecular mechanism underlying the gradual wetting to dewetting transition, we hypothesized that the permanent tethering of the grafted chains to the highly curved particle surface lead to the gradual wetting to dewetting transition, unlike the free graft and free matrix blend which exhibited a sharp transition. Our rationale behind the hypothesis was that with increasing distance from the (spherical) particle surface, each successive layer of grafted monomers has increased free volume available for mixing with matrix monomers. This creates a gradient in graft-matrix mixing entropy gain and conformational entropy loss upon wetting as the distance from the particle surface is increased. For example, the grafted region closest to the particle center has a higher conformational entropy loss and lower mixing entropy gain from graft- 
matrix wetting than the outer region in the grafted layer. Thus, as the temperature increases likely various regions of the grafted layers are dewet in a step by step fashion rather than sharply as in the case of untethered graft and matrix free blends. To test in particular the curvature effects we conducted simulations of flat, grafted-surfaces at the same total volume fraction, length of grafted and matrix chains, and number of grafted and matrix chains as the spherical grafted particle case at $\phi_{G}=0.13$. We chose to explore two grafting densities for the flat case as we found that using the same grafting density as the spherical grafted particles $\left(\sigma=0.76\right.$ chains $\left.\left./ d^{2}\right)\right)$ led to an extremely dense flat grafted layer that showed little wetting at all $T^{*}$ (shown as red triangles in Supplementary Figure 8.13). The data in the figure clearly show smooth wetting-dewetting profiles for all spherical and flat grafted cases. Considering that the sharp wetting to dewetting transition was only seen in the free graft and free matrix blends we conclude that inhomogeneity in the wetting from near the grafted surface to the top layer of the grafts leads to a gradual wetting to dewetting transition. 


\subsubsection{Experimental Methods}

\subsubsection{Materials.}

Colloidal silica (MIBK-ST, effective diameter $\sim 12 \mathrm{~nm}, \sim 30-31 \%$ silica in methyl isobutyl ketone) was kindly provided by Nissan Chemical Industries, Ltd. and used as received. Allyl 2-bromo-2methylpropionate (98\%, Sigma-Aldrich), chlorodimethylsilane (98\%, Sigma-Aldrich), 5\% platinum on activated charcoal (Sigma-Aldrich), hexamethyldisilazane (HMDZ, 99.9\%, SigmaAldrich), tetrahydrofuran (THF, 99.9+\%, Sigma-Aldrich), copper(II) bromide (Cu(II)Br, 99\%, Sigma-Aldrich), Tin (II) 2-ethylhexanoate $\left(\mathrm{Sn}(\mathrm{EH})_{2}, \quad \sim 95 \%, \quad\right.$ Aldrich), $N, N, N^{\prime}, N^{\prime \prime}, N^{\prime \prime}-$ Pentamethyldiethylenetriamine (PMDETA, 99\%, Macron Chemicals), 4,4'-dinonyl-2,2'dipyridyl (dNbpy, 97\%, Sigma-Aldrich), toluene (ACS grade, 99.5\%, Sigma-Aldrich), methanol (99.8\%, Mallinckrodt) and hydrofluoric acid (48 51\%, VWR) were used as received. Deuterated styrene (d-styrene, $\geq 98 \%$, Polymer Source) was passed through a column of alternating inhibitor remover (for hydroquinone and monomethyl ether hydroquinone, Sigma-Aldrich) and aluminum oxide (activated, neutral, Brockmann I, Sigma-Aldrich) to remove the inhibitor.

\subsubsection{Preparation of initiator-functionalized nanoparticles}

Surface modification of the silica nanoparticles (Figure 8.14a) was performed following a procedure by Pyun et al.[50] Briefly, this involved reacting the 1-(chlorodimethylsilyl)propyl 2bromoisobutyrate initiator with the silica dispersion in reflux overnight. HMDZ was then added as a capping agent for the remaining unfunctionalized hydroxyl groups on the silica surface. The initiator-functionalized nanoparticles were washed several times and separated from the unmodified ones by allowing them to settle in hexane for a few hours and decanting the solvent layer. This step was repeated at least ten times. Finally, the purified particles were recovered by centrifugation and dried. 
(a)

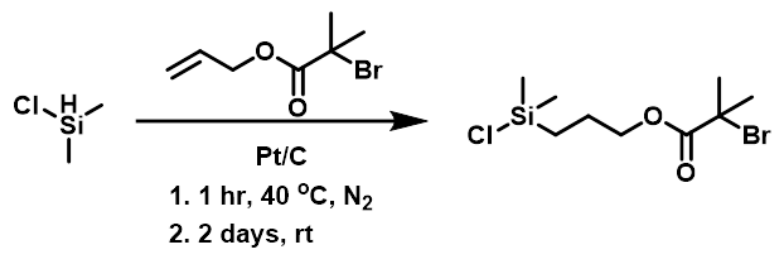

(b)

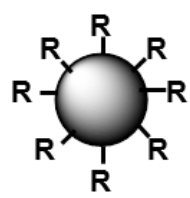

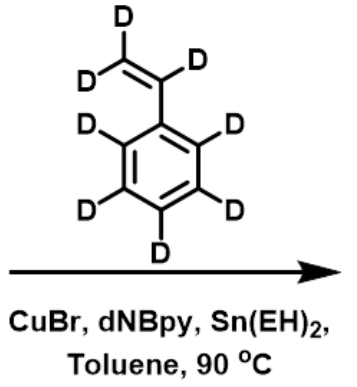
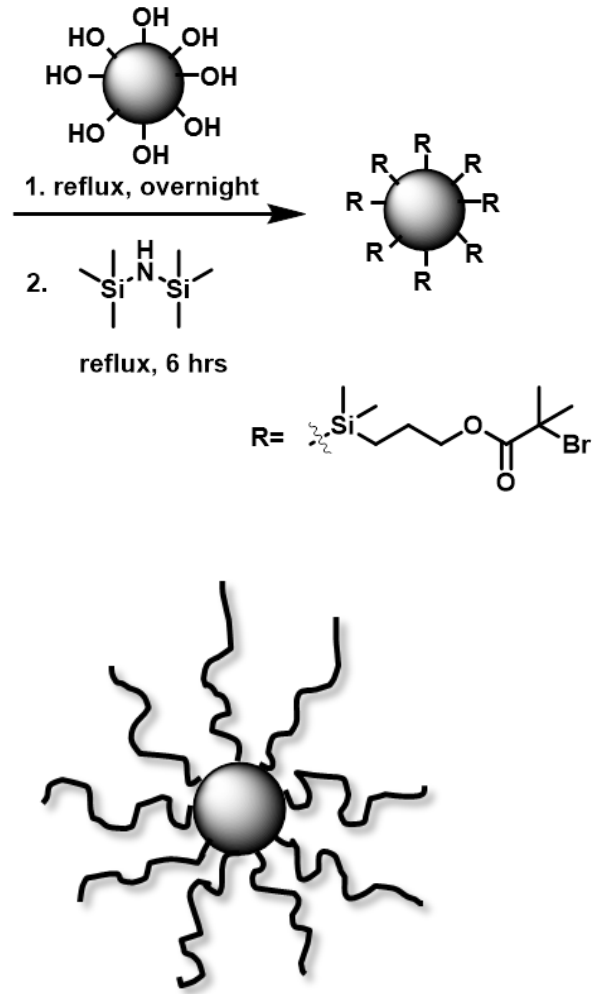

Figure 8.14: Synthesis procedure for the preparation of initiator-functionalized (a) and polymergrafted (b) silica nanoparticles.

\subsubsection{Preparation of polymer-grafted nanoparticles by AGET ATRP}

This work also demonstrated the preparation of deuterated polystyrene (dPS) using a modified AGET ATRP reaction procedure adopted from a previous work by Jakubowski et al.[51] A $\mathrm{Cu}(\mathrm{II}) \mathrm{Br}$ catalyst and dNbpy ligand were combined with d-styrene in one flask and bubbled with nitrogen for 30 minutes. In another flask, the $\mathrm{Sn}(\mathrm{EH})_{2}$ reducing agent and initiator-functionalized silica were dissolved in toluene, purged and transferred to the first flask. The reaction mixture was then placed in an oil bath at $90^{\circ} \mathrm{C}$ and allowed to proceed for 40.5 hours (Figure $8.14 \mathrm{~b}$ ). After polymerization, the reaction mixture was further diluted with THF, filtered through a column of neutral aluminum oxide to remove the catalyst, concentrated in vacuo and precipitated in excess amount of cold methanol. The recovered polymer-functionalized nanoparticles were further purified through a mixed solvent precipitation method using toluene and methanol. This step 
ensured the removal of unfunctionalized silica nanoparticles as well as free chains from the bulk sample. Prior to gel permeation chromatography experiments to determine the dPS brush molecular weight, the grafted polymers were cleaved from the silica surface by first dissolving the hybrid nanoparticles in $2 \mathrm{ml}$ THF and then adding $2 \mathrm{ml}$ of a $2 \%(\mathrm{v} / \mathrm{v})$ solution of aqueous HF. After stirring the solution overnight, the polymer was reprecipitated in excess amount of methanol and dried under vacuum for at least 24 hours. The grafted nanoparticles were also subjected to thermo-gravimetric analysis to calculate the polymer grafting density. This technique afforded particles with a brush molecular weight of 33,000 g/mol (dPS33k), PDI of 1.27 and polymer grafting density of 0.7 chain $/ \mathrm{nm}^{2}$.

\subsubsection{Purification of SiO-dPS nanoparticles.}

The purification of polymer-grafted nanoparticles followed a mixed solvent precipitation procedure to remove any remaining untethered or free chains. The dried sample was first redissolved in minimum amount of toluene and centrifuged for 10 minutes. Unfunctionalized silica nanoparticles remained in the bottom of the tube and were readily separated from the bulk of the sample. The supernatant was then transferred to another flask and placed in an oil bath at $50{ }^{\circ} \mathrm{C}$ for 30 minutes. This step ensured the homogenous dispersion of the $\mathrm{SiO}_{2}$-dPS nanoparticles as well as any unattached chains in the solvent. The flask was then cooled down to room temperature. Methanol was added dropwise to the solution until the cloudiness of the system persisted. After which, the flask was again heated to $50{ }^{\circ} \mathrm{C}$ which brought the solution back to a homogenous phase. The mixture was transferred to a separatory funnel and was left undisturbed for 2 days. The formation of two layers was observed. The bottom layer which contained the pure $\mathrm{SiO}_{2}-\mathrm{dPS}$ nanoparticles dispersed in toluene was collected and precipitated in excess amount of methanol. 
The solid sample was collected by centrifugation and dried at $60{ }^{\circ} \mathrm{C}$ for at least $24 \mathrm{hr}$. The process was repeated to ensure complete removal of all unwanted materials

\subsubsection{Preparation of nanocomposites}

The preparation of the polymer nanocomposite followed a simple solution mixing procedure. Initially, poly(vinyl methyl ether) (PVME) $\left(\mathrm{M}_{\mathrm{w}}=226,000 \mathrm{~g} / \mathrm{mol}, \mathrm{PVME} 226 \mathrm{k}\right)$ was dried at $70^{\circ} \mathrm{C}$ under vacuum overnight and cooled down to room temperature prior to use. A 20:80 (by weight) blend composition of $\mathrm{SiO}_{2}$-PS/PVME was prepared by co-dissolving the dPS33k and PVME226k in toluene and mixing at room temperature for at least 24 hours. The polymer blend was obtained by precipitating the solution in a large excess of hexane and collecting the solid by vacuum filtration. The resulting composite was allowed to dry in air for 2 days and annealed at $60^{\circ} \mathrm{C}$ under vacuum for at least 24 hours before the scattering measurements were performed. The samples were heated from room temperature to elevated temperature. In some cases the samples were also studied in cooling and we found reproducible data between heating and cooling as long as we did not exceed $145^{\circ} \mathrm{C}$

The resulting volume fractions, $\varphi$, were computed as follows:

Weight $\%$ of dPS33k tethered on $\mathrm{SiO}_{2}$ (from TGA, see Figure 8.16) $=89 \%$

Weight $\%$ of $\mathrm{SiO}_{2}=11 \%$

Composite preparation:

Mass of $\mathrm{SiO}_{2}-\mathrm{dPS} 33 \mathrm{k}$ hybrid nanoparticles: $0.0377 \mathrm{~g}$ (20\% by mass relative to PVME) Mass of $\mathrm{SiO}_{2}$ (11\% of hybrid nanoparticle mass): $0.004147 \mathrm{~g}$

Density of $\mathrm{SiO}_{2}=2.2 \mathrm{~g} / \mathrm{cm}^{3}$

Calculated $\mathrm{SiO}_{2}$ volume $=1.885 \times 10^{-3} \mathbf{c m}^{3}$

Mass of dPS33k (89\% of hybrid nanoparticle mass): $0.03353 \mathrm{~g}$ 
Density of dPS $=1.1307 \mathrm{~g} / \mathrm{cm}^{3}$

Calculated dPS volume $=\mathbf{0 . 0 2 9 7} \mathbf{c m}^{\mathbf{3}}$

Mass of PVME: $0.1506 \mathrm{~g}$ ( $80 \%$ by mass relative to $\mathrm{SiO}_{2}-\mathrm{dPS} 33 \mathrm{k}$ )

Density of PVME $=1.03 \mathrm{~g} / \mathrm{cm}^{3}$

Calculated PVME volume $=\mathbf{0 . 1 4 6 2} \mathbf{c m}^{3}$

\section{Total volume $=0.1778 \mathrm{~cm}^{3}$}

$\varphi_{\mathrm{SiO} 2}=\frac{\mathbf{0 . 0 0 1 8 8 5}}{\mathbf{0 . 1 7 7 8}}=0.0106$

$\varphi_{\mathrm{dPS}}=\frac{\mathbf{0 . 0 2 9 7}}{\mathbf{0 . 1 7 7 8}}=0.1670$

$\varphi_{\mathrm{SiO} 2-\mathrm{dPS}}=0.178$

$\varphi_{\mathrm{PVME}}=\frac{0.1462}{0.1778}=0.8223$

\subsubsection{Fourier transform infrared spectroscopy (FTIR)}

The presence of attached PS brushes was confirmed through FTIR measurements (Nicolet 4700

FTIR, Thermo Electron Corporation) which showed absorption bands corresponding to the different functional groups present in the sample. 


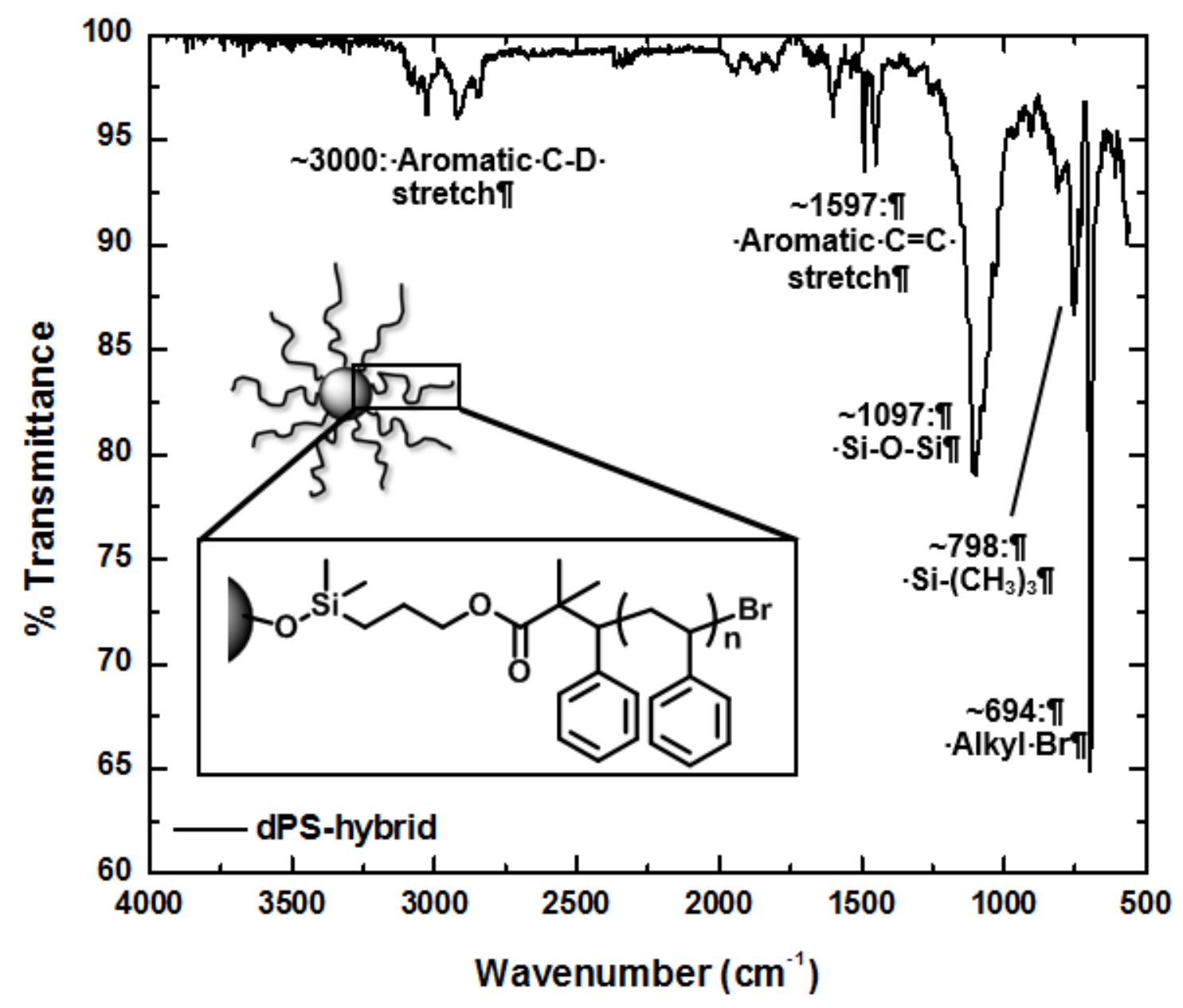

Figure 8.15: IR spectra of the hybrid nanoparticle showing signature absorption bands of the different functional groups of the tethered brush.

\subsubsection{Thermo-gravimetric analysis (TGA)}

Weight losses due to the tethered initiator and dPS brushes were determined by TGA (TGA Q500, TA Instruments). The experiments were conducted under argon atmosphere and the samples were heated from $25{ }^{\circ} \mathrm{C}$ to $800{ }^{\circ} \mathrm{C}$ at a heating rate of $10{ }^{\circ} \mathrm{C} / \mathrm{min}$. The resulting effective weight losses 


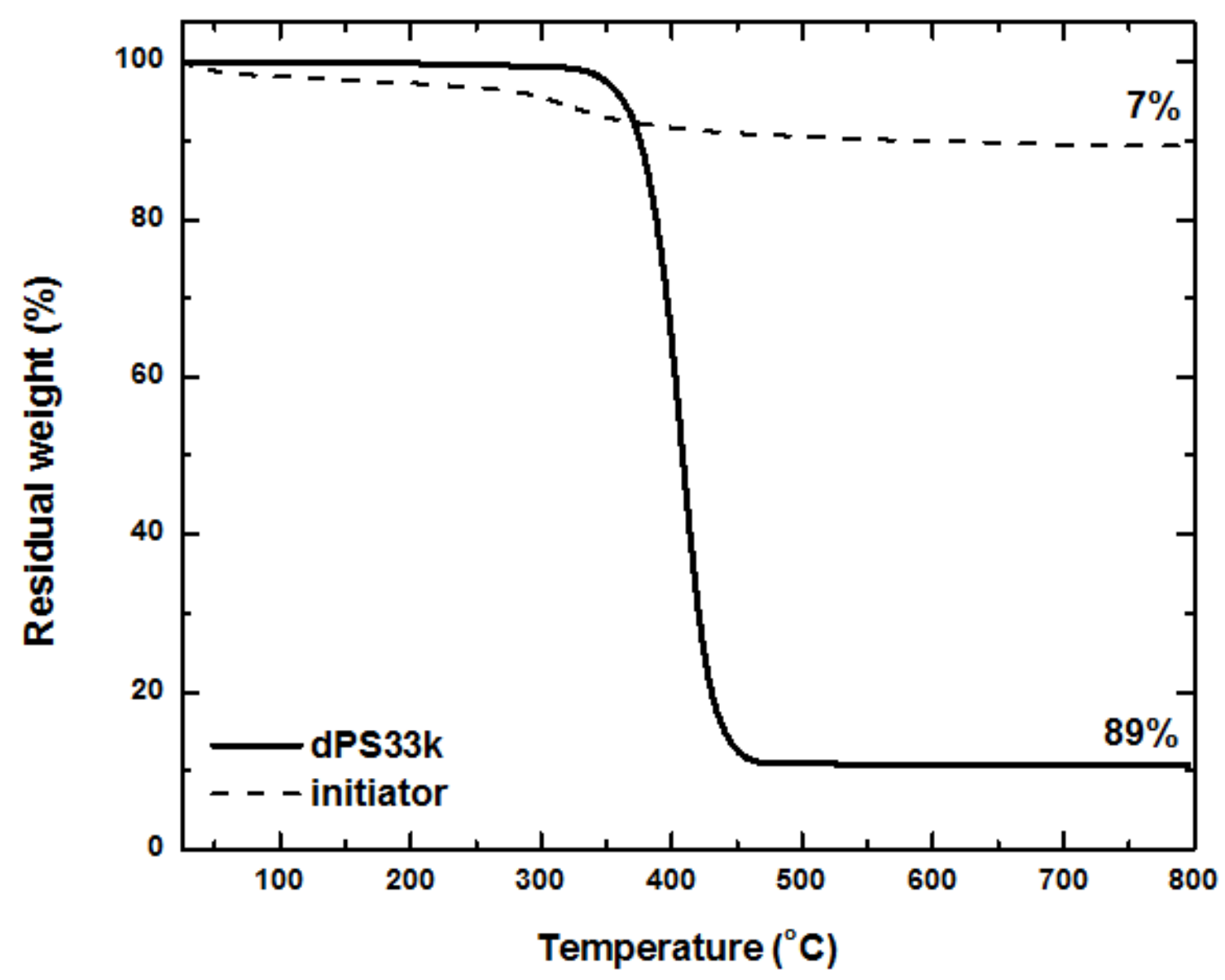

Figure 8.16: Thermogravimetric analysis plot of the dPS33k hybrid (solid line) and initiatorgrafted (dashed line) nanoparticle showing the weight loss due to the tethered brush and initiator, respectively.

\subsubsection{Gel permeation chromatography (GPC)}

Polystyrene chains cleaved from the surface of silica were dissolved in THF $(1 \mathrm{mg} / \mathrm{ml}$ concentration) and analyzed for molecular weight and molecular weight distribution (Viscotek 270, using PS standards). The obtained values were used to compute for the polymer grafting density. 


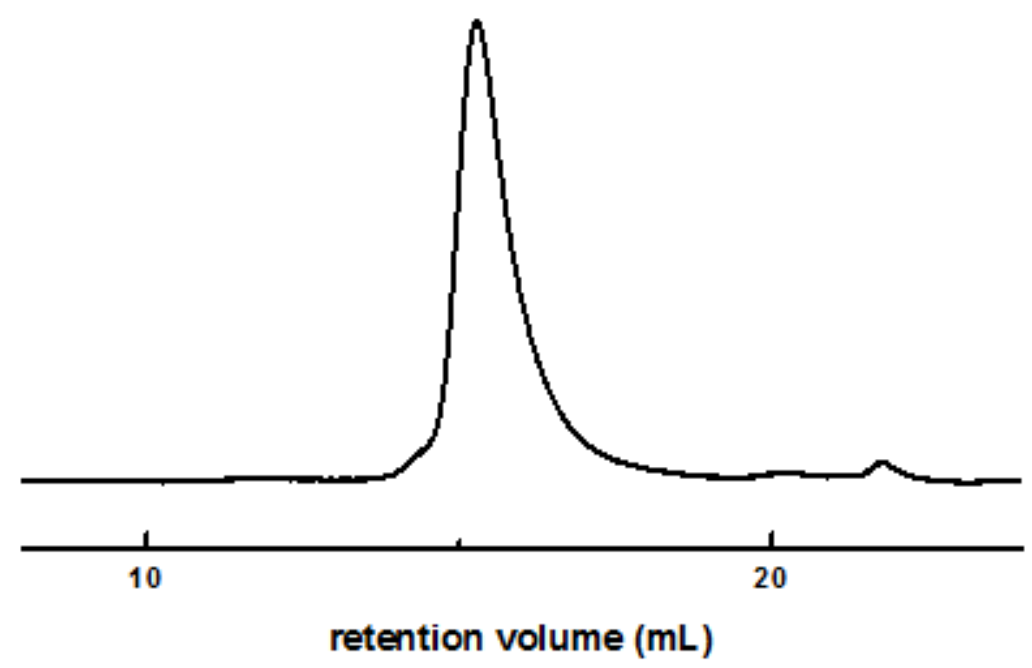

Figure 8.17: Gel permeation chromatogram of cleaved deuterated polystyrene brush.

\subsubsection{Calculation of polymer grafting density.}

$$
\text { Grafting density }=\frac{\frac{W \%_{\text {polymer+initiator }+ \text { silica }}}{100-W \%_{\text {polymer }}+\text { initiator }+ \text { silica }}-\frac{W \%_{\text {silica+initiator }}}{100-W \%_{\text {silica }}+\text { inititor }}}{\left(\mathrm{M}_{\mathrm{w}}\right)\left(\mathrm{S}_{\mathrm{sp}}\right)} \times \mathrm{N}_{\mathrm{A}}
$$

$\mathrm{W} \%=\mathrm{TGA}$ weight loss $\left(\right.$ from $\left.300{ }^{\circ} \mathrm{C}-800{ }^{\circ} \mathrm{C}\right)$

$\mathrm{M}_{\mathrm{w}}=$ molecular weight of grafted polymer from GPC

$\mathrm{S}_{\mathrm{sp}}=$ specific surface area $\left(1.93 \times 10^{20} \mathrm{~nm}^{2} / \mathrm{g}\right)$

$\mathrm{N}_{\mathrm{A}}=$ Avogadro's number $\left(6.022 \times 10^{23} \mathrm{molecules} / \mathrm{mol}\right)$

Grafting density $=\frac{\frac{88.77}{100-88.77}-\frac{7.168}{100-7.168}}{(32,717)\left(1.93 \times 10^{20} \mathrm{~nm}^{2} / \mathrm{g}\right)} \times\left(6.022 \times 10^{23} \mathrm{molecules} / \mathrm{mol}\right)$

$$
=0.74 \text { chain } / \mathrm{nm}^{2}
$$




\subsubsection{Small angle X-ray scattering (SAXS)}

We use a Rigaku SMax 3000 with a MicroMax-007HF rotating anode X-ray generator. The range of the magnitude of the scattering vector, $\mathrm{q}(=4 \pi / \lambda \operatorname{Sin}(\theta / 2))$, where $\lambda$ is the wavelength of the radiation and $\theta$ is the scattering angle) probed was $0.007-0.3 \AA^{-1}$. . The hybrid nanoparticle size in the bulk is inferred from the inter-particle distance in the matrix obtained from the first diffraction peak, $\mathrm{q}^{*}$.

\subsubsection{Analysis of hybrid nanoparticle size by SAXS}

The structural profile of the dPS-grafted silica nanoparticle was analyzed using SAXS. Supplementary Figure 8.10 shows the scattering intensity plot of the pure dPS33k hybrid which showed both first- and second-order intensity peaks denoted by $q_{1}{ }^{*}\left(=0.019 \mathrm{~A}^{-1}\right)$ and $q_{2}{ }^{*}(=0.033$ $\left.\mathrm{A}^{-1}\right)$, respectively. The ordered structure of the hybrid nanoparticles as confirmed by $\mathrm{q}_{1}{ }^{*}: \mathrm{q}_{2}:: 1: \sqrt{3}$ and gave an inter-particle distance, $d$, of $33.0 \mathrm{~nm}$ :

$$
d=\frac{2 \pi}{q^{*}} \times 1.22
$$

from this value and the known diameter of bare silica $(15.5 \mathrm{~nm})$, the brush height in the bulk system was estimated to be about $\sim 9 \mathrm{~nm}$. 


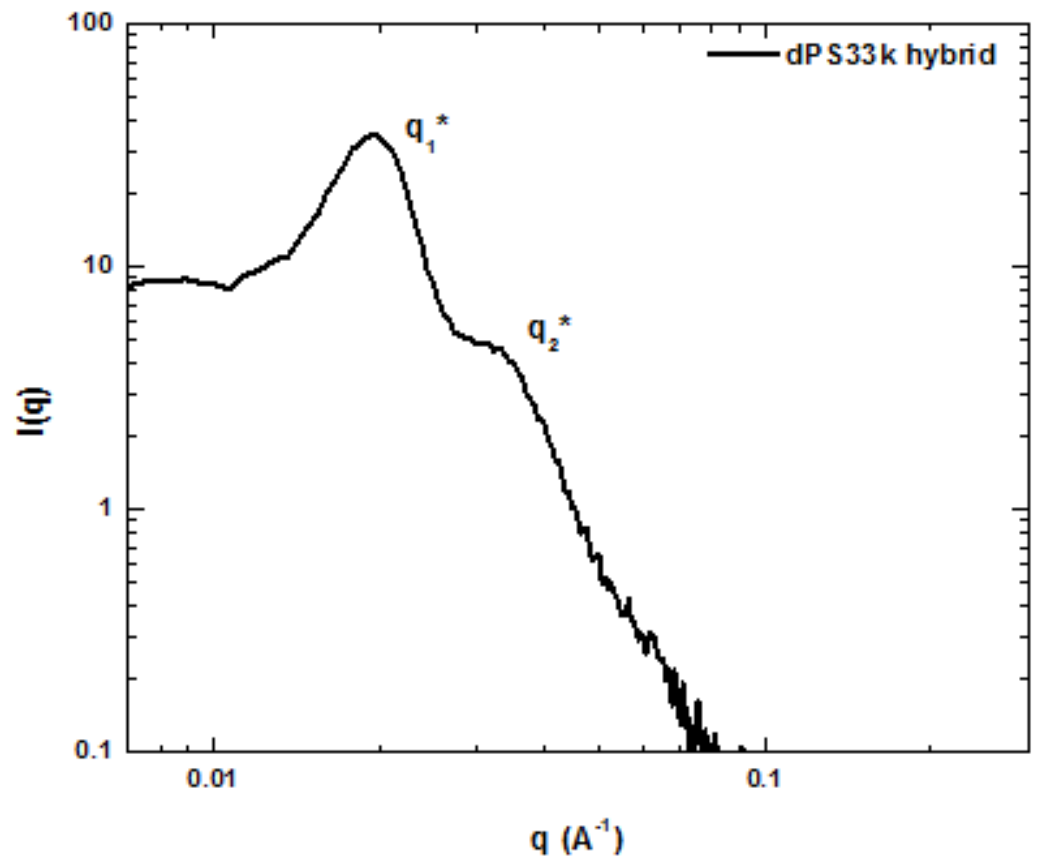

Figure 8.18: SAXS intensity profile of the pure dPS33k hybrid nanoparticle.

\subsubsection{Small-Angle Neutron Scattering (SANS)}

SANS measurements were taken on the NGB 30m SANS beamline at the National Institute of Standards and Technology Center for Neutron Research (NCNR) in Gaithersburg, MD. The measurements were performed with a neutron wavelength of $6 \AA$ and three sample-to-detector distances of $13 \mathrm{~m}, 4 \mathrm{~m}$ and $1 \mathrm{~m}$, thus accessing a q-range of $0.003 \AA^{-1}$ to $0.5 \AA^{-1}$. The nanocomposite samples were pressed into $1 \mathrm{~mm}$-thick pellets at $70{ }^{\circ} \mathrm{C}$ under high vacuum for $10 \mathrm{mins}$ to ensure the expulsion of trapped air bubbles. The pellets were sandwiched between two glass windows and secured in demountable titanium sample cells available at NCNR. The sample cells were then placed in a multiple-slot cell holder with a temperature-controlled fluid circulation system. The obtained raw data were corrected for detector sensitivity, background and empty cell contributions, as well as incoherent and coherent scattering from the pure PVME matrix component. The collected data were also normalized to an absolute scattering intensity. 
Table 8.1: Values of the Scattering Length Densities

\begin{tabular}{|c|c|c|}
\hline Compound & Neutron Coherent SLD $\left(\AA^{-2}\right)$ & $\mathrm{Cu} \operatorname{K} \alpha \operatorname{SLD}\left(\AA^{-2}\right)$ \\
\hline $\mathrm{SiO}_{2}$ & $3.48 \times 10^{-6}$ & $1.89 \times 10^{-5}$ \\
\hline Deuterated polystyrene (dPS) & $6.46 \times 10^{-6}$ & $9.60 \times 10^{-6}$ \\
\hline Polystyrene (PS) & $1.41 \times 10^{-6}$ & $9.61 \times 10^{-6}$ \\
\hline $\begin{array}{l}\text { Poly(vinyl methyl ether) } \\
\text { (PVME) }\end{array}$ & $3.53 \times 10^{-7}$ & $9.66 \times 10^{-6}$ \\
\hline
\end{tabular}

\subsubsection{Transmission Electron Microscopy (TEM)}

TEM measurements were performed at the National Institute of Standards and Technology in Gaithersburg, MD. The composite sample was thinned into a lamellar, electron transparent $(\sim 150$ $\mathrm{nm}$ ) section and mounted onto a copper half-grid by focused ion beam (FIB) milling, optimized for soft materials, as described elsewhere.[58] The spatial distribution of the nanoparticles in the composite material was imaged using an FEI Titan TEM in bright-field mode at $300 \mathrm{kV}$ with a 40 micrometer objective aperture inserted.

\subsubsection{Excluded Volume Model}

We employed the excluded volume model to quantitatively model the SANS data obtained for the mixtures of dPS and PVME. This model describes polymer chain conformations and accounts for excluded volume effects and uses the formalism developed by Hammouda.[57] For highly-grafted nanoparticles, it can be assumed that the inner region closest to the core consists of stretched chains (to avoid steric crowding), while the outer region has more flexible chains. These flexible chains are directly involved in the wetting-dewetting transition and dominate the intermediate and high $\mathrm{q}$ behavior of the coherent SANS intensity I(q). Using the excluded volume model, the changes in the conformation of these brushes during wetting/dewetting can thus be monitored as reflected in the extracted $R_{g}$ values of the corona. 


\section{Tuning the Wetting-DeWetting AND Dispersion-Aggregation TRANSITIONS IN POLYMER NANOCOMPOSITES USING COMPOSITION OF GRAFT AND MATRIX POLYMERS}

Adapted from: Submitted to Materials Research Express

\subsection{INTRODUCTION}

Polymer nanocomposites are a broad class of soft materials where nanoscale filler materials are added to a bulk polymer matrix in order to modify and improve the mechanical, optical, electrical, and/or thermal properties of the polymer matrix.[1-6] Controlling the spatial arrangement of the components within the nanocomposite (i.e. its morphology) has been the focus of many studies, as the macroscopic properties of the nanocomposites are a function of their microscopic morphology. [1-6] One strategy to promote nanoscale fillers' dispersion or aggregation in the matrix is to densely graft the surface of the nanofillers or nanoparticles with polymer chains that are chemically identical to the matrix polymer. In this way, the direct particle-particle and particlematrix interactions are screened by the grafted polymer layer, and as a result the effective fillerfiller interactions and the morphology of the nanocomposite, is tuned by varying the physical properties of the composite, such as the graft to matrix molecular weights ratio, particle curvature, grafting density, flexibility of graft and matrix polymers, dispersity in graft and matrix molecular weights. [5, 7-24]

Furthermore, these past studies have also established the connection between the particles' dispersion-aggregation and the extent of mixing of the graft and matrix polymer chains. When the graft and matrix chains are mixed (i.e. the grafted layer is wet by the matrix chains) the nanocomposite is driven towards dispersed morphologies. When the graft-matrix are demixed (i.e. the grafted layer is dewet by the matrix chains) the nanocomposite is driven towards aggregated 
morphologies. Based on this relationship, one can expect that tuning the wetting or dewetting of the grafted layer provides a way to control dispersion or aggregation of the filler material within the matrix. For composites with chemically identical graft and matrix chains at high grafting densities, since the direct particle-particle and particle-polymer interactions are screened by the dense grafted polymer layer and the graft and matrix chains are chemically identical, the wettingdewetting is driven purely by entropy. The free energy change going from wet to dewet state is dictated by the loss in graft-matrix mixing entropy and gain in graft and matrix chain conformational entropy. The nanocomposite design parameters (e.g. graft and matrix molecular weights, particle curvature, grafting density, flexibility) that increase the mixing entropy gain and decrease the conformational entropy loss will shift the nanocomposite morphology towards a wet grafted layer state and thus promote particle dispersion. For example, at high $\frac{N_{\text {graft }}}{N_{\text {matrix }}}$ ratio, the loss in conformational entropy of the matrix chains is smaller than the mixing entropy gain leading to a wet grafted layer and thus a dispersed morphology. Many applications where particle dispersion is desired, however, require matrix chains of fairly large molecular weights which then require long graft chains to achieve a high $\frac{N_{\text {graft }}}{N_{\text {matrix }}}$ ratio for wetting. [1-3, 25] Grafting large molecular weight polymer chains on the particle surface is a challenge synthetically and the focus of many recent studies [26-31], and furthermore, the large graft chain molecular weights also limit the amount of filler (i.e. polymer grafted particles) fraction in the matrix. Thus there is a need for approaches that achieve wetting of the grafted layer and in turn, particle dispersion at values of $\frac{N_{\text {graft }}}{N_{\text {matrix }}}<1$

One such approach is to consider nanocomposites where the graft and matrix chains are chemically dissimilar and, at the desired temperatures, have attractive enthalpic interactions 
between the graft and matrix chains. In addition to the entropic driving forces for wettingdewetting (and therefore dispersion-aggregation), these chemically dissimilar attractive graftmatrix composites also have enthalpic driving forces that favor grafted layer wetting. There are only a few studies that consider such systems with attractive, chemically-dissimilar graft and matrix chains. For a system of grafted colloids in a polymer matrix, theoretical studies have shown that as the interactions between the grafted and matrix chains became more attractive, the wetting of the grafted layer increased and therefore the effective interactions between two grafted layers became more repulsive.[32, 33] Two separate experimental studies have also demonstrated that the dispersed morphology is stabilized for small $\frac{N_{\text {graft }}}{N_{\text {matrix }}}$ either by using a graft-matrix pair with LCST phase behavior or by introducing hydrogen bond acceptors and donors into the graft and matrix chains respectively. $[3,34]$ In the latter case with hydrogen bonds, the nanocomposites were additionally shown to display thermo-reversible dispersion-aggregation phase behavior.

Recently, we used simulations and experiments to show that, for polymer grafted nanoparticles in a chemically different polymer matrix where the graft-matrix polymer pair exhibit LCST phase behavior, the wetting-dewetting and dispersion-aggregation transitions are distinct. [35] For these composites, while the dispersion-aggregation transition is a sharp transition that occurs over a narrow range of temperatures, the wetting-dewetting transition occurs gradually over a broader range of temperatures. Furthermore, for these chemically dissimilar graft-matrix composites there is a critical extent of wetting which marks the onset of dispersion-aggregation transition; this critical extent of wetting is equal to the extent of wetting of an equivalent chemically identical or athermal nanocomposite. Since the wetting-dewetting transition for chemically different graftmatrix composites occurs over a range temperatures rather than a distinct temperature, there are a range of "partially wet" and "partially dewet" states that occur between the "fully wet" and "fully 
dewet" states. This means that the extent of wetting, i.e. the extent of mixing between the grafted layer and matrix chains, can be tuned within the aggregated and dispersed morphologies by varying the chemical properties of the system, such as graft-matrix interactions or graft/matrix composition, at specific graft and matrix chain lengths. Furthermore, the presence of a critical degree of wetting suggests that the temperature at which the dispersion-aggregation transition occurs can be tuned by influencing the wetting-dewetting behavior of the composite using these composite design properties.

In this study, we show that, for nanocomposites with $\frac{N_{\text {graft }}}{N_{\text {matrix }}} \leq 1$, by using graft and matrix polymers which are random copolymers of attractive and athermal monomers, we tune the overall degree of wetting and the dispersion-aggregation transition by varying the fraction of attractive monomers in the graft $\left(f_{G}\right)$ and matrix $\left(f_{M}\right)$ chains and Flory-Huggins interaction parameter between the attractive graft and matrix monomers, $\chi_{G M}$. At constant $f_{G} / f_{M}$, simultaneously reducing the magnitude of $f_{G}$ and $f_{M}$ reduces the degree of wetting in the dispersed state while maintaining a constant dispersion-aggregation transition $\chi_{G M}$. In contrast, varying $f_{G} / f_{M}$ tunes both the wetting of the grafted layer and the dispersion-aggregation transition. Additionally, varying $f_{G}$ and/or $f_{M}$ tailors the chain conformations of the graft and matrix polymers in the wet and dewet states, where composites with higher $f_{G}, f_{M}$ values show larger variation in their ensemble averaged radius of gyration $\left\langle R_{g}^{2}\right\rangle$ with varying $\chi_{G M}$. Interestingly, at the dispersionaggregation transition, the graft and matrix chains in the chemically dissimilar composites adopt chain conformations identical to those of the graft and matrix chains in an equivalent chemically identical (athermal) composite. 


\subsection{APPROACH}

\subsubsection{Model}

We model polymer grafted spherical nanoparticles in a polymer matrix using a generic coarsegrained model. The nanoparticle (denoted as P) is modeled as a rigid-body of several 1d diameter beads (where $\mathrm{d} \approx 1 \mathrm{~nm}$ ). The nanoparticle consists of surface beads to preserve the excluded volume of the particle and grafting sites to anchor the graft chains. The surface and grafting site beads overlap in the rigid body of the particle, with the grafting site beads isotropically located on the spherical particle surface. Each graft $(\mathrm{G})$ or matrix $(\mathrm{M})$ polymer is modeled as a fully-flexible bead-spring chain $[36,37]$, with each polymer bead of size d representing a group of monomers or Kuhn segment on the polymer chain, and harmonic springs linking the beads having a force constant of $\mathrm{k}=50 \mathrm{k} \mathrm{B} / \mathrm{d}^{2}$ and a bond rest length of $\mathrm{r}_{0}=1 \mathrm{~d}$, mathematically represented as

$$
U_{\text {bond }}(r)=\frac{1}{2} k\left(r-r_{0}\right)^{2}
$$

where $r$ is the center to center distance between the bonded beads.

Any attractive graft or matrix monomer-monomer interactions are modeled using a Lennard-Jones (LJ) potential:

$$
U_{L J}(r)=4 \varepsilon\left[\left(\frac{\sigma}{r}\right)^{12}-\left(\frac{\sigma}{r}\right)^{6}\right]
$$

where $\varepsilon$ is the well depth, $\sigma$ is the contact distance, and $\mathrm{r}$ is the center-center distance of the interacting beads. Any athermal graft or matrix monomer-monomer interactions are modeled via a purely-repulsive Weeks-Chandler-Andersen (WCA) potential with $\varepsilon=1.0 k_{B} T$ :

$$
U_{W C A}(r)=\left\{\begin{array}{c}
4 \varepsilon\left[\left(\frac{\sigma}{r}\right)^{12}-\left(\frac{\sigma}{r}\right)^{6}\right]+\varepsilon, \quad r<2^{\frac{1}{6}} \sigma \\
0, \quad r \geq 2^{\frac{1}{6} \sigma}
\end{array}\right.
$$


The athermal particle-particle and particle- (graft or matrix) monomer interactions are always modeled using the WCA potential.

In this study the nanoparticle size is maintained at $\mathrm{D}=5 \mathrm{~d}$, grafting density at $\Sigma=0.76$ chains $/ \mathrm{d}^{2}$, and the graft length at $\mathrm{N}_{\text {graft }}=10$ and matrix length as $\mathrm{N}_{\text {matrix }}=10$ or 50 . The graft and matrix chains are random copolymers of attractive and athermal monomers, with each graft and matrix chain having an independently randomized sequence of monomers. The composition of the graft and matrix random copolymers are varied via $f_{G}$ and $f_{M}$, the fraction of attractive beads per chain of the graft and matrix chains respectively, over the range of 0.0 to 1.0. For example $f_{G}=f_{M}=1.0$ corresponds to the fully attractive homopolymer graft and matrix case and $f_{G}=$ $f_{M}=0.0$ corresponds to the fully athermal homopolymer case (i.e. chemically identical graftmatrix case). Figure 9.1 below describes some of the chain compositions studied in this work.

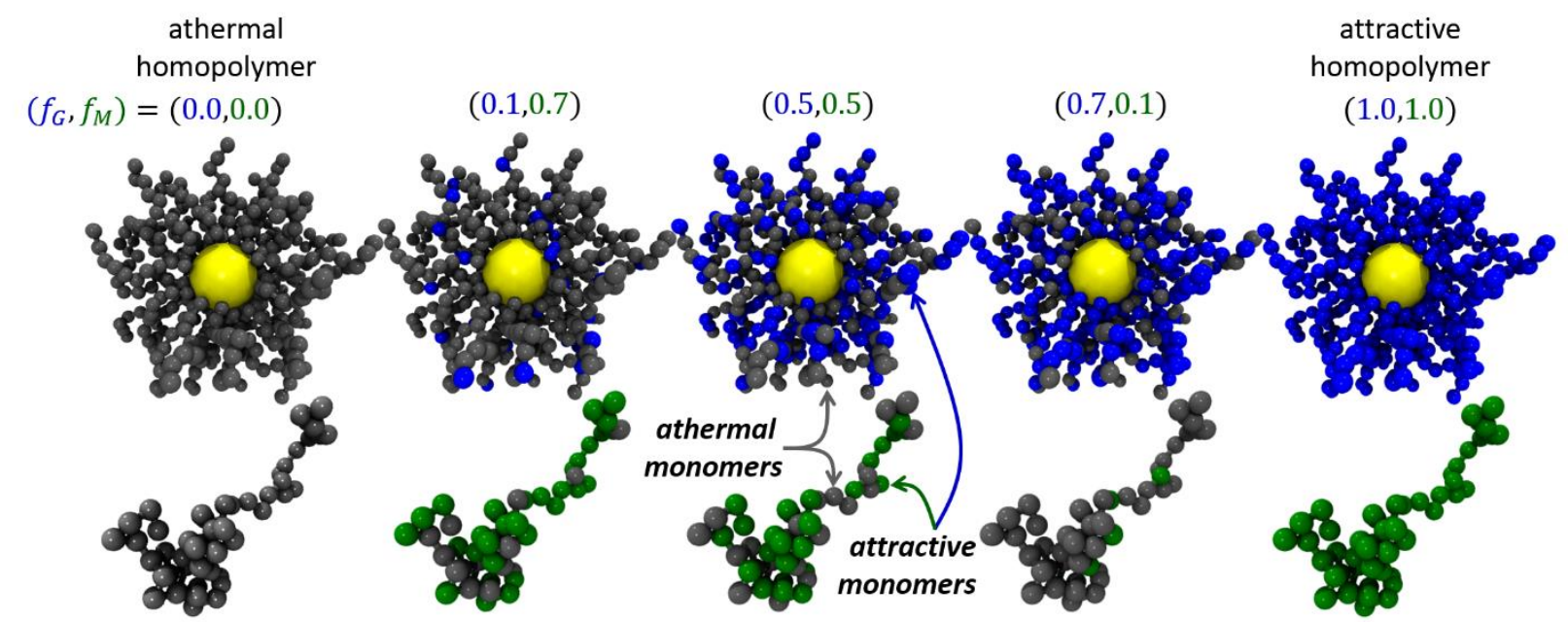

Figure 9.1: Schematic depiction of some of the graft and matrix chain compositions considered in this work. Note that the graft monomers are isotropically .grafted to the particle surface and that, in these images, some of the grafted chains are hidden for clarity. Grey beads represent athermal monomers, blue and green beads represent attractive monomers in graft and matrix chains respectively. This figure is best viewed in color. 
The attractive graft-graft (blue-blue) and matrix-matrix (green-green) interactions are maintained constant with $\mathrm{LJ}$ interaction strength $\varepsilon_{G G}=\varepsilon_{M M}=0.5 k_{B} T$, while the attractive graftmatrix $\mathrm{LJ}$ interaction strength $\varepsilon_{G M}$ is varied from 0.3 to $2.0 \mathrm{k}_{\mathrm{B}} \mathrm{T}$. All grey graft and matrix monomer-monomer or monomer particle interactions are treated as athermal. The overall graftmatrix Flory-Huggins interaction parameter $\chi_{G M}$ is defined as

$$
\chi_{G M}=\frac{\chi_{G M}^{*}}{Z}=\left(\varepsilon_{G M}-0.5\left(\varepsilon_{G G}+\varepsilon_{M M}\right)\right) / k_{B} T
$$

where $\chi_{G M}^{*}$ is the standard Flory-Huggins interactions parameter, scaled by lattice coordination number z. Supplementary Figure 9.7 shows how our simulated $\chi_{G M}$ parameter relates to real experimental temperatures for a specific blend of poly(styrene) and poly(vinyl-methyl-ether). We caution the reader that, while these data provide a useful connection between our simulations and experiments, we do not have access to experimental data for exactly the same graft lengths, matrix lengths, or compositions used in our study. We present the data in Supplementary Figure 9.7 only as a point of reference to the reader. We also note that the random copolymer composites in this study are similar to an experimental nanocomposite system studied (experimentally) by Hayward and coworkers. [38] In that study, the authors considered a polymer grafted nanoparticle with poly2-vinyl-pyridine-r-styrene (P2VP-r-PS) graft random copolymer in a poly-4-vinyl-phenol-rstyrene (P4VPh-r-PS) matrix. While PS, P2VP, and P4VPh are all structurally similar, P2VP and $\mathrm{P} 4 \mathrm{VPh}$ are hydrogen bond donor and acceptors (and therefore are "attracted" to one another) and PS in the graft and matrix chains act as relatively athermal comonomers. In our simulations, the attractive $\mathrm{G}$ and $\mathrm{M}$ beads are (qualitatively) similar to the attractive P2VP and P4VPh monomers with isotropic LJ interactions rather than directional H-bond interactions, and the athermal monomers in the graft and matrix chains in our model are similar to the PS comonomers. 


\subsubsection{Method}

Using the model described above, we conduct Brownian dynamics (BD) simulations in the canonical ensemble using the graphical processing unit based HOOMD-blue package $[39,40]$. We first create an initial configuration in the following manner: We generate a particle of a desired diameter with isotropically distributed graft points, with the chains extending radially from these graft points embedded on the particle surface. In order to make it easier to insert the grafted particle into the simulation box, a short simulation with strong Lennard-Jones monomer-monomer and monomer-particle attraction is run to compress the grafted chains from these extended conformations. Copies of this one compressed grafted nanoparticle are then randomly placed in a large cubic box to achieve the desired filler fraction along with the desired number of matrix chains. The filler fraction of the nanocomposite in this study is maintained at $\phi_{G}=0.13$ where $\phi_{G}$ is defined as

$$
\phi_{G}=\frac{V_{\text {grafts }}}{V_{\text {grafts }}+V_{\text {matrix }}}
$$

where $V_{X}$ is the total volume occupied by component $\mathrm{X}$ in the system.

At an integrator temperature of $\mathrm{T}_{\text {inital }}=5.0$, the initial configuration is then integrated using a Brownian dynamics integrator for $5 \mathrm{e} 6$ time steps to both mix and relax the graft and matrix chains. The box is then compressed to the desired volume fraction over $10 \mathrm{e} 6$ steps, and then mixed again for $15 \mathrm{e} 6$ steps at the compressed state. For all simulations, the total occupied volume fraction in the simulation box is maintained to be $\eta=0.35$, and we maintain 60,000 matrix beads to enforce a minimum box size of $\approx 44 \mathrm{dx} 44 \mathrm{dx} 44 \mathrm{~d}$. We then vary the integrator temperature $\mathrm{T}$ from $\mathrm{T}_{\text {inital }}=$ 5.0 to $\mathrm{T}_{\text {final }}=1.0$ using ten, geometrically sized quenches over a period of 50e6 time steps. After the annealing is completed, we run the simulations for an additional 50e6 time steps where we sample the equilibrium configurations of the system every $0.5 \mathrm{e} 6$ time steps to calculate the 
ensemble averages of various thermodynamic and structural properties. The starting temperature, ending temperature, number of quenches, quench temperatures, and number of time steps of each stage of the protocol are chosen after rigorously testing many protocols, and finding that this protocol is the most computationally efficient that produced statistically similar equilibrium states from different initial conditions. Our simulation protocol is visually depicted in Supplementary Figure 9.8 .

\subsubsection{Analyses}

The average radius of gyration of the graft (matrix) chains quantifies the chain conformations averaged over all of the graft (matrix) chains in the system.

$$
\left\langle R_{g}^{2}\right\rangle=\left(\frac{1}{n_{X C} * N_{X}}\right) \sum_{i=1}^{n_{X C}} \sum_{j=1}^{N_{X}}\left(r_{i, j}-r_{i, c o m}\right)^{2}
$$

where $\left\langle\mathrm{R}_{\mathrm{g}}{ }^{2}\right\rangle$ is the average squared radius of gyration, in units of $\mathrm{d}^{2}, \mathrm{n}_{\mathrm{XC}}$ is the number of graft or matrix chains in the system(across all particles), $N_{X}$ is the length of the graft or matrix chains, $r_{i, j}$ is the position of monomer $\mathrm{j}$ on chain $\mathrm{i}$, and $\mathrm{r}_{\mathrm{i}, \mathrm{com}}$ is the center of mass of chain $\mathrm{i}$.

The wet matrix fraction quantifies the extent of wetting of the grafted layer by matrix monomers:

$$
\text { wet matrix fraction }=\frac{n_{M, w e t}}{n_{M}}
$$

where $n_{M}$ is the total number of matrix monomers in the system and $n_{M \text {,wet }}$ is the number of matrix monomers that are wetting grafted layers. We find $\mathrm{n}_{\mathrm{M}}$,wet by first calculating the graft-monomer count field throughout the simulation box, then finding the spatial locations of the isosurfaces corresponding to a monomer count of 0.01 , and then counting the number of matrix monomers that are within this closed isosurface. For this study, we found that an isosurface corresponding to 
an average monomer count of 0.01 best captures the outer edge of the grafted layer of isolated particles or clusters of particles.

We calculate the particle-particle, graft-graft, and matrix-matrix pair correlation functions, $\left(g_{P P}(r) g_{G G}(r)\right.$, and $\left.g_{M M}(r)\right)$, to quantify the spatial correlation between these various species.[41] In order to better assess the extent of phase separation between the components of our simulation, we also convert the real space pair correlation functions to Fourier space structure factors via:

$$
S_{X X}(q)=1+\frac{4 \pi \rho_{X}}{k} \int r \cdot\left(g_{X X}(r)-1\right) \cdot \sin (q r) d r
$$

where $S_{X X}(q)$ is the value of the structure factor for pair $X X(P P, M M$, or GG) at wavenumber $q$, $\rho_{X}$ is the number density of beads of type $\mathrm{X}$, and $\mathrm{g}_{\mathrm{XX}}(\mathrm{r})$ is the pair correlation function for pair $\mathrm{XX}$ at a real-space distance $\mathrm{r}$.

In order to ensure that no unrealistic density fluctuations appear in our simulated composites, we calculate the average minimum and maximum local volume fraction along with the overall average local volume fraction. The local volume fraction is calculated by subdividing the simulation box at each timestep into 100 equal sized cuboids and calculating the occupied volume fraction separately for each small region. The average minimum and maximum local volume fractions are then calculated by averaging the values of the instantaneous minimum and maximum cuboid volume fractions, respectively, over the last 50e6 timesteps of the simulation. Similarly, the overall average local volume fraction is calculated by averaging all of the cuboid volume fractions over the last 50e6 timesteps. Based on these data shown in Supplementary Figure 9.9, we limit the data presented for the $f_{G}=f_{M}=1.0$ (homopolymer) and $f_{G}=f_{M}=0.7$ composites to $\chi_{G M}>-0.5$ as unrealistic density fluctuations appear below this $\chi_{G M}$. All other systems studied show near constant density fluctuations at all $\chi_{G M}$, while $f_{G}=f_{M}=1.0$ and 0.7 show systematically increasing density fluctuations as $\chi_{G M}$ decreases at all $\chi_{G M}<0.5$ 
9.3 RESULTS AND DISCUSSION
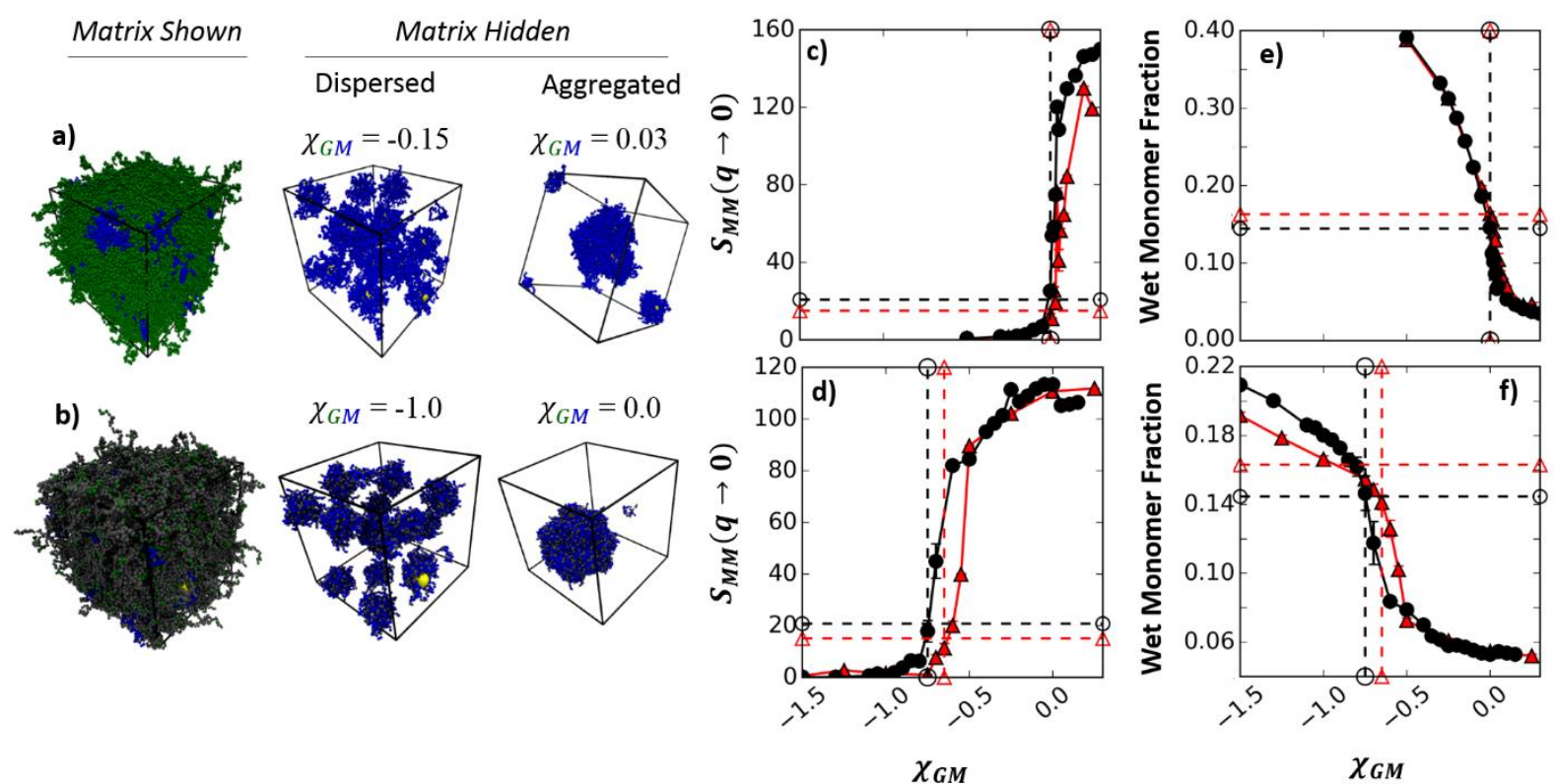

Figure 9.2: Simulation snapshots, low-q value of the matrix-matrix structure factor, and wet monomer fraction. In all parts, the particle diameter is $D=5 d$, the grafting density $=$ 0.76 chains $/ d^{2}$, the graft chain monomer volume fraction is $\phi_{G}=0.13$ and the total volume fraction in the simulation box is $\eta=0.35$. Parts $a$, $c$ and $e$ are for composites with $f_{G}=f_{M}=$ 1.0 and parts $b, d$ and fare for composites with $f_{G}=0.7, f_{M}=0.1$. The graft length is $N_{\text {graft }}=10$ in all parts, while a matrix of length $N_{\text {matrix }}=10$ is denoted with red triangles and $N_{\text {matrix }}=50$ is denoted with black circles. The horizontal dashed lines in parts $c, d$ and e,f represent the low- $q$ structure factor and wet monomer fraction (respectively) of the equivalent athermal ( $f_{G}=f_{M}=$ $0.0)$ composite. The vertical dashed lines in parts c-f mark the location of the dispersionaggregation transition. The error bars in part b-f are standard deviations calculated from 50 uncorrelated configurations for each system; the error bars when not visible are smaller than the size of the symbol.

In Figure 9.2 we present simulation snapshots and data which quantify the dispersionaggregation state and the extent of wetting-dewetting for two graft and matrix chain compositions: $f_{G}=1.0, f_{M}=1.0$ (Figure 9.2a, Figure 9.2c, and Figure 9.2e) and $f_{G}=0.7, f_{M}=0.1$ (Figure 9.2b, Figure 9.2d, and Figure 9.2f). Figure 9.2a and Figure 9.2b are representative simulation 
snapshots which visually depict the dispersed and aggregated morphologies for both the homopolymer and random copolymer $f_{G}=0.7, f_{M}=0.1$ composites. At negative $\chi_{G M}$, equivalent to lower temperatures in a composite with lower critical solution temperature (LCST) phase behavior (Supplementary Figure 9.7),-the grafted particles of both chain compositions show a dispersed morphology. At positive $\chi_{G M}$, equivalent to higher temperatures in the LCST phase diagram, the grafted particles phase separate from the matrix chains into an aggregated morphology. In Figure 9.2c and Figure 9.2d, we plot the value of the matrix-matrix structure factor $S_{M M}$ as $q \rightarrow 0$ as a function of $\chi_{G M}$ in order to quantify the extent of phase separation in the nanocomposite with $N_{\text {matrix }}=10$ and 50. Low values of $S_{M M}(q \rightarrow 0)$ are indicative of dispersed morphologies while an upturn in $S_{M M}(q)$ as $q \rightarrow 0$ indicates the onset of aggregation of matrix chains. The dispersion -aggregation transition (vertical lines in Figure 9.2c through Figure 9.2f) is identified as the $\chi_{G M}$ at which a sharp increase in $S_{M M}(q \rightarrow 0)$ occurs and we find good qualitative agreement between these data and the visual analysis. The dispersion-aggregation transition for the homopolymer graft-matrix pair at both values of $N_{\text {matrix }}$ is sharp and occurs at $\chi_{G M} \approx 0$ while the random copolymer composites go through dispersion to aggregation transition at $\chi_{G M} \approx$ -0.75 for $N_{\text {matrix }}=50$ and $\chi_{G M} \approx-0.65$ for $N_{\text {matrix }}=10$. We note that the particle-particle and graft-graft structure factors also show upturn at $q \rightarrow 0$ at approximately the same $\chi_{G M}$ as the matrix-matrix structure factor (Supplementary Figure 9.10). Figure 9.2c and Figure 9.2d also show that the dispersion-aggregation transition is slightly broader for the random copolymer $\left(f_{G}=\right.$ $\left.0.7, f_{M}=0.1\right)$ composites compared to the homopolymer composites. The increased breadth of the dispersion-aggregation transition for the random copolymer composite compared to the homopolymer composites is also seen in the graft-graft and matrix-matrix structure factors (Supplementary Figure 9.10). 
Interestingly, for the homopolymer composites, varying $\mathrm{N}_{\text {matrix }}$ shows insignificant differences in the dispersion-aggregation transition even though in chemically identical homopolymer graft and matrix composites, increasing $\mathrm{N}_{\text {matrix }}$ at constant $\mathrm{N}_{\text {graft }}$ has been shown to dictate dispersion/aggregation. $[5,7,19-21]$ For the random copolymer $f_{G}=0.7, f_{M}=0.1$ composites (Figure 9.2d), as $N_{\text {matrix }}$ decreases the dispersion-aggregation transition $\chi_{G M}$ shifts right to less negative values.

We present the extent of wetting of the grafted layer by the matrix chains using the wet monomer fraction as a function of Flory Huggins $\chi_{G M}$ in Figure 9.2e and Figure 9.2f for $f_{G}=$ $1.0, f_{M}=1.0$ and $f_{G}=0.7, f_{M}=0.1$, respectively. For all composites, the wet monomer fraction gradually decreases over the entire range of $\chi_{G M}$. The gradual wetting-dewetting transitions are in contrast to the comparatively sharp dispersion-aggregation transitions (characterized by the $S_{M M}(q \rightarrow 0)$ data in Figure 9.2c and Figure 9.2d) marked by vertical lines in Figure 9.2e and Figure 9.2f. Thus, wetting-dewetting and dispersion-aggregation transitions cannot be treated as synonymous as one occurs gradually over a broad range of $\chi_{G M}$ while the other occurs over a much smaller range of $\chi_{G M}$. This phenomena has also been shown experimentally in our recent work [35] for a PS (graft) grafted silica in PVME (matrix) analogous to the simulated homopolymer $\mathrm{N}_{\text {graft }}=10$ and $\mathrm{N}_{\text {matrix }}=50$ composites discussed here. Similar to the $S_{M M}(q \rightarrow 0)$ data, for the composites with fully attractive homopolymer graft and matrix chains, the $N_{\text {matrix }}=10$ and $N_{\text {matrix }}=50$ cases have nearly identical wetting at all $\chi_{G M}$, which is in line with their similar dispersion-aggregation behavior discussed in Figure 2c. For the random copolymer cases there are small differences in the wet monomer fraction profiles of $N_{\text {matrix }}=10$ and $N_{\text {matrix }}=50$ in line with their slightly different dispersion-aggregation transitions in Figure 9.2d. 
Strikingly, for both graft-matrix compositions and both matrix lengths, the dispersionaggregation transition (vertical lines in Figure 9.2c through Figure 9.2d) is approximately located where the wet monomer fraction of the attractive composite equals the wet monomer fraction of the equivalent athermal composite (horizontal lines). The presence of this "athermal critical wet monomer fraction" suggests that the dispersion-aggregation transition occurs when the enthalpic and entropic driving forces are in perfect balance, bringing about a system that is effectively athermal. This is equivalent to saying that the effective interaction parameter between the polymer grafted particles and the matrix chains $\chi_{F M}^{e f f}$ is equal to zero at the dispersion-aggregation transition $\chi_{G M}$.

We rationalize the thermodynamic driving forces that bring about the trends in Figure 9.2 as follows. The composites with chain compositions of $f_{G}=0.7, f_{M}=0.1$ have a more negative transition $\chi_{G M}$ than that of the homopolymer composites due to reduced enthalpic driving forces for wetting from the reduced number of possible attractive graft-matrix (G-M) interactions. These reduced enthalpic driving forces for wetting also explains why, at the same $\chi_{G M}$ the homopolymer composites have a higher extent of wetting when compared to the random copolymer composites. Since one effect of reducing the matrix length is to reduce the conformational entropy loss of the matrix wetting the grafted later, we would expect reducing matrix length to also increase the dispersion-aggregation transition $\chi_{G M}$. Indeed, for the random copolymer composites with $f_{G}=$ 0.7 and $f_{M}=0.1$, there is a small increase in the transition $\chi_{G M}$ when the matrix length is reduced from $N_{\text {matrix }}=50$ to 10 . Conversely, for the same reduction in matrix length, there is no change in the dispersion-aggregation $\chi_{G M}$ for the homopolymer composites. Based on this, we conclude that the dispersion-aggregation and wetting-dewetting transitions for the homopolymer composites are primarily enthalpically driven, while the random copolymer composites at $f_{G}=0.7, f_{M}=0.1$, 
due to the reduced number of attractive $\mathrm{G}$ and $\mathrm{M}$ monomers, show small effects from varying entropic driving forces.
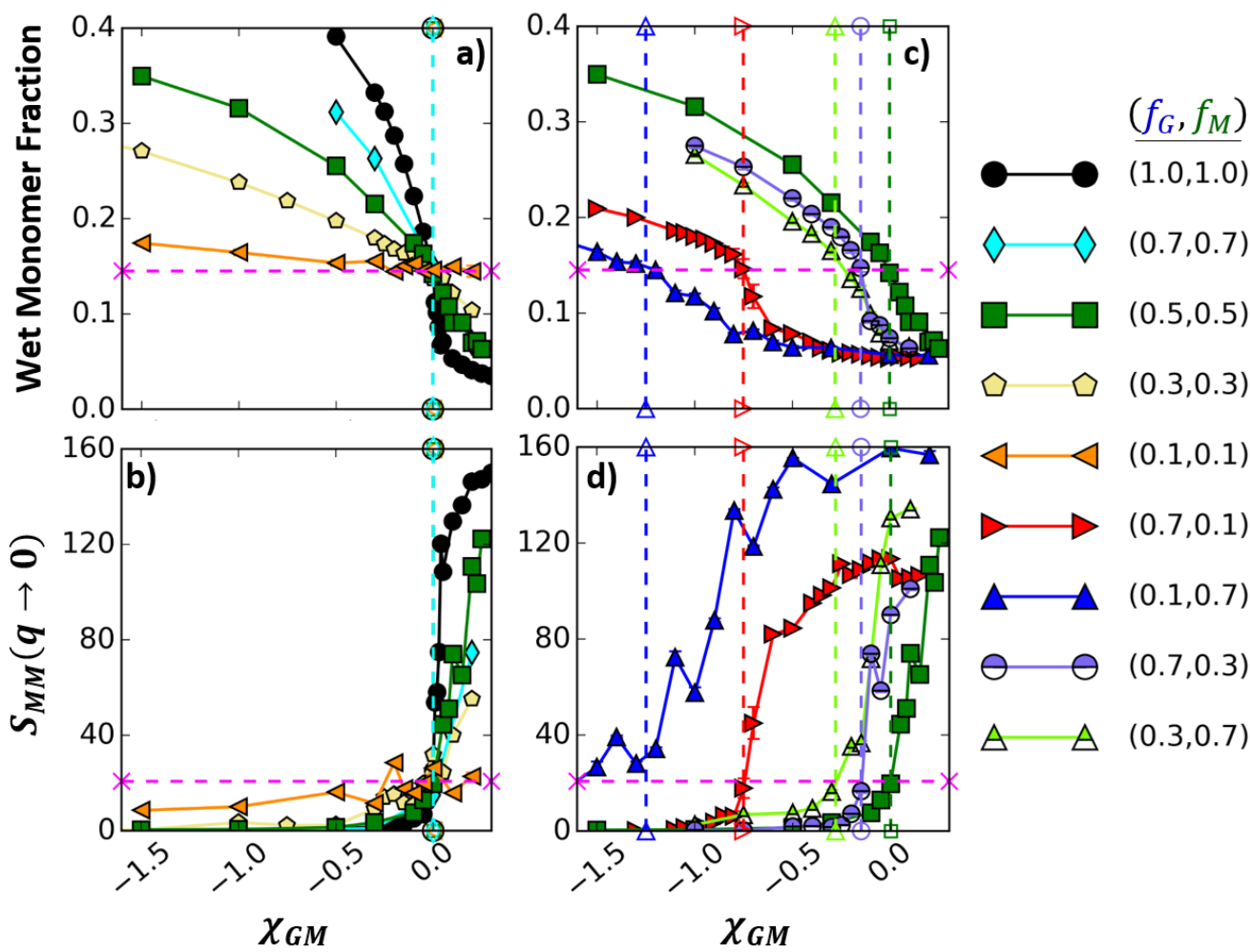

Figure 9.3: Wet monomer fraction $(a$ and $c)$ and $S_{M M}(q \rightarrow 0)(b$ and $d)$ versus FloryHuggins $\chi_{G M}$ at $N_{\text {matrix }}=50$ for varying symmetric $(a$ and $b)$ and asymmetric $(c$ and $d)$ attractive monomer graft and matrix chain compositions. Also shown are the dispersionaggregation transition $\chi_{G M}$ (vertical dashed lines) and the wet monomer fraction and $S_{M M}(q \rightarrow 0)$ for the equivalent athermal composite (horizontal dashed lines). The error bars are standard deviations calculated from 50 uncorrelated configurations for each system; the error bars when not visible are smaller than the size of the symbol.

While $\chi_{G M}$ describes the enthalpic driving forces for graft-matrix mixing, the effective interaction parameter, $\chi^{e f f}$, includes both entropic and enthalpic driving forces for mixing of various components in the composite. In particular, $\chi^{\text {eff }}$ serves as a proxy for the free energy of mixing between the various composite components and is equal to zero at a mixing-demixing transition between those components. If the athermal critical wet monomer fraction determines the 
onset of the dispersion-aggregation transition, and it occurs when the entropic and enthalpic driving forces in the composite are in balance, i.e. filler-matrix $\chi^{e f f}=0$, one should be able to tune the dispersion-aggregation transition by tailoring the graft and matrix chain composition which in turn impact the filler-matrix $\chi^{e f f}$.

In Figure 9.3a and Figure 9.3c, the wet monomer fraction as a function of $\chi_{G M}$ is shown for several different symmetric $\left(f_{G}=f_{M}\right.$, Figure 9.3a) and asymmetric $\left(f_{G} \neq f_{M}\right.$, Figure 9.3c) graft and matrix chain compositions. For all composites with $f_{G}$ or $f_{M}<1.0$, the attractive and athermal graft and matrix monomers are randomly located along the chains. For all graft-matrix compositions shown, with increasing $\chi_{G M}$, the wet monomer fraction gradually decreases until it reaches the wet monomer fraction of the corresponding athermal composite (horizontal line) at which point the onset of the dispersion-aggregation transition occurs (vertical lines). As was done in Figure 9.2 the dispersion-aggregation transition is determined independently from the wet monomer fraction calculation by using $S_{M M}(q \rightarrow 0)$ (Figure $9.3 \mathrm{~b}$ and Figure $\left.9.3 \mathrm{~d}\right)$, or $S_{G G}(q \rightarrow$ 0 ) and $S_{P P}(q \rightarrow 0)$ (Supplementary Figure 9.11) for each system. We note that, for the $f_{G}=$ $0.1, f_{M}=0.7$ composites, even though $S_{M M}(q \rightarrow 0)$ (blue upward triangles) does not intersect the athermal $S_{M M}(q \rightarrow 0)$ (horizontal line), the $S_{G G}(q \rightarrow 0)$ and $S_{P P}(q \rightarrow 0)$ in Supplementary Figure 9.11 along with visual analysis show that the marked transition point (vertical blue dashed line) is clearly where the composite transitions from dispersed to aggregated morphologies.

For the composites with symmetric graft and matrix chain compositions $\left(f_{G}=f_{M}\right)$ in Figure 9.3a and Figure 9.3b, the dispersion-aggregation transition occurs at $\chi_{G M} \approx 0$ with the magnitude of $f_{G}$ and $f_{M}$ tuning the overall extent of wetting of the grafted particles for $\chi_{G M}<0$ in the dispersed state. Conversely, Figure 9.3c and Figure 9.3d show that varying the asymmetry of the graft-matrix composition (i.e. $f_{G} / f_{M}$ ) tunes both the extent of wetting of the grafted layer 
and the $\chi_{G M}$ of the dispersion-aggregation transition simultaneously. Furthermore, by reducing the magnitude of $f_{G}$ and/or $f_{M}$ or varying the asymmetry ratio $f_{G} / f_{M}$ from 1 , we broaden the dispersion-aggregation transition (Figure 9.3b and Figure 9.3d). For example, while the homopolymer graft and matrix composites $\left(f_{G} / f_{M}=1\right)$ transition over a $\delta \chi_{G M}$ of $\approx 0.05$, the $f_{G}=0.7, f_{M}=0.1$ and $f_{G}=0.5, f_{M}=0.5$ composites both have slightly broader transitions at $\delta \chi_{G M} \approx 0.2$. The composites with the lowest $f_{G}$ and $f_{M}$ showed the broadest dispersion to aggregation transition. In the near-athermal limit of $f_{G}=f_{M}=0.1$, the extent of wetting (Figure 9.3a) and the characteristics of the dispersed and aggregated states (Figure 9.3b) are only marginally different than the equivalent athermal composite. We also note that reducing either $f_{G}$ or $f_{M}$ does not necessarily produce a symmetric reduction in the dispersion-aggregation transition $\chi_{G M}$ (compare $(0.7,0.1)$ and $\left.(0.1,0.7)\right)$, and composites with higher composition asymmetry show higher differences between reducing the graft or matrix composition.

All composites with symmetric chain composition $\left(f_{G}=f_{M}\right)$ reach the athermal critical wet monomer fraction at the same $\chi_{G M}$, leading to the dispersion-aggregation $\chi_{G M}$ being identical for these composites. This is despite the fact that, for the composites with symmetric chain compositions at $\chi_{G M}$ below the dispersion-aggregation $\chi_{G M}$, decreasing $f_{G}$ and/or $f_{M}$ decreases the extent of wetting. This means that the extent of wetting in the dispersed state can be tuned by decreasing $f_{G}$ and $f_{M}$ symmetrically, and that the wetting is tuned independently of the location of the dispersion-aggregation $\chi_{G M}$. In contrast, the composition asymmetry ratio $\left(f_{G} / f_{M}\right)$ tunes both the extent of wetting of the grafted layer and the dispersion-aggregation $\chi_{G M}$ simultaneously.

To explain the above trends, we first point to the fact that the number of like interactions (GG and $\mathrm{MM}$ ) are maximized in aggregated morphologies while the number of unlike interactions 
(GM) are maximized in dispersed morphologies, as shown by the number of like and unlike monomer-monomer contacts in Supplementary Figure 9.12. As discussed above, our data suggests that the composites with attractive $\mathrm{G}$ and $\mathrm{M}$ monomers are mostly dominated by enthalpic driving forces, therefore by promoting either like or unlike interactions, one is in turn driving the composite towards aggregated or dispersed morphologies, respectively. The values of $f_{G}$ and $f_{M}$ at constant graft and matrix chain lengths relate to the total number of attractive graft and matrix monomers in the composite, therefore the $f_{G} / f_{M}$ ratio is related to the stoichiometric balancing of like and unlike attractive monomer interactions. When, $f_{G} / f_{M}$ deviates from unity it increases the favorability of the aggregated morphology due to the fact that there are more possible like monomer interactions than unlike monomer interactions. Based on this, a stronger enthalpic driving force for graft-matrix mixing is then needed to stabilize the dispersed morphology resulting in a more-negative $\chi_{G M}$ for the asymmetric graft-matrix compositions than the symmetric ones. 

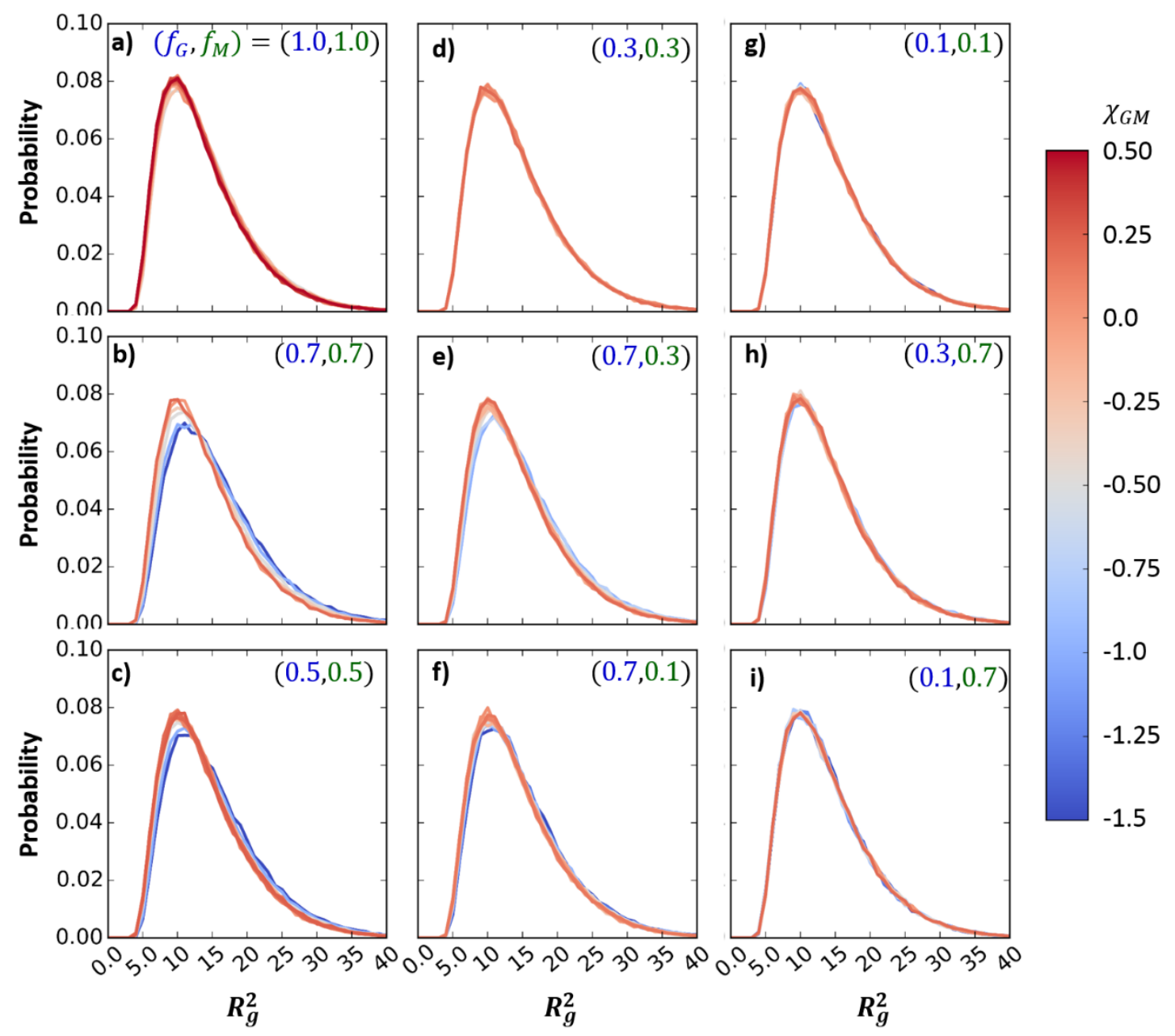

Figure 9.4: Matrix chain radius of gyration, $R_{g}^{2}$, distributions for varying $\chi_{G M}$, at $N_{\text {matrix }}=50$, and for varying graft and matrix chain compositions $\left(f_{G}, f_{M}\right)$ (as labeled).

The matrix polymer chain conformations presented in Figure 9.4, show that there is no strong effect of $\chi_{G M}$ or $f_{G}, f_{M}$ on the matrix $R_{g}^{2}$ distributions; for all $\chi_{G M}$ and chain compositions the mean of the distribution is approximately $\left\langle R_{g}^{2}\right\rangle \approx 14-16 \mathrm{~d}^{2}$ (Supplementary Figure 9.13). While Supplementary Figure 9.3 shows some systematic variation in the averages of the chain distributions this effect is small compared to the width of the distribution as shown in Figure 9.4. The lack of variation in the matrix chain distributions is surprising given that all systems in Figure 9.4 have a dispersion-aggregation transitions (Figure 9.3b and Figure 9.3d) in the range of $\chi_{G M}$ 
presented with varying extents of grafted layer wetting (Figure 9.3a and Figure 9.3c). In addition, as the matrix chain attractive monomer composition $f_{M}$ increases, the amount of effective intrachain attraction increases, which should increase the enthalpic driving forces for the matrix chains to assume compressed conformations. We attribute the lack of variation in the matrix chain conformations to the matrix chains being the bulk component in the composite $\left(\phi_{M}=1-\phi_{G}=\right.$ 0.87) and therefore, despite changes in dispersion-aggregation and wetting-dewetting, the majority of matrix chains are separated from graft chains and therefore unaffected by $f_{G}, f_{M}$ or $\chi_{G M}$.
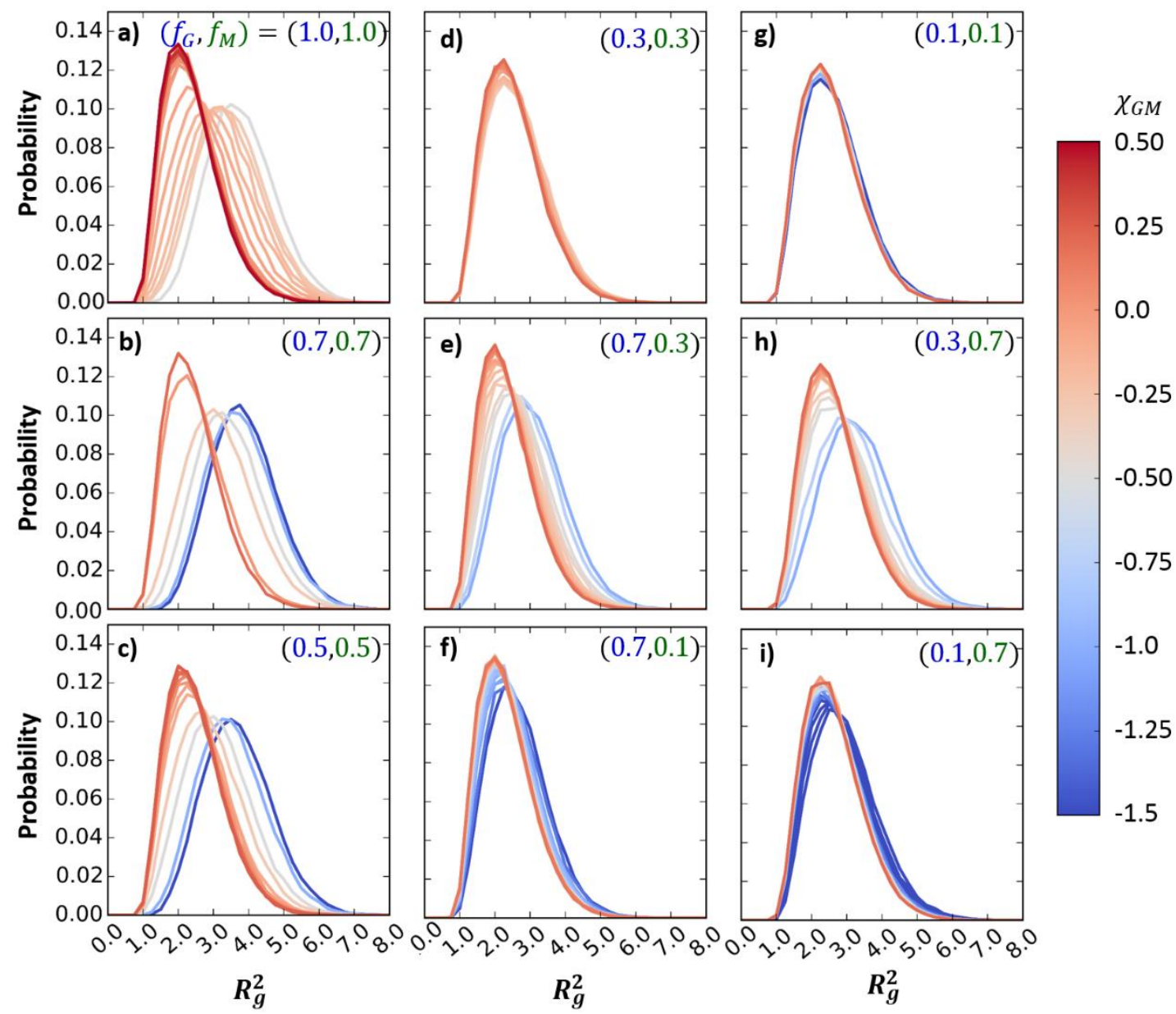

Figure 9.5: Graft chain radius of gyration, $R_{g}^{2}$, distributions for varying $\chi_{G M}$, at $N_{\text {matrix }}=50$, and for varying graft and matrix chain compositions $\left(f_{G}, f_{M}\right)$ (as labeled). 
In contrast to the matrix $R_{g}^{2}$ distributions, the graft $R_{g}^{2}$ distributions (Figure 9.5) show variations with both $\chi_{G M}$ and graft-matrix composition. The homopolymer (Figure 9.5a) and symmetric random copolymer composites with $f_{G}=f_{M}=0.7$ or 0.5 (Figure $9.5 \mathrm{~b}$ and Figure $9.5 \mathrm{c}$ ) show increase in mean $\operatorname{Rg}^{2}$ (Supplementary Figure 9.13) or extension of graft chains with decreasing $\chi_{G M}$. The $R_{g}^{2}$ distributions of the two composites with asymmetric or low $\left(f_{G}=f_{M}=\right.$ 0.1 or 0.3 ) graft and matrix compositions (Figure 9.5d through Figure 9.5i) are less sensitive to varying $\chi_{G M}$. In other words, the cases with the higher attractive graft and matrix compositions show the largest shifts in graft $R_{g}^{2}$. This trend in graft chain expansion mimics the trend in the wet monomer fractions in Figure 9.3a and Figure 9.3c. The composites with the largest variations wet monomer fraction values with changing $\chi_{G M}$ also show the largest changes in chain conformation. This follows logically as the composites with the higher extents of wetting must have more extended graft chains to accommodate the matrix chains which are penetrating grafted layers. These data demonstrate that by varying the composition of attractive monomers in the graft and matrix chains, we not only tune the extent of wetting-dewetting of the grafted layer and the dispersion-aggregation $\chi_{G M}$, but also the conformations of the graft chains. 


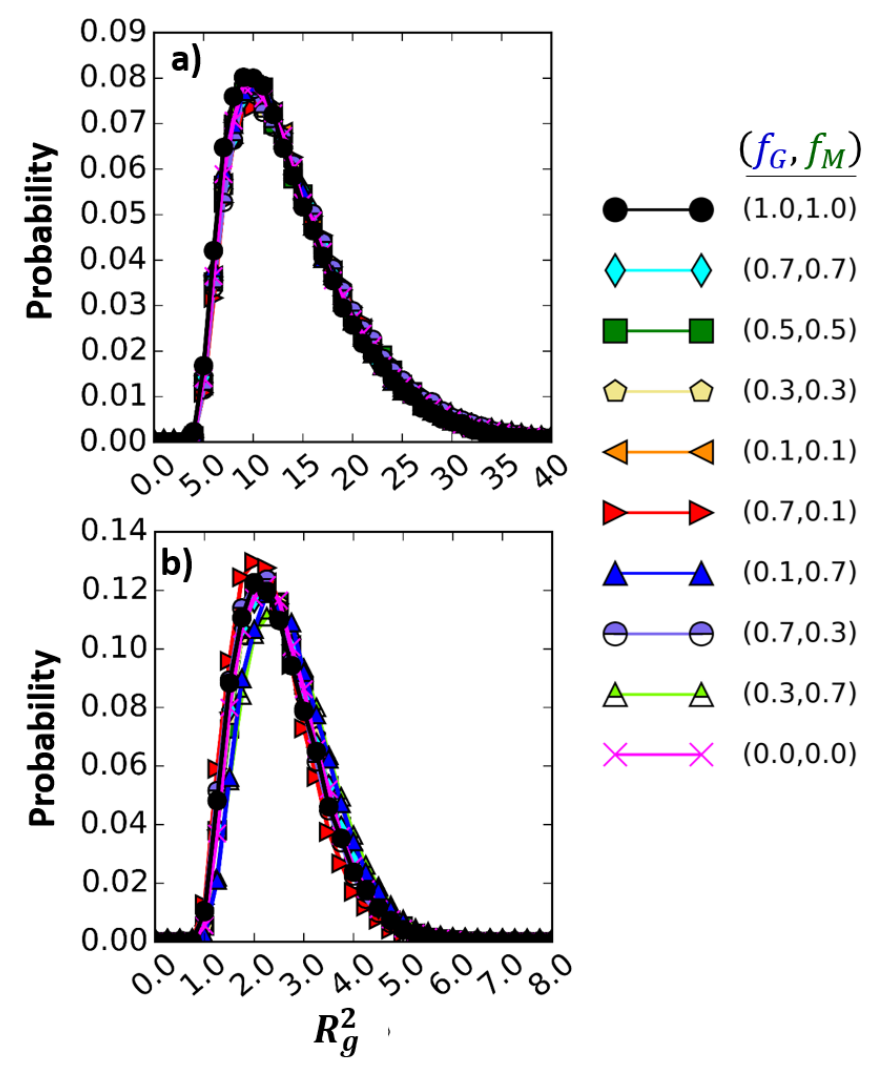

Figure 9.6: Matrix (a) and graft (b) chain radius of gyration, $R_{g}^{2}$, distributions at the dispersionaggregation transition $\chi_{G M}$ for each composite with varying graft and matrix chain compositions.

Figure 9.6 shows the $R_{g}^{2}$ distributions at the dispersion-aggregation $\chi_{G M}$ for the nine composite compositions along with the $R_{g}^{2}$ distribution of the equivalent athermal composite. Remarkably, the graft and matrix chain $R_{g}^{2}$ distributions at the dispersion-aggregation $\chi_{G M}$ for all composite are identical to their corresponding athermal chain $R_{g}^{2}$ distributions. This further highlights that, irrespective of the graft and matrix random copolymer composition at the dispersion-aggregation transition $\chi_{G M}$, these chemically-dissimilar graft-matrix composites assume a state that is effectively athermal. At the dispersion-aggregation $\chi_{G M}\left(\right.$ where $\left.\chi^{e f f}=0\right)$, both the wetting of the grafted layer (Figure 9.3a and Figure 9.3c) and the distribution in graft and matrix chain conformations match that of the equivalent, chemically identical (athermal) 
composite. The chain conformations and extent of wetting-dewetting of the athermal composite serve as baseline values for the attractive composites, and variations in $\chi_{G M}$ increase or decrease the chain extension and wetting of the grafted layer away from these baselines. The degree to which variations in $\chi_{G M}$ affect the extension of the graft chains or wetting-dewetting is tuned by the graft and matrix chain composition, as shown by the graft chain $R_{g}^{2}$ distributions in Figure 9.5.

\subsection{CONCLUSION}

For polymer grafted particle filled composites with chemically different graft and matrix chains, the extent of wetting-dewetting and/or the $\chi_{G M}$ or temperature of the dispersion-aggregation transition is tuned by varying the fraction of attractive monomers in the graft $\left(f_{G}\right)$ and matrix chains $\left(f_{M}\right)$. For all graft and matrix chain compositions studied, there is critical extent of wetting, equal to the extent of wetting of an equivalent athermal (chemically identical) composite that marks the onset of the dispersion-aggregation transition. At the dispersion-aggregation transition, not only does the extent of wetting in the chemically different composites match that of the chemically identical (athermal) composites, but the graft and matrix chain conformations at all chain compositions also match that of the athermal case.

In these chemically different composites, with increasing fraction of attractive monomers in the graft $\left(f_{G}\right)$ and matrix chains $\left(f_{M}\right)$, the enthalpic driving forces increasingly dominate over the entropic driving forces. As a result, the effect of matrix length at constant graft length on the wetting/dewetting and dispersion-aggregation is diminished for the fully attractive homopolymer composites with $f_{G}=f_{M}=1.0$ compared to those with $f_{G}<1.0$ and $f_{M}<1.0$. The magnitudes of $f_{G}$ and $f_{M}$ and the ratio $f_{G} / f_{M}$, to some degree, independently tune the extent of wetting and the dispersion-aggregation transition $\chi_{G M}$. When $f_{G}=f_{M}$, reducing the magnitude of $f_{G}$ and $f_{M}$ reduces the extent of wetting in the dispersed state while maintaining a constant dispersion- 
aggregation transition $\chi_{G M}$. In contrast, varying $f_{G} / f_{M}$ tunes both the wetting of the grafted layer and the dispersion-aggregation transition $\chi_{G M}$. We attribute the effect of $f_{G} / f_{M}$ on the dispersionaggregation transition $\chi_{G M}$ to the fact that deviations from unity $f_{G} / f_{M}$ will create interaction site stoichiometries that favor like interactions (GG and MM) over unlike interactions (GM) which increase the favorability of the aggregated state. Finally, we find that while the matrix chain conformations are relatively unaffected by varying $f_{G}, f_{M}$, or $\chi_{G M}$, the graft chain extension is tuned by varying these parameters. The composites with higher $f_{G}$ and $f_{M}$ magnitudes show the highest variability in graft chain extension with varying $\chi_{G M}$.

These results demonstrate the tunability of composite morphology with chemically different graft and matrix chains. While the wetting-dewetting and the dispersion-aggregation, of chemically identical graft-matrix composites is tuned by varying physical parameters (e.g. molecular weights of graft and matrix or grafting density) the morphology of chemically different composites is tuned by choosing the graft and matrix chemistries, or varying $\chi_{G M}$ via temperature or solvent selection. The presence of a broad wetting-dewetting transition in the chemically different composites also allows for control over the extent of wetting in both the dispersed and aggregated morphologies, a phenomena that has not been shown to exist in chemically identical composites. Fine control over the extent of wetting-dewetting in composites presents useful control knob for tuning rheological properties that are strongly dependent on the mixing/entanglement of the graft and matrix chains.[42-49]

\subsection{ACKNOWLEDGEMENTS}

AJ acknowledges financial support from U.S. Department of Energy under Grant DE-SC0003912 and TBM acknowledges financial support from NSF graduate fellowship (award number DGE 1144083). This work used NERSC supercomputing supported by the U.S. Department of Energy 
(DE-AC02- 05CH11231), Farber supercomputer at University of Delaware, and Opuntia supercomputer at University of Houston.

\subsection{REFERENCES}

1. Dang, A., et al., High-Transparency Polymer Nanocomposites Enabled by Polymer-Graft Modification of Particle Fillers. Langmuir, 2014. 30(48): p. 14434-14442.

2. Tao, P., et al., Bulk transparent epoxy nanocomposites filled with poly(glycidyl methacrylate) brush-grafted TiO2 nanoparticles. Polymer, 2013. 54(6): p. 1639-1646.

3. Ojha, S., et al., Strategies for the Synthesis of Thermoplastic Polymer Nanocomposite Materials with High Inorganic Filling Fraction. Langmuir, 2013. 29(28): p. 8989-8996.

4. Vaia, R.A. and J.F. Maguire, Polymer nanocomposites with prescribed morphology: Going beyond nanoparticle-filled polymers. Chemistry of Materials, 2007. 19(11): p. 2736-2751.

5. Krishnamoorti, R. and R.A. Vaia, Polymer nanocomposites. Journal of Polymer Science Part B-Polymer Physics, 2007. 45(24): p. 3252-3256.

6. Crosby, A.J. and J.Y. Lee, Polymer nanocomposites: The "nano" effect on mechanical properties. Polymer Reviews, 2007. 47(2): p. 217-229.

7. Green, P.F., The structure of chain end-grafted nanoparticle/homopolymer nanocomposites. Soft Matter, 2011. 7(18): p. 7914-7926.

8. Corbierre, M.K., et al., Gold nanoparticle/polymer nanocomposites: Dispersion of nanoparticles as a function of capping agent molecular weight and grafting density. Langmuir, 2005. 21: p. 6063-6072.

9. Goel, V., et al., Viscoelastic properties of silica-grafted poly(styrene-acrylonitrile) nanocomposites. Journal of Polymer Science Part B-Polymer Physics, 2006. 44(14): p. 2014-2023.

10. Tsubokawa, N., Surface grafting of polymers onto nanoparticles in a solvent-free drysystem and applications of polymer-grafted nanoparticles as novel functional hybrid materials. Polymer Journal, 2007. 39(10): p. 983-1000.

11. Kim, B.J., et al., Effect of areal chain density on the location of polymer-modified gold nanoparticles in a block copolymer template. Macromolecules, 2006. 39(12): p. 41084114.

12. Lan, Q., L.F. Francis, and F.S. Bates, Silica nanoparticles dispersions in homopolymer versus block copolymer. Journal of Polymer Science Part B-Polymer Physics, 2007. 45: p. 2284-2299.

13. Shull, K.R., THEORY OF END-ADSORBED POLYMER BRUSHES IN POLYMERIC MATRICES. Journal of Chemical Physics, 1991. 94(8): p. 5723-5738.

14. Harton, S.E. and S.K. Kumar, Mean-field theoretical analysis of brush-coated nanoparticle dispersion in polymer matrices. Journal of Polymer Science Part B-Polymer Physics, 2008. 46: p. 351-358. 
15. Akcora, P., et al., Anisotropic self-assembly of polymer-decorated spherical nanoparticles. Nature Materials, 2009. 8: p. 354-359.

16. Trombly, D.M. and V. Ganesan, Curvature effects upon interactions of polymer-grafted nanoparticles in chemically identical polymer matrices. Journal of Chemical Physics, 2010. 133(15).

17. $\mathrm{Xu}, \mathrm{C}$., et al., Dispersion of polymer-grafted magnetic nanoparticles in homopolymers and block copolymers. Polymer, 2008. 49(16): p. 3568-3577.

18. Leibler, L., Theory of Microphase Separation in Block Copolymers. Macromolecules, 1980. 13(6): p. 1602-1617.

19. Krishnamoorti, R., Strategies for Dispersing Nanoparticles in Polymers. MRS Bulletin, 2007. 32(4): p. 341-347.

20. Chevigny, C., et al., Polymer-Grafted-Nanoparticles Nanocomposites: Dispersion, Grafted Chain Conformation, and Rheological Behavior. Macromolecules, 2011. 44(1): p. 122133.

21. Frischknecht, A.L. and A. Yethiraj, Two- and three-body interactions among nanoparticles in a polymer melt. The Journal of Chemical Physics, 2011. 134(17): p. 174901-11.

22. Martin, T.B. and A. Jayaraman, Effect of matrix bidispersity on the morphology of polymer-grafted nanoparticle-filled polymer nanocomposites. Journal of Polymer Science Part B: Polymer Physics, 2014. 52(24): p. 1661-1668.

23. Lin, B., T.B. Martin, and A. Jayaraman, Decreasing Polymer Flexibility Improves Wetting and Dispersion of Polymer-Grafted Particles in a Chemically Identical Polymer Matrix. Acs Macro Letters, 2014. 3(7): p. 628-632.

24. Martin, T.B., P.M. Dodd, and A. Jayaraman, Polydispersity for Tuning the Potential of Mean Force between Polymer Grafted Nanoparticles in a Polymer Matrix. Physical Review Letters, 2013. 110(1): p. 018301.

25. Smith, G.D. and D. Bedrov, Dispersing Nanoparticles in a Polymer Matrix: Are Long, Dense Polymer Tethers Really Necessary? Langmuir, 2009. 25(19): p. 11239-11243.

26. Wong, M.H., et al., Synthesis and Fabrication of Multifunctional Nanocomposites: Stable Dispersions of Nanoparticles Tethered with Short, Dense and Polydisperse Polymer Brushes in Poly(methyl methacrylate). Advanced Functional Materials, 2012. 22(17): p. 3614-3624.

27. Wu, X.L., et al., Polystyrene grafted carbon black synthesis via in situ solution radical polymerization in ionic liquid. Journal of Polymer Research, 2013. 20(6): p. 7.

28. Chen, F., et al., Synthesis and characterization of copolymer grafted magnetic nanoparticles via surface-initiated nitroxide-mediated radical polymerization. Polymer Engineering and Science, 2013. 53(5): p. 956-962.

29. Rousseau, G., et al., Azido-Coated Nanoparticles: A Versatile Clickable Platform for the Preparation of Fluorescent Polystyrene Core-PAMAM Shell Nanoparticles. Macromolecules, 2012. 45(8): p. 3513-3522. 
30. Qin, J., et al., Controlled Bimodal Molecular-Weight-Distribution Polymers: Facile Synthesis by RAFT Polymerization. Chemistry-a European Journal, 2012. 18(19): p. 60156021.

31. Jiang, K., et al., One-Pot Controlled Synthesis of Homopolymers and Diblock Copolymers Grafted Graphene Oxide Using Couplable RAFT Agents. Macromolecules, 2012. 45(3): p. 1346-1355.

32. Borukhov, I. and L. Leibler, Enthalpic stabilization of brush-coated particles in a polymer melt. Macromolecules, 2002. 35(13): p. 5171-5182.

33. Borukhov, I. and L. Leibler, Stabilizing grafted colloids in a polymer melt: Favorable enthalpic interactions. Physical Review E, 2000. 62(1): p. R41-R44.

34. Cui, L., et al., A facile "graft from" method to prepare molecular-level dispersed graphenepolymer composites. Journal of Polymer Science Part a-Polymer Chemistry, 2012. 50(21): p. 4423-4432.

35. Martin, T.B., et al., Wetting-Dewetting and Dispersion-Aggregation Transitions Are Distinct for Polymer Grafted Nanoparticles in Chemically Dissimilar Polymer Matrix. Journal of the American Chemical Society, 2015. 137(33): p. 10624-10631.

36. Ceperley, D., M.H. Kalos, and J.L. Lebowitz, Computer Simulation of the Dynamics of a Single Polymer Chain. Physical Review Letters, 1978. 41(5): p. 313-316.

37. Grest, G.S. and K. Kremer, MOLECULAR-DYNAMICS SIMULATION FOR POLYMERS IN THE PRESENCE OF A HEAT BATH. Physical Review A, 1986. 33(5): p. 3628-3631.

38. Heo, K., et al., Thermally Reversible Aggregation of Gold Nanoparticles in Polymer Nanocomposites through Hydrogen Bonding. Nano Letters, 2013. 13(11): p. 5297-5302.

39. Kalb, J., et al., End grafted polymer nanoparticles in a polymeric matrix: Effect of coverage and curvature. Soft Matter, 2011. 7(4): p. 1418-1425.

40. http://codeblue.umich.edu/hoomd-blue/. HOOMD-Blue webpage. Available from: http://codeblue.umich.edu/hoomd-blue/ (2010).

41. Hansen, J.P.M., I.R., Theory of Simple Liquids. 3rd ed. 2006.

42. Kumar, S.K., et al., Nanocomposites with Polymer Grafted Nanoparticles. Macromolecules, 2013. 46(9): p. 3199-3214.

43. Kumar, S.K. and R. Krishnamoorti, Nanocomposites: Structure, Phase Behavior, and Properties, in Annual Review of Chemical and Biomolecular Engineering, Vol 1, J.M. Prausnitz, M.F. Doherty, and M.A. Segalman, Editors. 2010. p. 37-58.

44. Jancar, J., et al., Current issues in research on structure-property relationships in polymer nanocomposites. Polymer, 2010. 51(15): p. 3321-3343.

45. Madbouly, S.A. and J.U. Otaigbe, Recent advances in synthesis, characterization and rheological properties of polyurethanes and POSS/polyurethane nanocomposites dispersions and films. Progress in Polymer Science, 2009. 34(12): p. 1283-1332. 
46. Jouault, N., et al., Well-Dispersed Fractal Aggregates as Filler in Polymer-Silica Nanocomposites: Long-Range Effects in Rheology. Macromolecules, 2009. 42(6): p. 2031-2040.

47. Burgentzlé, D., et al., Solvent-based nanocomposite coatings: I. Dispersion of organophilic montmorillonite in organic solvents. Journal of Colloid and Interface Science, 2004. 278(1): p. 26-39.

48. Lee, K.M. and C.D. Han, Effect of hydrogen bonding on the rheology of polycarbonate/organoclay nanocomposites. Polymer, 2003. 44(16): p. 4573-4588.

49. Zhang, Q. and L.A. Archer, Poly(ethylene oxide)/Silica Nanocomposites: Structure and Rheology. Langmuir, 2002. 18(26): p. 10435-10442. 


\subsection{SUPPLEMENTARY}

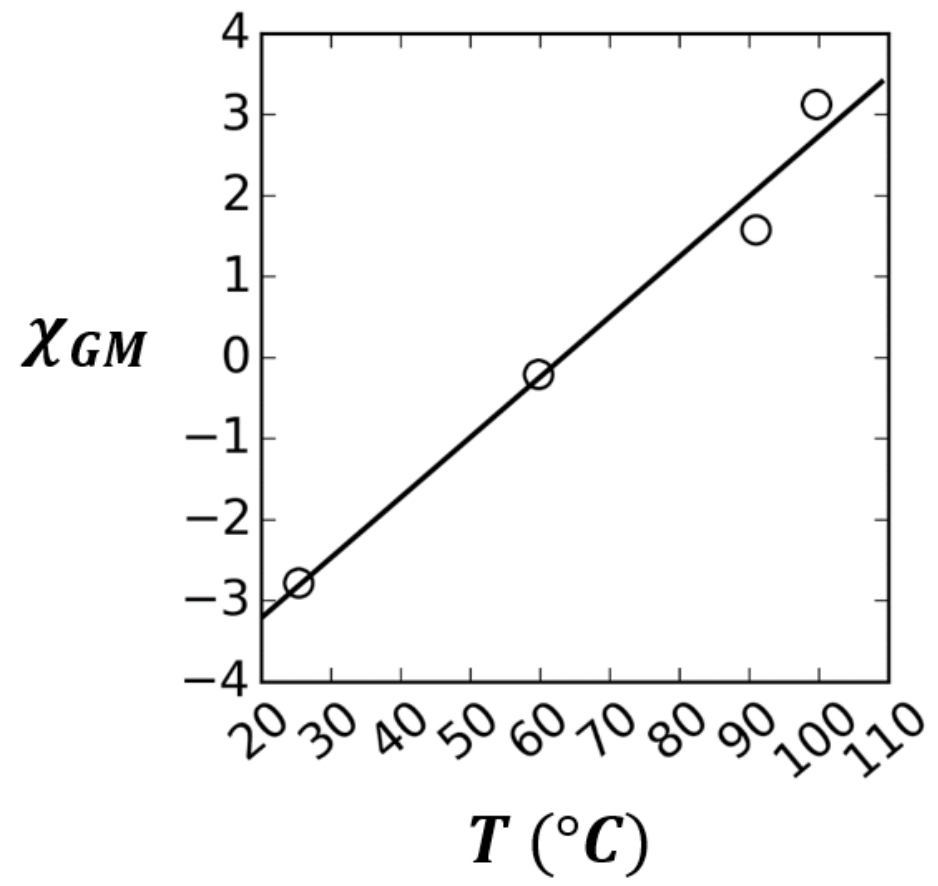

Figure 9.7: Simulated Flory-Huggins $\chi_{G M}$ versus temperature for a blend of deuterated polystyrene (dPS) at $N_{d P S}=465000$ and poly-vinyl-methyl-ether $(P V M E)$ at $N_{P V M E}=10,000$. The data points were extracted from Figure 4 of [1] while the line is a linear fit intended as a guide to the eye. To convert from real experimental units to reduced simulation units, a reference volume of $1148 \mathrm{~cm}^{3} / \mathrm{mol}$ (based on a PS Kuhn length of $1.24 \mathrm{~nm}$ [2]), and a coordination number of $z=26$ was used.

\section{Figure 9.7 References}

[1] Jelenič, J.; Kriste, R. G.; Oberthür, R. C.; Schmitt-Strecker, S.; Schmitt, B. J., Investigation of exothermic polymer blends by neutron scattering. Die Makromolekulare Chemie 1984, 185 (1), 129-156.

[2] (Anisotropy of Segments and Monomer Units of Polymer Molecules) Brandrup, J.; Immergut, Edmund H.; Grulke, Eric A.; Abe, Akihiro; Bloch, Daniel R. (1999; 2005). Polymer Handbook (4th Edition).. John Wiley \& Sons. 


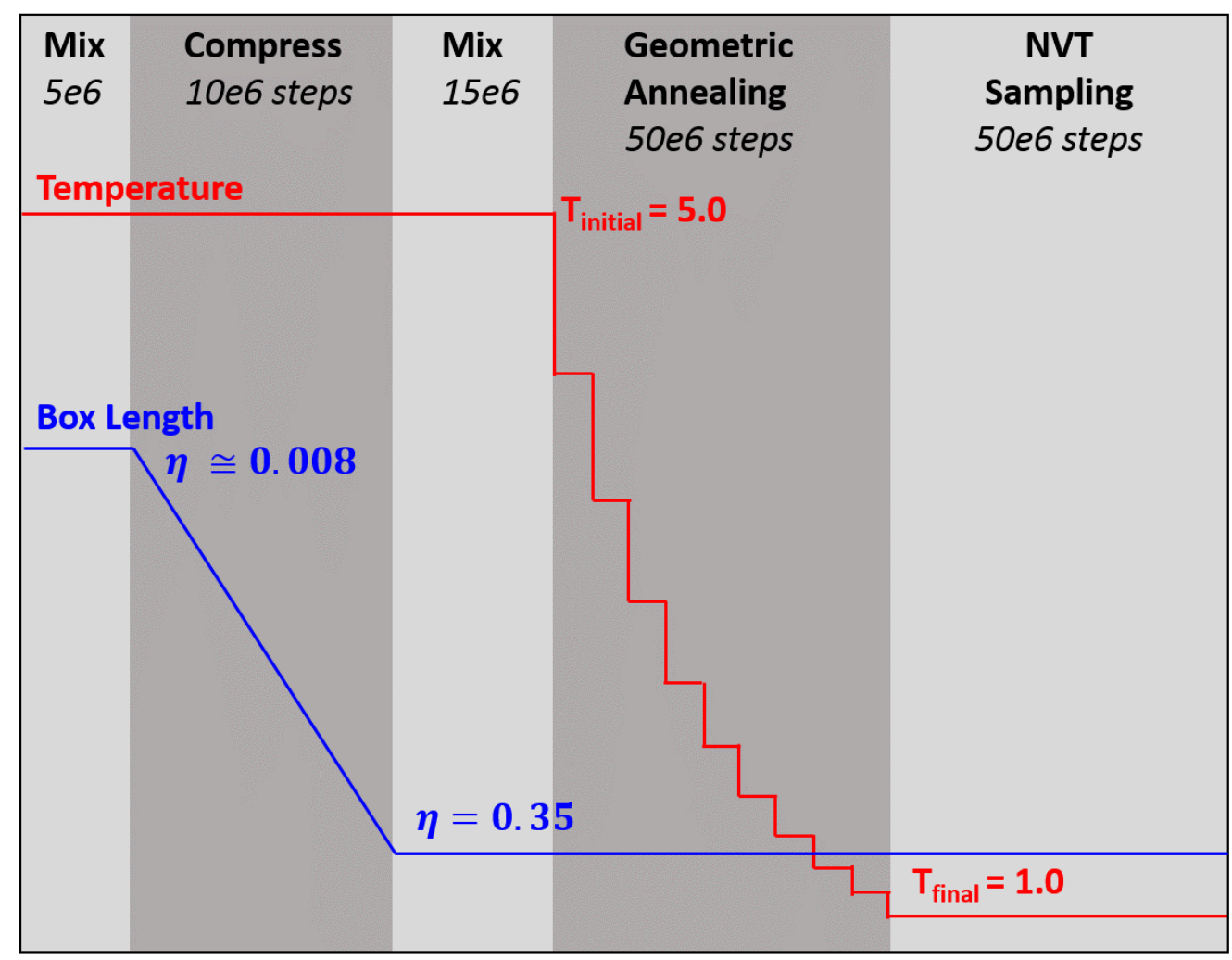

Simulation Time

Figure 9.8: Schematic diagram of the simulation protocol used in this study. 

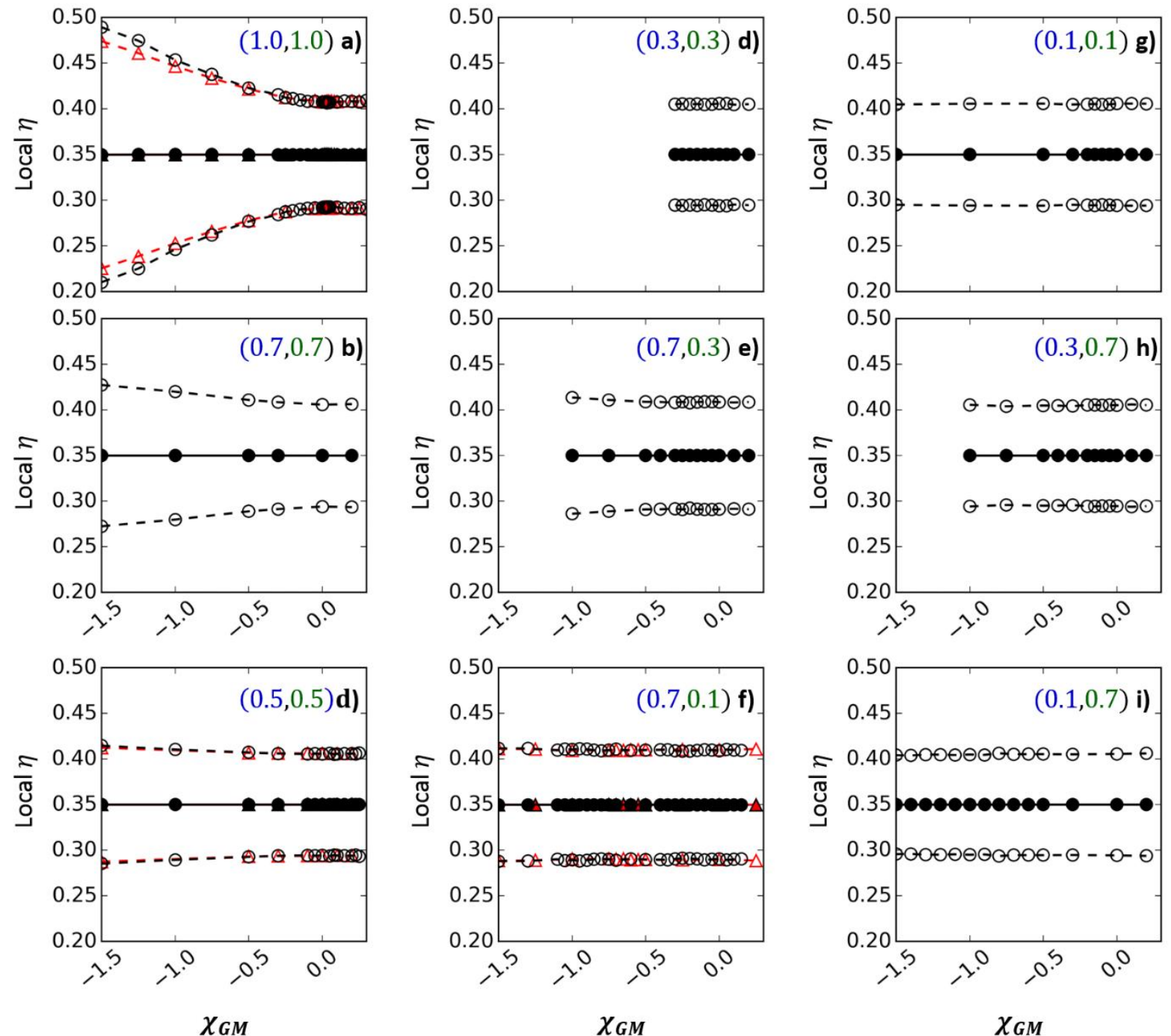

Figure 9.9: Overall average (solid), minimum and maximum (dashed) local volume fraction, $\eta$, of the simulated composites for $N_{\text {matrix }}=50$ (black circles) and 10 (red triangles) and for graft and matrix chain composition $\left(f_{G}, f_{M}\right)$ as marked. 

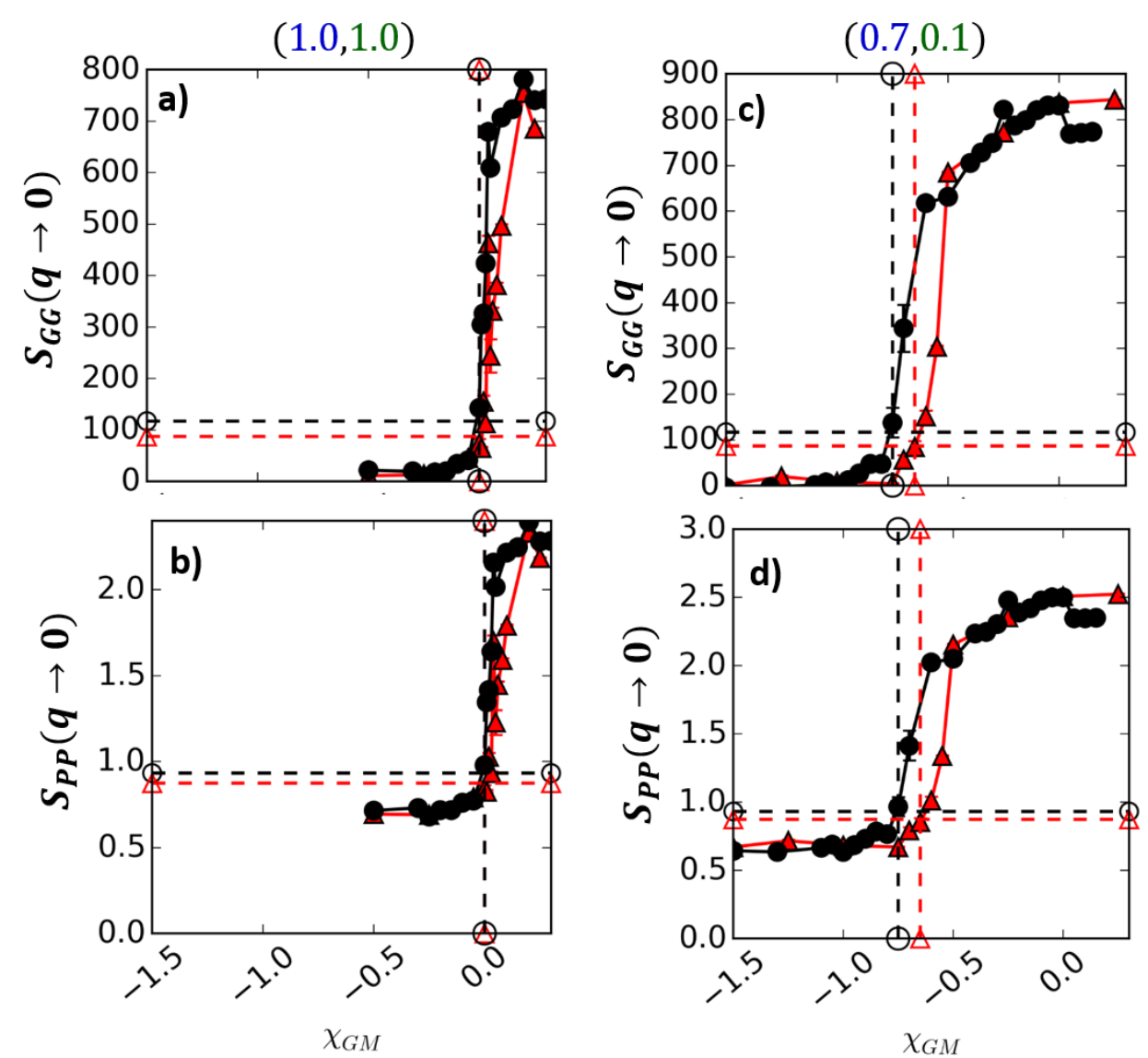

Figure 9.10: Low-q value of the graft-graft $(a, b)$ and particle-particle $(c, d)$ structure factors. In all parts, the particle diameter is $D=5 d$, the grafting density $=0.76$ chains $/ d^{2}$, the graft chain monomer volume fraction is $\phi_{G}=0.13$ and the total volume fraction is $\eta=0.35$. Parts $a$ and $b$ are for composites with $f_{G}=f_{M}=1.0$ and parts $c$ and $d$ are for composites with $f_{G}=0.7, f_{M}=$ 0.1. The graft length is $N_{\text {graft }}=10$ in all parts, while a matrix of length $N_{\text {matrix }}=10$ is denoted with red triangles and $N_{\text {matrix }}=50$ is denoted with black circles. The horizontal lines in represent the low-q structure factor of the equivalent athermal $\left(f_{G}=f_{M}=0.0\right)$ composite The error bars are standard deviations calculated from 50 uncorrelated configurations for each system; the error bars when not visible are smaller than the size of the symbol. 

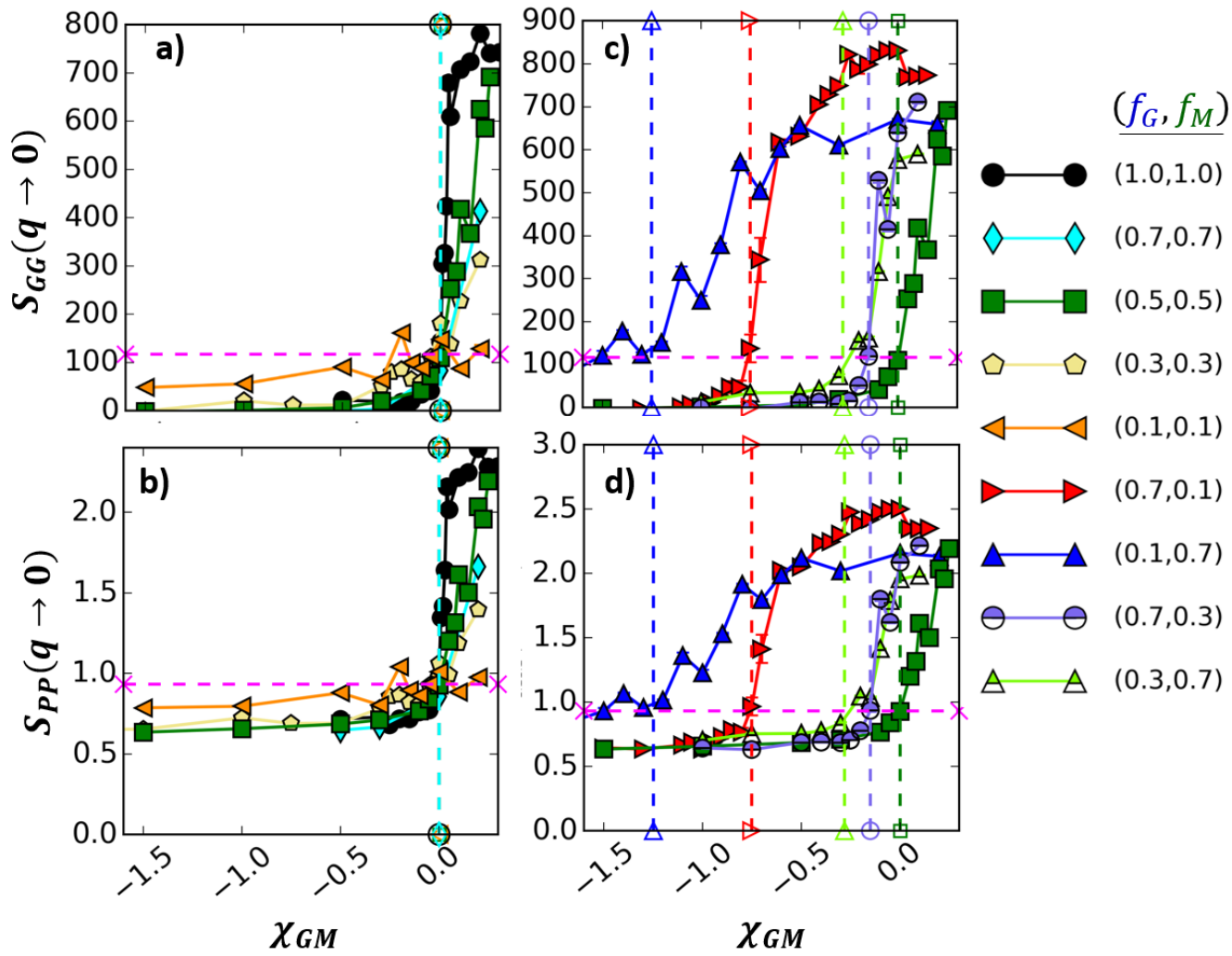

Figure 9.11: Graft-graft monomer structure factor $S_{G G}(q \rightarrow 0)(a$ and $c)$ and particle-particle structure factor $S_{P P}(q \rightarrow 0)(b$ and $d)$ versus Flory-Huggins $\chi_{G M}$ at $N_{\text {matrix }}=50$ for varying symmetric ( $a$ and $b$ ) and asymmetric $(c$ and $d)$ graft and matrix chain compositions. Also shown are the dispersion-aggregation transition $\chi_{G M}$ (vertical lines) and $S_{G G}(q \rightarrow 0)$ or $S_{P P}(q \rightarrow 0)$ for the equivalent athermal composite (horizontal lines). The error bars are standard deviations calculated from 50 uncorrelated configurations for each system; the error bars when not visible are smaller than the size of the symbol. 

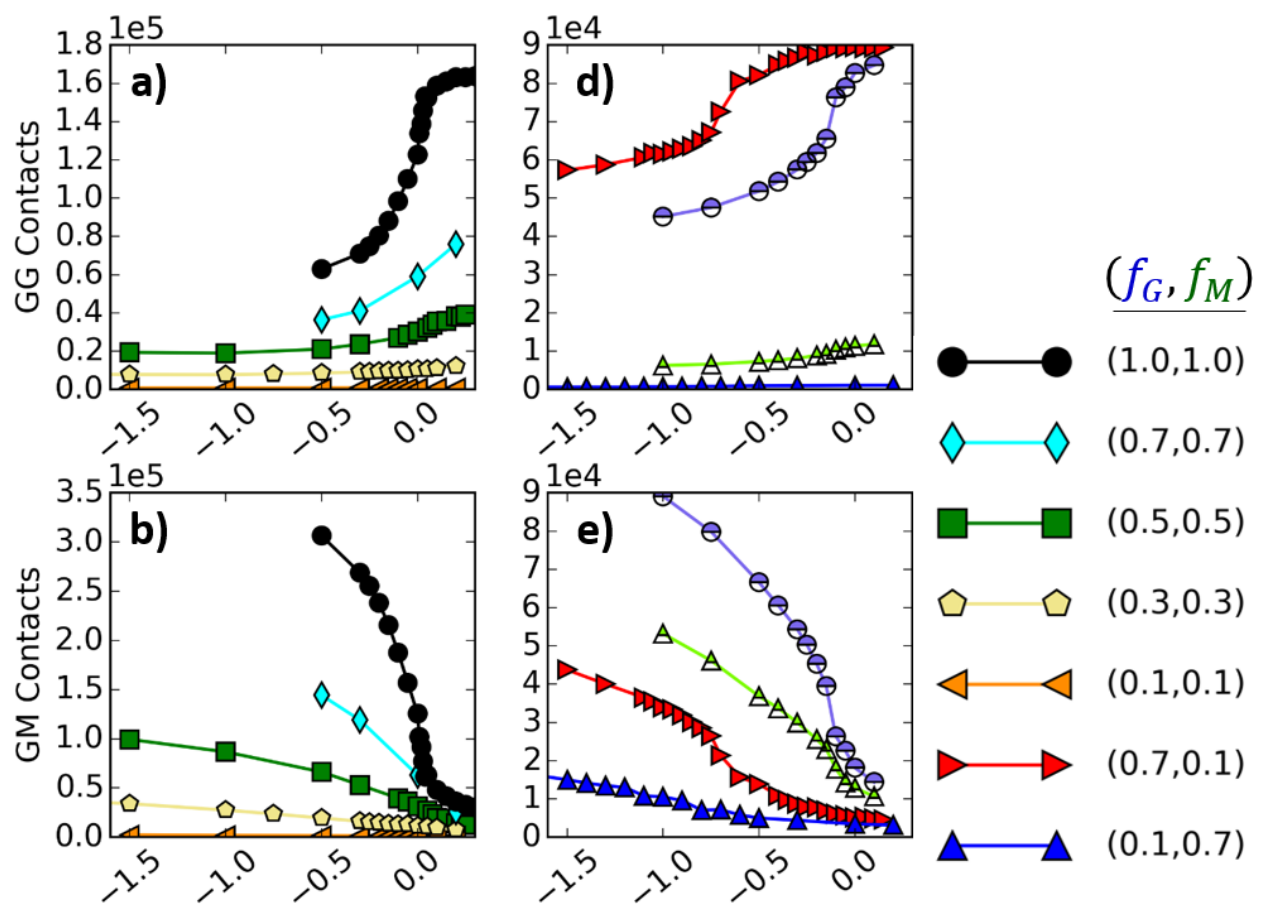

$\diamond \diamond(0.7,0.7)$

$\square \square(0.5,0.5)$

$\checkmark \quad \square(0.3,0.3)$

$\triangleleft \quad \triangleleft(0.1,0.1)$

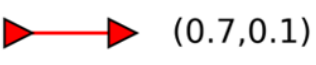

$\Delta(0.1,0.7)$
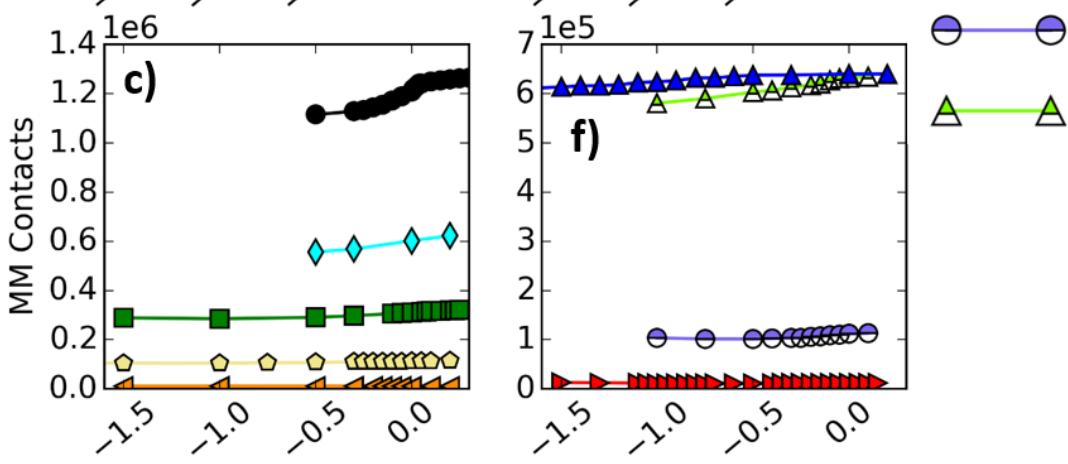

$(0.7,0.3)$

$(0.3,0.7)$

$\chi_{G M}$

$\chi_{G M}$

Figure 9.12: Average number of Graft-Graft $(a, d)$ Graft-Matrix $(b, e)$ and Matrix-Matrix $(c, f)$ monomer contacts versus Flory-Huggins $\chi_{G M}$ at $N_{\text {matrix }}=50$ for varying symmetric $(a, b$, and c) and asymmetric ( $c, d$, and $f$ ) graft and matrix chain compositions. Two monomers are in "contact" when they are within the cutoff distance for the attractive Lennard-Jones interactions (i.e. center to center distance $<2.5 d$ ). 

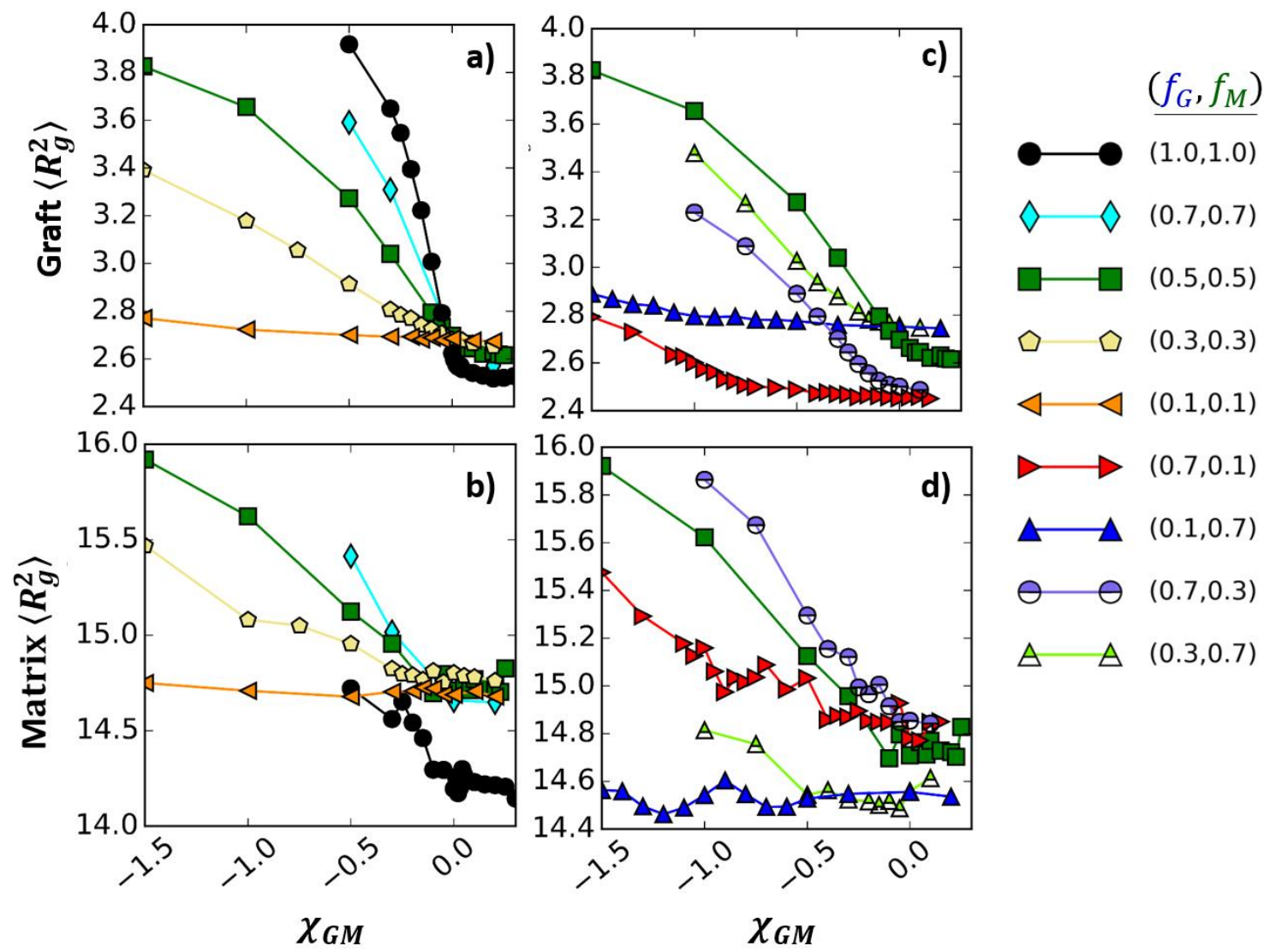

Figure 9.13: Average $R_{g}^{2}$ versus Flory-Huggins $\chi_{G M}$ at $N_{\text {matrix }}=50$ for graft $(a$ and $c)$ and matrix $(b$ and $d)$ chains at varying symmetric $(a$ and $b)$ and asymmetric $(c$ and $d)$ attractive monomer graft and matrix chain compositions as shown in the legend. 


\section{CONCLUSION}

\subsection{Copolymer GRAFTED NANOPARTICLES IN IMPLICIT SOLVENT}

Chapter 2 presented a study on the assembly of copolymer grafted nanoparticles in implicit solvent, with the goal of elucidating design rules to help materials scientists create prescribed nanostructures and particle morphologies. In this chapter, we explored a wide parameter space associated with the chemical and physical properties of the copolymer grafted nanoparticles and correlated these parameters with the resultant properties of the assembled nanostructure.

Specifically in Chapter 2, we elucidated the effect of blockiness in the grafted copolymer sequence at varying monomer-monomer and monomer-particle interactions on size, shape and structure of assembled copolymer grafted nanoparticles. The grafted chain conformations are closely linked to how monomer sequence facilitates the chain's ability to form energetically favorable attractive monomer contacts while minimizing unfavorable repulsive monomer contacts. As the graft sequence blockiness increases, attractive monomer contacts are brought together more easily due to the favorable topological placement of like monomers along the chain. Within the assembled nanocluster, the alternating sequence, $\left(\mathrm{A}_{1} \mathrm{~B}_{1}\right)_{12}$, forms "networks" of attractive monomer contacts in contrast to the distinct domains of attractive monomers formed by blockier monomer sequences. In the case of A-A or B-B monomer attraction without A-B repulsion, we observed decreasing cluster size and decreasing connectivity within the cluster with increasing graft sequence blockiness for particle sizes $\mathrm{D}=4 \mathrm{~d}$ and $\mathrm{D}=12 \mathrm{~d}$, with $\left(\mathrm{A}_{1} \mathrm{~B}_{1}\right)_{12}$ always being an outlier in these trends. The effect of graft sequence on cluster shape is reduced for $\mathrm{D}=12 \mathrm{~d}$ as compared to $\mathrm{D}=4 \mathrm{~d}$ at constant graft length. Lastly, monomer-particle interactions only weakly affect the assembly of copolymer grafted nanoparticles when the particle size is small relative to the graft length, and the strength of particle-monomer interactions are commensurate with the monomer- 
monomer interactions. The effects of monomer-particle interactions on the cluster size, shape, and structure are enhanced with significantly strong particle-monomer interactions, and/or with substantially large particle sizes relative to graft lengths.

\subsection{Homopolymer Grafted Nanoparticles in Chemically Identical Polymer Matrix}

Chapter 3-7 focused on systems of homopolymer grafted nanoparticles in a chemically identical homopolymer matrix. Due to the chemical similarity of the graft and matrix chains, the driving forces that control the nanocomposite morphology in these composites are purely entropic in nature. It is the balance of graft-matrix mixing entropy and the conformational entropy of the grafts and matrix chains which dictates the wetting of the grafted layer by matrix chains and, in turn, the dispersed or aggregated morphology adopted by the composite. In these chapters, we explored the effect of graft and matrix polydispersity and graft and matrix flexibility on the morphology of the composites using theory and simulations.

In Chapters 3, 4, and 5, we studied the effect of graft-length polydispersity on nanocomposite morphology. We showed that increasing graft-length polydispersity increases the wetting of the grafted layer over a monodisperse grafted layer. The increased wetting of the grafted layer results in an increasingly repulsive potential of mean force between the grafted particles in the explicit polymer matrix. This means that polymer nanocomposites with graft-length polydispersity have a stabilized dispersed morphology over those with monodisperse grafted layers due to increased grafted layer wetting by the matrix chains, and increased effective repulsion between the grafted particles. We also showed that to maximize the effective repulsion between the grafted particles, it is best to synthesize grafted particles at high grafting density with polymer graft length distributions that maximize monomer crowding near the particle surface to shield particle-particle attraction, and minimize crowding at farther distances from the particle to increase 
wetting of the grafted layer by matrix chains. Polydisperse (log-normal) graft length distributions and bidisperse graft length distributions with few long chains among many short chains satisfy this criterion, and better disperse grafted particles in a chemically identical matrix than monodisperse grafts or bidisperse graft length distributions with equal number of short and long chains, with equivalent average graft length.

In Chapter 6, we studied the effect of matrix bidispersity on the wetting of monodisperse grafted layers and grafted particle dispersion for chemically identical grafted and matrix chains. We found that short matrix chains in a bidisperse matrix have a higher correlation with the grafted particles at short distances than either than the long matrix chains in the same bidisperse matrix or short matrix chains in a monodisperse matrix. These results indicate that bidispersity in the matrix causes short matrix chains to preferentially wet the grafted layers over the long matrix chains. We showed that the preferential wetting of the grafted layer by the short matrix chains is likely due to their much higher mixing entropy when compared to the longer matrix chains wetting the grafted layer. Interestingly despite the increased wetting of the grafted layer, the particle-particle correlation functions show either no change with increasing matrix bidispersity or a slightly increased tendency for particle aggregation. This is due to the fact that, while the particle-short matrix monomer and particle-long matrix monomer correlation functions showed the effects of increasing matrix bidispersity, the particle-all matrix monomers correlation function, and therefore the total wetting, remained unaffected by the matrix bidispersity.

In Chapter 7, we studied the effect of graft and matrix flexibility on nanocomposite morphology for monodisperse graft and matrix chains. We found that the wetting of the grafted layer by chemically identical matrix chains is strongly dependent on the flexibility of the graft and matrix chains. Decreasing flexibility of the grafts more significantly improves the wetting of the 
grafted layer than decreasing flexibility of the matrix. Furthermore, finite filler fraction simulations show that decreasing flexibility improves dispersion due to increasing wetting of the grafted layer. These results suggest that polymer grafted nanoparticles filled polymer nanocomposites with larger persistence length polymers (graft and matrix), one would see a larger window in the phase space where the particles would be dispersed.

\subsection{Homopolymer Grafted Nanoparticles in Chemically Dissimilar Polymer Matrix}

Chapters 8 and 9 were focused on systems of polymer grafted particles in a chemically dissimilar polymer matrix. In contrast to the studies presented in Chapters 3-7, these composites have attractive interactions between the graft and matrix chains, mimicking the effective interactions of a lower-critical solution temperature (LCST) blend pair. Our goal in these studies was to understand the connection between wetting and dewetting in these composites and to leverage this connection to create highly tunable composite materials.

In Chapter 8, using coarse-grained simulations, along side x-ray and neutron scattering (conducted by experimental collaborator Prof. Ramanan Krishnamoorti and his research group), we demonstrated that, unlike chemically identical graft-matrix systems, a sharp dispersion to aggregation transition is distinct from the gradual continuous wetting to dewetting transition in a polymer nanocomposite with a chemically dissimilar graft-matrix polymer pair that exhibits LCST behavior. We showed that the dispersion to aggregation transition occurs when the extent of wetting in the attractive graft-matrix polymer pair approaches that of the analogous chemically similar graft-matrix polymer composite. Finally, we compared the behavior of grafted-particle based nanocomposites with chemically-dissimilar graft and matrix chains to a free polymer blend of the graft and matrix chains. We found that the mixing-demixing transition for the free blend was significantly sharper than the composite wetting-dewetting transition, suggesting that grafting 
the polymer chains to nanoparticles significantly alters the entropic driving forces for wetting dewetting. This study presented in Chapter 8 showed, for the first time, that chemically-dissimilar graft-matrix composites have distinct phase behavior from their more highly studied chemicallyidentical counterparts.

In Chapter 9, we showed that the unique phase behavior of the chemically dissimilar graftmatrix composites could be leveraged to create composites with highly tunable graft-matrix mixing and chain conformations. In these chemically different composites, with increasing fraction of attractive monomers in the random copolymer graft $\left(f_{G}\right)$ and matrix chains $\left(f_{M}\right)$, the enthalpic driving forces increasingly dominate over the entropic driving forces. As a result, the effect of matrix length at constant graft length on the wetting/dewetting and dispersion-aggregation is diminished for the fully attractive homopolymer composites with $f_{G}=f_{M}=1.0$ compared to those with $f_{G}<1.0$ and $f_{M}<1.0$. The magnitudes of $f_{G}$ and $f_{M}$ and the ratio $f_{G} / f_{M}$, to some degree, independently tune the extent of wetting and the dispersion-aggregation transition $\chi_{G M}$. When $f_{G}=f_{M}$, reducing the magnitude of $f_{G}$ and $f_{M}$ reduces the extent of wetting in the dispersed state while maintaining a constant dispersion-aggregation transition $\chi_{G M}$. In contrast, varying $f_{G} / f_{M}$ tunes both the wetting of the grafted layer and the dispersion-aggregation transition $\chi_{G M}$. We attributed the effect of $f_{G} / f_{M}$ on the dispersion-aggregation transition $\chi_{G M}$ to the fact that deviations from unity $f_{G} / f_{M}$ will create interaction site stoichiometries that favor like interactions (GG and $\mathrm{MM}$ ) over unlike interactions (GM) which increase the favorability of the aggregated state. Finally, we found that while the matrix chain conformations are relatively unaffected by varying $f_{G}, f_{M}$, or $\chi_{G M}$, the graft chain extension is tuned by varying these parameters. The composites with higher $f_{G}$ and $f_{M}$ magnitudes show the highest variability in graft chain extension with varying $\chi_{G M}$. These results demonstrated the tunability of composite 
morphology with chemically different graft and matrix chains. While the wetting-dewetting and the dispersion-aggregation, of chemically identical graft-matrix composites is tuned by varying physical parameters (e.g. molecular weights of graft and matrix or grafting density) the morphology of chemically different composites is tuned by choosing the graft and matrix chemistries, or varying $\chi_{G M}$ via temperature or solvent selection.

\subsection{FUTURE WORK}

In Chapter 2, we studied the assembly of copolymer grafted particles in an implicit solvent using Monte Carlo simulations. While these studies provide useful insight for experimentalists looking to synthesize nanoparticles into controlled nanostructures, one interesting next study would be to reduce the level of coarse graining in our model to study the effects of specific types of two- and three-body interactions that better capture the chemistry of specific systems. For example, there are many biological and synthetic polymers that interact strongly with water and other solvents, so a model that properly included enough chemical detail in the polymer and (possibly explicit) solvent to capture solvent effects would be needed for these systems. While this more detailed model would be greatly restricted in its ability to study large parameters spaces as we have in Chapter 2, it would instead allow researchers to explore particle assembly for specific systems of interest where our current, simple model might fail. Another interesting question involving the level of chemical detail in the model would be the effect of polymer flexibility on particle assembly. Much of our reasoning in these chapters centers on balancing the entropic loss associated with chain stretching with the enthalpic driving force for attractive monomer-monomer contacts. By decreasing the flexibility of the graft chains, we would be able to tune this entropic loss and it is currently unclear how this would affect assembly. While we studied the effect of flexibility for homopolymer grafted particles in an explicit homopolymer matrix in Chapter 7 , 
these results do not provide much insight into copolymer grafted particle assembly due to the inherent differences in the thermodynamics of the two systems. Understanding the role of graft flexibility on the assembly of copolymer grafted nanoparticles would provide materials scientists yet another avenue towards controlling particle assembly.

One question left unanswered is the effect of an explicit polymer matrix on the assembly of copolymer grafted particles with varying graft monomer sequence and blockiness. Recent work by Jayaraman and coworkers [1] have studied the effect of varying polymer interactions on the assembly of diblock copolymer grafted particles in homopolymer matrix. In line with the results of Chapters 2, they found that the chain conformations and assembly of the grafted particles largely depended on whether the inner or outer block was attractive to the homopolymer matrix. Furthermore, they also extended these studies to diblock grafted nanoparticles in homopolymer matrix blends with the focus on how the particles affected the interfacial configuration and energetics of the blends.[2] While these studies provide great insight into how copolymer grafted particles assemble in polymer matrix rather than implicit solvent, they do not study the effect of monomer sequence or blockiness on assembly in explicit matrix. Advances in radical polymerization techniques have made the synthesis of arbitrary chain sequences a reality and, based on our results in Chapter 2, we would expect blockiness to play a large role in determining the shape, size and structure of particle assemblies in explicit polymer matrix. Unfortunately, due to the vastly different thermodynamics, these results cannot be inferred from the implicit solvent results alone, therefore a separate detailed study is needed.

In Chapters 3-7, we presented studies on homopolymer grafted particles in a chemicallyidentical homopolymer matrix. While it is generally accepted that increased dispersion leads to better mechanical properties, and this thesis presents several new methods of stabilizing and tuning 
dispersion, we do not study the secondary effect of these techniques on the macroscopic material properties. For example, we found that graft length polydispersity stabilized dispersions of polymer grafted particles. It is not clear how graft-length polydispersity stabilized composites would differ from monodisperse grafted composites when considering the segmental dynamics, glass transition temperature, and rheology of the nanocomposite. While these properties were recently studied in experiments and simulations for monodisperse graft and matrix systems [3] [4, 5], we cannot immediately extrapolate their results to polydisperse systems. Furthermore, the increased wetting of the grafted layer by the matrix chains in the presence of graft polydispersity could enhance the entanglement of the long graft chains with the matrix chains, when graft and matrix molecular weights are greater than the entanglement molecular weight. Therefore, it would be worth investigating systematically how polydispersity in grafts impacts the mechanical reinforcement of the nanocomposite. Identical arguments to these can be made for studying the thermomechanical and rheological properties of flexibility stabilized particle dispersions. Studies into the secondary effects of polydispersity and flexibility stabilized dispersion are essential steps towards evaluating the industrial applicability of these techniques.

In Chapters 8 and 9, we studied homopolymer grafted nanoparticles in a chemically different polymer matrix. We found that, unlike the chemically-identical composites, the wettingdewetting transition was broad and independent of the dispersion-aggregation transition. Due to this difference, we showed that that the degree of wetting of the dispersed state can be tuned by varying the temperature, graft-matrix $\chi_{G M}$, and graft and matrix chain composition. The ability to tune the degree of wetting within the dispersed phase opens the possibility of directly tuning the material properties of the composite by controlling graft -matrix mixing. The thermomechanical, rheological, and mechanical properties will, to differing degrees, be connected to the extent of 
graft matrix mixing. A detailed study of one or more of these phenomena is needed to understand how these chemically-different graft-matrix systems can be leveraged to create highly tunable composite systems.

\subsection{REFERENCES}

1. Estridge, C.E. and A. Jayaraman, Assembly of diblock copolymer functionalized spherical nanoparticles as a function of copolymer composition. The Journal of Chemical Physics, 2014. 140(14): p. 144905.

2. Estridge, C.E. and A. Jayaraman, Diblock Copolymer Grafted Particles as Compatibilizers for Immiscible Binary Homopolymer Blends. ACS Macro Letters, 2015. 4(2): p. 155-159.

3. Chremos, A., A.Z. Panagiotopoulos, and D.L. Koch, Dynamics of solvent-free grafted nanoparticles. Journal of Chemical Physics, 2012. 136(4).

4. Akcora, P., et al., Segmental Dynamics in PMMA-Grafted Nanoparticle Composites. Macromolecules, 2010. 43(19): p. 8275-8281.

5. Goyal, S. and F.A. Escobedo, Structure and transport properties of polymer grafted nanoparticles. Journal of Chemical Physics, 2011. 135(18). 


\section{BIBLIOGRAPHY}

1. Agarwal, U. and F.A. Escobedo, Mesophase behaviour of polyhedral particles. Nature Materials, 2011. 10(3): p. 230-235.

2. Akcora, P., et al., Segmental Dynamics in PMMA-Grafted Nanoparticle Composites (vol 43, pg 8275, 2010). Macromolecules, 2011. 44(2): p. 416-416.

3. Akcora, P., et al., Segmental Dynamics in PMMA-Grafted Nanoparticle Composites. Macromolecules, 2010. 43(19): p. 8275-8281.

4. Akcora, P., et al., Anisotropic self-assembly of polymer-decorated spherical nanoparticles. Nature Materials, 2009. 8: p. 354-359.

5. Akcora, P., et al., Anisotropic self-assembly of spherical polymer-grafted nanoparticles. Nature Materials, 2009. 8(4): p. 354-359.

6. Altintas, O., et al., Combining Modular Ligation and Supramolecular Self-Assembly for the Construction of Star-Shaped Macromolecules. Macromolecular Rapid Communications, 2012. 33(11): p. 977-983.

7. Anderson, J.A., C.D. Lorenz, and A. Travesset, General purpose molecular dynamics simulations fully implemented on graphics processing units. Journal of Computational Physics, 2008. 227(10): p. 5342-5359.

8. Auhl, R., et al., Equilibration of long chain polymer melts in computer simulations. Journal of Chemical Physics, 2003. 119(24): p. 12718-12728.

9. Balazs, A.C., T. Emrick, and T.P. Russell, Nanoparticle polymer composites: Where two small worlds meet. Science, 2006. 314(5802): p. 1107-1110.

10. Barata, T., et al., Computational design principles for bioactive dendrimer based constructs as antagonists of the TLR4-MD-2-LPS complex. Biomaterials, 2011. 32(33): p. 8702-8711.

11. Bates, F.S., POLYMER-POLYMER PHASE-BEHAVIOR. Science, 1991. 251(4996): p. 898-905.

12. Bates, F.S., et al., Multiblock Polymers: Panacea or Pandora's Box? Science, 2012. 336(6080): p. 434-440.

13. Borukhov, I. and L. Leibler, Enthalpic stabilization of brush-coated particles in a polymer melt. Macromolecules, 2002. 35(13): p. 5171-5182.

14. Borukhov, I. and L. Leibler, Stabilizing grafted colloids in a polymer melt: Favorable enthalpic interactions. Physical Review E, 2000. 62(1): p. R41-R44.

15. Broseta, D., et al., MOLECULAR-WEIGHT AND POLYDISPERSITY EFFECTS AT POLYMER POLYMER INTERFACES. Macromolecules, 1990. 23(1): p. 132-139.

16. Brown, H.R., K. Char, and V.R. Deline, Enthalpy-driven swelling of a polymer brush. Macromolecules, 1990. 23(13): p. 3383-3385. 
17. Burgentzlé, D., et al., Solvent-based nanocomposite coatings: I. Dispersion of organophilic montmorillonite in organic solvents. Journal of Colloid and Interface Science, 2004. 278(1): p. 26-39.

18. Camargo, M., S.A. Egorov, and C.N. Likos, Cluster formation in star-linear polymer mixtures: equilibrium and dynamical properties. Soft Matter, 2012.

19. Ceperley, D., M.H. Kalos, and J.L. Lebowitz, Computer Simulation of the Dynamics of a Single Polymer Chain. Physical Review Letters, 1978. 41(5): p. 313-316.

20. Chan, E.R., L.C. Ho, and S.C. Glotzer, Computer simulations of block copolymer tethered nanoparticle self-assembly. Journal of Chemical Physics, 2006. 125(6): p. 9.

21. Chen, F., et al., Synthesis and characterization of copolymer grafted magnetic nanoparticles via surface-initiated nitroxide-mediated radical polymerization. Polymer Engineering and Science, 2013. 53(5): p. 956-962.

22. Chevigny, C., et al., Polymer-Grafted-Nanoparticles Nanocomposites: Dispersion, Grafted Chain Conformation, and Rheological Behavior. Macromolecules, 2011. 44(1): p. 122-133.

23. Chremos, A., A.Z. Panagiotopoulos, and D.L. Koch, Dynamics of solvent-free grafted nanoparticles. Journal of Chemical Physics, 2012. 136(4).

24. Chremos, A., et al., Structure of solvent-free grafted nanoparticles: Molecular dynamics and density-functional theory. Journal of Chemical Physics, 2011. 135(11).

25. Cifra, P., Differences and limits in estimates of persistence length for semi-flexible macromolecules. Polymer, 2004. 45(17): p. 5995-6002.

26. Clark, M.D. and R. Krishnamoorti, Dispersion of Functionalized Multiwalled Carbon Nanotubes. Journal of Physical Chemistry C, 2009. 113: p. 20861-20868.

27. Corbierre, M.K., et al., Gold nanoparticle/polymer nanocomposites: Dispersion of nanoparticles as a function of capping agent molecular weight and grafting density. Langmuir, 2005. 21: p. 6063-6072.

28. Crawford, M.K., et al., Chain Conformation in Polymer Nanocomposites with Uniformly Dispersed Nanoparticles. Physical Review Letters, 2013. 110(19): p. 5.

29. Crocker, J.C., Nanomaterials: Golden handshake. Nature, 2008. 451(7178): p. 528-529.

30. Crosby, A.J. and J.Y. Lee, Polymer nanocomposites: The "nano" effect on mechanical properties. Polymer Reviews, 2007. 47(2): p. 217-229.

31. Cui, L., et al., A facile "graft from" method to prepare molecular-level dispersed graphenepolymer composites. Journal of Polymer Science Part a-Polymer Chemistry, 2012. 50(21): p. 4423-4432.

32. Cui, S.T., P.T. Cummings, and H.D. Cochran, The calculation of the viscosity from the autocorrelation function using molecular and atomic stress tensors. Molecular Physics, 1996. 88(6): p. 1657-1664.

33. Dang, A., et al., High-Transparency Polymer Nanocomposites Enabled by Polymer-Graft Modification of Particle Fillers. Langmuir, 2014. 30(48): p. 14434-14442. 
34. Daoud, M., ADSORPTION FROM A BIDISPERSE POLYMER MIXTURE. Macromolecules, 1991. 24(25): p. 6748-6753.

35. de Espinosa, L.M., et al., Side-Chain Modification and "Grafting Onto" via Olefin CrossMetathesis. Macromolecular Rapid Communications, 2012. 33(23): p. 2023-2028.

36. de Vos, W.M. and F.A.M. Leermakers, Modeling the structure of a polydisperse polymer brush. Polymer, 2009. 50(1): p. 305-316.

37. Dijkstra, M. and D. Frenkel, SIMULATION STUDY OF THE ISOTROPIC-TO-NEMATIC TRANSITIONS OF SEMIFLEXIBLE POLYMERS. Physical Review E, 1995. 51(6): p. 5891-5898.

38. Dodd, P.M. and A. Jayaraman, Monte carlo simulations of polydisperse polymers grafted on spherical surfaces. Journal of Polymer Science Part B-Polymer Physics, 2012. 50(10): p. 694-705.

39. Dupre, D.B. and S.J. Yang, LIQUID-CRYSTALLINE PROPERTIES OF SOLUTIONS OF PERSISTENT POLYMER-CHAINS. Journal of Chemical Physics, 1991. 94(11): p. 74667477.

40. Egorov, S.A. and K. Binder, Effect of solvent quality on the dispersibility of polymergrafted spherical nanoparticles in polymer solutions. Journal of Chemical Physics, 2012. 137(9).

41. Eitouni, H. and N. Balsara, Thermodynamics of Polymer Blends, in Physical Properties of Polymers Handbook, J. Mark, Editor. 2007, Springer New York. p. 339-356.

42. Estridge, C.E. and A. Jayaraman, Assembly of diblock copolymer functionalized spherical nanoparticles as a function of copolymer composition. The Journal of Chemical Physics, 2014. 140(14): p. 144905.

43. Estridge, C.E. and A. Jayaraman, Diblock Copolymer Grafted Particles as Compatibilizers for Immiscible Binary Homopolymer Blends. ACS Macro Letters, 2015. 4(2): p. 155-159.

44. Faust, A.B., et al., Influence of star-core exclusion on polymer-polymer miscibility. Macromolecules, 1989. 22(3): p. 1250-1254.

45. Ferreira, P.G., A. Ajdari, and L. Leibler, Scaling law for entropic effects at interfaces between grafted layers and polymer melts. Macromolecules, 1998. 31(12): p. 3994-4003.

46. Flory, P.J., THE CONFIGURATION OF REAL POLYMER CHAINS. Journal of Chemical Physics, 1949. 17(3): p. 303-310.

47. Frenkel, D. and B. Smit, Understanding Molecular Simulation: From Algorithms to Applications. 2nd ed. 2002, San Diego: Academic Press.

48. Frischknecht, A.L., E.S. McGarrity, and M.E. Mackay, Expanded chain dimensions in polymer melts with nanoparticle fillers. Journal of Chemical Physics, 2010. 132: p. 204901.

49. Frischknecht, A.L. and A. Yethiraj, Two- and three-body interactions among nanoparticles in a polymer melt. The Journal of Chemical Physics, 2011. 134(17): p. 174901-11.

50. Fynewever, H. and A. Yethiraj, Phase behavior of semiflexible tangent hard sphere chains. Journal of Chemical Physics, 1998. 108(4): p. 1636-1644. 
51. Ganesan, V. and A. Jayaraman, Theory and simulation studies of effective interactions, phase behavior and morphology in polymer nanocomposites. Soft Matter, 2014. 10(1): p. 13-38.

52. Ganesan, V., L. Khounlavong, and V. Pryamitsyn, Equilibrium characteristics of semiflexible polymer solutions near probe particles. Physical Review E, 2008. 78(5).

53. Ghanbari, A., et al., Interphase Structure in Silica-Polystyrene Nanocomposites: A CoarseGrained Molecular Dynamics Study. Macromolecules, 2012. 45(1): p. 572-584.

54. Glotzer, S.C., Nanotechnology Shape Matters. Nature, 2012. 481(7382): p. 450-452.

55. Glotzer, S.C. and J.A. Anderson, NANOPARTICLE ASSEMBLY Made to order. Nature Materials, 2010. 9(11): p. 885-887.

56. Goel, V., et al., Viscoelastic properties of silica-grafted poly(styrene-acrylonitrile) nanocomposites. Journal of Polymer Science Part B-Polymer Physics, 2006. 44(14): p. 2014-2023.

57. Goel, V., et al., Structure of Polymer Tethered Highly Grafted Nanoparticles. Macromolecules, 2011. 44(20): p. 8129-8135.

58. Goel, V., et al., Structure of Polymer Tethered Highly Grafted Nanoparticles. Macromolecules, 2011. 44(20): p. 8129-8135.

59. Goyal, S. and F.A. Escobedo, Structure and transport properties of polymer grafted nanoparticles. Journal of Chemical Physics, 2011. 135(18).

60. Grayce, C.J. and K.S. Schweizer, Solvn potentials for macromolecules. Journal of Chemical Physics, 1994. 100: p. 6846-6856.

61. Grayce, C.J., A. Yethiraj, and K.S. Schweizer, Liquid state theory of the density dependent conformation of nonpolar linear polymers. Journal of Chemical Physics, 1994. 100: p. 6857-6872.

62. Green, P.F., The structure of chain end-grafted nanoparticle/homopolymer nanocomposites. Soft Matter, 2011. 7(18): p. 7914-7926.

63. Grest, G.S. and K. Kremer, MOLECULAR-DYNAMICS SIMULATION FOR POLYMERS IN THE PRESENCE OF A HEAT BATH. Physical Review A, 1986. 33(5): p. 3628-3631.

64. Hall, L.M., et al., Concentration Fluctuations, Local Order, and the Collective Structure of Polymer Nanocomposites. Macromolecules, 2009. 42(21): p. 8435-8442.

65. Hall, L.M., A. Jayaraman, and K.S. Schweizer, Molecular theories of polymer nanocomposites. Current Opinion in Solid State \& Materials Science, 2010. 14(2): p. 3848.

66. Hall, L.M. and K.S. Schweizer, Many body effects on the phase separation and structure of dense polymer-particle melts. Journal of Chemical Physics, 2008. 128: p. 234901.

67. Hall, L.M. and K.S. Schweizer, Structure, scattering patterns and phase behavior of polymer nanocomposites with nonspherical fillers. Soft Matter, 2010. 6(5): p. 1015-1025.

68. Hammouda, B., SANS from homogeneous polymer mixtures: A unified overview, in Polymer Characteristics. 1993, Springer Berlin Heidelberg. p. 87-133. 
69. Hansen, J.-P. and I.R. McDonald, Theory of Simple Liquids

3rd ed, ed. Elsevier. 2006.

70. $\quad$ Hansen, J.P.M., I.R., Theory of Simple Liquids. 3rd ed. 2006.

71. Harton, S.E. and S.K. Kumar, Mean-field theoretical analysis of brush-coated nanoparticle dispersion in polymer matrices. Journal of Polymer Science Part B-Polymer Physics, 2008. 46: p. 351-358.

72. Harton, S.E. and S.K. Kumar, Mean-field theoretical analysis of brush-coated nanoparticle dispersion in polymer matrices. Journal of Polymer Science Part B-Polymer Physics, 2008. 46(4): p. 351-358.

73. Hasegawa, R., Y. Aoki, and M. Doi, Optimum graft density for dispersing particles in polymer melts. Macromolecules, 1996. 29(20): p. 6656-6662.

74. Heo, K., et al., Thermally Reversible Aggregation of Gold Nanoparticles in Polymer Nanocomposites through Hydrogen Bonding. Nano Letters, 2013. 13(11): p. 5297-5302.

75. Himmi, M., et al., Interaction force between colloidal particles clothed by end-grafted polydisperse polymer chains in solution. Journal of Molecular Liquids, 2003. 102(1-3): p. 347-363.

76. Hindmarsh, A.C., et al., SUNDIALS: Suite of nonlinear and differential/algebraic equation solvers. Acm Transactions on Mathematical Software, 2005. 31(3): p. 363-396.

77. Hong, B.B. and A.Z. Panagiotopoulos, Molecular Dynamics Simulations of Silica Nanoparticles Grafted with Poly(ethylene oxide) Oligomer Chains. Journal of Physical Chemistry B, 2012. 116(8): p. 2385-2395.

78. HOOMD-blue. http://codeblue.umich.edu/hoomd-blue.

79. Hooper, J.B. and K.S. Schweizer, Contact aggregation, bridging, and steric stabilization in dense polymer-particle mixtures. Macromolecules, 2005. 38: p. 8858-8869.

80. Hooper, J.B. and K.S. Schweizer, Theory of phase separation in polymer nanocomposites. Macromolecules, 2006. 39: p. 5133-5142.

81. Hooper, J.B., et al., Structure, surface excess and effective interactions in polymer nanocomposite melts and concentrated solutions. Journal of Chemical Physics, 2004. 121(14): p. 6986-6997.

82. http://codeblue.umich.edu/hoomd-blue/. HOOMD-Blue webpage. Available from: http://codeblue.umich.edu/hoomd-blue/ (2010).

83. Hu, S.W., Y.J. Sheng, and H.K. Tsao, Self-Assembly of Organophilic Nanoparticles in a Polymer Matrix: Depletion Interactions. Journal of Physical Chemistry C, 2012. 116(2): p. 1789-1797.

84. Humphrey, W., A. Dalke, and K. Schulten, VMD - Visual Molecular Dynamics. J. Molec. Graphics, 1996. 14: p. 33-38.

85. Iacovella, C.R. and S.C. Glotzer, Phase behavior of ditethered nanospheres. Soft Matter, 2009. 5(22): p. 4492-4498. 
86. Ivanov, V.A., et al., Conformational Properties of Semiflexible Chains at Nematic Ordering Transitions in Thin Films: A Monte Carlo Simulation. Macromolecules, 2014. 47(3): p. 1206-1220.

87. Ivanov, V.A., et al., Structures of stiff macromolecules of finite chain length near the coilglobule transition: A Monte Carlo simulation. Macromolecular Theory and Simulations, 2000. 9(8): p. 488-499.

88. Jaffer, K.M., et al., The nematic-isotropic phase transition in semiflexible fused hardsphere chain fluids. Journal of Chemical Physics, 2001. 114(7): p. 3314-3324.

89. Jakubowski, W. and K. Matyjaszewski, Activator Generated by Electron Transfer for Atom Transfer Radical Polymerization. Macromolecules, 2005. 38(10): p. 4139-4146.

90. Jancar, J., et al., Current issues in research on structure-property relationships in polymer nanocomposites. Polymer, 2010. 51(15): p. 3321-3343.

91. Jayaraman, A., Polymer Grafted Nanoparticles: Effect of Chemical and Physical Heterogeneity in Polymer Grafts on Particle Assembly and Dispersion. Journal of Polymer Science Part B-Polymer Physics, 2013. 51(7): p. 524-534.

92. Jayaraman, A. and N. Nair, Integrating PRISM theory and Monte Carlo simulation to study polymer-functionalised particles and polymer nanocomposites. Molecular Simulation, 2012. 38(8-9): p. 751-761.

93. Jayaraman, A. and N. Nair, Integrating PRISM theory and Monte Carlo simulations for studying polymer functionalized particles and polymer nanocomposites. Molecular Simulations, 2012. in press(Special issue on New Developments in Molecular Simulations).

94. Jayaraman, A. and K.S. Schweizer, Effect of the number and placement of polymer tethers on the structure of concentrated solutions and melts of hybrid nanoparticles. Langmuir, 2008. 24(19): p. 11119-11130.

95. Jayaraman, A. and K.S. Schweizer, Effect of the number and placement of polymer tethers on the structure of dense solutions of hybrid nanoparticles. Langmuir, 2008. 24(19): p. 11119-11130.

96. Jayaraman, A. and K.S. Schweizer, Effective Interactions and Self-Assembly of Hybrid Polymer Grafted Nanoparticles in a Homopolymer Matrix. Macromolecules, 2009. 42 p. pp 8423-8434.

97. Jayaraman, A. and K.S. Schweizer, Effective Interactions and Self-Assembly of Hybrid Polymer Grafted Nanoparticles in a Homopolymer Matrix. Macromolecules, 2009. 42(21): p. 8423-8434.

98. Jayaraman, A. and K.S. Schweizer, Effective Interactions, Structure, and Phase Behavior of Lightly Tethered Nanoparticles in Polymer Melts. Macromolecules, 2008. 41(23): p. 9430-9438.

99. Jayaraman, A. and K.S. Schweizer, Liquid state theory of the structure and phase behaviour of polymer-tethered nanoparticles in dense suspensions, melts and nanocomposites. Molecular Simulation, 2009. 35(10-11): p. 835-848. 
100. Jayaraman, A. and K.S. Schweizer, Structure and Assembly of Dense Solutions and Melts of Single Tethered Nanoparticles. Journal of Chemical Physics, 2008. 128: p. 164904.

101. Jayaraman, A. and K.S. Schweizer, Structure and Assembly of Dense Solutions and Melts of Single Tethered Nanoparticles. J. Chem. Phys., 2008. 128: p. 164904.

102. Jiang, K., et al., One-Pot Controlled Synthesis of Homopolymers and Diblock Copolymers Grafted Graphene Oxide Using Couplable RAFT Agents. Macromolecules, 2012. 45(3): p. 1346-1355.

103. Jiao, Y. and P. Akcora, Assembly of Polymer-Grafted Magnetic Nanoparticles in Polymer Melts. Macromolecules, 2012. 45(8): p. 3463-3470.

104. Jones, M.R., et al., DNA-nanoparticle superlattices formed from anisotropic building blocks. Nature Materials, 2010. 9(11): p. 913-917.

105. Jouault, N., et al., Well-Dispersed Fractal Aggregates as Filler in Polymer-Silica Nanocomposites: Long-Range Effects in Rheology. Macromolecules, 2009. 42(6): p. 20312040.

106. Kalb, J., et al., End grafted polymer nanoparticles in a polymeric matrix: Effect of coverage and curvature. Soft Matter. 7(4): p. 1418-1425.

107. Kalb, J., et al., End grafted polymer nanoparticles in a polymeric matrix: Effect of coverage and curvature. Soft Matter, 2011. 7(4): p. 1418-1425.

108. Keller, K., A. Amirian, and P. Akcora, Elastic Properties of a Protein-Polymer-Grafted Surface. Langmuir, 2012. 28(8): p. 3807-3813.

109. Khalatur, P.G. and A.R. Khokhlov, Hybrid MC/RISM technique for simulation of polymer solutions: MC+RISM integral eqns. Molecular Physics, 1998. 93: p. 555-572.

110. Khalatur, P.G., L.V. Zherenkova, and A.R. Khokhlov, Entropy-driven polymer collapse: Application of the hybrid MC/RISM method to the study of conformational transitions in macromolecules interacting with hard colloidal particles. European Physical Journal B, 1998. 5(4): p. 881-897.

111. Kim, B., et al., Dewetting of PMMA on PS-Brush Substrates. Macromolecules, 2009. 42(20): p. 7919-7923.

112. Kim, B.J., et al., Effect of areal chain density on the location of polymer-modified gold nanoparticles in a block copolymer template. Macromolecules, 2006. 39(12): p. 41084114.

113. Kim, J., et al., Highly effective gold nanoparticle-enhanced biosensor array on the wettability controlled substrate by wiping. Journal of Applied Physics, 2011. 110(8): p. 7.

114. Kindt, J.T. and W.M. Gelbart, Chain self-assembly and phase transitions in semiflexible polymer systems. Journal of Chemical Physics, 2001. 114(3): p. 1432-1439.

115. Knorowski, C. and A. Travesset, Materials design by DNA programmed self-assembly. Current Opinion in Solid State \& Materials Science, 2011. 15(6): p. 262-270.

116. Kong, M., et al., Systematic Coarse-Graining of the Dynamics of Self-Attractive Semiflexible Polymers. Macromolecules, 2014. 47(4): p. 1494-1502. 
117. Krishnamoorti, R., Strategies for Dispersing Nanoparticles in Polymers. MRS Bulletin, 2007. 32(4): p. 341-347.

118. Krishnamoorti, R. and R.A. Vaia, Polymer nanocomposites. Journal of Polymer Science Part B-Polymer Physics, 2007. 45(24): p. 3252-3256.

119. Kumar, S.K., et al., Nanocomposites with Polymer Grafted Nanoparticles. Macromolecules, 2013. 46(9): p. 3199-3214.

120. Kumar, S.K. and R. Krishnamoorti, Nanocomposites: Structure, Phase Behavior, and Properties, in Annual Review of Chemical and Biomolecular Engineering, Vol 1, J.M. Prausnitz, M.F. Doherty, and M.A. Segalman, Editors. 2010. p. 37-58.

121. Lan, Q., L.F. Francis, and F.S. Bates, Silica nanoparticles dispersions in homopolymer versus block copolymer. Journal of Polymer Science Part B-Polymer Physics, 2007. 45: p. 2284-2299.

122. Laub, C.F. and J.T. Koberstein, EFFECT OF BRUSH POLYDISPERSITY ON THE INTERPHASE BETWEEN END-GRAFTED BRUSHES AND POLYMERIC MATRICES. Macromolecules, 1994. 27(18): p. 5016-5023.

123. Lee, K.M. and C.D. Han, Effect of hydrogen bonding on the rheology of polycarbonate/organoclay nanocomposites. Polymer, 2003. 44(16): p. 4573-4588.

124. Leibler, L., Theory of Microphase Separation in Block Copolymers. Macromolecules, 1980. 13(6): p. 1602-1617.

125. Li, Y., et al., Ligand Engineering of Polymer Nanocomposites: From the Simple to the Complex. Acs Applied Materials \& Interfaces, 2014.

126. Lin, B., T.B. Martin, and A. Jayaraman, Decreasing Polymer Flexibility Improves Wetting and Dispersion of Polymer-Grafted Particles in a Chemically Identical Polymer Matrix. Acs Macro Letters, 2014. 3(7): p. 628-632.

127. Lin, Y.L., et al., Self-Assembled Superstructures of Polymer-Grafted Nanoparticles: Effects of Particle Shape and Matrix Polymer. Journal of Physical Chemistry C, 2011. 115(13): p. 5566-5577.

128. Mackay, M.E., et al., General Strategies for Nanoparticle Dispersion. Science, 2006. 311(5768): p. 1740-1743.

129. Madbouly, S.A. and J.U. Otaigbe, Recent advances in synthesis, characterization and rheological properties of polyurethanes and POSS/polyurethane nanocomposites dispersions and films. Progress in Polymer Science, 2009. 34(12): p. 1283-1332.

130. Maillard, D., et al., Polymer-Grafted-Nanoparticle Surfactants. Nano Letters, 2011. 11(11): p. 4569-4573.

131. Martin, T.B., P.M. Dodd, and A. Jayaraman, Polydispersity for Tuning the Potential of Mean Force between Polymer Grafted Nanoparticles in a Polymer Matrix. Physical Review Letters, 2013. 110(1): p. 018301.

132. Martin, T.B., P.M. Dodd, and A. Jayaraman, Polydispersity for Tuning the Potential of Mean Force between Polymer Grafted Nanoparticles in a Polymer Matrix. Physical Review Letters, 2013. 110(1). 
133. Martin, T.B. and A. Jayaraman, Effect of matrix bidispersity on the morphology of polymergrafted nanoparticle-filled polymer nanocomposites. Journal of Polymer Science Part B: Polymer Physics, 2014. 52(24): p. 1661-1668.

134. Martin, T.B. and A. Jayaraman, Identifying the Ideal Characteristics of the Grafted Polymer Chain Length Distribution for Maximizing Dispersion of Polymer Grafted Nanoparticles in a Polymer Matrix. Macromolecules, 2013. 46(22): p. 9144-9150.

135. Martin, T.B. and A. Jayaraman, Polydisperse homopolymer grafts stabilize dispersions of nanoparticles in a chemically identical homopolymer matrix: an integrated theory and simulation study. Soft Matter, 2013. 9(29): p. 6876-6889.

136. Martin, T.B. and A. Jayaraman, Polydisperse homopolymer grafts stabilize dispersions of nanoparticles in a chemically identical homopolymer matrix: an integrated theory and simulation study. Soft Matter, 2013.

137. Martin, T.B., et al., Wetting-Dewetting and Dispersion-Aggregation Transitions Are Distinct for Polymer Grafted Nanoparticles in Chemically Dissimilar Polymer Matrix. Journal of the American Chemical Society, 2015. 137(33): p. 10624-10631.

138. Martin, T.B., A. Seifpour, and A. Jayaraman, Assembly of copolymer functionalized nanoparticles: a Monte Carlo simulation study. Soft Matter, 2011. 7(13): p. 5952-5964.

139. Matsen, M.W., Scaling behavior of a brush-homopolymer interface in the limit of high grafting density. Journal of Chemical Physics, 2005. 122(14).

140. McGarrity, E.S., et al., Surface-induced first-order transition in athermal polymernanoparticle blends. Physical Review Letters, 2007. 99(23).

141. McMaster, L.P., ASPECTS OF POLYMER-POLYMER THERMODYNAMICS. Macromolecules, 1973. 6(5): p. 760-773.

142. Melenkevitz, J., K.S. Schweizer, and J.G. Curro, Self consistent integral eqn theory for the eqbm properties of polymer solns. Macromolecules, 1993. 26: p. 6190-6196.

143. Mendez, S., et al., An integral equation theory for polymer solutions: explicit inclusion of the solvent molecules. Journal of Chemical Physics, 2001. 115: p. 5669-5678.

144. Meng, D., et al., Effective interactions between grafted nanoparticles in a polymer matrix. Soft Matter, 2012. 8(18): p. 5002-5010.

145. Metropolis, N., et al., EQUATION OF STATE CALCULATIONS BY FAST COMPUTING MACHINES. Journal of Chemical Physics, 1953. 21(6): p. 1087-1092.

146. Milchev, A., PHASE-TRANSITIONS IN POLYDISPERSE POLYMER MELTS. Polymer, 1993. 34(2): p. 362-368.

147. Milner, S.T., T.A. Witten, and M.E. Cates, EFFECTS OF POLYDISPERSITY IN THE END-GRAFTED POLYMER BRUSH. Macromolecules, 1989. 22(2): p. 853-861.

148. Min, K., et al., One-Pot Synthesis of Hairy Nanoparticles by Emulsion ATRP. Macromolecules, 2009. 42(5): p. 1597-1603.

149. Morse, D.C. and G.H. Fredrickson, SEMIFLEXIBLE POLYMERS NEAR INTERFACES. Physical Review Letters, 1994. 73(24): p. 3235-3238. 
150. Muller, M. and L.G. MacDowell, Wetting of a short chain liquid on a brush: First-order and critical wetting transitions. Europhysics Letters, 2001. 55: p. 221.

151. Muralidhar, A., et al., Interplay between chain stiffness and excluded volume of semiflexible polymers confined in nanochannels. Journal of Chemical Physics, 2014. 140(8).

152. Nair, N. and A. Jayaraman, Self-Consistent PRISM Theory-Monte Carlo Simulation Studies of Copolymer Grafted Nanoparticles in a Homopolymer Matrix. Macromolecules, 2010. 43(19): p. 8251-8263.

153. Nair, N., N. Wentzel, and A. Jayaraman, Effect of bidispersity in grafted chain length on grafted chain conformations and potential of mean force between polymer grafted nanoparticles in a homopolymer matrix. Journal of Chemical Physics, 2011. 134(19).

154. Nair, N., N. Wentzel, and A. Jayaraman, Effect of bidispersity in grafted chain length on grafted chain conformations and potential of mean force between polymer grafted nanoparticles in a homopolymer matrix. Journal of Chemical Physics, 2011. 134: p. 194906.

155. Natarajan, B., et al., The Evolution of Carbon Nanotube Network Structure in Unidirectional Nanocomposites Resolved by Quantitative Electron Tomography. ACS Nano, 2015. 9(6): p. 6050-6058.

156. Ndoro, T.V.M., et al., Interface of Grafted and Ungrafted Silica Nanoparticles with a Polystyrene Matrix: Atomistic Molecular Dynamics Simulations. Macromolecules, 2011. 44(7): p. 2316-2327.

157. Ojha, S., et al., Strategies for the Synthesis of Thermoplastic Polymer Nanocomposite Materials with High Inorganic Filling Fraction. Langmuir, 2013. 29(28): p. 8989-8996.

158. Padmanabhan, V., Effect of grafting on nanoparticle segregation in polymer/nanoparticle blends near a substrate. Journal of Chemical Physics, 2012. 137(9).

159. Padmanabhan, V., A.L. Frischknecht, and M.E. Mackay, Effect of Chain Stiffness on Nanoparticle Segregation in Polymer/Nanoparticle Blends Near a Substrate. Macromolecular Theory and Simulations, 2012. 21(2): p. 98-105.

160. Park, S.Y., et al., DNA programmable nanoparticle crystallization. Nature, 2008. 451: p. 553-556.

161. Paul, D.R. and C.B. Bucknall, Polymer Blends: Formulation and Performance (Two volume set). 2000: Wiley.

162. Pedersen, J.S., M. Laso, and P. Schurtenberger, Monte Carlo study of excluded volume effects in wormlike micelles and semiflexible polymers. Physical Review E, 1996. 54(6): p. R5917-R5920.

163. Popov, K.I., et al., Interacting nanoparticles with functional surface groups. Journal of Polymer Science Part B-Polymer Physics, 2012. 50(12): p. 852-862.

164. Pryamtisyn, V., et al., Modeling the anisotropic self-assembly of spherical polymer-grafted nanoparticles. Journal of Chemical Physics, 2009. 131(22). 
165. Putz, M., J.G. Curro, and G.S. Grest, Self-consistent integral equation theory for polyolefins: comparison to molecular dynamics simulations and $x$-ray scattering. Journal of Chemical Physics, 2001. 114: p. 2847-2860.

166. Pyun, J., et al., Synthesis and Characterization of Organic/Inorganic Hybrid Nanoparticles: Kinetics of Surface-Initiated Atom Transfer Radical Polymerization and Morphology of Hybrid Nanoparticle Ultrathin Films. Macromolecules, 2003. 36(14): p. 5094-5104.

167. Qin, J., et al., Controlled Bimodal Molecular-Weight-Distribution Polymers: Facile Synthesis by RAFT Polymerization. Chemistry-a European Journal, 2012. 18(19): p. 60156021.

168. Rousseau, G., et al., Azido-Coated Nanoparticles: A Versatile Clickable Platform for the Preparation of Fluorescent Polystyrene Core-PAMAM Shell Nanoparticles. Macromolecules, 2012. 45(8): p. 3513-3522.

169. Russell, T.P., et al., Concentration fluctuations in mixtures of linear and star-shaped polymers. Macromolecules, 1990. 23(2): p. 654-659.

170. Ryan, A.J., Polymer science: Designer polymer blends. Nat Mater, 2002. 1(1): p. 8-10.

171. Schnurr, B., F. Gittes, and F.C. MacKintosh, Metastable intermediates in the condensation of semiflexible polymers. Physical Review E, 2002. 65(6).

172. Schnurr, B., F.C. MacKintosh, and D.R.M. Williams, Dynamical intermediates in the collapse of semiflexible polymers in poor solvents. Europhysics Letters, 2000. 51(3): p. 279-285.

173. Schweizer, K.S. and J.G. Curro, INTEGRAL-EQUATION THEORY OF THE STRUCTURE OF POLYMER MELTS. Physical Review Letters, 1987. 58(3): p. 246-249.

174. Schweizer, K.S. and J.G. Curro, Integral equation theory of polymer melts: intramolecular structurem local order, and the correlation hole. Macromolecules, 1988. 21: p. 3070-3081.

175. Schweizer, K.S. and J.G. Curro, PRISM Theory of the Structure, Thermodynamics, and Phase-Transitions of Polymer Liquids and Alloys. Advances in Polymer Science, 1994. 116: p. 319-377.

176. Schweizer, K.S. and A. Yethiraj, POLYMER REFERENCE INTERACTION SITE MODEL-THEORY - NEW MOLECULAR CLOSURES FOR PHASE-SEPARATING FLUIDS AND ALLOYS. Journal of Chemical Physics, 1993. 98(11): p. 9053-9079.

177. Seifpour, A., et al., Effect of monomer sequences on conformations of copolymers grafted on spherical nanoparticles: A Monte Carlo simulation study. Journal of Chemical Physics, 2010. 132(16).

178. Sen, S., et al., Chain conformations and bound-layer correlations in polymer nanocomposites. Physical Review Letters, 2007. 98(12).

179. Shen, J.X., et al., Revisiting the Dispersion Mechanism of Grafted Nanoparticles in Polymer Matrix: A Detailed Molecular Dynamics Simulation. Langmuir, 2011. 27(24): p. 15213-15222. 
180. Shull, K.R., THEORY OF END-ADSORBED POLYMER BRUSHES IN POLYMERIC MATRICES. Journal of Chemical Physics, 1991. 94(8): p. 5723-5738.

181. Shull, K.R., Wetting behavior of polymer melts on polydisperse grafted polymer layers. Macromolecules, 1996. 29(26): p. 8487-8491.

182. Sintes, T., K. Sumithra, and E. Straube, Adsorption of semiflexible polymers on flat, homogeneous surfaces. Macromolecules, 2001. 34(5): p. 1352-1357.

183. Slota, J.E., X. He, and W.T.S. Huck, Controlling nanoscale morphology in polymer photovoltaic devices. Nano Today, 2010. 5(3): p. 231-242.

184. Smith, G.D. and D. Bedrov, Dispersing Nanoparticles in a Polymer Matrix: Are Long, Dense Polymer Tethers Really Necessary? Langmuir, 2009. 25(19): p. 11239-11243.

185. Srivastava, S., P. Agarwal, and L.A. Archer, Tethered Nanoparticle-Polymer Composites: Phase Stability and Curvature. Langmuir, 2012. 28(15): p. 6276-6281.

186. Steinhauser, M.O., Static and dynamic scaling of semiflexible polymer chains-a molecular dynamics simulation study of single chains and melts. Mechanics of Time-Dependent Materials, 2008. 12(4): p. 291-312.

187. Stukan, M.R., et al., Chain length dependence of the state diagram of a single stiff-chain macromolecule: Theory and Monte Carlo simulation. Journal of Chemical Physics, 2003. 118(7): p. 3392-3400.

188. Sunday, D., J. Ilavsky, and D.L. Green, A Phase Diagram for Polymer-Grafted Nanoparticles in Homopolymer Matrices. Macromolecules, 2012. 45(9): p. 4007-4011.

189. Tallury, S.S. and M.A. Pasquinelli, Molecular Dynamics Simulations of Polymers with Stiff Backbones Interacting with Single-Walled Carbon Nanotubes. Journal of Physical Chemistry B, 2010. 114(29): p. 9349-9355.

190. Tao, P., et al., Transparent luminescent silicone nanocomposites filled with bimodal PDMS-brush-grafted CdSe quantum dots. Journal of Materials Chemistry C, 2013. 1(1): p. 86-94.

191. Tao, P., et al., Bulk transparent epoxy nanocomposites filled with poly(glycidyl methacrylate) brush-grafted TiO2 nanoparticles. Polymer, 2013. 54(6): p. 1639-1646.

192. Theodorou, D.N. and U.W. Suter, SHAPE OF UNPERTURBED LINEAR-POLYMERS POLYPROPYLENE. Macromolecules, 1985. 18(6): p. 1206-1214.

193. Trombly, D.M. and V. Ganesan, Curvature effects upon interactions of polymer-grafted nanoparticles in chemically identical polymer matrices. Journal of Chemical Physics, 2010. 133(15).

194. Tsubokawa, N., Surface grafting of polymers onto nanoparticles in a solvent-free drysystem and applications of polymer-grafted nanoparticles as novel functional hybrid materials. Polymer Journal, 2007. 39(10): p. 983-1000.

195. Tuteja, A., P.M. Duxbury, and M.E. Mackay, Polymer chain swelling induced by dispersed nanoparticles. Physical Review Letters, 2008. 100(7).

196. Vaia, R.A. and J.F. Maguire, Polymer nanocomposites with prescribed morphology: Going beyond nanoparticle-filled polymers. Chemistry of Materials, 2007. 19(11): p. 2736-2751. 
197. van Anders, G. and S.C. Glotzer, DNA NANOTECHNOLOGY The world's smallest assembly line. Nature Chemistry, 2012. 4(2): p. 79-80.

198. Vorselaars, B., et al., Self-consistent field theory for diblock copolymers grafted to a sphere. Soft Matter, 2011. 7(11): p. 5128-5137.

199. Weeks, J.D., D. Chandler, and H.C. Andersen, ROLE OF REPULSIVE FORCES IN DETERMINING EQUILIBRIUM STRUCTURE OF SIMPLE LIQUIDS. Journal of Chemical Physics, 1971. 54(12): p. 5237-+.

200. Weeks, J.D., D. Chandler, and H.C. Andersen, Role of Repulsive Forces in Determining the Equilibrium Structure of Simple Liquids. The Journal of Chemical Physics, 1971. 54(12): p. 5237-5247.

201. Wiggins, P.A. and P.C. Nelson, Generalized theory of semiflexible polymers. Physical Review E, 2006. 73(3).

202. Wilhelm, J. and E. Frey, Radial distribution function of semiflexible polymers. Physical Review Letters, 1996. 77(12): p. 2581-2584.

203. Winey, K.I. and R.A. Vaia, Polymer nanocomposites. Mrs Bulletin, 2007. 32(4): p. 314319.

204. Winkler, R.G., P. Reineker, and L. Harnau, Models and equilibrium properties of stiff molecular chains. The Journal of Chemical Physics, 1994. 101(9): p. 8119-8129.

205. Witten, T.A. and P.A. Pincus, COLLOID STABILIZATION BY LONG GRAFTED POLYMERS. Macromolecules, 1986. 19(10): p. 2509-2513.

206. Wong, M.H., et al., Synthesis and Fabrication of Multifunctional Nanocomposites: Stable Dispersions of Nanoparticles Tethered with Short, Dense and Polydisperse Polymer Brushes in Poly(methyl methacrylate). Advanced Functional Materials, 2012. 22(17): p. 3614-3624.

207. Wu, X.L., et al., Polystyrene grafted carbon black synthesis via in situ solution radical polymerization in ionic liquid. Journal of Polymer Research, 2013. 20(6): p. 7.

208. Xu, C., et al., Dispersion of polymer-grafted magnetic nanoparticles in homopolymers and block copolymers. Polymer, 2008. 49(16): p. 3568-3577.

209. Xu, J., et al., Morphology and interactions of polymer brush-coated spheres in a polymer matrix. Journal of Polymer Science Part B-Polymer Physics, 2006. 44(19): p. 2811-2820.

210. Xu, M., et al., Integral Equation Prediction of Surface-Induced Layering Transition of Polymer Nanocomposites. Journal of Physical Chemistry C, 2013. 117(38): p. 1940919418.

211. Yethiraj, A. and H. Fynewever, Isotropic to nematic transition in semiflexible polymer melts. Molecular Physics, 1998. 93(5): p. 693-701.

212. Yethiraj, A. and C.K. Hall, Monte Carlo simulations and integral equation theory for microscopic correlations in polymeric fluids. Journal of Chemical Physics, 1991. 96: p. 797-807. 
213. Yethiraj, A. and K.S. Schweizer, INTEGRAL-EQUATION THEORY OF POLYMER BLENDS - NUMERICAL INVESTIGATION OF MOLECULAR CLOSURE APPROXIMATIONS. Journal of Chemical Physics, 1993. 98(11): p. 9080-9093.

214. Zhang, D., et al., Phase separation and crystallization of binary nanoparticles induced by polymer brushes. Soft Matter, 2013. 9(6): p. 1789-1797.

215. Zhang, Q. and L.A. Archer, Poly(ethylene oxide)/Silica Nanocomposites: Structure and Rheology. Langmuir, 2002. 18(26): p. 10435-10442.

216. Zhang, R. and K.S. Schweizer, Theory of nonlinear elasticity, stress-induced relaxation, and dynamic yielding in dense fluids of hard nonspherical colloids. Journal of Chemical Physics, 2012. 136(15).

217. Zhu, X., et al., Ordered Nanostructures Self-Assembled from Block Copolymer Tethered Nanoparticles. Acs Nano, 2010. 4(9): p. 4979-4988. 